\title{
Stereodivergent Synthesis of Tertiary Fluoride-Tethered Allenes via Copper and Palladium Dual Catalysis
}

\author{
Shao-Qian Yang, ${ }^{\#}$ Yi-Fan Wang, , Wei-Cheng Zhao, Guo-Qiang Lin* and Zhi-Tao He* \\ CAS Key Laboratory of Synthetic Chemistry of Natural Substances, Center for Excellence in Molecular Synthesis, Shanghai \\ Institute of Organic Chemistry, University of Chinese Academy of Sciences, Shanghai, 200032, China.
}

\section{Table of Contents}

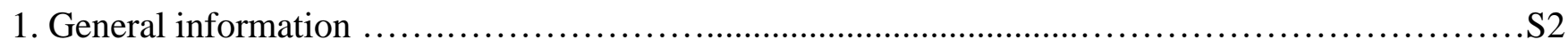

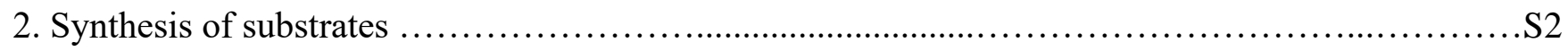

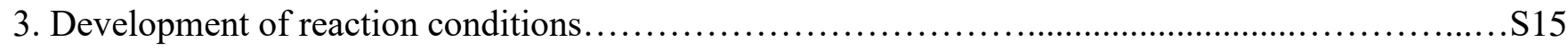

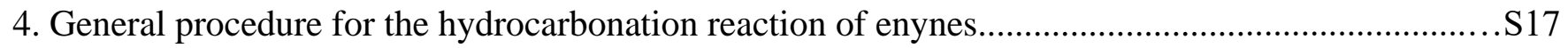

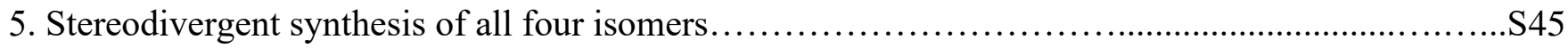

6. Kinetic studies on the diastereoselectivities and yields of reactions over time.......................S49

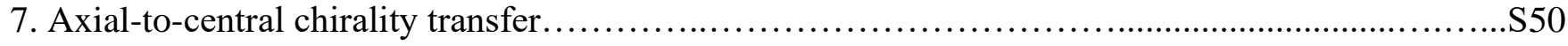

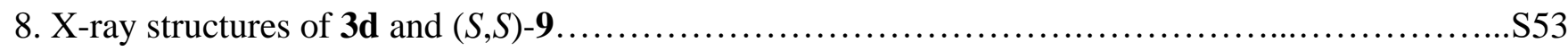

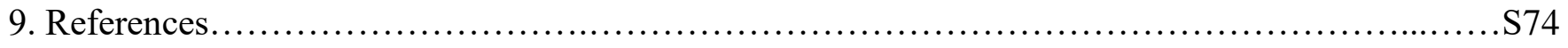

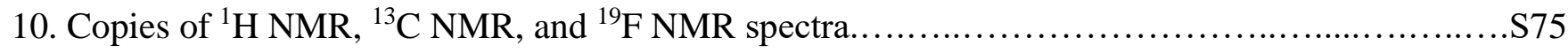




\section{General information}

All air-sensitive procedures were conducted by Schlenk techniques under argon. Unless otherwise indicated, all commercially available starting materials and dry solvents were purchased and used directly without further purification. ${ }^{1} \mathrm{H},{ }^{13} \mathrm{C}$ and ${ }^{19} \mathrm{~F}$ NMR spectra were acquired on $400 \mathrm{MHz}$ Bruker or $500 \mathrm{MHz}$ Agilent instruments at Shanghai Institute of Organic Chemistry. For High-resolution mass spectra: ESI mass spectra were recorded on Thermo Scientific Q Exactive HF Orbitrap-FTMS; MALDI was measured on Voyager-DE STR; EI mass spectra were recorded on Waters Premier GC-TOF MS; FI mass spectra were recorded on JEOL-AccuTOF-GCv4G-GCT MS. Optical rotation was measured using a $1 \mathrm{~mL}$ cell with $1.0 \mathrm{dm}$ path length on a JASCO P-1030 polarimeter. HPLC analysis was conducted on a Shimadzu HPLC system equipped with Daicel or Chiralpak chiral-stationary-phase columns $(\phi 4.6$ $\mathrm{mm} \times 250 \mathrm{~mm}$ ). Chemical shifts are reported in $\delta(\mathrm{ppm})$ referenced to an internal TMS standard or $\mathrm{CHCl}_{3}$ in $\mathrm{CDCl}_{3}(7.26 \mathrm{ppm})$ for ${ }^{1} \mathrm{H}$ NMR, $\mathrm{CDCl}_{3}(\delta=77.10 \mathrm{ppm})$ for ${ }^{13} \mathrm{C} \mathrm{NMR}$, and $\mathrm{CFCl}_{3}(0 \mathrm{ppm})$ for ${ }^{19} \mathrm{~F}$ NMR. Coupling constants $(J)$ are reported in Hz. Multiplicities are reported using the following abbreviations: $\mathrm{br}=$ broad, $\mathrm{s}=$ singlet, $\mathrm{d}=$ doublet, $\mathrm{t}=$ triplet, $\mathrm{q}=$ quartet, $\mathrm{m}=$ multiplet. Column chromatography was performed with 300-400 mesh silica gel using flash column chromatography technique. The X-ray crystal structure was measured on Bruker D8 VENTURE.

\section{Synthesis of substrates}

\subsection{Synthesis of fluorinated esters}

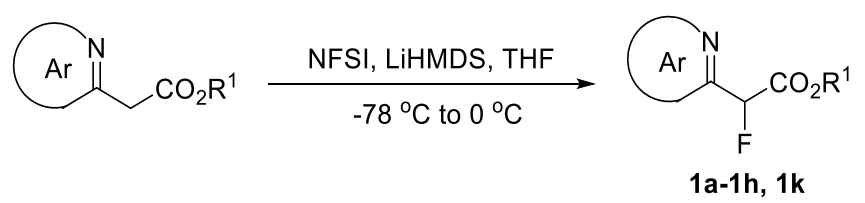

The preparation of 1a as an example: To a three-necked flask with LiHMDS solution (1 M in THF, 10 $\mathrm{mL})$ and dry THF $(20 \mathrm{~mL})$ was added the solution of methyl 2-(pyridin-2-yl)acetate $(10 \mathrm{mmol})$ dropwise in THF $(10 \mathrm{~mL})$ at $-78{ }^{\circ} \mathrm{C}$ under argon. The mixtures were stirred at $0{ }^{\circ} \mathrm{C}$ for $1 \mathrm{~h}$. After this time, the solution of NFSI $(10 \mathrm{mmol})$ in THF $(30 \mathrm{~mL})$ was added dropwise at $-78^{\circ} \mathrm{C}$ and the solution was stirred at $0{ }^{\circ} \mathrm{C}$ for another $1 \mathrm{~h}$. Then the reaction was quenched by saturated $\mathrm{NH}_{4} \mathrm{Cl}$ aqueous solution $(10 \mathrm{~mL})$ and water $(40 \mathrm{~mL})$, extracted by EtOAc $(40 \mathrm{~mL} \times 3)$. The combined organic phase was dried with sodium sulfate, filtered, concentrated and purified by flash column chromatography to provide the pure product 1a.<smiles>COC(=O)C(F)c1ccccn1</smiles>

\section{Methyl 2-fluoro-2-(pyridin-2-yl)acetate (1a) ${ }^{[1]}$}

Known compound. Yellow oil, 75\% yield. ${ }^{1} \mathrm{H}$ NMR $(500 \mathrm{MHz}$, chloroform- $d$ ) $\delta 8.63(\mathrm{~d}, J=4.7 \mathrm{~Hz}, 1 \mathrm{H})$, $7.79(\mathrm{td}, J=7.7,1.7 \mathrm{~Hz}, 1 \mathrm{H}), 7.54(\mathrm{~d}, J=7.8 \mathrm{~Hz}, 1 \mathrm{H}), 7.37-7.31(\mathrm{~m}, 1 \mathrm{H}), 5.94(\mathrm{~d}, J=47.6 \mathrm{~Hz}, 1 \mathrm{H})$, $3.82(\mathrm{~s}, 3 \mathrm{H})$. 


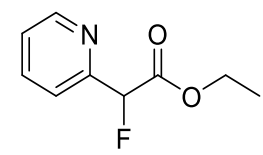

\section{Ethyl 2-fluoro-2-(pyridin-2-yl)acetate $(1 b)^{[1]}$}

Known compound. Yellow oil, 77\% yield. ${ }^{1} \mathrm{H}$ NMR $(500 \mathrm{MHz}$, chloroform- $d) \delta 8.66-8.61(\mathrm{~m}, 1 \mathrm{H})$, $7.82-7.75(\mathrm{~m}, 1 \mathrm{H}), 7.56-7.51(\mathrm{~m}, 1 \mathrm{H}), 7.36-7.31(\mathrm{~m}, 2 \mathrm{H}), 5.90(\mathrm{~d}, J=47.8 \mathrm{~Hz}, 1 \mathrm{H}), 4.29(\mathrm{q}, J=7.1$ $\mathrm{Hz}, 2 \mathrm{H}), 1.28(\mathrm{t}, J=7.1 \mathrm{~Hz}, 3 \mathrm{H})$.<smiles>COC(=O)C(F)c1cnccn1</smiles>

\section{Methyl 2-fluoro-2-(pyrazin-2-yl)acetate (1c) ${ }^{[1]}$}

Known compound. White solid, $21 \%$ yield. ${ }^{1} \mathrm{H}$ NMR $(400 \mathrm{MHz}$, chloroform- $d$ ) $\delta 8.82(\mathrm{~d}, J=1.5 \mathrm{~Hz}$, $1 \mathrm{H}), 8.65(\mathrm{~d}, J=2.5 \mathrm{~Hz}, 1 \mathrm{H}), 8.63-8.57(\mathrm{~m}, 1 \mathrm{H}), 5.98(\mathrm{~d}, J=47.2 \mathrm{~Hz}, 1 \mathrm{H}), 3.85(\mathrm{~s}, 3 \mathrm{H})$.<smiles>COC(=O)C(F)c1ccc2ccccc2n1</smiles>

\section{Methyl 2-fluoro-2-(quinolin-2-yl)acetate (1d $)^{[1]}$}

Known compound. Yellow solid, $41 \%$ yield. ${ }^{1} \mathrm{H}$ NMR (400 MHz, chloroform- $d$ ) $\delta 8.27$ (d, $J=8.5 \mathrm{~Hz}$, $1 \mathrm{H}), 8.15(\mathrm{~d}, J=8.5 \mathrm{~Hz}, 1 \mathrm{H}), 7.86(\mathrm{~d}, J=8.1 \mathrm{~Hz}, 1 \mathrm{H}), 7.76(\mathrm{ddd}, J=8.5,6.9,1.6 \mathrm{~Hz}, 1 \mathrm{H}), 7.67-7.56$ $(\mathrm{m}, 2 \mathrm{H}), 6.10(\mathrm{~d}, J=47.7 \mathrm{~Hz}, 1 \mathrm{H}), 3.82(\mathrm{~s}, 3 \mathrm{H})$.<smiles>COC(=O)C(F)c1ncccn1</smiles>

\section{Methyl 2-fluoro-2-(pyrimidin-2-yl)acetate (1e)}

Light yellow oil, 54\% yield. ${ }^{1} \mathrm{H}$ NMR (400 MHz, chloroform- $\left.d\right) \delta 8.83(\mathrm{~d}, J=4.9 \mathrm{~Hz}, 2 \mathrm{H}), 7.36(\mathrm{t}, J=$ $4.8 \mathrm{~Hz}, 1 \mathrm{H}), 6.00(\mathrm{~d}, J=47.7 \mathrm{~Hz}, 1 \mathrm{H}), 3.85(\mathrm{~s}, 3 \mathrm{H}) .{ }^{13} \mathrm{C} \mathrm{NMR}(101 \mathrm{MHz}$, chloroform- $d) \delta 167.1(\mathrm{~d}, J=$ $25.5 \mathrm{~Hz}), 162.6(\mathrm{~d}, J=19.8 \mathrm{~Hz}), 157.8,121.4(\mathrm{~d}, J=2.0 \mathrm{~Hz}), 89.9(\mathrm{~d}, J=188.9 \mathrm{~Hz}), 52.9(\mathrm{~d}, J=1.0$ $\mathrm{Hz}) .{ }^{19} \mathrm{~F}$ NMR $\left(376 \mathrm{MHz}\right.$, chloroform- $d$ ) $\delta-185.4(\mathrm{~d}, J=47.7 \mathrm{~Hz})$. HRMS (ESI): $[\mathrm{M}+\mathrm{H}]{ }^{\oplus}$ calcd for $\mathrm{C}_{7} \mathrm{H}_{8} \mathrm{O}_{2} \mathrm{~N}_{2} \mathrm{~F}^{\oplus} 171.0564$, found 171.0563 .<smiles>COC(=O)C(F)c1cc(OC)ccn1</smiles>

Methyl 2-fluoro-2-(4-methoxypyridin-2-yl)acetate (1f $)^{[1]}$ 
Known compound. Yellow oil, 53\% yield. ${ }^{1} \mathrm{H}$ NMR $(500 \mathrm{MHz}$, chloroform- $d$ ) $\delta 8.38(\mathrm{~d}, J=5.7 \mathrm{~Hz}, 1 \mathrm{H})$, $7.01(\mathrm{~d}, J=2.5 \mathrm{~Hz}, 1 \mathrm{H}), 6.81-6.76(\mathrm{~m}, 1 \mathrm{H}), 5.83(\mathrm{~d}, J=47.7 \mathrm{~Hz}, 1 \mathrm{H}), 3.83$ (s, 3H), 3.77 (s, 3H).<smiles>COC(=O)C(F)c1ccc(Cl)cn1</smiles>

\section{Methyl 2-(5-chloropyridin-2-yl)-2-fluoroacetate (1g)}

White solid, 68\% yield. ${ }^{1} \mathrm{H}$ NMR $(500 \mathrm{MHz}$, chloroform- $d$ ) $\delta 8.57(\mathrm{~d}, J=2.4 \mathrm{~Hz}, 1 \mathrm{H}), 7.76(\mathrm{dd}, J=8.4$, $2.4 \mathrm{~Hz}, 1 \mathrm{H}), 7.49(\mathrm{~d}, J=8.4 \mathrm{~Hz}, 1 \mathrm{H}), 5.90(\mathrm{~d}, J=47.4 \mathrm{~Hz}, 1 \mathrm{H}), 3.82(\mathrm{~s}, 3 \mathrm{H}) .{ }^{13} \mathrm{C} \mathrm{NMR}(101 \mathrm{MHz}$, chloroform- $d$ ) $\delta 167.7(\mathrm{~d}, J=26.4 \mathrm{~Hz}), 151.8(\mathrm{~d}, J=23.6 \mathrm{~Hz}), 148.7,137.1,133.0(\mathrm{~d}, J=1.9 \mathrm{~Hz}), 122.3$ $(\mathrm{d}, J=4.7 \mathrm{~Hz}), 89.4(\mathrm{~d}, J=186.3 \mathrm{~Hz}), 53.1 .{ }^{19} \mathrm{~F}$ NMR $(376 \mathrm{MHz}$, chloroform- $d$ ) $\delta-184.0$ (d, $J=47.1$ $\mathrm{Hz}$ ). HRMS (ESI): $[\mathrm{M}+\mathrm{H}]^{\oplus}$ calcd for $\mathrm{C}_{8} \mathrm{H}_{8} \mathrm{ClFNO}_{2}{ }^{\oplus}$ 204.0222, found 204.0224.<smiles>COC(=O)C(F)c1ccc(Br)cn1</smiles>

\section{Methyl 2-(5-bromopyridin-2-yl)-2-fluoroacetate (1h)}

Yellow solid, 69\% yield. ${ }^{1} \mathrm{H}$ NMR $(500 \mathrm{MHz}$, chloroform- $d) \delta 8.68(\mathrm{~d}, J=2.4 \mathrm{~Hz}, 1 \mathrm{H}), 7.92(\mathrm{dd}, J=8.3$, $2.3 \mathrm{~Hz}, 1 \mathrm{H}), 7.44(\mathrm{~d}, J=8.3 \mathrm{~Hz}, 1 \mathrm{H}), 5.88(\mathrm{~d}, J=47.4 \mathrm{~Hz}, 1 \mathrm{H}), 3.82(\mathrm{~s}, 3 \mathrm{H}) .{ }^{13} \mathrm{C} \mathrm{NMR}(101 \mathrm{MHz}$, chloroform- $d) \delta 167.6(\mathrm{~d}, J=26.3 \mathrm{~Hz}), 152.2(\mathrm{~d}, J=23.7 \mathrm{~Hz}), 150.9(\mathrm{~d}, J=1.1 \mathrm{~Hz}), 140.0,122.8(\mathrm{~d}, J=$ $4.6 \mathrm{~Hz}), 121.7(\mathrm{~d}, J=2.0 \mathrm{~Hz}), 89.5(\mathrm{~d}, J=186.5 \mathrm{~Hz}), 53.1(\mathrm{~d}, J=1.1 \mathrm{~Hz}) .{ }^{19} \mathrm{~F} \mathrm{NMR}(376 \mathrm{MHz}$, chloroform- $d$ ) $\delta-184.4\left(\mathrm{~d}, J=47.3 \mathrm{~Hz}\right.$ ). HRMS (ESI): $[\mathrm{M}+\mathrm{H}]^{\oplus}$ calcd for $\mathrm{C}_{8} \mathrm{H}_{8} \mathrm{BrFNO}_{2}{ }^{\oplus} 247.9717$, found 247.9719.

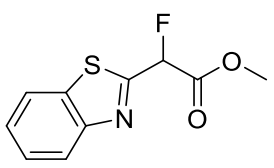

\section{Methyl 2-(benzo[d]thiazol-2-yl)-2-fluoroacetate (1k) ${ }^{[1]}$}

Known compound. Light yellow solid, $18 \%$ yield. ${ }^{1} \mathrm{H}$ NMR (400 MHz, chloroform- $d$ ) $\delta 8.11(\mathrm{~d}, J=8.2$ $\mathrm{Hz}, 1 \mathrm{H}), 7.94(\mathrm{~d}, J=8.0 \mathrm{~Hz}, 1 \mathrm{H}), 7.58-7.51(\mathrm{~m}, 1 \mathrm{H}), 7.50-7.43(\mathrm{~m}, 1 \mathrm{H}), 6.24(\mathrm{~d}, J=47.0 \mathrm{~Hz}, 1 \mathrm{H})$, $3.89(\mathrm{~s}, 3 \mathrm{H})$.

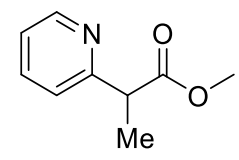

\section{Methyl 2-(pyridin-2-yl)propanoate $(11)^{[1]}$}

Known compound. Light yellow liquid, 58\% yield. ${ }^{1} \mathrm{H}$ NMR (400 MHz, chloroform- $d$ ) $\delta 8.59-8.52(\mathrm{~m}$, $1 \mathrm{H}), 7.71-7.61(\mathrm{~m}, 1 \mathrm{H}), 7.32-7.24(\mathrm{~m}, 1 \mathrm{H}), 7.21-7.13(\mathrm{~m}, 1 \mathrm{H}), 3.96(\mathrm{q}, \mathrm{J}=7.2 \mathrm{~Hz}, 1 \mathrm{H}), 3.69$ (s, 3H), $1.56(\mathrm{~d}, \mathrm{~J}=7.3 \mathrm{~Hz}, 3 \mathrm{H})$. 
<smiles>COC(=O)C(Cl)c1ccccn1</smiles>

\section{Methyl 2-chloro-2-(pyridin-2-yl)acetate (1m)}

Procedure: to a solution of methyl 2-2(pyridyl)acetate $(2.3 \mathrm{~g}, 15 \mathrm{mmol}, 1 \mathrm{eq})$ in $60 \mathrm{~mL}$ carbon tetrachloride was added dibenzoylperoxide $(0.36 \mathrm{~g}, 1.5 \mathrm{mmol}, 0.1 \mathrm{eq})$ and $N$-chloro-succinimide $(2.1 \mathrm{~g}$, $16 \mathrm{mmol}, 1.2 \mathrm{eq})$. The resulting solution was heated to reflux for 4 hours. After this time, the solution was filtered, concentrated and purified by flash column chromatography to achieve the pure product $\mathbf{1 m}$ as light-yellow liquid in $35 \%$ yield. ${ }^{1} \mathrm{H}$ NMR $(400 \mathrm{MHz}$, chloroform- $d) \delta 8.63-8.54(\mathrm{~m}, 1 \mathrm{H}), 7.82-$ $7.72(\mathrm{~m}, 1 \mathrm{H}), 7.65-7.57(\mathrm{~m}, 1 \mathrm{H}), 7.32-7.26(\mathrm{~m}, 1 \mathrm{H}), 5.54(\mathrm{~s}, 1 \mathrm{H}), 3.82(\mathrm{~s}, 3 \mathrm{H}) .{ }^{13} \mathrm{C} \mathrm{NMR}(126 \mathrm{MHz}$, $\left.\mathrm{CDCl}_{3}\right) \delta 168.1,155.1,149.2,137.6,123.9,122.8,59.4,53.5$. HRMS (ESI): $[\mathrm{M}+\mathrm{H}]^{\oplus}$ calcd for $\mathrm{C}_{8} \mathrm{H}_{9} \mathrm{ClNO}_{2}{ }^{\oplus} 186.0316$, found 186.0316 .

\subsection{Synthesis of substituted enynes}

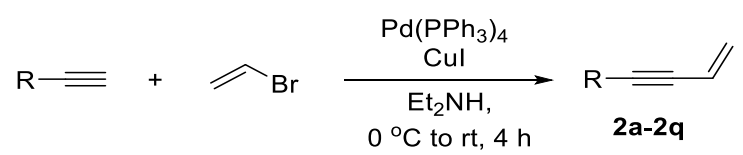

General procedure: To a three-necked flask with $\mathrm{Pd}\left(\mathrm{PPh}_{3}\right)_{4}(0.5 \mathrm{mmol})$ and $\mathrm{CuI}(1 \mathrm{mmol})$ was added $\mathrm{Et}_{2} \mathrm{NH}(5.0 \mathrm{~mL})$ under argon. Then alkyne $(10 \mathrm{mmol})$ and bromoethane $(1 \mathrm{M}$ in THF, $13 \mathrm{~mL})$ was added to above solution dropwise under ice bath. The resulting solution was stirred at room temperature for $4 \mathrm{~h}$. After this time, it was quenched by $\mathrm{HCl}$ aqueous solution $(1.0 \mathrm{M}, 15 \mathrm{ml})$, extracted by DCM $(50 \mathrm{~mL} \times$ 3 ), concentrated and purified by flash column chromatography to provide the pure product.

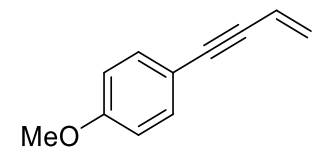

\section{1-(But-3-en-1-yn-1-yl)-4-methoxybenzene $(2 a)^{[2]}$}

Known compound. Yellow oil, 82\% yield. ${ }^{1} \mathrm{H}$ NMR $(400 \mathrm{MHz}$, chloroform- $d$ ) $\delta 7.38(\mathrm{~d}, J=8.6 \mathrm{~Hz}, 2 \mathrm{H})$, $6.84(\mathrm{~d}, J=8.5 \mathrm{~Hz}, 2 \mathrm{H}), 6.00(\mathrm{dd}, J=17.5,11.1 \mathrm{~Hz}, 1 \mathrm{H}), 5.69(\mathrm{dd}, J=17.5,2.1 \mathrm{~Hz}, 1 \mathrm{H}), 5.49(\mathrm{dd}, J=$ 11.1, $2.1 \mathrm{~Hz}, 1 \mathrm{H}), 3.81(\mathrm{~s}, 3 \mathrm{H})$.

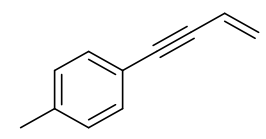

\section{1-(But-3-en-1-yn-1-yl)-4-methylbenzene $(2 b)^{[3]}$}

Known compound. Yellow oil, 77\% yield. ${ }^{1} \mathrm{H}$ NMR (500 MHz, chloroform- $d$ ) $\delta 7.40-7.34(\mathrm{~m}, 2 \mathrm{H})$, $7.15(\mathrm{~d}, J=7.8 \mathrm{~Hz}, 2 \mathrm{H}), 6.05(\mathrm{dd}, J=17.5,11.2 \mathrm{~Hz}, 1 \mathrm{H}), 5.75(\mathrm{dd}, J=17.5,2.1 \mathrm{~Hz}, 1 \mathrm{H}), 5.54(\mathrm{dd}, J=$ $11.1,2.1 \mathrm{~Hz}, 1 \mathrm{H}), 2.37$ (s, 3H). 


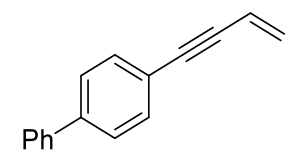

\section{4-(But-3-en-1-yn-1-yl)-1,1'-biphenyl (2c) ${ }^{[2]}$}

Known compound. White solid, $71 \%$ yield. ${ }^{1} \mathrm{H}$ NMR $(500 \mathrm{MHz}$, chloroform- $d$ ) $\delta 7.63-7.50(\mathrm{~m}, 6 \mathrm{H})$, $7.46(\mathrm{dd}, J=8.5,6.9 \mathrm{~Hz}, 2 \mathrm{H}), 7.41-7.33(\mathrm{~m}, 1 \mathrm{H}), 6.06(\mathrm{dd}, J=17.5,11.2 \mathrm{~Hz}, 1 \mathrm{H}), 5.77(\mathrm{dd}, J=17.5$, $2.0 \mathrm{~Hz}, 1 \mathrm{H}), 5.57(\mathrm{dd}, J=11.1,2.0 \mathrm{~Hz}, 1 \mathrm{H})$.

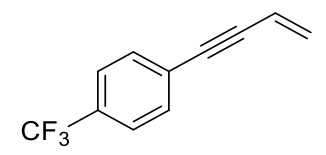

\section{1-(But-3-en-1-yn-1-yl)-4-(trifluoromethyl)benzene $(2 \mathrm{~d})^{[4]}$}

Known compound. Colorless oil, 89\% yield. ${ }^{1} \mathrm{H}$ NMR (400 MHz, chloroform- $d$ ) $\delta 7.60-7.50(\mathrm{~m}, 4 \mathrm{H})$, $6.03(\mathrm{dd}, J=17.6,11.2 \mathrm{~Hz}, 1 \mathrm{H}), 5.79(\mathrm{dd}, J=17.5,2.1 \mathrm{~Hz}, 1 \mathrm{H}), 5.62(\mathrm{dd}, J=11.2,2.0 \mathrm{~Hz}, 1 \mathrm{H})$.

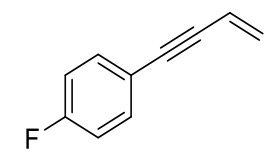

\section{1-(But-3-en-1-yn-1-yl)-4-fluorobenzene $(2 e)^{[2]}$}

Known compound. Colorless oil, $41 \%$ yield. ${ }^{1} \mathrm{H}$ NMR (400 MHz, chloroform- $d$ ) $\delta 7.47-7.37$ (m, 2H), $7.09-6.96(\mathrm{~m}, 2 \mathrm{H}), 6.00(\mathrm{dd}, J=17.5,11.1 \mathrm{~Hz}, 1 \mathrm{H}), 5.73(\mathrm{dd}, J=17.6,2.0 \mathrm{~Hz}, 1 \mathrm{H}), 5.55(\mathrm{dd}, J=11.2$, $2.1 \mathrm{~Hz}, 1 \mathrm{H})$.

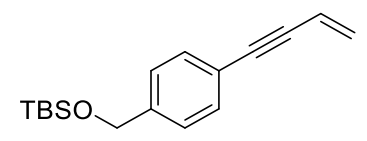

\section{((4-(But-3-en-1-yn-1-yl)benzyl)oxy)(tert-butyl)dimethylsilane (2f)}

Yellow oil, 77\% yield. ${ }^{1} \mathrm{H}$ NMR (500 MHz, chloroform- $d$ ) $\delta 7.48-7.39(\mathrm{~m}, 2 \mathrm{H}), 7.35-7.27(\mathrm{~m}, 2 \mathrm{H})$, $6.04(\mathrm{dd}, J=17.5,11.2,1 \mathrm{H}), 5.75$ (dd, $J=17.5,1.6 \mathrm{~Hz}, 1 \mathrm{H}), 5.55$ (dd,J=11.3, $1.6 \mathrm{~Hz}, 1 \mathrm{H}), 4.75$ (s, $2 \mathrm{H}), 0.97$ (s, 9H), $0.12(\mathrm{~s}, 6 \mathrm{H}) .{ }^{13} \mathrm{C}$ NMR $(126 \mathrm{MHz}$, chloroform- $d) \delta 141.9,131.5,126.7,126.0,121.6$, 117.3, 90.2, 87.8, 64.7, 26.0, 18.5, -5.2. HRMS (EI): $[\mathrm{M}]^{\oplus} \mathrm{Calcd}$ for $\mathrm{C}_{17} \mathrm{H}_{24} \mathrm{OSi} 272.1591$, found 272.1593.

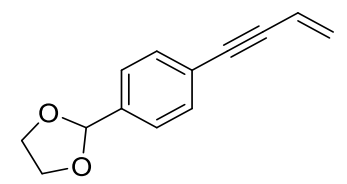

\section{2-(4-(But-3-en-1-yn-1-yl)phenyl)-1,3-dioxolane (2g)}

Yellow oil, 70\% yield. ${ }^{1} \mathrm{H}$ NMR (400 MHz, chloroform- $d$ ) $\delta 7.50-7.39(\mathrm{~m}, 4 \mathrm{H}), 6.02(\mathrm{dd}, J=17.5$, $11.1 \mathrm{~Hz}, 1 \mathrm{H}), 5.80(\mathrm{~s}, 1 \mathrm{H}), 5.74(\mathrm{dd}, J=17.6,2.0 \mathrm{~Hz}, 1 \mathrm{H}), 5.55(\mathrm{dd}, J=11.1,2.0 \mathrm{~Hz}, 1 \mathrm{H}), 4.18-3.97$ $(\mathrm{m}, 4 \mathrm{H}) .{ }^{13} \mathrm{C}$ NMR $(126 \mathrm{MHz}$, chloroform- $d) \delta 138.0,131.6,127.2,126.5,124.0,117.1,103.3,89.7$, 88.6, 65.3. HRMS (EI): [M] ${ }^{\oplus}$ Calcd for $\mathrm{C}_{13} \mathrm{H}_{12} \mathrm{O}_{2} 200.0832$, found 200.0838 . 


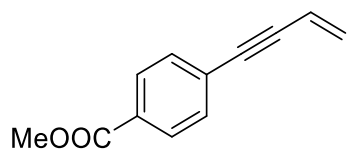

Methyl 4-(but-3-en-1-yn-1-yl)benzoate (2h) $)^{[5]}$

Known compound. White solid, $90 \%$ yield. ${ }^{1} \mathrm{H}$ NMR $(500 \mathrm{MHz}$, chloroform- $d$ ) $\delta 8.0-8.0$ (m, 2H), 7.5 $-7.5(\mathrm{~m}, 2 \mathrm{H}), 6.0(\mathrm{dd}, J=17.5,11.2 \mathrm{~Hz}, 1 \mathrm{H}), 5.8(\mathrm{dd}, J=17.5,1.9 \mathrm{~Hz}, 1 \mathrm{H}), 5.6(\mathrm{dd}, J=11.2,2.0 \mathrm{~Hz}$, $1 \mathrm{H}), 3.9$ (s, 3H).

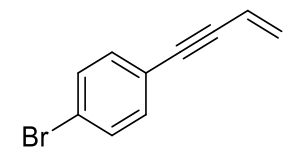

\section{1-Bromo-4-(but-3-en-1-yn-1-yl)benzene $(2 \mathrm{i})^{[3]}$}

Known compound. Yellow oil, 77\% yield. ${ }^{1} \mathrm{H}$ NMR (500 MHz, chloroform- $d$ ) $\delta 7.45(\mathrm{~d}, J=8.3 \mathrm{~Hz}, 2 \mathrm{H})$, $7.30(\mathrm{~d}, J=8.3 \mathrm{~Hz}, 2 \mathrm{H}), 6.00(\mathrm{dd}, J=17.6,11.2 \mathrm{~Hz}, 1 \mathrm{H}), 5.75(\mathrm{dd}, J=17.6,1.9 \mathrm{~Hz}, 1 \mathrm{H}), 5.57(\mathrm{dd}, J=$ $11.2,2.0 \mathrm{~Hz}, 1 \mathrm{H})$

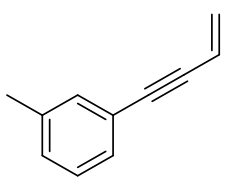

\section{1-(But-3-en-1-yn-1-yl)-3-methylbenzene $(2 \mathbf{j})^{[2]}$}

Known compound. Colorless oil, $62 \%$ yield. ${ }^{1} \mathrm{H}$ NMR $(500 \mathrm{MHz}$, chloroform- $d) \delta 7.34-7.27$ (m, 2H), $7.24(\mathrm{t}, J=7.6 \mathrm{~Hz}, 1 \mathrm{H}), 7.18-7.12(\mathrm{~m}, 1 \mathrm{H}), 6.06(\mathrm{dd}, J=17.5,11.1 \mathrm{~Hz}, 1 \mathrm{H}), 5.77(\mathrm{dd}, J=17.5,2.1 \mathrm{~Hz}$, $1 \mathrm{H}), 5.57(\mathrm{dd}, J=11.2,2.0 \mathrm{~Hz}, 1 \mathrm{H}), 2.36(\mathrm{~s}, 3 \mathrm{H})$.

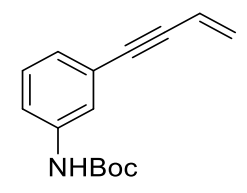

\section{tert-Butyl (3-(but-3-en-1-yn-1-yl)phenyl)carbamate (2k)}

White solid, 60\% yield. ${ }^{1} \mathrm{H}$ NMR (400 MHz, chloroform- $d$ ) $\delta 7.51(\mathrm{~s}, 1 \mathrm{H}), 7.32-7.19(\mathrm{~m}, 2 \mathrm{H}), 7.15-$ $7.08(\mathrm{~m}, 1 \mathrm{H}), 6.47(\mathrm{~s}, 1 \mathrm{H}), 6.07-5.96(\mathrm{~m}, 1 \mathrm{H}), 5.80-5.70(\mathrm{~m}, 1 \mathrm{H}), 5.60-5.52(\mathrm{~m}, 1 \mathrm{H}), 1.54(\mathrm{~s}$, 9H). ${ }^{13} \mathrm{C}$ NMR (101 MHz, chloroform- $d$ ) $\delta$ 152.6, 138.4, 129.0, 127.1, 126.3, 123.9, 121.3, 118.5, 117.2, 89.8, 88.2, 80.9, 28.4. HRMS (ESI): $[\mathrm{M}+\mathrm{Na}]^{\oplus}$ calcd for $\mathrm{C}_{15} \mathrm{H}_{17} \mathrm{O}_{2} \mathrm{NNa}^{\oplus} 266.1152$, found 266.1150.

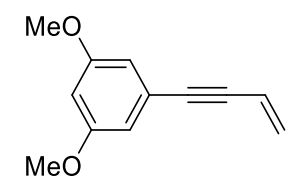

\section{1-(But-3-en-1-yn-1-yl)-3,5-dimethoxybenzene (2l)}

Colorless oil, 82\% yield. ${ }^{1} \mathrm{H}$ NMR $(500 \mathrm{MHz}$, chloroform- $d$ ) $\delta 6.61(\mathrm{~d}, J=2.4 \mathrm{~Hz}, 2 \mathrm{H}), 6.44(\mathrm{t}, J=2.3$ $\mathrm{Hz}, 1 \mathrm{H}), 6.01(\mathrm{dd}, J=17.5,11.2 \mathrm{~Hz}, 1 \mathrm{H}), 5.75(\mathrm{dd}, J=17.5,2.1 \mathrm{~Hz}, 1 \mathrm{H}), 5.56(\mathrm{dd}, J=11.1,2.0 \mathrm{~Hz}$, 
1H), $3.78(\mathrm{~s}, 6 \mathrm{H}) .{ }^{13} \mathrm{C}$ NMR (126 MHz, chloroform- $\left.d\right) \delta 160.5,127.2,124.4,117.1,109.3,101.9,90.0$, 87.7, 55.4. HRMS (EI): [M] ${ }^{\oplus}$ Calcd for $\mathrm{C}_{12} \mathrm{H}_{12} \mathrm{O}_{2} 188.0832$, found 188.0834.

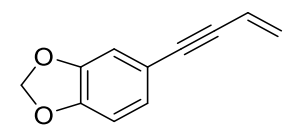

\section{5-(But-3-en-1-yn-1-yl)benzo[d][1,3]dioxole $(2 \mathrm{~m})^{[6]}$}

Known compound. Colorless oil, $69 \%$ yield. ${ }^{1} \mathrm{H}$ NMR (400 MHz, chloroform- $d$ ) $\delta 6.97$ (dd, $J=8.1,1.6$ $\mathrm{Hz}, 1 \mathrm{H}), 6.92-6.86(\mathrm{~m}, 1 \mathrm{H}), 6.76(\mathrm{~d}, J=8.0 \mathrm{~Hz}, 1 \mathrm{H}), 6.05-5.93(\mathrm{~m}, 3 \mathrm{H}), 5.70(\mathrm{dd}, J=17.5,2.1 \mathrm{~Hz}$, $1 \mathrm{H}), 5.51(\mathrm{dd}, J=11.1,2.1 \mathrm{~Hz}, 1 \mathrm{H})$.

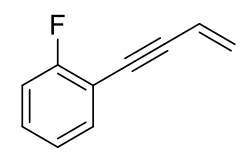

\section{1-(But-3-en-1-yn-1-yl)-2-fluorobenzene $(2 n)^{[2]}$}

Known compound. Yellow oil, 29\% yield. ${ }^{1} \mathrm{H}$ NMR $(500 \mathrm{MHz}$, chloroform- $d) \delta 7.45-7.39$ (m, 1H), $7.31-7.21(\mathrm{~m}, 1 \mathrm{H}), 7.11-7.01(\mathrm{~m}, 2 \mathrm{H}), 6.04(\mathrm{dd}, J=17.5,11.2 \mathrm{~Hz}, 1 \mathrm{H}), 5.77(\mathrm{dd}, J=17.5,1.9 \mathrm{~Hz}$, $1 \mathrm{H}), 5.57(\mathrm{dd}, J=11.2,1.9 \mathrm{~Hz}, 1 \mathrm{H})$.

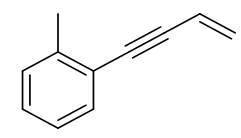

\section{1-(But-3-en-1-yn-1-yl)-2-methylbenzene $(20)^{[5]}$}

Known compound. Yellow oil, 59\% yield. ${ }^{1} \mathrm{H}$ NMR (400 MHz, chloroform- $\left.d\right) \delta 7.45-7.37(\mathrm{~m}, 1 \mathrm{H})$, $7.25-7.17(\mathrm{~m}, 2 \mathrm{H}), 7.16-7.10(\mathrm{~m}, 1 \mathrm{H}), 6.07(\mathrm{dd}, J=17.5,11.1 \mathrm{~Hz}, 1 \mathrm{H}), 5.73(\mathrm{dd}, J=17.6,2.0 \mathrm{~Hz}$, $1 \mathrm{H}), 5.54(\mathrm{dd}, J=11.1,2.1 \mathrm{~Hz}, 1 \mathrm{H}), 2.45(\mathrm{~s}, 3 \mathrm{H})$.

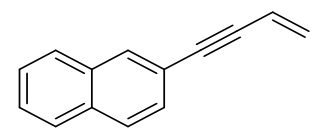

\section{2-(But-3-en-1-yn-1-yl)naphthalene $(2 p)^{[2]}$}

Known compound. Yellow oil, 86\% yield. ${ }^{1} \mathrm{H}$ NMR (500 MHz, chloroform-d) $\delta 8.04$ (s, 1H), $7.87-$ $7.79(\mathrm{~m}, 3 \mathrm{H}), 7.60-7.48(\mathrm{~m}, 3 \mathrm{H}), 6.15(\mathrm{dd}, J=17.5,11.2 \mathrm{~Hz}, 1 \mathrm{H}), 5.86(\mathrm{dd}, J=17.5,2.0 \mathrm{~Hz}, 1 \mathrm{H}), 5.64$ (dd, $J=11.2,2.0 \mathrm{~Hz}, 1 \mathrm{H})$.

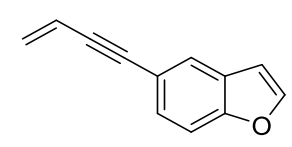

\section{5-(But-3-en-1-yn-1-yl)benzofuran (2q)}

Yellow oil, 76\% yield. ${ }^{1} \mathrm{H}$ NMR $(500 \mathrm{MHz}$, chloroform- $d) \delta 7.74(\mathrm{~d}, J=1.6 \mathrm{~Hz}, 1 \mathrm{H}), 7.63(\mathrm{~d}, J=2.2$ $\mathrm{Hz}, 1 \mathrm{H}), 7.50-7.39(\mathrm{~m}, 2 \mathrm{H}), 6.74(\mathrm{dd}, J=2.2,0.9 \mathrm{~Hz}, 1 \mathrm{H}), 6.08(\mathrm{dd}, J=17.5,11.1 \mathrm{~Hz}, 1 \mathrm{H}), 5.78(\mathrm{dd}$, $J=17.5,2.0 \mathrm{~Hz}, 1 \mathrm{H}), 5.56(\mathrm{dd}, J=11.2,2.1 \mathrm{~Hz}, 1 \mathrm{H}) .{ }^{13} \mathrm{C}$ NMR $(126 \mathrm{MHz}$, chloroform- $d$ ) $\delta 154.6$, 
145.8, 128.0, 127.6, 126.5, 124.7, 117.7, 117.4, 111.6, 106.5, 90.4, 86.8. HRMS (EI): [M] ${ }^{\oplus}$ Calcd for $\mathrm{C}_{12} \mathrm{H}_{8} \mathrm{O} 168.0570$, found 168.0572 .

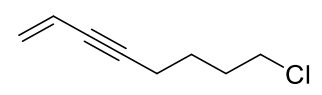

\section{8-Chlorooct-1-en-3-yne (2r) ${ }^{[2]}$}

Known compound. Colorless oil, 37\% yield. ${ }^{1} \mathrm{H}$ NMR $(400 \mathrm{MHz}$, chloroform- $d$ ) $\delta 5.79$ (dd, $J=17.4$, $10.9,1 \mathrm{H}), 5.58(\mathrm{dd}, J=17.5,2.2 \mathrm{~Hz}, 1 \mathrm{H}), 5.41(\mathrm{dd}, J=11.0,2.2 \mathrm{~Hz}, 1 \mathrm{H}), 3.60(\mathrm{t}, J=6.5 \mathrm{~Hz}, 2 \mathrm{H}), 2.45$ $-2.31(\mathrm{~m}, 2 \mathrm{H}), 2.00-1.86(\mathrm{~m}, 2 \mathrm{H}), 1.78-1.66(\mathrm{~m}, 2 \mathrm{H})$.

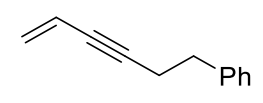

\section{Hex-5-en-3-yn-1-ylbenzene $(2 s)^{[2]}$}

Known compound. Colorless oil, 67\% yield. ${ }^{1} \mathrm{H}$ NMR (400 MHz, chloroform-d) $\delta 7.34-7.31$ (m, 2H), 7.26-7.22 (m, 3H), $5.86-5.74(\mathrm{~m}, 1 \mathrm{H}), 5.57(\mathrm{dd}, J=17.6,2.2 \mathrm{~Hz}, 1 \mathrm{H}), 5.41(\mathrm{dd}, J=11.0,2.2 \mathrm{~Hz}, 1 \mathrm{H})$, $2.88(\mathrm{t}, J=7.6 \mathrm{~Hz}, 2 \mathrm{H}), 2.62(\mathrm{td}, J=7.7,2.1 \mathrm{~Hz}, 2 \mathrm{H})$.

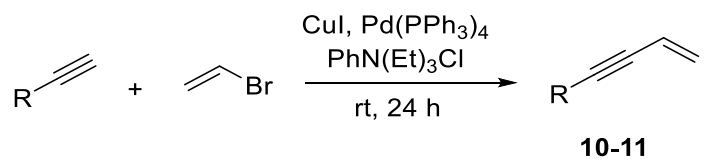

To a three-necked flask equipped with a magnetic stir bar was added $\mathrm{Pd}\left(\mathrm{PPh}_{3}\right)_{4}(1 \mathrm{mmol}), \mathrm{CuI}(1$ $\mathrm{mmol})$, alkyne $(20 \mathrm{mmol}), \mathrm{PhN}(\mathrm{Et})_{3} \mathrm{Cl}(2 \mathrm{mmol})$ and dry toluene $(5.4 \mathrm{~mL})$ under argon at room temperature. Then bromoethane $(1 \mathrm{M}$ in THF, $26 \mathrm{~mL})$ and $10 \% \mathrm{NaOH}$ aqueous solution $(24 \mathrm{~mL})$ was added sequentially to the flask. The resulting solution continued to stir at room temperature for $16 \mathrm{~h}$. after this time, the reaction was quenched with saturated $\mathrm{NH}_{4} \mathrm{Cl}$ solution $(50 \mathrm{~mL})$, extracted by EtOAc $(40 \mathrm{~mL} \times 3)$, concentrated and purified by flash column chromatography to provide the pure product.

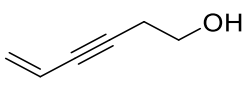

\section{Hex-5-en-3-yn-1-ol (10) ${ }^{[7]}$}

Known compound. Colorless oil, $48 \%$ yield. ${ }^{1} \mathrm{H}$ NMR $(500 \mathrm{MHz}$, chloroform- $d$ ) $\delta 5.76$ (dd, $J=17.5$, $11.1,1 \mathrm{H}), 5.57(\mathrm{dd}, J=17.5,2.2 \mathrm{~Hz}, 1 \mathrm{H}), 5.41(\mathrm{dd}, J=11.1,2.2 \mathrm{~Hz}, 1 \mathrm{H}), 3.71(\mathrm{t}, J=6.3 \mathrm{~Hz}, 2 \mathrm{H}), 2.56$ (td, $J=6.3,2.1 \mathrm{~Hz}, 2 \mathrm{H}), 2.23$ (br s, 1H).

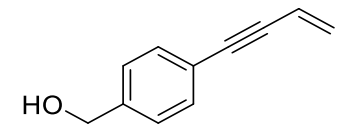

\section{(4-(But-3-en-1-yn-1-yl)phenyl)methanol (11)}

Yellow solid, 77\% yield. ${ }^{1} \mathrm{H}$ NMR (400 MHz, chloroform- $d$ ) $\delta 7.44(\mathrm{~d}, J=7.9 \mathrm{~Hz}, 2 \mathrm{H}), 7.32(\mathrm{~d}, J=7.9$ $\mathrm{Hz}, 2 \mathrm{H}), 6.02(\mathrm{dd}, J=17.5,11.1 \mathrm{~Hz}, 1 \mathrm{H}), 5.74(\mathrm{dd}, J=17.5,2.1 \mathrm{~Hz}, 1 \mathrm{H}), 5.55(\mathrm{dd}, J=11.1,2.1 \mathrm{~Hz}$, 1H), 4.70 (s, 2H). ${ }^{13} \mathrm{C}$ NMR (101 MHz, chloroform- $d$ ) $\delta 141.1,131.8,127.1,126.9,122.4,117.2,89.8$, 88.2, 65.0. HRMS (EI): [M] ${ }^{\oplus}$ Calcd for $\mathrm{C}_{11} \mathrm{H}_{10} \mathrm{O} 158.0726$, found 158.0727. 


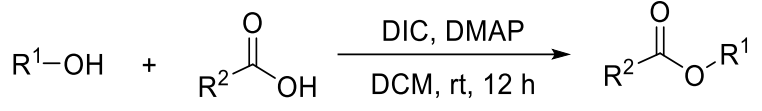

$$
\begin{aligned}
& \text { 10-11 2t-2z, 2aa-2ee }
\end{aligned}
$$

To a solution of carboxylic acid $(6 \mathrm{mmol})$, alcohol $(5 \mathrm{mmol})$ and DMAP $(1 \mathrm{mmol})$ in dry DCM (30 $\mathrm{mL})$ was added diisopropylcarbodiimide (DIC, $6.5 \mathrm{mmol})$ in DCM $(5 \mathrm{~mL})$ dropwise. The resulting mixture was stirred at room temperature overnight. After this time, the reaction was quenched with water $(50 \mathrm{~mL})$, extracted by EtOAc $(40 \mathrm{~mL} \times 3)$, concentrated and purified by flash column chromatography to provide the pure product.

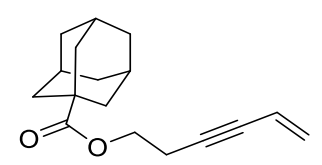

\section{Hex-5-en-3-yn-1-yl $(3 r, 5 r, 7 r)$-adamantane-1-carboxylate $(2 \mathrm{t})$}

White solid, $40 \%$ yield. ${ }^{1} \mathrm{H}$ NMR (400 MHz, Chloroform- $d$ ) $\delta 5.76(\mathrm{dd}, J=17.6,11.0 \mathrm{~Hz}, 1 \mathrm{H}), 5.57$ (dd, $J=17.6,2.2 \mathrm{~Hz}, 1 \mathrm{H}), 5.41(\mathrm{dd}, J=11.0,2.2 \mathrm{~Hz}, 1 \mathrm{H}), 4.16(\mathrm{t}, J=6.9 \mathrm{~Hz}, 2 \mathrm{H}), 2.63(\mathrm{t}, J=6.9 \mathrm{~Hz}, 2 \mathrm{H})$, $2.07-1.98(\mathrm{~m}, 3 \mathrm{H}), 1.93-1.87(\mathrm{~m}, 6 \mathrm{H}), 1.76-1.67(\mathrm{~m}, 6 \mathrm{H}) .{ }^{13} \mathrm{C} \mathrm{NMR}\left(126 \mathrm{MHz}, \mathrm{CDCl}_{3}\right) \delta 177.5$, 126.4, 117.3, 86.4, 80.7, 61.9, 40.8, 38.8, 36.6, 28.0, 19.9. HRMS (EI): $[\mathrm{M}]^{\oplus}$ Calcd for $\mathrm{C}_{17} \mathrm{H}_{22} \mathrm{O}_{2} 258.1614$, found 258.1619.

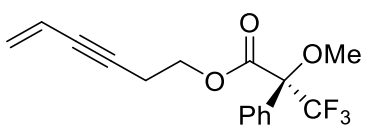

\section{Hex-5-en-3-yn-1-yl (S)-3,3,3-trifluoro-2-methoxy-2-phenylpropanoate (2u)}

Yellow oil, 62\% yield. ${ }^{1} \mathrm{H}$ NMR (500 MHz, chloroform- $d$ ) $\delta 7.62-7.54(\mathrm{~m}, 2 \mathrm{H}), 7.46-7.40(\mathrm{~m}, 3 \mathrm{H})$, $5.75(\mathrm{dd}, J=17.5,11.0,1 \mathrm{H}), 5.58(\mathrm{dd}, J=17.5,2.1 \mathrm{~Hz}, 1 \mathrm{H}), 5.45(\mathrm{dd}, J=11.1,2.2 \mathrm{~Hz}, 1 \mathrm{H}), 4.55-$ $4.46(\mathrm{~m}, 1 \mathrm{H}), 4.45-4.37(\mathrm{~m}, 1 \mathrm{H}), 3.61(\mathrm{~s}, 3 \mathrm{H}), 2.76(\mathrm{t}, J=6.6,2 \mathrm{H}) .{ }^{13} \mathrm{C} \mathrm{NMR}\left(126 \mathrm{MHz}, \mathrm{CDCl}_{3}\right) \delta$ 166.4, 132.2, 129.7, 128.5, 127.4, 126.8, 123.3 (q, $J=288.4 \mathrm{~Hz}$ ), 117.0, 85.5, 84.7 (q, $J=27.8 \mathrm{~Hz}$ ), 81.2, 64.1, 55.5, 19.7. ${ }^{19} \mathrm{~F}$ NMR (376 MHz, chloroform- $d$ ) $\delta-71.8$ (s, 3F). HRMS (ESI): $[\mathrm{M}+\mathrm{Na}]^{\oplus}$ calcd for $\mathrm{C}_{16} \mathrm{H}_{15} \mathrm{O}_{3} \mathrm{~F}_{3} \mathrm{Na}^{\oplus} 335.0866$, found 335.0866.

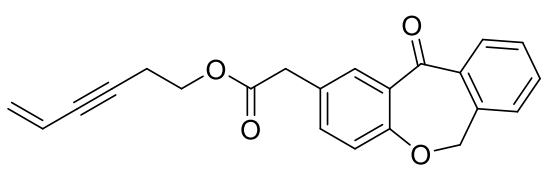

\section{Hex-5-en-3-yn-1-yl 2-(11-oxo-6,11-dihydrodibenzo[b,e]oxepin-2-yl)acetate (2v)}

White solid, 84\% yield. ${ }^{1} \mathrm{H}$ NMR (400 MHz, chloroform- $d$ ) $\delta 8.11(\mathrm{~d}, J=2.4 \mathrm{~Hz}, 1 \mathrm{H}), 7.94-7.82(\mathrm{~m}$, $1 \mathrm{H}), 7.61-7.51(\mathrm{~m}, 1 \mathrm{H}), 7.50-7.42(\mathrm{~m}, 2 \mathrm{H}), 7.39-7.33(\mathrm{~m}, 1 \mathrm{H}), 7.02(\mathrm{~d}, J=8.4 \mathrm{~Hz}, 1 \mathrm{H}), 5.75(\mathrm{ddt}, J$ $=17.6,11.0,2.1 \mathrm{~Hz}, 1 \mathrm{H}), 5.57(\mathrm{dd}, J=17.6,2.3 \mathrm{~Hz}, 1 \mathrm{H}), 5.41(\mathrm{dd}, J=11.0,2.3 \mathrm{~Hz}, 1 \mathrm{H}), 5.18(\mathrm{~s}, 2 \mathrm{H})$, $4.21(\mathrm{t}, J=6.9 \mathrm{~Hz}, 2 \mathrm{H}), 3.67(\mathrm{~s}, 2 \mathrm{H}), 2.65(\mathrm{td}, J=6.9,2.1 \mathrm{~Hz}, 2 \mathrm{H}) .{ }^{13} \mathrm{C}$ NMR $(126 \mathrm{MHz}$, chloroform- $d$ ) $\delta 190.9,171.2,160.5,140.5,136.4,135.6,132.8,132.5,129.5,129.3,127.9,127.7,126.6,125.2,121.1$, 117.2, 86.1, 80.8, 73.7, 62.8, 40.2, 19.9. HRMS (ESI): $[\mathrm{M}+\mathrm{H}]^{\oplus}$ calcd for $\mathrm{C}_{22} \mathrm{H}_{19} \mathrm{O}_{4}{ }^{\oplus} 347.1278$, found 347.1277. 


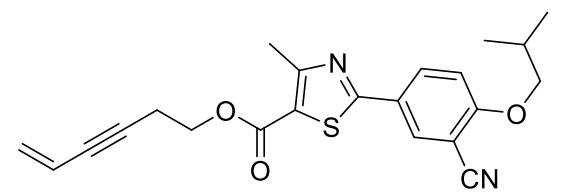

\section{Hex-5-en-3-yn-1-yl 2-(3-cyano-4-isobutoxyphenyl)-4-methylthiazole-5-carboxylate (2w)}

White solid, $88 \%$ yield. ${ }^{1} \mathrm{H}$ NMR (500 MHz, chloroform- $d$ ) $\delta 8.17(\mathrm{~d}, J=2.2 \mathrm{~Hz}, 1 \mathrm{H}$ ), 8.09 (dd, $J=8.9$, $2.2 \mathrm{~Hz}, 1 \mathrm{H}), 7.02(\mathrm{~d}, J=8.8 \mathrm{~Hz}, 1 \mathrm{H}), 5.83-5.73(\mathrm{~m}, 1 \mathrm{H}), 5.60(\mathrm{dd}, J=17.6,2.1 \mathrm{~Hz}, 1 \mathrm{H}), 5.45(\mathrm{dd}, J=$ $11.1,2.1 \mathrm{~Hz}, 1 \mathrm{H}), 4.41(\mathrm{t}, J=6.8 \mathrm{~Hz}, 2 \mathrm{H}), 3.90(\mathrm{~d}, J=6.4 \mathrm{~Hz}, 2 \mathrm{H}), 2.81-2.75(\mathrm{~m}, 5 \mathrm{H}), 2.21$ (hept, $J=$ $6.6 \mathrm{~Hz}, 1 \mathrm{H}), 1.10(\mathrm{~s}, 3 \mathrm{H}), 1.09$ (s, 3H). ${ }^{13} \mathrm{C}$ NMR $(126 \mathrm{MHz}$, chloroform- $d$ ) $\delta 167.5,162.6,161.8,161.5$, 132.6, 132.1, 126.8, 126.0, 121.6, 117.1, 115.4, 112.7, 103.0, 85.9, 81.1, 75.7, 63.1, 28.2, 20.0, 19.1, 17.6. HRMS (ESI): $[\mathrm{M}+\mathrm{H}]^{\oplus}$ calcd for $\mathrm{C}_{22} \mathrm{H}_{23} \mathrm{~N}_{2} \mathrm{O}_{3} \mathrm{~S}{ }^{\oplus} 395.1423$, found 395.1422 .

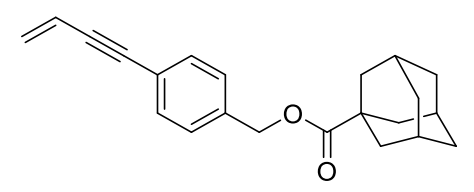

\section{4-(But-3-en-1-yn-1-yl)benzyl $(3 r, 5 r, 7 r)$-adamantane-1-carboxylate (2x)}

White solid, 57\% yield. ${ }^{1} \mathrm{H}$ NMR $(400 \mathrm{MHz}$, chloroform- $d$ ) $\delta 7.43(\mathrm{~d}, J=8.1 \mathrm{~Hz}, 2 \mathrm{H}), 7.28(\mathrm{~d}, J=8.0$ $\mathrm{Hz}, 2 \mathrm{H}), 6.02(\mathrm{dd}, J=17.5,11.2 \mathrm{~Hz}, 1 \mathrm{H}), 5.74(\mathrm{dd}, J=17.5,2.1 \mathrm{~Hz}, 1 \mathrm{H}), 5.55(\mathrm{dd}, J=11.2,2.1 \mathrm{~Hz}$, $1 \mathrm{H}), 5.08(\mathrm{~s}, 2 \mathrm{H}), 2.07-1.98(\mathrm{~m}, 3 \mathrm{H}), 1.96-1.84(\mathrm{~m}, 6 \mathrm{H}), 1.78-1.65(\mathrm{~m}, 6 \mathrm{H}) .{ }^{13} \mathrm{C}$ NMR $(126 \mathrm{MHz}$, $\left.\mathrm{CDCl}_{3}\right) \delta 177.4,136.8,131.7,127.6,127.1,122.8,117.2,89.7,88.4,65.4,40.9,38.9,36.5$, 28.0. HRMS (EI): $[\mathrm{M}]^{\oplus}$ Calcd for $\mathrm{C}_{22} \mathrm{H}_{24} \mathrm{O}_{2} 320.1771$, found 320.1766 .

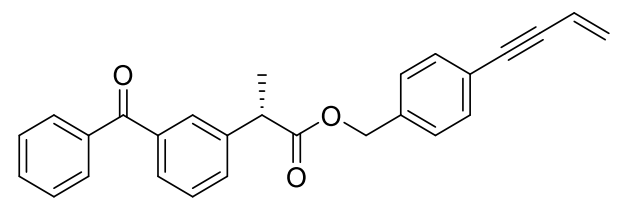

\section{4-(But-3-en-1-yn-1-yl)benzyl (S)-2-(3-benzoylphenyl)propanoate (2y)}

Yellow oil, 90\% yield. ${ }^{1} \mathrm{H}$ NMR (500 MHz, chloroform- $d$ ) $\delta 7.80-7.73(\mathrm{~m}, 3 \mathrm{H}), 7.68(\mathrm{~d}, J=7.6 \mathrm{~Hz}$, $1 \mathrm{H}), 7.62-7.56(\mathrm{~m}, 1 \mathrm{H}), 7.53(\mathrm{~d}, J=7.8 \mathrm{~Hz}, 1 \mathrm{H}), 7.50-7.41(\mathrm{~m}, 3 \mathrm{H}), 7.38(\mathrm{~d}, J=7.8 \mathrm{~Hz}, 2 \mathrm{H}), 7.18(\mathrm{~d}$, $J=7.7 \mathrm{~Hz}, 2 \mathrm{H}), 6.01$ (ddd, $J=17.6,11.1,1.8 \mathrm{~Hz}, 1 \mathrm{H}), 5.73(\mathrm{dd}, J=17.6,2.1 \mathrm{~Hz}, 1 \mathrm{H}), 5.55(\mathrm{dd}, J=$ $11.2,1.8 \mathrm{~Hz}, 1 \mathrm{H}), 5.10(\mathrm{~s}, 2 \mathrm{H}), 3.86(\mathrm{q}, J=7.2 \mathrm{~Hz}, 1 \mathrm{H}), 1.56(\mathrm{~d}, J=7.2 \mathrm{~Hz}, 3 \mathrm{H}) .{ }^{13} \mathrm{C}$ NMR $(126 \mathrm{MHz}$, $\left.\mathrm{CDCl}_{3}\right) \delta 196.5,173.8,140.6,138.0,137.5,135.9,132.6,131.7,131.5,130.1,129.3,129.1,128.6,128.4$, 127.8, 127.2, 123.1, 117.1, 89.6, 88.6, 66.2, 45.4, 18.4. HRMS (EI): $[\mathrm{M}]^{\oplus}$ Calcd for $\mathrm{C}_{27} \mathrm{H}_{22} \mathrm{O}_{3} 394.1563$, found 394.1570 . 


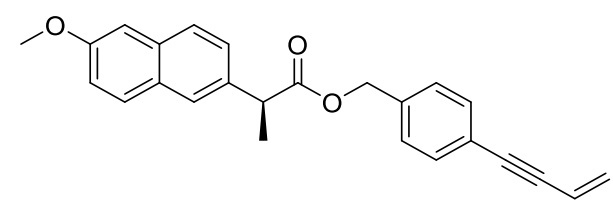

4-(But-3-en-1-yn-1-yl)benzyl (S)-2-(6-methoxynaphthalen-2-yl)propanoate (2z)

White solid, $87 \%$ yield. ${ }^{1} \mathrm{H}$ NMR $(500 \mathrm{MHz}$, chloroform- $d) \delta 7.73-7.62(\mathrm{~m}, 3 \mathrm{H}), 7.42-7.30(\mathrm{~m}, 3 \mathrm{H})$, $7.20-7.10(\mathrm{~m}, 4 \mathrm{H}), 6.01(\mathrm{dd}, J=17.5,11.2 \mathrm{~Hz}, 1 \mathrm{H}), 5.74(\mathrm{dd}, J=17.6,2.0 \mathrm{~Hz}, 1 \mathrm{H}), 5.55(\mathrm{dd}, J=11.2$, $2.0 \mathrm{~Hz}, 1 \mathrm{H}), 5.15-5.05(\mathrm{~m}, 2 \mathrm{H}), 3.96-3.87(\mathrm{~m}, 4 \mathrm{H}), 1.60(\mathrm{~d}, J=7.2 \mathrm{~Hz}, 3 \mathrm{H}) .{ }^{13} \mathrm{C} \mathrm{NMR}(101 \mathrm{MHz}$, chloroform- $d$ ) $\delta 174.4,157.7,136.2,135.5,133.8,131.7,129.3,129.0,127.8,127.24,127.18,126.3$, $126.1,122.9,119.1,117.2,105.6,89.7,88.5,66.0,55.4,45.5,18.5$. HRMS (EI): $[\mathrm{M}]^{\oplus}$ Calcd for $\mathrm{C}_{25} \mathrm{H}_{22} \mathrm{O}_{3} 370.1563$, found 370.1560 .

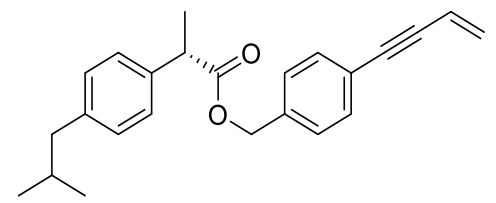

\section{4-(But-3-en-1-yn-1-yl)benzyl (S)-2-(4-isobutylphenyl)propanoate (2aa)}

White solid, 92\% yield. ${ }^{1} \mathrm{H}$ NMR (500 MHz, chloroform- $d$ ) $\delta 7.42-7.35(\mathrm{~m}, 2 \mathrm{H}), 7.25-7.20(\mathrm{~m}, 2 \mathrm{H})$, $7.19-7.15(\mathrm{~m}, 2 \mathrm{H}), 7.13-7.09(\mathrm{~m}, 2 \mathrm{H}), 6.03(\mathrm{dd}, J=17.5,11.2 \mathrm{~Hz}, 1 \mathrm{H}), 5.76(\mathrm{dd}, J=17.5,2.1 \mathrm{~Hz}$, $1 \mathrm{H}), 5.56(\mathrm{dd}, J=11.2,2.0 \mathrm{~Hz}, 1 \mathrm{H}), 5.11(\mathrm{~s}, 2 \mathrm{H}), 3.78(\mathrm{q}, J=7.1 \mathrm{~Hz}, 1 \mathrm{H}), 2.48(\mathrm{~d}, J=7.2 \mathrm{~Hz}, 2 \mathrm{H})$, $1.94-1.82(\mathrm{~m}, 1 \mathrm{H}), 1.54(\mathrm{~d}, J=7.2 \mathrm{~Hz}, 3 \mathrm{H}), 0.93(\mathrm{~d}, J=6.6 \mathrm{~Hz}, 6 \mathrm{H}) .{ }^{13} \mathrm{C} \mathrm{NMR}\left(126 \mathrm{MHz}, \mathrm{CDCl}_{3}\right) \delta$ $174.4,140.7,137.5,136.3,131.6,129.4,127.6,127.2$, 127.1, 122.8, 117.1, 89.7, 88.4, 65.8, 45.1, 45.1, 30.2, 22.4, 18.4. HRMS (EI): [M] ${ }^{\oplus}$ Calcd for $\mathrm{C}_{24} \mathrm{H}_{26} \mathrm{O}_{2} 346.1927$, found 346.1930.

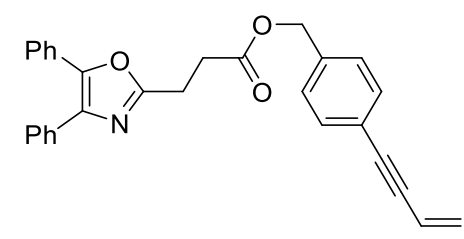

\section{4-(But-3-en-1-yn-1-yl)benzyl 3-(4,5-diphenyloxazol-2-yl)propanoate (2bb)}

White solid, 91\% yield. ${ }^{1} \mathrm{H}$ NMR (500 MHz, chloroform- $\left.d\right) \delta 7.65-7.58(\mathrm{~m}, 2 \mathrm{H}), 7.58-7.51(\mathrm{~m}, 2 \mathrm{H})$, $7.41-7.31(\mathrm{~m}, 8 \mathrm{H}), 7.30-7.26(\mathrm{~m}, 2 \mathrm{H}), 6.02(\mathrm{dd}, J=17.5,11.2 \mathrm{~Hz}, 1 \mathrm{H}), 5.75(\mathrm{dd}, J=17.5,2.0 \mathrm{~Hz}$, $1 \mathrm{H}), 5.56(\mathrm{dd}, J=11.1,2.0 \mathrm{~Hz}, 1 \mathrm{H}), 5.16(\mathrm{~s}, 2 \mathrm{H}), 3.21(\mathrm{t}, J=7.4 \mathrm{~Hz}, 2 \mathrm{H}), 2.98(\mathrm{t}, J=7.4 \mathrm{~Hz}, 2 \mathrm{H}) .{ }^{13} \mathrm{C}$ NMR $\left(126 \mathrm{MHz}, \mathrm{CDCl}_{3}\right) \delta 171.8,161.6,145.5,135.9,135.2,132.5,131.8,129.0,128.7,128.6,128.5$, 128.1, 128.0, 127.9, 127.2, 126.5, 123.1, 117.2, 89.6, 88.6, 66.1, 31.2, 23.6. HRMS (ESI): $[\mathrm{M}+\mathrm{H}]^{\oplus}$ calcd for $\mathrm{C}_{29} \mathrm{H}_{24} \mathrm{NO}_{3}{ }^{\oplus} 434.1751$, found 434.1752. 


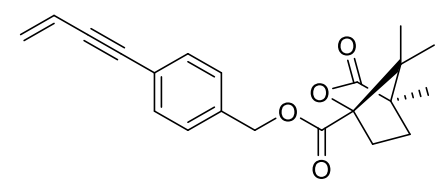

4-(But-3-en-1-yn-1-yl)benzyl carboxylate (2cc)

White solid, 77\% yield. ${ }^{1} \mathrm{H}$ NMR $(500 \mathrm{MHz}$, chloroform- $d) \delta 7.43(\mathrm{~d}, J=8.1 \mathrm{~Hz}, 2 \mathrm{H}), 7.33(\mathrm{~d}, J=8.1$ $\mathrm{Hz}, 2 \mathrm{H}), 6.01(\mathrm{dd}, J=17.5,11.1 \mathrm{~Hz}, 1 \mathrm{H}), 5.74(\mathrm{dd}, J=17.5,2.0 \mathrm{~Hz}, 1 \mathrm{H}), 5.56(\mathrm{dd}, J=11.2,2.0 \mathrm{~Hz}$, $1 \mathrm{H}), 5.24(\mathrm{~s}, 2 \mathrm{H}), 2.42(\mathrm{ddd}, J=13.4,10.8,4.2 \mathrm{~Hz}, 1 \mathrm{H}), 2.04$ (ddd, $J=13.9,9.4,4.5 \mathrm{~Hz}, 1 \mathrm{H}), 1.91$ (ddd, $J=13.2,10.8,4.6 \mathrm{~Hz}, 1 \mathrm{H}), 1.68(\mathrm{ddd}, J=13.4,9.3,4.2 \mathrm{~Hz}, 1 \mathrm{H}), 1.10$ (s, 3H), 1.01 (s, 3H), 0.89 (s, 3H). ${ }^{13} \mathrm{C}$ NMR $(101 \mathrm{MHz}$, chloroform- $d) \delta 178.1,167.4,135.2,131.9,128.4,127.4,123.5,117.0,91.0,89.4$, 88.8, 66.8, 54.8, 54.4, 30.7, 29.0, 16.83, 16.75, 9.8. HRMS (EI): $[\mathrm{M}]^{\oplus}$ Calcd for $\mathrm{C}_{21} \mathrm{H}_{22} \mathrm{O}_{4} 338.1513$, found 338.1507 .

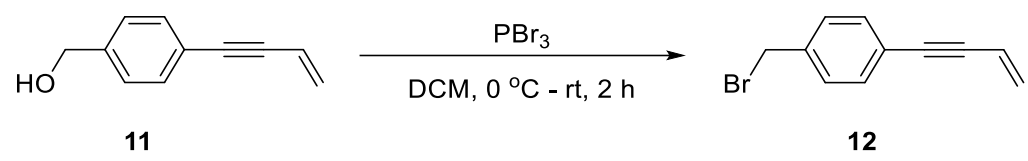

To a solution of enyne $(4 \mathrm{mmol})$ in dry DCM $(15 \mathrm{~mL})$ was added $\mathrm{PBr}_{3}(1.6 \mathrm{mmol})$ under ice bath. The reaction was stirred at room temperature for $2 \mathrm{~h}$. After this time, the reaction was quenched with saturated $\mathrm{NaHCO}_{3}$ aqueous solution, extracted with DCM $(50 \mathrm{~mL} \times 3)$, condensed and purified by flash column chromatography to provide the pure product.

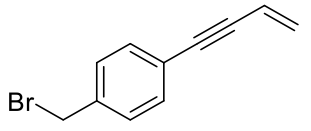

\section{1-(Bromomethyl)-4-(but-3-en-1-yn-1-yl)benzene (12)}

Yellow oil, 65\% yield. ${ }^{1} \mathrm{H}$ NMR (500 MHz, chloroform- $\left.d\right) \delta 7.45-7.39(\mathrm{~m}, 2 \mathrm{H}), 7.37-7.31(\mathrm{~m}, 2 \mathrm{H})$, $6.02(\mathrm{dd}, J=17.5,11.2 \mathrm{~Hz}, 1 \mathrm{H}), 5.76(\mathrm{dd}, J=17.5,2.0 \mathrm{~Hz}, 1 \mathrm{H}), 5.57$ (dd, $J=11.2,2.0 \mathrm{~Hz}, 1 \mathrm{H}), 4.47$ (s, 2H). ${ }^{13} \mathrm{C}$ NMR (126 MHz, chloroform- $d$ ) $\delta 137.8,132.0,129.1,127.4,123.3,117.1,89.5,89.0,33.1$. HRMS (EI): $[\mathrm{M}]^{\oplus}$ Calcd for $\mathrm{C}_{11} \mathrm{H}_{9} \mathrm{Br} 219.9882$, found 219.9879 .

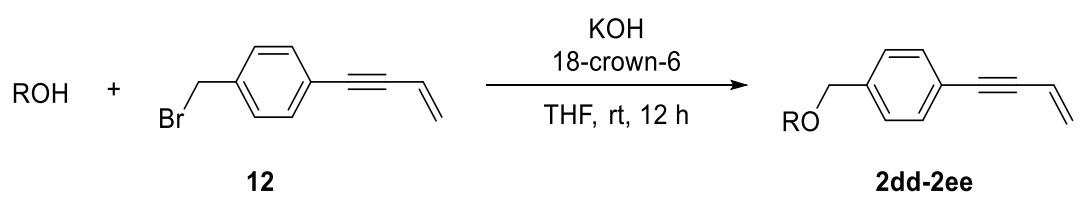

To a solution of alcohol $(4.5 \mathrm{mmol}), 18$-crown-6 $(0.3 \mathrm{mmol})$ in dry THF $(20 \mathrm{~mL})$ was added the $\mathrm{KOH}$ $(18 \mathrm{mmol})$. The reaction was stirred at room temperature for $3 \mathrm{~h}$. Then 1-(bromomethyl)-4-(but-3-en-1yn-1-yl)benzene 12 in THF (5 ml) was added to above solution dropwise. The resulting mixture continued to stir at room temperature for $12 \mathrm{~h}$. After this time, the reaction was quenched with water (50 $\mathrm{mL})$, extracted by EtOAc $(40 \mathrm{~mL} \times 3)$, concentrated and purified by flash column chromatography to provide the pure product. 


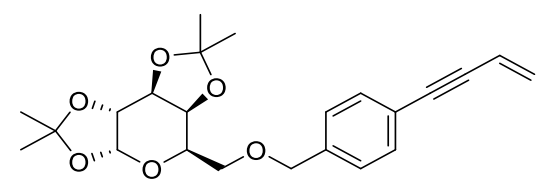

$(3 \mathrm{a} R, 5 R, 5 \mathrm{a} S, 8 \mathrm{a} S, 8 \mathrm{~b} R)-5-(((4-(B u t-3-\mathrm{en}-1-\mathrm{yn}-1-\mathrm{yl}) \mathrm{benzyl}) \mathrm{xxy})$ methyl)-2,2,7,7tetramethyltetrahydro-5H-bis([1,3]dioxolo)[4,5-b:4',5'-d]pyran (2dd)

Yellow solid, 79\% yield. ${ }^{1} \mathrm{H}$ NMR (500 MHz, chloroform- $d$ ) $\delta 7.40$ (d, $\left.J=8.0 \mathrm{~Hz}, 2 \mathrm{H}\right), 7.29$ (d, $J=7.9$ $\mathrm{Hz}, 2 \mathrm{H}), 6.01(\mathrm{dd}, J=17.5,11.2 \mathrm{~Hz}, 1 \mathrm{H}), 5.72(\mathrm{dd}, J=17.5,2.1 \mathrm{~Hz}, 1 \mathrm{H}), 5.57-5.49(\mathrm{~m}, 2 \mathrm{H}), 4.64-$ $4.58(\mathrm{~m}, 2 \mathrm{H}), 4.53(\mathrm{~d}, J=12.5 \mathrm{~Hz}, 1 \mathrm{H}), 4.31(\mathrm{dd}, J=5.0,2.4 \mathrm{~Hz}, 1 \mathrm{H}), 4.26(\mathrm{dd}, J=8.0,1.9 \mathrm{~Hz}, 1 \mathrm{H})$, $4.00(\mathrm{td}, J=6.3,1.9 \mathrm{~Hz}, 1 \mathrm{H}), 3.72-3.59(\mathrm{~m}, 2 \mathrm{H}), 1.54(\mathrm{~s}, 3 \mathrm{H}), 1.43(\mathrm{~s}, 3 \mathrm{H}), 1.33(\mathrm{~d}, J=2.1 \mathrm{~Hz}, 6 \mathrm{H})$. ${ }^{13} \mathrm{C}$ NMR $\left(126 \mathrm{MHz}, \mathrm{CDCl}_{3}\right) \delta 138.7,131.6,127.5,126.9,122.3,117.2,109.3,108.6,96.4,90.0,88.0$, $72.9,71.2,70.7,70.6,69.1,67.0,26.1,26.0,25.0,24.5$. HRMS (ESI): $[\mathrm{M}+\mathrm{Na}]^{\oplus}$ calcd for $\mathrm{C}_{23} \mathrm{H}_{28} \mathrm{O}_{2} \mathrm{Na}^{\oplus} 423.1778$, found 423.1776 .

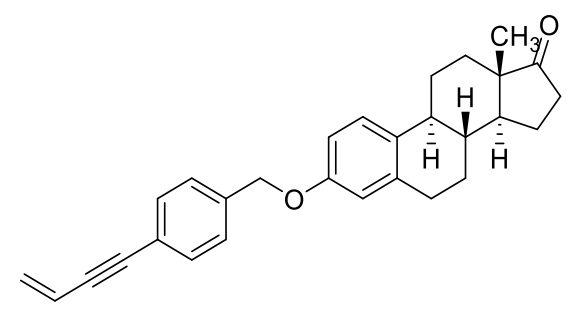

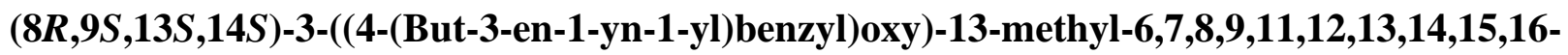 decahydro-17H-cyclopenta[a]phenanthren-17-one (2ee)}

White solid, 50\% yield. ${ }^{1} \mathrm{H}$ NMR $(500 \mathrm{MHz}$, chloroform- $d) \delta 7.46(\mathrm{~d}, J=8.2 \mathrm{~Hz}, 2 \mathrm{H}), 7.38(\mathrm{~d}, J=8.0$ $\mathrm{Hz}, 2 \mathrm{H}), 7.20(\mathrm{~d}, J=8.6 \mathrm{~Hz}, 1 \mathrm{H}), 6.77(\mathrm{dd}, J=8.6,2.8 \mathrm{~Hz}, 1 \mathrm{H}), 6.71(\mathrm{~d}, J=2.8 \mathrm{~Hz}, 1 \mathrm{H}), 6.02(\mathrm{dd}, J=$ $17.5,11.2 \mathrm{~Hz}, 1 \mathrm{H}), 5.74(\mathrm{dd}, J=17.5,2.0 \mathrm{~Hz}, 1 \mathrm{H}), 5.56(\mathrm{dd}, J=11.2,2.0 \mathrm{~Hz}, 1 \mathrm{H}), 5.03(\mathrm{~s}, 2 \mathrm{H}), 2.97-$ $2.84(\mathrm{~m}, 2 \mathrm{H}), 2.51(\mathrm{dd}, J=19.0,8.7 \mathrm{~Hz}, 1 \mathrm{H}), 2.45-2.33(\mathrm{~m}, 1 \mathrm{H}), 2.30-2.22(\mathrm{~m}, 1 \mathrm{H}), 2.20-1.90(\mathrm{~m}$, $4 \mathrm{H}), 1.69-1.37(\mathrm{~m}, 6 \mathrm{H}), 0.91(\mathrm{~s}, 3 \mathrm{H}) .{ }^{13} \mathrm{C}$ NMR $\left(126 \mathrm{MHz}, \mathrm{CDCl}_{3}\right) \delta 221.0,156.7,137.9,137.6,132.5$, 131.8, 127.3, 127.1, 126.5, 122.6, 117.2, 115.0, 112.4, 89.8, 88.3, 69.6, 50.5, 48.1, 44.0, 38.4, 35.9, 31.6, 29.7, 26.6, 26.0, 21.7, 13.9. HRMS (ESI): $[\mathrm{M}+\mathrm{H}]^{\oplus}$ calcd for $\mathrm{C}_{29} \mathrm{H}_{31} \mathrm{O}_{2}{ }^{\oplus} 411.2319$, found 411.2322. 


\section{Development of reaction conditions}

\subsection{Evaluation of different ligands for Pd catalyst}

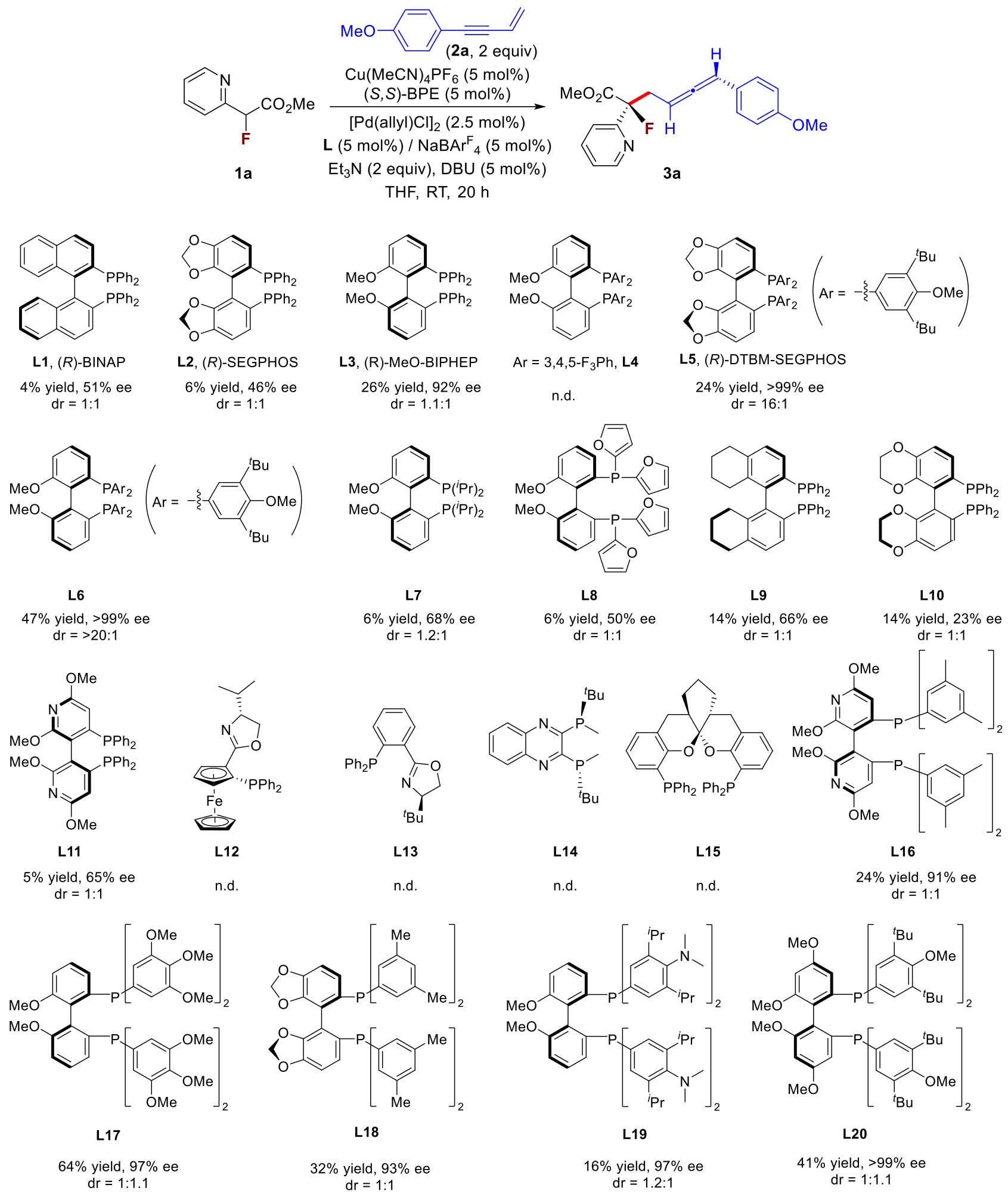




\subsection{Evaluation of solvents and other conditions}

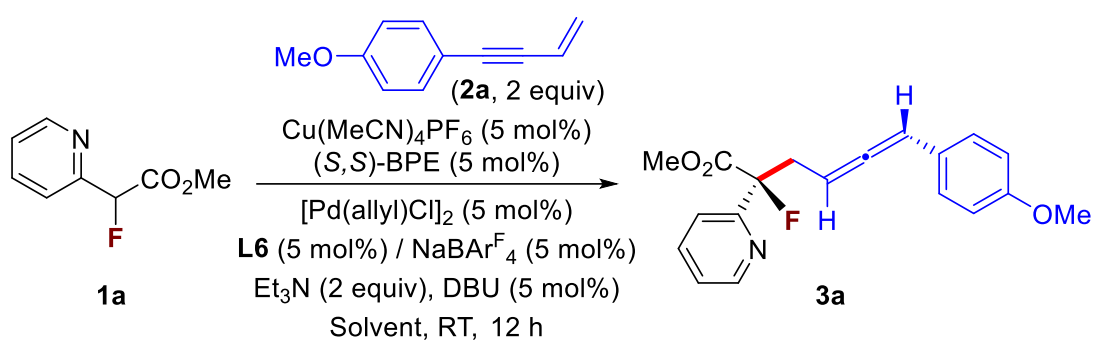

\begin{tabular}{|c|c|c|c|c|}
\hline Entry & Solvent & Yield $(\%)^{d}$ & $\mathrm{dr}^{f}$ & ee $(\%)^{g}$ \\
\hline 1 & $\mathrm{MeCN}$ & 10 & $1.1: 1$ & 83 \\
\hline 2 & DCM & 20 & $7: 1$ & $>99$ \\
\hline 3 & DMF & 20 & $1.1: 1$ & 95 \\
\hline 4 & Toluene & 38 & $>20: 1$ & $>99$ \\
\hline 5 & Dioxane & 16 & $>20: 1$ & $>99$ \\
\hline 6 & DCE & 8 & $16: 1$ & $>99$ \\
\hline 7 & MTBE & 4 & $12: 1$ & $>99$ \\
\hline 8 & EA & 41 & $5: 1$ & $>99$ \\
\hline 9 & 2-MeTHF & 77 & $7: 1$ & $>99$ \\
\hline 10 & DME & 34 & $1.1: 1$ & $>99$ \\
\hline 11 & $\mathrm{MeOH}$ & 4 & $1: 1$ & \\
\hline 12 & Hexane & 22 & $1.1: 1$ & 97 \\
\hline 13 & HFIP & 0 & & \\
\hline 14 & CPME & 62 & $>20: 1$ & $>99$ \\
\hline $15^{a}$ & CPME & $82^{e}$ & $16: 1$ & $>99$ \\
\hline $16^{a, b}$ & CPME & 0 & & \\
\hline $17^{a, c}$ & CPME & 36 & $>20: 1$ & $>99$ \\
\hline
\end{tabular}

${ }^{a}$ Reaction time was 20 h. ${ }^{b} \mathrm{DBU}$ (1 equiv). ${ }^{c}$ Without DBU. ${ }^{d}$ Crude ${ }^{1} \mathrm{H}$ NMR yield. ${ }^{e}$ Isolated yield. ${ }^{f}$ Determined by ${ }^{1} \mathrm{H}$ NMR or ${ }^{19} \mathrm{~F}$ NMR. ${ }^{g}$ Determiend by chiral HPLC analysis. 


\section{General procedure for the hydrocarbonation reaction of enynes}
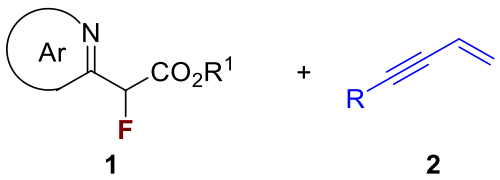
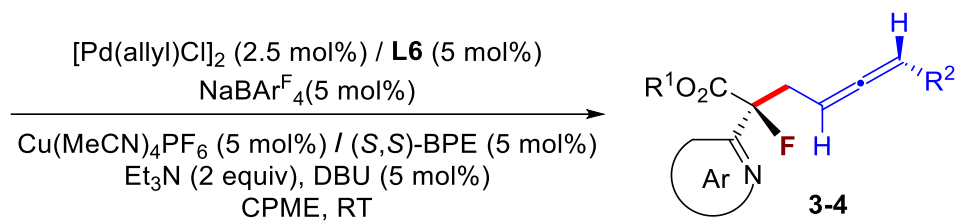

General procedure (3a as an example, standard condition): In the $\mathrm{N}_{2}$-filled glovebox, $\mathrm{Cu}(\mathrm{MeCN})_{4} \mathrm{PF}_{6}$ $(1.9 \mathrm{mg}, 0.0050 \mathrm{mmol}),(S, S)$-BPE $(2.5 \mathrm{mg}, 0.0050 \mathrm{mmol})$ and dry CPME $(0.2 \mathrm{~mL})$ were added to a 4 $\mathrm{mL}$ vial and stirred at room temperature for $0.5 \mathrm{~h}$. To a second vial, $\left[\mathrm{Pd}\left(\eta^{3} \text {-allyl }\right) \mathrm{Cl}\right]_{2}(0.9 \mathrm{mg}, 0.0050$ $\mathrm{mmol})$, L6 $(5.8 \mathrm{mg}, 0.0050 \mathrm{mmol})$ and dry DCM $(0.2 \mathrm{~mL})$ was added and the mixture was stirred at room temperature for $15 \mathrm{~min}$. Then sodium tetrakis[3,5-bis(trifluoromethyl)phenyl]borate ( $\mathrm{NaBAr}_{4}{ }_{4}, 4.4$ $\mathrm{mg}, 0.0050 \mathrm{mmol}$ ) was added to the DCM solution above and the reaction continued to stir for another $0.5 \mathrm{~h}$. After this time, the DCM solvent was removed under vacuum and the residue was re-dissolved in CPME $(0.2 \mathrm{~mL})$ for use. To the vial with copper complex above was added $\mathrm{Et}_{3} \mathrm{~N}(28 \mu \mathrm{L}, 0.20 \mathrm{mmol})$, DBU $(0.0050 \mathrm{~mol}$ in $0.1 \mathrm{~mL}$ CPME) and $\alpha$-fluoro ester $\mathbf{1 a}(17 \mathrm{mg}, 0.10 \mathrm{mmol})$ sequentially. The resulting mixture was stirred at room temperature for $5 \mathrm{~min}$. Finally, the freshly prepared palladium complex solution and enyne $\mathbf{2 a}(32 \mathrm{mg}, 0.20 \mathrm{mmol})$ were added to the above solution. The reaction was then stirred at room temperature for $20 \mathrm{~h}$. After this time, the reaction was diluted with hexane $(2 \mathrm{~mL})$, filtered through a 0.5-inch plug of silica gel, and eluted with EtOAc $(2 \mathrm{~mL} \times 3)$. The filtrate was further condensed and purified by flash column chromatography (Hexane/ EtOAc $=5: 1$ ) to afford product 3a as a mixture of diastereoisomers.

Note: the racemic product was prepared by using rac-L6 and rac-BPE ligand under standard condition. By means of this method, the product was obtained as a mixture of four stereoisomers with one pair of enantiomers as the major isomers (i.e., the $\mathrm{dr}$ is $>1: 1$ ). By using chiral HPLC analysis, we could therefore determine the relationship between corresponding four stereoisomers.

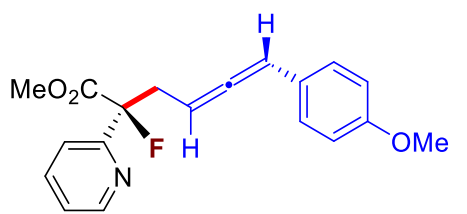

\section{Methyl (2R,5R)-2-fluoro-6-(4-methoxyphenyl)-2-(pyridin-2-yl)hexa-4,5-dienoate (3a)}

Yellow oil, $20 \mathrm{~h}, 82 \%$ yield, $\left.16: 1 \mathrm{dr},[\alpha]_{\mathrm{D}}{ }^{25}-174.2(c) 1.0, \mathrm{CHCl}_{3}\right)$ for $>99 \%$ ee; ${ }^{1} \mathrm{H}$ NMR $(400 \mathrm{MHz}$, chloroform- $d$ ) $\delta 8.59(\mathrm{~d}, J=4.4 \mathrm{~Hz}, 1 \mathrm{H}), 7.77(\mathrm{td}, J=7.8,1.8 \mathrm{~Hz}, 1 \mathrm{H}), 7.65(\mathrm{~d}, J=7.9 \mathrm{~Hz}, 1 \mathrm{H}), 7.33-$ $7.24(\mathrm{~m}, 2 \mathrm{H}), 7.07-6.97(\mathrm{~m}, 2 \mathrm{H}), 6.85-6.69(\mathrm{~m}, 2 \mathrm{H}), 6.09(\mathrm{dt}, J=6.4,2.4 \mathrm{~Hz}, 1 \mathrm{H}), 5.52-5.46(\mathrm{~m}$, $1 \mathrm{H}), 3.79(\mathrm{~s}, 3 \mathrm{H}), 3.77(\mathrm{~s}, 3 \mathrm{H}), 3.42-3.23(\mathrm{~m}, 1 \mathrm{H}), 3.25-3.08(\mathrm{~m}, 1 \mathrm{H}) .{ }^{13} \mathrm{C}$ NMR $(126 \mathrm{MHz}$, chloroform- $d$ ) $\delta 207.0,169.2(\mathrm{~d}, J=26.3 \mathrm{~Hz}), 158.8,156.5(\mathrm{~d}, J=26.9 \mathrm{~Hz}), 149.3(\mathrm{~d}, J=2.3 \mathrm{~Hz}), 137.1$, 128.0, 126.3, 123.6, $120.3(\mathrm{~d}, J=8.3 \mathrm{~Hz}), 114.1,97.2(\mathrm{~d}, J=189.2 \mathrm{~Hz}), 94.3,87.6(\mathrm{~d}, J=3.7 \mathrm{~Hz}), 55.4$, 53.1, $36.6(\mathrm{~d}, J=20.5 \mathrm{~Hz}) .{ }^{19} \mathrm{~F}$ NMR $(376 \mathrm{MHz}$, chloroform- $d$ ) $\delta-151.4(\mathrm{t}, J=24.5 \mathrm{~Hz}, 1 \mathrm{~F})$. HRMS (EI): $[\mathrm{M}]^{\oplus}$ calcd for $\mathrm{C}_{19} \mathrm{H}_{18} \mathrm{FNO}_{3}{ }^{\oplus}$ 327.1265, found 327.1262. HPLC analysis: Chiracel AD-H column; detected at $254 \mathrm{~nm}, 40{ }^{\circ} \mathrm{C} ; 3 \%{ }^{i} \mathrm{PrOH}$ in $n$-hexane; flow $=0.7 \mathrm{~mL} / \mathrm{min}$. Retention time: $19.6 \mathrm{~min}, 22.7$ min, $24.5 \mathrm{~min}, 26.6 \mathrm{~min}$. The ee value was determined based on peaks at $19.6 \mathrm{~min}$ (minor) and $24.5 \mathrm{~min}$ (major). 

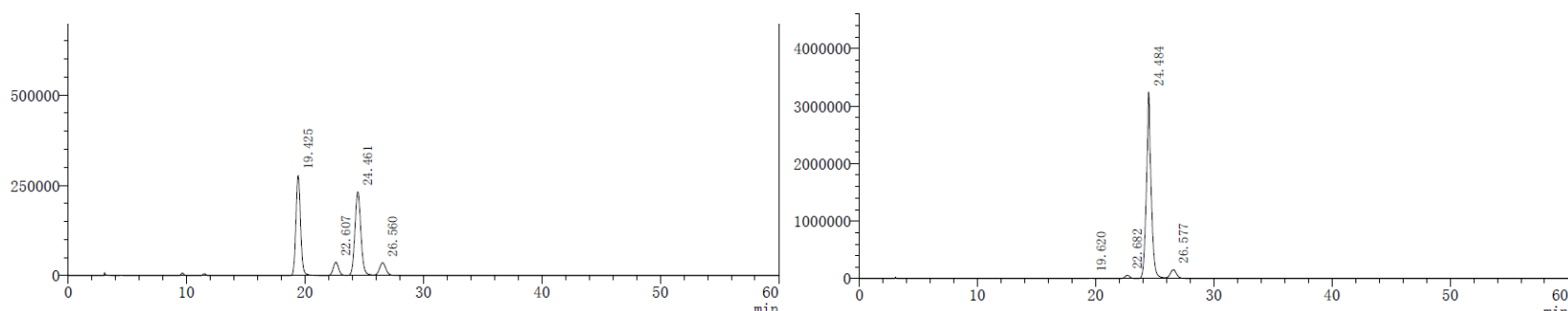

\begin{tabular}{|c|c|c|}
\hline Peak & retension time/min & area\% \\
\hline 1 & 19.425 & 41.289 \\
\hline 2 & 22.607 & 6.499 \\
\hline 3 & 24.461 & 44.714 \\
\hline 4 & 26.560 & 7.498 \\
\hline
\end{tabular}

\begin{tabular}{|c|c|c|}
\hline Peak & retension time/min & area\% \\
\hline 1 & 19.620 & 0.169 \\
\hline 2 & 22.682 & 1.841 \\
\hline 3 & 24.484 & 91.791 \\
\hline 4 & 26.577 & 6.199 \\
\hline
\end{tabular}<smiles>COC(=O)C(F)(/C=C/c1ccc(C)cc1)c1ccccn1</smiles>

\section{Methyl (2R,5R)-2-fluoro-2-(pyridin-2-yl)-6-(p-tolyl)hexa-4,5-dienoate (3b)}

Yellow oil, $22 \mathrm{~h}, 71 \%$ yield, $17: 1 \mathrm{dr},[\alpha]_{\mathrm{D}}{ }^{25}-207.5\left(c 0.2, \mathrm{CHCl}_{3}\right)$ for $>99 \%$ ee; ${ }^{1} \mathrm{H} \mathrm{NMR}(500 \mathrm{MHz}$, chloroform- $d$ ) $\delta 8.60(\mathrm{~s}, 1 \mathrm{H}), 7.77(\mathrm{td}, J=7.8,1.6 \mathrm{~Hz}, 1 \mathrm{H}), 7.65(\mathrm{~d}, J=7.9 \mathrm{~Hz}, 1 \mathrm{H}), 7.30-7.28(\mathrm{~m}, 1 \mathrm{H})$, $7.04(\mathrm{~d}, J=7.8 \mathrm{~Hz}, 2 \mathrm{H}), 6.97(\mathrm{~d}, J=8.1 \mathrm{~Hz}, 2 \mathrm{H}), 6.10(\mathrm{dt}, J=6.6,2.3 \mathrm{~Hz}, 1 \mathrm{H}), 5.52-5.47(\mathrm{~m}, 1 \mathrm{H})$, $3.77(\mathrm{~s}, 3 \mathrm{H}), 3.40-3.26(\mathrm{~m}, 1 \mathrm{H}), 3.25-3.09(\mathrm{~m}, 1 \mathrm{H}), 2.31(\mathrm{~s}, 3 \mathrm{H}) .{ }^{13} \mathrm{C}$ NMR $(126 \mathrm{MHz}$, chloroform-d) $\delta 207.2,169.2(\mathrm{~d}, J=26.3 \mathrm{~Hz}), 156.5(\mathrm{~d}, J=26.6 \mathrm{~Hz}), 149.2,137.1,136.7,131.0,129.3,126.8,123.6$, $120.3(\mathrm{~d}, J=7.0 \mathrm{~Hz}), 97.2(\mathrm{~d}, J=189.0 \mathrm{~Hz}), 94.7,87.6(\mathrm{~d}, J=3.5 \mathrm{~Hz}), 53.1,36.5(\mathrm{~d}, J=20.6 \mathrm{~Hz}), 21.2$. ${ }^{19} \mathrm{~F}$ NMR (376 MHz, chloroform- $d$ ) $\delta-161.12(\mathrm{t}, J=24.5 \mathrm{~Hz}, 1 \mathrm{~F})$. HRMS (FI): [M] ${ }^{\oplus}$ calcd for $\mathrm{C}_{19} \mathrm{H}_{18} \mathrm{FNO}_{2}{ }^{\oplus}$ 311.1316, found 311.1310. HPLC analysis: Chiralpak IC column; detected at $254 \mathrm{~nm}, 40$ ${ }^{\circ} \mathrm{C} ; 9 \%{ }^{i} \mathrm{PrOH}$ in $n$-hexane; flow $=0.7 \mathrm{~mL} / \mathrm{min}$. Retention time: $16.3 \mathrm{~min}, 17.3 \mathrm{~min}, 18.3 \mathrm{~min}, 24.2 \mathrm{~min}$. The ee value was determined based on peaks at $16.3 \mathrm{~min}$ (major) and $17.3 \mathrm{~min}$ (minor).
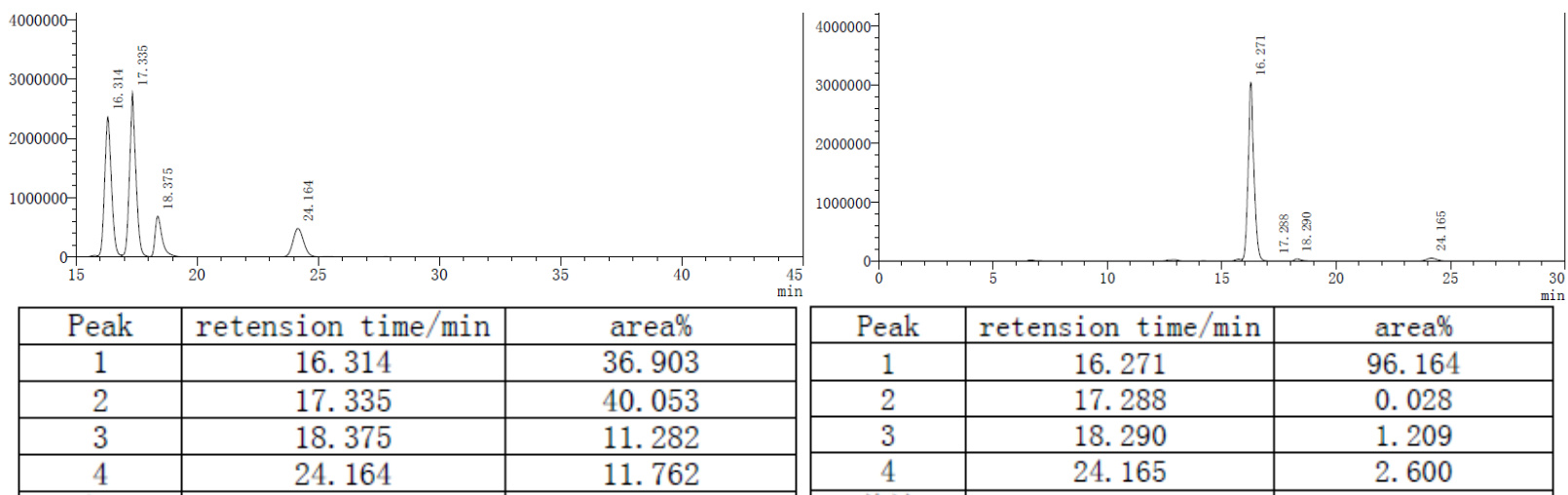


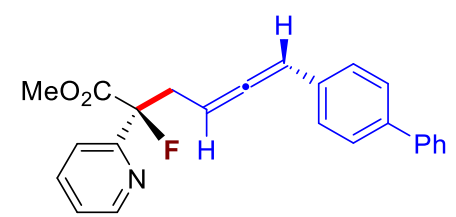

\section{Methyl (2R,5R)-6-([1,1'-biphenyl]-4-yl)-2-fluoro-2-(pyridin-2-yl)hexa-4,5-dienoate (3c)}

White solid, $18 \mathrm{~h}, 78 \%$ yield, $15: 1 \mathrm{dr},[\alpha]_{\mathrm{D}}{ }^{25}-182.3\left(c 1.0, \mathrm{CHCl}_{3}\right)$ for $>99 \%$ ee; ${ }^{1} \mathrm{H} \mathrm{NMR}(500 \mathrm{MHz}$, chloroform- $d$ ) $\delta 8.59(\mathrm{~d}, J=4.7 \mathrm{~Hz}, 1 \mathrm{H}), 7.77(\mathrm{td}, J=7.7,1.8 \mathrm{~Hz}, 1 \mathrm{H}), 7.66(\mathrm{~d}, J=7.8 \mathrm{~Hz}, 1 \mathrm{H}), 7.57(\mathrm{~d}$, $J=7.6 \mathrm{~Hz}, 2 \mathrm{H}), 7.47(\mathrm{~d}, J=8.3 \mathrm{~Hz}, 2 \mathrm{H}), 7.44-7.41(\mathrm{~m}, 2 \mathrm{H}), 7.37-7.30(\mathrm{~m}, 1 \mathrm{H}), 7.30-7.26(\mathrm{~m}, 1 \mathrm{H})$, $7.15(\mathrm{~d}, J=8.0 \mathrm{~Hz}, 2 \mathrm{H}), 6.17(\mathrm{dt}, J=6.7,2.4 \mathrm{~Hz}, 1 \mathrm{H}), 5.58-5.53(\mathrm{~m}, 1 \mathrm{H}), 3.78(\mathrm{~s}, 3 \mathrm{H}), 3.45-3.29(\mathrm{~m}$, $1 \mathrm{H}), 3.29-3.13(\mathrm{~m}, 1 \mathrm{H}) .{ }^{13} \mathrm{C}$ NMR $(126 \mathrm{MHz}$, chloroform- $d) \delta 207.7,169.2(\mathrm{~d}, J=26.2 \mathrm{~Hz}), 156.4(\mathrm{~d}$, $J=27.0 \mathrm{~Hz}), 149.3(\mathrm{~d}, J=2.2 \mathrm{~Hz}), 140.8,139.7,137.1,133.1,128.8,127.27,127.26,127.25,127.0$, 123.6, $120.3(\mathrm{~d}, J=8.4 \mathrm{~Hz}), 97.2(\mathrm{~d}, J=189.2 \mathrm{~Hz}), 94.6,87.8(\mathrm{~d}, J=3.9 \mathrm{~Hz}), 53.1,36.4(\mathrm{~d}, J=20.4$ $\mathrm{Hz}$ ). ${ }^{19} \mathrm{~F}$ NMR (376 MHz, chloroform- $d$ ) $\delta-164.5$ (t, $\left.J=24.1 \mathrm{~Hz}, 1 \mathrm{~F}\right)$. HRMS (FI): $[\mathrm{M}]{ }^{\oplus}$ calcd for $\mathrm{C}_{24} \mathrm{H}_{20} \mathrm{FNO}_{2}{ }^{\oplus}$ 373.1473, found 373.1477. HPLC analysis: Chiralpak IC column; detected at $254 \mathrm{~nm}, 40$ ${ }^{\circ} \mathrm{C}$; 9\% ${ }^{i} \mathrm{PrOH}$ in $n$-hexane; flow = $0.7 \mathrm{~mL} / \mathrm{min}$. Retention time: $19.2 \mathrm{~min}, 20.7 \mathrm{~min}, 21.8 \mathrm{~min}, 29.7 \mathrm{~min}$.

The ee value was determined based on peaks at $19.2 \mathrm{~min}$ (major and $21.8 \mathrm{~min}$ (minor).
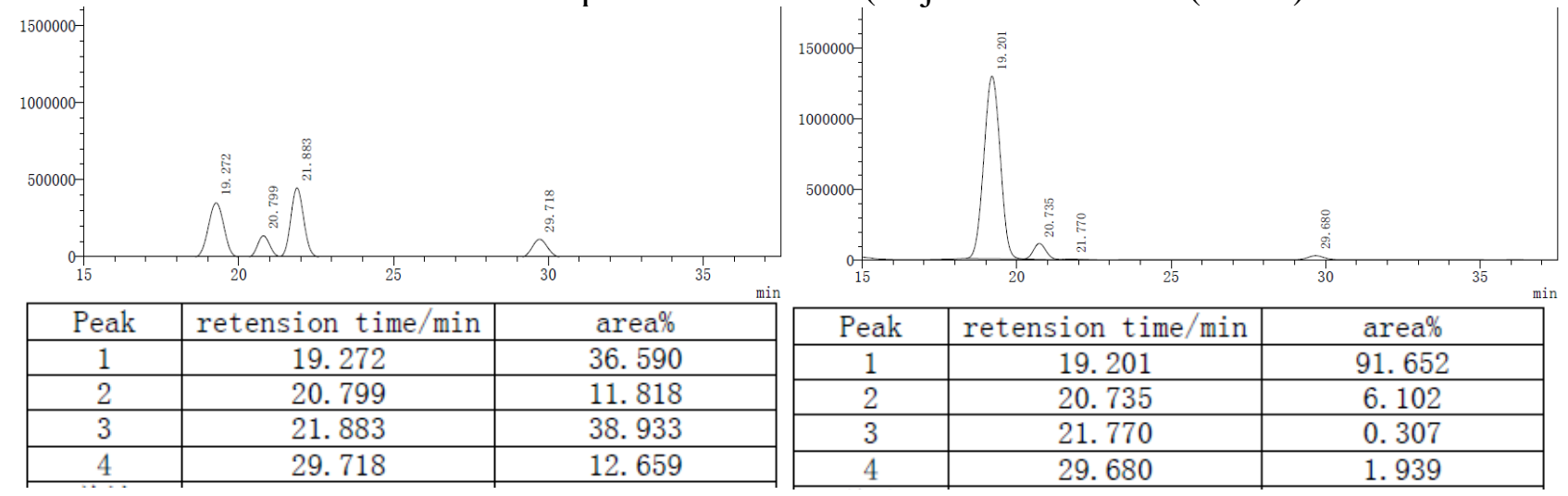

\begin{tabular}{|c|c|c|}
\hline Peak & retension time/min & area\% \\
\hline 1 & 19.272 & 36.590 \\
\hline 2 & 20.799 & 11.818 \\
\hline 3 & 21.883 & 38.933 \\
\hline 4 & 29.718 & 12.659 \\
\hline
\end{tabular}

\begin{tabular}{|c|c|c|}
\hline Peak & retension time/min & area\% \\
\hline 1 & 19.201 & 91.652 \\
\hline 2 & 20.735 & 6.102 \\
\hline 3 & 21.770 & 0.307 \\
\hline 4 & 29.680 & 1.939 \\
\hline
\end{tabular}

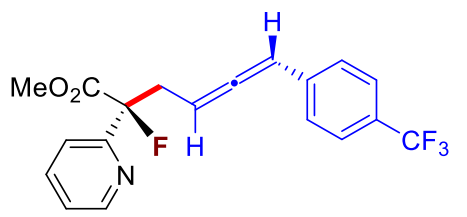

\section{Methyl (2R,5R)-2-fluoro-2-(pyridin-2-yl)-6-(4-(trifluoromethyl)phenyl)hexa-4,5-dienoate (3d)}

Yellow solid, $24 \mathrm{~h}, 96 \%$ yield, $>20: 1 \mathrm{dr},[\alpha]_{\mathrm{D}}{ }^{25}-172.3\left(c 0.2, \mathrm{CHCl}_{3}\right)$ for $>99 \%$ ee; ${ }^{1} \mathrm{H} \mathrm{NMR}(500 \mathrm{MHz}$, chloroform- $d$ ) $\delta 8.58(\mathrm{~d}, J=4.6,1 \mathrm{H}), 7.79(\mathrm{td}, J=7.7,1.8 \mathrm{~Hz}, 1 \mathrm{H}), 7.65(\mathrm{~d}, J=7.8 \mathrm{~Hz}, 1 \mathrm{H}), 7.46(\mathrm{~d}, J$ $=8.1 \mathrm{~Hz}, 2 \mathrm{H}), 7.32-7.27(\mathrm{~m}, 1 \mathrm{H}), 7.16(\mathrm{~d}, J=8.0 \mathrm{~Hz}, 2 \mathrm{H}), 6.18-6.10(\mathrm{~m}, 1 \mathrm{H}), 5.62-5.55(\mathrm{~m}, 1 \mathrm{H})$,

$3.78(\mathrm{~s}, 3 \mathrm{H}), 3.41-3.16(\mathrm{~m}, 2 \mathrm{H}) .{ }^{13} \mathrm{C}$ NMR $(126 \mathrm{MHz}$, chloroform- $d$ ) $\delta 208.3,169.0(\mathrm{~d}, J=26.1 \mathrm{~Hz})$, $156.3(\mathrm{~d}, J=27.1 \mathrm{~Hz}), 149.4(\mathrm{~d}, J=2.3 \mathrm{~Hz}), 138.1,137.2,128.8(\mathrm{q}, J=32.5 \mathrm{~Hz}), 126.9,125.5(\mathrm{q}, J=$ $3.9 \mathrm{~Hz}), 124.3(\mathrm{q}, J=271.8 \mathrm{~Hz}), 123.7,120.3(\mathrm{~d}, J=8.5 \mathrm{~Hz}), 97.1(\mathrm{~d}, J=189.4 \mathrm{~Hz}), 94.1,88.4(\mathrm{~d}, J=$ $3.7 \mathrm{~Hz}), 53.2,36.1(\mathrm{~d}, J=20.5 \mathrm{~Hz}) .{ }^{19} \mathrm{~F}$ NMR $(376 \mathrm{MHz}$, chloroform- $d) \delta-62.5(\mathrm{~s}, 3 \mathrm{~F}),-164.7(\mathrm{t}, J=$ $23.9 \mathrm{~Hz}, 1 \mathrm{~F})$. HRMS (FI): [M] ${ }^{\oplus}$ calcd for $\mathrm{C}_{19} \mathrm{H}_{15} \mathrm{~F}_{4} \mathrm{NO}_{2}{ }^{\oplus}$ 365.1033, found 365.1031. HPLC analysis: Chiracel AD-H column; detected at $254 \mathrm{~nm}, 40{ }^{\circ} \mathrm{C} ; 3 \%{ }^{i} \mathrm{PrOH}$ in $n$-hexane; flow $=0.7 \mathrm{~mL} / \mathrm{min}$. 
Retention time: $9.8 \mathrm{~min}, 10.7 \mathrm{~min}, 11.3 \mathrm{~min}, 11.9 \mathrm{~min}$. The ee value was determined based on peaks at $9.8 \mathrm{~min}$ (minor) and $11.9 \mathrm{~min}$ (major).
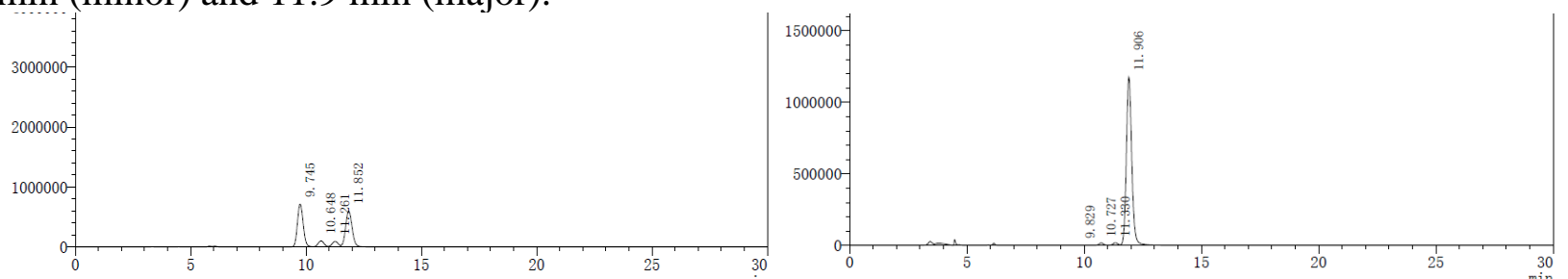

\begin{tabular}{|c|c|c|}
\hline Peak & retension time/min & area\% \\
\hline 1 & 9.745 & 44.577 \\
\hline 2 & 10.648 & 6.788 \\
\hline 3 & 11.261 & 6.309 \\
\hline 4 & 11.852 & 42.326 \\
\hline
\end{tabular}

\begin{tabular}{|c|c|c|}
\hline Peak & retension time/min & area\% \\
\hline 1 & 9.829 & 0.064 \\
\hline 2 & 10.727 & 1.189 \\
\hline 3 & 11.330 & 1.344 \\
\hline 4 & 11.906 & 97.403 \\
\hline
\end{tabular}<smiles>COC(=O)C(F)(C/C=C/c1ccc(F)cc1)c1ccccn1</smiles>

\section{Methyl (2R,5R)-6-(4-bromophenyl)-2-fluoro-2-(pyridin-2-yl)hexa-4,5-dienoate (3e)}

Yellow oil, $17 \mathrm{~h}, 92 \%$ yield, $>20: 1 \mathrm{dr},[\alpha]_{\mathrm{D}}{ }^{25}-140.2\left(c 0.2, \mathrm{CHCl}_{3}\right)$ for $>99 \%$ ee; ${ }^{1} \mathrm{H} \mathrm{NMR}(500 \mathrm{MHz}$, acetone- $\left.d_{6}\right) \delta 8.57(\mathrm{~d}, J=4.6 \mathrm{~Hz}, 1 \mathrm{H}), 7.95(\mathrm{td}, J=7.6,1.8 \mathrm{~Hz}, 1 \mathrm{H}), 7.68-7.66(\mathrm{~m}, 1 \mathrm{H}), 7.46-7.44(\mathrm{~m}$, $1 \mathrm{H}), 7.15-7.08(\mathrm{~m}, 2 \mathrm{H}), 7.07-6.99(\mathrm{~m}, 2 \mathrm{H}), 6.24(\mathrm{dt}, J=6.9,2.4 \mathrm{~Hz}, 1 \mathrm{H}), 5.58-5.54(\mathrm{~m}, 1 \mathrm{H}), 3.74$ (s, 3H), $3.40-3.06(\mathrm{~m}, 2 \mathrm{H}) .{ }^{13} \mathrm{C}$ NMR $\left(126 \mathrm{MHz}\right.$, acetone- $\left.d_{6}\right) \delta 207.0(\mathrm{~d}, J=2.5 \mathrm{~Hz}), 168.6(\mathrm{~d}, J=26.1$ $\mathrm{Hz}), 161.8(\mathrm{~d}, J=244.2 \mathrm{~Hz}), 156.5(\mathrm{~d}, J=26.7 \mathrm{~Hz}), 149.2(\mathrm{~d}, J=2.2 \mathrm{~Hz}), 137.3,130.4(\mathrm{~d}, J=3.2 \mathrm{~Hz})$, $128.4(\mathrm{~d}, J=8.1 \mathrm{~Hz}), 123.9,120.2(\mathrm{~d}, J=7.5 \mathrm{~Hz}), 115.3(\mathrm{~d}, J=21.9 \mathrm{~Hz}), 97.2(\mathrm{~d}, J=187.6 \mathrm{~Hz}), 93.3$, $88.0(\mathrm{~d}, J=4.1 \mathrm{~Hz}), 52.2,35.8(\mathrm{~d}, J=20.6 \mathrm{~Hz}) .{ }^{19} \mathrm{~F}$ NMR $\left(376 \mathrm{MHz}\right.$, acetone- $d_{6} \delta-115.9(\mathrm{~s}, 1 \mathrm{~F}),-161.7$ (t, $J=24.0 \mathrm{~Hz}, 1 \mathrm{~F})$. HRMS (EI): $[\mathrm{M}]^{\oplus}$ calcd for $\mathrm{C}_{18} \mathrm{H}_{15} \mathrm{~F}_{2} \mathrm{NO}_{2}{ }^{\oplus}$ 315.1065, found 315.1068. HPLC analysis: Chiralpak IC column; detected at $254 \mathrm{~nm}, 40{ }^{\circ} \mathrm{C} ; 3 \%{ }^{i} \mathrm{PrOH}$ in $n$-hexane; flow $=0.7 \mathrm{~mL} / \mathrm{min}$. Retention time: $24.0 \mathrm{~min}, 26.8 \mathrm{~min}, 28.3 \mathrm{~min}, 49.1 \mathrm{~min}$. The ee value was determined based on peaks at $24.0 \mathrm{~min}$ (major) and $28.3 \mathrm{~min}$ (minor).

\begin{tabular}{|c|c|c|c|c|c|}
\hline Peak & retension time/min & area\% \\
\hline 1 & 23.828 & 43.281 \\
\hline 2 & 26.514 & 8.459 \\
\hline 3 & 27.877 & 41.076 \\
\hline 4 & 49.638 & 7.184 \\
\hline
\end{tabular}




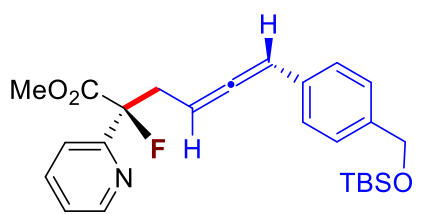

Methyl (2R,5R)-6-(4-(((tert-butyldimethylsilyl)oxy)methyl)phenyl)-2-fluoro-2-(pyridin-2-yl)hexa4,5-dienoate (3f)

Yellow oil, $20 \mathrm{~h}, 90 \%$ yield, $12: 1 \mathrm{dr},[\alpha]_{\mathrm{D}}{ }^{25}-165.7\left(c 0.3, \mathrm{CHCl}_{3}\right)$ for $>99 \%$ ee; ${ }^{1} \mathrm{H}$ NMR $(500 \mathrm{MHz}$, chloroform- $d$ ) $\delta 8.59(\mathrm{~d}, J=4.6 \mathrm{~Hz}, 1 \mathrm{H}), 7.77(\mathrm{td}, J=7.7,1.8 \mathrm{~Hz}, 1 \mathrm{H}), 7.65(\mathrm{~d}, J=7.6 \mathrm{~Hz}, 1 \mathrm{H}), 7.31-$ $7.26(\mathrm{~m}, 1 \mathrm{H}), 7.18(\mathrm{~d}, J=7.7 \mathrm{~Hz}, 2 \mathrm{H}), 7.03(\mathrm{~d}, J=7.9 \mathrm{~Hz}, 2 \mathrm{H}), 6.12(\mathrm{dt}, J=6.5,2.4 \mathrm{~Hz}, 1 \mathrm{H}), 5.53-$ $5.49(\mathrm{~m}, 1 \mathrm{H}), 4.70(\mathrm{~s}, 2 \mathrm{H}), 3.77(\mathrm{~s}, 3 \mathrm{H}), 3.39-3.26(\mathrm{~m}, 1 \mathrm{H}), 3.26-3.11(\mathrm{~m}, 1 \mathrm{H}), 0.94(\mathrm{~s}, 9 \mathrm{H}), 0.09(\mathrm{~s}$, $6 \mathrm{H}) .{ }^{13} \mathrm{C}$ NMR $(126 \mathrm{MHz}$, chloroform- $d$ ) $\delta 207.4,169.2(\mathrm{~d}, J=26.2 \mathrm{~Hz}), 156.4(\mathrm{~d}, J=27.1 \mathrm{~Hz}), 149.3$ (d, $J=2.2 \mathrm{~Hz}), 140.3,137.1,132.6,126.7,126.3,123.6,120.3(\mathrm{~d}, J=8.4 \mathrm{~Hz}), 97.2(\mathrm{~d}, J=189.2 \mathrm{~Hz})$, 94.7, $87.6(\mathrm{~d}, J=4.0 \mathrm{~Hz}), 64.9,53.1,36.5(\mathrm{~d}, J=20.4 \mathrm{~Hz}), 26.0,18.5,-5.1 .{ }^{19} \mathrm{~F}$ NMR $(376 \mathrm{MHz}$, chloroform- $d$ ) $\delta-165.7$ (t, $J=24.4 \mathrm{~Hz}, 1 \mathrm{~F}$ ). HRMS (EI): $[\mathrm{M}]^{\oplus}$ calcd for $\mathrm{C}_{25} \mathrm{H}_{32} \mathrm{FNO}_{3} \mathrm{Si}^{\oplus}$ 441.2130, found 441.2123. HPLC analysis: Chiralpak OD-H + Chiralpak IG-3 column (The two columns were connected to each other in this order); detected at $254 \mathrm{~nm}, 40{ }^{\circ} \mathrm{C} ; 3 \%{ }^{i} \mathrm{PrOH}$ in $n$-hexane; flow $=0.5$ $\mathrm{mL} / \mathrm{min}$. Retention time: $29.8 \mathrm{~min}, 32.4 \mathrm{~min}, 48.6 \mathrm{~min}, 58.5 \mathrm{~min}$. The ee value was determined based on peaks at $29.8 \mathrm{~min}$ (minor) and $48.6 \mathrm{~min}$ (major).
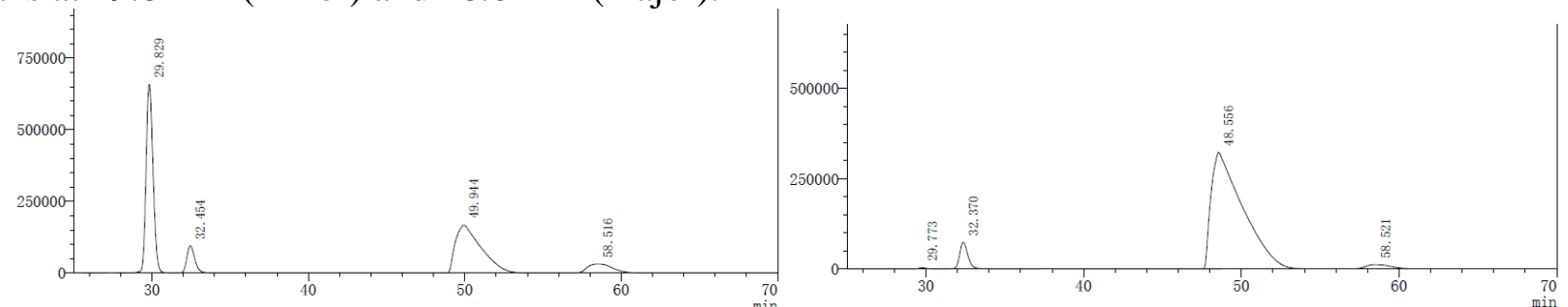

\begin{tabular}{|c|c|c|}
\hline Peak & retension time/min & area\% \\
\hline 1 & 29.829 & 44.156 \\
\hline 2 & 32.454 & 7.283 \\
\hline 3 & 49.944 & 41.039 \\
\hline 4 & 58.516 & 7.522 \\
\hline
\end{tabular}

\begin{tabular}{|c|c|c|}
\hline Peak & retension time/min & area\% \\
\hline 1 & 29.773 & 0.178 \\
\hline 2 & 32.370 & 5.402 \\
\hline 3 & 48.556 & 92.029 \\
\hline 4 & 58.521 & 2.391 \\
\hline
\end{tabular}

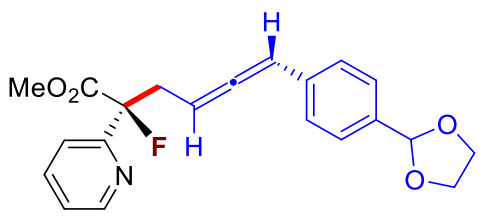

Methyl (2R,5R)-6-(4-(1,3-dioxolan-2-yl)phenyl)-2-fluoro-2-(pyridin-2-yl)hexa-4,5-dienoate (3g)

Yellow oil, $22 \mathrm{~h}, 99 \%$ yield, $>20: 1 \mathrm{dr},[\alpha]_{\mathrm{D}}{ }^{25}-191.9\left(c\right.$ 0.2, $\left.\mathrm{CHCl}_{3}\right)$ for $>99 \%$ ee; ${ }^{1} \mathrm{H}$ NMR $(400 \mathrm{MHz}$, chloroform- $d$ ) $\delta 8.58(\mathrm{~d}, J=4.7 \mathrm{~Hz}, 1 \mathrm{H}), 7.77(\mathrm{td}, J=7.8,1.8 \mathrm{~Hz}, 1 \mathrm{H}), 7.64(\mathrm{~d}, J=7.9 \mathrm{~Hz}, 1 \mathrm{H}), 7.34(\mathrm{~d}$, $J=7.8 \mathrm{~Hz}, 2 \mathrm{H}), 7.31-7.27(\mathrm{~m}, 1 \mathrm{H}), 7.08(\mathrm{~d}, J=7.8 \mathrm{~Hz}, 2 \mathrm{H}), 6.20-6.06(\mathrm{~m}, 1 \mathrm{H}), 5.77(\mathrm{~s}, 1 \mathrm{H}), 5.53$ $(\mathrm{m}, 1 \mathrm{H}), 4.18-3.92(\mathrm{~m}, 4 \mathrm{H}), 3.77(\mathrm{~s}, 3 \mathrm{H}), 3.43-3.10(\mathrm{~m}, 2 \mathrm{H}) .{ }^{13} \mathrm{C}$ NMR (126 MHz, chloroform- $d$ ) $\delta$ 207.7, 169.1 (d, $J=26.2 \mathrm{~Hz}), 156.3(\mathrm{~d}, J=27.1 \mathrm{~Hz}), 149.3(\mathrm{~d}, J=2.3 \mathrm{~Hz}), 137.1,136.5,135.1,126.8$, 126.7, 123.6, $120.2(\mathrm{~d}, J=8.4 \mathrm{~Hz}), 103.6,97.2(\mathrm{~d}, J=189.2 \mathrm{~Hz}), 94.5,87.8(\mathrm{~d}, J=3.7 \mathrm{~Hz}), 65.3,53.1$, $36.3(\mathrm{~d}, J=20.5 \mathrm{~Hz}) .{ }^{19} \mathrm{~F}$ NMR $(376 \mathrm{MHz}$, chloroform- $d$ ) $\delta-164.6(\mathrm{t}, J=24.0 \mathrm{~Hz}, 1 \mathrm{~F})$. HRMS (EI): [M] 
${ }^{\oplus}$ calcd for $\mathrm{C}_{21} \mathrm{H}_{20} \mathrm{FNO}_{4}{ }^{\oplus}$ 369.1371, found 369.1370. HPLC analysis: Chiracel OD-H column; detected at $254 \mathrm{~nm}, 40{ }^{\circ} \mathrm{C} ; 3 \%{ }^{i} \mathrm{PrOH}$ in $n$-hexane; flow $=0.7 \mathrm{~mL} / \mathrm{min}$. Retention time: $29.2 \mathrm{~min}, 31.6 \mathrm{~min}, 37.7$ $\mathrm{min}, 39.4 \mathrm{~min}$. The ee value was determined based on peaks at $29.2 \mathrm{~min}$ (minor) and $37.7 \mathrm{~min}$ (major).
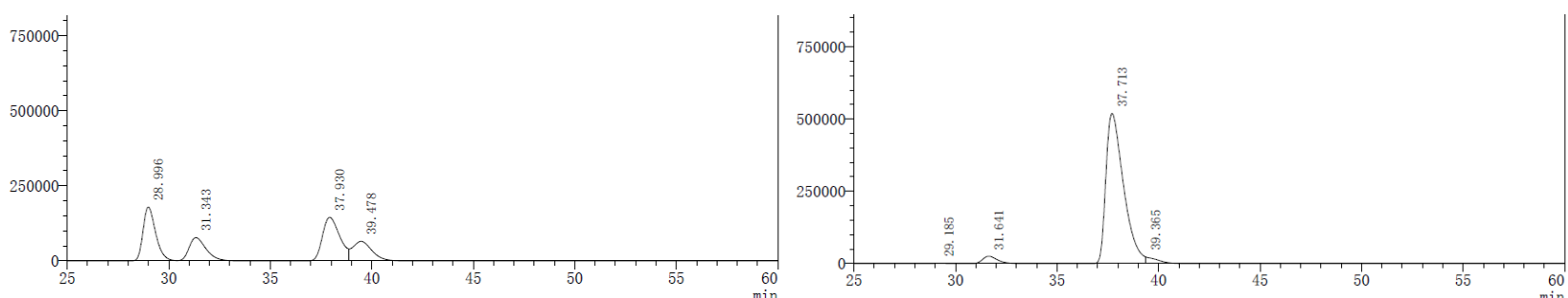

\begin{tabular}{|c|c|c|}
\hline Peak & retension time/min & area\% \\
\hline 1 & 28.996 & 30.682 \\
\hline 2 & 31.343 & 18.000 \\
\hline 3 & 37.930 & 34.247 \\
\hline 4 & 39.478 & 17.071 \\
\hline
\end{tabular}

\begin{tabular}{|c|c|c|}
\hline Peak & retension time/min & area\% \\
\hline 1 & 29.185 & 0.171 \\
\hline 2 & 31.641 & 3.958 \\
\hline 3 & 37.713 & 93.034 \\
\hline 4 & 39.365 & 2.838 \\
\hline
\end{tabular}<smiles>COC(=O)c1ccc(C=C=CC(F)(C(C)=O)c2ccccn2)cc1</smiles>

\section{Methyl 4-((2R,5R)-5-fluoro-6-methoxy-6-oxo-5-(pyridin-2-yl)hexa-1,2-dien-1-yl)benzoate (3h)}

White solid, $17 \mathrm{~h}, 96 \%$ yield, $>20: 1 \mathrm{dr},[\alpha]_{\mathrm{D}}{ }^{25}-211.0\left(c 0.2, \mathrm{CHCl}_{3}\right)$ for $>99 \%$ ee; ${ }^{1} \mathrm{H}$ NMR $(500 \mathrm{MHz}$, chloroform- $d$ ) $\delta 8.58(\mathrm{~d}, J=4.4 \mathrm{~Hz}, 1 \mathrm{H}), 7.89(\mathrm{~d}, J=8.3 \mathrm{~Hz}, 2 \mathrm{H}), 7.79(\mathrm{td}, J=7.8,1.8 \mathrm{~Hz}, 1 \mathrm{H}), 7.66(\mathrm{~d}$, $J=7.9 \mathrm{~Hz}, 1 \mathrm{H}), 7.32-7.29(\mathrm{~m}, 1 \mathrm{H}), 7.10(\mathrm{~d}, J=8.0 \mathrm{~Hz}, 2 \mathrm{H}), 6.15(\mathrm{dt}, J=6.6,2.4 \mathrm{~Hz}, 1 \mathrm{H}), 5.60-5.56$ $(\mathrm{m}, 1 \mathrm{H}), 3.90(\mathrm{~s}, 3 \mathrm{H}), 3.77(\mathrm{~s}, 3 \mathrm{H}), 3.51-2.99(\mathrm{~m}, 2 \mathrm{H}) .{ }^{13} \mathrm{C}$ NMR $(126 \mathrm{MHz}$, chloroform- $d$ ) $\delta 208.4$, $169.0(\mathrm{~d}, J=26.1 \mathrm{~Hz}), 167.0,156.2(\mathrm{~d}, J=27.1 \mathrm{~Hz}), 149.4(\mathrm{~d}, J=2.3 \mathrm{~Hz}), 139.1,137.1,129.8,128.4$, 126.7, 123.7, $120.3(\mathrm{~d}, J=8.5 \mathrm{~Hz}), 97.1(\mathrm{~d}, J=189.2 \mathrm{~Hz}), 94.4,88.2(\mathrm{~d}, J=3.7 \mathrm{~Hz}), 53.1,52.1,36.1(\mathrm{~d}$, $J=20.3 \mathrm{~Hz}) .{ }^{19} \mathrm{~F}$ NMR $(376 \mathrm{MHz}$, chloroform- $d$ ) $\delta-164.8$ (t, $J=24.2 \mathrm{~Hz}, 1 \mathrm{~F})$. HRMS (EI): $[\mathrm{M}]^{\oplus}$ calcd for $\mathrm{C}_{20} \mathrm{H}_{18} \mathrm{FNO}_{4}{ }^{\oplus} 355.1214$, found 355.1208. HPLC analysis: Chiralpak IA column; detected at $254 \mathrm{~nm}$, $40{ }^{\circ} \mathrm{C} ; 3 \%{ }^{i} \mathrm{PrOH}$ in $n$-hexane; flow $=0.7 \mathrm{~mL} / \mathrm{min}$. Retention time: $41.9 \mathrm{~min}, 47.5 \mathrm{~min}, 50.3 \mathrm{~min}, 62.5$ $\min$. The ee value was determined based on peaks at $41.9 \mathrm{~min}$ (minor) and $47.5 \mathrm{~min}$ (major).
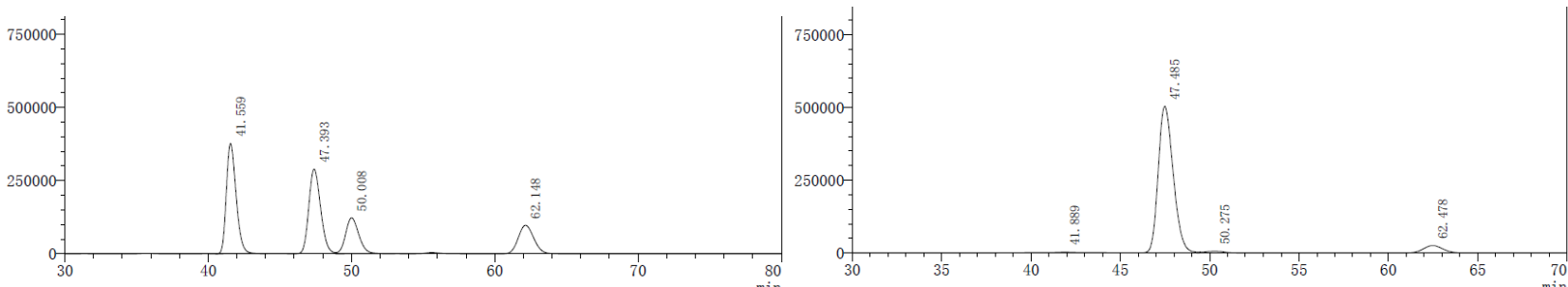

\begin{tabular}{|c|c|c|}
\hline Peak & retension time/min & area\% \\
\hline 1 & 41.559 & 35.974 \\
\hline 2 & 47.393 & 33.376 \\
\hline 3 & 50.008 & 15.900 \\
\hline 4 & 62.148 & 14.750 \\
\hline
\end{tabular}

\begin{tabular}{|c|c|c|}
\hline Peak & retension time/min & area\% \\
\hline 1 & 41.889 & 0.183 \\
\hline 2 & 47.485 & 92.581 \\
\hline 3 & 50.275 & 1.260 \\
\hline 4 & 62.478 & 5.976 \\
\hline
\end{tabular}




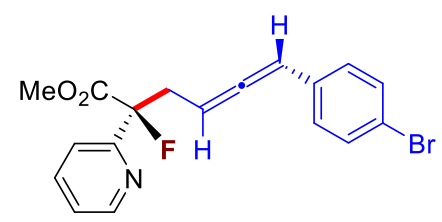

Methyl (2R,5R)-6-(4-bromophenyl)-2-fluoro-2-(pyridin-2-yl)hexa-4,5-dienoate (3i)

Yellow oil, $20 \mathrm{~h}, 99 \%$ yield, $>20: 1 \mathrm{dr},[\alpha]_{\mathrm{D}}{ }^{25}-187.6\left(c 0.2, \mathrm{CHCl}_{3}\right)$ for $>99 \%$ ee; ${ }^{1} \mathrm{H} \mathrm{NMR}(400 \mathrm{MHz}$, chloroform- $d) \delta 8.58(\mathrm{~d}, J=4.8 \mathrm{~Hz}, 1 \mathrm{H}), 7.78(\mathrm{td}, J=7.9,1.9 \mathrm{~Hz}, 1 \mathrm{H}), 7.65(\mathrm{~d}, J=7.9 \mathrm{~Hz}, 1 \mathrm{H}), 7.34(\mathrm{~d}$, $J=8.1 \mathrm{~Hz}, 2 \mathrm{H}), 7.31-7.28(\mathrm{~m}, 1 \mathrm{H}), 6.92(\mathrm{~d}, J=8.2 \mathrm{~Hz}, 2 \mathrm{H}), 6.07-6.05(\mathrm{~m}, 1 \mathrm{H}), 5.55-5.49(\mathrm{~m}, 1 \mathrm{H})$, 3.77 (s, 3H), $3.45-3.04(\mathrm{~m}, 2 \mathrm{H}) .{ }^{13} \mathrm{C} \mathrm{NMR}\left(126 \mathrm{MHz} \mathrm{CDCl}_{3}\right) \delta 207.5,169.0(\mathrm{~d}, J=26.1 \mathrm{~Hz}), 156.3(\mathrm{~d}$, $J=27.3 \mathrm{~Hz}), 149.3,137.1,133.1,131.5,128.3,123.6,120.5,120.2(\mathrm{~d}, J=8.5 \mathrm{~Hz}), 97.1(\mathrm{~d}, J=189.1$ $\mathrm{Hz}), 94.0,88.2(\mathrm{~d}, J=3.8 \mathrm{~Hz}), 53.1,36.2(\mathrm{~d}, J=20.4 \mathrm{~Hz}) .{ }^{19} \mathrm{~F}$ NMR $(376 \mathrm{MHz}$, chloroform- $d$ ) $\delta-164.7$ (t, $J=24.4 \mathrm{~Hz}, 1 \mathrm{~F})$. HRMS (EI): [M] ${ }^{\oplus}$ calcd for $\mathrm{C}_{18} \mathrm{H}_{15} \mathrm{BrFNO}_{2}{ }^{\oplus}$ 375.0265, found 375.0261. HPLC analysis: Chiralpak IC column; detected at $254 \mathrm{~nm}, 40{ }^{\circ} \mathrm{C} ; 3 \%{ }^{i} \mathrm{PrOH}$ in $n$-hexane; flow $=0.7 \mathrm{~mL} / \mathrm{min}$. Retention time: $24.4 \mathrm{~min}, 26.3 \mathrm{~min}, 28.6 \mathrm{~min}, 49.6 \mathrm{~min}$. The ee value was determined based on peaks at $24.4 \mathrm{~min}$ (major) and $28.6 \mathrm{~min}$ (minor).
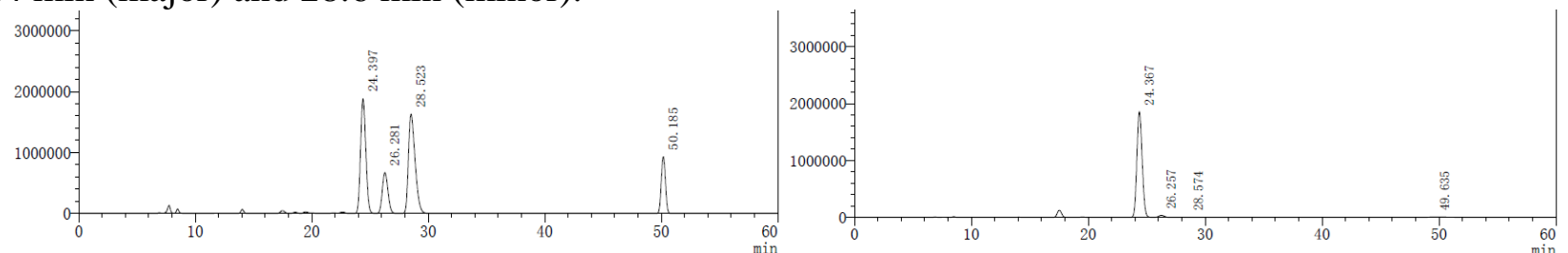

\begin{tabular}{|c|c|c|}
\hline Peak & retension time/min & area\% \\
\hline 1 & 24.397 & 34.724 \\
\hline 2 & 26.281 & 13.370 \\
\hline 3 & 28.523 & 38.010 \\
\hline 4 & 50.185 & 13.896 \\
\hline
\end{tabular}

\begin{tabular}{|c|c|c|}
\hline Peak & retension time/min & area\% \\
\hline 1 & 24.367 & 95.814 \\
\hline 2 & 26.257 & 2.053 \\
\hline 3 & 28.574 & 0.086 \\
\hline 4 & 49.635 & 2.046 \\
\hline
\end{tabular}<smiles>CC(=O)CC(F)(/C=C/c1cccc([N+](=O)[O-])c1)c1ccccn1</smiles>

\section{Methyl (2R,5R)-2-fluoro-2-(pyridin-2-yl)-6-(m-tolyl)hexa-4,5-dienoate (3j)}

Yellow oil, $20 \mathrm{~h}, 99 \%$ yield, $11: 1 \mathrm{dr},[\alpha]_{\mathrm{D}}{ }^{25}-206.8\left(c 0.2, \mathrm{CHCl}_{3}\right)$ for $>99 \%$ ee; ${ }^{1} \mathrm{H} \mathrm{NMR}(500 \mathrm{MHz}$, chloroform- $d$ ) $\delta 8.59(\mathrm{~d}, J=5.0 \mathrm{~Hz}, 1 \mathrm{H}), 7.77(\mathrm{td}, J=7.8,1.8 \mathrm{~Hz}, 1 \mathrm{H}), 7.66-7.64(\mathrm{~m}, 1 \mathrm{H}), 7.29-7.26$ $(\mathrm{m}, 1 \mathrm{H}), 7.14-7.11(\mathrm{~m}, 1 \mathrm{H}), 7.01-6.93(\mathrm{~m}, 2 \mathrm{H}), 6.89(\mathrm{~d}, J=7.6 \mathrm{~Hz}, 1 \mathrm{H}), 6.10(\mathrm{dt}, J=6.4,2.4 \mathrm{~Hz}$, $1 \mathrm{H}), 5.53-5.48(\mathrm{~m}, 1 \mathrm{H}), 3.77(\mathrm{~s}, 3 \mathrm{H}), 3.42-3.11(\mathrm{~m}, 2 \mathrm{H}), 2.29(\mathrm{~s}, 3 \mathrm{H}) .{ }^{13} \mathrm{C} \mathrm{NMR}(126 \mathrm{MHz}$ chloroform- $d$ ) $\delta 207.4,169.2(\mathrm{~d}, J=26.2 \mathrm{~Hz}), 156.4(\mathrm{~d}, J=27.0 \mathrm{~Hz}), 149.3(\mathrm{~d}, J=2.2 \mathrm{~Hz}), 138.0,137.1$, 133.9, 128.4, 127.8, 127.5, 124.0, 123.6, $120.3(\mathrm{~d}, J=8.3 \mathrm{~Hz}), 97.2(\mathrm{~d}, J=189.3 \mathrm{~Hz}), 94.9,87.6(\mathrm{~d}, J=$ $3.8 \mathrm{~Hz}), 53.1,36.4(\mathrm{~d}, J=20.5 \mathrm{~Hz}), 21.4 .{ }^{19} \mathrm{~F}$ NMR $(376 \mathrm{MHz}$, chloroform- $d) \delta-160.3(\mathrm{t}, J=24.3 \mathrm{~Hz}$, 1F). HRMS (EI): $[\mathrm{M}]^{\oplus}$ calcd for $\mathrm{C}_{19} \mathrm{H}_{18} \mathrm{FNO}_{2}{ }^{\oplus}$ 311.1316, found 311.1319. HPLC analysis: Chiralpak IC column; detected at $254 \mathrm{~nm}, 40{ }^{\circ} \mathrm{C} ; 3 \%{ }^{i} \mathrm{PrOH}$ in $n$-hexane; flow $=0.7 \mathrm{~mL} / \mathrm{min}$. Retention time: 19.9 $\min , 21.3 \mathrm{~min}, 24.5 \mathrm{~min}, 47.2 \mathrm{~min}$. The ee value was determined based on peaks at $19.9 \mathrm{~min}$ (major) and 21.3 min (minor). 

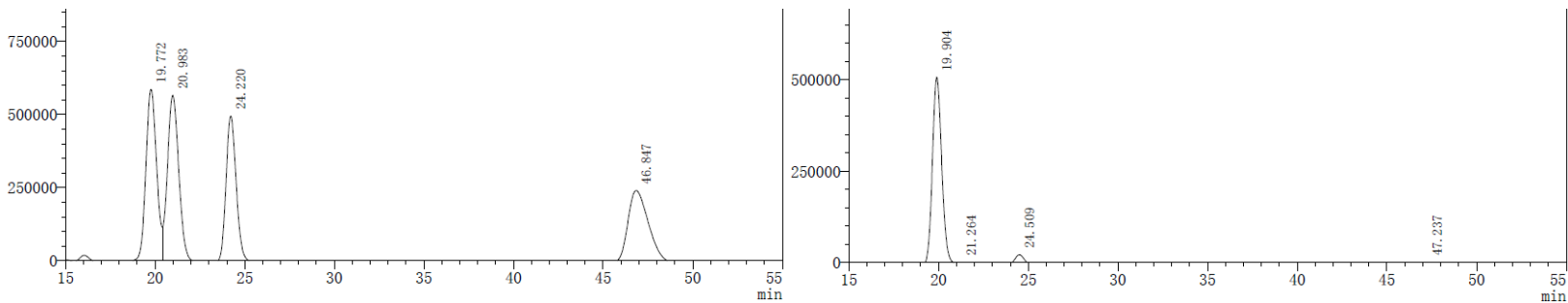

\begin{tabular}{|c|c|c|}
\hline Peak & retension time/min & area\% \\
\hline 1 & 19.772 & 27.441 \\
\hline 2 & 20.983 & 28.291 \\
\hline 3 & 24.220 & 21.973 \\
\hline 4 & 46.847 & 22.296 \\
\hline
\end{tabular}

\begin{tabular}{|c|c|c|}
\hline Peak & retension time/min & area\% \\
\hline 1 & 19.904 & 92.420 \\
\hline 2 & 21.264 & 0.247 \\
\hline 3 & 24.509 & 5.202 \\
\hline 4 & 47.237 & 2.131 \\
\hline
\end{tabular}<smiles>COC(=O)c1cccc(/C=C/CC(F)(C/C=C/c2ccccn2)c2ccccn2)c1</smiles>

\section{Methyl (2R,5R)-6-(3-((tert-butoxycarbonyl)amino)phenyl)-2-fluoro-2-(pyridin-2-yl)hexa-4,5- dienoate $(3 \mathbf{k})$}

Yellow oil, $20 \mathrm{~h}, 85 \%$ yield, $10: 1 \mathrm{dr},[\alpha]_{\mathrm{D}}{ }^{25}-149.6\left(c 0.2, \mathrm{CHCl}_{3}\right)$ for $>99 \%$ ee; ${ }^{1} \mathrm{H} \mathrm{NMR}(500 \mathrm{MHz}$, chloroform- $d) \delta 8.58(\mathrm{~d}, J=4.6 \mathrm{~Hz}, 1 \mathrm{H}), 7.77(\mathrm{td}, J=7.8,1.8 \mathrm{~Hz}, 1 \mathrm{H}), 7.65(\mathrm{~d}, J=7.8 \mathrm{~Hz}, 1 \mathrm{H}), 7.36-$ $7.25(\mathrm{~m}, 2 \mathrm{H}), 7.15(\mathrm{t}, J=7.9 \mathrm{~Hz}, 1 \mathrm{H}), 7.00(\mathrm{~s}, 1 \mathrm{H}), 6.76(\mathrm{~d}, J=7.6,1 \mathrm{H}), 6.47(\mathrm{~s}, 1 \mathrm{H}), 6.08(\mathrm{dt}, J=6.5$, $2.4 \mathrm{~Hz}, 1 \mathrm{H}), 5.53-5.49(\mathrm{~m}, 1 \mathrm{H}), 3.77(\mathrm{~s}, 3 \mathrm{H}), 3.40-3.26(\mathrm{~m}, 1 \mathrm{H}), 3.26-3.11(\mathrm{~m}, 1 \mathrm{H}), 1.52(\mathrm{~s}, 9 \mathrm{H})$. ${ }^{13} \mathrm{C}$ NMR $(126 \mathrm{MHz}$, chloroform- $d$ ) $\delta 207.5,169.1(\mathrm{~d}, J=26.2 \mathrm{~Hz}), 156.3(\mathrm{~d}, J=26.8 \mathrm{~Hz}), 152.7,149.3$ $(\mathrm{d}, J=2.3 \mathrm{~Hz}), 138.6,137.1,134.9,129.1,123.6,121.6,120.3(\mathrm{~d}, J=8.2 \mathrm{~Hz}), 116.9(\mathrm{~d}, J=56.5 \mathrm{~Hz})$, $97.2(\mathrm{~d}, J=189.2 \mathrm{~Hz}), 94.8,80.6,87.8(\mathrm{~d}, J=3.8 \mathrm{~Hz}), 53.1,36.3(\mathrm{~d}, J=20.4 \mathrm{~Hz}), 28.4$ (One aromatic carbon signal was not observed because of overlapping). ${ }^{19} \mathrm{~F}$ NMR (376 MHz, chloroform- $d$ ) $\delta-162.6-$ -167.8 (m, 1F). HRMS (ESI): $[\mathrm{M}+\mathrm{H}]^{\oplus}$ calcd for $\mathrm{C}_{23} \mathrm{H}_{26} \mathrm{FN}_{2} \mathrm{O}_{4}{ }^{\oplus}$ 413.1871, found 413.1874. HPLC analysis: Chiracel AD-H column; detected at $254 \mathrm{~nm}, 40{ }^{\circ} \mathrm{C} ; 9 \%{ }^{i} \mathrm{PrOH}$ in $n$-hexane; flow $=0.7 \mathrm{~mL} / \mathrm{min}$. Retention time: $33.6 \mathrm{~min}, 37.5 \mathrm{~min}, 38.7 \mathrm{~min}, 48.3 \mathrm{~min}$. The ee value was determined based on peaks at $33.6 \mathrm{~min}$ (minor) and $48.3 \mathrm{~min}$ (major).
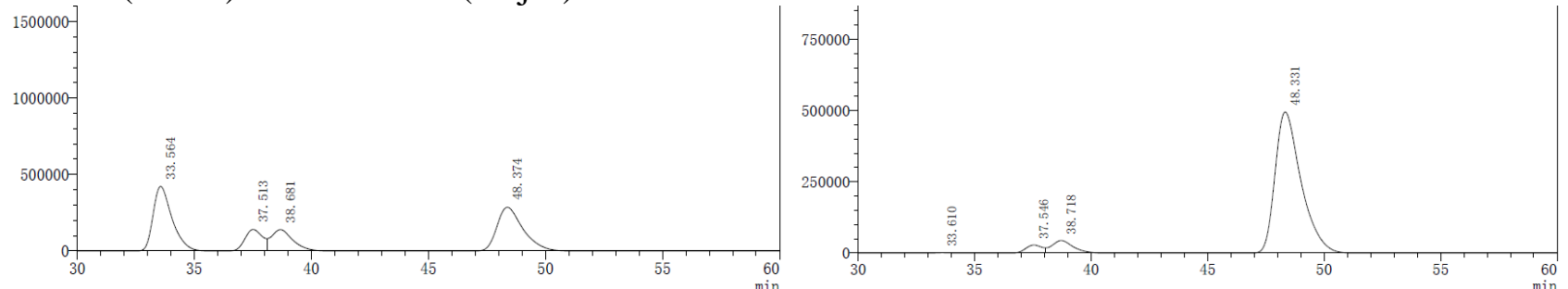

\begin{tabular}{|c|c|c|}
\hline Peak & retension time/min & area\% \\
\hline 1 & 33.564 & 38.042 \\
\hline 2 & 37.513 & 11.783 \\
\hline 3 & 38.681 & 14.574 \\
\hline 4 & 48.374 & 35.600 \\
\hline
\end{tabular}

\begin{tabular}{|c|c|c|}
\hline Peak & retension time/min & area\% \\
\hline 1 & 33.610 & 0.216 \\
\hline 2 & 37.546 & 3.343 \\
\hline 3 & 38.718 & 6.701 \\
\hline 4 & 48.331 & 89.739 \\
\hline
\end{tabular}


<smiles>COc1cc(/C=C/CC(F)(C(C)=O)c2ccccn2)cc(OC)c1</smiles>

Methyl (2R,5R)-6-(3,5-dimethoxyphenyl)-2-fluoro-2-(pyridin-2-yl)hexa-4,5-dienoate (3I)

Yellow oil, $20 \mathrm{~h}, 62 \%$ yield, $11: 1 \mathrm{dr},[\alpha]_{\mathrm{D}}{ }^{25}-219.2\left(c 0.2, \mathrm{CHCl}_{3}\right)$ for $>99 \%$ ee; ${ }^{1} \mathrm{H} \mathrm{NMR}(500 \mathrm{MHz}$, chloroform- $d$ ) $\delta 8.59(\mathrm{~d}, J=4.8 \mathrm{~Hz}, 1 \mathrm{H}), 7.76(\mathrm{td}, J=7.8,1.8 \mathrm{~Hz}, 1 \mathrm{H}), 7.63(\mathrm{~d}, J=7.9 \mathrm{~Hz}, 1 \mathrm{H}), 7.36-$ $7.18(\mathrm{~m}, 1 \mathrm{H}), 6.46-6.35(\mathrm{~m}, 2 \mathrm{H}), 6.32-6.30(\mathrm{~m}, 1 \mathrm{H}), 6.08(\mathrm{dt}, J=6.4,2.5 \mathrm{~Hz}, 1 \mathrm{H}), 5.59-5.46(\mathrm{~m}, 1 \mathrm{H})$, $3.78(\mathrm{~s}, 6 \mathrm{H}), 3.77(\mathrm{~s}, 3 \mathrm{H}), 3.45-3.28(\mathrm{~m}, 1 \mathrm{H}), 3.22-3.03(\mathrm{~m}, 1 \mathrm{H}) .{ }^{13} \mathrm{C}$ NMR $(126 \mathrm{MHz}$, chloroform- $d$ ) $\delta 207.7,169.1(\mathrm{~d}, J=26.6 \mathrm{~Hz}), 160.9,156.4(\mathrm{~d}, J=26.9 \mathrm{~Hz}), 149.3(\mathrm{~d}, J=2.1 \mathrm{~Hz}), 137.1,136.2,123.7$, $120.2(\mathrm{~d}, J=8.2 \mathrm{~Hz}), 104.9,99.6,97.1(\mathrm{~d}, J=189.6 \mathrm{~Hz}), 95.2,88.0(\mathrm{~d}, J=3.4 \mathrm{~Hz}), 55.4,53.1,36.4(\mathrm{~d}$, $J=20.8 \mathrm{~Hz}) .{ }^{19} \mathrm{~F}$ NMR (376 MHz, chloroform- $d$ ) $\delta-164.57--164.70$ (m, 1F). HRMS (EI): [M] ${ }^{\oplus}$ calcd for $\mathrm{C}_{20} \mathrm{H}_{20} \mathrm{FNO}_{4}{ }^{\oplus}$ 357.1371, found 357.1368. HPLC analysis: Chiralpak IA column; detected at $254 \mathrm{~nm}$, $40{ }^{\circ} \mathrm{C} ; 3 \%{ }^{i} \mathrm{PrOH}$ in $n$-hexane; flow $=0.7 \mathrm{~mL} / \mathrm{min}$. Retention time: $26.9 \mathrm{~min}, 29.0 \mathrm{~min}, 29.8 \mathrm{~min}, 35.1$ min. The ee value was determined based on peaks at 26.9 min (minor) and 35.1 min (major).
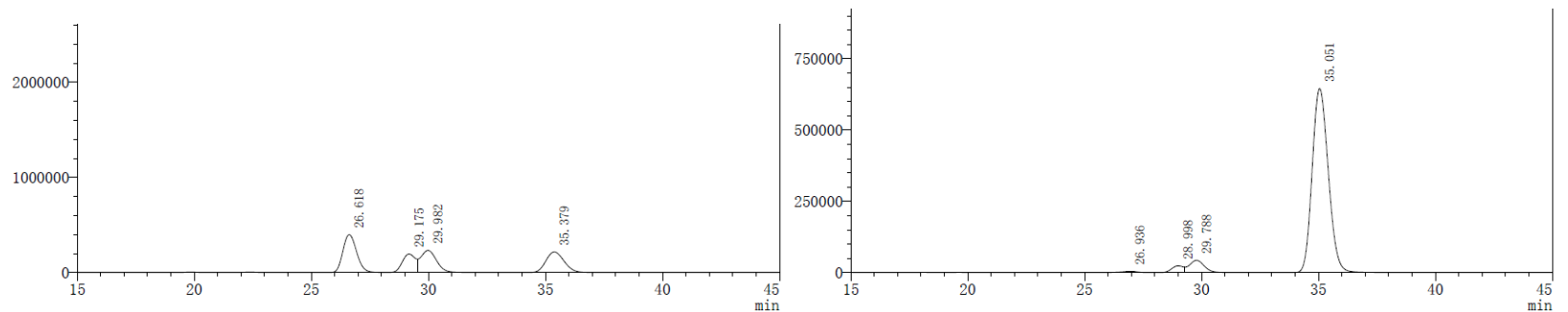

\begin{tabular}{|c|c|c|}
\hline Peak & retension time/min & area\% \\
\hline 1 & 26.618 & 34.941 \\
\hline 2 & 29.175 & 16.250 \\
\hline 3 & 29.982 & 23.693 \\
\hline 4 & 35.379 & 25.116 \\
\hline
\end{tabular}

\begin{tabular}{|c|c|c|}
\hline Peak & retension time/min & area\% \\
\hline 1 & 26.936 & 0.598 \\
\hline 2 & 28.998 & 2.465 \\
\hline 3 & 29.788 & 5.647 \\
\hline 4 & 35.051 & 91.290 \\
\hline
\end{tabular}<smiles>CC(=O)[C@](F)(/C=C/C=C/c1ccc2c(c1)OCO2)c1ccccn1</smiles>

\section{Methyl (2R,5R)-6-(benzo[d][1,3]dioxol-5-yl)-2-fluoro-2-(pyridin-2-yl)hexa-4,5-dienoate (3m)}

Yellow oil, $20 \mathrm{~h}, 84 \%$ yield, $10: 1 \mathrm{dr},[\alpha]_{\mathrm{D}}{ }^{25}-185.6\left(c 1.0, \mathrm{CHCl}_{3}\right)$ for $>99 \%$ ee; ${ }^{1} \mathrm{H} \mathrm{NMR}(400 \mathrm{MHz}$, chloroform- $d$ ) $\delta 8.59(\mathrm{~d}, J=4.8 \mathrm{~Hz}, 1 \mathrm{H}), 7.78(\mathrm{td}, J=7.8,1.8 \mathrm{~Hz}, 1 \mathrm{H}), 7.65(\mathrm{~d}, J=7.9 \mathrm{~Hz}, 1 \mathrm{H}), 7.37-$ $7.22(\mathrm{~m}, 1 \mathrm{H}), 6.68(\mathrm{~d}, J=7.8 \mathrm{~Hz}, 1 \mathrm{H}), 6.56(\mathrm{~d}, J=7.9 \mathrm{~Hz}, 1 \mathrm{H}), 6.51(\mathrm{~s}, 1 \mathrm{H}), 6.05(\mathrm{dt}, J=6.6,2.4 \mathrm{~Hz}$, 1H), $5.93-5.92(\mathrm{~m}, 2 \mathrm{H}), 5.52-5.47(\mathrm{~m}, 1 \mathrm{H}), 3.78(\mathrm{~s}, 3 \mathrm{H}), 3.40-3.08(\mathrm{~m}, 2 \mathrm{H}) .{ }^{13} \mathrm{C} \mathrm{NMR}(126 \mathrm{MHz}$, chloroform- $d$ ) $\delta 207.0,169.2(\mathrm{~d}, J=26.1 \mathrm{~Hz}), 156.3(\mathrm{~d}, J=27.0 \mathrm{~Hz}), 149.3(\mathrm{~d}, J=2.3 \mathrm{~Hz}), 147.9,146.8$, 137.1, 128.1, 123.7, 120.6, 120.2 (d, $J=8.4 \mathrm{~Hz}), 108.2,106.7,101.0,97.2$ (d, $J=189.2 \mathrm{~Hz}), 94.7,87.9$ $(\mathrm{d}, J=3.8 \mathrm{~Hz}), 53.1,36.5(\mathrm{~d}, J=20.4 \mathrm{~Hz}) .{ }^{19} \mathrm{~F}$ NMR $(376 \mathrm{MHz}$, chloroform- $d) \delta-164.7(\mathrm{t}, J=24.5 \mathrm{~Hz}$, 1F). HRMS (FI): [M] ${ }^{\oplus}$ calcd for $\mathrm{C}_{19} \mathrm{H}_{16} \mathrm{FNO}_{4}{ }^{\oplus}$ 341.1058, found 341.1057. HPLC analysis: Chiralpak IA column; detected at $254 \mathrm{~nm}, 40{ }^{\circ} \mathrm{C} ; 3 \%{ }^{i} \mathrm{PrOH}$ in $n$-hexane; flow $=0.7 \mathrm{~mL} / \mathrm{min}$. Retention time: 27.3 min, $31.1 \mathrm{~min}, 33.5 \mathrm{~min}, 34.8 \mathrm{~min}$. The ee value was determined based on peaks at $27.3 \mathrm{~min}$ (minor) and 34.8 min (major). 

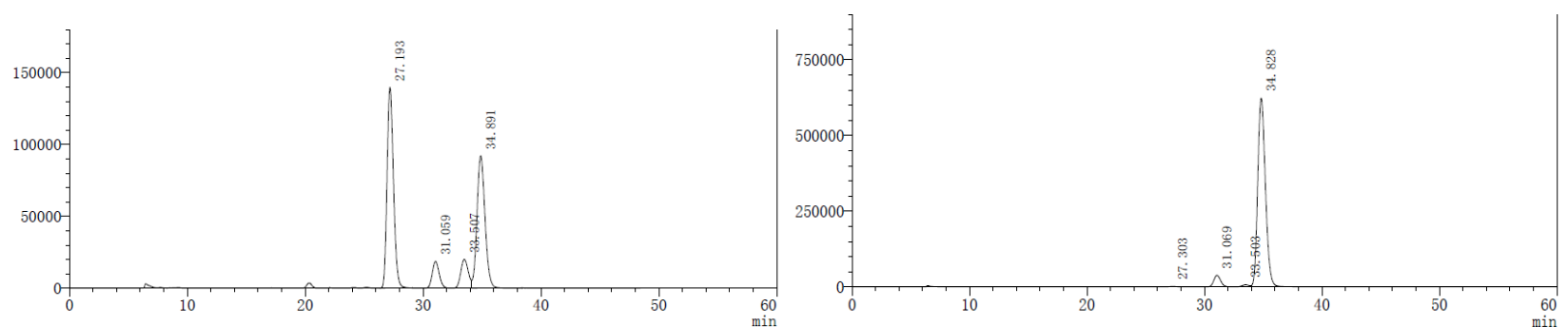

\begin{tabular}{|c|c|c|}
\hline Peak & retension time/min & area\% \\
\hline 1 & 27.193 & 46.787 \\
\hline 2 & 31.059 & 6.772 \\
\hline 3 & 33.507 & 7.844 \\
\hline 4 & 34.891 & 38.597 \\
\hline
\end{tabular}

\begin{tabular}{|c|c|c|}
\hline Peak & retension time/min & area\% \\
\hline 1 & 27.303 & 0.185 \\
\hline 2 & 31.069 & 5.091 \\
\hline 3 & 33.503 & 0.874 \\
\hline 4 & 34.828 & 93.851 \\
\hline
\end{tabular}<smiles>COC(=O)C(F)(C/C=C/c1ccccc1F)c1ccccn1</smiles>

\section{Methyl (2R,5R)-2-fluoro-6-(2-fluorophenyl)-2-(pyridin-2-yl)hexa-4,5-dienoate (3n)}

Yellow oil, $17 \mathrm{~h}, 99 \%$ yield, $13: 1 \mathrm{dr},[\alpha]_{\mathrm{D}}{ }^{25}-199.2\left(c 0.2, \mathrm{CHCl}_{3}\right)$ for $>99 \%$ ee; ${ }^{1} \mathrm{H} \mathrm{NMR}(500 \mathrm{MHz}$, chloroform- $d) \delta 8.58(\mathrm{~d}, J=4.2 \mathrm{~Hz}, 1 \mathrm{H}), 7.77(\mathrm{td}, J=7.7,1.8 \mathrm{~Hz}, 1 \mathrm{H}), 7.65(\mathrm{~d}, J=7.7 \mathrm{~Hz}, 1 \mathrm{H}), 7.35-$ $7.27(\mathrm{~m}, 1 \mathrm{H}), 7.17-7.10(\mathrm{~m}, 1 \mathrm{H}), 7.07(\mathrm{td}, J=7.8,1.9 \mathrm{~Hz}, 1 \mathrm{H}), 7.02-6.94(\mathrm{~m}, 2 \mathrm{H}), 6.36(\mathrm{dt}, J=6.6$, $2.4 \mathrm{~Hz}, 1 \mathrm{H}), 5.57-5.53(\mathrm{~m}, 1 \mathrm{H}), 3.79(\mathrm{~s}, 3 \mathrm{H}), 3.44-3.27(\mathrm{~m}, 1 \mathrm{H}), 3.27-3.13(\mathrm{~m}, 1 \mathrm{H}) .{ }^{13} \mathrm{C}$ NMR $(126$ $\mathrm{MHz}$, chloroform- $d) \delta 207.9,169.1(\mathrm{~d}, J=26.4 \mathrm{~Hz}), 159.5(\mathrm{~d}, J=249.2 \mathrm{~Hz}), 156.3(\mathrm{~d}, J=27.0 \mathrm{~Hz})$, $149.3(\mathrm{~d}, J=2.3 \mathrm{~Hz}), 137.1,128.3(\mathrm{~d}, J=2.3 \mathrm{~Hz}), 128.2,124.1(\mathrm{~d}, J=3.5 \mathrm{~Hz}), 123.7,121.6(\mathrm{~d}, J=12.1$ $\mathrm{Hz}), 120.3(\mathrm{~d}, J=8.4 \mathrm{~Hz}), 115.5(\mathrm{~d}, J=21.5 \mathrm{~Hz}), 97.2(\mathrm{~d}, J=189.2 \mathrm{~Hz}), 87.9(\mathrm{~d}, J=3.7 \mathrm{~Hz}), 87.2(\mathrm{~d}, J$ $=6.9 \mathrm{~Hz}), 53.1,36.2(\mathrm{~d}, J=20.5 \mathrm{~Hz}) .{ }^{19} \mathrm{~F}$ NMR $(376 \mathrm{MHz}$, chloroform- $d$ ) $\delta-119.7(\mathrm{~s}, 1 \mathrm{~F}),-164.1(\mathrm{~d}, J$ $=22.3 \mathrm{~Hz}, 1 \mathrm{~F})$. HRMS (FI): $[\mathrm{M}]^{\oplus}$ calcd for $\mathrm{C}_{18} \mathrm{H}_{15} \mathrm{~F}_{2} \mathrm{NO}_{2}{ }^{\oplus}$ 315.1065, found 315.1068. HPLC analysis: Chiralpak IC-3 column; detected at $254 \mathrm{~nm}, 40{ }^{\circ} \mathrm{C} ; 3 \%{ }^{i} \mathrm{PrOH}$ in $n$-hexane; flow $=0.5 \mathrm{~mL} / \mathrm{min}$. Retention time: $25.3 \mathrm{~min}, 29.2 \mathrm{~min}, 30.2 \mathrm{~min}, 37.6 \mathrm{~min}$. The ee value was determined based on peaks at 25.3 min (major) and 29.2 min (minor).
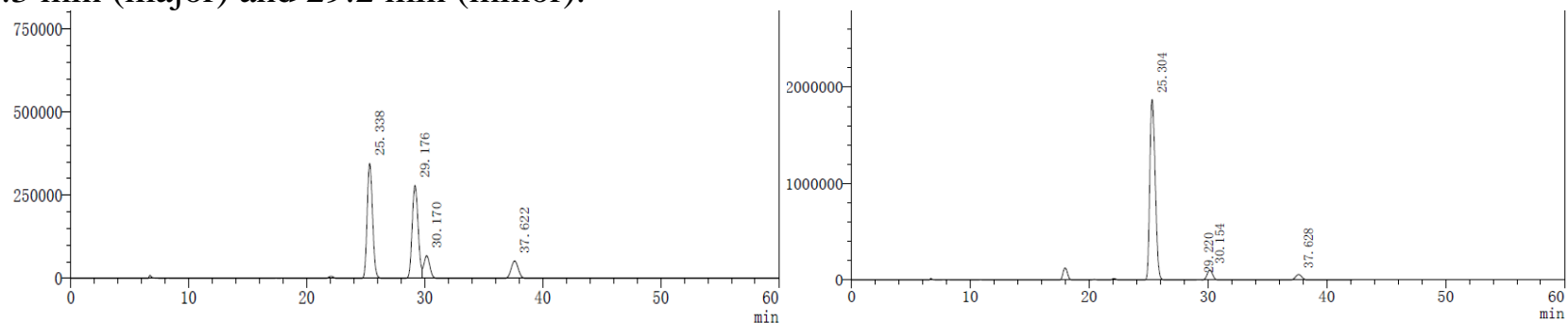

\begin{tabular}{|c|c|c|}
\hline Peak & retension time/min & area\% \\
\hline 1 & 25.338 & 43.170 \\
\hline 2 & 29.176 & 38.717 \\
\hline 3 & 30.170 & 9.410 \\
\hline 4 & 37.622 & 8.703 \\
\hline
\end{tabular}

\begin{tabular}{|c|c|c|}
\hline Peak & retension time/min & area\% \\
\hline 1 & 25.304 & 90.955 \\
\hline 2 & 29.220 & 0.245 \\
\hline 3 & 30.154 & 5.283 \\
\hline 4 & 37.628 & 3.517 \\
\hline
\end{tabular}




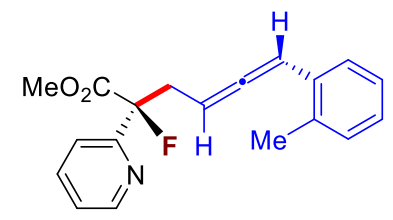

\section{Methyl (2R,5R)-2-fluoro-2-(pyridin-2-yl)-6-(o-tolyl)hexa-4,5-dienoate (3o)}

Yellow oil, $19 \mathrm{~h}, 62 \%$ yield, $11: 1 \mathrm{dr},[\alpha]_{\mathrm{D}}{ }^{25}-213.9\left(c 0.2, \mathrm{CHCl}_{3}\right)$ for $>99 \%$ ee; ${ }^{1} \mathrm{H} \mathrm{NMR}(500 \mathrm{MHz}$, chloroform- $d) \delta 8.59(\mathrm{~m}, 1 \mathrm{H}), 7.76(\mathrm{t}, J=7.7 \mathrm{~Hz}, 1 \mathrm{H}), 7.64(\mathrm{~d}, J=7.9 \mathrm{~Hz}, 1 \mathrm{H}), 7.31-7.27(\mathrm{~m}, 1 \mathrm{H})$, $7.15-7.00(\mathrm{~m}, 4 \mathrm{H}), 6.31(\mathrm{dt}, J=6.6,2.3 \mathrm{~Hz}, 1 \mathrm{H}), 5.50-5.46(\mathrm{~m}, 1 \mathrm{H}), 3.77(\mathrm{~s}, 3 \mathrm{H}), 3.41-3.27(\mathrm{~m}$, $1 \mathrm{H}), 3.27-3.12(\mathrm{~m}, 1 \mathrm{H}), 2.30(\mathrm{~s}, 3 \mathrm{H}) .{ }^{13} \mathrm{C}$ NMR $(126 \mathrm{MHz}$, chloroform- $d$ ) $\delta 208.1,169.2(\mathrm{~d}, J=26.2$ $\mathrm{Hz}), 156.4$ (d, $J=26.4 \mathrm{~Hz}), 149.2,137.1,135.0,132.2,130.4,127.4,126.9,126.1,123.6,120.3(\mathrm{~d}, J=$ $6.0 \mathrm{~Hz}), 97.3(\mathrm{~d}, J=189.4 \mathrm{~Hz}), 92.1,86.8(\mathrm{~d}, J=3.5 \mathrm{~Hz}), 53.1,36.5(\mathrm{~d}, J=20.3 \mathrm{~Hz}), 19.8 .{ }^{19} \mathrm{~F}$ NMR (376 MHz, chloroform- $d$ ) $\delta-164.4(\mathrm{t}, J=24.9 \mathrm{~Hz}, 1 \mathrm{~F})$. HRMS (EI): $[\mathrm{M}]^{\oplus}$ calcd for $\mathrm{C}_{19} \mathrm{H}_{18} \mathrm{FNO}_{2}{ }^{\oplus}$ 311.1316, found 311.1320. HPLC analysis: Chiralpak IC column; detected at $254 \mathrm{~nm}, 40{ }^{\circ} \mathrm{C} ; 9 \%{ }^{i} \mathrm{PrOH}$ in $n$-hexane; flow $=0.7 \mathrm{~mL} / \mathrm{min}$. Retention time: $27.8 \mathrm{~min}, 28.7 \mathrm{~min}, 31.8 \mathrm{~min}, 51.4 \mathrm{~min}$. The ee value was determined based on peaks at $27.8 \mathrm{~min}$ (major) and $28.7 \mathrm{~min}$ (minor).
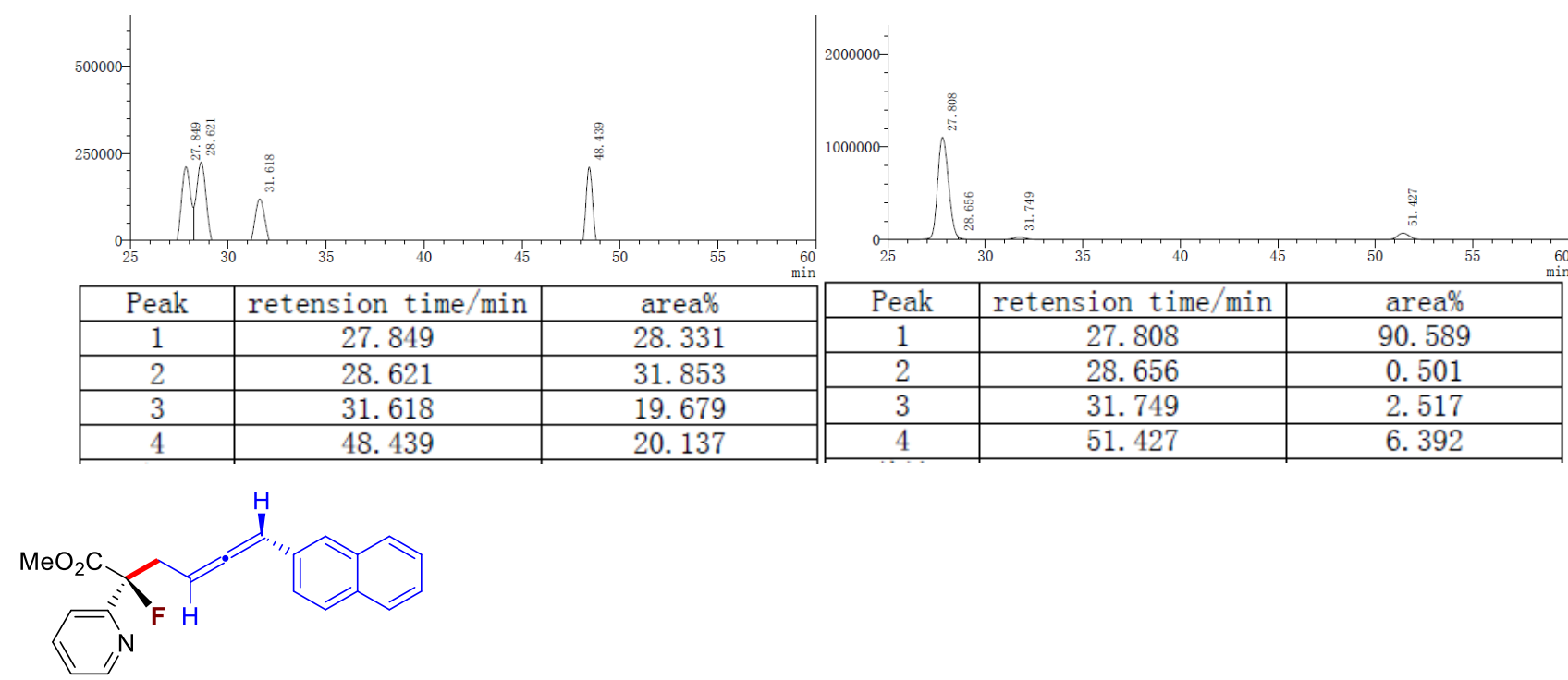

\section{Methyl (2R,5R)-2-fluoro-6-(naphthalen-2-yl)-2-(pyridin-2-yl)hexa-4,5-dienoate (3p)}

Yellow oil, $22 \mathrm{~h}, 94 \%$ yield, $>20: 1 \mathrm{dr},[\alpha]_{\mathrm{D}}{ }^{25}-188.8\left(c 0.2, \mathrm{CHCl}_{3}\right)$ for $>99 \%$ ee; ${ }^{1} \mathrm{H} \mathrm{NMR}(500 \mathrm{MHz}$, chloroform- $d) \delta 8.58(\mathrm{~d}, J=4.2 \mathrm{~Hz}, 1 \mathrm{H}), 7.81-7.70(\mathrm{~m}, 3 \mathrm{H}), 7.67(\mathrm{~d}, J=8.3 \mathrm{~Hz}, 2 \mathrm{H}), 7.54(\mathrm{~s}, 1 \mathrm{H})$, $7.47-7.38(\mathrm{~m}, 2 \mathrm{H}), 7.30-7.24(\mathrm{~m}, 1 \mathrm{H}), 7.19(\mathrm{dd}, J=8.5,1.7 \mathrm{~Hz}, 1 \mathrm{H}), 6.30(\mathrm{dt}, J=6.5,2.5 \mathrm{~Hz}, 1 \mathrm{H})$, $5.63-5.57(\mathrm{~m}, 1 \mathrm{H}), 3.77(\mathrm{~s}, 3 \mathrm{H}), 3.48-3.14(\mathrm{~m}, 2 \mathrm{H}) .{ }^{13} \mathrm{C}$ NMR $(126 \mathrm{MHz}$, chloroform-d) $\delta 208.0$, $169.2(\mathrm{~d}, J=26.3 \mathrm{~Hz}), 156.4(\mathrm{~d}, J=27.0 \mathrm{~Hz}), 149.3(\mathrm{~d}, J=2.2 \mathrm{~Hz}), 137.1,133.6,132.7,131.6,128.2$, 127.75, 127.72, 126.2, 125.67, 125.63, 124.8, 123.7, $120.3(\mathrm{~d}, J=8.4 \mathrm{~Hz}), 97.2(\mathrm{~d}, J=189.2 \mathrm{~Hz}), 95.3$, $88.0(\mathrm{~d}, J=3.7 \mathrm{~Hz}), 53.1,36.4(\mathrm{~d}, J=20.5 \mathrm{~Hz}) .{ }^{19} \mathrm{~F}$ NMR $(376 \mathrm{MHz}$, chloroform- $d$ ) $\delta-164.4(\mathrm{t}, J=24.3$ $\mathrm{Hz}, 1 \mathrm{~F}$ ). HRMS (FI): [M] ${ }^{\oplus}$ calcd for $\mathrm{C}_{22} \mathrm{H}_{18} \mathrm{FNO}_{2}{ }^{\oplus}$ 347.1316, found 347.1321. HPLC analysis: Chiracel AD-H column; detected at $254 \mathrm{~nm}, 40{ }^{\circ} \mathrm{C} ; 3 \%{ }^{i} \mathrm{PrOH}$ in $n$-hexane; flow $=0.7 \mathrm{~mL} / \mathrm{min}$. Retention time: $17.9 \mathrm{~min}, 20.8 \mathrm{~min}, 21.6 \mathrm{~min}, 23.9 \mathrm{~min}$. The ee value was determined based on peaks at $17.9 \mathrm{~min}$ (minor) and 23.9 min (major). 

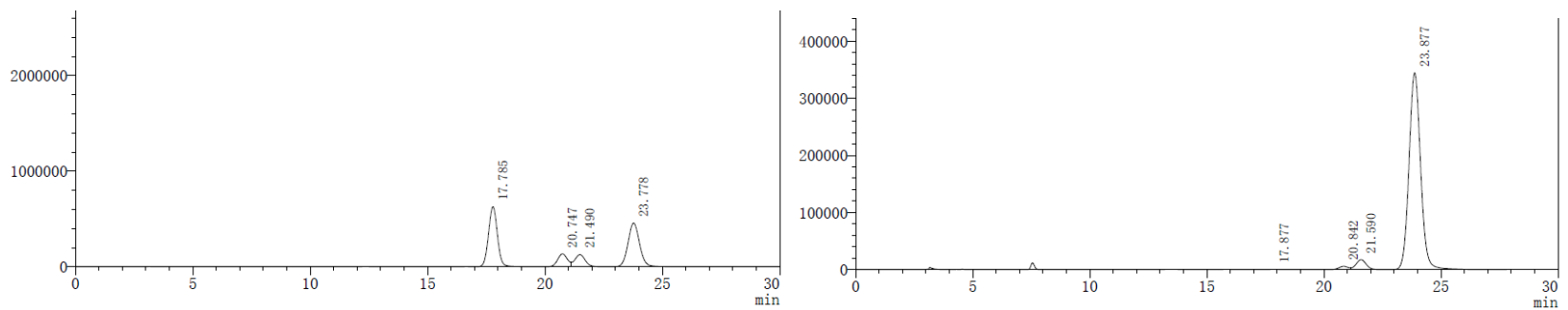

\begin{tabular}{|c|c|c|}
\hline Peak & retension time/min & area\% \\
\hline 1 & 17.785 & 41.270 \\
\hline 2 & 20.747 & 9.831 \\
\hline 3 & 21.490 & 9.874 \\
\hline 4 & 23.778 & 39.025 \\
\hline
\end{tabular}

\begin{tabular}{|c|c|c|}
\hline Peak & retension time/min & area\% \\
\hline 1 & 17.877 & 0.161 \\
\hline 2 & 20.842 & 1.183 \\
\hline 3 & 21.590 & 4.084 \\
\hline 4 & 23.877 & 94.572 \\
\hline
\end{tabular}<smiles>CC(=O)C(F)(C/C=C/c1ccc2occc2c1)c1ccccn1</smiles>

\section{Methyl (2R,5R)-6-(benzofuran-5-yl)-2-fluoro-2-(pyridin-2-yl)hexa-4,5-dienoate (3q)}

Yellow oil, $19 \mathrm{~h}, 94 \%$ yield, $>20: 1 \mathrm{dr},[\alpha]_{\mathrm{D}}{ }^{25}-202.3\left(c 0.5, \mathrm{CHCl}_{3}\right)$ for $>99 \%$ ee; ${ }^{1} \mathrm{H} \mathrm{NMR}(500 \mathrm{MHz}$, chloroform- $d$ ) $\delta 8.58(\mathrm{~d}, J=4.4 \mathrm{~Hz}, 1 \mathrm{H}), 7.77(\mathrm{td}, J=7.8,1.8 \mathrm{~Hz}, 1 \mathrm{H}), 7.66(\mathrm{~d}, J=7.8 \mathrm{~Hz}, 1 \mathrm{H}), 7.58(\mathrm{~d}$, $J=2.3 \mathrm{~Hz}, 1 \mathrm{H}), 7.35(\mathrm{~d}, J=8.5 \mathrm{~Hz}, 1 \mathrm{H}), 7.31(\mathrm{~s}, 1 \mathrm{H}), 7.30-7.23(\mathrm{~m}, 1 \mathrm{H}), 7.03(\mathrm{dd}, J=8.5,1.8 \mathrm{~Hz}$, $1 \mathrm{H}), 6.68(\mathrm{~d}, J=2.3 \mathrm{~Hz}, 1 \mathrm{H}), 6.22(\mathrm{dt}, J=6.6,2.4 \mathrm{~Hz}, 1 \mathrm{H}), 5.56-5.50(\mathrm{~m}, 1 \mathrm{H}), 3.77(\mathrm{~s}, 3 \mathrm{H}), 3.44-$ $3.29(\mathrm{~m}, 1 \mathrm{H}), 3.29-3.15(\mathrm{~m}, 1 \mathrm{H}) .{ }^{13} \mathrm{C}$ NMR $(126 \mathrm{MHz}$, chloroform- $d) \delta 207.2,169.2(\mathrm{~d}, J=26.4 \mathrm{~Hz})$, $156.4(\mathrm{~d}, J=27.0 \mathrm{~Hz}), 154.3,149.3(\mathrm{~d}, J=2.3 \mathrm{~Hz}), 145.4,137.1,128.7,127.7,123.6,123.4,120.3(\mathrm{~d}, J$ $=8.3 \mathrm{~Hz}), 119.3,111.4,106.5,97.2(\mathrm{~d}, J=189.0 \mathrm{~Hz}), 95.0,87.7(\mathrm{~d}, J=3.8 \mathrm{~Hz}), 53.1,36.5(\mathrm{~d}, J=20.5$ Hz). ${ }^{19} \mathrm{~F}$ NMR (376 MHz, chloroform- $d$ ) $\delta-164.5(\mathrm{t}, J=24.5 \mathrm{~Hz}, 1 \mathrm{~F})$. HRMS (FI): [M] ${ }^{\oplus}$ calcd for $\mathrm{C}_{20} \mathrm{H}_{16} \mathrm{FNO}_{3}{ }^{\oplus}$ 337.1109, found 337.1113. HPLC analysis: Chiracel OD-H + Chiralpak ID-3 column (The two columns were connected to each other in this order); detected at $254 \mathrm{~nm}, 40{ }^{\circ} \mathrm{C} ; 3 \%{ }^{i} \mathrm{PrOH}$ in $n$-hexane; flow $=0.5 \mathrm{~mL} / \mathrm{min}$. Retention time: $60.9 \mathrm{~min}, 68.6 \mathrm{~min}, 73.3 \mathrm{~min}, 77.1 \mathrm{~min}$. The ee value was determined based on peaks at 60.9 min (minor) and 73.3 min (major).
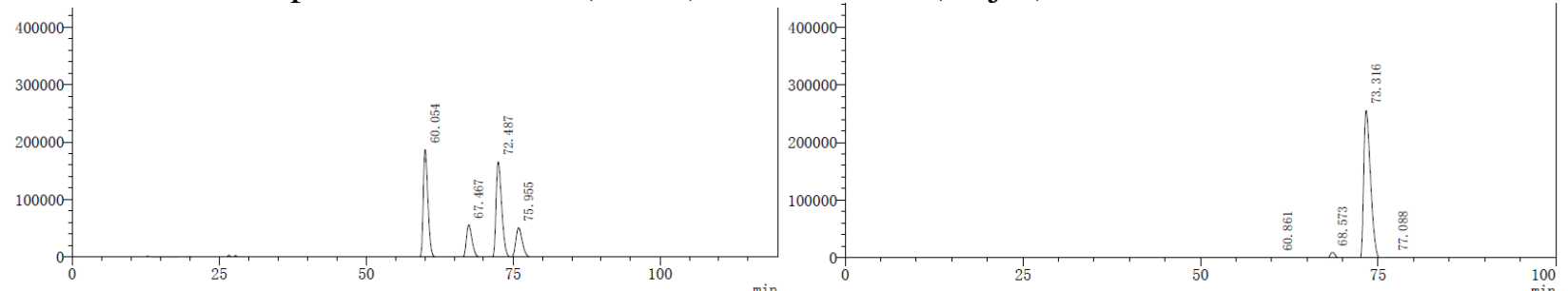

\begin{tabular}{|c|c|c|}
\hline Peak & retension time/min & area\% \\
\hline 1 & 60.054 & 34.988 \\
\hline 2 & 67.467 & 13.214 \\
\hline 3 & 72.487 & 37.946 \\
\hline 4 & 75.955 & 13.853 \\
\hline
\end{tabular}

\begin{tabular}{|c|c|c|}
\hline Peak & retension time/min & area\% \\
\hline 1 & 60.861 & 0.175 \\
\hline 2 & 68.573 & 5.396 \\
\hline 3 & 73.316 & 92.581 \\
\hline 4 & 77.088 & 1.848 \\
\hline
\end{tabular}


<smiles>COC(=O)C(F)(/C=C/[CH]Cl)c1ccccn1</smiles>

\section{Methyl (2S,5R)-10-chloro-2-fluoro-2-(pyridin-2-yl)deca-4,5-dienoate (3r)}

Yellow oil, $20 \mathrm{~h}, 89 \%$ yield, $11: 1 \mathrm{dr},[\alpha]_{\mathrm{D}}{ }^{25}-59.8\left(c 1.0, \mathrm{CHCl}_{3}\right)$ for $96 \%$ ee; ${ }^{1} \mathrm{H} \mathrm{NMR}(500 \mathrm{MHz}$, chloroform- $d) \delta 8.72-8.55(\mathrm{~m}, 1 \mathrm{H}), 7.79-7.75(\mathrm{~m}, 1 \mathrm{H}), 7.63(\mathrm{~d}, J=7.9 \mathrm{~Hz}, 1 \mathrm{H}), 7.32-7.28(\mathrm{~m}, 1 \mathrm{H})$, $5.09-5.05(\mathrm{~m}, 2 \mathrm{H}), 3.79(\mathrm{~s}, 3 \mathrm{H}), 3.51(\mathrm{t}, J=6.8 \mathrm{~Hz}, 2 \mathrm{H}), 3.29-3.11(\mathrm{~m}, 1 \mathrm{H}), 3.14-2.93(\mathrm{~m}, 1 \mathrm{H})$, $1.91-1.87(\mathrm{~m}, 2 \mathrm{H}), 1.75(\mathrm{p}, J=6.8 \mathrm{~Hz}, 2 \mathrm{H}), 1.45(\mathrm{p}, J=7.4 \mathrm{~Hz}, 2 \mathrm{H}) .{ }^{13} \mathrm{C} \mathrm{NMR}(126 \mathrm{MHz}$, chloroform- $d$ ) $\delta 206.4,169.3(\mathrm{~d}, J=26.4 \mathrm{~Hz}), 156.6(\mathrm{~d}, J=26.6 \mathrm{~Hz}), 149.2,137.0,123.6,120.3,97.4(\mathrm{~d}$, $J=188.9 \mathrm{~Hz}), 90.5,83.9,53.0,44.9,36.8(\mathrm{~d}, J=20.6 \mathrm{~Hz}), 31.9,27.7,26.0 .{ }^{19} \mathrm{~F} \mathrm{NMR}(376 \mathrm{MHz}$, chloroform- $d$ ) $\delta-164.5\left(\mathrm{t}, J=24.8 \mathrm{~Hz}\right.$ ). HRMS (FI): $[\mathrm{M}]^{\oplus}$ calcd for $\mathrm{C}_{16} \mathrm{H}_{19} \mathrm{O}_{2} \mathrm{NClF}^{\oplus} 311.1083$, found 311.1086. HPLC analysis: Chiracel OD-H column; detected at $254 \mathrm{~nm}, 40{ }^{\circ} \mathrm{C} ; 3 \%{ }^{i} \mathrm{PrOH}$ in $n$-hexane; flow $=0.7 \mathrm{~mL} / \mathrm{min}$. Retention time: $8.5 \mathrm{~min}, 9.9 \mathrm{~min}, 10.8 \mathrm{~min}, 11.2 \mathrm{~min}$. The ee value was determined based on peaks at 8.5 min (minor) and 11.2 min (major).
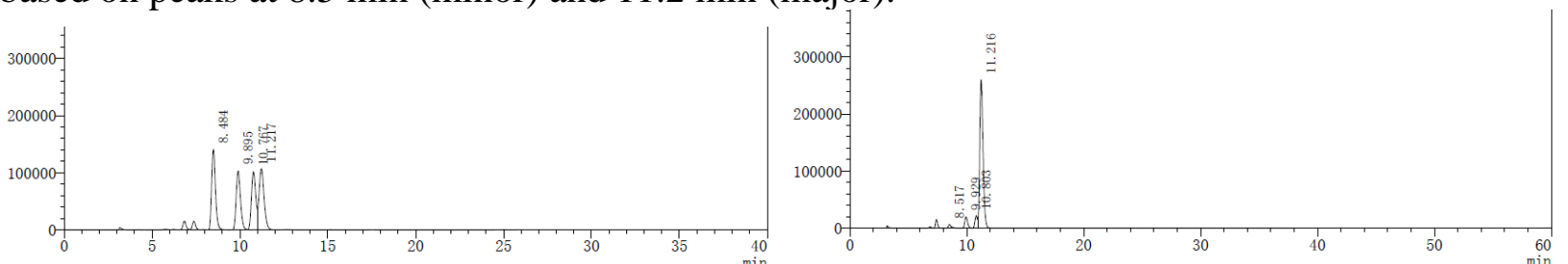

\begin{tabular}{|c|c|c|}
\hline Peak & retension time/min & area\% \\
\hline 1 & 8.484 & 27.480 \\
\hline 2 & 9.895 & 22.553 \\
\hline 3 & 10.767 & 22.676 \\
\hline 4 & 11.217 & 27.292 \\
\hline
\end{tabular}

\begin{tabular}{|c|c|c|}
\hline Peak & retension time/min & area\% \\
\hline 1 & 8.517 & 1.872 \\
\hline 2 & 9.929 & 6.169 \\
\hline 3 & 10.803 & 5.606 \\
\hline 4 & 11.216 & 86.354 \\
\hline
\end{tabular}<smiles>CC(=O)C(F)(C/C=C/CCc1ccccc1)c1ccccn1</smiles>

\section{Methyl (2R,5R)-2-fluoro-8-phenyl-2-(pyridin-2-yl)octa-4,5-dienoate (3s)}

For the synthesis of 3s, $(R)$-DTBM-SEGPHOS (5 mol\%), $10 \mathrm{~mol} \%[\mathrm{Cu}] /(S, S)$-BPE, Et ${ }_{3} \mathrm{~N}(3 \mathrm{~mol} \%)$, without DBU, toluene as the solvent were used instead. Yellow oil, $20 \mathrm{~h}, 89 \%$ yield, 9:1 dr, $[\alpha]_{\mathrm{D}}{ }^{25}-56.7$ $\left(c 1.0, \mathrm{CHCl}_{3}\right)$ for $92 \%$ ee; The ee value of this compound was determined by the reduced derivative 3s' from 3s. ${ }^{1} \mathrm{H}$ NMR $(500 \mathrm{MHz}$, chloroform- $d$ ) $\delta 8.61(\mathrm{~d}, J=4.8 \mathrm{~Hz}, 1 \mathrm{H}), 7.74(\mathrm{t}, J=7.3 \mathrm{~Hz}, 1 \mathrm{H}), 7.61(\mathrm{~d}$, $J=7.9 \mathrm{~Hz}, 1 \mathrm{H}), 7.30-7.22(\mathrm{~m}, 3 \mathrm{H}), 7.20-7.11(\mathrm{~m}, 3 \mathrm{H}), 5.18-5.09(\mathrm{~m}, 1 \mathrm{H}), 5.10-5.01(\mathrm{~m}, 1 \mathrm{H})$, $3.78(\mathrm{~s}, 3 \mathrm{H}), 3.25-3.09(\mathrm{~m}, 1 \mathrm{H}), 3.09-2.94(\mathrm{~m}, 1 \mathrm{H}), 2.58(\mathrm{t}, J=7.8 \mathrm{~Hz}, 2 \mathrm{H}), 2.30-2.07(\mathrm{~m}, 2 \mathrm{H}) .{ }^{13} \mathrm{C}$ NMR (126 MHz, chloroform- $d$ ) $\delta 206.4,169.3(\mathrm{~d}, J=26.4 \mathrm{~Hz}), 156.6(\mathrm{~d}, J=26.9 \mathrm{~Hz}), 149.2,141.8$, 137.0, 128.5, 128.3, 125.9, 123.6, $120.3(\mathrm{~d}, J=8.3 \mathrm{~Hz}), 97.4(\mathrm{~d}, J=188.8 \mathrm{~Hz}), 90.5,84.0,53.0,36.8$ (d, $J=20.5 \mathrm{~Hz}), 35.2,30.2 .{ }^{19} \mathrm{~F}$ NMR $(376 \mathrm{MHz} \text {, chloroform- } d \text { ) } \delta-164.5(\mathrm{t}, J=24.7 \mathrm{~Hz}) \text {. HRMS (FI): [M] }]^{\oplus}$ calcd for $\mathrm{C}_{20} \mathrm{H}_{20} \mathrm{O}_{2} \mathrm{NF}^{\oplus} 325.1473$, found 325.1468 . 


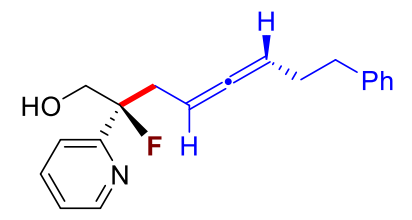

\section{(2R,5R)-2-fluoro-8-phenyl-2-(pyridin-2-yl)octa-4,5-dien-1-ol (3s')}

This compound was prepared via the reduction of $\mathbf{3 s}$ according to the procedure described below in part 7 “Axial-to-Central Chirality Transfer". Colorless oil, $92 \%$ yield, 9:1 dr, $[\alpha]_{\mathrm{D}}{ }^{25}-6.2\left(c\right.$ 1.0, $\left.\mathrm{CHCl}_{3}\right)$ for $92 \%$ ee; ${ }^{1} \mathrm{H}$ NMR $(400 \mathrm{MHz}$, chloroform- $d$ ) $\delta 8.52(\mathrm{~d}, J=3.0 \mathrm{~Hz}, 1 \mathrm{H}), 7.81-7.66(\mathrm{~m}, 1 \mathrm{H}), 7.67-7.49$ $(\mathrm{m}, 1 \mathrm{H}), 7.32-7.21(\mathrm{~m}, 1 \mathrm{H}), 7.25-7.23(\mathrm{~m}, 1 \mathrm{H}), 7.23-7.13(\mathrm{~m}, 2 \mathrm{H}), 7.15-7.07(\mathrm{~m}, 2 \mathrm{H}), 5.14-5.03$ $(\mathrm{m}, 1 \mathrm{H}), 5.06-4.91(\mathrm{~m}, 1 \mathrm{H}), 4.11-3.95(\mathrm{~m}, 2 \mathrm{H}), 2.83-2.62(\mathrm{~m}, 2 \mathrm{H}), 2.56-2.39(\mathrm{~m}, 2 \mathrm{H}), 2.29-2.00$ $(\mathrm{m}, 2 \mathrm{H}) .{ }^{13} \mathrm{C}$ NMR $\left(126 \mathrm{MHz}\right.$, acetonitrile- $\left.d_{3}\right) \delta 200.0,154.8(\mathrm{~d}, J=27.4 \mathrm{~Hz}), 142.4(\mathrm{~d}, J=2.7 \mathrm{~Hz})$, $130.9(\mathrm{~d}, J=1.7 \mathrm{~Hz}), 135.8,122.5,122.3,119.9,116.8,114.6(\mathrm{~d}, J=9.1 \mathrm{~Hz}), 92.1(\mathrm{~d}, J=177.7 \mathrm{~Hz})$, 84.2, $78.6(\mathrm{~d}, J=4.7 \mathrm{~Hz}), 61.3(\mathrm{~d}, J=26.5 \mathrm{~Hz}), 31.0(\mathrm{~d}, J=21.6 \mathrm{~Hz}), 29.1,24.2 .{ }^{19} \mathrm{~F} \mathrm{NMR}(376 \mathrm{MHz}$, chloroform- $d$ ) $\delta-170.6$ - -170.9 (m). HRMS (EI): $[\mathrm{M}]^{\oplus}$ calcd for $\mathrm{C}_{19} \mathrm{H}_{20} \mathrm{ONF}{ }^{\oplus}$ 297.1523, found 297.1519. HPLC analysis: Chiracel AY-3 column; detected at $254 \mathrm{~nm}, 40{ }^{\circ} \mathrm{C} ; 9 \%{ }^{i} \mathrm{PrOH}$ in $n$-hexane; flow $=0.5 \mathrm{~mL} / \mathrm{min}$. Retention time: $15.2 \mathrm{~min}, 16.4 \mathrm{~min}, 17.0 \mathrm{~min}, 18.3 \mathrm{~min}$. The ee value was determined based on peaks at $18.3 \mathrm{~min}$ (minor) and $15.2 \mathrm{~min}$ (major).
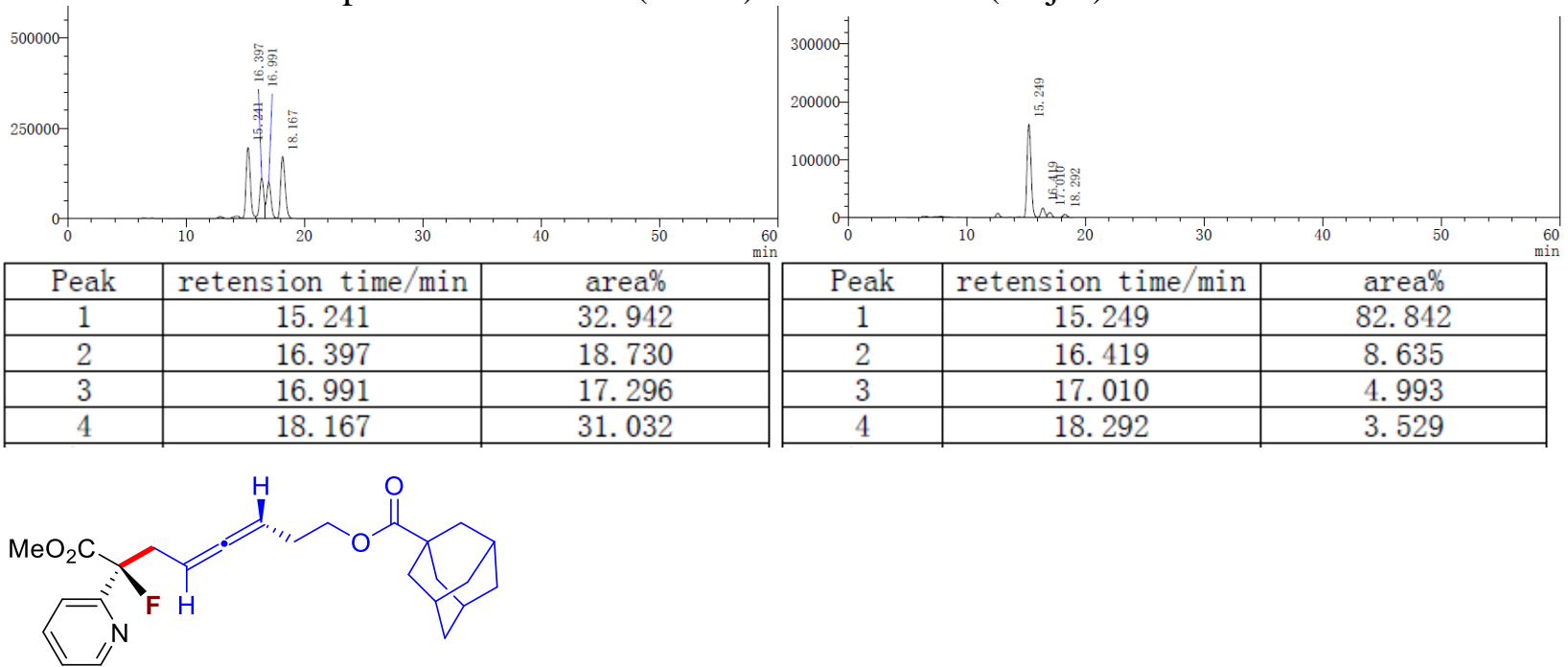

\section{$(4 R, 7 R)-7-F l u o r o-8-m e t h o x y-8-0 x 0-7-(p y r i d i n-2-y l) o c t a-3,4-d i e n-1-y l ~(1 R, 3 S)$-adamantane-1- carboxylate $(3 \mathrm{t})$}

For the synthesis of 3t, $10 \mathrm{~mol} \%[\mathrm{Cu}] /(S, S)-\mathrm{BPE}, \mathrm{Et}_{3} \mathrm{~N}(5 \mathrm{~mol} \%)$, without DBU, toluene as the solvent were used instead. Yellow oil, $21 \mathrm{~h}, 77 \%$ yield, 9:1 dr, $[\alpha]_{\mathrm{D}}{ }^{25}-36.8\left(c 1.0, \mathrm{CHCl}_{3}\right)$ for $95 \%$ ee; ${ }^{1} \mathrm{H} \mathrm{NMR}$ $(500 \mathrm{MHz}$, chloroform- $d) \delta 8.63(\mathrm{~d}, J=4.7 \mathrm{~Hz}, 1 \mathrm{H}), 7.79(\mathrm{td}, J=7.8,1.6 \mathrm{~Hz}, 1 \mathrm{H}), 7.62(\mathrm{~d}, J=7.9 \mathrm{~Hz}$, $1 \mathrm{H}), 7.33-7.26(\mathrm{~m}, 1 \mathrm{H}), 5.15-4.99(\mathrm{~m}, 2 \mathrm{H}), 4.12-3.96(\mathrm{~m}, 2 \mathrm{H}), 3.79(\mathrm{~s}, 3 \mathrm{H}), 3.29-3.13(\mathrm{~m}, 1 \mathrm{H})$, $3.14-2.96(\mathrm{~m}, 1 \mathrm{H}), 2.19-2.14(\mathrm{~m}, 2 \mathrm{H}), 2.04-1.95(\mathrm{~m}, 3 \mathrm{H}), 1.88-1.85(\mathrm{~m}, 6 \mathrm{H}), 1.78-1.62(\mathrm{~m}, 6 \mathrm{H})$. ${ }^{13} \mathrm{C}$ NMR $(126 \mathrm{MHz}$, chloroform- $d$ ) $\delta 207.0,177.6,169.1(\mathrm{~d}, J=26.4 \mathrm{~Hz}), 156.3(\mathrm{~d}, J=27.1 \mathrm{~Hz}), 149.0$ $(\mathrm{d}, J=2.3 \mathrm{~Hz}), 137.3,123.7,120.4(\mathrm{~d}, J=8.3 \mathrm{~Hz}), 97.3(\mathrm{~d}, J=189.0 \mathrm{~Hz}), 87.1,84.2(\mathrm{~d}, J=3.7 \mathrm{~Hz})$, 63.1, 53.1, 40.7, 38.9, 36.7, 36.6, 28.2, 28.0. ${ }^{19} \mathrm{~F}$ NMR (376 MHz, chloroform- $d$ ) $\delta-164.4(\mathrm{t}, J=24.6 \mathrm{~Hz}$, 1F). HRMS (EI): [M] ${ }^{\oplus}$ calcd for $\mathrm{C}_{25} \mathrm{H}_{30} \mathrm{FNO}_{4}{ }^{\oplus}$ 427.2153, found 427.2157. HPLC analysis: Chiracel OD-H + Chiralpak IG-3 column (the two columns were connected to each other in this order); detected 
at $254 \mathrm{~nm}, 40{ }^{\circ} \mathrm{C} ; 3 \%{ }^{i} \mathrm{PrOH}$ in $n$-hexane; flow $=0.5 \mathrm{~mL} / \mathrm{min}$. Retention time: $87.7 \mathrm{~min}, 92.0 \mathrm{~min}, 105.3$ min, $118.1 \mathrm{~min}$. The ee value was determined based on peaks at $92.0 \mathrm{~min}$ (minor) and $118.1 \mathrm{~min}$ (major).
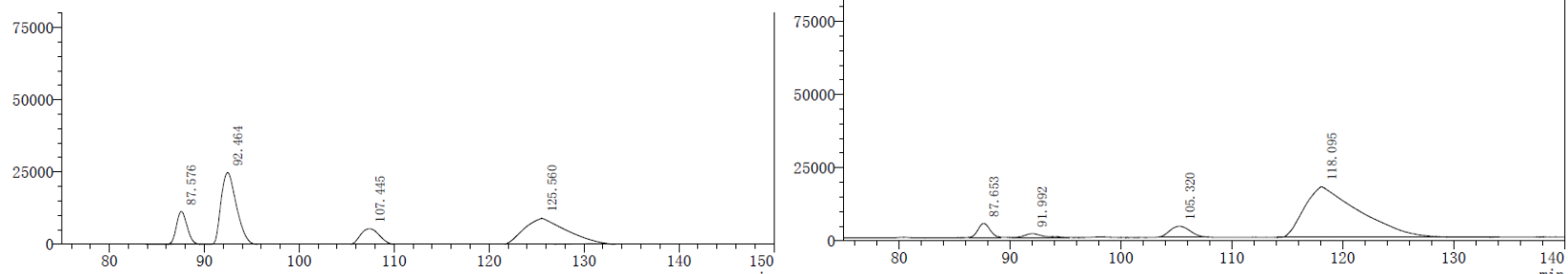

\begin{tabular}{|c|c|c|}
\hline Peak & retension time/min & area\% \\
\hline 1 & 87.576 & 12.069 \\
\hline 2 & 92.464 & 38.590 \\
\hline 3 & 107.445 & 10.905 \\
\hline 4 & 125.560 & 38.435 \\
\hline
\end{tabular}

\begin{tabular}{|c|c|c|}
\hline Peak & retension time/min & area\% \\
\hline 1 & 87.653 & 5.090 \\
\hline 2 & 91.992 & 2.065 \\
\hline 3 & 105.320 & 6.528 \\
\hline 4 & 118.095 & 86.316 \\
\hline
\end{tabular}<smiles>COC(=O)C(F)(Cc1ccccn1)C(F)(F)c1ccccn1</smiles>

\section{(4R,7R)-7-fluoro-8-hydroxy-7-(pyridin-2-yl)octa-3,4-dien-1-yl (S)-3,3,3-trifluoro-2-methoxy-2- phenylpropanoate $(3 \mathbf{u})$}

For the synthesis of 3u, $(R)$-DTBM-SEGPHOS (5 mol\%), $10 \mathrm{~mol} \%[\mathrm{Cu}] /(S, S)-\mathrm{BPE}, \mathrm{Et}_{3} \mathrm{~N}(5 \mathrm{~mol} \%)$, without DBU, toluene as the solvent were used instead. Yellow oil, $20 \mathrm{~h}, 90 \%$ yield, 11:1 dr, $[\alpha]_{\mathrm{D}}{ }^{25}-$ $72.3\left(c\right.$ 1.0, $\left.\mathrm{CHCl}_{3}\right) ;{ }^{1} \mathrm{H} \mathrm{NMR}(400 \mathrm{MHz}$, chloroform- $d) \delta 8.60(\mathrm{~d}, J=4.9 \mathrm{~Hz}, 1 \mathrm{H}), 7.74(\mathrm{td}, J=7.8,1.8$ $\mathrm{Hz}, 1 \mathrm{H}), 7.61-7.58(\mathrm{~m}, 1 \mathrm{H}), 7.57-7.46(\mathrm{~m}, 2 \mathrm{H}), 7.42-7.38(\mathrm{~m}, 3 \mathrm{H}), 7.26-7.22(\mathrm{~m}, 1 \mathrm{H}), 5.16-4.94$ $(\mathrm{m}, 2 \mathrm{H}), 4.25(\mathrm{t}, J=6.8 \mathrm{~Hz}, 2 \mathrm{H}), 3.77(\mathrm{~s}, 3 \mathrm{H}), 3.54(\mathrm{~d}, J=1.2 \mathrm{~Hz}, 3 \mathrm{H}), 3.30-2.95(\mathrm{~m}, 2 \mathrm{H}), 2.26-2.21$ $(\mathrm{m}, 2 \mathrm{H}) .{ }^{13} \mathrm{C}$ NMR $(126 \mathrm{MHz}$, chloroform- $d) \delta 207.0,169.2(\mathrm{~d}, J=26.2 \mathrm{~Hz}), 166.5,156.4(\mathrm{~d}, J=26.9$ $\mathrm{Hz}), 149.2(\mathrm{~d}, J=2.3 \mathrm{~Hz}), 137.0(\mathrm{~d}, J=1.6 \mathrm{~Hz}), 132.3,129.7,128.5,127.4,123.6,123.3(\mathrm{q}, J=288.4$ Hz), $120.2(\mathrm{~d}, J=8.4 \mathrm{~Hz}), 97.3(\mathrm{~d}, J=188.8 \mathrm{~Hz}), 86.3,84.8(\mathrm{~d}, J=3.8 \mathrm{~Hz}), 84.6(\mathrm{q}, J=27.7 \mathrm{~Hz}), 65.3$, 55.5, 53.0, $36.4(\mathrm{~d}, J=20.5 \mathrm{~Hz}), 27.7 .{ }^{19} \mathrm{~F}$ NMR $(376 \mathrm{MHz}$, chloroform- $d$ ) $\delta-71.7,-164.7(\mathrm{t}, J=24.6$ $\mathrm{Hz}$ ). HRMS (ESI): $[\mathrm{M}+\mathrm{H}]^{\oplus}$ calcd for $\mathrm{C}_{24} \mathrm{H}_{23} \mathrm{O}_{5} \mathrm{NF}_{4}{ }^{\oplus} 482.1585$, found 482.1580 .<smiles>CC(=O)C[C@@](F)(/C=C/C=C/OC(=O)Cc1ccc2c(c1)C(=O)c1ccccc1CO2)c1ccccn1</smiles>

\section{Methyl (2R,5R)-2-fluoro-8-(2-(11-oxo-6,11-dihydrodibenzo[b,e]oxepin-2-yl)acetoxy)-2-(pyridin-2- yl)octa-4,5-dienoate $(3 \mathrm{v})$}

For the synthesis of 3v, $(R)$-DTBM-SEGPHOS (5 mol\%), $10 \mathrm{~mol} \%[\mathrm{Cu}] /(S, S)-\mathrm{BPE}, \mathrm{Et}_{3} \mathrm{~N}(5 \mathrm{~mol} \%)$, without DBU, toluene as the solvent were used instead. Yellow oil, $20 \mathrm{~h}, 80 \%$ yield, 10:1 dr, $[\alpha]_{\mathrm{D}}{ }^{25}-$ $29.3\left(c\right.$ 1.0, $\left.\mathrm{CHCl}_{3}\right)$ for $90 \%$ ee; ${ }^{1} \mathrm{H} \mathrm{NMR}(400 \mathrm{MHz}$, chloroform- $d) \delta 8.60(\mathrm{~d}, J=4.9 \mathrm{~Hz}, 1 \mathrm{H}), 8.11(\mathrm{~d}$, $J=2.4 \mathrm{~Hz}, 1 \mathrm{H}), 7.88(\mathrm{~d}, J=7.6 \mathrm{~Hz}, 1 \mathrm{H}), 7.82-7.70(\mathrm{~m}, 1 \mathrm{H}), 7.63-7.51(\mathrm{~m}, 2 \mathrm{H}), 7.51-7.39(\mathrm{~m}, 2 \mathrm{H})$, $7.36(\mathrm{~d}, J=8.7 \mathrm{~Hz}, 1 \mathrm{H}), 7.28-7.21(\mathrm{~m}, 1 \mathrm{H}), 7.03(\mathrm{~d}, J=8.4 \mathrm{~Hz}, 1 \mathrm{H}), 5.19(\mathrm{~s}, 2 \mathrm{H}), 5.13-4.98(\mathrm{~m}, 2 \mathrm{H})$, $4.20-3.98(\mathrm{~m}, 2 \mathrm{H}), 3.77(\mathrm{~s}, 3 \mathrm{H}), 3.63(\mathrm{~s}, 2 \mathrm{H}), 3.27-2.91(\mathrm{~m}, 2 \mathrm{H}), 2.40-2.09(\mathrm{~m}, 2 \mathrm{H}) .{ }^{13} \mathrm{C} \mathrm{NMR}(126$ MHz, chloroform- $d$ ) $\delta 207.0,190.8,171.3,169.2(\mathrm{~d}, J=26.5 \mathrm{~Hz}), 160.5,156.4(\mathrm{~d}, J=26.9 \mathrm{~Hz}), 149.2$ $(\mathrm{d}, J=2.3 \mathrm{~Hz}), 140.5,137.0,136.4,135.6,132.8,132.5,129.5,129.3,127.9$, 127.8, 125.1, 123.6, 121.1, 
$120.2(\mathrm{~d}, J=8.3 \mathrm{~Hz}), 97.3(\mathrm{~d}, J=188.8 \mathrm{~Hz}), 86.8,84.4(\mathrm{~d}, J=3.7 \mathrm{~Hz}), 73.7,64.0,53.0,40.2,36.5(\mathrm{~d}, J$ $=20.6 \mathrm{~Hz}), 27.9 .{ }^{19} \mathrm{~F}$ NMR $\left(376 \mathrm{MHz}\right.$, chloroform- $d$ ) $\delta-164.5(\mathrm{t}, J=24.7 \mathrm{~Hz})$. HRMS (ESI): $[\mathrm{M}]^{\oplus}$ calcd for $\mathrm{C}_{30} \mathrm{H}_{27} \mathrm{FNO}_{6}{ }^{\oplus} 516.1817$, found 516.1811. HPLC analysis: Chiracel OD-H, detected at $254 \mathrm{~nm}, 40{ }^{\circ} \mathrm{C}$; $9 \%{ }^{i} \mathrm{PrOH}$ in $n$-hexane; flow $=0.7 \mathrm{~mL} / \mathrm{min}$. Retention time: $68.5 \mathrm{~min}, 80.5 \mathrm{~min}, 85.3 \mathrm{~min}, 97.9 \mathrm{~min}$. The ee value was determined based on peaks at $68.5 \mathrm{~min}$ (minor) and $85.3 \mathrm{~min}$ (major).
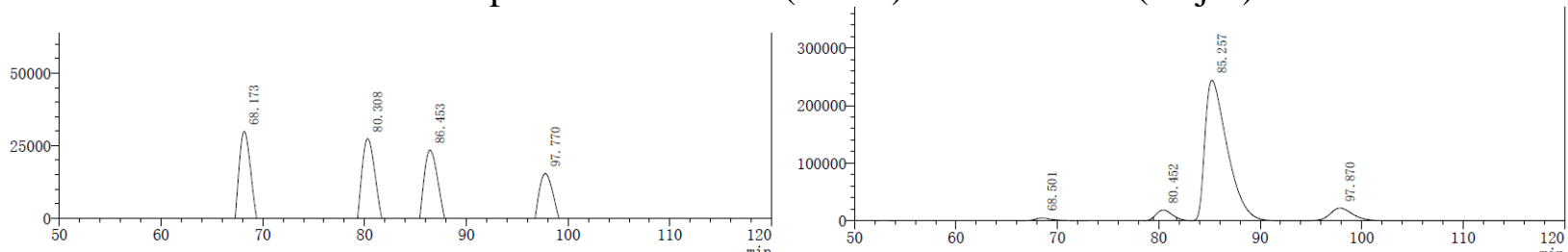

\begin{tabular}{|c|c|c|}
\hline Peak & retension time/min & area\% \\
\hline 1 & 68.173 & 24.273 \\
\hline 2 & 80.308 & 26.357 \\
\hline 3 & 86.453 & 26.123 \\
\hline 4 & 97.770 & 23.248 \\
\hline
\end{tabular}

\begin{tabular}{|c|c|c|}
\hline Peak & retension time/min & area\% \\
\hline 1 & 68.501 & 1.679 \\
\hline 2 & 80.452 & 4.295 \\
\hline 3 & 85.257 & 85.786 \\
\hline 4 & 97.870 & 8.240 \\
\hline
\end{tabular}<smiles>COC(=O)[C@](F)(C/C=C\COC(=O)c1sc(-c2ccc(OCC(C)C)c(C#N)c2)nc1C)c1ccccn1</smiles>

(4R,7R)-7-fluoro-8-methoxy-8-oxo-7-(pyridin-2-yl)octa-3,4-dien-1-yl 2-(3-cyano-4isobutoxyphenyl)-4-methylthiazole-5-carboxylate $(3 \mathrm{w})$

For the synthesis of 3w, $(R)$-DTBM-SEGPHOS (5 mol\%), $10 \mathrm{~mol} \%[\mathrm{Cu}] /(S, S)$-BPE, Et $3 \mathrm{~N}(5 \mathrm{~mol} \%)$, without DBU, toluene as the solvent were used instead. White solid, $19 \mathrm{~h}, 82 \%$ yield, $11: 1 \mathrm{dr},[\alpha]_{\mathrm{D}}{ }^{25}$. $29.6\left(c 1.0, \mathrm{CHCl}_{3}\right)$ for $98 \%$ ee; ${ }^{1} \mathrm{H}$ NMR $(400 \mathrm{MHz}$, chloroform- $d) \delta 8.59(\mathrm{~d}, J=6.1 \mathrm{~Hz}, 1 \mathrm{H}), 8.18(\mathrm{~d}, J$ $=2.3 \mathrm{~Hz}, 1 \mathrm{H}), 8.09(\mathrm{dd}, J=8.9,2.3 \mathrm{~Hz}, 1 \mathrm{H}), 7.75(\mathrm{td}, J=7.7,1.8 \mathrm{~Hz}, 1 \mathrm{H}), 7.58(\mathrm{~d}, J=8.0 \mathrm{~Hz}, 1 \mathrm{H})$, $7.33-7.22(\mathrm{~m}, 2 \mathrm{H}), 7.02(\mathrm{~d}, J=8.9 \mathrm{~Hz}, 1 \mathrm{H}), 5.22-4.99(\mathrm{~m}, 2 \mathrm{H}), 4.27(\mathrm{t}, J=6.5 \mathrm{~Hz}, 2 \mathrm{H}), 3.91(\mathrm{~d}, J=$ $6.5 \mathrm{~Hz}, 2 \mathrm{H}), 3.78(\mathrm{~s}, 3 \mathrm{H}), 3.33-2.95(\mathrm{~m}, 2 \mathrm{H}), 2.75(\mathrm{~s}, 3 \mathrm{H}), 2.42-2.27(\mathrm{~m}, 2 \mathrm{H}), 2.27-2.12(\mathrm{~m}, 1 \mathrm{H})$, $1.10(\mathrm{~d}, J=6.7 \mathrm{~Hz}, 6 \mathrm{H}) .{ }^{13} \mathrm{C}$ NMR $(126 \mathrm{MHz}$, chloroform- $d$ ) $\delta 207.0,169.1(\mathrm{~d}, J=26.5 \mathrm{~Hz}), 167.3$, $162.5,161.9,161.2,156.4(\mathrm{~d}, J=26.9 \mathrm{~Hz}), 149.2(\mathrm{~d}, J=2.2 \mathrm{~Hz}), 137.0,132.6,132.1,126.0,123.6$, 121.8, $120.2(\mathrm{~d}, J=8.2 \mathrm{~Hz}), 115.4,112.6,103.0,97.3(\mathrm{~d}, J=188.9 \mathrm{~Hz}), 86.8,84.6(\mathrm{~d}, J=3.7 \mathrm{~Hz}), 75.7$, 64.2, 53.0, $36.5(\mathrm{~d}, J=20.6 \mathrm{~Hz}), 28.2,28.1,19.1,17.5 .{ }^{19} \mathrm{~F}$ NMR $(376 \mathrm{MHz}$, chloroform- $d) \delta-166.9(\mathrm{t}$, $J=24.5 \mathrm{~Hz}$ ). HRMS (ESI): [M] $]^{\oplus}$ calcd for $\mathrm{C}_{30} \mathrm{H}_{31} \mathrm{FSN}_{3} \mathrm{O}_{5}{ }^{\oplus}$ 564.1963, found 564.1953. HPLC analysis: Chiracel OD-H, detected at $254 \mathrm{~nm}, 40{ }^{\circ} \mathrm{C} ; 3 \%{ }^{i} \mathrm{PrOH}$ in $n$-hexane; flow $=0.7 \mathrm{~mL} / \mathrm{min}$. Retention time: $36.7 \mathrm{~min}, 39.1 \mathrm{~min}, 41.2 \mathrm{~min}, 42.4 \mathrm{~min}$. The ee value was determined based on peaks at $36.7 \mathrm{~min}$ (minor) and 42.4 min (major).
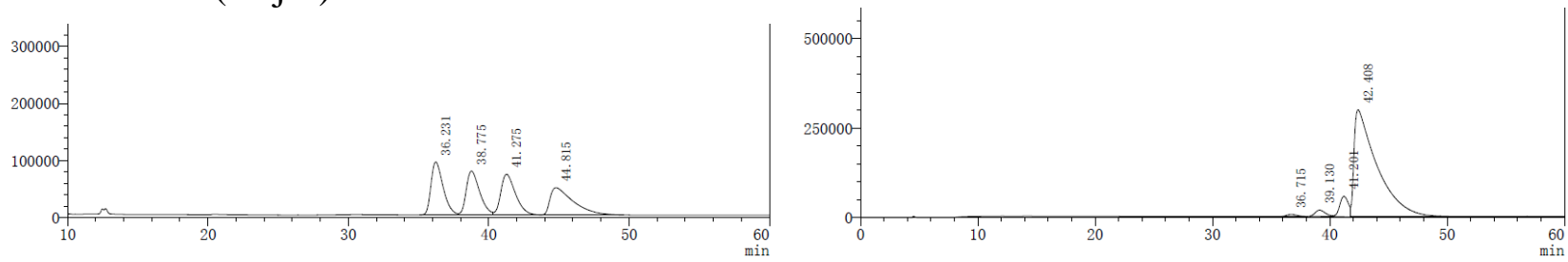


\begin{tabular}{|c|c|c|}
\hline Peak & retension time/min & area\% \\
\hline 1 & 36.231 & 26.209 \\
\hline 2 & 38.775 & 23.890 \\
\hline 3 & 41.275 & 24.305 \\
\hline 4 & 44.815 & 25.596 \\
\hline
\end{tabular}

\begin{tabular}{|c|c|c|}
\hline Peak & retension time/min & area\% \\
\hline 1 & 36.715 & 0.745 \\
\hline 2 & 39.130 & 2.380 \\
\hline 3 & 41.201 & 6.531 \\
\hline 4 & 42.408 & 90.345 \\
\hline
\end{tabular}<smiles>CC(=O)OC(F)(CC=C=Cc1ccc(COC(=O)C23CC4CC(CC(C4)C2)C3)cc1)c1ccccn1</smiles>

\section{4-((2R,5R)-5-Fluoro-6-methoxy-6-oxo-5-(pyridin-2-yl)hexa-1,2-dien-1-yl)benzyl $(3 R, 5 R, 7 R)$ - adamantane-1-carboxylate $(3 \mathbf{x})$}

Yellow oil, $20 \mathrm{~h}, 99 \%$ yield, $13: 1 \mathrm{dr},[\alpha]_{\mathrm{D}}{ }^{25}-151.3\left(c 0.2, \mathrm{CHCl}_{3}\right)$ for $>99 \%$ ee; ${ }^{1} \mathrm{H} \mathrm{NMR}(500 \mathrm{MHz}$, chloroform- $d$ ) $\delta 8.58(\mathrm{~d}, J=4.7 \mathrm{~Hz}, 1 \mathrm{H}), 7.78(\mathrm{td}, J=7.8,1.8 \mathrm{~Hz}, 1 \mathrm{H}), 7.65(\mathrm{~d}, J=7.9 \mathrm{~Hz}, 1 \mathrm{H}), 7.32-$ $7.25(\mathrm{~m}, 1 \mathrm{H}), 7.20(\mathrm{~d}, J=7.9 \mathrm{~Hz}, 2 \mathrm{H}), 7.07(\mathrm{~d}, J=7.9 \mathrm{~Hz}, 2 \mathrm{H}), 6.12(\mathrm{dt}, J=6.7,2.4 \mathrm{~Hz}, 1 \mathrm{H}), 5.55-$ $5.50(\mathrm{~m}, 1 \mathrm{H}), 5.05(\mathrm{~s}, 2 \mathrm{H}), 3.77(\mathrm{~s}, 3 \mathrm{H}), 3.43-3.27(\mathrm{~m}, 1 \mathrm{H}), 3.27-3.06(\mathrm{~m}, 1 \mathrm{H}), 2.09-1.97(\mathrm{~m}, 3 \mathrm{H})$, 1.93-1.89 (m, 6H), $1.76-1.66(\mathrm{~m}, 6 \mathrm{H}) .{ }^{13} \mathrm{C}$ NMR $(101 \mathrm{MHz}$, chloroform- $d$ ) $\delta 207.6,177.5,169.1(\mathrm{~d}, J$ $=26.2 \mathrm{~Hz}), 156.3(\mathrm{~d}, J=26.9 \mathrm{~Hz}), 149.3(\mathrm{~d}, J=2.3 \mathrm{~Hz}), 137.1(\mathrm{~d}, J=1.5 \mathrm{~Hz}), 135.2,133.8,128.1$, 126.9, 123.6, 120.2 (d, $J=8.4 \mathrm{~Hz}), 97.1(\mathrm{~d}, J=189.2 \mathrm{~Hz}), 94.5,87.8(\mathrm{~d}, J=3.8 \mathrm{~Hz}), 65.6,53.1,40.8$, 38.9, 36.53, 36.48, 28.0. ${ }^{19} \mathrm{~F}$ NMR (376 MHz, chloroform- $d$ ) $\delta-165.7$ (t, $\left.J=24.4 \mathrm{~Hz}, 1 \mathrm{~F}\right)$. HRMS (EI): $[\mathrm{M}]^{\oplus}$ calcd for $\mathrm{C}_{30} \mathrm{H}_{33} \mathrm{FNO}_{4}{ }^{\oplus}$ 490.2388, found 490.2387. HPLC analysis: Chiracel OD-H column; detected at $254 \mathrm{~nm}, 40{ }^{\circ} \mathrm{C} ; 9 \%{ }^{i} \mathrm{PrOH}$ in $n$-hexane; flow $=0.7 \mathrm{~mL} / \mathrm{min}$. Retention time: $12.0 \mathrm{~min}, 13.0$ min, $18.1 \mathrm{~min}, 19.7 \mathrm{~min}$. The ee value was determined based on peaks at $12.0 \mathrm{~min}$ (minor) and $18.1 \mathrm{~min}$ (major).
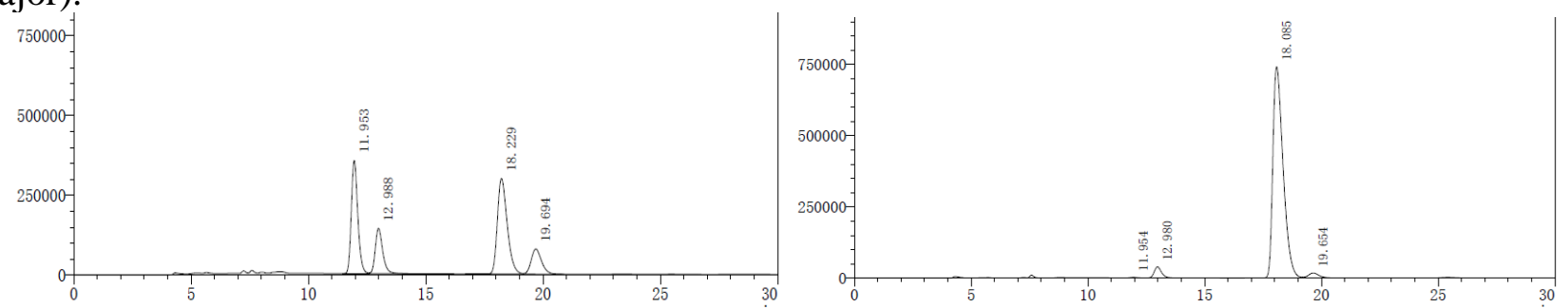

\begin{tabular}{|c|c|c|}
\hline Peak & retension time/min & area\% \\
\hline 1 & 11.953 & 32.403 \\
\hline 2 & 12.988 & 14.789 \\
\hline 3 & 18.229 & 41.329 \\
\hline 4 & 19.694 & 11.479 \\
\hline
\end{tabular}

\begin{tabular}{|c|c|c|}
\hline Peak & retension time/min & area\% \\
\hline 1 & 11.954 & 0.169 \\
\hline 2 & 12.980 & 3.499 \\
\hline 3 & 18.085 & 94.102 \\
\hline 4 & 19.654 & 2.231 \\
\hline
\end{tabular}<smiles>[Y6]C(F)(C(=O)OCc1ccc(C=CCC(F)(C(=O)OC)c2cccc(C(=O)c3ccccc3)c2)cc1)c1ccccn1</smiles>

Methyl (2R,5R)-6-(4-((((S)-2-(3-benzoylphenyl)propanoyl)oxy)methyl)phenyl)-2-fluoro-2-(pyridin2-yl)hexa-4,5-dienoate $(3 y)$

Yellow oil, $20 \mathrm{~h}, 68 \%$ yield, $>20: 1 \mathrm{dr},[\alpha]_{\mathrm{D}}{ }^{25}-100.4\left(c\right.$ 1.1, $\left.\mathrm{CHCl}_{3}\right)$ for $>99 \%$ de; ${ }^{1} \mathrm{H} \mathrm{NMR}(400 \mathrm{MHz}$, chloroform- $d$ ) $\delta 8.57(\mathrm{~d}, J=4.9 \mathrm{~Hz}, 1 \mathrm{H}), 7.81-7.72(\mathrm{~m}, 4 \mathrm{H}), 7.72-7.50(\mathrm{~m}, 4 \mathrm{H}), 7.50-7.39(\mathrm{~m}, 3 \mathrm{H})$, 7.29 (s, 1H), $7.12(\mathrm{~d}, J=7.9 \mathrm{~Hz}, 2 \mathrm{H}), 7.02(\mathrm{~d}, J=7.9 \mathrm{~Hz}, 2 \mathrm{H}), 6.09$ (dt, $J=6.6,2.4 \mathrm{~Hz}, 1 \mathrm{H}), 5.58-5.36$ $(\mathrm{m}, 1 \mathrm{H}), 5.13-4.94(\mathrm{~m}, 2 \mathrm{H}), 3.84(\mathrm{q}, J=7.1 \mathrm{~Hz}, 1 \mathrm{H}), 3.77(\mathrm{~s}, 3 \mathrm{H}), 3.43-3.06(\mathrm{~m}, 2 \mathrm{H}), 1.55(\mathrm{~d}, J=7.2$ 
$\mathrm{Hz}, 3 \mathrm{H}) .{ }^{13} \mathrm{C}$ NMR $(126 \mathrm{MHz}$, chloroform- $d) \delta 207.7,196.5,173.9,169.0(\mathrm{~d}, J=26.3 \mathrm{~Hz}), 156.2(\mathrm{~d}, J=$ $27.1 \mathrm{~Hz}), 149.2(\mathrm{~d}, J=2.2 \mathrm{~Hz}), 140.7,138.0,137.5,137.3,134.4,134.1,132.6,131.6,130.1,129.3$, 129.1, 128.6, 128.4, 126.9, 123.7, 120.3 (d, $J=8.3 \mathrm{~Hz}), 97.1(\mathrm{~d}, J=189.6 \mathrm{~Hz}), 94.5,87.8(\mathrm{~d}, J=3.7$ $\mathrm{Hz}), 66.5,53.1,45.5,36.3(\mathrm{~d}, J=20.5 \mathrm{~Hz}), 18.5$ (one aromatic carbon signal was not observed because of overlapping). ${ }^{19} \mathrm{~F}$ NMR (376 MHz, chloroform- $d$ ) $\delta-164.5$ (t, $\left.J=24.6 \mathrm{~Hz}, 1 \mathrm{~F}\right)$. HRMS (ESI): [M+H] ${ }^{\oplus}$ calcd for $\mathrm{C}_{35} \mathrm{H}_{31} \mathrm{FNO}_{5}{ }^{\oplus} 564.2181$, found 564.2183.

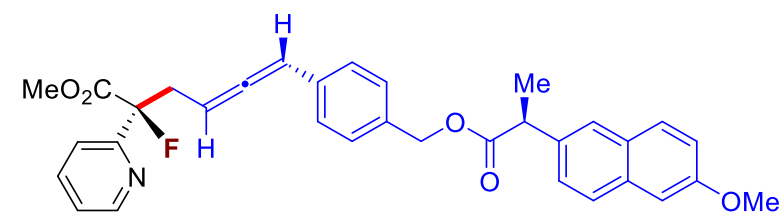

Methyl (2R,5R)-2-fluoro-6-(4-((((S)-2-(6-methoxynaphthalen-2-yl)propanoyl)oxy)methyl)phenyl)2-(pyridin-2-yl)hexa-4,5-dienoate $(3 z)$

White solid, $20 \mathrm{~h}, 93 \%$ yield, $11: 1 \mathrm{dr},[\alpha]_{\mathrm{D}}{ }^{25}-136.8\left(c 0.2, \mathrm{CHCl}_{3}\right)$ for $>99 \%$ de; ${ }^{1} \mathrm{H} \mathrm{NMR}(500 \mathrm{MHz}$, chloroform- $d$ ) $\delta 8.54(\mathrm{~d}, J=4.9 \mathrm{~Hz}, 1 \mathrm{H}), 7.76-7.58(\mathrm{~m}, 5 \mathrm{H}), 7.39(\mathrm{dd}, J=8.4,1.9 \mathrm{~Hz}, 1 \mathrm{H}), 7.22-7.19$ $(\mathrm{m}, 1 \mathrm{H}), 7.17-7.06(\mathrm{~m}, 4 \mathrm{H}), 6.97(\mathrm{~d}, J=8.0 \mathrm{~Hz}, 2 \mathrm{H}), 6.08(\mathrm{dt}, J=6.6,2.3 \mathrm{~Hz}, 1 \mathrm{H}), 5.53-5.49(\mathrm{~m}$, $1 \mathrm{H}), 5.10-5.02(\mathrm{~m}, 2 \mathrm{H}), 3.91-3.87(\mathrm{~m}, 4 \mathrm{H}), 3.76(\mathrm{~s}, 3 \mathrm{H}), 3.42-3.06(\mathrm{~m}, 2 \mathrm{H}), 1.58(\mathrm{~d}, J=7.2 \mathrm{~Hz}$, $3 \mathrm{H}) .{ }^{13} \mathrm{C}$ NMR $(126 \mathrm{MHz}$, chloroform- $d) \delta 207.6,174.5,169.1(\mathrm{~d}, J=26.1 \mathrm{~Hz}), 157.7,156.3(\mathrm{~d}, J=$ $27.1 \mathrm{~Hz}), 149.3(\mathrm{~d}, J=2.3 \mathrm{~Hz}), 137.1(\mathrm{~d}, J=1.7 \mathrm{~Hz}), 135.6,134.6,134.0,133.7,129.3,128.9,128.3$, 127.2, 126.9, 126.3, 126.0, 123.6, 120.2 (d, $J=8.4 \mathrm{~Hz}), 119.0,105.6,97.2(\mathrm{~d}, J=189.2 \mathrm{~Hz}), 94.4,87.8$ $(\mathrm{d}, J=3.7 \mathrm{~Hz}), 66.4,55.3,53.1,45.5,36.3(\mathrm{~d}, J=20.3 \mathrm{~Hz}), 18.6 .{ }^{19} \mathrm{~F}$ NMR $(376 \mathrm{MHz}$, chloroform- $d$ ) $\delta$ $-164.6(\mathrm{t}, J=24.3 \mathrm{~Hz}, 1 \mathrm{~F})$.

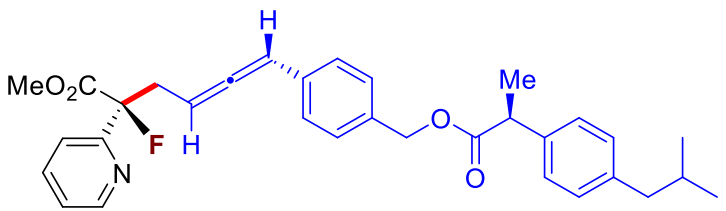

Methyl (2R,5R)-2-fluoro-6-(4-((((S)-2-(4-isobutylphenyl)propanoyl)oxy)methyl)phenyl)-2-(pyridin2-yl)hexa-4,5-dienoate (3aa)

Yellow oil, $20 \mathrm{~h}, 70 \%$ yield, >20:1 dr, $[\alpha]_{\mathrm{D}}{ }^{25}-122.0\left(c\right.$ 1.3, $\left.\mathrm{CHCl}_{3}\right)$ for $>99 \%$ de; ${ }^{1} \mathrm{H} \mathrm{NMR}(500 \mathrm{MHz}$, chloroform- $d$ ) $\delta 8.57(\mathrm{~d}, J=4.1 \mathrm{~Hz}, 1 \mathrm{H}), 7.76(\mathrm{td}, J=7.8,1.8 \mathrm{~Hz}, 1 \mathrm{H}), 7.65(\mathrm{~d}, J=7.9 \mathrm{~Hz}, 1 \mathrm{H}), 7.27-$ $7.25(\mathrm{~m}, 1 \mathrm{H}), 7.20(\mathrm{~d}, J=7.7 \mathrm{~Hz}, 2 \mathrm{H}), 7.13-7.06(\mathrm{~m}, 4 \mathrm{H}), 7.00(\mathrm{~d}, J=7.9 \mathrm{~Hz}, 2 \mathrm{H}), 6.09(\mathrm{dt}, J=6.6$, $2.4 \mathrm{~Hz}, 1 \mathrm{H}), 5.54-5.49(\mathrm{~m}, 1 \mathrm{H}), 5.11-4.96(\mathrm{~m}, 2 \mathrm{H}), 3.77(\mathrm{~s}, 3 \mathrm{H}), 3.74(\mathrm{q}, J=6.9 \mathrm{~Hz}, 1 \mathrm{H}), 3.39-3.13$ $(\mathrm{m}, 2 \mathrm{H}), 2.45(\mathrm{~d}, J=7.1 \mathrm{~Hz}, 2 \mathrm{H}), 1.88-1.80(\mathrm{~m}, 1 \mathrm{H}), 1.50(\mathrm{~d}, J=7.1 \mathrm{~Hz}, 3 \mathrm{H}), 0.90(\mathrm{~s}, 3 \mathrm{H}), 0.89$ (s, $3 \mathrm{H}) .{ }^{13} \mathrm{C}$ NMR $(126 \mathrm{MHz}$, chloroform- $d) \delta 207.7,174.6,169.0(\mathrm{~d}, J=26.2 \mathrm{~Hz}), 156.2(\mathrm{~d}, J=27.0 \mathrm{~Hz})$, $149.1(\mathrm{~d}, J=2.2 \mathrm{~Hz}), 140.6,137.7,137.3,134.7,133.9,129.4,128.2,127.3,126.9,123.7,120.3(\mathrm{~d}, J=$ $8.5 \mathrm{~Hz}), 97.1(\mathrm{~d}, J=189.4 \mathrm{~Hz}), 94.5,87.8(\mathrm{~d}, J=3.7 \mathrm{~Hz}), 66.2,53.1,45.2,45.1,36.4(\mathrm{~d}, J=20.5 \mathrm{~Hz})$, 30.3, 22.5, 18.5. ${ }^{19} \mathrm{~F}$ NMR (376 MHz, chloroform- $d$ ) $\delta-160.4(\mathrm{t}, J=24.6 \mathrm{~Hz}, 1 \mathrm{~F})$. HRMS (ESI): [M+H] ${ }^{\oplus}$ calcd for $\mathrm{C}_{32} \mathrm{H}_{34} \mathrm{FNO}_{4}{ }^{\oplus} 516.2545$, found 516.2550. 
<smiles>CC(=O)CC(F)(/C=C/c1ccc(COC(=O)CCc2nc(-c3ccccc3)c(-c3ccccc3)o2)cc1)c1ccccn1</smiles>

Methyl (2R,5R)-6-(4-(((3-(4,5-diphenyloxazol-2-yl)propanoyl)oxy)methyl)phenyl)-2-fluoro-2(pyridin-2-yl)hexa-4,5-dienoate (3bb)

Yellow solid, $9 \mathrm{~h}, 62 \%$ yield, $>20: 1 \mathrm{dr},[\alpha]_{\mathrm{D}}{ }^{25}-124.7\left(c 0.2, \mathrm{CHCl}_{3}\right)$ for $>99 \%$ ee; ${ }^{1} \mathrm{H} \mathrm{NMR}(500 \mathrm{MHz}$, chloroform- $d$ ) $\delta 8.58(\mathrm{~d}, J=4.4 \mathrm{~Hz}, 1 \mathrm{H}), 7.76(\mathrm{td}, J=7.8,1.9 \mathrm{~Hz}, 1 \mathrm{H}), 7.67-7.58(\mathrm{~m}, 3 \mathrm{H}), 7.58-7.50$ $(\mathrm{m}, 2 \mathrm{H}), 7.39-7.23(\mathrm{~m}, 7 \mathrm{H}), 7.21(\mathrm{~d}, J=7.8 \mathrm{~Hz}, 2 \mathrm{H}), 7.03(\mathrm{~d}, J=7.8 \mathrm{~Hz}, 2 \mathrm{H}), 6.09(\mathrm{dt}, J=6.5,2.4 \mathrm{~Hz}$, $1 \mathrm{H}), 5.54-5.50(\mathrm{~m}, 1 \mathrm{H}), 5.12(\mathrm{~s}, 2 \mathrm{H}), 3.77(\mathrm{~s}, 3 \mathrm{H}), 3.40-3.26(\mathrm{~m}, 1 \mathrm{H}), 3.3-3.1(\mathrm{~m}, 3 \mathrm{H}), 2.96(\mathrm{t}, J=$ $7.4 \mathrm{~Hz}, 2 \mathrm{H}) .{ }^{13} \mathrm{C}$ NMR $(126 \mathrm{MHz}$, chloroform- $d$ ) $\delta 207.7,171.9,169.1(\mathrm{~d}, J=26.2 \mathrm{~Hz}), 161.7,156.2$, $149.3(\mathrm{~d}, J=2.3 \mathrm{~Hz}), 145.5,137.1,135.1,134.4,134.2,132.5,129.0,128.7,128.6,128.53,128.48$, $128.1,127.9,127.0,126.5,123.6,120.2(\mathrm{~d}, J=8.4 \mathrm{~Hz}), 97.1(\mathrm{~d}, J=189.2 \mathrm{~Hz}), 94.5,87.9(\mathrm{~d}, J=3.7$ $\mathrm{Hz}), 66.4,53.1,36.3(\mathrm{~d}, J=20.3 \mathrm{~Hz}), 31.2,23.6 .{ }^{19} \mathrm{~F}$ NMR $(376 \mathrm{MHz}$, chloroform- $d) \delta-169.7(\mathrm{t}, J=$ 24.4 Hz, 1F). HRMS (ESI): $[\mathrm{M}+\mathrm{H}]^{\oplus}$ calcd for $\mathrm{C}_{37} \mathrm{H}_{32} \mathrm{FN}_{2} \mathrm{O}_{5}{ }^{\oplus}$ 603.2290, found 603.2293. HPLC analysis: Chiralpak IC column; detected at $254 \mathrm{~nm}, 40{ }^{\circ} \mathrm{C} ; 20 \%{ }^{i} \mathrm{PrOH}$ in $n$-hexane; flow $=0.7 \mathrm{~mL} / \mathrm{min}$. Retention time: $35.2 \mathrm{~min}, 37.2 \mathrm{~min}, 38.7 \mathrm{~min}, 51.7 \mathrm{~min}$. The ee value was determined based on peaks at $35.2 \mathrm{~min}$ (major) and $37.2 \mathrm{~min}$ (minor).
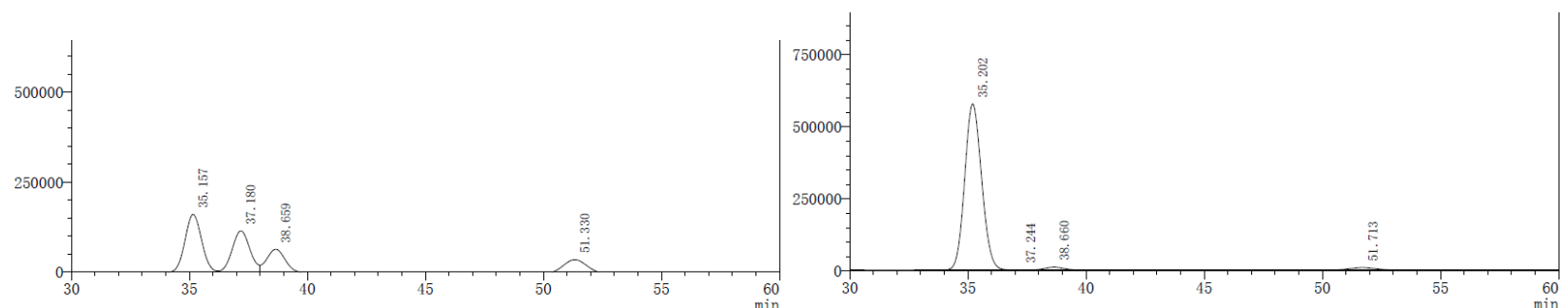

\begin{tabular}{|c|c|c|}
\hline Peak & retension time/min & area\% \\
\hline 1 & 35.157 & 38.816 \\
\hline 2 & 37.180 & 29.832 \\
\hline 3 & 38.659 & 17.363 \\
\hline 4 & 51.330 & 13.989 \\
\hline
\end{tabular}

\begin{tabular}{|c|c|c|}
\hline Peak & retension time/min & area\% \\
\hline 1 & 35.202 & 96.447 \\
\hline 2 & 37.244 & 0.022 \\
\hline 3 & 38.660 & 1.630 \\
\hline 4 & 51.713 & 1.900 \\
\hline
\end{tabular}<smiles>CC(=O)CC(F)(/C=C/c1ccc(COC(=O)C23CC[C@@H](C(=O)O2)C(C)C3(C)C)cc1)c1ccccn1</smiles>

\section{4-((2R,5R)-5-Fluoro-6-methoxy-6-oxo-5-(pyridin-2-yl)hexa-1,2-dien-1-yl)benzyl (1R,4R)-4,7,7- trimethyl-3-oxo-2-oxabicyclo[2.2.1]heptane-1-carboxylate (3cc)}

Yellow oil, $15 \mathrm{~h}, 84 \%$ yield, $9: 1 \mathrm{dr},[\alpha]_{\mathrm{D}}{ }^{25}-122.2\left(c 1.0, \mathrm{CHCl}_{3}\right)$ for $>99 \%$ de; ${ }^{1} \mathrm{H} \mathrm{NMR}(400 \mathrm{MHz}$, chloroform- $d) \delta 8.59(\mathrm{~d}, J=4.8 \mathrm{~Hz}, 1 \mathrm{H}), 7.89-7.75(\mathrm{~m}, 1 \mathrm{H}), 7.67(\mathrm{~d}, J=7.9 \mathrm{~Hz}, 1 \mathrm{H}), 7.39-7.28(\mathrm{~m}$, $1 \mathrm{H}), 7.25(\mathrm{~d}, J=7.9 \mathrm{~Hz}, 2 \mathrm{H}), 7.07(\mathrm{~d}, J=7.8 \mathrm{~Hz}, 2 \mathrm{H}), 6.19-5.98(\mathrm{~m}, 1 \mathrm{H}), 5.59-5.39(\mathrm{~m}, 1 \mathrm{H}), 5.22(\mathrm{~s}$, 2H), 3.78 (s, 3H), $3.44-3.09$ (m, 2H), $2.43(\mathrm{ddd}, J=14.2,10.8,4.2 \mathrm{~Hz}, 1 \mathrm{H}), 2.04$ (ddd, $J=13.6,9.3$, $4.5 \mathrm{~Hz}, 1 \mathrm{H}), 1.91$ (ddd, $J=12.5,10.8,4.5 \mathrm{~Hz}, 1 \mathrm{H}), 1.69$ (ddd, $J=13.4,9.3,4.2 \mathrm{~Hz}, 1 \mathrm{H}), 1.10(\mathrm{~s}, 3 \mathrm{H})$, $1.01(\mathrm{~s}, 3 \mathrm{H}), 0.90(\mathrm{~s}, 3 \mathrm{H}) .{ }^{13} \mathrm{C}$ NMR $(101 \mathrm{MHz}$, chloroform-d) $\delta 207.8,178.2,169.0(\mathrm{~d}, J=26.2 \mathrm{~Hz})$, 167.4, $156.0(\mathrm{~d}, J=27.6 \mathrm{~Hz}), 149.0,137.6,134.5,133.7,128.9,127.0,123.8,120.4(\mathrm{~d}, J=8.3 \mathrm{~Hz}), 97.0$ 
$(\mathrm{d}, J=189.4 \mathrm{~Hz}), 94.5,91.1,87.9(\mathrm{~d}, J=3.7 \mathrm{~Hz}), 67.1,54.9,54.4,53.2,36.3(\mathrm{~d}, J=20.5 \mathrm{~Hz}), 30.7$, 29.0, 16.9, 16.8, 9.8,. ${ }^{19} \mathrm{~F}$ NMR $(376 \mathrm{MHz}$, chloroform- $d$ ) $\delta-160.4(\mathrm{t}, J=24.2 \mathrm{~Hz}, 1 \mathrm{~F})$. HRMS (ESI): $[\mathrm{M}+\mathrm{H}]^{\oplus}$ calcd for $\mathrm{C}_{29} \mathrm{H}_{31} \mathrm{FNO}_{6}{ }^{\oplus} 508.2130$, found 508.2135.

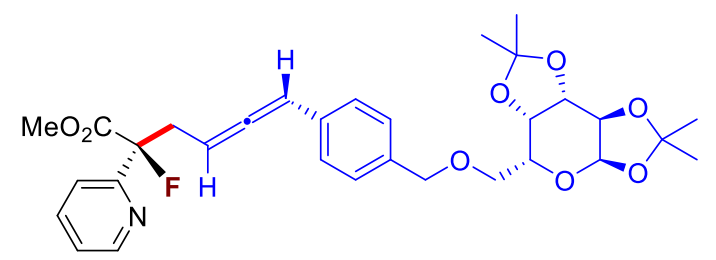

\section{Methyl (2R,5R)-2-fluoro-2-(pyridin-2-yl)-6-(4-((((3ak,5R,5aS,8aS,8bR)-2,2,7,7-} tetramethyltetrahydro-5H-bis([1,3]dioxolo $)\left[4,5-\mathrm{b}: 4^{\prime}, 5^{\prime}-\mathrm{d}\right]$ pyran-5-yl)methoxy)methyl)phenyl)hexa4,5-dienoate (3dd)

Yellow solid, $20 \mathrm{~h}, 84 \%$ yield, $10: 1 \mathrm{dr},[\alpha]_{\mathrm{D}}{ }^{25}-143.7\left(c \mathrm{c} .8, \mathrm{CHCl}_{3}\right)$ for $>99 \% \mathrm{de} ;{ }^{1} \mathrm{H}$ NMR $(500 \mathrm{MHz}$, chloroform- $d$ ) $\delta 8.58(\mathrm{~d}, J=4.4 \mathrm{~Hz}, 1 \mathrm{H}), 7.78(\mathrm{td}, J=7.7,1.8 \mathrm{~Hz}, 1 \mathrm{H}), 7.65(\mathrm{~d}, J=7.8 \mathrm{~Hz}, 1 \mathrm{H}), 7.33-$ $7.26(\mathrm{~m}, 1 \mathrm{H}), 7.21(\mathrm{~d}, J=7.8 \mathrm{~Hz}, 2 \mathrm{H}), 7.03(\mathrm{~d}, J=7.8 \mathrm{~Hz}, 2 \mathrm{H}), 6.11(\mathrm{dt}, J=6.5,2.4 \mathrm{~Hz}, 1 \mathrm{H}), 5.59-$ $5.44(\mathrm{~m}, 2 \mathrm{H}), 4.66-4.47(\mathrm{~m}, 3 \mathrm{H}), 4.35-4.20(\mathrm{~m}, 2 \mathrm{H}), 4.03-3.95(\mathrm{~m}, 1 \mathrm{H}), 3.78(\mathrm{~s}, 3 \mathrm{H}), 3.71-3.63$ (m, 1H), $3.65-3.57(\mathrm{~m}, 1 \mathrm{H}), 3.40-3.26(\mathrm{~m}, 1 \mathrm{H}), 3.27-3.13(\mathrm{~m}, 1 \mathrm{H}), 1.54(\mathrm{~s}, 3 \mathrm{H}), 1.44(\mathrm{~s}, 3 \mathrm{H}), 1.34$ (s, 6H). ${ }^{13} \mathrm{C}$ NMR (126 MHz, chloroform- $d$ ) $\delta 207.6,169.2(\mathrm{~d}, J=26.3 \mathrm{~Hz}), 156.4(\mathrm{~d}, J=27.0 \mathrm{~Hz})$, $149.3(\mathrm{~d}, J=2.3 \mathrm{~Hz}), 137.1,137.0,133.4,128.0,126.8,123.7,120.3$ (d, $J=8.3 \mathrm{~Hz}), 109.3,108.6,97.2$ $(\mathrm{d}, J=189.4 \mathrm{~Hz}), 96.4,94.6,87.7(\mathrm{~d}, J=3.7 \mathrm{~Hz}), 73.1,71.3,70.7,70.6,68.9,67.0,53.1,36.4(\mathrm{~d}, J=$ $20.5 \mathrm{~Hz}$ ), 26.2, 26.1, 25.0, 24.5. ${ }^{19} \mathrm{~F}$ NMR (376 MHz, chloroform- $d$ ) $\delta-164.7$ (t, $\left.J=24.4 \mathrm{~Hz}, 1 \mathrm{~F}\right)$. HRMS (ESI): $[\mathrm{M}+\mathrm{H}]^{\oplus}$ calcd for $\mathrm{C}_{31} \mathrm{H}_{37} \mathrm{FNO}_{8}{ }^{\oplus} 570.2498$, found 570.2499 .

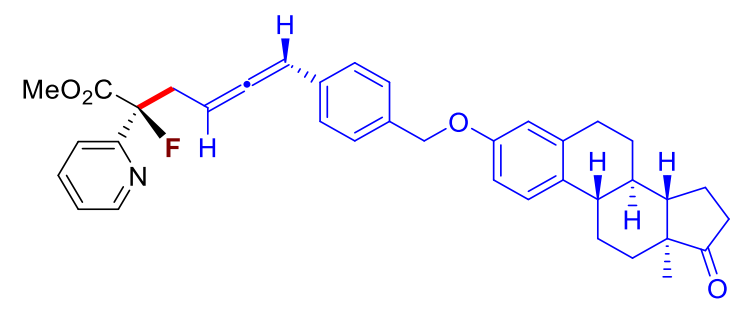

Methyl (2R,5R)-2-fluoro-6-(4-((((8R,9S,13S,14S)-13-methyl-17-oxo-7,8,9,11,12,13,14,15,16,17decahydro-6H-cyclopenta[a]phenanthren-3-yl)oxy)methyl)phenyl)-2-(pyridin-2-yl)hexa-4,5dienoate (3ee)

Yellow solid, $20 \mathrm{~h}, 58 \%$ yield, $>20: 1 \mathrm{dr},[\alpha]_{\mathrm{D}}{ }^{25}-41.5\left(c 0.7, \mathrm{CHCl}_{3}\right)$ for $>99 \%$ de; ${ }^{1} \mathrm{H}$ NMR $(400 \mathrm{MHz}$, chloroform- $d$ ) $\delta 8.59(\mathrm{~d}, J=4.8 \mathrm{~Hz}, 1 \mathrm{H}), 7.91-7.74(\mathrm{~m}, 1 \mathrm{H}), 7.67(\mathrm{~d}, J=7.9 \mathrm{~Hz}, 1 \mathrm{H}), 7.29-7.26(\mathrm{~m}$, $3 \mathrm{H}), 7.20(\mathrm{~d}, J=8.7 \mathrm{~Hz}, 1 \mathrm{H}), 7.08(\mathrm{~d}, J=7.8 \mathrm{~Hz}, 2 \mathrm{H}), 6.77(\mathrm{dd}, J=8.6,2.7 \mathrm{~Hz}, 1 \mathrm{H}), 6.72(\mathrm{~s}, 1 \mathrm{H}), 6.12$ $(\mathrm{dt}, J=6.0,2.3 \mathrm{~Hz}, 1 \mathrm{H}), 5.55-5.50(\mathrm{~m}, 1 \mathrm{H}), 5.00(\mathrm{~s}, 2 \mathrm{H}), 3.78(\mathrm{~s}, 3 \mathrm{H}), 3.47-3.09(\mathrm{~m}, 2 \mathrm{H}), 2.92-2.88$ $(\mathrm{m}, 2 \mathrm{H}), 2.57-2.21(\mathrm{~m}, 3 \mathrm{H}), 2.21-1.87(\mathrm{~m}, 4 \mathrm{H}), 1.70-1.31(\mathrm{~m}, 6 \mathrm{H}), 0.91(\mathrm{~s}, 3 \mathrm{H}) .{ }^{13} \mathrm{C}$ NMR $(101$ MHz, chloroform- $d$ ) $\delta 221.1,207.7,169.0(\mathrm{~d}, J=26.0 \mathrm{~Hz}), 156.8,156.0(\mathrm{~d}, J=27.4 \mathrm{~Hz}), 148.9,137.9$, 137.6, 135.9, 133.7, 132.4, 127.7, 127.0, 126.4, 123.8, 120.5 (d, $J=8.0 \mathrm{~Hz}), 115.0,112.5,97.1(\mathrm{~d}, J=$ $189.7 \mathrm{~Hz}), 94.6,87.7(\mathrm{~d}, J=3.5 \mathrm{~Hz}), 69.8,53.2,50.4,48.1,44.0,38.4,36.4(\mathrm{~d}, J=20.5 \mathrm{~Hz}), 36.0,31.6$, 29.7, 26.6, 26.0, 21.7, 13.9. ${ }^{19} \mathrm{~F}$ NMR (376 MHz, chloroform- $d$ ) $\delta-164.6(\mathrm{t}, J=25.3 \mathrm{~Hz}, 1 \mathrm{~F})$. HRMS (ESI): $[\mathrm{M}+\mathrm{H}]^{\oplus}$ calcd for $\mathrm{C}_{37} \mathrm{H}_{39} \mathrm{FNO}_{4}{ }^{\oplus} 580.2858$, found 580.2861 . 
<smiles>CCOC(=O)C(F)(/C=C/c1ccc(OC)cc1)c1ccccn1</smiles>

\section{Ethyl (2R,5R)-2-fluoro-6-(4-methoxyphenyl)-2-(pyridin-2-yl)hexa-4,5-dienoate (4b)}

Yellow oil, $20 \mathrm{~h}, 60 \%$ yield, $20: 1 \mathrm{dr},[\alpha]_{\mathrm{D}}{ }^{25}-216.9\left(c\right.$ 1.0, $\left.\mathrm{CHCl}_{3}\right)$ for $>99 \%$ ee; ${ }^{1} \mathrm{H} \mathrm{NMR}(400 \mathrm{MHz}$, chloroform- $d) \delta 8.61(\mathrm{~d}, J=4.9 \mathrm{~Hz}, 1 \mathrm{H}), 7.85-7.72(\mathrm{~m}, 1 \mathrm{H}), 7.66(\mathrm{~d}, J=7.9 \mathrm{~Hz}, 1 \mathrm{H}), 7.34-7.27(\mathrm{~m}$, $1 \mathrm{H}), 7.01(\mathrm{~d}, J=8.3 \mathrm{~Hz}, 2 \mathrm{H}), 6.78(\mathrm{~d}, J=8.3 \mathrm{~Hz}, 2 \mathrm{H}), 6.15-6.01(\mathrm{~m}, 1 \mathrm{H}), 5.57-5.42(\mathrm{~m}, 1 \mathrm{H}), 4.37-$ $4.04(\mathrm{~m}, 2 \mathrm{H}), 3.79(\mathrm{~s}, 3 \mathrm{H}), 3.44-3.07(\mathrm{~m}, 2 \mathrm{H}), 1.26(\mathrm{t}, J=7.1 \mathrm{~Hz}, 3 \mathrm{H}) .{ }^{13} \mathrm{C} \mathrm{NMR}(126 \mathrm{MHz}$, chloroform- $d$ ) $\delta 207.1,168.6(\mathrm{~d}, J=25.6 \mathrm{~Hz}), 158.8,156.4(\mathrm{~d}, J=25.3 \mathrm{~Hz}), 149.0,137.3,128.0,126.4$, 123.6, $120.5(\mathrm{~d}, J=9.2 \mathrm{~Hz}), 114.1,97.0(\mathrm{~d}, J=189.4 \mathrm{~Hz}), 94.3,87.7(\mathrm{~d}, J=3.7 \mathrm{~Hz}), 62.3,55.4,36.5(\mathrm{~d}$, $J=20.6 \mathrm{~Hz}), 14.1 .{ }^{19} \mathrm{~F}$ NMR $(376 \mathrm{MHz}$, chloroform- $d) \delta-164.2(\mathrm{t}, J=24.5 \mathrm{~Hz}, 1 \mathrm{~F})$. HRMS (FI): [M] ${ }^{\oplus}$ calcd for $\mathrm{C}_{20} \mathrm{H}_{20} \mathrm{FNO}_{3}{ }^{\oplus} 341.1422$, found 341.1425. HPLC analysis: Chiralpak IC column; detected at $254 \mathrm{~nm}, 40{ }^{\circ} \mathrm{C} ; 9 \%{ }^{i} \mathrm{PrOH}$ in $n$-hexane; flow $=0.7 \mathrm{~mL} / \mathrm{min}$. Retention time: $21.6 \mathrm{~min}, 24.1 \mathrm{~min}, 26.4$ $\mathrm{min}, 41.6 \mathrm{~min}$. The ee value was determined based on peaks at $21.6 \mathrm{~min}$ (major) and $26.4 \mathrm{~min}$ (minor).
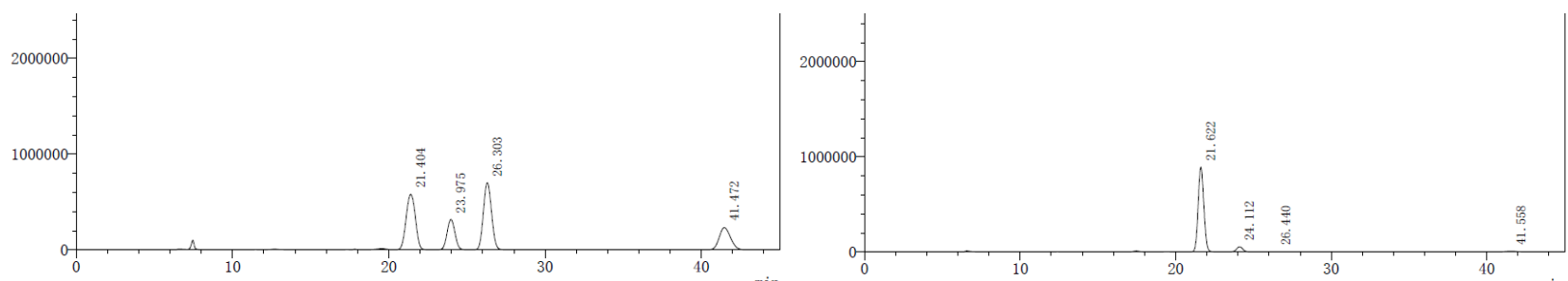

\begin{tabular}{|c|c|c|}
\hline Peak & retension time/min & area\% \\
\hline 1 & 21.404 & 33.051 \\
\hline 2 & 23.975 & 15.377 \\
\hline 3 & 26.303 & 35.331 \\
\hline 4 & 41.472 & 16.241 \\
\hline
\end{tabular}

\begin{tabular}{|c|c|c|}
\hline Peak & retension time/min & area\% \\
\hline 1 & 21.622 & 92.393 \\
\hline 2 & 24.112 & 6.150 \\
\hline 3 & 26.440 & 0.139 \\
\hline 4 & 41.558 & 1.318 \\
\hline
\end{tabular}<smiles>COc1ccc(/C=C/CC(F)(C(C)=O)c2cnccn2)cc1</smiles>

\section{Methyl (2R,5R)-2-fluoro-6-(4-methoxyphenyl)-2-(pyrazin-2-yl)hexa-4,5-dienoate (4c)}

Yellow solid, $19 \mathrm{~h}, 79 \%$ yield, $>20: 1 \mathrm{dr},[\alpha]_{\mathrm{D}}{ }^{25}-242.0\left(c 0.2, \mathrm{CHCl}_{3}\right)$ for $>99 \%$ ee; ${ }^{1} \mathrm{H} \mathrm{NMR}(400 \mathrm{MHz}$, chloroform- $d) \delta 8.97(\mathrm{~s}, 1 \mathrm{H}), 8.61-8.54(\mathrm{~m}, 2 \mathrm{H}), 7.17-6.95(\mathrm{~m}, 2 \mathrm{H}), 6.88-6.74(\mathrm{~m}, 2 \mathrm{H}), 6.10(\mathrm{dt}, J$ $=6.5,2.4 \mathrm{~Hz}, 1 \mathrm{H}), 5.52-5.45(\mathrm{~m}, 1 \mathrm{H}), 3.79(\mathrm{~s}, 3 \mathrm{H}), 3.78(\mathrm{~s}, 3 \mathrm{H}), 3.39-3.09(\mathrm{~m}, 2 \mathrm{H}) .{ }^{13} \mathrm{C}$ NMR $(126$ $\mathrm{MHz}$, chloroform- $d) \delta 207.2,168.5(\mathrm{~d}, J=26.0 \mathrm{~Hz}), 158.9,144.7,143.7,142.3(\mathrm{~d}, J=9.5 \mathrm{~Hz}), 128.1$, 127.9, 126.0, 114.1, $96.6(\mathrm{~d}, J=189.6 \mathrm{~Hz}), 94.6,87.0(\mathrm{~d}, J=3.7 \mathrm{~Hz}), 55.4,53.3,36.4(\mathrm{~d}, J=20.2 \mathrm{~Hz})$. ${ }^{19} \mathrm{~F}$ NMR (376 MHz, chloroform- $d$ ) $\delta-166.9(\mathrm{t}, J=24.0 \mathrm{~Hz}, 1 \mathrm{~F})$. HRMS (FI): $[\mathrm{M}]{ }^{\oplus}$ calcd for $\mathrm{C}_{18} \mathrm{H}_{17} \mathrm{FN}_{2} \mathrm{O}_{3}{ }^{\oplus} 328.1218$, found 328.1213. HPLC analysis: Chiralpak IA column; detected at $254 \mathrm{~nm}, 40$ ${ }^{\circ} \mathrm{C} ; 3 \%{ }^{i} \mathrm{PrOH}$ in $n$-hexane; flow $=0.7 \mathrm{~mL} / \mathrm{min}$. Retention time: $31.3 \mathrm{~min}, 32.4 \mathrm{~min}, 36.0 \mathrm{~min}, 37.4 \mathrm{~min}$. The ee value was determined based on peaks at $31.3 \mathrm{~min}$ (minor) and $32.4 \mathrm{~min}$ (major). 

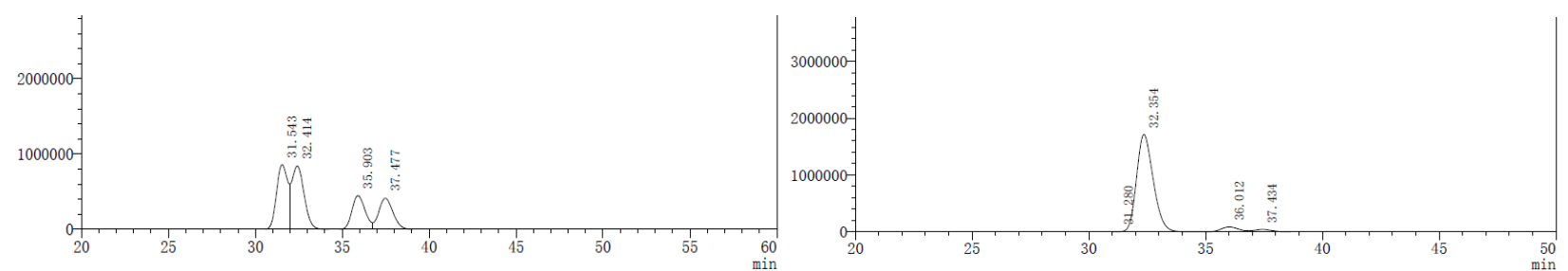

\begin{tabular}{|c|c|c|}
\hline Peak & retension time/min & area\% \\
\hline 1 & 31.543 & 29.845 \\
\hline 2 & 32.414 & 32.986 \\
\hline 3 & 35.903 & 18.551 \\
\hline 4 & 37.477 & 18.618 \\
\hline
\end{tabular}

\begin{tabular}{|c|c|c|}
\hline Peak & retension time/min & area\% \\
\hline 1 & 31.280 & 0.078 \\
\hline 2 & 32.354 & 91.756 \\
\hline 3 & 36.012 & 5.322 \\
\hline 4 & 37.434 & 2.844 \\
\hline
\end{tabular}<smiles>COc1ccc(C=C=CC[C@](F)(C(C)=O)c2ccc3ccccc3n2)cc1</smiles>

\section{Methyl (2R,5R)-2-fluoro-6-(4-methoxyphenyl)-2-(quinolin-2-yl)hexa-4,5-dienoate (4d)}

Yellow oil, $16 \mathrm{~h}, 98 \%$ yield, $13: 1 \mathrm{dr},[\alpha]_{\mathrm{D}}{ }^{25}-153.9\left(c 0.2, \mathrm{CHCl}_{3}\right)$ for $>99 \%$ ee; ${ }^{1} \mathrm{H} \mathrm{NMR}(500 \mathrm{MHz}$, chloroform- $d$ ) $\delta 8.23(\mathrm{~d}, J=8.6 \mathrm{~Hz}, 1 \mathrm{H}), 8.08(\mathrm{~d}, J=8.6 \mathrm{~Hz}, 1 \mathrm{H}), 7.85(\mathrm{~d}, J=8.0 \mathrm{~Hz}, 1 \mathrm{H}), 7.75-7.69$ $(\mathrm{m}, 2 \mathrm{H}), 7.64-7.51(\mathrm{~m}, 1 \mathrm{H}), 7.01-6.85(\mathrm{~m}, 2 \mathrm{H}), 6.67-6.48(\mathrm{~m}, 2 \mathrm{H}), 6.08(\mathrm{dt}, J=6.6,2.4 \mathrm{~Hz}, 1 \mathrm{H})$, $5.59-5.55(\mathrm{~m}, 1 \mathrm{H}), 3.76(\mathrm{~s}, 3 \mathrm{H}), 3.74(\mathrm{~s}, 3 \mathrm{H}), 3.54-3.25(\mathrm{~m}, 2 \mathrm{H}) .{ }^{13} \mathrm{C}$ NMR $(126 \mathrm{MHz}$, chloroform-d) $\delta 207.0,169.2(\mathrm{~d}, J=26.4 \mathrm{~Hz}), 158.6,156.4(\mathrm{~d}, J=27.1 \mathrm{~Hz}), 147.5(\mathrm{~d}, J=2.0 \mathrm{~Hz}), 137.2,129.9(\mathrm{~d}, J=$ 9.5 Hz), 128.0, 127.84, 127.78, 127.6, 127.2, 126.2, $117.7(\mathrm{~d}, J=7.6 \mathrm{~Hz}), 113.9,97.8(\mathrm{~d}, J=189.1 \mathrm{~Hz})$, 94.3, $87.8(\mathrm{~d}, J=3.8 \mathrm{~Hz}), 55.3,53.1,36.5(\mathrm{~d}, J=20.3 \mathrm{~Hz}) .{ }^{19} \mathrm{~F}$ NMR $(376 \mathrm{MHz}$, chloroform- $d$ ) $\delta-163.4$ (t, $J=24.4 \mathrm{~Hz}, 1 \mathrm{~F})$. HRMS (EI): $[\mathrm{M}]^{\oplus}$ calcd for $\mathrm{C}_{23} \mathrm{H}_{20} \mathrm{FNO}_{3}{ }^{\oplus} 377.1422$, found 377.1424. HPLC analysis: Chiracel AD-H column; detected at $254 \mathrm{~nm}, 40{ }^{\circ} \mathrm{C} ; 3 \%{ }^{i} \mathrm{PrOH}$ in $n$-hexane; flow $=0.7 \mathrm{~mL} / \mathrm{min}$. Retention time: $20.4 \mathrm{~min}, 22.7 \mathrm{~min}, 24.4 \mathrm{~min}, 32.2 \mathrm{~min}$. The ee value was determined based on peaks at 20.4 min (minor) and 24.4 min (major).
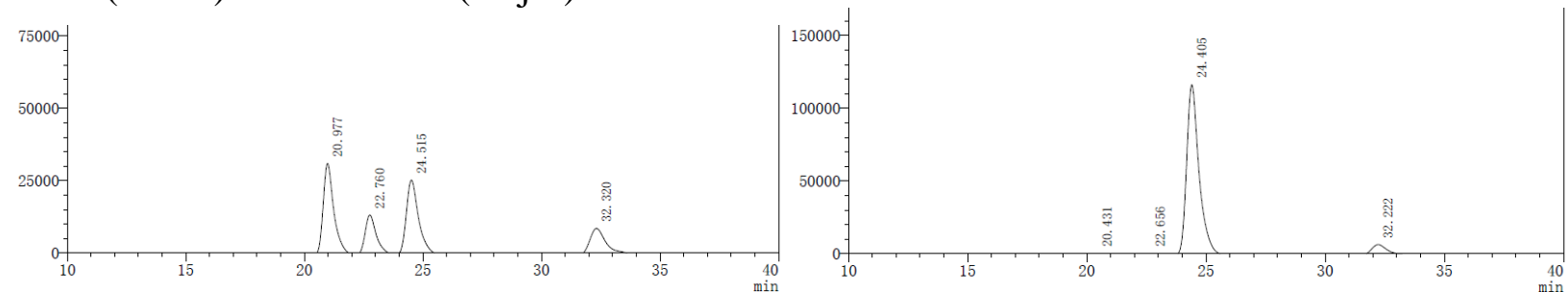

\begin{tabular}{|c|c|c|}
\hline Peak & retension time/min & area\% \\
\hline 1 & 20.977 & 34.738 \\
\hline 2 & 22.760 & 16.222 \\
\hline 3 & 24.515 & 32.677 \\
\hline 4 & 32.320 & 16.363 \\
\hline
\end{tabular}

\begin{tabular}{|c|c|c|}
\hline Peak & retension time/min & area\% \\
\hline 1 & 20.431 & 0.200 \\
\hline 2 & 22.656 & 0.633 \\
\hline 3 & 24.405 & 91.103 \\
\hline 4 & 32.222 & 8.064 \\
\hline
\end{tabular}


<smiles>COc1ccc(/C=C/CC(F)(C(C)=O)c2ncccn2)cc1</smiles>

\section{Methyl (2R,5R)-2-fluoro-6-(4-methoxyphenyl)-2-(pyrimidin-2-yl)hexa-4,5-dienoate (4e)}

For the synthesis of $\mathbf{4 e}, 10 \mathrm{~mol} \%[\mathrm{Cu}] /(S, S)-\mathrm{BPE}, \mathrm{Et}_{3} \mathrm{~N}$ (2 equiv), DBU (2 mol\%), THF as the solvent were used instead. Yellow oil, $9 \mathrm{~h}, 52 \%$ yield, 8:1 dr, $[\alpha]_{\mathrm{D}}{ }^{25}-172.6\left(c 0.2, \mathrm{CHCl}_{3}\right)$ for $>99 \%$ ee; ${ }^{1} \mathrm{H}$ NMR (500 MHz, chloroform- $d) \delta 8.80(\mathrm{~m}, 2 \mathrm{H}), 7.31(\mathrm{t}, J=4.8 \mathrm{~Hz}, 1 \mathrm{H}), 7.22-7.08(\mathrm{~m}, 2 \mathrm{H}), 6.90-$ $6.72(\mathrm{~m}, 2 \mathrm{H}), 6.12(\mathrm{dt}, J=6.4,2.4 \mathrm{~Hz}, 1 \mathrm{H}), 5.62-5.57(\mathrm{~m}, 1 \mathrm{H}), 3.83(\mathrm{~s}, 3 \mathrm{H}), 3.79(\mathrm{~s}, 3 \mathrm{H}), 3.44-3.18$ $(\mathrm{m}, 2 \mathrm{H}) .{ }^{13} \mathrm{C}$ NMR $(126 \mathrm{MHz}$, chloroform- $d) \delta 207.1,168.9(\mathrm{~d}, J=26.1 \mathrm{~Hz}), 164.8(\mathrm{~d}, J=22.0 \mathrm{~Hz})$, 158.8, 157.6, 128.1, 126.3, $121.1(\mathrm{~d}, J=1.7 \mathrm{~Hz}), 114.1,97.1(\mathrm{~d}, J=192.8 \mathrm{~Hz}), 94.4,87.5(\mathrm{~d}, J=3.7$ $\mathrm{Hz}), 55.4,53.1,35.9(\mathrm{~d}, J=21.6 \mathrm{~Hz}) .{ }^{19} \mathrm{~F}$ NMR $(376 \mathrm{MHz}$, chloroform- $d$ ) $\delta-160.3(\mathrm{dd}, J=28.4,18.8$ $\mathrm{Hz}, 1 \mathrm{~F}$ ). HRMS (FI): [M] ${ }^{\oplus}$ calcd for $\mathrm{C}_{18} \mathrm{H}_{17} \mathrm{FN}_{2} \mathrm{O}_{3}{ }^{\oplus}$ 328.1218, found 328.1214. HPLC analysis: Chiracel OD-H + Chiracel AD-H column (the two columns were connected to each other in this order); detected at $254 \mathrm{~nm}, 40{ }^{\circ} \mathrm{C} ; 9 \%{ }^{i} \mathrm{PrOH}$ in $n$-hexane; flow $=0.7 \mathrm{~mL} / \mathrm{min}$. Retention time: $67.3 \mathrm{~min}, 70.4 \mathrm{~min}, 72.0$ min, $78.8 \mathrm{~min}$. The ee value was determined based on peaks at $67.3 \mathrm{~min}$ (minor) and $72.0 \mathrm{~min}$ (major).
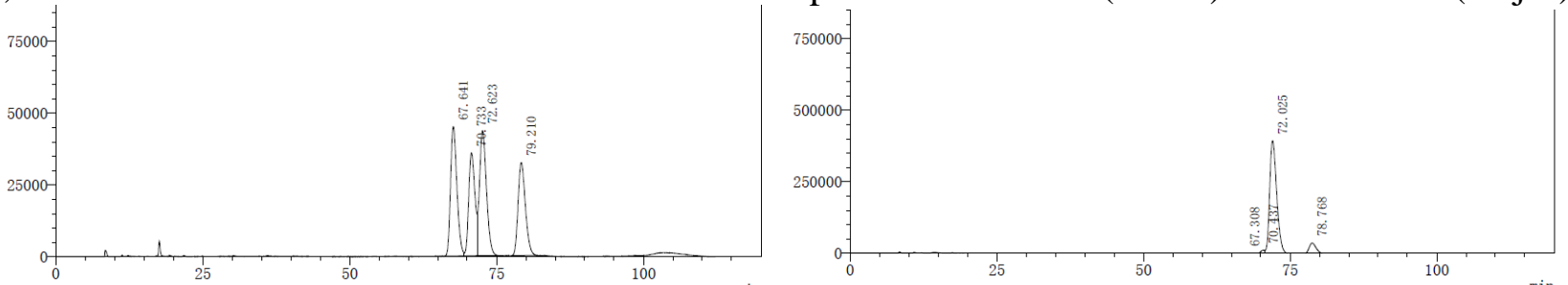

\begin{tabular}{|c|c|c|}
\hline Peak & retension time/min & area\% \\
\hline 1 & 67.641 & 27.372 \\
\hline 2 & 70.733 & 21.043 \\
\hline 3 & 72.623 & 29.035 \\
\hline 4 & 79.210 & 22.550 \\
\hline
\end{tabular}

\begin{tabular}{|c|c|c|}
\hline Peak & retension time/min & area\% \\
\hline 1 & 67.308 & 0.214 \\
\hline 2 & 70.437 & 1.697 \\
\hline 3 & 72.025 & 89.163 \\
\hline 4 & 78.768 & 8.926 \\
\hline
\end{tabular}<smiles>COc1ccc(/C=C/CC(F)(C(C)=O)c2cc(OC)ccn2)cc1</smiles>

\section{Methyl (2R,5R)-2-fluoro-6-(4-methoxyphenyl)-2-(4-methoxypyridin-2-yl)hexa-4,5-dienoate (4f)}

Yellow oil, $16 \mathrm{~h}, 73 \%$ yield, $20: 1 \mathrm{dr},[\alpha]_{\mathrm{D}}{ }^{25}-174.8\left(c 0.2, \mathrm{CHCl}_{3}\right)$ for $>99 \%$ ee; ${ }^{1} \mathrm{H} \mathrm{NMR}(500 \mathrm{MHz}$, chloroform- $d) \delta 8.39(\mathrm{~d}, J=5.7 \mathrm{~Hz}, 1 \mathrm{H}), 7.17-7.15(\mathrm{~m}, 1 \mathrm{H}), 7.11-6.94(\mathrm{~m}, 2 \mathrm{H}), 6.87-6.77(\mathrm{~m}, 3 \mathrm{H})$, $6.09(\mathrm{dt}, J=6.6,2.4 \mathrm{~Hz}, 1 \mathrm{H}), 5.51-5.46(\mathrm{~m}, 1 \mathrm{H}), 3.86(\mathrm{~s}, 3 \mathrm{H}), 3.79(\mathrm{~s}, 3 \mathrm{H}), 3.77(\mathrm{~s}, 3 \mathrm{H}), 3.41-3.24$ $(\mathrm{m}, 1 \mathrm{H}), 3.22-3.08(\mathrm{~m}, 1 \mathrm{H}) .{ }^{13} \mathrm{C}$ NMR $(126 \mathrm{MHz}$, chloroform-d) $\delta 207.0,169.1(\mathrm{~d}, J=26.2 \mathrm{~Hz}), 166.7$ $(\mathrm{d}, J=1.6 \mathrm{~Hz}), 158.8,158.2(\mathrm{~d}, J=26.5 \mathrm{~Hz}), 150.5(\mathrm{~d}, J=2.3 \mathrm{~Hz}), 128.0,126.3,114.0,110.1,106.0$ (d, $J=9.5 \mathrm{~Hz}), 97.2(\mathrm{~d}, J=189.5 \mathrm{~Hz}), 94.3,87.6(\mathrm{~d}, J=3.7 \mathrm{~Hz}), 55.41,55.35,53.1,36.6(\mathrm{~d}, J=20.4 \mathrm{~Hz})$. ${ }^{19} \mathrm{~F}$ NMR $\left(376 \mathrm{MHz}\right.$, chloroform- $d$ ) $\delta-165.9(\mathrm{~d}, J=24.5 \mathrm{~Hz}, 1 \mathrm{~F})$. HRMS (ESI): $[\mathrm{M}+\mathrm{H}]{ }^{\oplus}$ calcd for $\mathrm{C}_{20} \mathrm{H}_{21} \mathrm{FNO}_{4}{ }^{\oplus} 358.1449$, found 358.1448. HPLC analysis: Chiracel AD-H column; detected at $254 \mathrm{~nm}$, $40{ }^{\circ} \mathrm{C} ; 3 \%{ }^{i} \mathrm{PrOH}$ in $n$-hexane; flow $=0.7 \mathrm{~mL} / \mathrm{min}$. Retention time: $35.2 \mathrm{~min}, 40.4 \mathrm{~min}, 43.3 \mathrm{~min}, 51.4$ min. The ee value was determined based on peaks at $35.2 \mathrm{~min}$ (minor) and $40.4 \mathrm{~min}$ (major). 

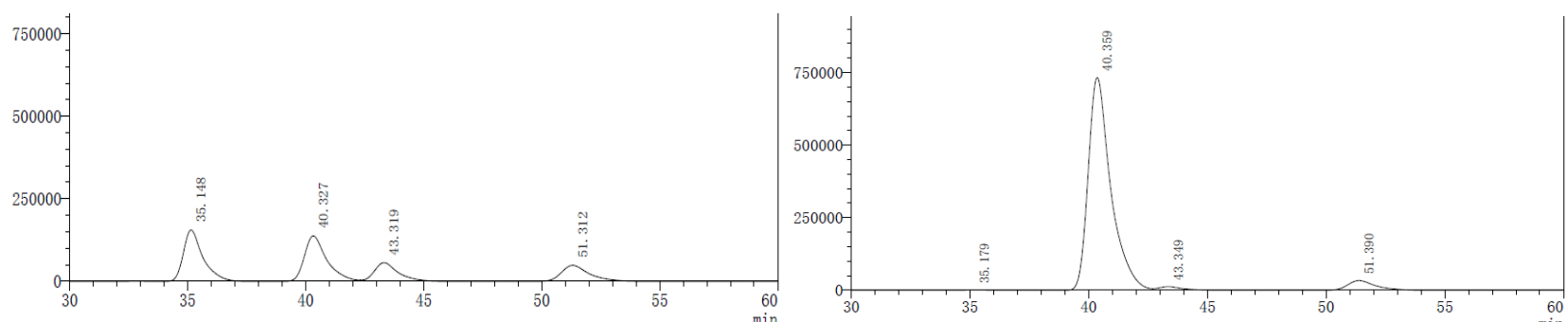

\begin{tabular}{|c|c|c|}
\hline Peak & retension time/min & area\% \\
\hline 1 & 35.148 & 34.457 \\
\hline 2 & 40.327 & 35.007 \\
\hline 3 & 43.319 & 15.342 \\
\hline 4 & 51.312 & 15.193 \\
\hline
\end{tabular}

\begin{tabular}{|c|c|c|}
\hline Peak & retension time/min & area\% \\
\hline 1 & 35.179 & 0.153 \\
\hline 2 & 40.359 & 92.901 \\
\hline 3 & 43.349 & 1.721 \\
\hline 4 & 51.390 & 5.225 \\
\hline
\end{tabular}<smiles>COc1ccc(/C=C/CC(F)(C(C)=O)c2ccc(Cl)cn2)cc1</smiles>

Methyl (2R,5R)-2-(5-chloropyridin-2-yl)-2-fluoro-6-(4-methoxyphenyl)hexa-4,5-dienoate (4g)

Yellow solid, $7 \mathrm{~h}, 83 \%$ yield, $11: 1 \mathrm{dr},[\alpha]_{\mathrm{D}}{ }^{25}-194.1\left(c 0.2, \mathrm{CHCl}_{3}\right)$ for $>99 \%$ ee; ${ }^{1} \mathrm{H} \mathrm{NMR}(500 \mathrm{MHz}$, chloroform- $d$ ) $\delta 8.51(\mathrm{~d}, J=2.4 \mathrm{~Hz}, 1 \mathrm{H}), 7.75(\mathrm{dd}, J=8.5,2.4 \mathrm{~Hz}, 1 \mathrm{H}), 7.60(\mathrm{dd}, J=8.5,1.6 \mathrm{~Hz}, 1 \mathrm{H})$, $7.02-6.92(\mathrm{~m}, 2 \mathrm{H}), 6.89-6.73(\mathrm{~m}, 2 \mathrm{H}), 6.08(\mathrm{dt}, J=6.7,2.4 \mathrm{~Hz}, 1 \mathrm{H}), 5.48-5.44(\mathrm{~m}, 1 \mathrm{H}), 3.79(\mathrm{~s}$, $3 \mathrm{H}), 3.77$ (s, 3H), $3.39-3.09(\mathrm{~m}, 2 \mathrm{H}) .{ }^{13} \mathrm{C}$ NMR $(126 \mathrm{MHz}$, chloroform- $d$ ) $\delta 207.1,168.8(\mathrm{~d}, J=26.2$ $\mathrm{Hz}), 158.8,154.5(\mathrm{~d}, J=27.9 \mathrm{~Hz}), 148.3(\mathrm{~d}, J=2.1 \mathrm{~Hz}), 136.8,132.2,127.9,126.1,121.3(\mathrm{~d}, J=8.4$ $\mathrm{Hz}), 114.1,97.0(\mathrm{~d}, J=189.4 \mathrm{~Hz}), 94.4,87.3(\mathrm{~d}, J=3.9 \mathrm{~Hz}), 55.4,53.2,36.5(\mathrm{~d}, J=20.2 \mathrm{~Hz}) .{ }^{19} \mathrm{~F}$ NMR (376 MHz, chloroform- $d$ ) $\delta-165.78$ (t, $J=24.5 \mathrm{~Hz}, 1 \mathrm{~F})$. HRMS (ESI): $[\mathrm{M}+\mathrm{H}]^{\oplus}$ calcd for $\mathrm{C}_{19} \mathrm{H}_{18} \mathrm{O}_{3} \mathrm{NClF}$ ${ }^{\oplus}$ 362.0954, found 362.0954. HPLC analysis: Chiralpak IC column; detected at $254 \mathrm{~nm}, 40{ }^{\circ} \mathrm{C} ; 9 \%$ ${ }^{i} \mathrm{PrOH}$ in $n$-hexane; flow $=0.7 \mathrm{~mL} / \mathrm{min}$. Retention time: $14.1 \mathrm{~min}, 14.6 \mathrm{~min}, 15.4 \mathrm{~min}, 15.7 \mathrm{~min}$. The ee value was determined based on peaks at $14.1 \mathrm{~min}$ (minor) and $14.6 \mathrm{~min}$ (major).
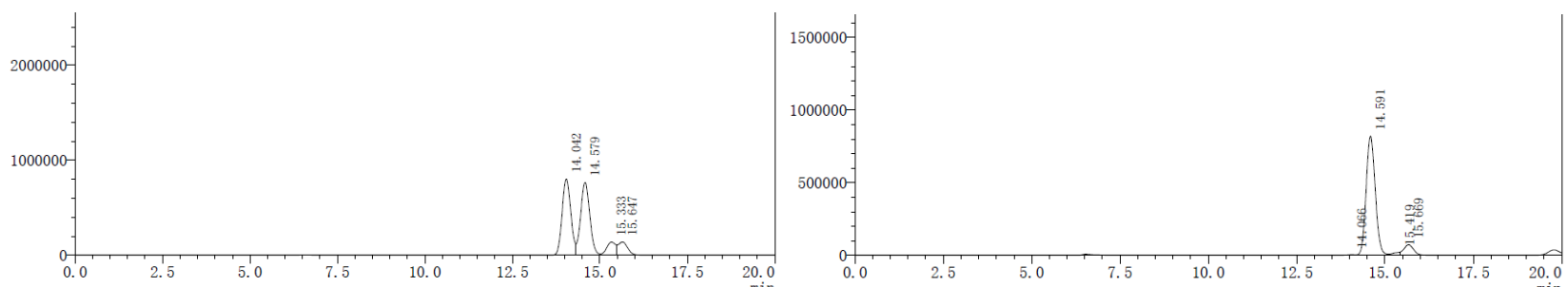

\begin{tabular}{|c|c|c|}
\hline Peak & retension time/min & area\% \\
\hline 1 & 14.042 & 42.146 \\
\hline 2 & 14.579 & 42.348 \\
\hline 3 & 15.333 & 7.571 \\
\hline 4 & 15.647 & 7.935 \\
\hline
\end{tabular}

\begin{tabular}{|c|c|c|}
\hline Peak & retension time/min & area\% \\
\hline 1 & 14.066 & 0.304 \\
\hline 2 & 14.591 & 89.532 \\
\hline 3 & 15.419 & 1.698 \\
\hline 4 & 15.669 & 8.466 \\
\hline
\end{tabular}




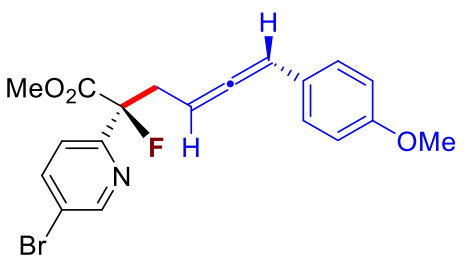

\section{Methyl (2R,5R)-2-(5-bromopyridin-2-yl)-2-fluoro-6-(4-methoxyphenyl)hexa-4,5-dienoate (4h)}

Yellow solid, $12 \mathrm{~h}, 89 \%$ yield, $10: 1 \mathrm{dr},[\alpha]_{\mathrm{D}}{ }^{25}-278.9\left(c 0.1, \mathrm{CHCl}_{3}\right)$ for $>99 \%$ ee; ${ }^{1} \mathrm{H} \mathrm{NMR}(500 \mathrm{MHz}$, chloroform- $d) \delta 8.61(\mathrm{~d}, J=2.3 \mathrm{~Hz}, 1 \mathrm{H}), 7.90(\mathrm{dd}, J=8.4,2.4 \mathrm{~Hz}, 1 \mathrm{H}), 7.55(\mathrm{dd}, J=8.5,1.7 \mathrm{~Hz}, 1 \mathrm{H})$, $7.16-6.87(\mathrm{~m}, 2 \mathrm{H}), 6.85-6.75(\mathrm{~m}, 2 \mathrm{H}), 6.07(\mathrm{dt}, J=6.7,2.4 \mathrm{~Hz}, 1 \mathrm{H}), 5.47-5.73(\mathrm{~m}, 1 \mathrm{H}), 3.79(\mathrm{~s}$, $3 \mathrm{H}), 3.76(\mathrm{~s}, 3 \mathrm{H}), 3.36-3.06(\mathrm{~m}, 2 \mathrm{H}) .{ }^{13} \mathrm{C} \mathrm{NMR}(126 \mathrm{MHz}$, chloroform- $d$ ) $\delta 207.1,168.7(\mathrm{~d}, J=26.1$ $\mathrm{Hz}), 158.8,154.9(\mathrm{~d}, J=27.8 \mathrm{~Hz}), 150.5(\mathrm{~d}, J=2.3 \mathrm{~Hz}), 139.7,127.9,126.1,121.8(\mathrm{~d}, J=8.4 \mathrm{~Hz})$, 120.9, 114.1, 97.1 (d, $J=189.4 \mathrm{~Hz}), 94.4,87.3(\mathrm{~d}, J=3.9 \mathrm{~Hz}), 55.4,53.2,36.4(\mathrm{~d}, J=20.1 \mathrm{~Hz}) .{ }^{19} \mathrm{~F}$ NMR (376 MHz, chloroform- $d$ ) $\delta-164.82(\mathrm{t}, J=24.5 \mathrm{~Hz}, 1 \mathrm{~F})$. HRMS (ESI): $[\mathrm{M}+\mathrm{H}]^{\oplus}$ calcd for $\mathrm{C}_{19} \mathrm{H}_{18} \mathrm{O}_{3} \mathrm{NBrF}^{\oplus}$ 406.0449, found 406.0450. HPLC analysis: Chiralpak IC column; detected at $254 \mathrm{~nm}$, $40{ }^{\circ} \mathrm{C} ; 3 \%{ }^{i} \mathrm{PrOH}$ in $n$-hexane; flow $=0.7 \mathrm{~mL} / \mathrm{min}$. Retention time: $16.8 \mathrm{~min}, 18.5 \mathrm{~min}, 20.5 \mathrm{~min}, 21.0$ min. The ee value was determined based on peaks at $16.8 \mathrm{~min}$ (minor) and $18.5 \mathrm{~min}$ (major).
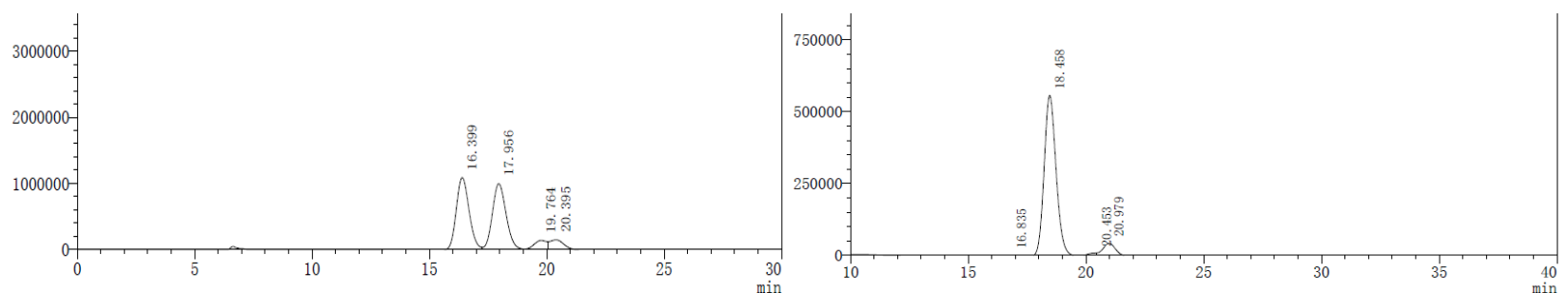

\begin{tabular}{|c|c|c|}
\hline Peak & retension time/min & area\% \\
\hline 1 & 16.399 & 43.914 \\
\hline 2 & 17.956 & 43.313 \\
\hline 3 & 19.764 & 5.802 \\
\hline 4 & 20.395 & 6.971 \\
\hline
\end{tabular}

\begin{tabular}{|c|c|c|}
\hline Peak & retension time/min & area\% \\
\hline 1 & 16.835 & 0.326 \\
\hline 2 & 18.458 & 90.028 \\
\hline 3 & 20.453 & 1.488 \\
\hline 4 & 20.979 & 8.159 \\
\hline
\end{tabular}<smiles>CC(=O)C(F)(CC=Cc1ccc(C(F)(F)F)cc1)c1cnccn1</smiles>

Methyl (2R,5R)-2-fluoro-2-(pyrazin-2-yl)-6-(4-(trifluoromethyl)phenyl)hexa-4,5-dienoate (4i)

White solid, $20 \mathrm{~h}, 72 \%$ yield, $>20: 1 \mathrm{dr},[\alpha]_{\mathrm{D}}{ }^{25}-196.2\left(c 0.2, \mathrm{CHCl}_{3}\right)$ for $>99 \%$ ee; ${ }^{1} \mathrm{H} \mathrm{NMR}(500 \mathrm{MHz}$, chloroform- $d$ ) $\delta 8.94(\mathrm{~s}, 1 \mathrm{H}), 8.59(\mathrm{~d}, J=2.5 \mathrm{~Hz}, 1 \mathrm{H}), 8.55-8.45(\mathrm{~m}, 1 \mathrm{H}), 7.49(\mathrm{~d}, J=8.0 \mathrm{~Hz}, 2 \mathrm{H})$, $7.18(\mathrm{~d}, J=8.0 \mathrm{~Hz}, 2 \mathrm{H}), 6.17(\mathrm{dt}, J=6.6,2.4 \mathrm{~Hz}, 1 \mathrm{H}), 5.64-5.50(\mathrm{~m}, 1 \mathrm{H}), 3.79(\mathrm{~s}, 3 \mathrm{H}), 3.42-3.28(\mathrm{~m}$, $1 \mathrm{H}), 3.28-3.15(\mathrm{~m}, 1 \mathrm{H}) .{ }^{13} \mathrm{C}$ NMR $(126 \mathrm{MHz}$, chloroform- $d) \delta 208.4,168.3(\mathrm{~d}, J=25.9 \mathrm{~Hz}), 151.8(\mathrm{~d}$, $J=26.9 \mathrm{~Hz}), 144.9,143.7(\mathrm{~d}, J=2.1 \mathrm{~Hz}), 142.4(\mathrm{~d}, J=9.2 \mathrm{~Hz}), 137.8,129.0(\mathrm{q}, J=32.4 \mathrm{~Hz}), 126.9$, $125.6(\mathrm{q}, J=3.8 \mathrm{~Hz}), 124.2(\mathrm{q}, J=271.9 \mathrm{~Hz}), 96.4(\mathrm{~d}, J=190.0 \mathrm{~Hz}), 94.3,87.8(\mathrm{~d}, J=3.7 \mathrm{~Hz}), 53.4$, $35.8(\mathrm{~d}, J=20.2 \mathrm{~Hz}) .{ }^{19} \mathrm{~F}$ NMR $(376 \mathrm{MHz}$, chloroform- $d) \delta-62.5(\mathrm{~s}, 3 \mathrm{~F}),-167.0(\mathrm{t}, J=23.8 \mathrm{~Hz}, 1 \mathrm{~F})$. HRMS (EI): $[\mathrm{M}]^{\oplus}$ calcd for $\mathrm{C}_{18} \mathrm{H}_{14} \mathrm{~F}_{4} \mathrm{~N}_{2} \mathrm{O}_{2}{ }^{\oplus}$ 366.0986, found 366.0992. HPLC analysis: Chiracel OD-H column; detected at $254 \mathrm{~nm}, 40{ }^{\circ} \mathrm{C} ; 3 \%{ }^{i} \mathrm{PrOH}$ in $n$-hexane; flow $=0.7 \mathrm{~mL} / \mathrm{min}$. Retention time: 10.3 
min, $11.0 \mathrm{~min}, 12.6 \mathrm{~min}, 14.1 \mathrm{~min}$. The ee value was determined based on peaks at $10.3 \mathrm{~min}$ (minor) and 12.6 min (major).
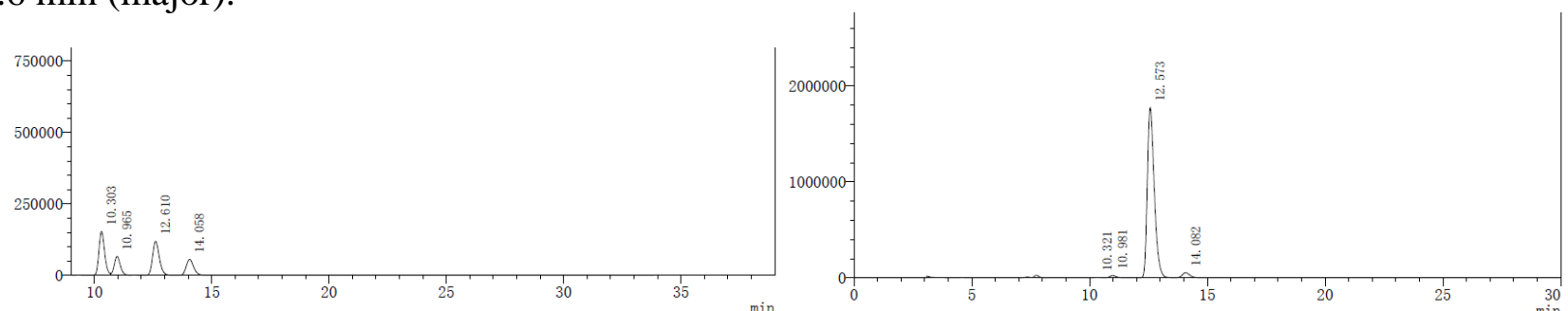

\begin{tabular}{|c|c|c|}
\hline Peak & retension time/min & area\% \\
\hline 1 & 10.303 & 34.972 \\
\hline 2 & 10.965 & 16.129 \\
\hline 3 & 12.610 & 32.279 \\
\hline 4 & 14.058 & 16.619 \\
\hline
\end{tabular}

\begin{tabular}{|c|c|c|}
\hline Peak & retension time/min & area\% \\
\hline 1 & 10.321 & 0.120 \\
\hline 2 & 10.981 & 1.073 \\
\hline 3 & 12.573 & 95.705 \\
\hline 4 & 14.082 & 3.102 \\
\hline
\end{tabular}<smiles>COC(=O)C(F)(CC=C=Cc1ccc(C(F)(F)F)cc1)c1ccc2ccccc2n1</smiles>

\section{Methyl (2R,5R)-2-fluoro-2-(quinolin-2-yl)-6-(4-(trifluoromethyl)phenyl)hexa-4,5-dienoate (4j)}

Yellow solid, $20 \mathrm{~h}, 96 \%$ yield, $>20: 1 \mathrm{dr},[\alpha]_{\mathrm{D}}{ }^{25}-224.2\left(c 0.2, \mathrm{CHCl}_{3}\right)$ for $>99 \%$ ee; ${ }^{1} \mathrm{H} \mathrm{NMR}(500 \mathrm{MHz}$, chloroform- $d$ ) $\delta 8.24(\mathrm{~d}, J=8.6 \mathrm{~Hz}, 1 \mathrm{H}), 8.02(\mathrm{~d}, J=8.4 \mathrm{~Hz}, 1 \mathrm{H}), 7.84(\mathrm{~d}, J=8.3 \mathrm{~Hz}, 1 \mathrm{H}), 7.75(\mathrm{dd}, J=$ 8.7, $1.9 \mathrm{~Hz}, 1 \mathrm{H}), 7.71-7.61(\mathrm{~m}, 1 \mathrm{H}), 7.59-7.56(\mathrm{~m}, 1 \mathrm{H}), 7.21(\mathrm{~d}, J=8.0 \mathrm{~Hz}, 2 \mathrm{H}), 6.97(\mathrm{~d}, J=7.9 \mathrm{~Hz}$, $2 \mathrm{H}), 6.20-5.98(\mathrm{~m}, 1 \mathrm{H}), 5.66-5.62(\mathrm{~m}, 1 \mathrm{H}), 3.77(\mathrm{~s}, 3 \mathrm{H}), 3.52-3.45(\mathrm{~m}, 1 \mathrm{H}), 3.45-3.39(\mathrm{~m}, 1 \mathrm{H})$. ${ }^{13} \mathrm{C}$ NMR $(126 \mathrm{MHz}$, chloroform- $d) \delta 208.4,169.1(\mathrm{~d}, J=26.2 \mathrm{~Hz}), 156.0(\mathrm{~d}, J=27.2 \mathrm{~Hz}), 147.5(\mathrm{~d}, J=$ $2.2 \mathrm{~Hz}), 137.8,137.3(\mathrm{~d}, J=1.6 \mathrm{~Hz}), 130.0,129.8,128.6(\mathrm{q}, J=32.4 \mathrm{~Hz}), 127.7,127.6,127.4,126.7$, $125.3(\mathrm{t}, J=271.8 \mathrm{~Hz}), 125.2(\mathrm{q}, J=3.8 \mathrm{~Hz}), 117.6(\mathrm{~d}, J=7.8 \mathrm{~Hz}), 97.7(\mathrm{~d}, J=189.2 \mathrm{~Hz}), 93.9,88.5(\mathrm{~d}$, $J=3.9 \mathrm{~Hz}), 53.2,35.9(\mathrm{~d}, J=20.2 \mathrm{~Hz}) .{ }^{19} \mathrm{~F} \mathrm{NMR}(376 \mathrm{MHz}$, chloroform- $d$ ) $\delta-62.5(\mathrm{~s}, 3 \mathrm{~F}),-167.0(\mathrm{t}, J$ $=23.8 \mathrm{~Hz}, 1 \mathrm{~F})$. HRMS (FI): $[\mathrm{M}]^{\oplus}$ calcd for $\mathrm{C}_{23} \mathrm{H}_{17} \mathrm{~F}_{4} \mathrm{NO}_{2}{ }^{\oplus}$ 415.1190, found 415.1194. HPLC analysis: Chiracel OD-H column; detected at $254 \mathrm{~nm}, 40{ }^{\circ} \mathrm{C} ; 3 \%{ }^{i} \mathrm{PrOH}$ in $n$-hexane; flow $=0.7 \mathrm{~mL} / \mathrm{min}$. Retention time: $7.3 \mathrm{~min}, 8.1 \mathrm{~min}, 9.5 \mathrm{~min}, 15.8 \mathrm{~min}$. The ee value was determined based on peaks at 7.3 min (minor) and $9.5 \mathrm{~min}$ (minor).
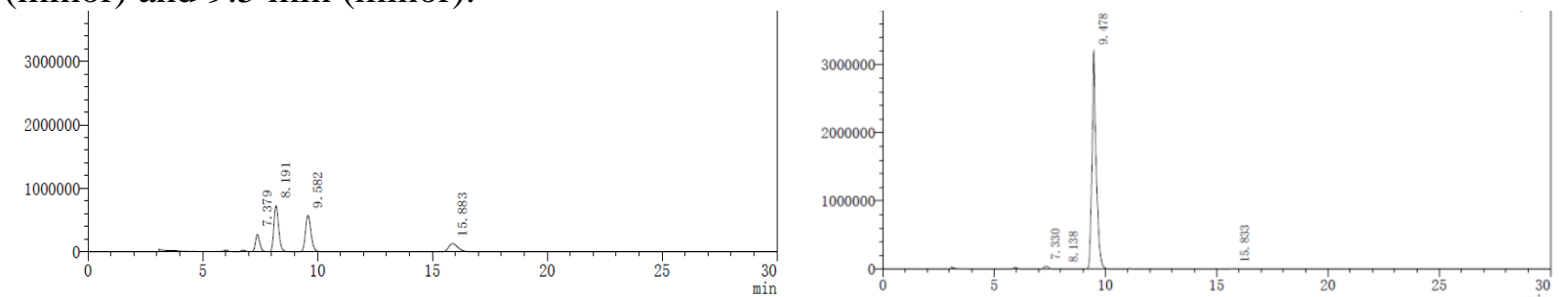

\begin{tabular}{|c|c|c|}
\hline Peak & retension time/min & area\% \\
\hline 1 & 7.379 & 13.434 \\
\hline 2 & 8.191 & 38.083 \\
\hline 3 & 9.582 & 35.128 \\
\hline 4 & 15.883 & 13.355 \\
\hline
\end{tabular}

\begin{tabular}{|c|c|c|}
\hline Peak & retension time/min & area\% \\
\hline 1 & 7.330 & 1.256 \\
\hline 2 & 8.138 & 0.045 \\
\hline 3 & 9.478 & 98.334 \\
\hline 4 & 15.833 & 0.365 \\
\hline
\end{tabular}


<smiles></smiles>

\section{Methyl (2S,5R)-2-(benzo[d]thiazol-2-yl)-2-fluoro-6-(4-(trifluoromethyl)phenyl)hexa-4,5-dienoate} $(4 \mathbf{k})$

For the synthesis of $\mathbf{4 k}, 10 \mathrm{~mol} \%[\mathrm{Cu}] /(S, S)-\mathrm{BPE}, \mathrm{Et}_{3} \mathrm{~N}(5 \mathrm{~mol} \%)$, without DBU, toluene as the solvent were used instead. Yellow solid, $12 \mathrm{~h}, 98 \%$ yield, $18: 1 \mathrm{dr},[\alpha]_{\mathrm{D}}{ }^{25}-175.9\left(c 1.0, \mathrm{CHCl}_{3}\right)$ for $>99 \%$ ee; ${ }^{1} \mathrm{H}$ NMR (400 MHz, chloroform- $d$ ) $\delta 8.02-7.87(\mathrm{~m}, 2 \mathrm{H}), 7.52-7.43(\mathrm{~m}, 2 \mathrm{H}), 7.22(\mathrm{~d}, J=8.0 \mathrm{~Hz}, 2 \mathrm{H})$, $7.03(\mathrm{~d}, J=8.0 \mathrm{~Hz}, 2 \mathrm{H}), 6.17(\mathrm{dt}, J=6.5,2.3 \mathrm{~Hz}, 1 \mathrm{H}), 5.67-5.61(\mathrm{~m}, 1 \mathrm{H}), 3.84(\mathrm{~s}, 3 \mathrm{H}), 3.47-3.33(\mathrm{~m}$, $2 \mathrm{H}) .{ }^{13} \mathrm{C}$ NMR (101 MHz, chloroform- $d$ ) $\delta 208.7,167.2(\mathrm{~d}, J=26.4 \mathrm{~Hz}), 166.2(\mathrm{~d}, J=38.8 \mathrm{~Hz}), 153.2$, $137.5,135.2,128.8(\mathrm{q}, J=32.6 \mathrm{~Hz}), 126.9,126.7,126.1,125.3(\mathrm{q}, J=3.9 \mathrm{~Hz}), 124.8(\mathrm{q}, J=150.5 \mathrm{~Hz})$, 124.0, 121.9, $96.4(\mathrm{~d}, J=189.3 \mathrm{~Hz}), 94.4,87.5(\mathrm{~d}, J=3.6 \mathrm{~Hz}), 53.7,36.9(\mathrm{~d}, J=19.9 \mathrm{~Hz}) .{ }^{19} \mathrm{~F}$ NMR (376 MHz, chloroform- $d$ ) $\delta-58.4(\mathrm{~s}, 3 \mathrm{~F}),-151.4(\mathrm{t}, J=22.6 \mathrm{~Hz}, 1 \mathrm{~F})$. HRMS (EI): [M] ${ }^{\oplus}$ calcd for $\mathrm{C}_{21} \mathrm{H}_{15} \mathrm{~F}_{4} \mathrm{NO}_{2} \mathrm{~S}^{\oplus}$ 421.0754, found 421.0756. HPLC analysis: Chiracel AD-H + Chiralpak IG-3 column (the two columns were connected to each other in this order); detected at $254 \mathrm{~nm}, 40{ }^{\circ} \mathrm{C} ; 1 \%{ }^{i} \mathrm{PrOH}$ in $n$ Hexane; flow = $0.7 \mathrm{~mL} / \mathrm{min}$. Retention time: $36.8 \mathrm{~min}, 44.9 \mathrm{~min}, 50.1 \mathrm{~min}, 92.4 \mathrm{~min}$. The ee value was determined based on peaks at $36.8 \mathrm{~min}$ (minor) and $50.1 \mathrm{~min}$ (major).
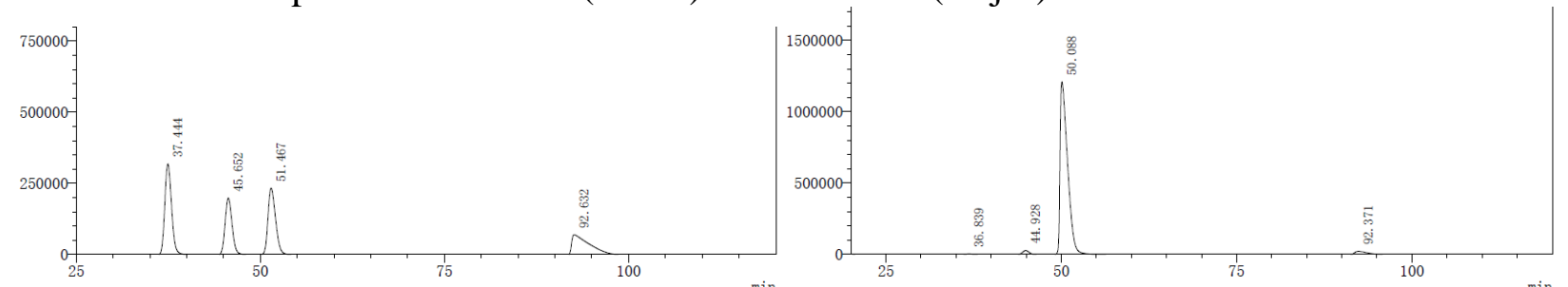

\begin{tabular}{|c|c|c|}
\hline Peak & retension time/min & area\% \\
\hline 1 & 37.444 & 32.789 \\
\hline 2 & 45.652 & 21.291 \\
\hline 3 & 51.467 & 26.967 \\
\hline 4 & 92.632 & 18.953 \\
\hline
\end{tabular}

\begin{tabular}{|c|c|c|}
\hline Peak & retension time/min & area\% \\
\hline 1 & 36.839 & 0.416 \\
\hline 2 & 44.928 & 1.959 \\
\hline 3 & 50.088 & 94.666 \\
\hline 4 & 92.371 & 2.958 \\
\hline
\end{tabular}

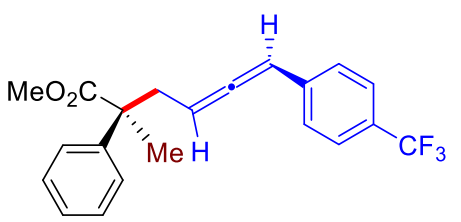

\section{Methyl (2S,5R)-2-methyl-2-phenyl-6-(4-(trifluoromethyl)phenyl)hexa-4,5-dienoate (4l)}

For the synthesis of $\mathbf{4 l},(S)$-L6 $(5 \mathrm{~mol} \%)$, $[\mathrm{Cu}] /(R, R)$-BPE (5 mol\%) were used instead. Yellow solid, 20 h, 34\% yield, $13: 1 \mathrm{dr},[\alpha]_{\mathrm{D}}{ }^{25} 118.0\left(c 0.7, \mathrm{CHCl}_{3}\right)$ for $98 \%$ ee; ${ }^{1} \mathrm{H}$ NMR $(500 \mathrm{MHz}$, Chloroform- $d) \delta$ $8.62-8.50(\mathrm{~m}, 1 \mathrm{H}), 7.66(\mathrm{td}, J=7.7,1.9 \mathrm{~Hz}, 1 \mathrm{H}), 7.49(\mathrm{~d}, J=8.1 \mathrm{~Hz}, 2 \mathrm{H}), 7.32-7.26(\mathrm{~m}, 1 \mathrm{H}), 7.25-$ $7.14(\mathrm{~m}, 3 \mathrm{H}), 6.16-6.03(\mathrm{~m}, 1 \mathrm{H}), 5.58-5.41(\mathrm{~m}, 1 \mathrm{H}), 3.69(\mathrm{~s}, 3 \mathrm{H}), 3.00-2.87(\mathrm{~m}, 2 \mathrm{H}), 1.70(\mathrm{~s}, 3 \mathrm{H})$. ${ }^{13} \mathrm{C}$ NMR (126 MHz, chloroform-d) $\delta$ 207.6, 175.5, 161.9, 149.2, 138.6, 136.6, $128.7(\mathrm{~d}, J=32.2 \mathrm{~Hz})$, $126.8,125.5(\mathrm{q}, J=3.8 \mathrm{~Hz}), 124.3(\mathrm{q}, J=271.5 \mathrm{~Hz}), 122.0,120.8,93.4,91.1,53.3,52.4,38.1,22.3 .{ }^{19} \mathrm{~F}$ NMR (376 MHz, Chloroform-d) $\delta-62.2$ (s, 3F). HRMS (FI): [M] ${ }^{\oplus}$ calcd for $\mathrm{C}_{20} \mathrm{H}_{18} \mathrm{~F}_{3} \mathrm{NO}_{2}{ }^{\oplus} 361.1284$, 
found 361.1278. HPLC analysis: Chiralpak IC column; detected at $254 \mathrm{~nm}, 40{ }^{\circ} \mathrm{C} ; 3 \%{ }^{i} \mathrm{PrOH}$ in $n$ Hexane; flow $=0.5 \mathrm{~mL} / \mathrm{min}$. Retention time: $17.3 \mathrm{~min}, 19.1 \mathrm{~min}, 20.6 \mathrm{~min}, 22.9 \mathrm{~min}$. The ee value was determined based on peaks at $19.1 \mathrm{~min}$ (major) and $22.9 \mathrm{~min}$ (minor).
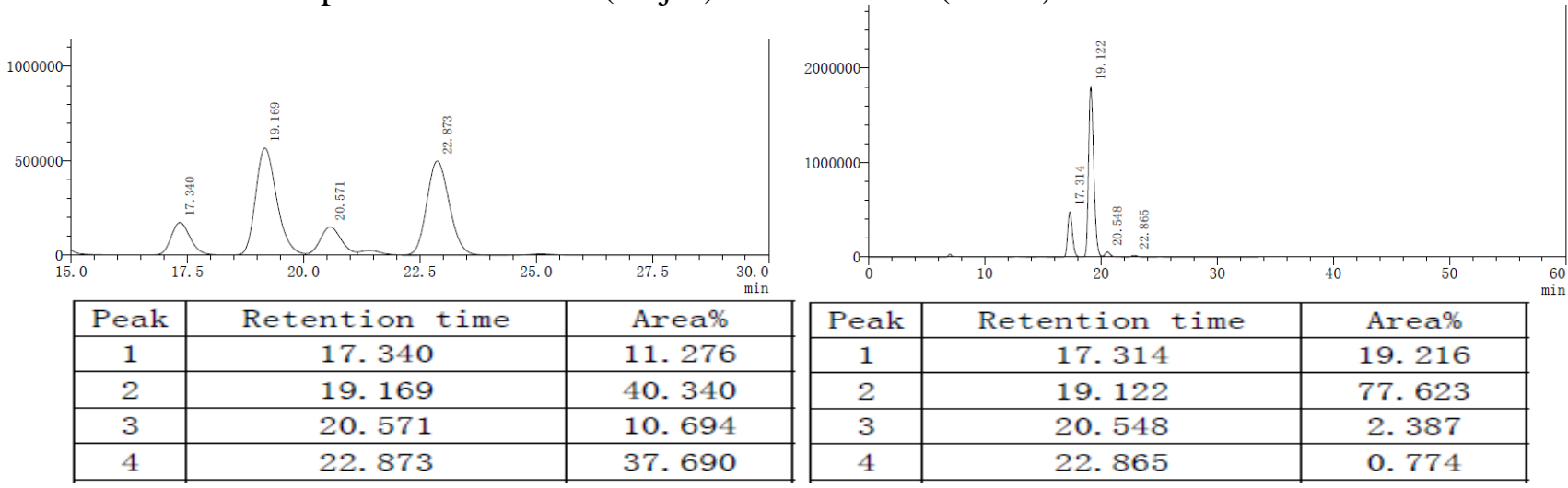<smiles>CC(=O)CC(O)(CC=Cc1ccc(C(F)(F)F)cc1)c1ccccc1</smiles>

\section{Methyl (2R,5R)-2-chloro-2-phenyl-6-(4-(trifluoromethyl)phenyl)hexa-4,5-dienoate $(4 \mathrm{~m})$}

For the synthesis of $\mathbf{4 m},(R)$-DTBM-SEGPHOS (5 mol\%), [Cu]/(S,S)-BPE (5 mol\%) were used instead. Yellow solid, $18 \mathrm{~h}, 68 \%$ yield, $>20: 1 \mathrm{dr},[\alpha]_{\mathrm{D}}{ }^{25}-189.6\left(c 1.0, \mathrm{CHCl}_{3}\right)$ for $>99 \%$ ee; ${ }^{1} \mathrm{H} \mathrm{NMR}(500 \mathrm{MHz}$, Chloroform- $d$ ) $\delta 8.57-8.46(\mathrm{~m}, 1 \mathrm{H}), 7.86-7.74(\mathrm{~m}, 2 \mathrm{H}), 7.45(\mathrm{~d}, J=8.1 \mathrm{~Hz}, 2 \mathrm{H}), 7.29-7.23(\mathrm{~m}, 1 \mathrm{H})$, $7.10(\mathrm{~d}, J=8.0 \mathrm{~Hz}, 2 \mathrm{H}), 6.15-6.01(\mathrm{~m}, 1 \mathrm{H}), 5.71-5.53(\mathrm{~m}, 1 \mathrm{H}), 3.78(\mathrm{~s}, 3 \mathrm{H}), 3.44-3.30(\mathrm{~m}, 2 \mathrm{H}) .{ }^{13} \mathrm{C}$ NMR (126 MHz, chloroform- $d$ ) $\delta 208.2,169.8,157.8,148.9,138.1,137.2,128.8$ (q, $J=32.3 \mathrm{~Hz}), 126.9$, $125.4(\mathrm{q}, J=3.7 \mathrm{~Hz}), 124.3(\mathrm{q}, J=271.5 \mathrm{~Hz}), 123.1,121.9,93.9,89.8,73.2,53.6,40.2 .{ }^{19} \mathrm{~F}$ NMR $(376$ $\mathrm{MHz}$, chloroform- $d$ ) $\delta-67.2$ (s, 3F). HRMS (ESI): $[\mathrm{M}+\mathrm{H}]^{\oplus}$ calcd for $\mathrm{C}_{19} \mathrm{H}_{15} \mathrm{~F}_{3} \mathrm{NO}_{2} \mathrm{Cl}^{\oplus} 382.0816$, found 382.0814. HPLC analysis: Chiracel OD-H + Chiracel OD-H column (the two columns were connected to each other in this order); detected at $254 \mathrm{~nm}, 40{ }^{\circ} \mathrm{C} ; 3 \%{ }^{i} \mathrm{PrOH}$ in $n$-Hexane; flow $=0.5 \mathrm{~mL} / \mathrm{min}$. Retention time: $30.9 \mathrm{~min}, 33.8 \mathrm{~min}, 41.5 \mathrm{~min}, 43.0 \mathrm{~min}$. The ee value was determined based on peaks at 30.9 min (minor) and 41.5 min (major).
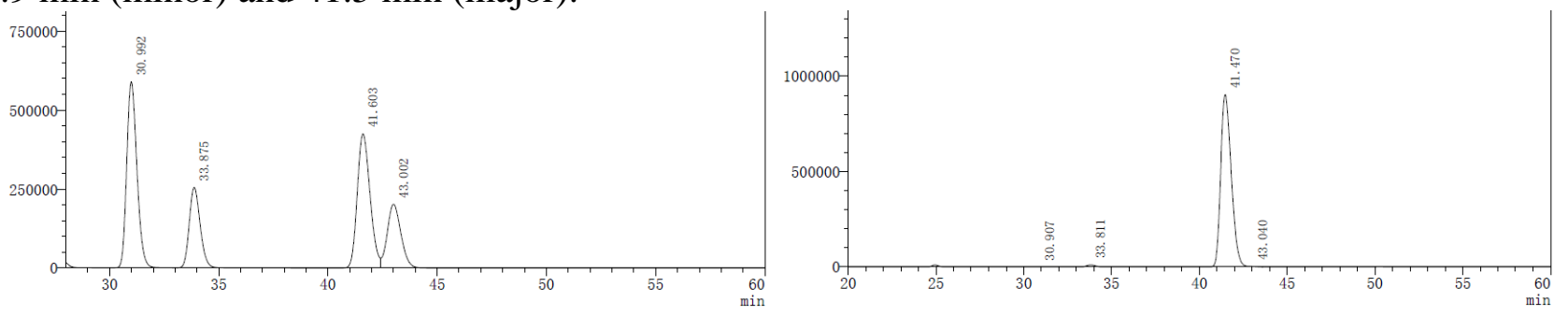

\begin{tabular}{|c|c|c|}
\hline Peak & Retention time & Area\% \\
\hline 1 & 30.992 & 35.363 \\
\hline 2 & 33.875 & 16.307 \\
\hline 3 & 41.603 & 32.083 \\
\hline 4 & 43.002 & 16.246 \\
\hline
\end{tabular}

\begin{tabular}{|c|c|c|}
\hline Peak & Retention time & Area\% \\
\hline 1 & 30.907 & 0.041 \\
\hline 2 & 33.811 & 1.039 \\
\hline 3 & 41.470 & 98.893 \\
\hline 4 & 43.040 & 0.027 \\
\hline
\end{tabular}




\section{Stereodivergent synthesis of all four isomers}

The procedure for the stereodivergent synthesis was the same as the standard condition described in the part "General procedure for the hydroalkylation reaction of enynes".

Notice: the synthesis of $\mathbf{3 d}, \mathbf{3 i}, \mathbf{6 d}$ and $\mathbf{6 i}$ were conducted under standard conditions. For the synthesis of $\mathbf{5 d}, \mathbf{5 i}, 7 \mathbf{d}$ and $\mathbf{7 i}$, the difference was that the reactions were conducted with $10 \mathrm{~mol} \%$ $[\mathrm{Pd}] / \mathbf{L 6} / \mathrm{NaBAr}_{4}{ }_{4}, 10 \mathrm{~mol} \%$ [Cu]/BPE, Et $3 \mathrm{~N}$ (2 equiv), DBU (5 mol\%) in toluene at RT.<smiles>COC(=O)C(F)(C/C=C/c1ccc(C(F)(F)F)cc1)c1ccccn1</smiles>

\section{Methyl (2R,5S)-2-fluoro-2-(pyridin-2-yl)-6-(4-(trifluoromethyl)phenyl)hexa-4,5-dienoate (5d)}

Yellow oil, $15 \mathrm{~h}, 69 \%$ yield, $8: 1 \mathrm{dr},[\alpha]_{\mathrm{D}}{ }^{25}+82.1\left(c 1.3, \mathrm{CHCl}_{3}\right)$ for $>99 \%$ ee; When $(S)$-L5 was used instead of original $(S)$-L6, the product $\mathbf{5 d}$ was observed in $82 \%$ yield, $18: 1 \mathrm{dr}$ and $>99 \%$ ee. ${ }^{1} \mathrm{H}$ NMR $(500 \mathrm{MHz}$, chloroform- $d$ ) $\delta 8.64(\mathrm{~d}, J=4.7 \mathrm{~Hz}, 1 \mathrm{H}), 7.79(\mathrm{td}, J=7.8,1.8 \mathrm{~Hz}, 1 \mathrm{H}), 7.66(\mathrm{~d}, J=8.0 \mathrm{~Hz}$, $1 \mathrm{H}), 7.52(\mathrm{~d}, J=8.0 \mathrm{~Hz}, 2 \mathrm{H}), 7.33-7.30(\mathrm{~m}, 3 \mathrm{H}), 6.16-6.10(\mathrm{~m}, 1 \mathrm{H}), 5.59-5.55(\mathrm{~m}, 1 \mathrm{H}), 3.76(\mathrm{~s}, 3 \mathrm{H})$, $3.40-3.21(\mathrm{~m}, 2 \mathrm{H}) .{ }^{13} \mathrm{C}$ NMR $(126 \mathrm{MHz}$, chloroform- $d$ ) $\delta 208.2,169.0(\mathrm{~d}, J=26.4 \mathrm{~Hz}), 156.0(\mathrm{~d}, J=$ $27.0 \mathrm{~Hz}), 149.1,138.1,137.5,128.9(\mathrm{q}, J=32.3 \mathrm{~Hz}), 127.0,125.5(\mathrm{q}, J=3.8 \mathrm{~Hz}), 124.3(\mathrm{q}, J=271.9$ $\mathrm{Hz}), 123.9,120.5(\mathrm{~d}, J=8.2 \mathrm{~Hz}), 97.0(\mathrm{~d}, J=189.8 \mathrm{~Hz}), 94.2,88.4(\mathrm{~d}, J=3.6 \mathrm{~Hz}), 53.3,36.1(\mathrm{~d}, J=$ $20.6 \mathrm{~Hz}) .{ }^{19} \mathrm{~F}$ NMR $(376 \mathrm{MHz}$, chloroform- $d$ ) $\delta-62.4$ (s, 3F), -163.8 (t, $J=23.9 \mathrm{~Hz}, 1 \mathrm{~F})$. HRMS (FI): $[\mathrm{M}]^{\oplus}$ calcd for $\mathrm{C}_{19} \mathrm{H}_{15} \mathrm{~F}_{4} \mathrm{NO}_{2}{ }^{\oplus}$ 365.1033, found 365.1031. HPLC analysis: Chiracel AD-H column; detected at $254 \mathrm{~nm}, 40{ }^{\circ} \mathrm{C} ; 3 \%{ }^{i} \mathrm{PrOH}$ in $n$-Hexane; flow $=0.7 \mathrm{~mL} / \mathrm{min}$. Retention time: $9.7 \mathrm{~min}, 10.6$ min, $11.2 \mathrm{~min}, 11.8 \mathrm{~min}$. The ee value was determined based on peaks at $10.6 \mathrm{~min}$ (minor) and $11.2 \mathrm{~min}$ (major).
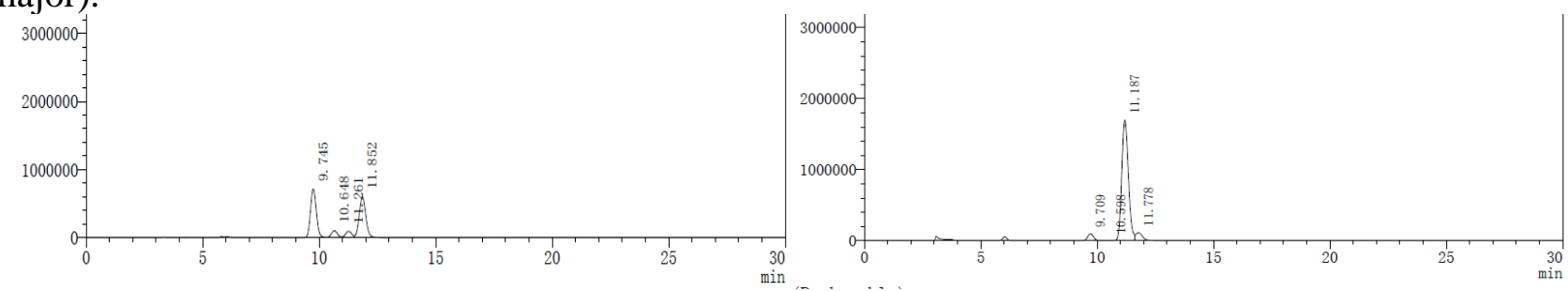

\begin{tabular}{|c|c|c|}
\hline Peak & retension time/min & area\% \\
\hline 1 & 9.745 & 44.577 \\
\hline 2 & 10.648 & 6.788 \\
\hline 3 & 11.261 & 6.309 \\
\hline 4 & 11.852 & 42.326 \\
\hline
\end{tabular}

\begin{tabular}{|c|c|c|}
\hline Peak & retension time/min & area\% \\
\hline 1 & 9.709 & 4.649 \\
\hline 2 & 10.598 & 0.114 \\
\hline 3 & 11.187 & 89.108 \\
\hline 4 & 11.778 & 6.130 \\
\hline
\end{tabular}


<smiles>CC(=O)C(F)(C/C=C/c1ccc(Br)cc1)c1ccccn1</smiles>

\section{Methyl (2R,5S)-6-(4-bromophenyl)-2-fluoro-2-(pyridin-2-yl)hexa-4,5-dienoate (5i)}

Yellow oil, $18 \mathrm{~h}, 88 \%$ yield, $8: 1 \mathrm{dr},[\alpha]_{\mathrm{D}}{ }^{25}+69.3\left(c 1.0, \mathrm{CHCl}_{3}\right)$ for $>99 \%$ ee; When $(S)-\mathbf{L 5}$ was used instead of original $(S)$-L6, the product 5i was observed in $85 \%$ yield, $20: 1 \mathrm{dr}$ and $>99 \%$ ee. ${ }^{1} \mathrm{H}$ NMR $(400 \mathrm{MHz}$, chloroform- $d) \delta 8.63(\mathrm{~d}, J=4.8 \mathrm{~Hz}, 1 \mathrm{H}), 7.78(\mathrm{td}, J=7.7,1.6 \mathrm{~Hz}, 1 \mathrm{H}), 7.67-7.63(\mathrm{~m}, 1 \mathrm{H})$, $7.43-7.28(\mathrm{~m}, 3 \mathrm{H}), 7.09(\mathrm{~d}, J=8.1 \mathrm{~Hz}, 2 \mathrm{H}), 6.06-6.03(\mathrm{~m}, 1 \mathrm{H}), 5.53-5.48(\mathrm{~m}, 1 \mathrm{H}), 3.75(\mathrm{~s}, 3 \mathrm{H})$, $3.43-3.10(\mathrm{~m}, 2 \mathrm{H}) .{ }^{13} \mathrm{C}$ NMR $(126 \mathrm{MHz}$, chloroform- $d) \delta 207.5,169.1(\mathrm{~d}, J=26.4 \mathrm{~Hz}), 156.2(\mathrm{~d}, J=$ $26.9 \mathrm{~Hz}), 149.2,137.3,133.2,131.7,128.5,123.8,120.7,120.4(\mathrm{~d}, J=7.8 \mathrm{~Hz}), 97.1(\mathrm{~d}, J=189.3 \mathrm{~Hz})$, 94.2, 88.2 (d, $J=3.7 \mathrm{~Hz}), 53.2,36.2(\mathrm{~d}, J=20.7 \mathrm{~Hz}) .{ }^{19} \mathrm{~F}$ NMR $(376 \mathrm{MHz}$, chloroform- $d) \delta-163.7(\mathrm{t}, J$ $=23.8 \mathrm{~Hz}, 1 \mathrm{~F})$. HRMS (EI): $[\mathrm{M}]^{\oplus}$ calcd for $\mathrm{C}_{18} \mathrm{H}_{15} \mathrm{BrFNO}_{2}{ }^{\oplus}$ 375.0265, found 375.0261. HPLC analysis: Chiralpak IC column; detected at $254 \mathrm{~nm}, 40{ }^{\circ} \mathrm{C} ; 3 \%{ }^{i} \mathrm{PrOH}$ in $n$-hexane; flow $=0.7 \mathrm{~mL} / \mathrm{min}$. Retention time: $24.5 \mathrm{~min}, 26.3 \mathrm{~min}, 28.7 \mathrm{~min}, 56.9 \mathrm{~min}$. The ee value was determined based on peaks at $26.3 \mathrm{~min}$ (major) and $56.9 \mathrm{~min}$ (minor).
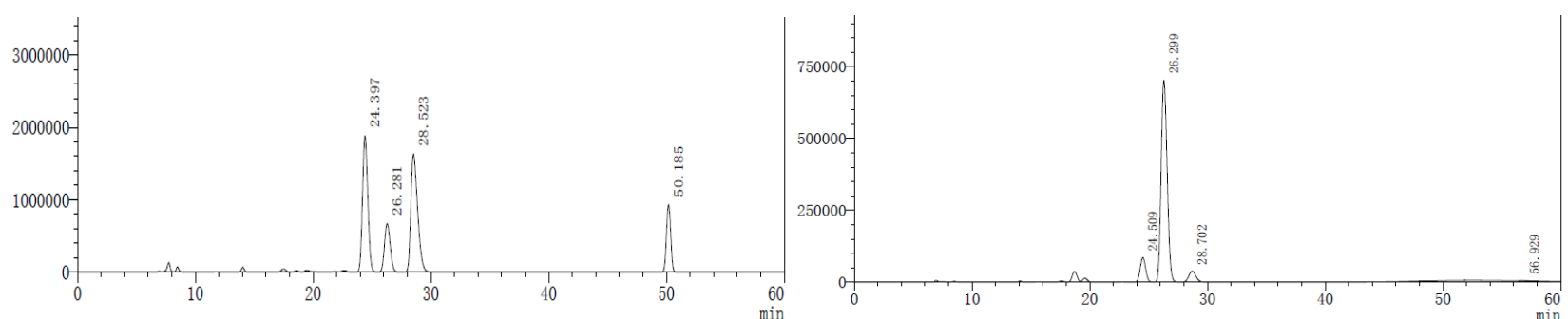

\begin{tabular}{|c|c|c|}
\hline Peak & retension time/min & area\% \\
\hline 1 & 24.397 & 34.724 \\
\hline 2 & 26.281 & 13.370 \\
\hline 3 & 28.523 & 38.010 \\
\hline 4 & 50.185 & 13.896 \\
\hline
\end{tabular}

\begin{tabular}{|c|c|c|}
\hline Peak & retension time/min & area\% \\
\hline 1 & 24.509 & 9.553 \\
\hline 2 & 26.299 & 84.812 \\
\hline 3 & 28.702 & 5.504 \\
\hline 4 & 56.929 & 0.131 \\
\hline
\end{tabular}

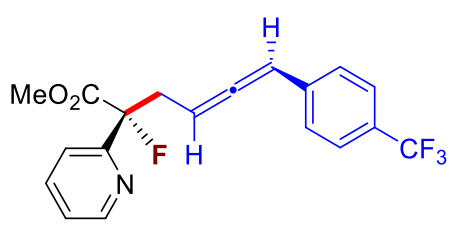

Methyl (2S,5S)-2-fluoro-2-(pyridin-2-yl)-6-(4-(trifluoromethyl)phenyl)hexa-4,5-dienoate (6d)

Yellow solid, $22 \mathrm{~h}, 87 \%$ yield, $>20: 1 \mathrm{dr},[\alpha]_{\mathrm{D}}{ }^{25}+128.0\left(c 1.0, \mathrm{CHCl}_{3}\right)$ for $>99 \%$ ee; The spectroscopic data of $\mathbf{6 d}$ is the same as that of 3d. HPLC analysis: Chiracel AD-H column; detected at $254 \mathrm{~nm}, 40{ }^{\circ} \mathrm{C}$; $3 \%{ }^{i} \mathrm{PrOH}$ in $n$-hexane; flow $=0.7 \mathrm{~mL} / \mathrm{min}$. Retention time: $9.7 \mathrm{~min}, 10.6 \mathrm{~min}, 11.3 \mathrm{~min}, 11.8 \mathrm{~min}$. The ee value was determined based on peaks at $9.7 \mathrm{~min}$ (major) and $11.8 \mathrm{~min}$ (minor). 

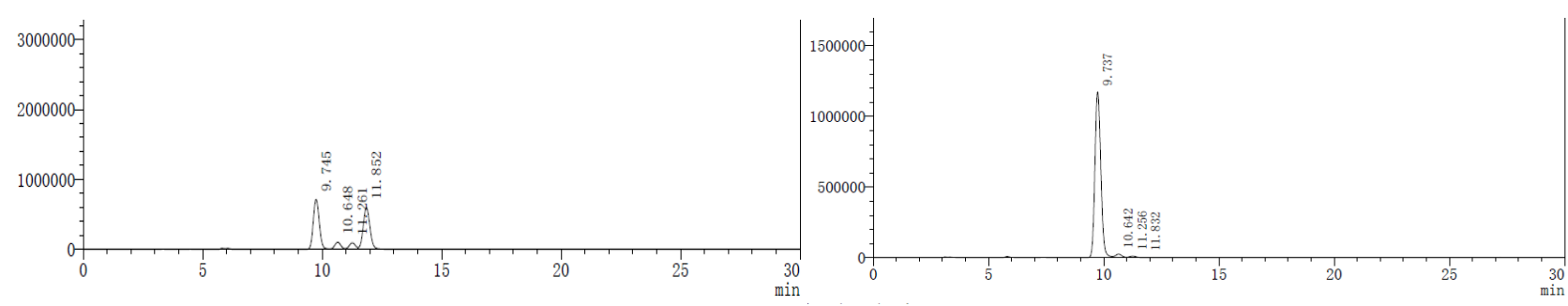

\begin{tabular}{|c|c|c|}
\hline Peak & retension time/min & area\% \\
\hline 1 & 9.745 & 44.577 \\
\hline 2 & 10.648 & 6.788 \\
\hline 3 & 11.261 & 6.309 \\
\hline 4 & 11.852 & 42.326 \\
\hline
\end{tabular}

\begin{tabular}{|c|c|c|}
\hline Peak & retension time/min & area\% \\
\hline 1 & 9.737 & 96.510 \\
\hline 2 & 10.642 & 2.365 \\
\hline 3 & 11.256 & 1.047 \\
\hline 4 & 11.832 & 0.078 \\
\hline
\end{tabular}<smiles>CC(=O)C(F)(C/C=C/c1ccc(Br)cc1)c1ccccn1</smiles>

\section{Methyl (2S,5S)-6-(4-bromophenyl)-2-fluoro-2-(pyridin-2-yl)hexa-4,5-dienoate (6i)}

Yellow oil, $20 \mathrm{~h}, 96 \%$ yield, $>20: 1 \mathrm{dr},[\alpha]_{\mathrm{D}}{ }^{25}+175.8\left(c 1.0, \mathrm{CHCl}_{3}\right)$ for $>99 \%$ ee; The spectroscopic data of $\mathbf{6 i}$ is the same as that of 3i. HPLC analysis: Chiralpak IC column; detected at $254 \mathrm{~nm}, 40{ }^{\circ} \mathrm{C} ; 3 \%$ ${ }^{i} \mathrm{PrOH}$ in $n$-hexane; flow $=0.7 \mathrm{~mL} / \mathrm{min}$. Retention time: $24.7 \mathrm{~min}, 26.6 \mathrm{~min}, 28.8 \mathrm{~min}, 50.6 \mathrm{~min}$. The ee value was determined based on peaks at 24.7 min (minor) and 28.8 min (major).
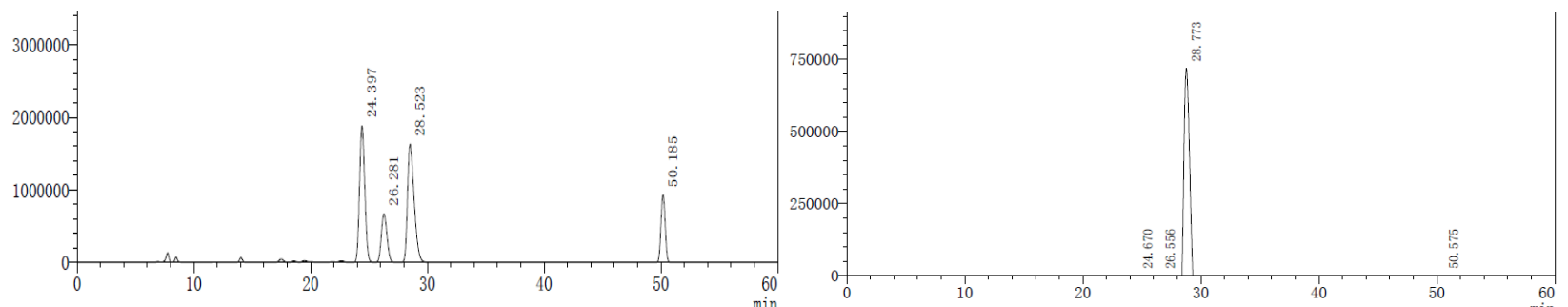

\begin{tabular}{|c|c|c|}
\hline Peak & retension time/min & area\% \\
\hline 1 & 24.397 & 34.724 \\
\hline 2 & 26.281 & 13.370 \\
\hline 3 & 28.523 & 38.010 \\
\hline 4 & 50.185 & 13.896 \\
\hline
\end{tabular}

\begin{tabular}{|c|c|c|}
\hline Peak & retension time/min & area\% \\
\hline 1 & 24.670 & 0.086 \\
\hline 2 & 26.556 & 1.444 \\
\hline 3 & 28.773 & 98.393 \\
\hline 4 & 50.575 & 0.077 \\
\hline
\end{tabular}<smiles>CC(=O)C(F)(C/C=C/c1ccc(C(F)(F)F)cc1)c1ccccn1</smiles>

\section{Methyl (2S,5R)-2-fluoro-2-(pyridin-2-yl)-6-(4-(trifluoromethyl)phenyl)hexa-4,5-dienoate (7d)}

Yellow oil, $22 \mathrm{~h}, 60 \%$ yield, $9: 1 \mathrm{dr},[\alpha]_{\mathrm{D}}{ }^{25}-82.6\left(c 0.8, \mathrm{CHCl}_{3}\right)$ for $>99 \%$ ee; The spectroscopic data of $\mathbf{7 d}$ is the same as that of $\mathbf{5 d}$. HPLC analysis: Chiracel AD-H column; detected at $254 \mathrm{~nm}, 40{ }^{\circ} \mathrm{C} ; 3 \%$ ${ }^{i} \mathrm{PrOH}$ in $n$-hexane; flow $=0.7 \mathrm{~mL} / \mathrm{min}$. Retention time: $9.7 \mathrm{~min}, 10.6 \mathrm{~min}, 11.3 \mathrm{~min}, 11.8 \mathrm{~min}$. The ee value was determined based on peaks at $10.6 \mathrm{~min}$ (major) and $11.3 \mathrm{~min}$ (minor). 

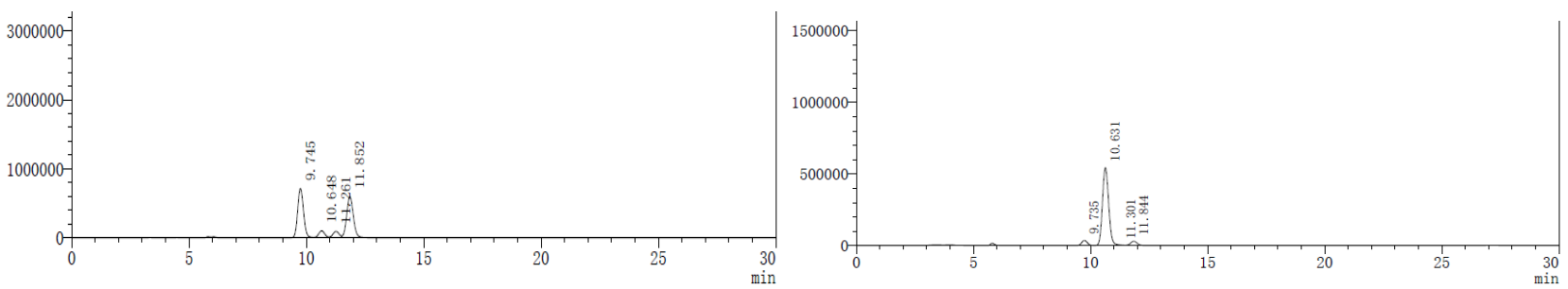

\begin{tabular}{|c|c|c|}
\hline Peak & retension time/min & area\% \\
\hline 1 & 9.745 & 44.577 \\
\hline 2 & 10.648 & 6.788 \\
\hline 3 & 11.261 & 6.309 \\
\hline 4 & 11.852 & 42.326 \\
\hline
\end{tabular}

\begin{tabular}{|c|c|c|}
\hline Peak & retension time/min & area\% \\
\hline 1 & 9.735 & 5.520 \\
\hline 2 & 10.631 & 88.759 \\
\hline 3 & 11.301 & 0.304 \\
\hline 4 & 11.844 & 5.417 \\
\hline
\end{tabular}<smiles>CC(=O)C(C/C=C/c1ccc(Br)cc1)c1ccccn1</smiles>

\section{Methyl (2S,5R)-6-(4-bromophenyl)-2-fluoro-2-(pyridin-2-yl)hexa-4,5-dienoate (7i)}

Yellow oil, $20 \mathrm{~h}, 80 \%$ yield, $9: 1 \mathrm{dr},[\alpha]_{\mathrm{D}}^{25}-74.3\left(c 1.0, \mathrm{CHCl}_{3}\right)$ for $>99 \%$ ee; The spectroscopic data of $7 \mathbf{i}$ is the same as that of 5i. HPLC analysis: Chiralpak IC column; detected at $254 \mathrm{~nm}, 40{ }^{\circ} \mathrm{C} ; 3 \%{ }^{i} \mathrm{PrOH}$ in $n$-hexane; flow $=0.7 \mathrm{~mL} / \mathrm{min}$. Retention time: $24.6 \mathrm{~min}, 26.5 \mathrm{~min}, 28.8 \mathrm{~min}, 56.9 \mathrm{~min}$. The ee value was determined based on peaks at $26.5 \mathrm{~min}$ (minor) and $56.9 \mathrm{~min}$ (major).
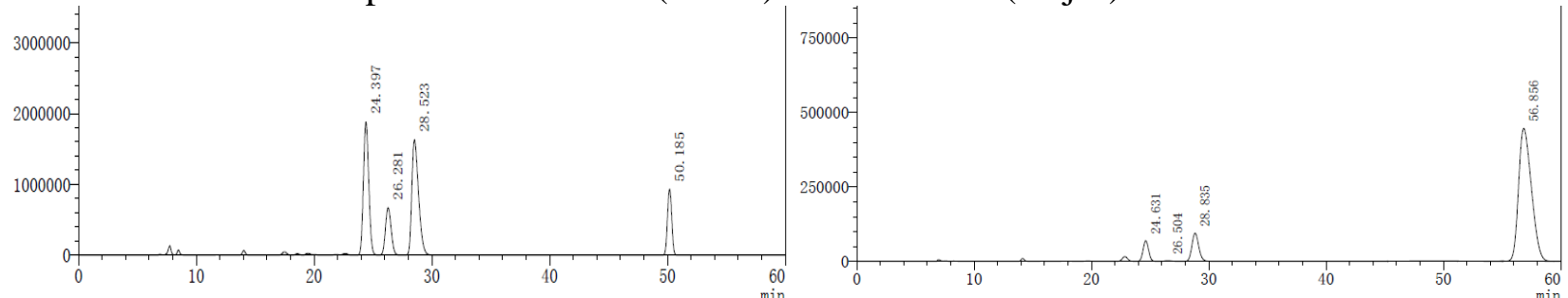

\begin{tabular}{|c|c|c|}
\hline Peak & retension time/min & area\% \\
\hline 1 & 24.397 & 34.724 \\
\hline 2 & 26.281 & 13.370 \\
\hline 3 & 28.523 & 38.010 \\
\hline 4 & 50.185 & 13.896 \\
\hline
\end{tabular}

\begin{tabular}{|c|c|c|}
\hline Peak & retension time/min & area\% \\
\hline 1 & 24.631 & 5.598 \\
\hline 2 & 26.504 & 0.234 \\
\hline 3 & 28.835 & 9.613 \\
\hline 4 & 56.856 & 84.555 \\
\hline
\end{tabular}




\section{Kinetic studies on the diastereoselectivities and yields of reactions over time}

The procedure for the kinetic study was the same as the standard condition described in the part "General procedure for the hydrocarbonation reaction of enynes", except that the reaction was run in 2.0 mmol scale. At certain reaction time, $100 \mu \mathrm{L}$ of reaction solution was removed from the reaction. The yield was analyzed by crude ${ }^{1} \mathrm{H}$ NMR. The de and ee values were analyzed by chiral HPLC to give the general corresponding trend.

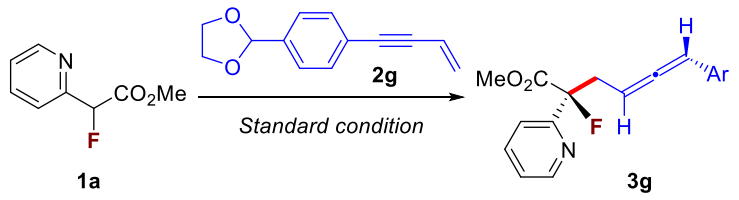

\begin{tabular}{cccc}
\hline Time/h & Yield $(\%)$ & de $(\%)$ & ee $(\%)$ \\
\hline 3 & 36 & 93.6 & $>99$ \\
11 & 70 & 92.4 & $>99$ \\
14 & 79 & 92.6 & $>99$ \\
20 & 89 & 92.2 & $>99$ \\
27 & 95 & 91.4 & $>99$ \\
38 & 95 & 90.6 & $>99$ \\
44 & 95 & 86.0 & $>99$ \\
50 & 95 & 84.8 & $>99$ \\
54 & 95 & 85.4 & $>99$ \\
57 & 95 & 84.2 & $>99$ \\
69 & 95 & 81.2 & $>99$ \\
83 & 95 & 72.2 & $>99$ \\
\hline
\end{tabular}

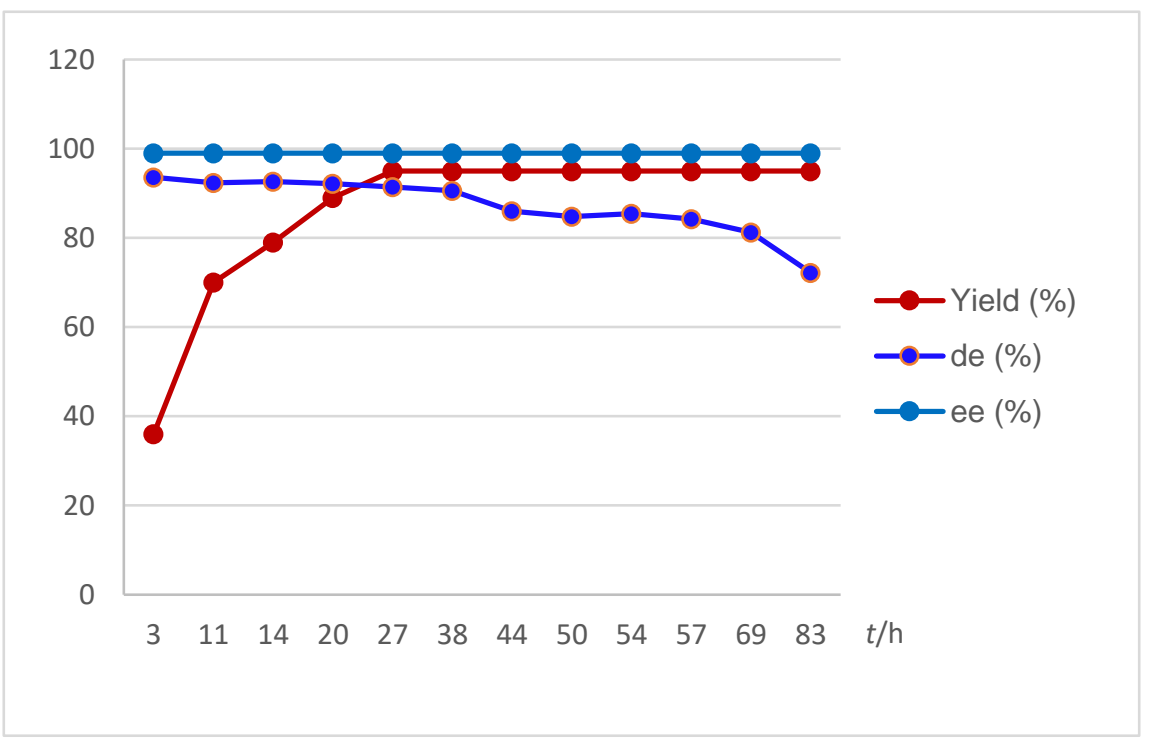




\section{Axial-to-central chirality transfer}

General procedure for the reduction of ester: To a solution of the fluorinated allene ( $34 \mathrm{mg}, 0.1$ $\mathrm{mmol})$ in dry $\mathrm{EtOH}(1.0 \mathrm{~mL})$ was added $\mathrm{NaBH}_{4}(11 \mathrm{mg}, 0.3 \mathrm{mmol})$. The resulting mixture was stirred at room temperature and monitored by TLC until the complete consumption of allene substrate. Then the reaction solvent was removed under reduced pressure and the residue was purified by flash column chromatography (hexane/ethyl acetate $=1: 1$ ) to give the pure product $\mathbf{8}$ as a white solid.

General procedure for the cyclization: To a $4 \mathrm{ml}$ vial with $t$ - $\mathrm{Bu} 3 \mathrm{PAuCl}(24 \mathrm{mg}, 0.050 \mathrm{mmol})$ and AgOTs $(14 \mathrm{mg}, 0.050 \mathrm{mmol})$ under nitrogen was added $\mathrm{PhCF}_{3}(0.5 \mathrm{ml})$. The reaction was stirred at room temperature for $15 \mathrm{~min}$ under dark. Then $8(17 \mathrm{mg}, 0.050 \mathrm{mmol})$ was added to above solution and the resulting mixture continued to stir at $80{ }^{\circ} \mathrm{C}$ for $10 \mathrm{~h}$ under dark. After this time, the reaction solvent was removed under reduced pressure and the residue was purified by flash column chromatography (hexane/ethyl acetate $=5: 1$ ) to give the cyclization product 9 as a white solid.

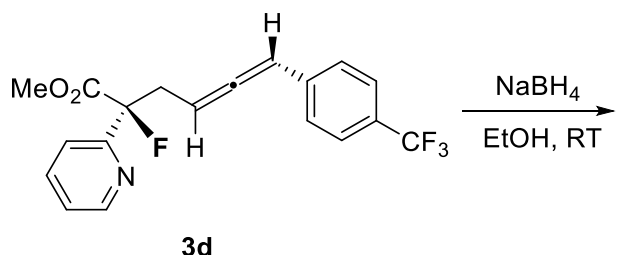

$3 d$

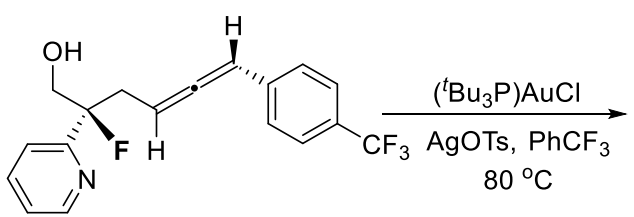

$(S, R)-8$

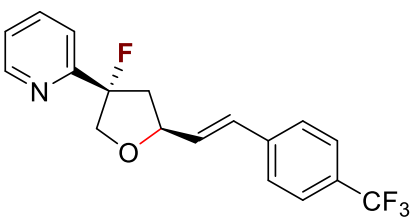

$(S, S)-\mathbf{9}$

\section{(2R,5R)-2-Fluoro-6-(4-methoxyphenyl)-2-(pyridin-2-yl)hexa-4,5-dien-1-ol [(S,R)-8]}

White solid, 83\% yield, $[\alpha]_{\mathrm{D}}{ }^{25}-127.7\left(c 1.0, \mathrm{CHCl}_{3}\right) ;{ }^{1} \mathrm{H}$ NMR $(400 \mathrm{MHz}$, chloroform- $d) \delta 8.52(\mathrm{~d}, J=$ $4.8 \mathrm{~Hz}, 1 \mathrm{H}), 7.88-7.74(\mathrm{~m}, 1 \mathrm{H}), 7.63(\mathrm{~d}, J=7.9 \mathrm{~Hz}, 1 \mathrm{H}), 7.42(\mathrm{~d}, J=8.0 \mathrm{~Hz}, 2 \mathrm{H}), 7.35-7.28(\mathrm{~m}, 1 \mathrm{H})$, $7.00(\mathrm{~d}, J=8.0 \mathrm{~Hz}, 2 \mathrm{H}), 6.20-6.01(\mathrm{~m}, 1 \mathrm{H}), 5.71-5.26(\mathrm{~m}, 1 \mathrm{H}), 4.31-3.95(\mathrm{~m}, 2 \mathrm{H}), 3.55(\mathrm{~s}, 1 \mathrm{H})$, $3.20-2.83(\mathrm{~m}, 2 \mathrm{H}) .{ }^{13} \mathrm{C}$ NMR $(126 \mathrm{MHz}$, chloroform- $d) \delta 207.9,160.2(\mathrm{~d}, J=27.9 \mathrm{~Hz}), 148.4,138.2$, $137.5,128.7(\mathrm{~d}, J=32.3 \mathrm{~Hz}), 126.8,125.4(\mathrm{q}, J=4.0 \mathrm{~Hz}), 124.2(\mathrm{q}, J=271.9 \mathrm{~Hz}), 123.1,120.7(\mathrm{~d}, J=$ $9.3 \mathrm{~Hz}), 98.0(\mathrm{~d}, J=179.0 \mathrm{~Hz}), 93.7,88.8(\mathrm{~d}, J=4.6 \mathrm{~Hz}), 67.4(\mathrm{~d}, J=26.4 \mathrm{~Hz}), 36.1(\mathrm{~d}, J=21.1 \mathrm{~Hz})$. ${ }^{19} \mathrm{~F}$ NMR $\left(376 \mathrm{MHz}\right.$, chloroform- $d$ ) $\delta-62.4$ (s, 3F), -171.1 (s, 1F). HRMS (EI): [M] ${ }^{\oplus}$ calcd for $\mathrm{C}_{18} \mathrm{H}_{15} \mathrm{~F}_{4} \mathrm{NO}^{\oplus} 337.1084$, found 337.1087.

\section{2-((3S,5S)-3-Fluoro-5-((E)-4-(trifluoromethyl)styryl)tetrahydrofuran-3-yl)pyridine [(S,S)-9]}

White solid, 60\% yield, $>20: 1 \mathrm{dr},[\alpha]_{\mathrm{D}}{ }^{25}+2.7\left(c 1.0, \mathrm{CHCl}_{3}\right)$ for $>99 \%$ ee; ${ }^{1} \mathrm{H}$ NMR $(400 \mathrm{MHz}$, chloroform- $d$ ) $\delta 8.63(\mathrm{~s}, 1 \mathrm{H}), 7.80(\mathrm{td}, J=7.7,1.4 \mathrm{~Hz}, 1 \mathrm{H}), 7.67(\mathrm{~d}, J=8.1 \mathrm{~Hz}, 1 \mathrm{H}), 7.58(\mathrm{~d}, J=8.2 \mathrm{~Hz}$, $2 \mathrm{H}), 7.51(\mathrm{~d}, J=8.2 \mathrm{~Hz}, 2 \mathrm{H}), 7.31-7.26(\mathrm{~m}, 1 \mathrm{H}), 6.75(\mathrm{~d}, J=15.9 \mathrm{~Hz}, 1 \mathrm{H}), 6.48(\mathrm{dd}, J=15.9,7.0 \mathrm{~Hz}$, $1 \mathrm{H}), 5.10-4.93(\mathrm{~m}, 1 \mathrm{H}), 4.50(\mathrm{dd}, J=35.4,10.5 \mathrm{~Hz}, 1 \mathrm{H}), 4.28(\mathrm{dd}, J=25.6,10.5 \mathrm{~Hz}, 1 \mathrm{H}), 2.76-2.57$ $(\mathrm{m}, 2 \mathrm{H}) .{ }^{13} \mathrm{C}$ NMR $(101 \mathrm{MHz}$, chloroform- $d$ ) $\delta 157.1(\mathrm{~d}, J=29.0 \mathrm{~Hz}), 149.0,140.0(\mathrm{~d}, J=1.5 \mathrm{~Hz})$, 137.3, 131.4, 130.6, 129.6 (q, $J=32.4 \mathrm{~Hz}), 126.8,125.6(\mathrm{q}, J=3.8 \mathrm{~Hz}), 124.2(\mathrm{q}, J=271.8 \mathrm{~Hz}), 123.2$, $120.1(\mathrm{~d}, J=12.5 \mathrm{~Hz}), 105.5(\mathrm{~d}, J=180.9 \mathrm{~Hz}), 80.2,78.3(\mathrm{~d}, J=25.0 \mathrm{~Hz}), 46.1(\mathrm{~d}, J=21.6 \mathrm{~Hz}) .{ }^{19} \mathrm{~F}$ NMR (376 MHz, chloroform- $d$ ) $\delta-57.9$ (s, 3F), -148.9 - -149.0 (m, 1F). HRMS (EI): $[\mathrm{M}]^{\oplus}$ calcd for $\mathrm{C}_{18} \mathrm{H}_{15} \mathrm{~F}_{4} \mathrm{NO}^{\oplus}$ 337.1084, found 337.1082. HPLC analysis: Chiracel AD-H column; detected at $254 \mathrm{~nm}$, $40{ }^{\circ} \mathrm{C} ; 3 \%{ }^{i} \mathrm{PrOH}$ in $n$-hexane; flow $=0.7 \mathrm{~mL} / \mathrm{min}$. Retention time: $12.6 \mathrm{~min}$ (major), $15.7 \mathrm{~min}$ (minor). 

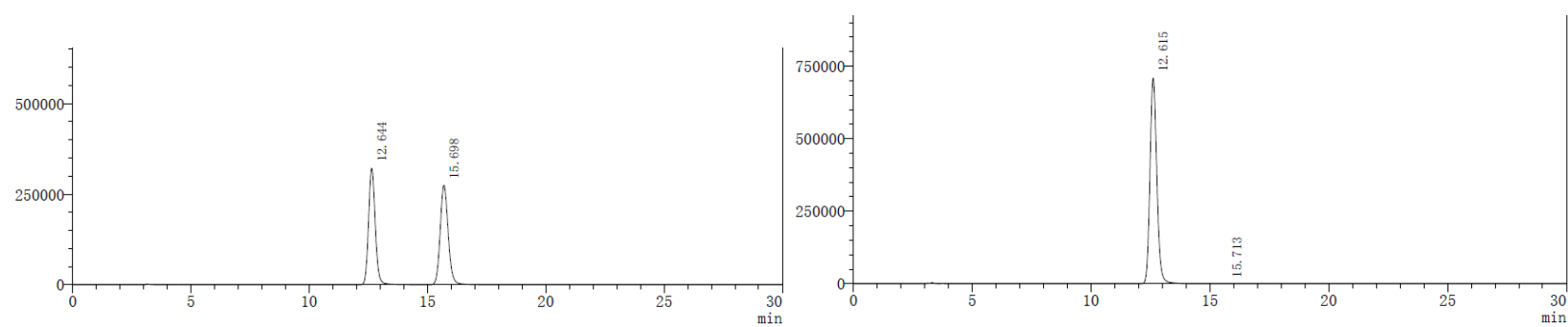

\begin{tabular}{|c|c|c|c|c|c|}
\hline Peak & retension time/min & area\% & Peak & retension time/min & area\% \\
\hline 1 & 12.644 & 49.485 & 1 & 12.615 & 99.889 \\
\hline 2 & 15.698 & 50.515 & 2 & 15.713 & 0.111 \\
\hline
\end{tabular}

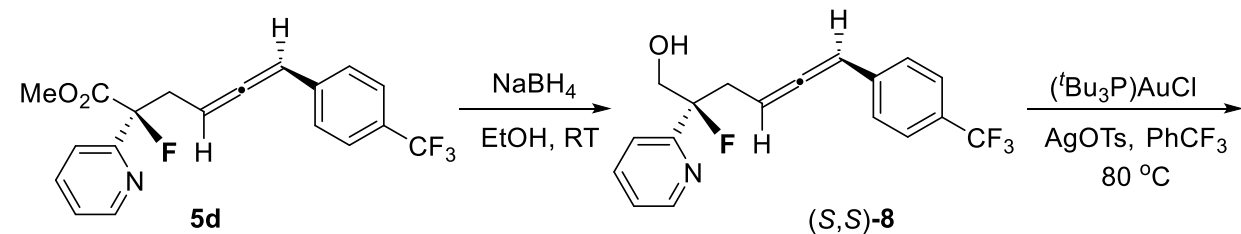

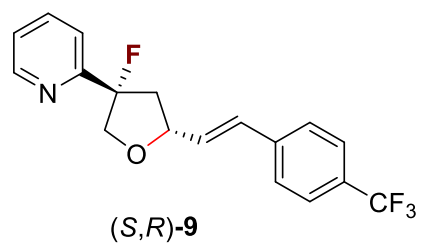

\section{(2R,5S)-2-Fluoro-6-(4-methoxyphenyl)-2-(pyridin-2-yl)hexa-4,5-dien-1-ol [(S,S)-8]}

White solid, $[\alpha]_{\mathrm{D}}{ }^{25}+89.5\left(c 1.0, \mathrm{CHCl}_{3}\right) ;{ }^{1} \mathrm{H}$ NMR $(500 \mathrm{MHz}$, chloroform- $d) \delta 8.57(\mathrm{~d}, J=4.8 \mathrm{~Hz}, 1 \mathrm{H})$, $7.80(\mathrm{td}, J=7.8,1.6 \mathrm{~Hz}, 1 \mathrm{H}), 7.62(\mathrm{~d}, J=7.9 \mathrm{~Hz}, 1 \mathrm{H}), 7.52(\mathrm{~d}, J=8.0 \mathrm{~Hz}, 2 \mathrm{H}), 7.30(\mathrm{~d}, J=8.2 \mathrm{~Hz}, 3 \mathrm{H})$, $6.11-5.97(\mathrm{~m}, 1 \mathrm{H}), 5.55-5.38(\mathrm{~m}, 1 \mathrm{H}), 4.20-3.96(\mathrm{~m}, 2 \mathrm{H}), 3.12-2.86(\mathrm{~m}, 2 \mathrm{H}) .{ }^{13} \mathrm{C} \mathrm{NMR}(126 \mathrm{MHz}$, chloroform- $d$ ) $\delta 207.9,159.9(\mathrm{~d}, J=27.6 \mathrm{~Hz}), 148.1,138.3,137.8,128.8(\mathrm{q}, J=32.3 \mathrm{~Hz}), 126.9,125.5$ $(\mathrm{q}, J=3.8 \mathrm{~Hz}), 124.3(\mathrm{q}, J=271.9 \mathrm{~Hz}), 123.2,120.9(\mathrm{~d}, J=8.7 \mathrm{~Hz}), 97.7(\mathrm{~d}, J=179.4 \mathrm{~Hz}), 93.8,88.8$ $(\mathrm{d}, J=4.2 \mathrm{~Hz}), 67.3(\mathrm{~d}, J=26.5 \mathrm{~Hz}), 36.1(\mathrm{~d}, J=21.8 \mathrm{~Hz}) .{ }^{19} \mathrm{~F}$ NMR $(376 \mathrm{MHz}$, chloroform- $d$ ) $\delta-62.5$ (s, 3F), -169.7 (s, 1F). HRMS (EI): [M] ${ }^{\oplus}$ calcd for $\mathrm{C}_{18} \mathrm{H}_{15} \mathrm{~F}_{4} \mathrm{NO}^{\oplus} 337.1084$, found 337.1087.

\section{2-((3S,5R)-3-Fluoro-5-((E)-4-(trifluoromethyl)styryl)tetrahydrofuran-3-yl)pyridine $[(S, R)-9]$}

White solid, 52\% yield, >20:1 dr, $[\alpha]_{\mathrm{D}}{ }^{25}-26.6\left(c\right.$ 1.0, $\left.\mathrm{CHCl}_{3}\right)$ for $>99 \%$ ee; ${ }^{1} \mathrm{H} \mathrm{NMR}(400 \mathrm{MHz}$, chloroform- $d$ ) $\delta 8.61(\mathrm{~d}, J=4.8 \mathrm{~Hz}, 1 \mathrm{H}), 7.75(\mathrm{td}, J=7.7,1.8 \mathrm{~Hz}, 1 \mathrm{H}), 7.64(\mathrm{~d}, J=8.0 \mathrm{~Hz}, 1 \mathrm{H}), 7.58(\mathrm{~d}$, $J=8.1 \mathrm{~Hz}, 2 \mathrm{H}), 7.52(\mathrm{~d}, J=8.2 \mathrm{~Hz}, 2 \mathrm{H}), 7.27-7.24(\mathrm{~m}, 1 \mathrm{H}), 6.71(\mathrm{~d}, J=15.9 \mathrm{~Hz}, 1 \mathrm{H}), 6.55-6.49(\mathrm{~m}$, 1H), $5.03-4.83(\mathrm{~m}, 1 \mathrm{H}), 4.43-4.09(\mathrm{~m}, 2 \mathrm{H}), 3.19-2.91(\mathrm{~m}, 1 \mathrm{H}), 2.46-2.29(\mathrm{~m}, 1 \mathrm{H}) .{ }^{13} \mathrm{C}$ NMR $(126$ MHz, chloroform- $d$ ) $\delta 157.9(\mathrm{~d}, J=29.0 \mathrm{~Hz}), 149.2(\mathrm{~d}, J=2.6 \mathrm{~Hz}), 140.1,136.8(\mathrm{~d}, J=1.7 \mathrm{~Hz}), 132.3$, $130.2,129.6(\mathrm{q}, J=32.3 \mathrm{~Hz}), 126.9,125.6(\mathrm{q}, J=3.8 \mathrm{~Hz}), 124.2(\mathrm{q}, J=272.0 \mathrm{~Hz}), 122.9,119.8(\mathrm{~d}, J=$ $9.7 \mathrm{~Hz}), 105.3(\mathrm{~d}, J=182.0 \mathrm{~Hz}), 80.8,78.8(\mathrm{~d}, J=25.0 \mathrm{~Hz}), 46.0(\mathrm{~d}, J=22.2 \mathrm{~Hz}) .{ }^{19} \mathrm{~F} \mathrm{NMR}(376 \mathrm{MHz}$, chloroform- $d$ ) $\delta-62.5(\mathrm{~s}, 3 \mathrm{~F}),-150.3--151.0(\mathrm{~m}, 1 \mathrm{~F})$. HRMS (EI): $[\mathrm{M}]^{\oplus}$ calcd for $\mathrm{C}_{18} \mathrm{H}_{15} \mathrm{~F}_{4} \mathrm{NO}^{\oplus}$ 337.1084, found 337.1082. HPLC analysis: Chiracel AD-H column; detected at $254 \mathrm{~nm}, 40{ }^{\circ} \mathrm{C} ; 3 \%$ ${ }^{i} \mathrm{PrOH}$ in $n$-hexane; flow $=0.7 \mathrm{~mL} / \mathrm{min}$. Retention time: $31.6 \mathrm{~min}$ (minor), $33.5 \mathrm{~min}$ (major).
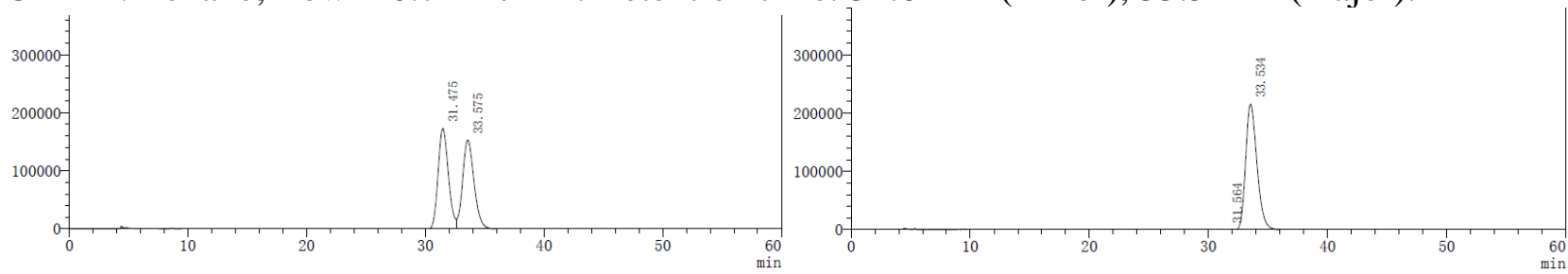

\begin{tabular}{|c|c|c|c|c|c|}
\hline Peak & retension time/min & area\% & Peak & retension time/min & area\% \\
\hline 1 & 31.475 & 50.005 & 1 & 31.564 & 0.247 \\
\hline 2 & 33.575 & 49.995 & 2 & 33.534 & 99.753 \\
\hline
\end{tabular}




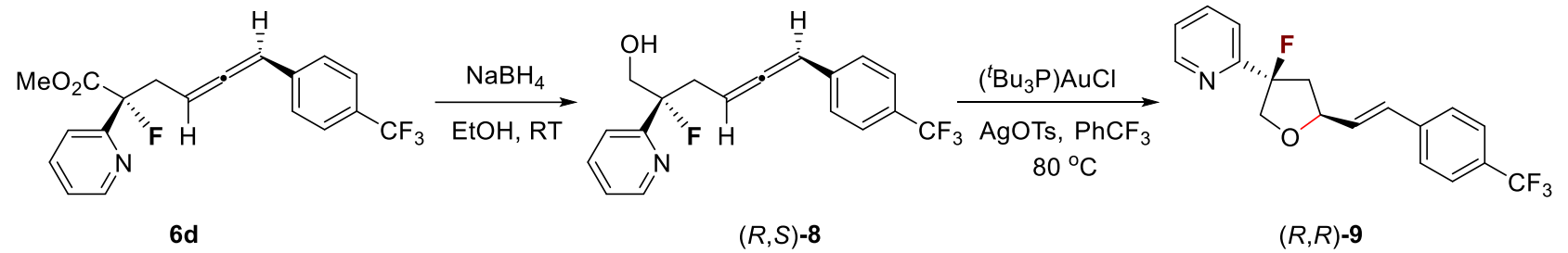

(2S,5S)-2-Fluoro-6-(4-methoxyphenyl)-2-(pyridin-2-yl)hexa-4,5-dien-1-ol [ $(R, S)$-8]

White solid, $[\alpha]_{\mathrm{D}}{ }^{25}+122.9\left(c 1.0, \mathrm{CHCl}_{3}\right)$; The spectroscopic data of $(R, S)-\mathbf{8}$ is the same as that of $(S, R)$ 8.

\section{2-((3R,5S)-3-Fluoro-5-((E)-4-(trifluoromethyl)styryl)tetrahydrofuran-3-yl)pyridine $[(R, R)-9]$}

White solid, 50\% yield, $>20: 1 \mathrm{dr},[\alpha]_{\mathrm{D}}{ }^{25}-3.4\left(c 1.0, \mathrm{CHCl}_{3}\right)$ for $>99 \%$ ee; The spectroscopic data of $(R, R)-9$ is the same as that of $(S, S)-9$. HPLC analysis: Chiracel AD-H column; detected at $254 \mathrm{~nm}, 40^{\circ} \mathrm{C}$; $3 \%{ }^{i} \mathrm{PrOH}$ in $n$-hexane; flow $=0.7 \mathrm{~mL} / \mathrm{min}$. Retention time: $31.8 \mathrm{~min}$ (major), $34.0 \mathrm{~min}$ (minor).
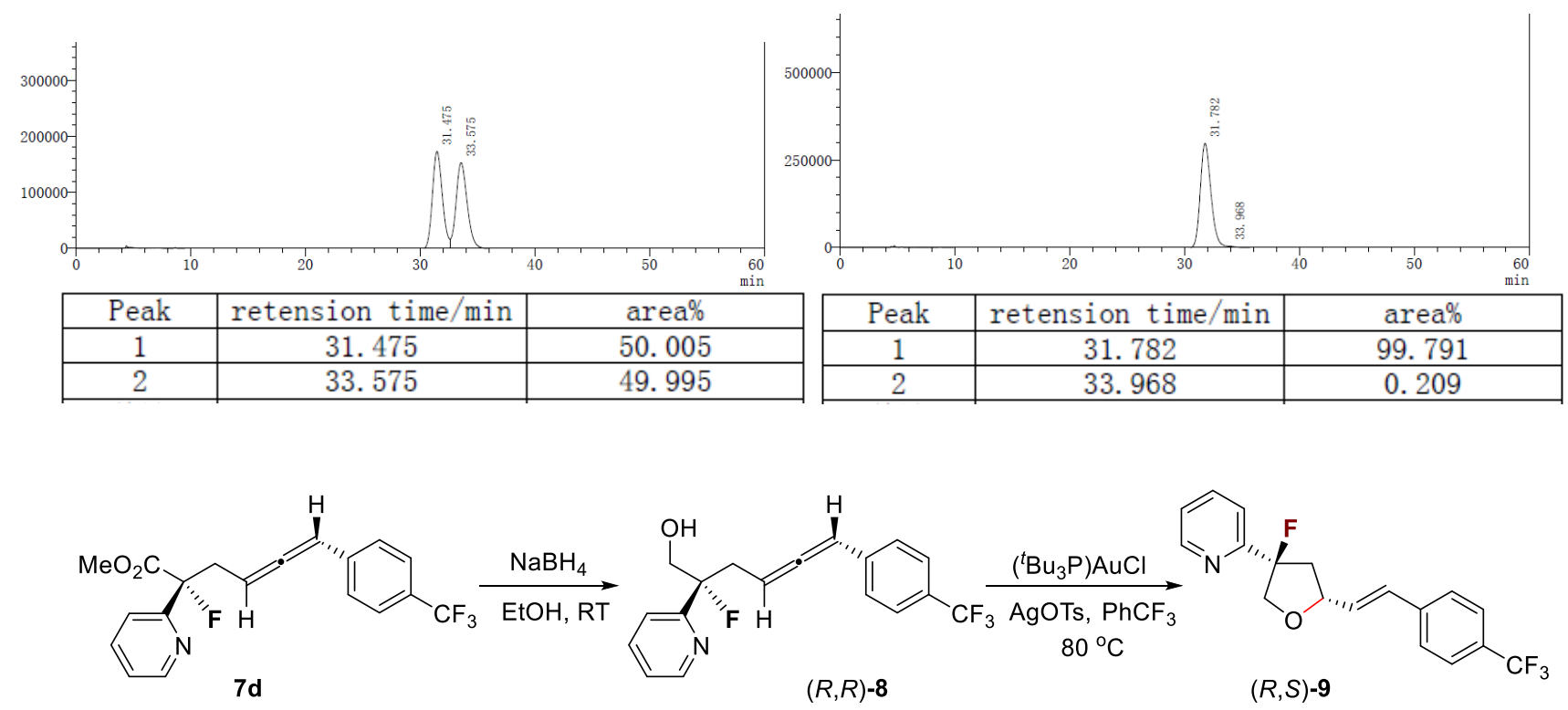

(2S,5R)-2-Fluoro-6-(4-methoxyphenyl)-2-(pyridin-2-yl)hexa-4,5-dien-1-ol $[(R, R)-8]$

White solid, $[\alpha]_{\mathrm{D}}{ }^{25}-80.5\left(c 1.0, \mathrm{CHCl}_{3}\right)$; The spectroscopic data of $(R, R)-8$ is the same as that of $(S, S)-8$.

\section{2-((3R,5R)-3-Fluoro-5-((E)-4-(trifluoromethyl)styryl)tetrahydrofuran-3-yl)pyridine [(R,S)-9]}

White solid, $72 \%$ yield, $>20: 1 \mathrm{dr},[\alpha]_{\mathrm{D}}{ }^{25}+23.0\left(c 1.0, \mathrm{CHCl}_{3}\right)$ for $>99 \%$ ee; The spectroscopic data of $(R, S)-9$ is the same as that of $(S, R)-9$. HPLC analysis: Chiracel AD-H column; detected at $254 \mathrm{~nm}, 40^{\circ} \mathrm{C}$; $3 \%{ }^{i} \mathrm{PrOH}$ in $n$-hexane; flow $=0.7 \mathrm{~mL} / \mathrm{min}$. Retention time: $12.6 \mathrm{~min}$ (minor), $15.7 \mathrm{~min}$ (major). 

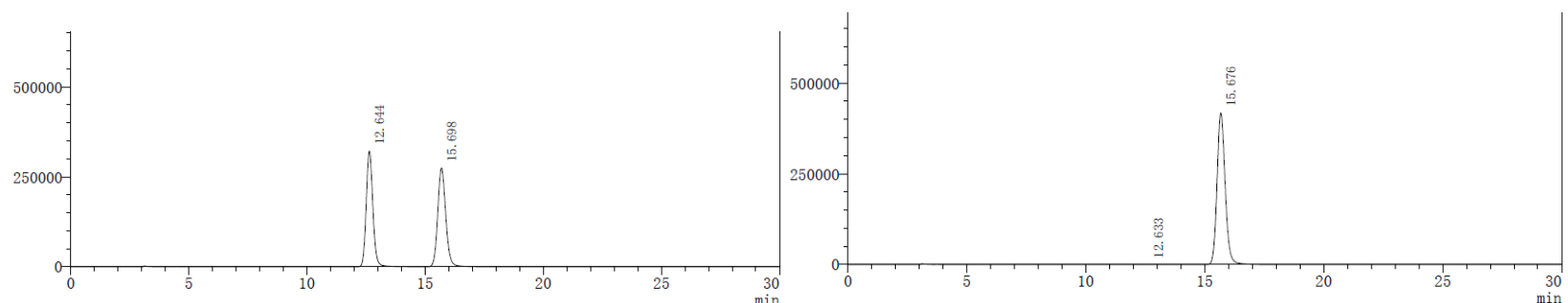

\begin{tabular}{|c|c|c|}
\hline Peak & retension time/min & area\% \\
\hline 1 & 12.644 & 49.485 \\
\hline 2 & 15.698 & 50.515 \\
\hline
\end{tabular}

\begin{tabular}{|c|c|c|}
\hline Peak & retension time/min & area\% \\
\hline 1 & 12.633 & 0.102 \\
\hline 2 & 15.676 & 99.898 \\
\hline
\end{tabular}

\section{X-ray structures of $3 d$ and $(S, S)-9$}

The X-ray crystallographic data for 3d (CCDC 2067635) and (S,S)-9 (CCDC 2067636), have been deposited at the Cambridge Crystallographic Data Center (www.ccdc.cam.ac.uk/data_request/cif).

For 3d: the data was collected by using copper irradiation source for the determination of absolute configuration of $\mathbf{3 d}$.

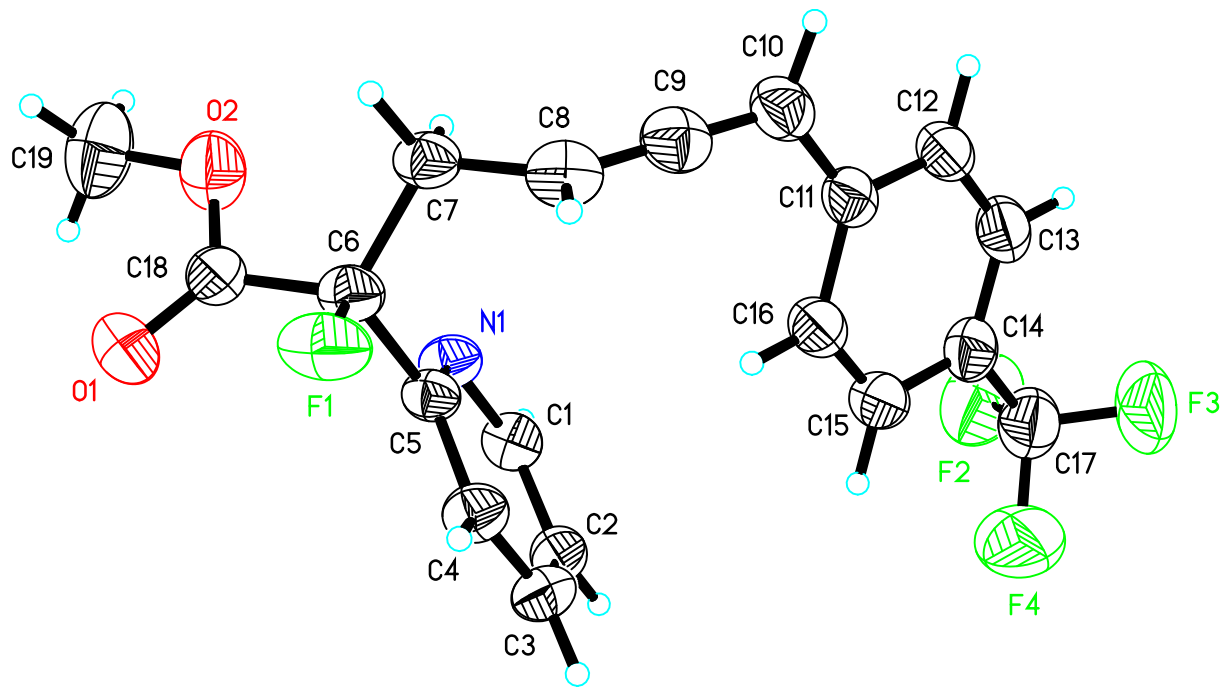




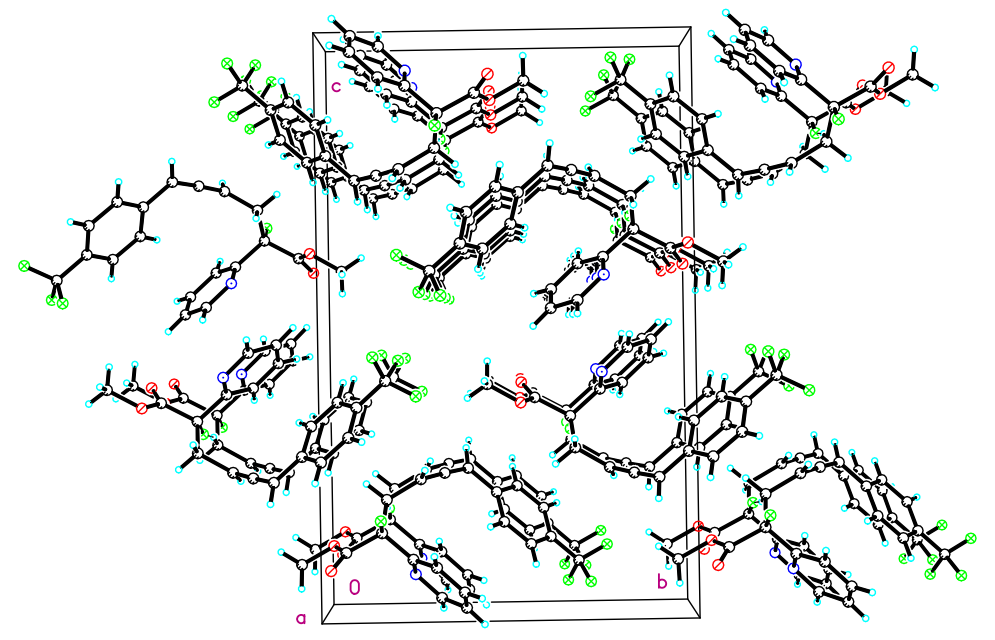

Table 1. Crystal data and structure refinement for $\mathbf{3 d}$ Identification code Empirical formula 3d Formula weight C19 H15 F4 N O2

Temperature

Wavelength

Crystal system

Space group

Unit cell dimensions

Volume

Z

Density (calculated)

Absorption coefficient

$\mathrm{F}(000)$

Crystal size

Theta range for data collection Index ranges

Reflections collected

Independent reflections

Completeness to theta $=67.679^{\circ}$

Absorption correction

Max. and min. transmission

Refinement method

Data / restraints / parameters

Goodness-of-fit on $\mathrm{F}^{2}$

Final $R$ indices [I>2sigma(I)]

$\mathrm{R}$ indices (all data)

Absolute structure parameter

Extinction coefficient

365.32

293(2) K

$1.54178 \AA$

Orthorhombic

P 212121

$\begin{array}{ll}\mathrm{a}=5.6946(2) \AA & \alpha=90^{\circ} . \\ \mathrm{b}=14.1225(4) \AA & \beta=90^{\circ} . \\ \mathrm{c}=22.0782(7) \AA & \gamma=90^{\circ} .\end{array}$

1775.57(10) $\AA^{3}$

4

$1.367 \mathrm{Mg} / \mathrm{m}^{3}$

$1.012 \mathrm{~mm}^{-1}$

752

$0.200 \times 0.150 \times 0.100 \mathrm{~mm}^{3}$

5.085 to $67.467^{\circ}$.

$-6<=\mathrm{h}<=6,-16<=\mathrm{k}<=15,-26<=1<=24$

7651

$3091[\mathrm{R}($ int $)=0.0809]$

$96.3 \%$

Semi-empirical from equivalents

0.7533 and 0.5033

Full-matrix least-squares on $\mathrm{F}^{2}$

3091 / 36 / 264

1.037

$\mathrm{R} 1=0.0578, \mathrm{wR} 2=0.1602$

$\mathrm{R} 1=0.0596, \mathrm{wR} 2=0.1631$

$-0.07(16)$

$0.038(9)$

Largest diff. peak and hole 
Table 2. Atomic coordinates (x $\left.10^{4}\right)$ and equivalent isotropic displacement parameters $\left(\AA^{2} \times 10^{3}\right)$ for 3d. $U(e q)$ is defined as one third of the trace of the orthogonalized $U^{\mathrm{ij}}$ tensor.

\begin{tabular}{lrrrr}
\hline & $\mathrm{x}$ & $\mathrm{y}$ & $\mathrm{z}$ & $\mathrm{U}(\mathrm{eq})$ \\
\hline $\mathrm{F}(1)$ & $744(4)$ & $8301(2)$ & $6814(1)$ & $98(1)$ \\
$\mathrm{F}(2)$ & $11520(20)$ & $3399(6)$ & $5485(4)$ & $158(2)$ \\
$\mathrm{F}(3)$ & $10670(20)$ & $2330(6)$ & $6127(4)$ & $158(3)$ \\
$\mathrm{F}(4)$ & $8040(20)$ & $2826(7)$ & $5555(5)$ & $173(3)$ \\
$\mathrm{F}\left(2^{\prime}\right)$ & $9240(30)$ & $3256(9)$ & $5329(5)$ & $147(3)$ \\
$\mathrm{F}\left(3^{\prime}\right)$ & $12170(30)$ & $2837(11)$ & $5892(7)$ & $168(3)$ \\
$\mathrm{F}\left(4^{\prime}\right)$ & $8890(40)$ & $2336(9)$ & $6041(8)$ & $168(3)$ \\
$\mathrm{O}(1)$ & $1631(10)$ & $9626(3)$ & $5989(3)$ & $174(3)$ \\
$\mathrm{O}(2)$ & $5289(6)$ & $9656(2)$ & $6306(2)$ & $100(1)$ \\
$\mathrm{N}(1)$ & $5203(5)$ & $7502(2)$ & $5807(1)$ & $65(1)$ \\
$\mathrm{C}(1)$ & $5456(6)$ & $6817(2)$ & $5395(1)$ & $67(1)$ \\
$\mathrm{C}(2)$ & $3763(6)$ & $6151(2)$ & $5276(1)$ & $69(1)$ \\
$\mathrm{C}(3)$ & $1731(7)$ & $6178(2)$ & $5594(2)$ & $74(1)$ \\
$\mathrm{C}(4)$ & $1400(6)$ & $6888(2)$ & $6022(2)$ & $68(1)$ \\
$\mathrm{C}(5)$ & $3182(5)$ & $7524(2)$ & $6110(1)$ & $57(1)$ \\
$\mathrm{C}(6)$ & $2993(5)$ & $8327(3)$ & $6567(2)$ & $69(1)$ \\
$\mathrm{C}(7)$ & $4747(6)$ & $8228(2)$ & $7086(1)$ & $68(1)$ \\
$\mathrm{C}(8)$ & $4445(7)$ & $7312(3)$ & $7430(1)$ & $77(1)$ \\
$\mathrm{C}(9)$ & $6110(7)$ & $6705(3)$ & $7503(2)$ & $78(1)$ \\
$\mathrm{C}(10)$ & $7826(7)$ & $6094(3)$ & $7552(2)$ & $78(1)$ \\
$\mathrm{C}(11)$ & $8319(6)$ & $5332(2)$ & $7120(2)$ & $69(1)$ \\
$\mathrm{C}(12)$ & $10306(7)$ & $4780(3)$ & $7187(2)$ & $78(1)$ \\
$\mathrm{C}(13)$ & $10790(7)$ & $4055(3)$ & $6784(2)$ & $84(1)$ \\
$\mathrm{C}(14)$ & $9315(8)$ & $3882(2)$ & $6312(2)$ & $80(1)$ \\
$\mathrm{C}(15)$ & $7303(7)$ & $4426(3)$ & $6240(2)$ & $80(1)$ \\
$\mathrm{C}(16)$ & $6805(6)$ & $5142(3)$ & $6638(2)$ & $74(1)$ \\
$\mathrm{C}(17)$ & $9881(13)$ & $3121(4)$ & $5877(3)$ & $107(2)$ \\
$\mathrm{C}(18)$ & $3219(9)$ & $9279(3)$ & $6238(2)$ & $88(1)$ \\
$\mathrm{C}(19)$ & $5782(14)$ & $10539(4)$ & $5987(3)$ & $127(2)$ \\
& & & & \\
\hline & & & &
\end{tabular}


Table 3. Bond lengths $[\AA]$ and angles $\left[{ }^{\circ}\right]$ for $\mathbf{3 d}$.

\begin{tabular}{|c|c|}
\hline $\mathrm{F}(1)-\mathrm{C}(6)$ & $1.393(4)$ \\
\hline$F(2)-C(17)$ & $1.331(10)$ \\
\hline $\mathrm{F}(3)-\mathrm{C}(17)$ & $1.325(8)$ \\
\hline $\mathrm{F}(4)-\mathrm{C}(17)$ & $1.333(11)$ \\
\hline $\mathrm{F}\left(2^{\prime}\right)-\mathrm{C}(17)$ & $1.278(13)$ \\
\hline $\mathrm{F}\left(3^{\prime}\right)-\mathrm{C}(17)$ & $1.362(16)$ \\
\hline F(4')-C(17) & $1.296(14)$ \\
\hline $\mathrm{O}(1)-\mathrm{C}(18)$ & $1.167(6)$ \\
\hline $\mathrm{O}(2)-\mathrm{C}(18)$ & $1.302(6)$ \\
\hline $\mathrm{O}(2)-\mathrm{C}(19)$ & $1.460(6)$ \\
\hline $\mathrm{N}(1)-\mathrm{C}(5)$ & $1.331(4)$ \\
\hline $\mathrm{N}(1)-\mathrm{C}(1)$ & $1.336(4)$ \\
\hline $\mathrm{C}(1)-\mathrm{C}(2)$ & $1.373(5)$ \\
\hline $\mathrm{C}(1)-\mathrm{H}(1)$ & 0.9300 \\
\hline $\mathrm{C}(2)-\mathrm{C}(3)$ & $1.354(5)$ \\
\hline $\mathrm{C}(2)-\mathrm{H}(2)$ & 0.9300 \\
\hline $\mathrm{C}(3)-\mathrm{C}(4)$ & $1.390(5)$ \\
\hline $\mathrm{C}(3)-\mathrm{H}(3)$ & 0.9300 \\
\hline $\mathrm{C}(4)-\mathrm{C}(5)$ & $1.369(4)$ \\
\hline $\mathrm{C}(4)-\mathrm{H}(4)$ & 0.9300 \\
\hline$C(5)-C(6)$ & $1.522(4)$ \\
\hline$C(6)-C(7)$ & $1.526(5)$ \\
\hline C(6)-C(18) & $1.534(6)$ \\
\hline$C(7)-C(8)$ & $1.510(5)$ \\
\hline $\mathrm{C}(7)-\mathrm{H}(7 \mathrm{~A})$ & 0.9700 \\
\hline $\mathrm{C}(7)-\mathrm{H}(7 \mathrm{~B})$ & 0.9700 \\
\hline $\mathrm{C}(8)-\mathrm{C}(9)$ & $1.289(6)$ \\
\hline $\mathrm{C}(8)-\mathrm{H}(8)$ & 0.9300 \\
\hline $\mathrm{C}(9)-\mathrm{C}(10)$ & $1.308(6)$ \\
\hline $\mathrm{C}(10)-\mathrm{C}(11)$ & $1.465(5)$ \\
\hline $\mathrm{C}(10)-\mathrm{H}(10)$ & 0.9300 \\
\hline$C(11)-C(12)$ & $1.381(5)$ \\
\hline $\mathrm{C}(11)-\mathrm{C}(16)$ & $1.395(5)$ \\
\hline$C(12)-C(13)$ & $1.384(6)$ \\
\hline $\mathrm{C}(12)-\mathrm{H}(12)$ & 0.9300 \\
\hline $\mathrm{C}(13)-\mathrm{C}(14)$ & $1.361(6)$ \\
\hline C(13)-H(13) & 0.9300 \\
\hline $\mathrm{C}(14)-\mathrm{C}(15)$ & $1.388(6)$ \\
\hline $\mathrm{C}(14)-\mathrm{C}(17)$ & $1.478(7)$ \\
\hline$C(15)-C(16)$ & $1.371(6)$ \\
\hline $\mathrm{C}(15)-\mathrm{H}(15)$ & 0.9300 \\
\hline C(16)-H(16) & 0.9300 \\
\hline C(19)-H(19A) & 0.9600 \\
\hline C(19)-H(19B) & 0.9600 \\
\hline $\mathrm{C}(19)-\mathrm{H}(19 \mathrm{C})$ & 0.9600 \\
\hline $\mathrm{C}(18)-\mathrm{O}(2)-\mathrm{C}(19)$ & $117.9(5)$ \\
\hline $\mathrm{C}(5)-\mathrm{N}(1)-\mathrm{C}(1)$ & $116.9(3)$ \\
\hline $\mathrm{N}(1)-\mathrm{C}(1)-\mathrm{C}(2)$ & $123.4(3)$ \\
\hline $\mathrm{N}(1)-\mathrm{C}(1)-\mathrm{H}(1)$ & 118.3 \\
\hline $\mathrm{C}(2)-\mathrm{C}(1)-\mathrm{H}(1)$ & 118.3 \\
\hline$C(3)-C(2)-C(1)$ & $118.8(3)$ \\
\hline $\mathrm{C}(3)-\mathrm{C}(2)-\mathrm{H}(2)$ & 120.6 \\
\hline $\mathrm{C}(1)-\mathrm{C}(2)-\mathrm{H}(2)$ & 120.6 \\
\hline
\end{tabular}




\begin{tabular}{|c|c|}
\hline$C(2)-C(3)-C(4)$ & 119.3(3) \\
\hline $\mathrm{C}(2)-\mathrm{C}(3)-\mathrm{H}(3)$ & 120.4 \\
\hline $\mathrm{C}(4)-\mathrm{C}(3)-\mathrm{H}(3)$ & 120.4 \\
\hline$C(5)-C(4)-C(3)$ & $118.0(3)$ \\
\hline $\mathrm{C}(5)-\mathrm{C}(4)-\mathrm{H}(4)$ & 121.0 \\
\hline $\mathrm{C}(3)-\mathrm{C}(4)-\mathrm{H}(4)$ & 121.0 \\
\hline $\mathrm{N}(1)-\mathrm{C}(5)-\mathrm{C}(4)$ & $123.7(3)$ \\
\hline $\mathrm{N}(1)-\mathrm{C}(5)-\mathrm{C}(6)$ & $114.3(3)$ \\
\hline$C(4)-C(5)-C(6)$ & $122.1(3)$ \\
\hline $\mathrm{F}(1)-\mathrm{C}(6)-\mathrm{C}(5)$ & $107.7(3)$ \\
\hline $\mathrm{F}(1)-\mathrm{C}(6)-\mathrm{C}(7)$ & $107.8(3)$ \\
\hline$C(5)-C(6)-C(7)$ & $112.5(3)$ \\
\hline $\mathrm{F}(1)-\mathrm{C}(6)-\mathrm{C}(18)$ & $106.6(3)$ \\
\hline$C(5)-C(6)-C(18)$ & $109.5(3)$ \\
\hline $\mathrm{C}(7)-\mathrm{C}(6)-\mathrm{C}(18)$ & $112.4(3)$ \\
\hline$C(8)-C(7)-C(6)$ & $112.4(3)$ \\
\hline $\mathrm{C}(8)-\mathrm{C}(7)-\mathrm{H}(7 \mathrm{~A})$ & 109.1 \\
\hline $\mathrm{C}(6)-\mathrm{C}(7)-\mathrm{H}(7 \mathrm{~A})$ & 109.1 \\
\hline $\mathrm{C}(8)-\mathrm{C}(7)-\mathrm{H}(7 \mathrm{~B})$ & 109.1 \\
\hline $\mathrm{C}(6)-\mathrm{C}(7)-\mathrm{H}(7 \mathrm{~B})$ & 109.1 \\
\hline $\mathrm{H}(7 \mathrm{~A})-\mathrm{C}(7)-\mathrm{H}(7 \mathrm{~B})$ & 107.8 \\
\hline $\mathrm{C}(9)-\mathrm{C}(8)-\mathrm{C}(7)$ & $123.3(3)$ \\
\hline $\mathrm{C}(9)-\mathrm{C}(8)-\mathrm{H}(8)$ & 118.4 \\
\hline $\mathrm{C}(7)-\mathrm{C}(8)-\mathrm{H}(8)$ & 118.4 \\
\hline $\mathrm{C}(8)-\mathrm{C}(9)-\mathrm{C}(10)$ & $177.6(4)$ \\
\hline$C(9)-C(10)-C(11)$ & $125.0(4)$ \\
\hline $\mathrm{C}(9)-\mathrm{C}(10)-\mathrm{H}(10)$ & 117.5 \\
\hline $\mathrm{C}(11)-\mathrm{C}(10)-\mathrm{H}(10)$ & 117.5 \\
\hline $\mathrm{C}(12)-\mathrm{C}(11)-\mathrm{C}(16)$ & 118.7(3) \\
\hline $\mathrm{C}(12)-\mathrm{C}(11)-\mathrm{C}(10)$ & $120.1(3)$ \\
\hline $\mathrm{C}(16)-\mathrm{C}(11)-\mathrm{C}(10)$ & $121.3(3)$ \\
\hline $\mathrm{C}(11)-\mathrm{C}(12)-\mathrm{C}(13)$ & $120.8(3)$ \\
\hline $\mathrm{C}(11)-\mathrm{C}(12)-\mathrm{H}(12)$ & 119.6 \\
\hline $\mathrm{C}(13)-\mathrm{C}(12)-\mathrm{H}(12)$ & 119.6 \\
\hline $\mathrm{C}(14)-\mathrm{C}(13)-\mathrm{C}(12)$ & $120.1(4)$ \\
\hline $\mathrm{C}(14)-\mathrm{C}(13)-\mathrm{H}(13)$ & 120.0 \\
\hline $\mathrm{C}(12)-\mathrm{C}(13)-\mathrm{H}(13)$ & 120.0 \\
\hline $\mathrm{C}(13)-\mathrm{C}(14)-\mathrm{C}(15)$ & $120.0(4)$ \\
\hline $\mathrm{C}(13)-\mathrm{C}(14)-\mathrm{C}(17)$ & $119.6(4)$ \\
\hline $\mathrm{C}(15)-\mathrm{C}(14)-\mathrm{C}(17)$ & $120.4(4)$ \\
\hline$C(16)-C(15)-C(14)$ & $120.3(4)$ \\
\hline $\mathrm{C}(16)-\mathrm{C}(15)-\mathrm{H}(15)$ & 119.9 \\
\hline $\mathrm{C}(14)-\mathrm{C}(15)-\mathrm{H}(15)$ & 119.9 \\
\hline $\mathrm{C}(15)-\mathrm{C}(16)-\mathrm{C}(11)$ & $120.2(4)$ \\
\hline $\mathrm{C}(15)-\mathrm{C}(16)-\mathrm{H}(16)$ & 119.9 \\
\hline $\mathrm{C}(11)-\mathrm{C}(16)-\mathrm{H}(16)$ & 119.9 \\
\hline $\mathrm{F}\left(2^{\prime}\right)-\mathrm{C}(17)-\mathrm{F}\left(4^{\prime}\right)$ & $105.6(12)$ \\
\hline $\mathrm{F}(3)-\mathrm{C}(17)-\mathrm{F}(2)$ & $106.4(9)$ \\
\hline $\mathrm{F}(3)-\mathrm{C}(17)-\mathrm{F}(4)$ & $102.9(8)$ \\
\hline $\mathrm{F}(2)-\mathrm{C}(17)-\mathrm{F}(4)$ & $107.3(8)$ \\
\hline $\mathrm{F}\left(2^{\prime}\right)-\mathrm{C}(17)-\mathrm{F}\left(3^{\prime}\right)$ & $109.8(11)$ \\
\hline $\mathrm{F}\left(4^{\prime}\right)-\mathrm{C}(17)-\mathrm{F}\left(3^{\prime}\right)$ & $99.0(11)$ \\
\hline $\mathrm{F}\left(2^{\prime}\right)-\mathrm{C}(17)-\mathrm{C}(14)$ & $116.5(7)$ \\
\hline $\mathrm{F}\left(4^{\prime}\right)-\mathrm{C}(17)-\mathrm{C}(14)$ & $110.2(8)$ \\
\hline $\mathrm{F}(3)-\mathrm{C}(17)-\mathrm{C}(14)$ & $114.6(5)$ \\
\hline $\mathrm{F}(2)-\mathrm{C}(17)-\mathrm{C}(14)$ & $111.2(5)$ \\
\hline
\end{tabular}




$\begin{array}{ll}\mathrm{F}(4)-\mathrm{C}(17)-\mathrm{C}(14) & 113.7(6) \\ \mathrm{F}\left(3^{\prime}\right)-\mathrm{C}(17)-\mathrm{C}(14) & 114.0(7) \\ \mathrm{O}(1)-\mathrm{C}(18)-\mathrm{O}(2) & 125.8(5) \\ \mathrm{O}(1)-\mathrm{C}(18)-\mathrm{C}(6) & 121.8(5) \\ \mathrm{O}(2)-\mathrm{C}(18)-\mathrm{C}(6) & 112.3(3) \\ \mathrm{O}(2)-\mathrm{C}(19)-\mathrm{H}(19 \mathrm{~A}) & 109.5 \\ \mathrm{O}(2)-\mathrm{C}(19)-\mathrm{H}(19 \mathrm{~B}) & 109.5 \\ \mathrm{H}(19 \mathrm{~A})-\mathrm{C}(19)-\mathrm{H}(19 \mathrm{~B}) & 109.5 \\ \mathrm{O}(2)-\mathrm{C}(19)-\mathrm{H}(19 \mathrm{C}) & 109.5 \\ \mathrm{H}(19 \mathrm{~A})-\mathrm{C}(19)-\mathrm{H}(19 \mathrm{C}) & 109.5 \\ \mathrm{H}(19 \mathrm{~B})-\mathrm{C}(19)-\mathrm{H}(19 \mathrm{C}) & 109.5\end{array}$

Symmetry transformations used to generate equivalent atoms: 
Table 4. Anisotropic displacement parameters $\left(\AA^{2} \times 10^{3}\right)$ for 3d. The anisotropic displacement factor exponent takes the form: $-2 \pi^{2}\left[\mathrm{~h}^{2} \mathrm{a}^{* 2} \mathrm{U}^{11}+\ldots+2 \mathrm{~h} \mathrm{k} \mathrm{a}^{*} \mathrm{~b}^{*} \mathrm{U}^{12}\right]$

\begin{tabular}{lcccccc}
\hline & $\mathrm{U}^{11}$ & $\mathrm{U}^{22}$ & $\mathrm{U}^{33}$ & $\mathrm{U}^{23}$ & $\mathrm{U}^{13}$ & $\mathrm{U}^{12}$ \\
\hline $\mathrm{F}(1)$ & $57(1)$ & $136(2)$ & $102(2)$ & $-48(2)$ & $4(1)$ & $14(1)$ \\
$\mathrm{F}(2)$ & $210(7)$ & $132(4)$ & $132(4)$ & $-31(4)$ & $51(5)$ & $8(5)$ \\
$\mathrm{F}(3)$ & $221(8)$ & $89(3)$ & $164(5)$ & $4(3)$ & $-26(6)$ & $57(5)$ \\
$\mathrm{F}(4)$ & $205(7)$ & $143(6)$ & $170(6)$ & $-63(5)$ & $-53(5)$ & $16(5)$ \\
$\mathrm{F}\left(2^{\prime}\right)$ & $217(9)$ & $122(6)$ & $102(5)$ & $-22(4)$ & $-4(6)$ & $27(6)$ \\
$\mathrm{F}\left(3^{\prime}\right)$ & $190(8)$ & $139(6)$ & $174(7)$ & $-33(6)$ & $3(7)$ & $35(7)$ \\
$\mathrm{F}\left(4^{\prime}\right)$ & $227(9)$ & $90(5)$ & $187(7)$ & $-24(5)$ & $-10(8)$ & $-8(7)$ \\
$\mathrm{O}(1)$ & $182(4)$ & $88(2)$ & $251(6)$ & $17(3)$ & $-138(4)$ & $6(3)$ \\
$\mathrm{O}(2)$ & $94(2)$ & $85(2)$ & $121(2)$ & $15(2)$ & $3(2)$ & $3(2)$ \\
$\mathrm{N}(1)$ & $54(1)$ & $72(1)$ & $70(1)$ & $-11(1)$ & $5(1)$ & $-5(1)$ \\
$\mathrm{C}(1)$ & $62(2)$ & $77(2)$ & $61(2)$ & $-5(1)$ & $9(1)$ & $5(1)$ \\
$\mathrm{C}(2)$ & $79(2)$ & $64(2)$ & $63(2)$ & $-8(1)$ & $-5(1)$ & $7(2)$ \\
$\mathrm{C}(3)$ & $73(2)$ & $65(2)$ & $83(2)$ & $-7(2)$ & $-5(2)$ & $-14(2)$ \\
$\mathrm{C}(4)$ & $57(2)$ & $74(2)$ & $73(2)$ & $-4(1)$ & $7(1)$ & $-9(1)$ \\
$\mathrm{C}(5)$ & $50(1)$ & $64(1)$ & $57(1)$ & $-5(1)$ & $-2(1)$ & $0(1)$ \\
$\mathrm{C}(6)$ & $53(2)$ & $77(2)$ & $76(2)$ & $-21(2)$ & $-4(1)$ & $9(2)$ \\
$\mathrm{C}(7)$ & $63(2)$ & $77(2)$ & $64(2)$ & $-17(1)$ & $-3(1)$ & $4(2)$ \\
$\mathrm{C}(8)$ & $73(2)$ & $95(2)$ & $64(2)$ & $-5(2)$ & $11(2)$ & $-6(2)$ \\
$\mathrm{C}(9)$ & $83(2)$ & $91(2)$ & $60(2)$ & $6(2)$ & $4(2)$ & $-10(2)$ \\
$\mathrm{C}(10)$ & $82(2)$ & $86(2)$ & $66(2)$ & $13(2)$ & $-6(2)$ & $-5(2)$ \\
$\mathrm{C}(11)$ & $67(2)$ & $71(2)$ & $67(2)$ & $21(1)$ & $-3(1)$ & $-13(2)$ \\
$\mathrm{C}(12)$ & $74(2)$ & $81(2)$ & $77(2)$ & $17(2)$ & $-16(2)$ & $-7(2)$ \\
$\mathrm{C}(13)$ & $80(2)$ & $78(2)$ & $94(2)$ & $20(2)$ & $-7(2)$ & $7(2)$ \\
$\mathrm{C}(14)$ & $91(3)$ & $68(2)$ & $82(2)$ & $16(2)$ & $2(2)$ & $-5(2)$ \\
$\mathrm{C}(15)$ & $80(2)$ & $79(2)$ & $80(2)$ & $11(2)$ & $-15(2)$ & $-13(2)$ \\
$\mathrm{C}(16)$ & $66(2)$ & $78(2)$ & $77(2)$ & $15(2)$ & $-8(2)$ & $-4(2)$ \\
$\mathrm{C}(17)$ & $139(4)$ & $82(3)$ & $100(3)$ & $-1(2)$ & $-7(3)$ & $10(3)$ \\
$\mathrm{C}(18)$ & $104(3)$ & $70(2)$ & $90(2)$ & $-24(2)$ & $-31(2)$ & $23(2)$ \\
$\mathrm{C}(19)$ & $159(5)$ & $89(3)$ & $134(4)$ & $23(3)$ & $27(4)$ & $10(3)$ \\
& & & & & &
\end{tabular}


Table 5. Hydrogen coordinates $\left(\mathrm{x} 10^{4}\right)$ and isotropic displacement parameters $\left(\AA^{2} \times 10^{3}\right)$ for 3d.

\begin{tabular}{lrrrr}
\hline & $\mathrm{x}$ & $\mathrm{y}$ & $\mathrm{z}$ & $\mathrm{U}(\mathrm{eq})$ \\
\hline & & & & \\
$\mathrm{H}(1)$ & 6852 & 6791 & 5177 & 80 \\
$\mathrm{H}(2)$ & 4007 & 5689 & 4982 & 83 \\
$\mathrm{H}(3)$ & 570 & 5727 & 5527 & 89 \\
$\mathrm{H}(4)$ & 10 & 6930 & 6241 & 82 \\
$\mathrm{H}(7 \mathrm{~A})$ & 6329 & 8257 & 6924 & 81 \\
$\mathrm{H}(7 \mathrm{~B})$ & 4552 & 8756 & 7363 & 81 \\
$\mathrm{H}(8)$ & 2984 & 7180 & 7599 & 93 \\
$\mathrm{H}(10)$ & 8808 & 6147 & 7887 & 94 \\
$\mathrm{H}(12)$ & 11329 & 4898 & 7507 & 93 \\
$\mathrm{H}(13)$ & 12126 & 3685 & 6837 & 101 \\
$\mathrm{H}(15)$ & 6289 & 4303 & 5919 & 96 \\
$\mathrm{H}(16)$ & 5454 & 5503 & 6587 & 88 \\
$\mathrm{H}(19 \mathrm{~A})$ & 4636 & 10632 & 5674 & 191 \\
$\mathrm{H}(19 \mathrm{~B})$ & 7320 & 10509 & 5811 & 191 \\
$\mathrm{H}(19 \mathrm{C})$ & 5714 & 11058 & 6268 & 191 \\
& & & & \\
\hline
\end{tabular}


Table 6. Torsion angles $\left[{ }^{\circ}\right]$ for $\mathbf{3 d}$.

\begin{tabular}{|c|c|}
\hline $\mathrm{C}(5)-\mathrm{N}(1)-\mathrm{C}(1)-\mathrm{C}(2)$ & $-0.3(5)$ \\
\hline $\mathrm{N}(1)-\mathrm{C}(1)-\mathrm{C}(2)-\mathrm{C}(3)$ & $-0.5(5)$ \\
\hline$C(1)-C(2)-C(3)-C(4)$ & $1.2(5)$ \\
\hline$C(2)-C(3)-C(4)-C(5)$ & $-1.1(5)$ \\
\hline $\mathrm{C}(1)-\mathrm{N}(1)-\mathrm{C}(5)-\mathrm{C}(4)$ & $0.4(4)$ \\
\hline $\mathrm{C}(1)-\mathrm{N}(1)-\mathrm{C}(5)-\mathrm{C}(6)$ & $-179.3(3)$ \\
\hline $\mathrm{C}(3)-\mathrm{C}(4)-\mathrm{C}(5)-\mathrm{N}(1)$ & $0.3(5)$ \\
\hline$C(3)-C(4)-C(5)-C(6)$ & $180.0(3)$ \\
\hline $\mathrm{N}(1)-\mathrm{C}(5)-\mathrm{C}(6)-\mathrm{F}(1)$ & $175.8(3)$ \\
\hline $\mathrm{C}(4)-\mathrm{C}(5)-\mathrm{C}(6)-\mathrm{F}(1)$ & $-3.9(4)$ \\
\hline $\mathrm{N}(1)-\mathrm{C}(5)-\mathrm{C}(6)-\mathrm{C}(7)$ & $-65.5(4)$ \\
\hline $\mathrm{C}(4)-\mathrm{C}(5)-\mathrm{C}(6)-\mathrm{C}(7)$ & $114.8(3)$ \\
\hline $\mathrm{N}(1)-\mathrm{C}(5)-\mathrm{C}(6)-\mathrm{C}(18)$ & $60.2(4)$ \\
\hline$C(4)-C(5)-C(6)-C(18)$ & $-119.5(4)$ \\
\hline $\mathrm{F}(1)-\mathrm{C}(6)-\mathrm{C}(7)-\mathrm{C}(8)$ & $59.9(4)$ \\
\hline$C(5)-C(6)-C(7)-C(8)$ & $-58.8(4)$ \\
\hline $\mathrm{C}(18)-\mathrm{C}(6)-\mathrm{C}(7)-\mathrm{C}(8)$ & 177.1(3) \\
\hline$C(6)-C(7)-C(8)-C(9)$ & $123.5(4)$ \\
\hline $\mathrm{C}(9)-\mathrm{C}(10)-\mathrm{C}(11)-\mathrm{C}(12)$ & 174.1(3) \\
\hline $\mathrm{C}(9)-\mathrm{C}(10)-\mathrm{C}(11)-\mathrm{C}(16)$ & $-6.4(5)$ \\
\hline$C(16)-C(11)-C(12)-C(13)$ & $0.1(5)$ \\
\hline $\mathrm{C}(10)-\mathrm{C}(11)-\mathrm{C}(12)-\mathrm{C}(13)$ & $179.7(3)$ \\
\hline$C(11)-C(12)-C(13)-C(14)$ & $0.6(6)$ \\
\hline $\mathrm{C}(12)-\mathrm{C}(13)-\mathrm{C}(14)-\mathrm{C}(15)$ & $-0.9(6)$ \\
\hline$C(12)-C(13)-C(14)-C(17)$ & $178.5(4)$ \\
\hline$C(13)-C(14)-C(15)-C(16)$ & $0.6(5)$ \\
\hline $\mathrm{C}(17)-\mathrm{C}(14)-\mathrm{C}(15)-\mathrm{C}(16)$ & $-178.8(4)$ \\
\hline$C(14)-C(15)-C(16)-C(11)$ & $0.1(5)$ \\
\hline$C(12)-C(11)-C(16)-C(15)$ & $-0.4(5)$ \\
\hline$C(10)-C(11)-C(16)-C(15)$ & $180.0(3)$ \\
\hline $\mathrm{C}(13)-\mathrm{C}(14)-\mathrm{C}(17)-\mathrm{F}\left(2^{\prime}\right)$ & $-147.2(11)$ \\
\hline $\mathrm{C}(15)-\mathrm{C}(14)-\mathrm{C}(17)-\mathrm{F}\left(2^{\prime}\right)$ & $32.2(13)$ \\
\hline $\mathrm{C}(13)-\mathrm{C}(14)-\mathrm{C}(17)-\mathrm{F}\left(4^{\prime}\right)$ & $92.6(12)$ \\
\hline $\mathrm{C}(15)-\mathrm{C}(14)-\mathrm{C}(17)-\mathrm{F}\left(4^{\prime}\right)$ & $-88.0(11)$ \\
\hline $\mathrm{C}(13)-\mathrm{C}(14)-\mathrm{C}(17)-\mathrm{F}(3)$ & $42.5(10)$ \\
\hline $\mathrm{C}(15)-\mathrm{C}(14)-\mathrm{C}(17)-\mathrm{F}(3)$ & $-138.0(9)$ \\
\hline $\mathrm{C}(13)-\mathrm{C}(14)-\mathrm{C}(17)-\mathrm{F}(2)$ & $-78.2(8)$ \\
\hline $\mathrm{C}(15)-\mathrm{C}(14)-\mathrm{C}(17)-\mathrm{F}(2)$ & $101.2(8)$ \\
\hline $\mathrm{C}(13)-\mathrm{C}(14)-\mathrm{C}(17)-\mathrm{F}(4)$ & $160.6(8)$ \\
\hline $\mathrm{C}(15)-\mathrm{C}(14)-\mathrm{C}(17)-\mathrm{F}(4)$ & $-20.0(9)$ \\
\hline $\mathrm{C}(13)-\mathrm{C}(14)-\mathrm{C}(17)-\mathrm{F}\left(3^{\prime}\right)$ & $-17.7(11)$ \\
\hline $\mathrm{C}(15)-\mathrm{C}(14)-\mathrm{C}(17)-\mathrm{F}\left(3^{\prime}\right)$ & $161.8(10)$ \\
\hline $\mathrm{C}(19)-\mathrm{O}(2)-\mathrm{C}(18)-\mathrm{O}(1)$ & $-7.5(8)$ \\
\hline $\mathrm{C}(19)-\mathrm{O}(2)-\mathrm{C}(18)-\mathrm{C}(6)$ & $176.7(4)$ \\
\hline $\mathrm{F}(1)-\mathrm{C}(6)-\mathrm{C}(18)-\mathrm{O}(1)$ & $-34.9(6)$ \\
\hline $\mathrm{C}(5)-\mathrm{C}(6)-\mathrm{C}(18)-\mathrm{O}(1)$ & $81.3(6)$ \\
\hline $\mathrm{C}(7)-\mathrm{C}(6)-\mathrm{C}(18)-\mathrm{O}(1)$ & $-152.8(5)$ \\
\hline $\mathrm{F}(1)-\mathrm{C}(6)-\mathrm{C}(18)-\mathrm{O}(2)$ & $141.1(3)$ \\
\hline $\mathrm{C}(5)-\mathrm{C}(6)-\mathrm{C}(18)-\mathrm{O}(2)$ & $-102.6(4)$ \\
\hline $\mathrm{C}(7)-\mathrm{C}(6)-\mathrm{C}(18)-\mathrm{O}(2)$ & $23.2(5)$ \\
\hline
\end{tabular}

Symmetry transformations used to generate equivalent atoms: 
Table 7. Hydrogen bonds for $\mathbf{3 d}\left[\AA\right.$ and $\left.^{\circ}\right]$.

\begin{tabular}{|c|c|c|c|c|}
\hline D-H...A & $d(D-H)$ & $\mathrm{d}(\mathrm{H} \ldots \mathrm{A})$ & $\mathrm{d}(\mathrm{D} \ldots \mathrm{A})$ & $<(\mathrm{DHA})$ \\
\hline $\mathrm{C}(19)-\mathrm{H}(19 \mathrm{C}) \ldots \mathrm{F}\left(4^{\prime}\right) \# 1$ & 0.96 & 2.60 & $3.097(15)$ & 112.1 \\
\hline $\mathrm{C}(7)-\mathrm{H}(7 \mathrm{~A}) \ldots \mathrm{F}(1) \# 2$ & 0.97 & 2.53 & $3.469(4)$ & 163.9 \\
\hline $\mathrm{C}(2)-\mathrm{H}(2) \ldots \mathrm{O}(1) \# 3$ & 0.93 & 2.65 & $3.415(6)$ & 139.9 \\
\hline $\mathrm{C}(19)-\mathrm{H}(19 \mathrm{C}) \ldots \mathrm{F}\left(4^{\prime}\right) \# 1$ & 0.96 & 2.60 & $3.097(15)$ & 112.1 \\
\hline $\mathrm{C}(7)-\mathrm{H}(7 \mathrm{~A}) \ldots \mathrm{F}(1) \# 2$ & 0.97 & 2.53 & $3.469(4)$ & 163.9 \\
\hline $\mathrm{C}(2)-\mathrm{H}(2) \ldots \mathrm{O}(1) \# 3$ & 0.93 & 2.65 & $3.415(6)$ & 139.9 \\
\hline $\mathrm{C}(19)-\mathrm{H}(19 \mathrm{C}) \ldots \mathrm{F}\left(4^{\prime}\right) \# 1$ & 0.96 & 2.60 & $3.097(15)$ & 112.1 \\
\hline $\mathrm{C}(7)-\mathrm{H}(7 \mathrm{~A}) \ldots \mathrm{F}(1) \# 2$ & 0.97 & 2.53 & $3.469(4)$ & 163.9 \\
\hline $\mathrm{C}(2)-\mathrm{H}(2) \ldots \mathrm{O}(1) \# 3$ & 0.93 & 2.65 & $3.415(6)$ & 139.9 \\
\hline $\mathrm{C}(19)-\mathrm{H}(19 \mathrm{C}) \ldots \mathrm{F}\left(4^{\prime}\right) \# 1$ & 0.96 & 2.60 & $3.097(15)$ & 112.1 \\
\hline $\mathrm{C}(7)-\mathrm{H}(7 \mathrm{~A}) \ldots \mathrm{F}(1) \# 2$ & 0.97 & 2.53 & $3.469(4)$ & 163.9 \\
\hline $\mathrm{C}(2)-\mathrm{H}(2) \ldots \mathrm{O}(1) \# 3$ & 0.93 & 2.65 & $3.415(6)$ & 139.9 \\
\hline $\mathrm{C}(7)-\mathrm{H}(7 \mathrm{~A}) \ldots \mathrm{F}(1) \# 2$ & 0.97 & 2.53 & $3.469(4)$ & 163.9 \\
\hline $\mathrm{C}(2)-\mathrm{H}(2) \ldots \mathrm{O}(1) \# 3$ & 0.93 & 2.65 & $3.415(6)$ & 139.9 \\
\hline $\mathrm{C}(19)-\mathrm{H}(19 \mathrm{C}) \ldots \mathrm{F}\left(4^{\prime}\right) \# 1$ & 0.96 & 2.60 & $3.097(15)$ & 112.1 \\
\hline $\mathrm{C}(7)-\mathrm{H}(7 \mathrm{~A}) \ldots \mathrm{F}(1) \# 2$ & 0.97 & 2.53 & $3.469(4)$ & 163.9 \\
\hline $\mathrm{C}(2)-\mathrm{H}(2) \ldots \mathrm{O}(1) \# 3$ & 0.93 & 2.65 & $3.415(6)$ & 139.9 \\
\hline $\mathrm{C}(19)-\mathrm{H}(19 \mathrm{C}) \ldots \mathrm{F}\left(4^{\prime}\right) \# 1$ & 0.96 & 2.60 & $3.097(15)$ & 112.1 \\
\hline $\mathrm{C}(7)-\mathrm{H}(7 \mathrm{~A}) \ldots \mathrm{F}(1) \# 2$ & 0.97 & 2.53 & $3.469(4)$ & 163.9 \\
\hline $\mathrm{C}(2)-\mathrm{H}(2) \ldots \mathrm{O}(1) \# 3$ & 0.93 & 2.65 & $3.415(6)$ & 139.9 \\
\hline $\mathrm{C}(7)-\mathrm{H}(7 \mathrm{~A}) \ldots \mathrm{F}(1) \# 2$ & 0.97 & 2.53 & $3.469(4)$ & 163.9 \\
\hline $\mathrm{C}(2)-\mathrm{H}(2) \ldots \mathrm{O}(1) \# 3$ & 0.93 & 2.65 & $3.415(6)$ & 139.9 \\
\hline $\mathrm{C}(2)-\mathrm{H}(2) \ldots \mathrm{O}(1) \# 3$ & 0.93 & 2.65 & $3.415(6)$ & 139.9 \\
\hline $\mathrm{C}(7)-\mathrm{H}(7 \mathrm{~A}) \ldots \mathrm{F}(1) \# 2$ & 0.97 & 2.53 & $3.469(4)$ & 163.9 \\
\hline $\mathrm{C}(2)-\mathrm{H}(2) \ldots \mathrm{O}(1) \# 3$ & 0.93 & 2.65 & $3.415(6)$ & 139.9 \\
\hline $\mathrm{C}(7)-\mathrm{H}(7 \mathrm{~A}) \ldots \mathrm{F}(1) \# 2$ & 0.97 & 2.53 & $3.469(4)$ & 163.9 \\
\hline $\mathrm{C}(19)-\mathrm{H}(19 \mathrm{C}) \ldots \mathrm{F}\left(4^{\prime}\right) \# 1$ & 0.96 & 2.60 & $3.097(15)$ & 112.1 \\
\hline $\mathrm{C}(2)-\mathrm{H}(2) \ldots \mathrm{O}(1) \# 3$ & 0.93 & 2.65 & $3.415(6)$ & 139.9 \\
\hline $\mathrm{C}(7)-\mathrm{H}(7 \mathrm{~A}) \ldots \mathrm{F}(1) \# 2$ & 0.97 & 2.53 & $3.469(4)$ & 163.9 \\
\hline $\mathrm{C}(2)-\mathrm{H}(2) \ldots \mathrm{O}(1) \# 3$ & 0.93 & 2.65 & $3.415(6)$ & 139.9 \\
\hline $\mathrm{C}(7)-\mathrm{H}(7 \mathrm{~A}) \ldots \mathrm{F}(1) \# 2$ & 0.97 & 2.53 & $3.469(4)$ & 163.9 \\
\hline $\mathrm{C}(19)-\mathrm{H}(19 \mathrm{C}) \ldots \mathrm{F}\left(4^{\prime}\right) \# 1$ & 0.96 & 2.60 & $3.097(15)$ & 112.1 \\
\hline
\end{tabular}

Symmetry transformations used to generate equivalent atoms:

$\# 1 \mathrm{x}, \mathrm{y}+1, \mathrm{z} \quad \# 2 \mathrm{x}+1, \mathrm{y}, \mathrm{z} \quad \# 3 \mathrm{x}+1 / 2,-\mathrm{y}+3 / 2,-\mathrm{z}+1$ 
For $(S, S)$-9: the data was collected by using copper irradiation source for the determination of absolute configuration of $(S, S)-9$.

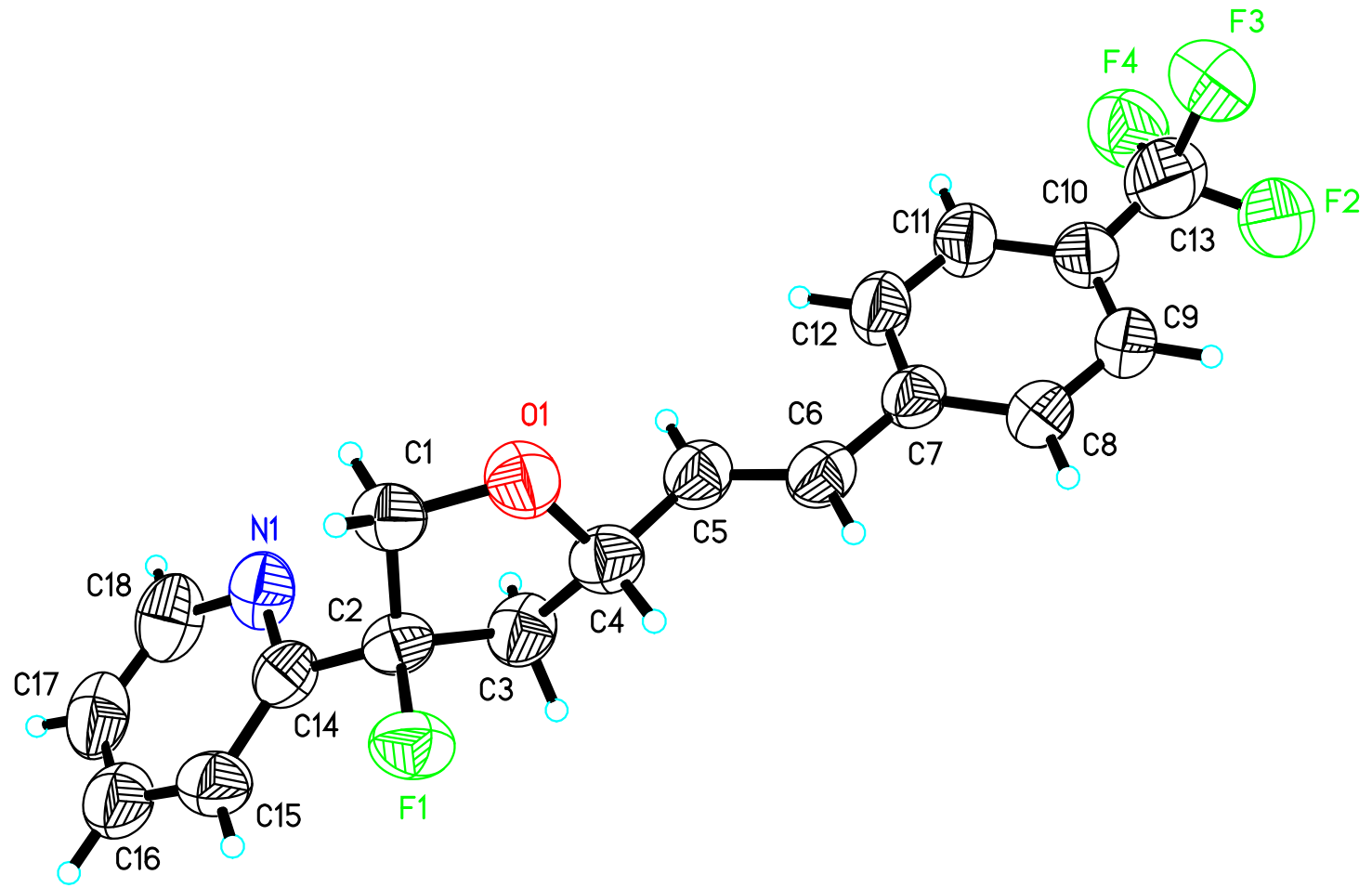




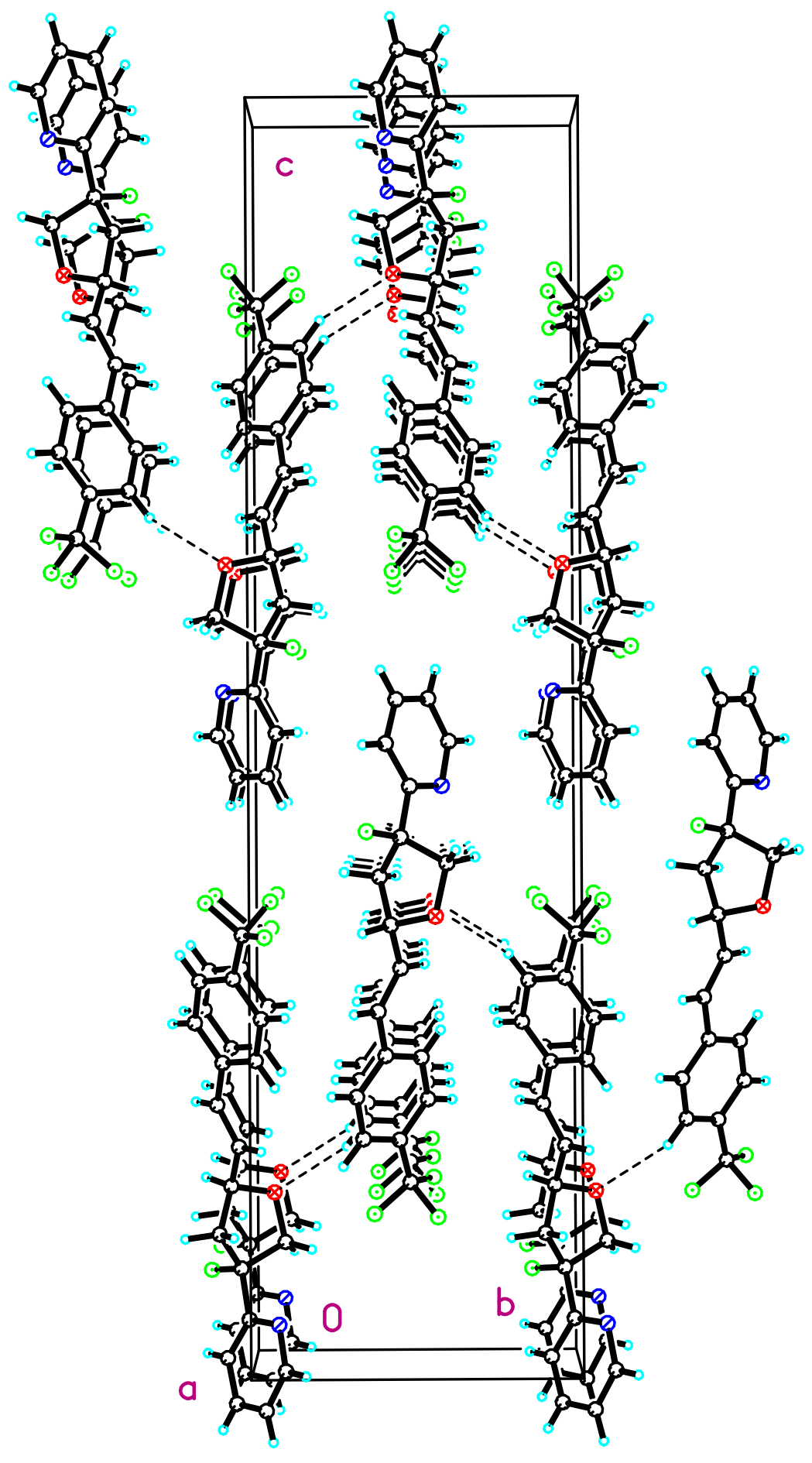


Table 1. Crystal data and structure refinement for $(S, S)-\mathbf{9}$.

Identification code

Empirical formula

Formula weight

Temperature

Wavelength

Crystal system

Space group

Unit cell dimensions

Volume

Z

Density (calculated)

Absorption coefficient

$\mathrm{F}(000)$

Crystal size

Theta range for data collection

Index ranges

Reflections collected

Independent reflections

Completeness to theta $=67.679^{\circ}$

Absorption correction

Max. and min. transmission

Refinement method

Data / restraints / parameters

Goodness-of-fit on $\mathrm{F}^{2}$

Final R indices [I $>2 \operatorname{sigma}(\mathrm{I})]$

$\mathrm{R}$ indices (all data)

Absolute structure parameter

Extinction coefficient

Largest diff. peak and hole
$(S, S)-9$

C18 H15 F4 N O

337.31

293(2) K

$1.54178 \AA$

Orthorhombic

P 212121

$\mathrm{a}=5.1435(6) \AA \quad \alpha=90^{\circ}$.

$\mathrm{b}=9.1413(11) \AA \quad \beta=90^{\circ}$.

$\mathrm{c}=35.202(4) \AA \quad \gamma=90^{\circ}$.

1655.1(3) $\AA^{3}$

4

$1.354 \mathrm{Mg} / \mathrm{m}^{3}$

$0.990 \mathrm{~mm}^{-1}$

696

$0.180 \times 0.110 \times 0.060 \mathrm{~mm}^{3}$

2.510 to $66.486^{\circ}$.

$-5<=\mathrm{h}<=6,-10<=\mathrm{k}<=10,-41<=\mathrm{l}<=36$

9796

$2836[\mathrm{R}(\mathrm{int})=0.0468]$

$95.3 \%$

Semi-empirical from equivalents

0.7533 and 0.4955

Full-matrix least-squares on $\mathrm{F}^{2}$

$2836 / 134$ / 254

1.204

$\mathrm{R} 1=0.0671, \mathrm{wR} 2=0.1977$

$\mathrm{R} 1=0.1192, \mathrm{wR} 2=0.2637$

$0.10(10)$

$0.043(12)$

0.221 and -0.264 e. $\AA^{-3}$ 
Table 2. Atomic coordinates $\left(\mathrm{x} 10^{4}\right)$ and equivalent isotropic displacement parameters $\left(\AA^{2} \mathrm{x} 10^{3}\right)$ for $(S, S)-9$. $\mathrm{U}(\mathrm{eq})$ is defined as one third of the trace of the orthogonalized $U^{i j}$ tensor.

\begin{tabular}{lrlrr}
\hline & $\mathrm{x}$ & $\mathrm{y}$ & $\mathrm{z}$ & $\mathrm{U}(\mathrm{eq})$ \\
\hline $\mathrm{F}(1)$ & $8144(8)$ & $3596(4)$ & $4302(1)$ & $107(1)$ \\
$\mathrm{F}(2)$ & $-1180(40)$ & $3702(18)$ & $1258(4)$ & $141(3)$ \\
$\mathrm{F}(3)$ & $430(20)$ & $5640(13)$ & $1274(3)$ & $149(2)$ \\
$\mathrm{F}(4)$ & $-3115(17)$ & $5514(9)$ & $1525(2)$ & $149(2)$ \\
$\mathrm{C}(13)$ & $-920(40)$ & $4750(20)$ & $1509(6)$ & $139(3)$ \\
$\mathrm{F}\left(2^{\prime}\right)$ & $-2210(20)$ & $3445(12)$ & $1408(3)$ & $146(3)$ \\
$\mathrm{F}\left(3^{\prime}\right)$ & $900(30)$ & $4571(19)$ & $1217(4)$ & $145(3)$ \\
$\mathrm{F}\left(4^{\prime}\right)$ & $-1250(80)$ & $6090(30)$ & $1433(9)$ & $144(3)$ \\
$\mathrm{C}\left(13^{\prime}\right)$ & $-900(50)$ & $4670(20)$ & $1500(6)$ & $138(3)$ \\
$\mathrm{O}(1)$ & $7319(10)$ & $5578(5)$ & $3663(1)$ & $108(2)$ \\
$\mathrm{N}(1)$ & $2859(11)$ & $5786(6)$ & $4633(2)$ & $102(2)$ \\
$\mathrm{C}(1)$ & $7126(12)$ & $5916(6)$ & $4059(2)$ & $89(2)$ \\
$\mathrm{C}(2)$ & $6051(10)$ & $4577(6)$ & $4250(2)$ & $82(2)$ \\
$\mathrm{C}(3)$ & $4326(14)$ & $3945(7)$ & $3946(2)$ & $98(2)$ \\
$\mathrm{C}(4)$ & $5799(13)$ & $4270(7)$ & $3585(2)$ & $94(2)$ \\
$\mathrm{C}(5)$ & $4163(13)$ & $4559(6)$ & $3246(2)$ & $92(2)$ \\
$\mathrm{C}(6)$ & $4469(13)$ & $3888(6)$ & $2917(2)$ & $89(2)$ \\
$\mathrm{C}(7)$ & $2981(12)$ & $4101(5)$ & $2562(2)$ & $83(2)$ \\
$\mathrm{C}(8)$ & $3527(16)$ & $3238(6)$ & $2249(2)$ & $101(2)$ \\
$\mathrm{C}(9)$ & $2241(16)$ & $3429(7)$ & $1911(2)$ & $105(2)$ \\
$\mathrm{C}(10)$ & $382(13)$ & $4504(7)$ & $1872(2)$ & $96(2)$ \\
$\mathrm{C}(11)$ & $-204(16)$ & $5353(8)$ & $2186(2)$ & $106(2)$ \\
$\mathrm{C}(12)$ & $1061(14)$ & $5150(7)$ & $2524(2)$ & $100(2)$ \\
$\mathrm{C}(14)$ & $4827(12)$ & $4839(6)$ & $4633(2)$ & $83(2)$ \\
$\mathrm{C}(15)$ & $5684(16)$ & $4147(7)$ & $4958(2)$ & $103(2)$ \\
$\mathrm{C}(16)$ & $4450(20)$ & $4457(8)$ & $5296(3)$ & $119(3)$ \\
$\mathrm{C}(17)$ & $2470(20)$ & $5395(10)$ & $5303(3)$ & $121(3)$ \\
$\mathrm{C}(18)$ & $1698(16)$ & $6020(10)$ & $4975(3)$ & $122(3)$ \\
& & & & \\
\hline & & & &
\end{tabular}


Table 3. Bond lengths $[\AA]$ and angles $\left[{ }^{\circ}\right]$ for $(S, S)-9$.

\begin{tabular}{|c|c|}
\hline $\mathrm{F}(1)-\mathrm{C}(2)$ & $1.413(6)$ \\
\hline $\mathrm{F}(2)-\mathrm{C}(13)$ & $1.31(2)$ \\
\hline $\mathrm{F}(3)-\mathrm{C}(13)$ & $1.35(2)$ \\
\hline $\mathrm{F}(4)-\mathrm{C}(13)$ & $1.33(2)$ \\
\hline$C(13)-C(10)$ & $1.461(19)$ \\
\hline $\mathrm{F}\left(2^{\prime}\right)-\mathrm{C}\left(13^{\prime}\right)$ & $1.35(2)$ \\
\hline $\mathrm{F}\left(3^{\prime}\right)-\mathrm{C}\left(13^{\prime}\right)$ & $1.37(2)$ \\
\hline $\mathrm{F}\left(4^{\prime}\right)-\mathrm{C}\left(13^{\prime}\right)$ & $1.33(2)$ \\
\hline$C\left(13^{\prime}\right)-C(10)$ & $1.47(2)$ \\
\hline $\mathrm{O}(1)-\mathrm{C}(1)$ & $1.433(8)$ \\
\hline $\mathrm{O}(1)-\mathrm{C}(4)$ & $1.454(7)$ \\
\hline $\mathrm{N}(1)-\mathrm{C}(14)$ & $1.332(8)$ \\
\hline $\mathrm{N}(1)-\mathrm{C}(18)$ & $1.359(10)$ \\
\hline $\mathrm{C}(1)-\mathrm{C}(2)$ & $1.502(8)$ \\
\hline $\mathrm{C}(1)-\mathrm{H}(1 \mathrm{~A})$ & 0.9700 \\
\hline $\mathrm{C}(1)-\mathrm{H}(1 \mathrm{~B})$ & 0.9700 \\
\hline$C(2)-C(3)$ & $1.506(9)$ \\
\hline $\mathrm{C}(2)-\mathrm{C}(14)$ & $1.507(9)$ \\
\hline$C(3)-C(4)$ & $1.507(9)$ \\
\hline $\mathrm{C}(3)-\mathrm{H}(3 \mathrm{~A})$ & 0.9700 \\
\hline $\mathrm{C}(3)-\mathrm{H}(3 \mathrm{~B})$ & 0.9700 \\
\hline$C(4)-C(5)$ & $1.486(9)$ \\
\hline $\mathrm{C}(4)-\mathrm{H}(4)$ & 0.9800 \\
\hline$C(5)-C(6)$ & $1.320(9)$ \\
\hline $\mathrm{C}(5)-\mathrm{H}(5)$ & 0.9300 \\
\hline$C(6)-C(7)$ & $1.475(9)$ \\
\hline $\mathrm{C}(6)-\mathrm{H}(6)$ & 0.9300 \\
\hline$C(7)-C(12)$ & $1.383(9)$ \\
\hline $\mathrm{C}(7)-\mathrm{C}(8)$ & $1.385(9)$ \\
\hline $\mathrm{C}(8)-\mathrm{C}(9)$ & $1.373(11)$ \\
\hline $\mathrm{C}(8)-\mathrm{H}(8)$ & 0.9300 \\
\hline C(9)-C(10) & $1.378(9)$ \\
\hline $\mathrm{C}(9)-\mathrm{H}(9)$ & 0.9300 \\
\hline $\mathrm{C}(10)-\mathrm{C}(11)$ & $1.382(9)$ \\
\hline $\mathrm{C}(11)-\mathrm{C}(12)$ & $1.369(10)$ \\
\hline $\mathrm{C}(11)-\mathrm{H}(11)$ & 0.9300 \\
\hline $\mathrm{C}(12)-\mathrm{H}(12)$ & 0.9300 \\
\hline$C(14)-C(15)$ & $1.381(9)$ \\
\hline$C(15)-C(16)$ & $1.377(11)$ \\
\hline $\mathrm{C}(15)-\mathrm{H}(15)$ & 0.9300 \\
\hline$C(16)-C(17)$ & $1.332(12)$ \\
\hline $\mathrm{C}(16)-\mathrm{H}(16)$ & 0.9300 \\
\hline $\mathrm{C}(17)-\mathrm{C}(18)$ & $1.349(12)$ \\
\hline C(17)-H(17) & 0.9300 \\
\hline $\mathrm{C}(18)-\mathrm{H}(18)$ & 0.9300 \\
\hline $\mathrm{F}(2)-\mathrm{C}(13)-\mathrm{F}(4)$ & $109.3(18)$ \\
\hline $\mathrm{F}(2)-\mathrm{C}(13)-\mathrm{F}(3)$ & $94.6(16)$ \\
\hline $\mathrm{F}(4)-\mathrm{C}(13)-\mathrm{F}(3)$ & $98.4(15)$ \\
\hline $\mathrm{F}(2)-\mathrm{C}(13)-\mathrm{C}(10)$ & $121.6(17)$ \\
\hline $\mathrm{F}(4)-\mathrm{C}(13)-\mathrm{C}(10)$ & $115.6(15)$ \\
\hline $\mathrm{F}(3)-\mathrm{C}(13)-\mathrm{C}(10)$ & $112.9(16)$ \\
\hline $\mathrm{F}\left(4^{\prime}\right)-\mathrm{C}\left(13^{\prime}\right)-\mathrm{F}\left(2^{\prime}\right)$ & $134(2)$ \\
\hline $\mathrm{F}\left(4^{\prime}\right)-\mathrm{C}\left(13^{\prime}\right)-\mathrm{F}\left(3^{\prime}\right)$ & $92(2)$ \\
\hline
\end{tabular}




\begin{tabular}{|c|c|}
\hline $\mathrm{F}\left(2^{\prime}\right)-\mathrm{C}\left(13^{\prime}\right)-\mathrm{F}\left(3^{\prime}\right)$ & $96.1(16)$ \\
\hline $\mathrm{F}\left(4^{\prime}\right)-\mathrm{C}\left(13^{\prime}\right)-\mathrm{C}(10)$ & 108.8(19) \\
\hline $\mathrm{F}\left(2^{\prime}\right)-\mathrm{C}\left(13^{\prime}\right)-\mathrm{C}(10)$ & $110.5(15)$ \\
\hline $\mathrm{F}\left(3^{\prime}\right)-\mathrm{C}\left(13^{\prime}\right)-\mathrm{C}(10)$ & $109.8(17)$ \\
\hline $\mathrm{C}(1)-\mathrm{O}(1)-\mathrm{C}(4)$ & $108.8(5)$ \\
\hline $\mathrm{C}(14)-\mathrm{N}(1)-\mathrm{C}(18)$ & $115.9(7)$ \\
\hline $\mathrm{O}(1)-\mathrm{C}(1)-\mathrm{C}(2)$ & $106.6(5)$ \\
\hline $\mathrm{O}(1)-\mathrm{C}(1)-\mathrm{H}(1 \mathrm{~A})$ & 110.4 \\
\hline $\mathrm{C}(2)-\mathrm{C}(1)-\mathrm{H}(1 \mathrm{~A})$ & 110.4 \\
\hline $\mathrm{O}(1)-\mathrm{C}(1)-\mathrm{H}(1 \mathrm{~B})$ & 110.4 \\
\hline $\mathrm{C}(2)-\mathrm{C}(1)-\mathrm{H}(1 \mathrm{~B})$ & 110.4 \\
\hline $\mathrm{H}(1 \mathrm{~A})-\mathrm{C}(1)-\mathrm{H}(1 \mathrm{~B})$ & 108.6 \\
\hline $\mathrm{F}(1)-\mathrm{C}(2)-\mathrm{C}(1)$ & $107.1(4)$ \\
\hline $\mathrm{F}(1)-\mathrm{C}(2)-\mathrm{C}(3)$ & $107.3(5)$ \\
\hline $\mathrm{C}(1)-\mathrm{C}(2)-\mathrm{C}(3)$ & $102.1(5)$ \\
\hline $\mathrm{F}(1)-\mathrm{C}(2)-\mathrm{C}(14)$ & $107.7(5)$ \\
\hline $\mathrm{C}(1)-\mathrm{C}(2)-\mathrm{C}(14)$ & $115.1(5)$ \\
\hline $\mathrm{C}(3)-\mathrm{C}(2)-\mathrm{C}(14)$ & $116.8(5)$ \\
\hline$C(2)-C(3)-C(4)$ & $103.2(5)$ \\
\hline $\mathrm{C}(2)-\mathrm{C}(3)-\mathrm{H}(3 \mathrm{~A})$ & 111.1 \\
\hline $\mathrm{C}(4)-\mathrm{C}(3)-\mathrm{H}(3 \mathrm{~A})$ & 111.1 \\
\hline $\mathrm{C}(2)-\mathrm{C}(3)-\mathrm{H}(3 \mathrm{~B})$ & 111.1 \\
\hline $\mathrm{C}(4)-\mathrm{C}(3)-\mathrm{H}(3 \mathrm{~B})$ & 111.1 \\
\hline $\mathrm{H}(3 \mathrm{~A})-\mathrm{C}(3)-\mathrm{H}(3 \mathrm{~B})$ & 109.1 \\
\hline $\mathrm{O}(1)-\mathrm{C}(4)-\mathrm{C}(5)$ & $108.0(5)$ \\
\hline $\mathrm{O}(1)-\mathrm{C}(4)-\mathrm{C}(3)$ & $105.9(5)$ \\
\hline $\mathrm{C}(5)-\mathrm{C}(4)-\mathrm{C}(3)$ & $115.3(6)$ \\
\hline $\mathrm{O}(1)-\mathrm{C}(4)-\mathrm{H}(4)$ & 109.1 \\
\hline $\mathrm{C}(5)-\mathrm{C}(4)-\mathrm{H}(4)$ & 109.1 \\
\hline $\mathrm{C}(3)-\mathrm{C}(4)-\mathrm{H}(4)$ & 109.1 \\
\hline$C(6)-C(5)-C(4)$ & $123.8(6)$ \\
\hline $\mathrm{C}(6)-\mathrm{C}(5)-\mathrm{H}(5)$ & 118.1 \\
\hline $\mathrm{C}(4)-\mathrm{C}(5)-\mathrm{H}(5)$ & 118.1 \\
\hline$C(5)-C(6)-C(7)$ & $128.2(6)$ \\
\hline $\mathrm{C}(5)-\mathrm{C}(6)-\mathrm{H}(6)$ & 115.9 \\
\hline $\mathrm{C}(7)-\mathrm{C}(6)-\mathrm{H}(6)$ & 115.9 \\
\hline$C(12)-C(7)-C(8)$ & $117.4(7)$ \\
\hline$C(12)-C(7)-C(6)$ & $123.1(5)$ \\
\hline$C(8)-C(7)-C(6)$ & $119.5(6)$ \\
\hline $\mathrm{C}(9)-\mathrm{C}(8)-\mathrm{C}(7)$ & $121.4(7)$ \\
\hline $\mathrm{C}(9)-\mathrm{C}(8)-\mathrm{H}(8)$ & 119.3 \\
\hline $\mathrm{C}(7)-\mathrm{C}(8)-\mathrm{H}(8)$ & 119.3 \\
\hline $\mathrm{C}(8)-\mathrm{C}(9)-\mathrm{C}(10)$ & $120.7(6)$ \\
\hline $\mathrm{C}(8)-\mathrm{C}(9)-\mathrm{H}(9)$ & 119.6 \\
\hline $\mathrm{C}(10)-\mathrm{C}(9)-\mathrm{H}(9)$ & 119.6 \\
\hline $\mathrm{C}(9)-\mathrm{C}(10)-\mathrm{C}(11)$ & $118.2(7)$ \\
\hline $\mathrm{C}(9)-\mathrm{C}(10)-\mathrm{C}(13)$ & $121.0(10)$ \\
\hline $\mathrm{C}(11)-\mathrm{C}(10)-\mathrm{C}(13)$ & $120.9(10)$ \\
\hline $\mathrm{C}(9)-\mathrm{C}(10)-\mathrm{C}\left(13^{\prime}\right)$ & $118.3(11)$ \\
\hline $\mathrm{C}(11)-\mathrm{C}(10)-\mathrm{C}\left(13^{\prime}\right)$ & $123.5(11)$ \\
\hline $\mathrm{C}(12)-\mathrm{C}(11)-\mathrm{C}(10)$ & $121.0(7)$ \\
\hline $\mathrm{C}(12)-\mathrm{C}(11)-\mathrm{H}(11)$ & 119.5 \\
\hline $\mathrm{C}(10)-\mathrm{C}(11)-\mathrm{H}(11)$ & 119.5 \\
\hline$C(11)-C(12)-C(7)$ & $121.3(6)$ \\
\hline $\mathrm{C}(11)-\mathrm{C}(12)-\mathrm{H}(12)$ & 119.3 \\
\hline $\mathrm{C}(7)-\mathrm{C}(12)-\mathrm{H}(12)$ & 119.3 \\
\hline
\end{tabular}




$\begin{array}{ll}\mathrm{N}(1)-\mathrm{C}(14)-\mathrm{C}(15) & 122.7(6) \\ \mathrm{N}(1)-\mathrm{C}(14)-\mathrm{C}(2) & 114.9(5) \\ \mathrm{C}(15)-\mathrm{C}(14)-\mathrm{C}(2) & 122.4(6) \\ \mathrm{C}(16)-\mathrm{C}(15)-\mathrm{C}(14) & 118.4(7) \\ \mathrm{C}(16)-\mathrm{C}(15)-\mathrm{H}(15) & 120.8 \\ \mathrm{C}(14)-\mathrm{C}(15)-\mathrm{H}(15) & 120.8 \\ \mathrm{C}(17)-\mathrm{C}(16)-\mathrm{C}(15) & 120.0(8) \\ \mathrm{C}(17)-\mathrm{C}(16)-\mathrm{H}(16) & 120.0 \\ \mathrm{C}(15)-\mathrm{C}(16)-\mathrm{H}(16) & 120.0 \\ \mathrm{C}(16)-\mathrm{C}(17)-\mathrm{C}(18) & 118.8(8) \\ \mathrm{C}(16)-\mathrm{C}(17)-\mathrm{H}(17) & 120.6 \\ \mathrm{C}(18)-\mathrm{C}(17)-\mathrm{H}(17) & 120.6 \\ \mathrm{C}(17)-\mathrm{C}(18)-\mathrm{N}(1) & 124.2(8) \\ \mathrm{C}(17)-\mathrm{C}(18)-\mathrm{H}(18) & 117.9 \\ \mathrm{~N}(1)-\mathrm{C}(18)-\mathrm{H}(18) & 117.9\end{array}$

Symmetry transformations used to generate equivalent atoms: 
Table 4. Anisotropic displacement parameters $\left(\AA^{2} \mathrm{x} 10^{3}\right)$ for $(S, S)-9$. The anisotropic displacement factor exponent takes the form: $-2 \pi^{2}\left[h^{2} a^{* 2} U^{11}+\ldots+2 h k a^{*} b^{*} U^{12}\right]$

\begin{tabular}{lcccccc}
\hline & $\mathrm{U}^{11}$ & $\mathrm{U}^{22}$ & $\mathrm{U}^{33}$ & $\mathrm{U}^{23}$ & $\mathrm{U}^{13}$ & $\mathrm{U}^{12}$ \\
\hline $\mathrm{F}(1)$ & $109(3)$ & $104(2)$ & $109(3)$ & $18(2)$ & $13(2)$ & $26(2)$ \\
$\mathrm{F}(2)$ & $179(7)$ & $156(6)$ & $90(5)$ & $-1(5)$ & $-23(5)$ & $-13(5)$ \\
$\mathrm{F}(3)$ & $180(6)$ & $169(5)$ & $98(4)$ & $20(4)$ & $-9(4)$ & $-13(5)$ \\
$\mathrm{F}(4)$ & $165(5)$ & $169(4)$ & $113(4)$ & $18(3)$ & $-35(4)$ & $7(4)$ \\
$\mathrm{C}(13)$ & $168(5)$ & $154(5)$ & $93(4)$ & $5(4)$ & $-23(4)$ & $-7(5)$ \\
$\mathrm{F}\left(2^{\prime}\right)$ & $171(6)$ & $160(5)$ & $105(5)$ & $-9(4)$ & $-24(4)$ & $-19(5)$ \\
$\mathrm{F}\left(3^{\prime}\right)$ & $176(6)$ & $167(6)$ & $92(5)$ & $9(5)$ & $-11(5)$ & $-7(6)$ \\
$\mathrm{F}\left(4^{\prime}\right)$ & $174(7)$ & $160(6)$ & $99(6)$ & $13(5)$ & $-21(5)$ & $1(6)$ \\
$\mathrm{C}\left(13^{\prime}\right)$ & $168(5)$ & $154(5)$ & $93(4)$ & $5(4)$ & $-23(4)$ & $-7(5)$ \\
$\mathrm{O}(1)$ & $121(3)$ & $111(3)$ & $91(3)$ & $8(2)$ & $3(3)$ & $-36(3)$ \\
$\mathrm{N}(1)$ & $88(3)$ & $114(4)$ & $105(4)$ & $-21(3)$ & $-10(3)$ & $12(3)$ \\
$\mathrm{C}(1)$ & $80(3)$ & $89(3)$ & $99(5)$ & $9(3)$ & $-8(3)$ & $-4(3)$ \\
$\mathrm{C}(2)$ & $73(3)$ & $72(2)$ & $99(5)$ & $5(3)$ & $2(3)$ & $5(2)$ \\
$\mathrm{C}(3)$ & $99(4)$ & $95(3)$ & $102(5)$ & $-7(3)$ & $20(4)$ & $-19(3)$ \\
$\mathrm{C}(4)$ & $95(4)$ & $88(3)$ & $100(5)$ & $4(3)$ & $-1(3)$ & $-6(3)$ \\
$\mathrm{C}(5)$ & $96(4)$ & $84(3)$ & $98(5)$ & $-8(3)$ & $11(3)$ & $5(3)$ \\
$\mathrm{C}(6)$ & $91(4)$ & $77(3)$ & $100(5)$ & $-4(3)$ & $11(3)$ & $2(3)$ \\
$\mathrm{C}(7)$ & $91(3)$ & $73(3)$ & $85(4)$ & $-2(3)$ & $17(3)$ & $-5(3)$ \\
$\mathrm{C}(8)$ & $127(5)$ & $84(3)$ & $92(5)$ & $-2(3)$ & $9(4)$ & $18(3)$ \\
$\mathrm{C}(9)$ & $139(5)$ & $92(4)$ & $83(5)$ & $-10(3)$ & $13(4)$ & $2(4)$ \\
$\mathrm{C}(10)$ & $106(4)$ & $91(4)$ & $90(5)$ & $-5(3)$ & $5(4)$ & $-13(3)$ \\
$\mathrm{C}(11)$ & $113(5)$ & $107(4)$ & $98(5)$ & $-17(4)$ & $-14(4)$ & $16(4)$ \\
$\mathrm{C}(12)$ & $111(5)$ & $102(4)$ & $88(5)$ & $-21(3)$ & $4(4)$ & $15(3)$ \\
$\mathrm{C}(14)$ & $79(3)$ & $75(3)$ & $95(4)$ & $-6(3)$ & $1(3)$ & $-11(2)$ \\
$\mathrm{C}(15)$ & $120(5)$ & $85(3)$ & $103(6)$ & $14(3)$ & $9(4)$ & $-5(4)$ \\
$\mathrm{C}(16)$ & $158(7)$ & $97(4)$ & $102(6)$ & $0(4)$ & $20(5)$ & $-22(5)$ \\
$\mathrm{C}(17)$ & $138(7)$ & $126(5)$ & $100(6)$ & $-28(5)$ & $22(5)$ & $-33(6)$ \\
$\mathrm{C}(18)$ & $95(4)$ & $138(6)$ & $134(8)$ & $-44(6)$ & $14(5)$ & $8(5)$ \\
& & & & & & \\
\hline & & & & & &
\end{tabular}


Table 5. Hydrogen coordinates (x 104) and isotropic displacement parameters $\left(\AA^{2} \times 10^{3}\right)$ for $(S, S)-9$.

\begin{tabular}{lrrrr}
\hline & $\mathrm{x}$ & $\mathrm{y}$ & $\mathrm{z}$ & $\mathrm{U}(\mathrm{eq})$ \\
\hline $\mathrm{H}(1 \mathrm{~A})$ & 5977 & 6744 & 4098 & 107 \\
$\mathrm{H}(1 \mathrm{~B})$ & 8823 & 6155 & 4162 & 107 \\
$\mathrm{H}(3 \mathrm{~A})$ & 2636 & 4416 & 3945 & 118 \\
$\mathrm{H}(3 \mathrm{~B})$ & 4095 & 2901 & 3980 & 118 \\
$\mathrm{H}(4)$ & 6981 & 3456 & 3530 & 113 \\
$\mathrm{H}(5)$ & 2848 & 5253 & 3266 & 111 \\
$\mathrm{H}(6)$ & 5790 & 3194 & 2907 & 107 \\
$\mathrm{H}(8)$ & 4790 & 2514 & 2268 & 121 \\
$\mathrm{H}(9)$ & 2629 & 2827 & 1706 & 126 \\
$\mathrm{H}(11)$ & -1476 & 6072 & 2167 & 127 \\
$\mathrm{H}(12)$ & 620 & 5728 & 2731 & 120 \\
$\mathrm{H}(15)$ & 7060 & 3488 & 4950 & 123 \\
$\mathrm{H}(16)$ & 5003 & 4013 & 5520 & 143 \\
$\mathrm{H}(17)$ & 1628 & 5614 & 5530 & 145 \\
$\mathrm{H}(18)$ & 283 & 6652 & 4982 & 147 \\
& & & & \\
\hline
\end{tabular}


Table 6. Torsion angles $\left[^{\circ}\right]$ for $(S, S)-9$.

\begin{tabular}{|c|c|}
\hline $\mathrm{C}(4)-\mathrm{O}(1)-\mathrm{C}(1)-\mathrm{C}(2)$ & $15.0(6)$ \\
\hline $\mathrm{O}(1)-\mathrm{C}(1)-\mathrm{C}(2)-\mathrm{F}(1)$ & $80.6(6)$ \\
\hline $\mathrm{O}(1)-\mathrm{C}(1)-\mathrm{C}(2)-\mathrm{C}(3)$ & $-32.0(6)$ \\
\hline $\mathrm{O}(1)-\mathrm{C}(1)-\mathrm{C}(2)-\mathrm{C}(14)$ & $-159.6(5)$ \\
\hline $\mathrm{F}(1)-\mathrm{C}(2)-\mathrm{C}(3)-\mathrm{C}(4)$ & $-76.5(6)$ \\
\hline$C(1)-C(2)-C(3)-C(4)$ & $36.0(6)$ \\
\hline$C(14)-C(2)-C(3)-C(4)$ & $162.6(5)$ \\
\hline $\mathrm{C}(1)-\mathrm{O}(1)-\mathrm{C}(4)-\mathrm{C}(5)$ & $132.3(6)$ \\
\hline $\mathrm{C}(1)-\mathrm{O}(1)-\mathrm{C}(4)-\mathrm{C}(3)$ & $8.3(7)$ \\
\hline $\mathrm{C}(2)-\mathrm{C}(3)-\mathrm{C}(4)-\mathrm{O}(1)$ & $-27.9(6)$ \\
\hline$C(2)-C(3)-C(4)-C(5)$ & $-147.3(5)$ \\
\hline $\mathrm{O}(1)-\mathrm{C}(4)-\mathrm{C}(5)-\mathrm{C}(6)$ & $113.1(7)$ \\
\hline$C(3)-C(4)-C(5)-C(6)$ & $-128.7(7)$ \\
\hline$C(4)-C(5)-C(6)-C(7)$ & $-179.3(5)$ \\
\hline$C(5)-C(6)-C(7)-C(12)$ & $3.9(10)$ \\
\hline$C(5)-C(6)-C(7)-C(8)$ & $-177.1(7)$ \\
\hline$C(12)-C(7)-C(8)-C(9)$ & $1.0(10)$ \\
\hline $\mathrm{C}(6)-\mathrm{C}(7)-\mathrm{C}(8)-\mathrm{C}(9)$ & $-178.0(6)$ \\
\hline $\mathrm{C}(7)-\mathrm{C}(8)-\mathrm{C}(9)-\mathrm{C}(10)$ & $0.8(11)$ \\
\hline $\mathrm{C}(8)-\mathrm{C}(9)-\mathrm{C}(10)-\mathrm{C}(11)$ & $-1.9(10)$ \\
\hline $\mathrm{C}(8)-\mathrm{C}(9)-\mathrm{C}(10)-\mathrm{C}(13)$ & $177.7(12)$ \\
\hline $\mathrm{C}(8)-\mathrm{C}(9)-\mathrm{C}(10)-\mathrm{C}\left(13^{\prime}\right)$ & $179.0(12)$ \\
\hline $\mathrm{F}(2)-\mathrm{C}(13)-\mathrm{C}(10)-\mathrm{C}(9)$ & $26(3)$ \\
\hline $\mathrm{F}(4)-\mathrm{C}(13)-\mathrm{C}(10)-\mathrm{C}(9)$ & $162.7(12)$ \\
\hline $\mathrm{F}(3)-\mathrm{C}(13)-\mathrm{C}(10)-\mathrm{C}(9)$ & $-85.1(17)$ \\
\hline $\mathrm{F}(2)-\mathrm{C}(13)-\mathrm{C}(10)-\mathrm{C}(11)$ & $-154.2(18)$ \\
\hline $\mathrm{F}(4)-\mathrm{C}(13)-\mathrm{C}(10)-\mathrm{C}(11)$ & $-18(2)$ \\
\hline $\mathrm{F}(3)-\mathrm{C}(13)-\mathrm{C}(10)-\mathrm{C}(11)$ & $94.5(17)$ \\
\hline $\mathrm{F}(2)-\mathrm{C}(13)-\mathrm{C}(10)-\mathrm{C}\left(13^{\prime}\right)$ & $4(32)$ \\
\hline$F(4)-C(13)-C(10)-C\left(13^{\prime}\right)$ & 141(34) \\
\hline $\mathrm{F}(3)-\mathrm{C}(13)-\mathrm{C}(10)-\mathrm{C}\left(13^{\prime}\right)$ & $-107(34)$ \\
\hline $\mathrm{F}\left(4^{\prime}\right)-\mathrm{C}\left(13^{\prime}\right)-\mathrm{C}(10)-\mathrm{C}(9)$ & $-142(2)$ \\
\hline $\mathrm{F}\left(2^{\prime}\right)-\mathrm{C}\left(13^{\prime}\right)-\mathrm{C}(10)-\mathrm{C}(9)$ & $62(2)$ \\
\hline $\mathrm{F}\left(3^{\prime}\right)-\mathrm{C}\left(13^{\prime}\right)-\mathrm{C}(10)-\mathrm{C}(9)$ & $-43(2)$ \\
\hline $\mathrm{F}\left(4^{\prime}\right)-\mathrm{C}\left(13^{\prime}\right)-\mathrm{C}(10)-\mathrm{C}(11)$ & $39(3)$ \\
\hline $\mathrm{F}\left(2^{\prime}\right)-\mathrm{C}\left(13^{\prime}\right)-\mathrm{C}(10)-\mathrm{C}(11)$ & $-117.1(15)$ \\
\hline $\mathrm{F}\left(3^{\prime}\right)-\mathrm{C}\left(13^{\prime}\right)-\mathrm{C}(10)-\mathrm{C}(11)$ & 138.1(14) \\
\hline $\mathrm{F}\left(4^{\prime}\right)-\mathrm{C}\left(13^{\prime}\right)-\mathrm{C}(10)-\mathrm{C}(13)$ & $17(32)$ \\
\hline $\mathrm{F}\left(2^{\prime}\right)-\mathrm{C}\left(13^{\prime}\right)-\mathrm{C}(10)-\mathrm{C}(13)$ & $-139(34)$ \\
\hline $\mathrm{F}\left(3^{\prime}\right)-\mathrm{C}\left(13^{\prime}\right)-\mathrm{C}(10)-\mathrm{C}(13)$ & $116(34)$ \\
\hline $\mathrm{C}(9)-\mathrm{C}(10)-\mathrm{C}(11)-\mathrm{C}(12)$ & $1.2(11)$ \\
\hline$C(13)-C(10)-C(11)-C(12)$ & $-178.4(12)$ \\
\hline$C\left(13^{\prime}\right)-C(10)-C(11)-C(12)$ & $-179.7(13)$ \\
\hline $\mathrm{C}(10)-\mathrm{C}(11)-\mathrm{C}(12)-\mathrm{C}(7)$ & $0.6(11)$ \\
\hline$C(8)-C(7)-C(12)-C(11)$ & $-1.7(10)$ \\
\hline $\mathrm{C}(6)-\mathrm{C}(7)-\mathrm{C}(12)-\mathrm{C}(11)$ & $177.3(7)$ \\
\hline $\mathrm{C}(18)-\mathrm{N}(1)-\mathrm{C}(14)-\mathrm{C}(15)$ & $-1.0(9)$ \\
\hline $\mathrm{C}(18)-\mathrm{N}(1)-\mathrm{C}(14)-\mathrm{C}(2)$ & $179.0(6)$ \\
\hline $\mathrm{F}(1)-\mathrm{C}(2)-\mathrm{C}(14)-\mathrm{N}(1)$ & $178.0(4)$ \\
\hline $\mathrm{C}(1)-\mathrm{C}(2)-\mathrm{C}(14)-\mathrm{N}(1)$ & $58.5(7)$ \\
\hline $\mathrm{C}(3)-\mathrm{C}(2)-\mathrm{C}(14)-\mathrm{N}(1)$ & $-61.3(7)$ \\
\hline $\mathrm{F}(1)-\mathrm{C}(2)-\mathrm{C}(14)-\mathrm{C}(15)$ & $-2.1(7)$ \\
\hline$C(1)-C(2)-C(14)-C(15)$ & $-121.5(6)$ \\
\hline $\mathrm{C}(3)-\mathrm{C}(2)-\mathrm{C}(14)-\mathrm{C}(15)$ & $118.7(7)$ \\
\hline
\end{tabular}


$\mathrm{N}(1)-\mathrm{C}(14)-\mathrm{C}(15)-\mathrm{C}(16)$

$\mathrm{C}(2)-\mathrm{C}(14)-\mathrm{C}(15)-\mathrm{C}(16)$

C(14)-C(15)-C(16)-C(17)

$-0.2(10)$

$179.8(6)$

$0.6(11)$

$\mathrm{C}(15)-\mathrm{C}(16)-\mathrm{C}(17)-\mathrm{C}(18)$

$0.3(12)$

$\mathrm{C}(16)-\mathrm{C}(17)-\mathrm{C}(18)-\mathrm{N}(1)$

$-1.6(13)$

$\mathrm{C}(14)-\mathrm{N}(1)-\mathrm{C}(18)-\mathrm{C}(17)$

$2.0(11)$

Symmetry transformations used to generate equivalent atoms: 
Table 7. Hydrogen bonds for $(S, S)-9$ [ $\left.\AA^{\text {and }}{ }^{\circ}\right]$.

\begin{tabular}{|c|c|c|c|c|}
\hline D-H...A & $\mathrm{d}(\mathrm{D}-\mathrm{H})$ & $\mathrm{d}(\mathrm{H} \ldots \mathrm{A})$ & $\mathrm{d}(\mathrm{D} \ldots \mathrm{A})$ & $<(\mathrm{DHA})$ \\
\hline $\mathrm{C}(1)-\mathrm{H}(1 \mathrm{~B}) \ldots \mathrm{N}(1) \# 1$ & 0.97 & 2.68 & $3.577(8)$ & 154.1 \\
\hline $\mathrm{C}(3)-\mathrm{H}(3 \mathrm{~B}) \ldots \mathrm{F}\left(4^{\prime}\right) \# 2$ & 0.97 & 2.64 & $3.33(3)$ & 128.0 \\
\hline $\mathrm{C}(1)-\mathrm{H}(1 \mathrm{~B}) \ldots \mathrm{N}(1) \# 1$ & 0.97 & 2.68 & $3.577(8)$ & 154.1 \\
\hline $\mathrm{C}(1)-\mathrm{H}(1 \mathrm{~B}) \ldots \mathrm{N}(1) \# 1$ & 0.97 & 2.68 & $3.577(8)$ & 154.1 \\
\hline $\mathrm{C}(3)-\mathrm{H}(3 \mathrm{~B}) \ldots \mathrm{F}\left(4^{\prime}\right) \# 2$ & 0.97 & 2.64 & $3.33(3)$ & 128.0 \\
\hline $\mathrm{C}(1)-\mathrm{H}(1 \mathrm{~B}) \ldots \mathrm{N}(1) \# 1$ & 0.97 & 2.68 & $3.577(8)$ & 154.1 \\
\hline
\end{tabular}

Symmetry transformations used to generate equivalent atoms:

$\# 1 \mathrm{x}+1, \mathrm{y}, \mathrm{z} \quad \# 2-\mathrm{x}, \mathrm{y}-1 / 2,-\mathrm{z}+1 / 2$

\section{References}

1. He, Z.-T.; Jiang, X.; Hartwig, J. F. J. Am. Chem. Soc. 2019, 141, 13066-13073.

2. Liao, Y.; Yin, X.; Wang, X.; Yu, W.; Fang, D.; Hu, L.; Wang, M.; Liao, J. Angew. Chem. Int. Ed. 2020, 59, 1176-1180.

3. Picher, M.-I.; Plietker, B. Org. Lett. 2020, 22, 340-344.

4. Meng, F.; Haeffner, F.; Hoveyda, A. H. J. Am. Chem. Soc. 2014, 136, 11304-11307.

5. Adamson, N. J.; Jeddi, H.; Malcolmson, S. J. J. Am. Chem. Soc. 2019, 141, $8574-8583$.

6. Davi, M.; Lebel, H., Org. Lett. 2009, 11, 41-44.

7. Yadav, J. S.; Valli, M. Y.; Prasad, A. R. Tetrahedron 1998, 54, 7551-7562. 
10. Copies of ${ }^{1} \mathrm{H}$ NMR, ${ }^{13} \mathrm{C}$ NMR and ${ }^{19} \mathrm{~F}$ NMR spectra 
ఈ゙

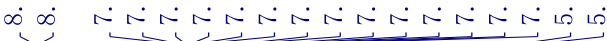

$\iint 1$
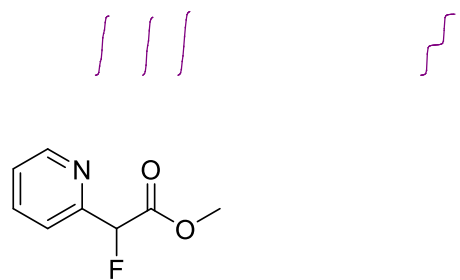

1a, ${ }^{1} \mathrm{H}$ NMR

$\mathrm{CDCl}_{3}$

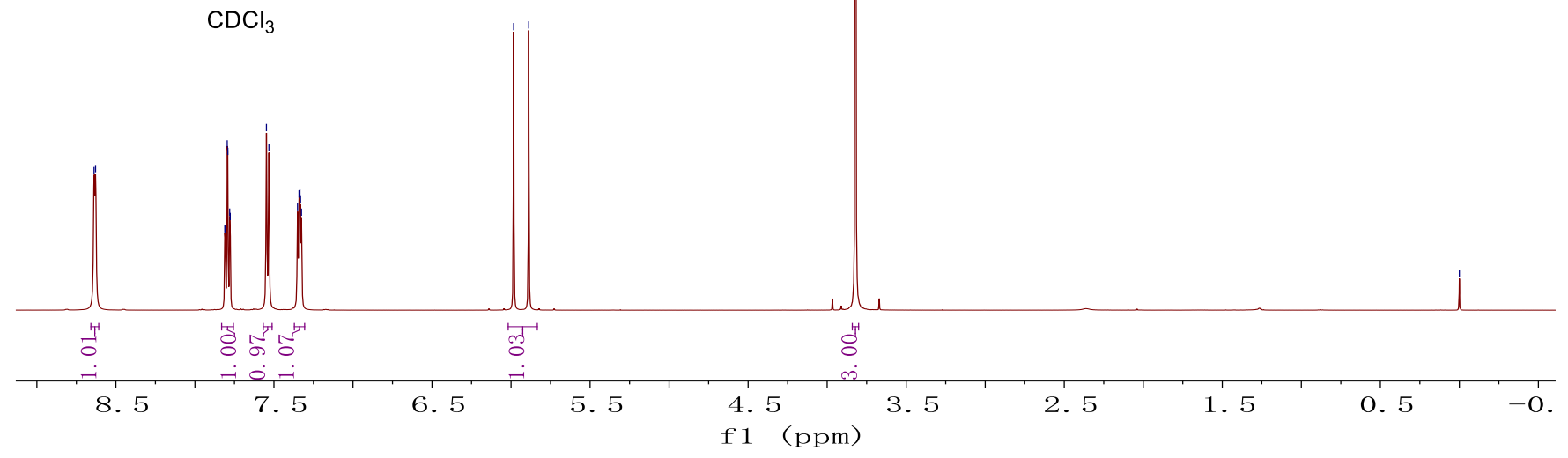

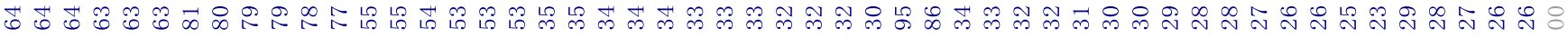

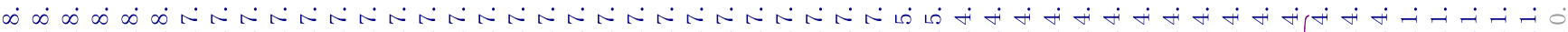
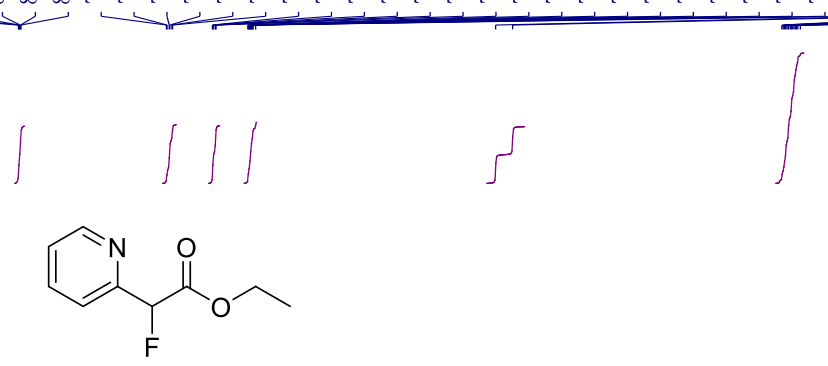

1b, ${ }^{1} \mathrm{H}$ NMR $\mathrm{CDCl}_{3}$

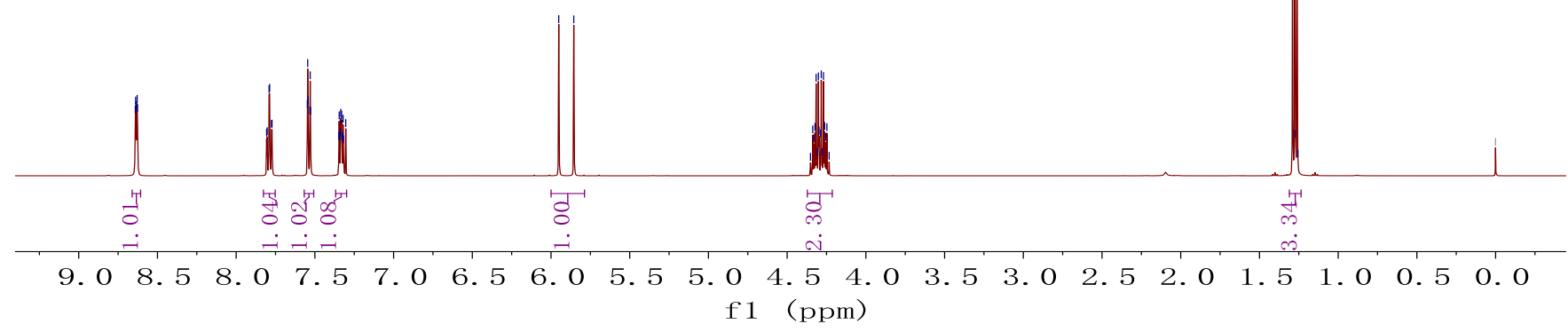




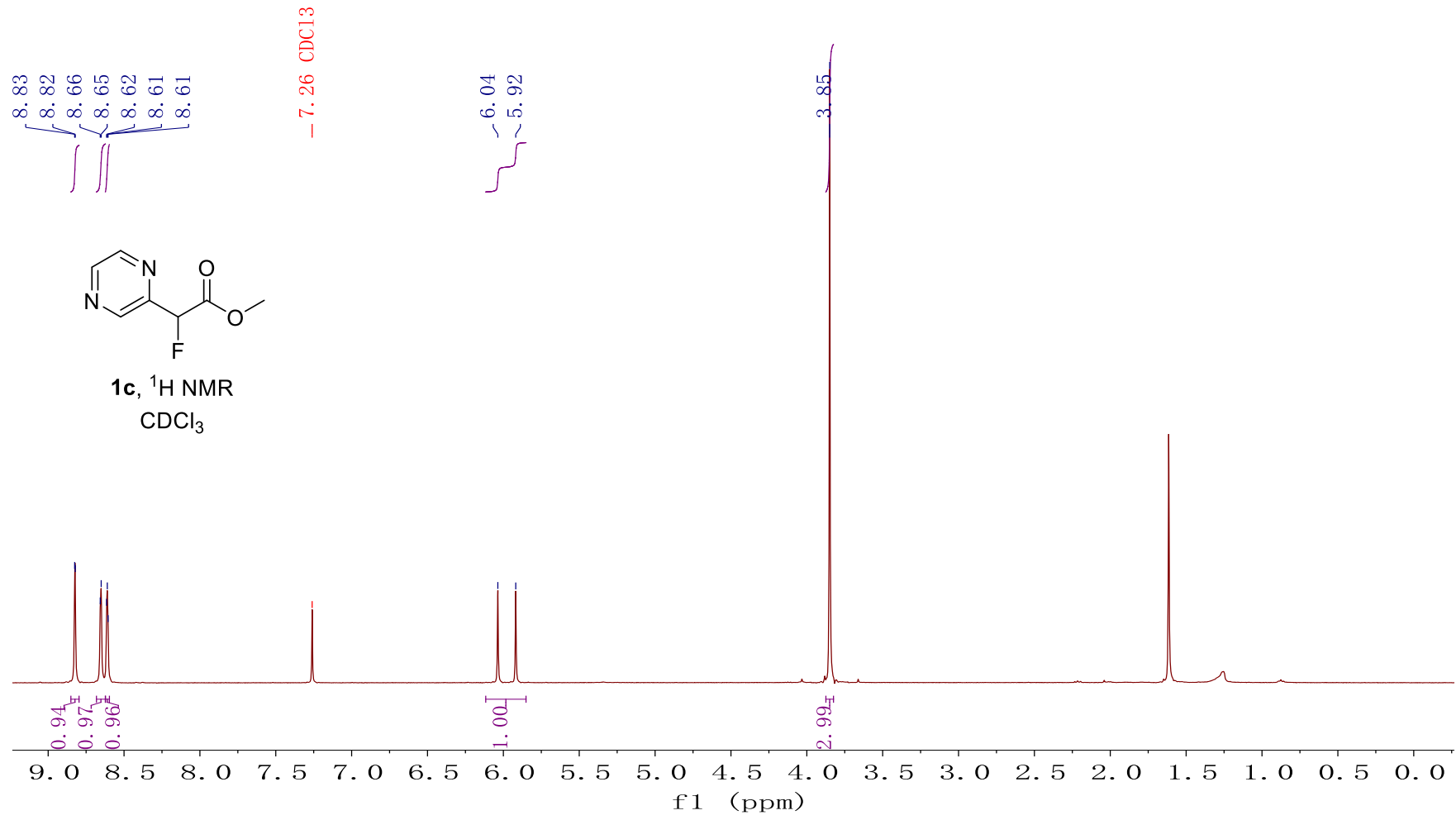

己

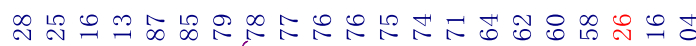

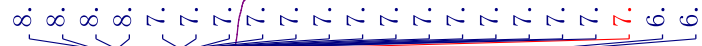

$\iiint \sqrt{1}$

1 (ppm)

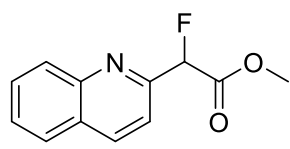

1d, ${ }^{1} \mathrm{H}$ NMR

$\mathrm{CDCl}_{3}$

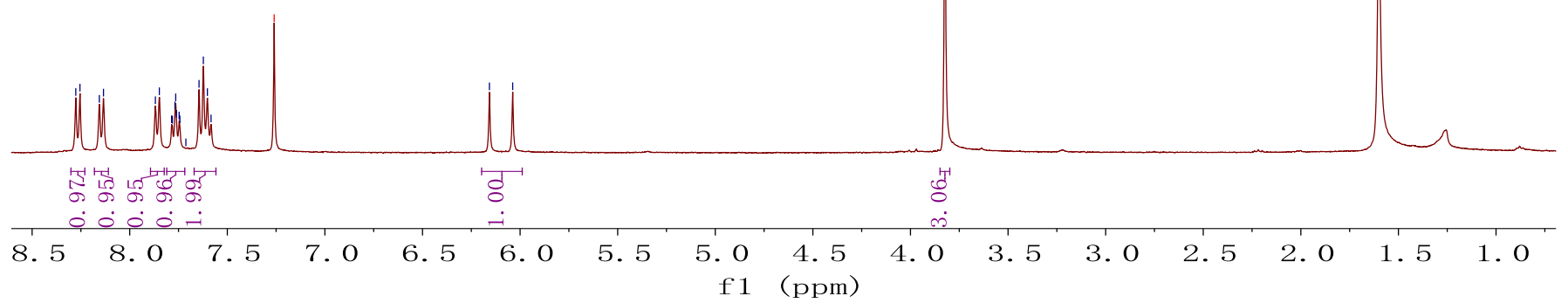




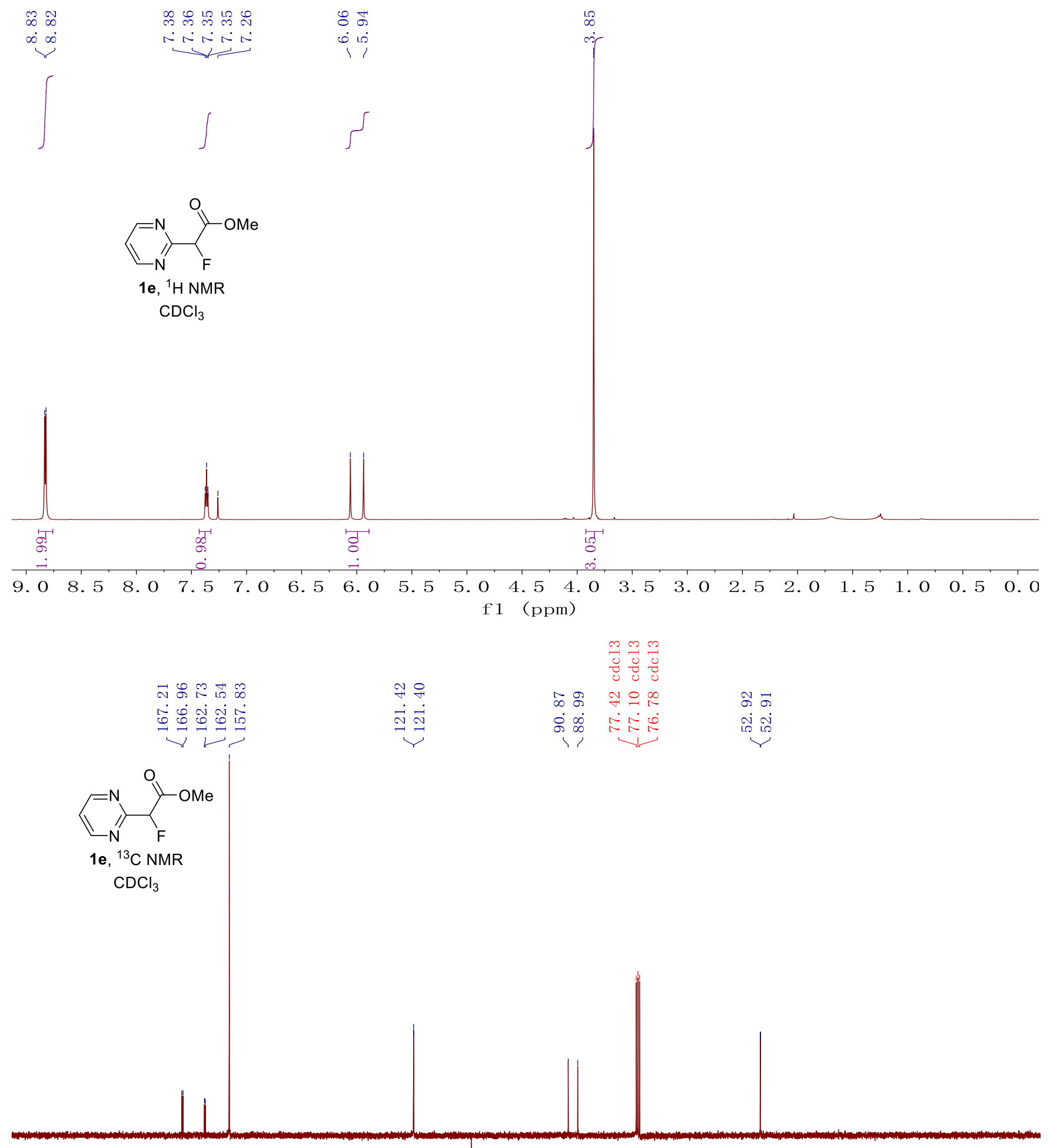

\begin{tabular}{llllllllllllllllllllllllll}
\hline 00 & 190 & 180 & 170 & 160 & 150 & 140 & 130 & 120 & 110 & 100 & 90 & 80 & 70 & 60 & 50 & 40 & 30 & 20 & 10 & 0
\end{tabular} f1 (ppm) 


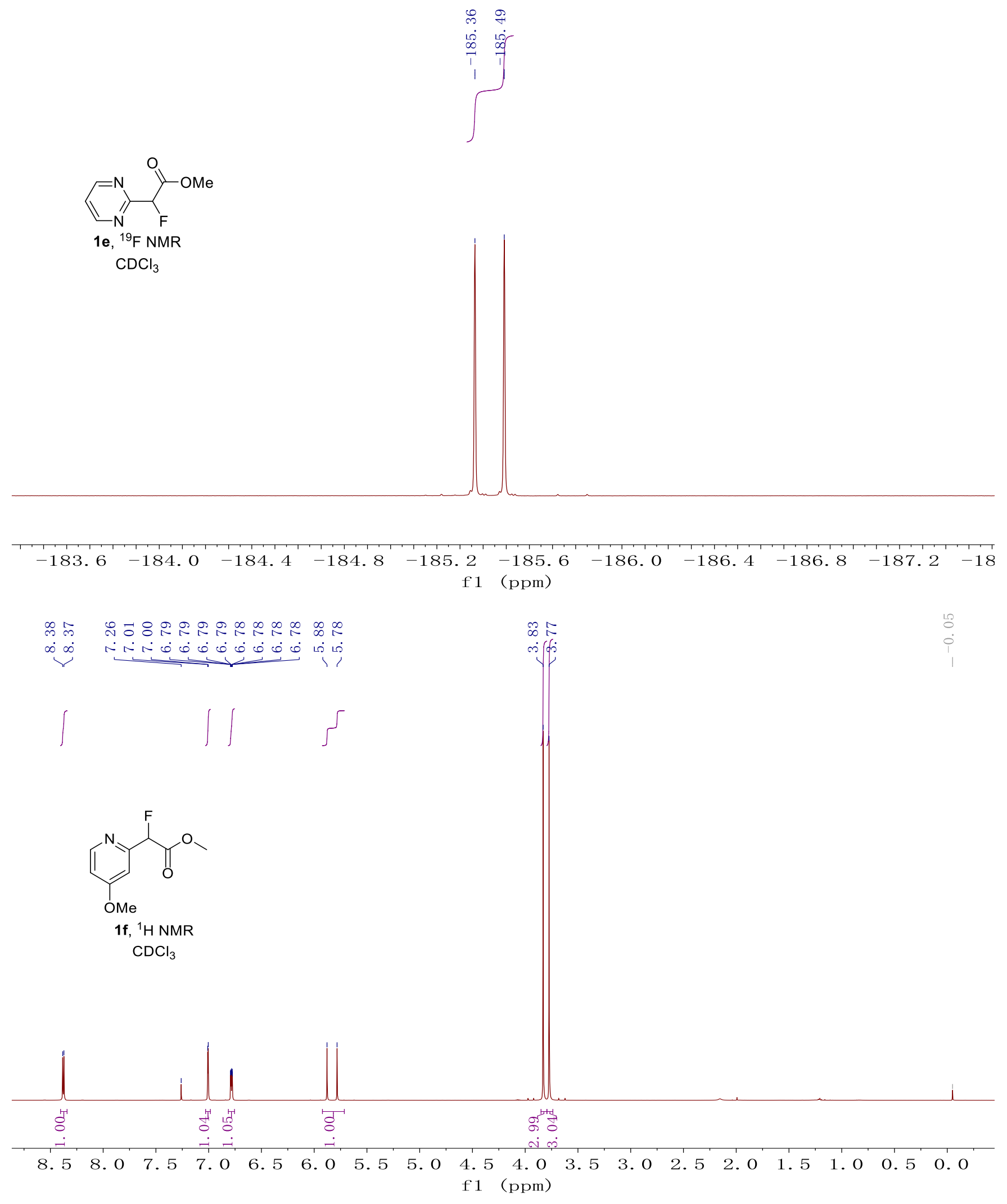



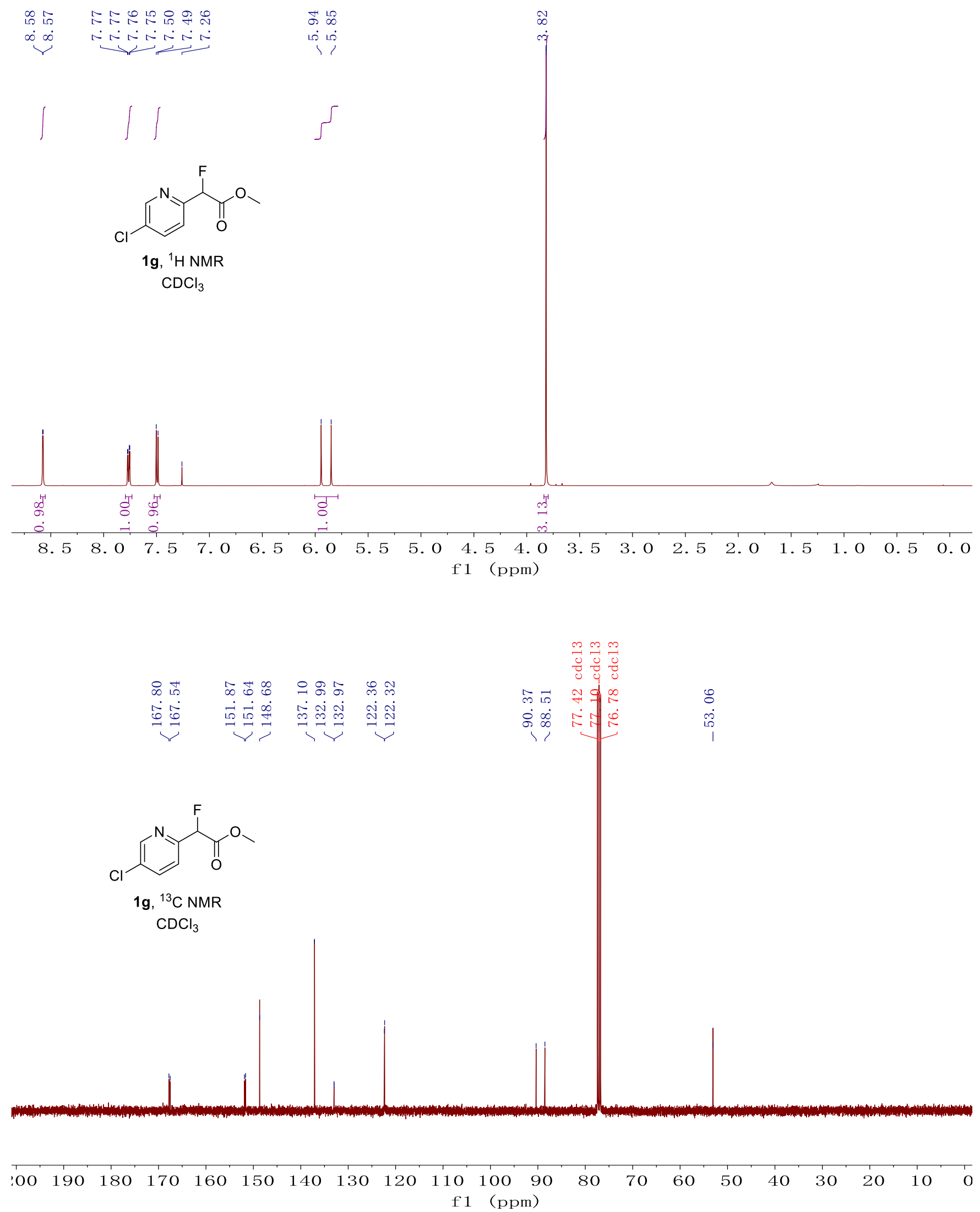


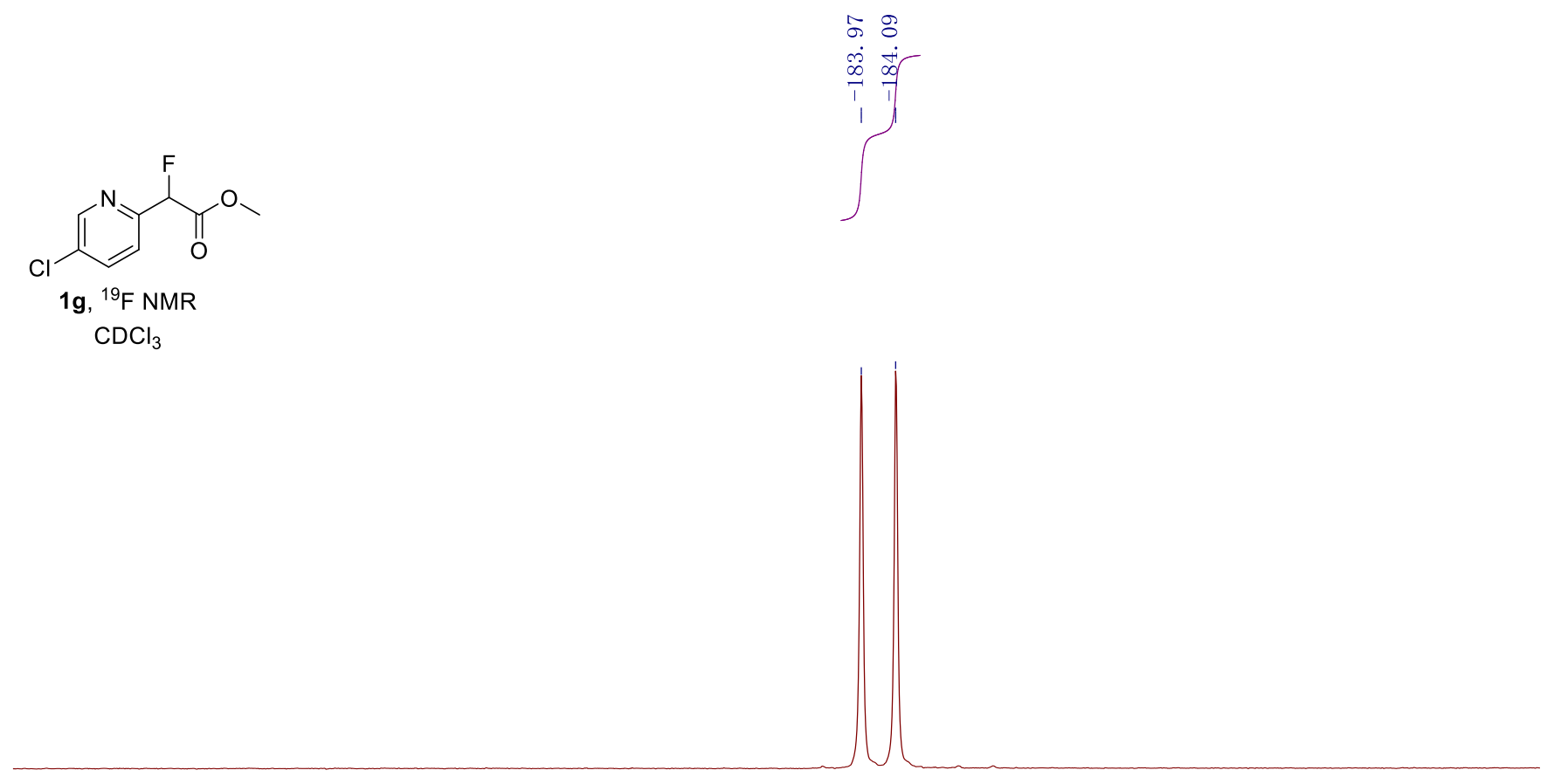

\begin{tabular}{|c|c|c|c|c|c|c|c|c|c|}
\hline-181.0 & -181.6 & -182.2 & -182.8 & $\begin{array}{c}-183.4 \\
f 1\end{array}$ & $\begin{array}{l}-184.0 \\
\mathrm{pm})\end{array}$ & -184.6 & -185.2 & -185.8 & -181 \\
\hline
\end{tabular}



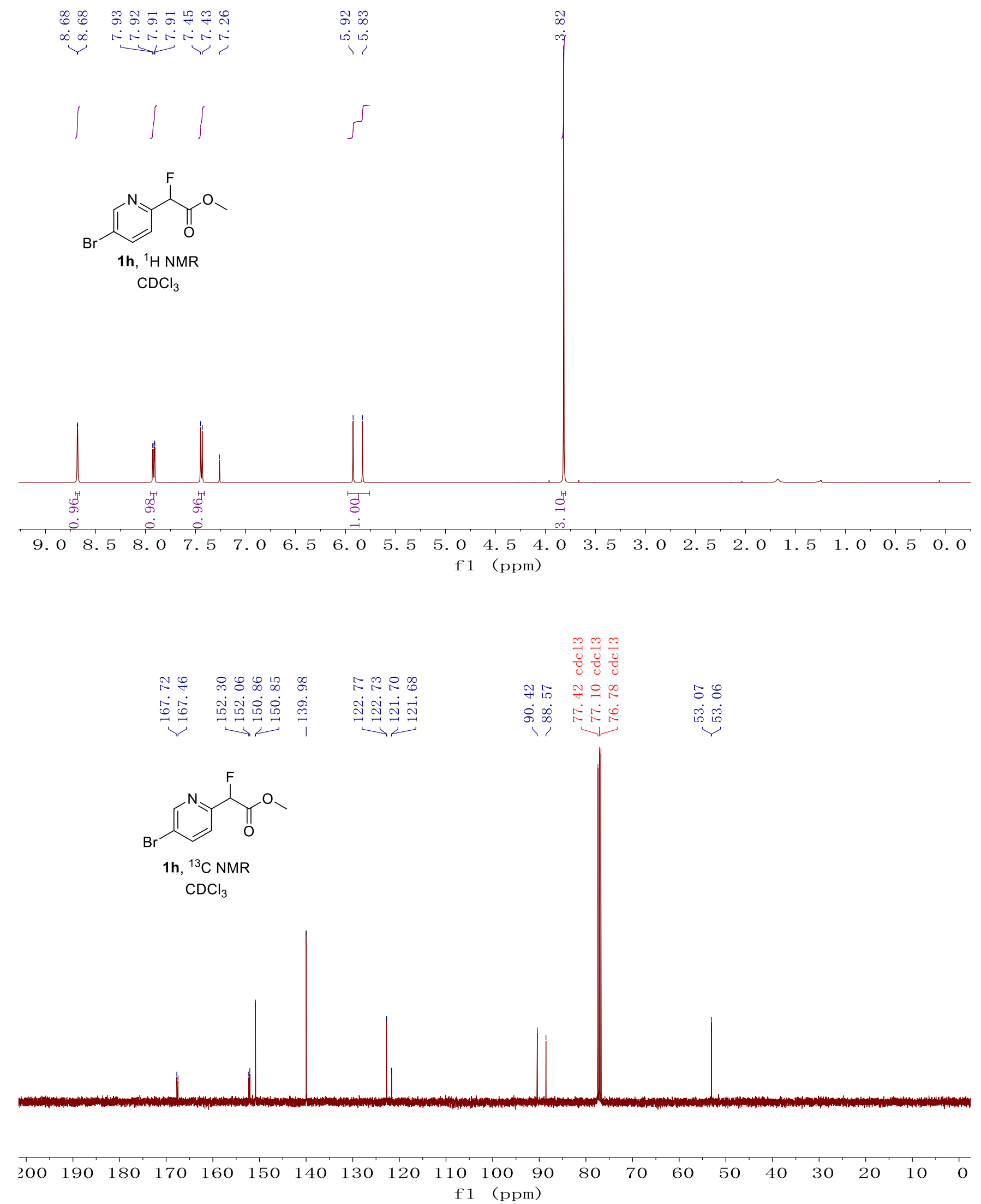


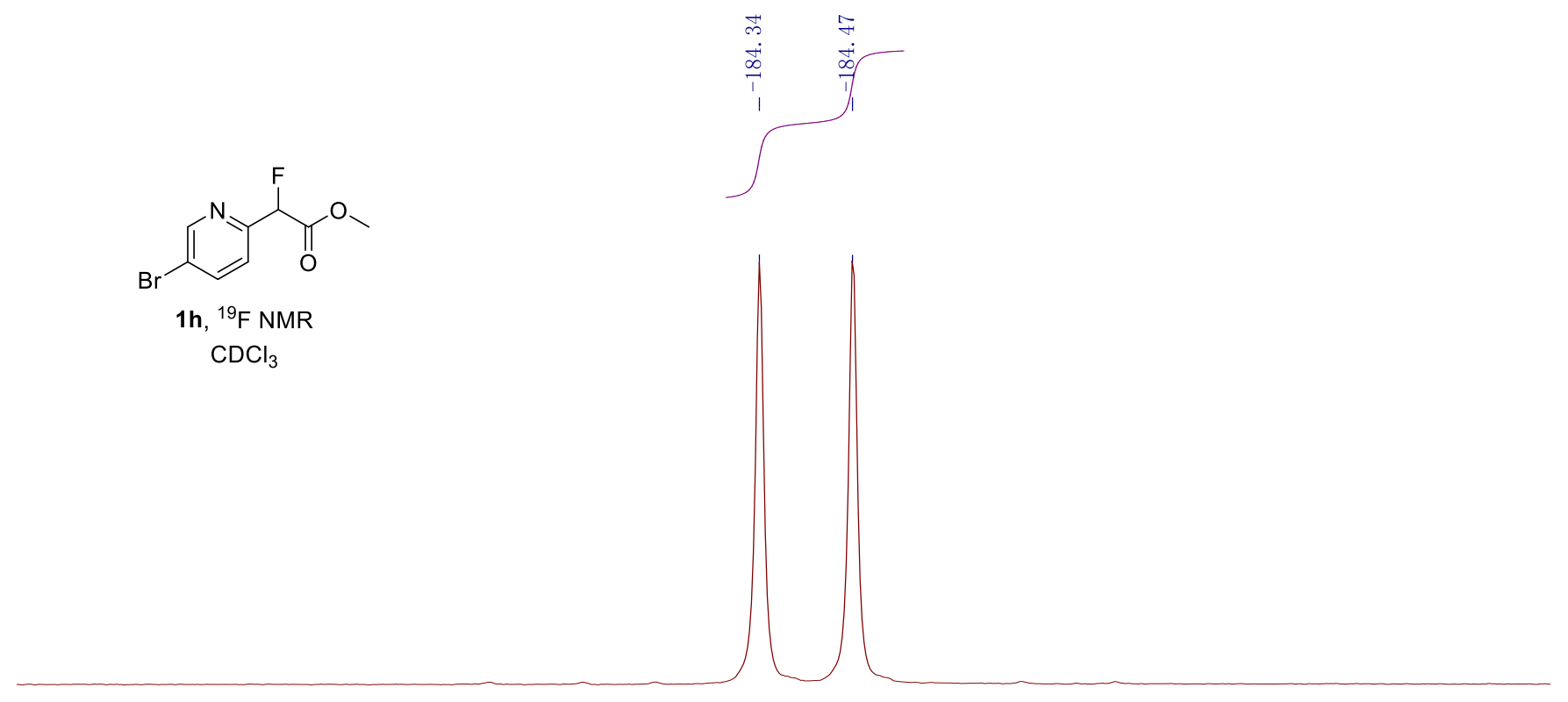

\begin{tabular}{|c|c|c|c|c|c|c|c|c|}
\hline-183.4 & -183.6 & -183.8 & -184.0 & $\begin{array}{rr}-184.2 & -184.4 \\
\mathrm{f} 1 & (\mathrm{ppm})\end{array}$ & -184.6 & -184.8 & -185.0 & $-185.2-18$ \\
\hline
\end{tabular}

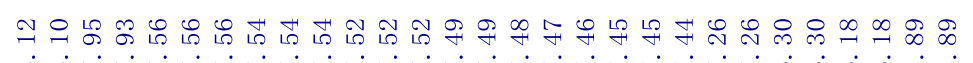

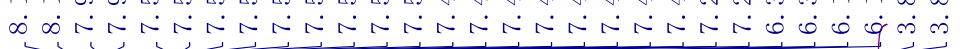
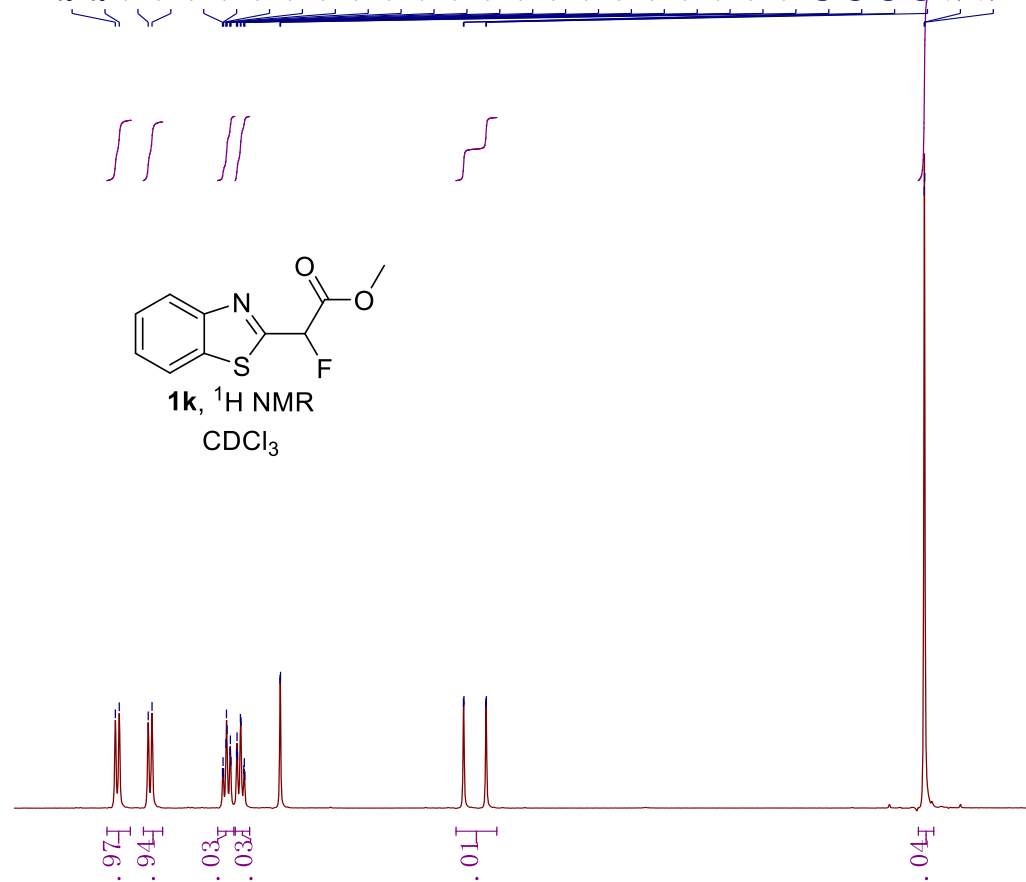

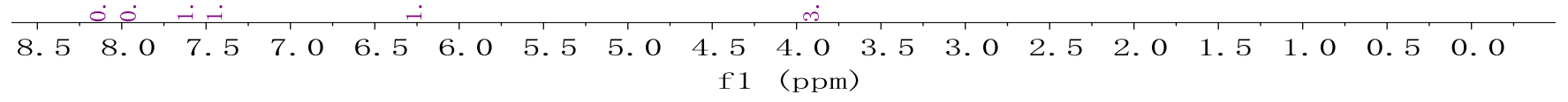



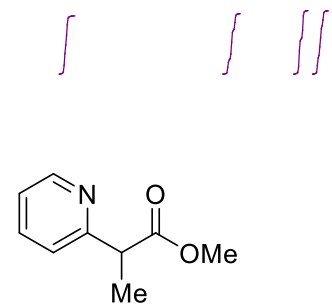

1I, ${ }^{1} \mathrm{H}$ NMR $\mathrm{CDCl}_{3}$

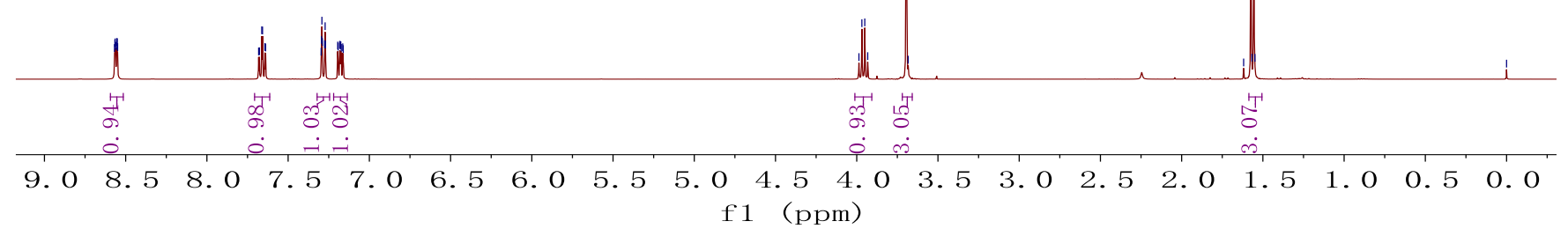




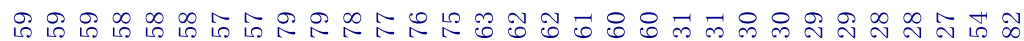

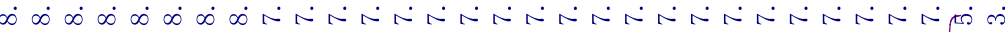
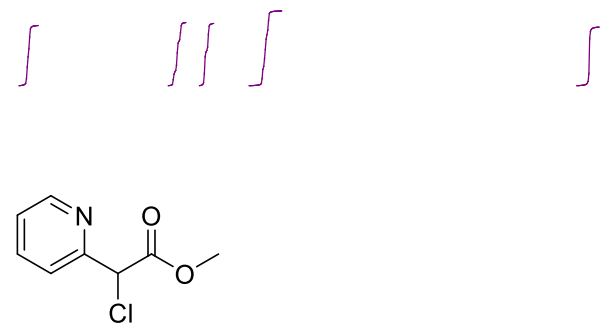

$1 \mathrm{~m},{ }^{1} \mathrm{H}$ NMR $\mathrm{CDCl}_{3}$

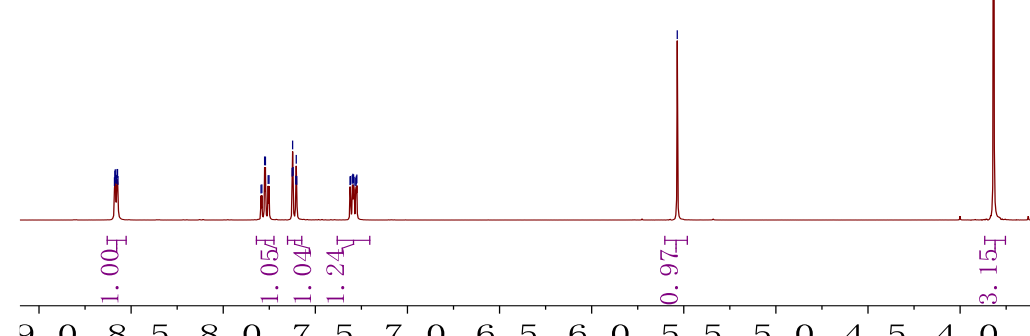

$\begin{array}{llllllllllllllllllllllllllll}9.0 & 8.5 & 8.0 & 7.5 & 7.0 & 6.5 & 6.0 & 5.5 & 5.0 & 4.5 & 4.0 & 3.5 & 3.0 & 2.5 & 2.0 & 1.5 & 1.0 & 0.5 & 0.0\end{array}$ f1 (ppm)

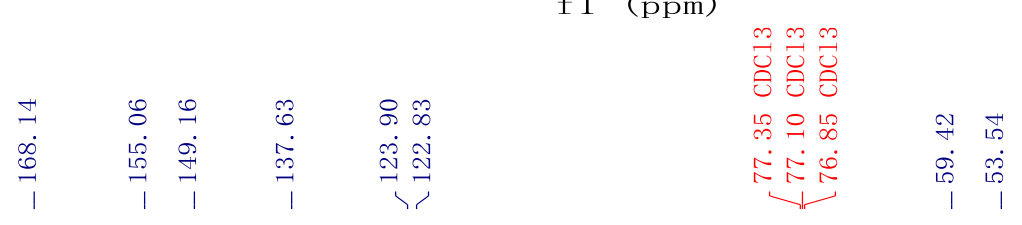

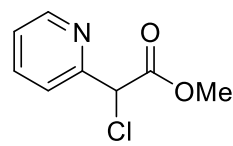

$1 \mathrm{~m},{ }^{1} \mathrm{H}$ NMR $\mathrm{CDCl}_{3}$
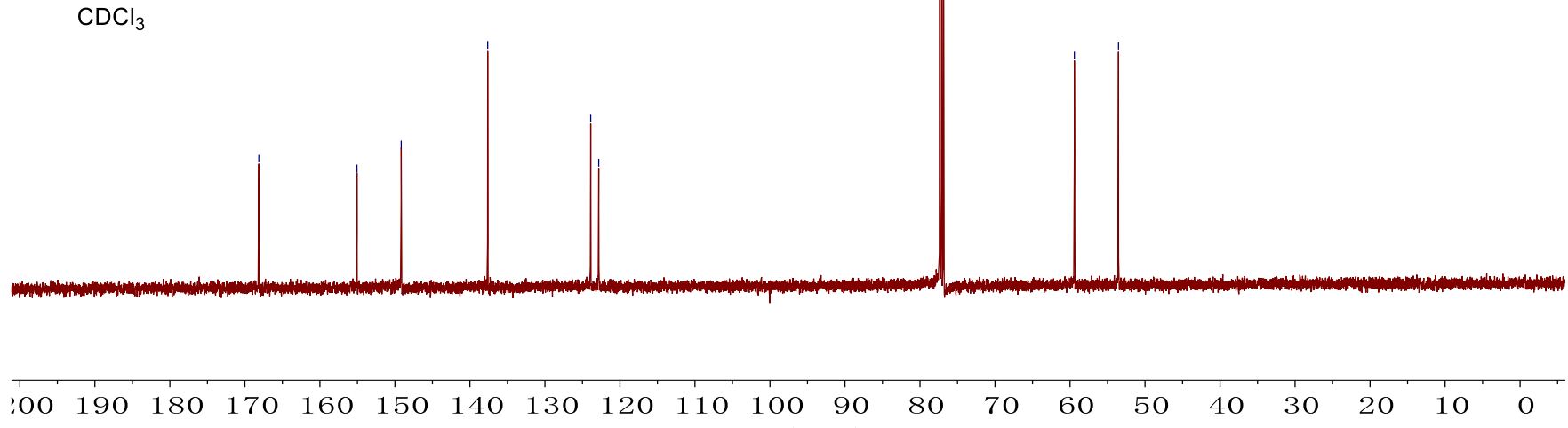

f1 (ppm) 


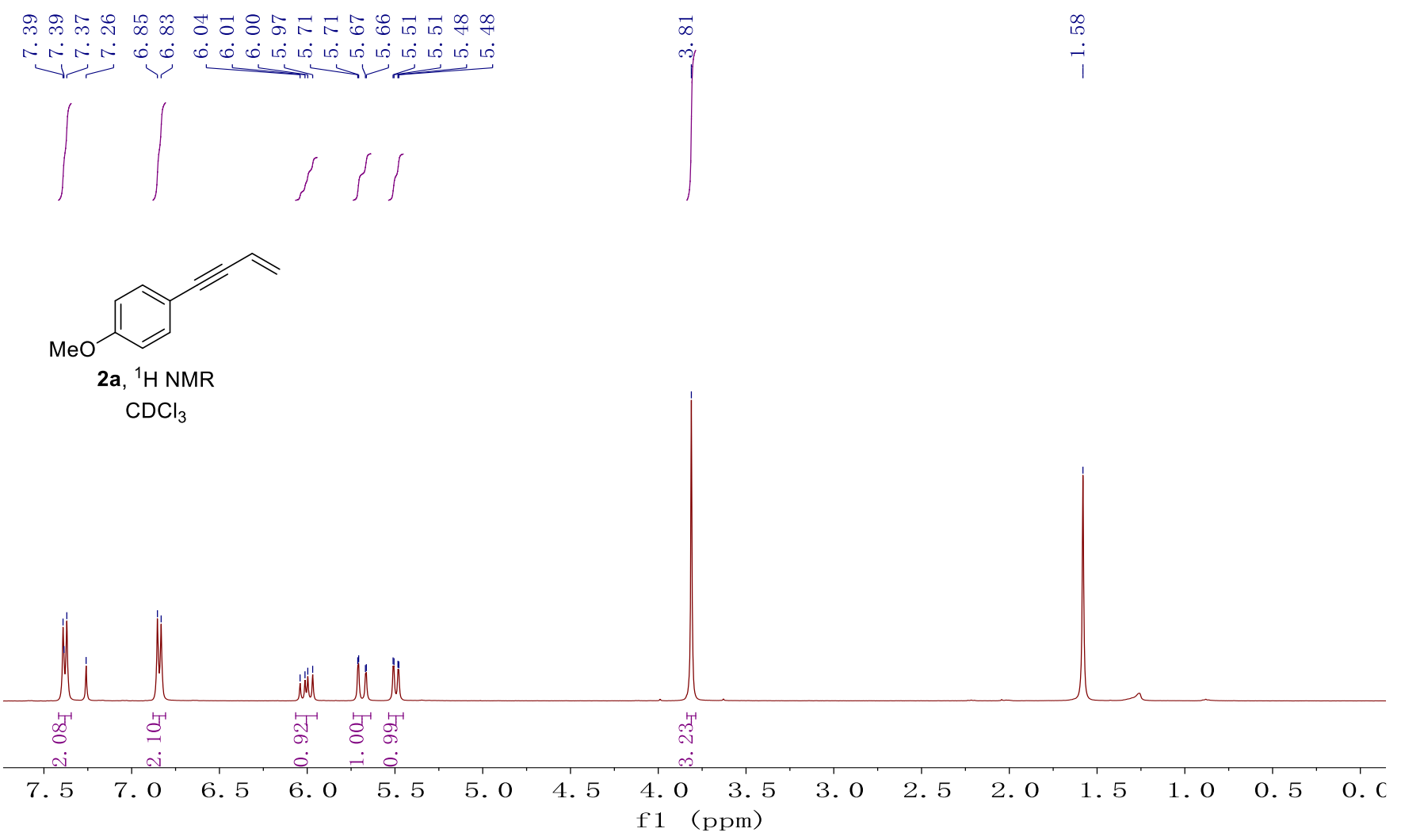

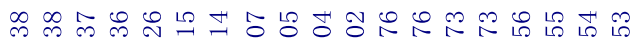

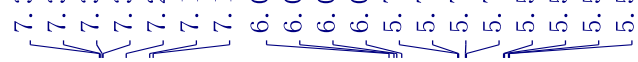
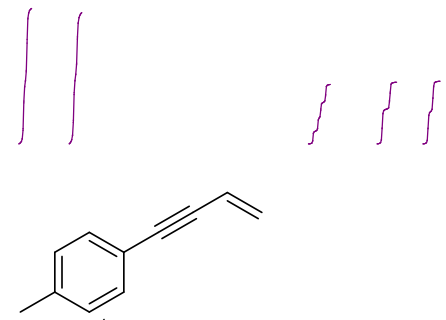

2b, ${ }^{1} \mathrm{H}$ NMR

$\mathrm{CDCl}_{3}$

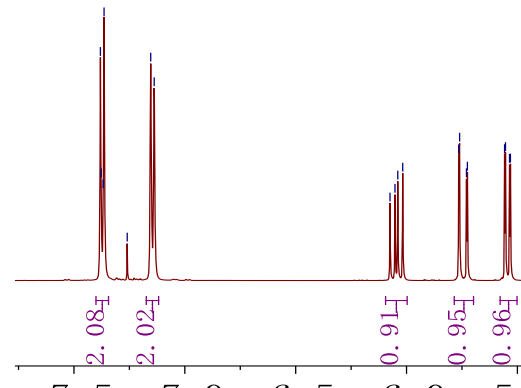

7. 5 7.0 6.5

6. 0 5. 5

5. 0

4. 5

4. 0 3.5 3.0 3.5

$\begin{array}{llllll}2.0 & 1.5 & 1.0 & 0.5 & 0.0\end{array}$ f1 (ppm) 


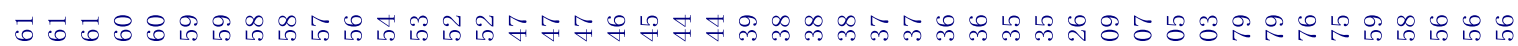

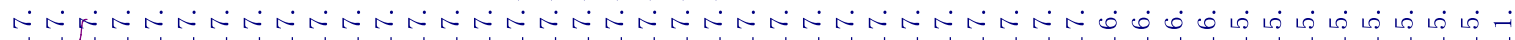
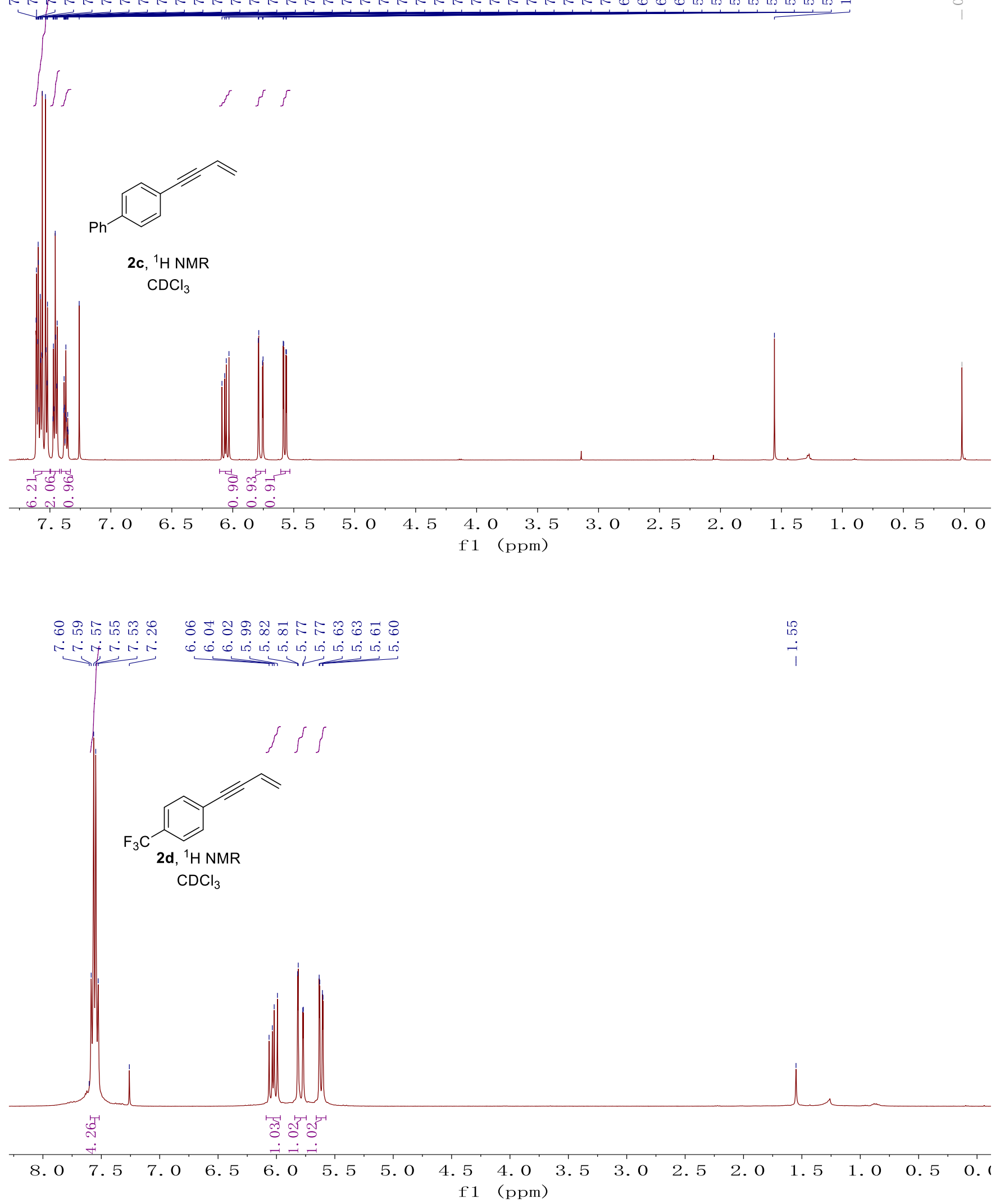


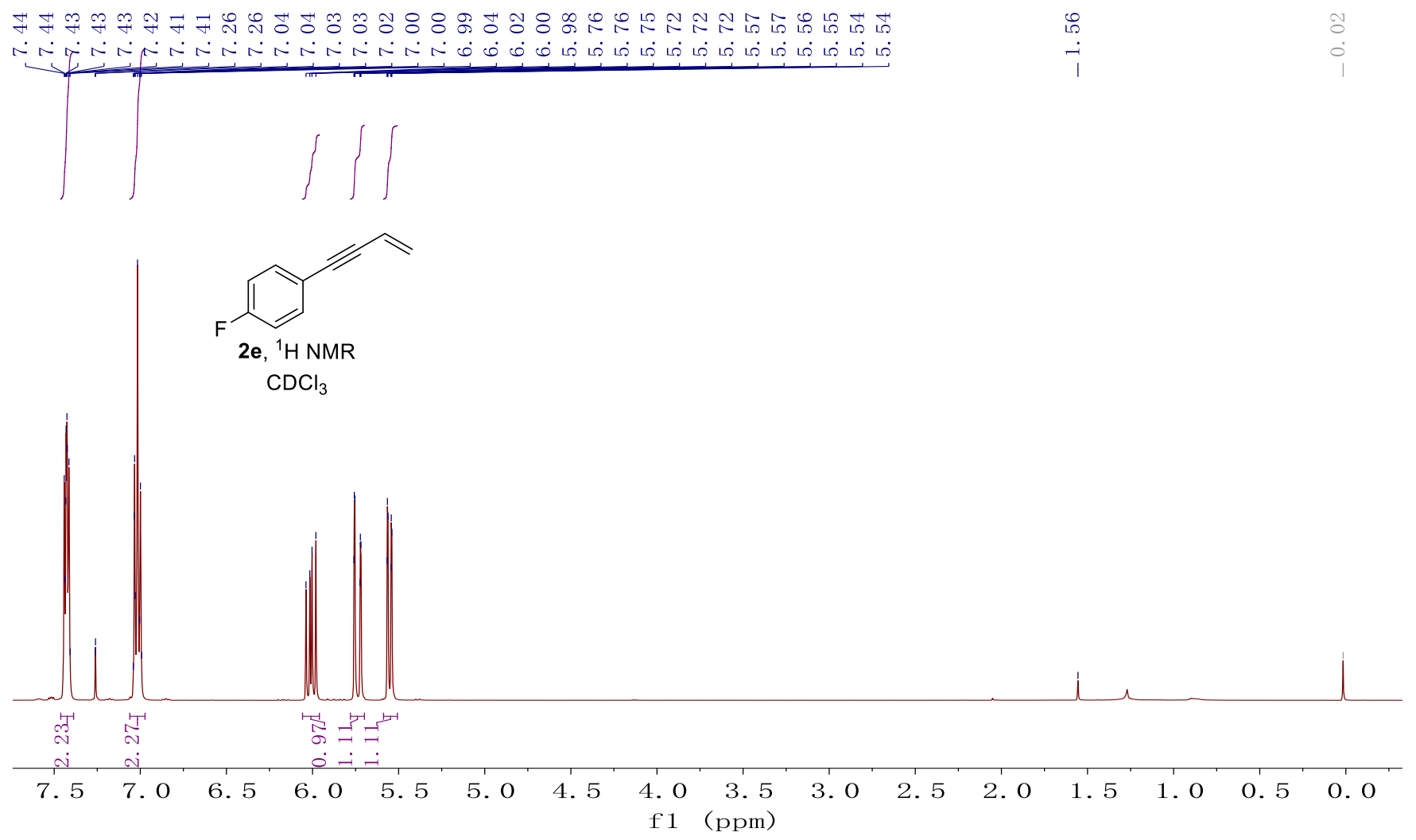




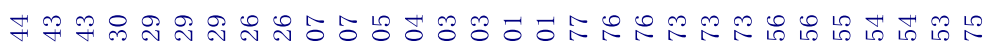

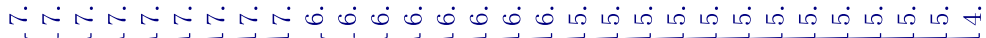
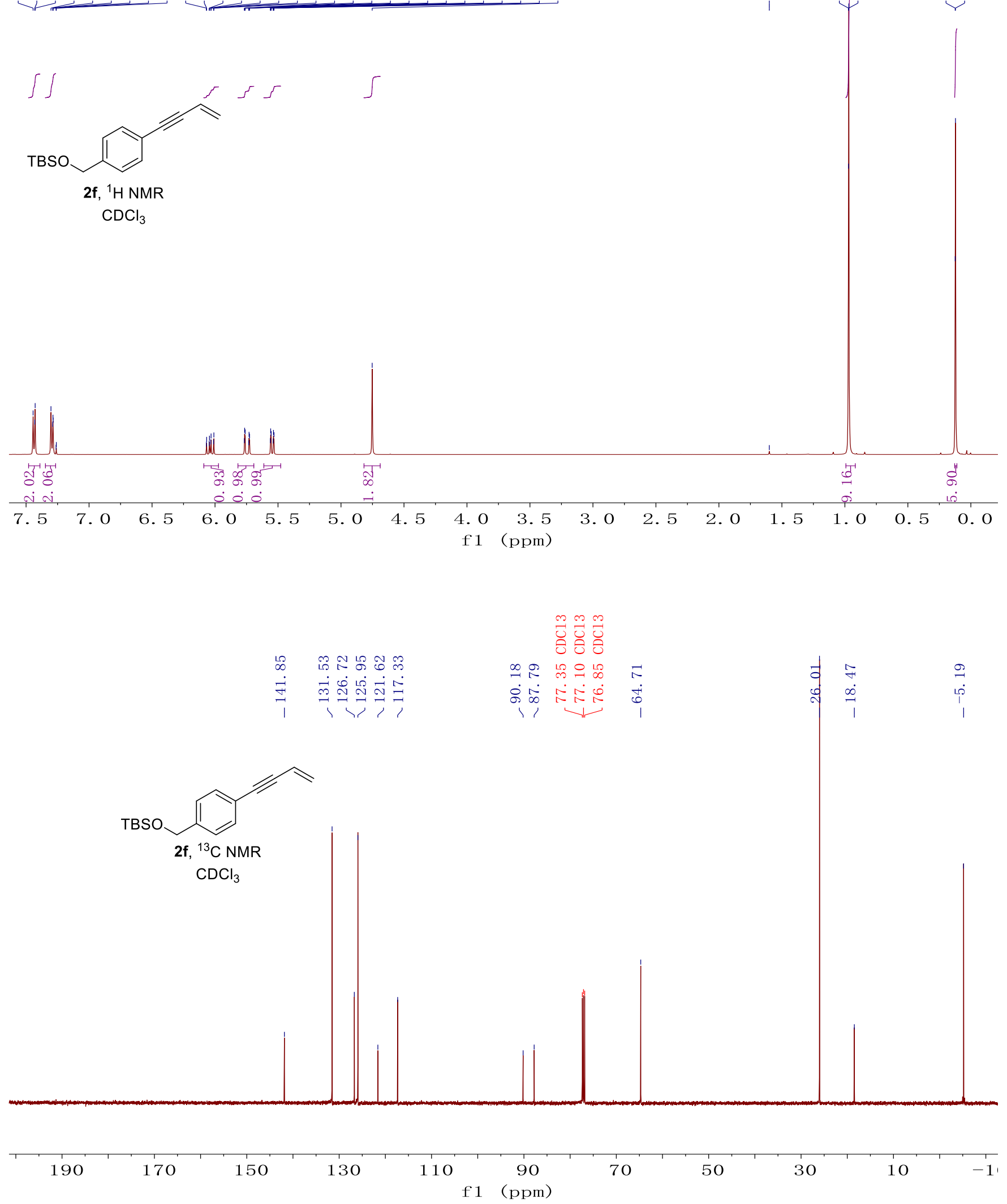

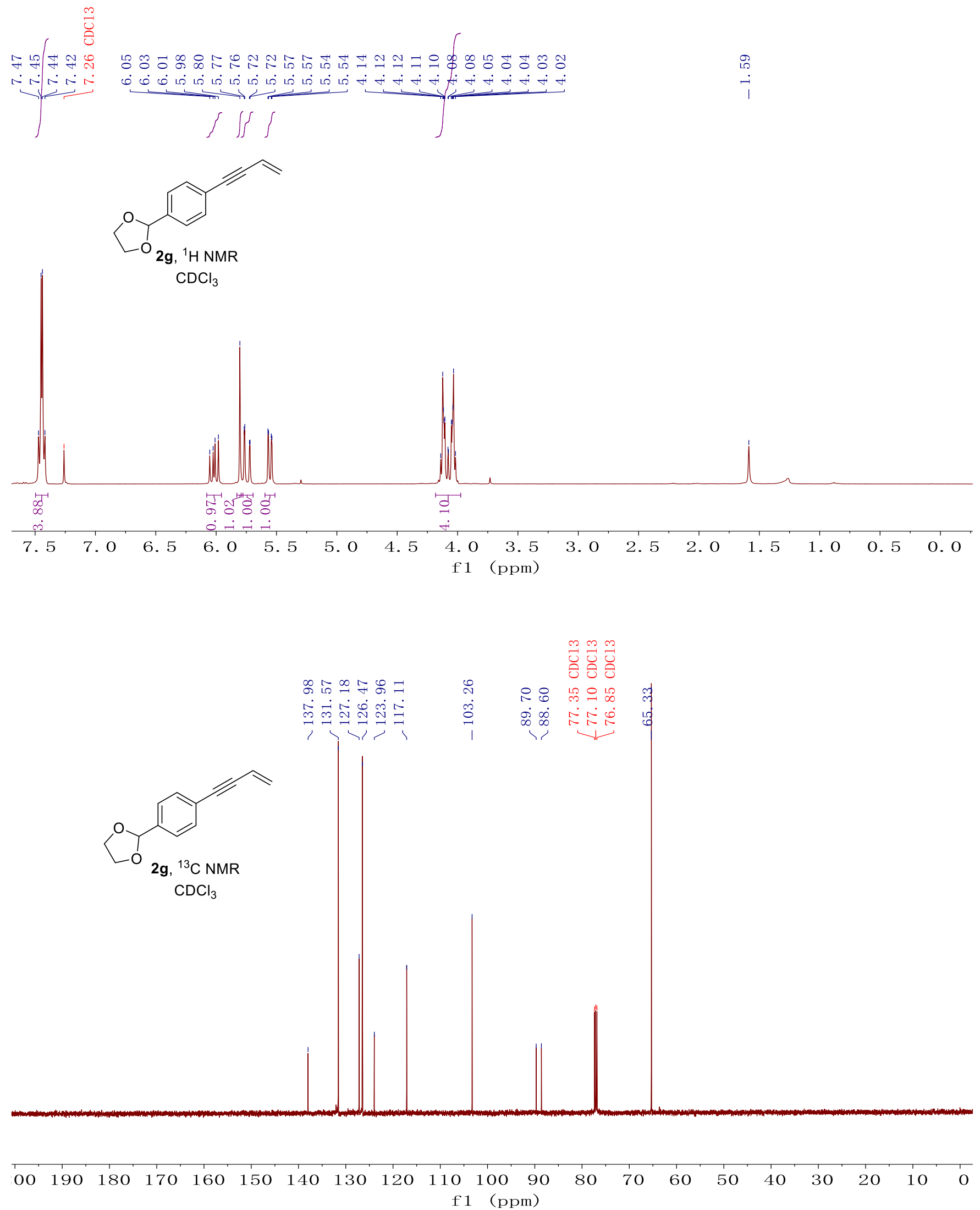

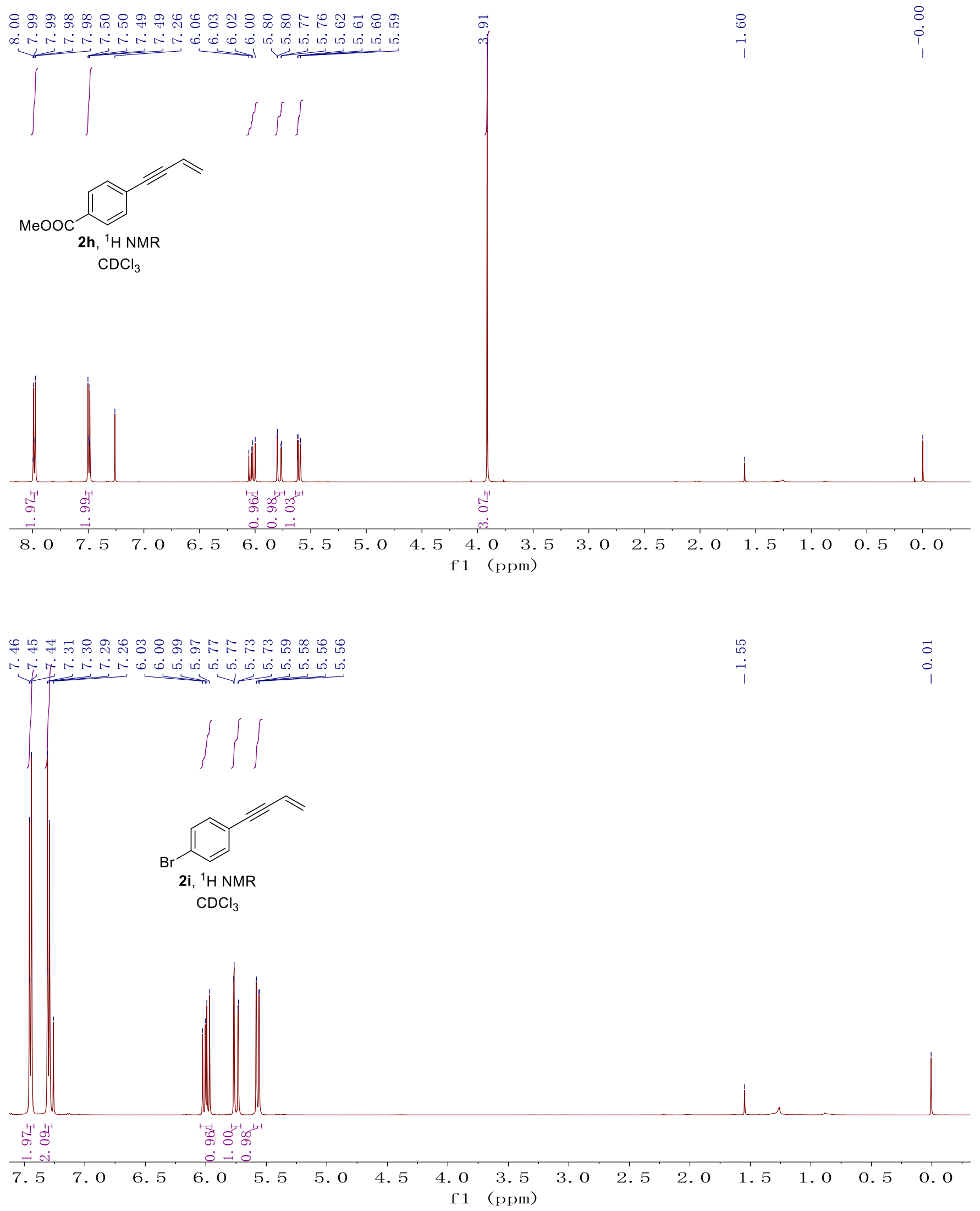


\section{范}

그 m

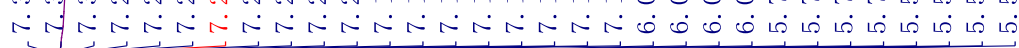
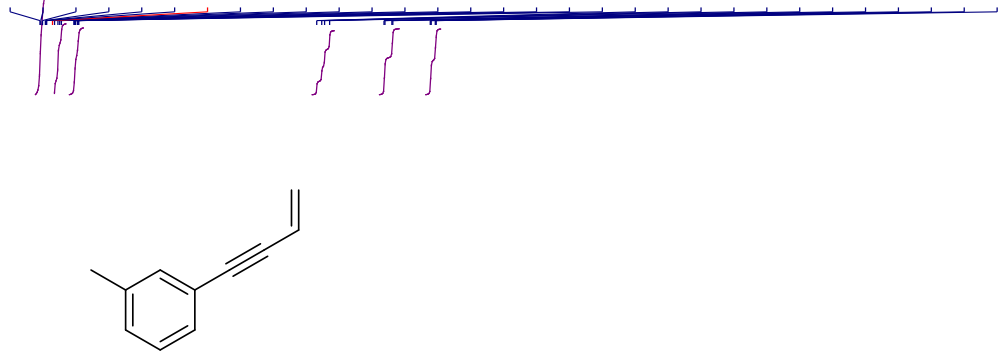

2j, ${ }^{1} \mathrm{H}$ NMR

$\mathrm{CDCl}_{3}$

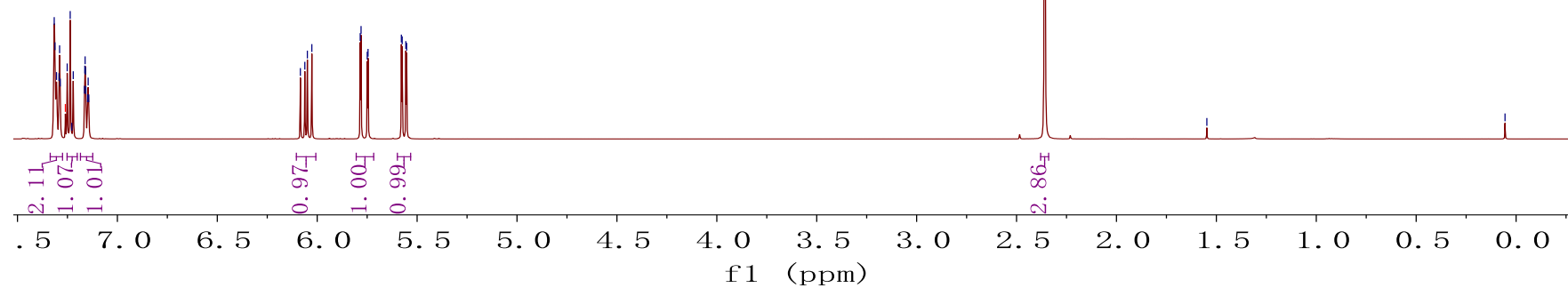




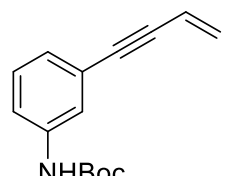

2k, ${ }^{1} \mathrm{H}$ NMR

$\mathrm{CDCl}_{3}$
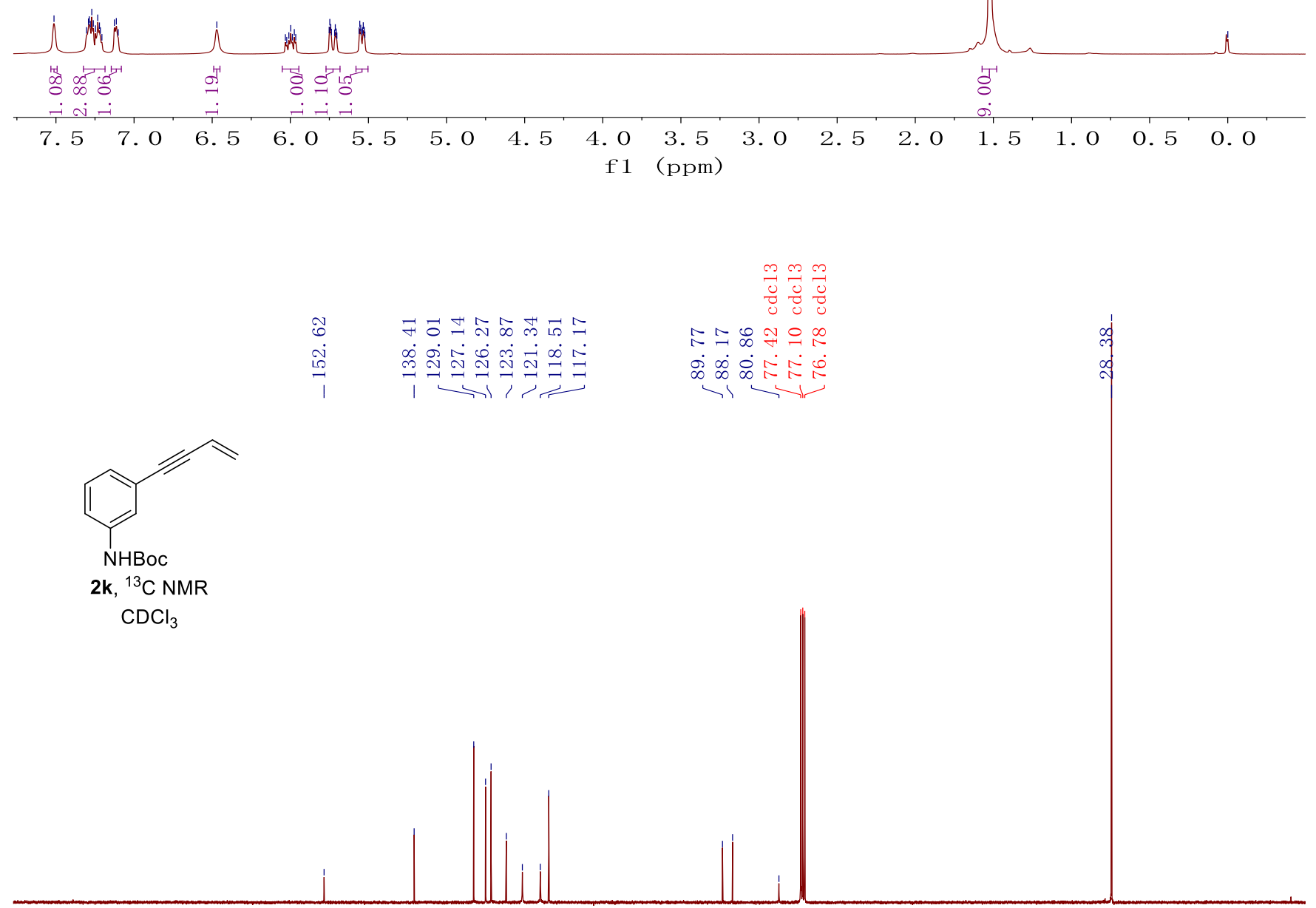

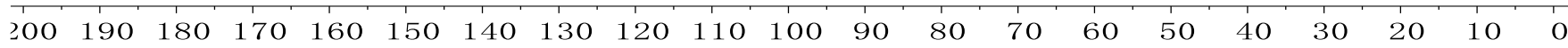
f1 (ppm) 

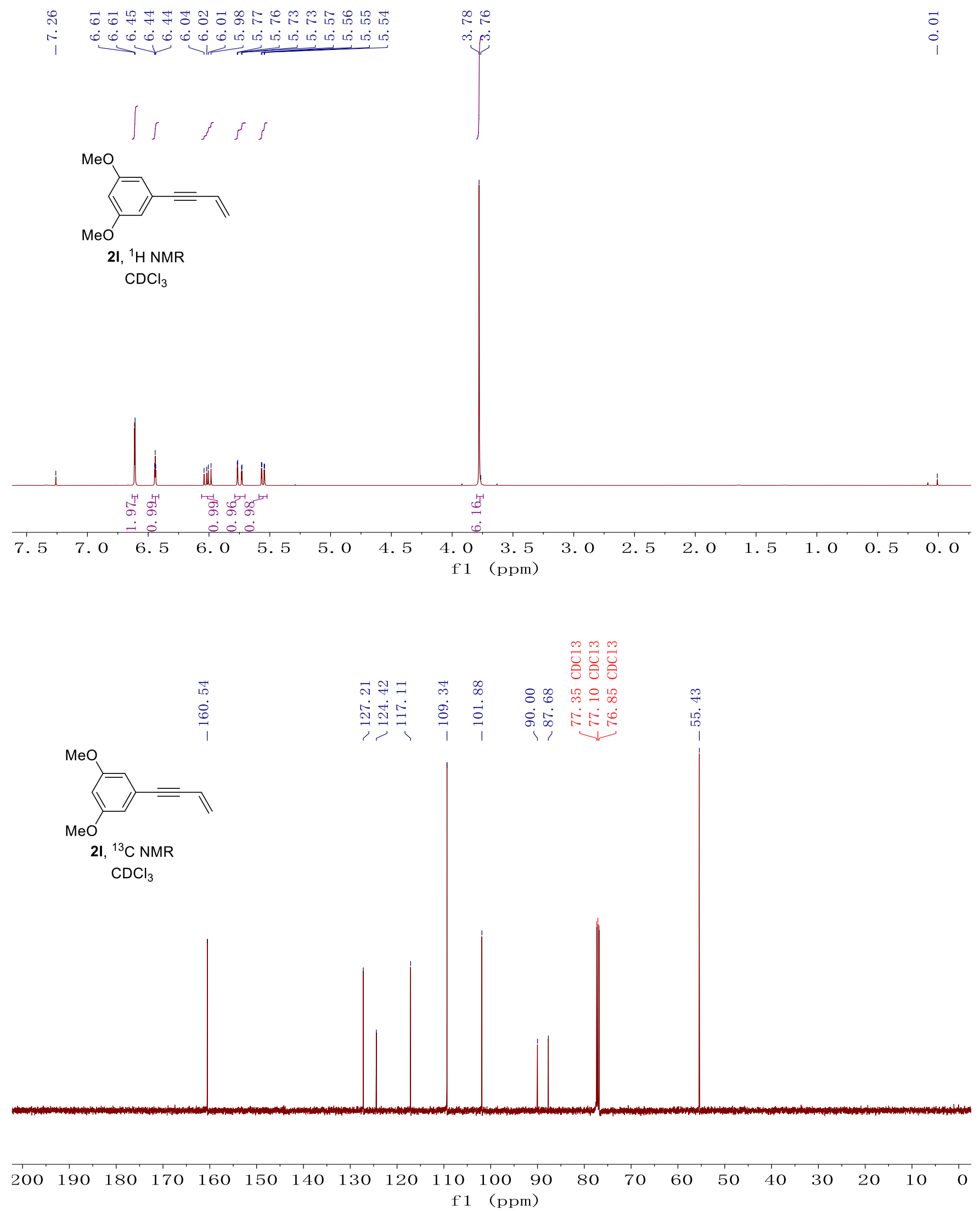


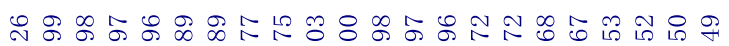

$\therefore$
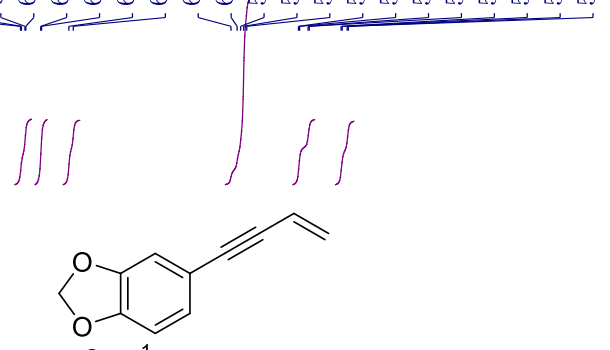

2m, ${ }^{1} \mathrm{H}$ NMR

$\mathrm{CDCl}_{3}$

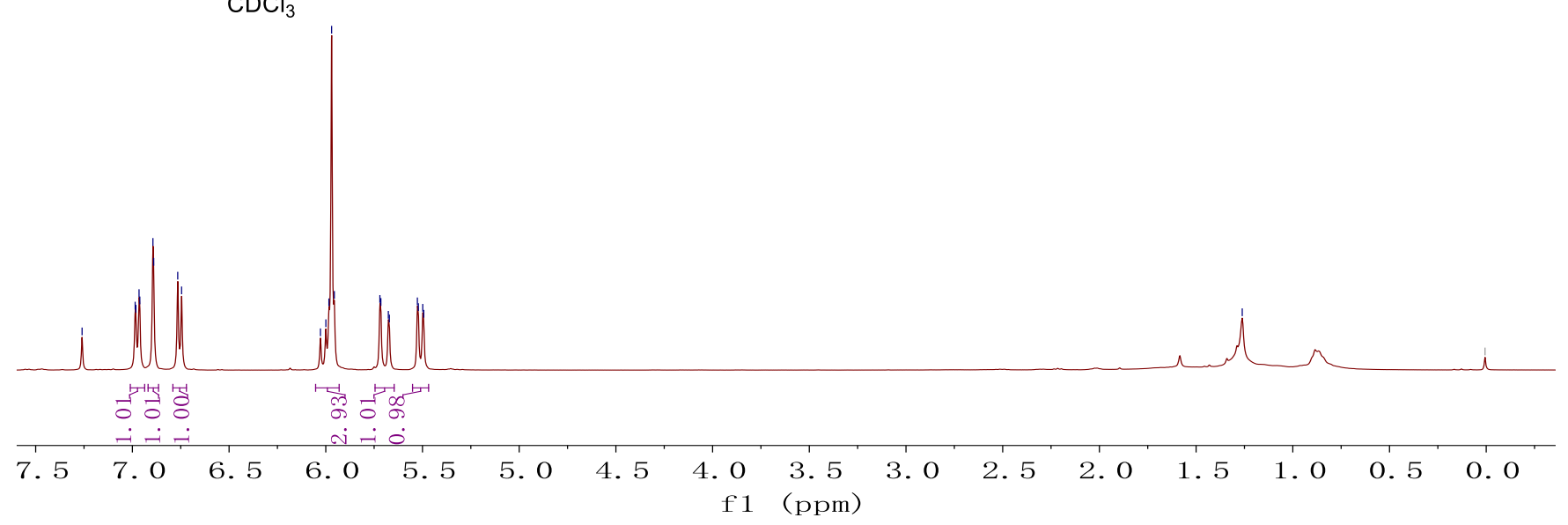

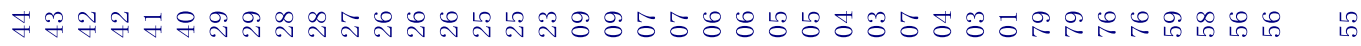

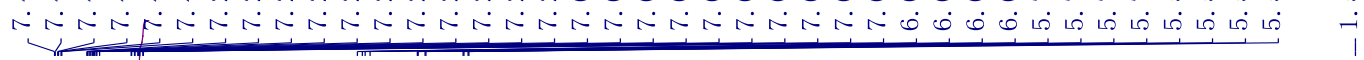

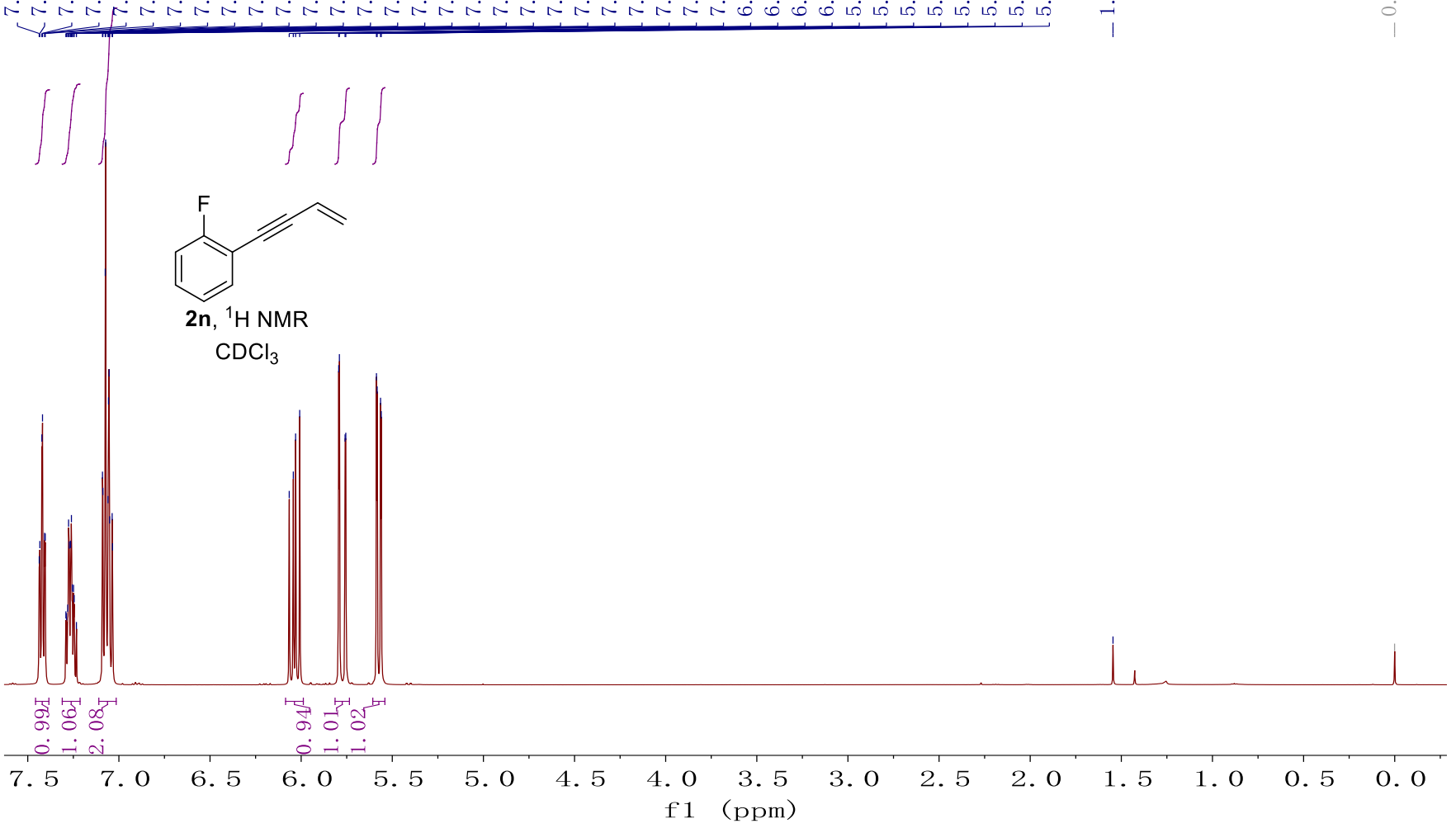




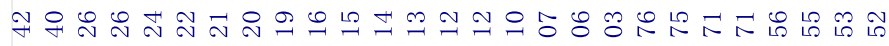

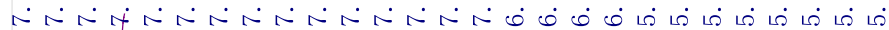

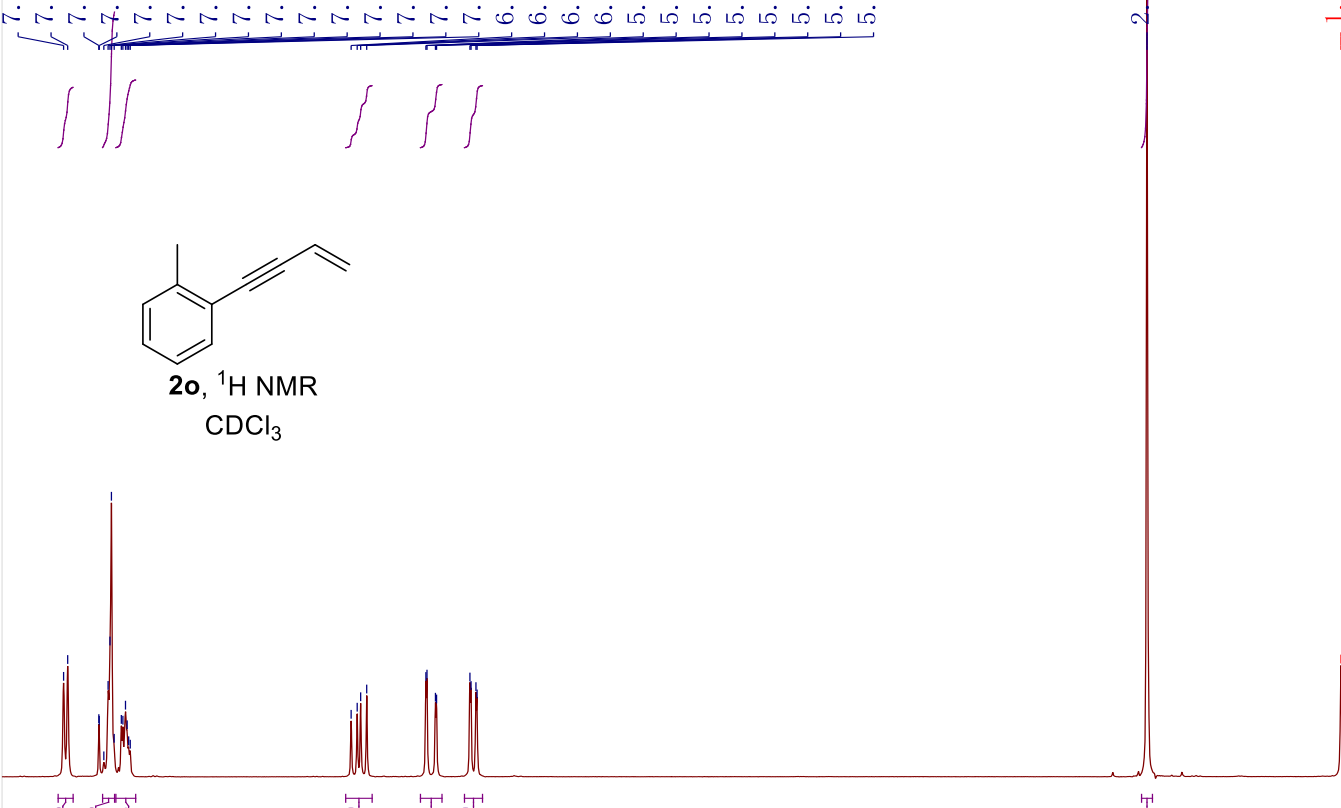

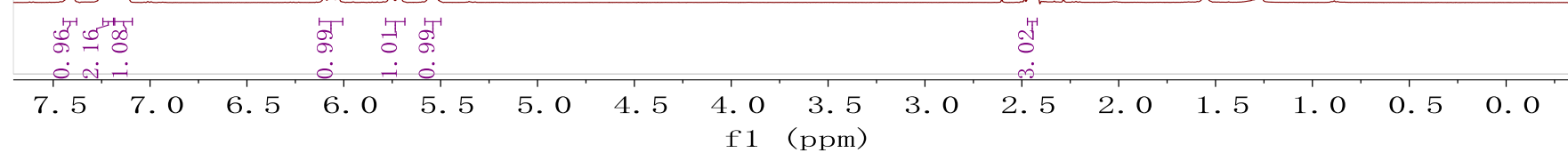

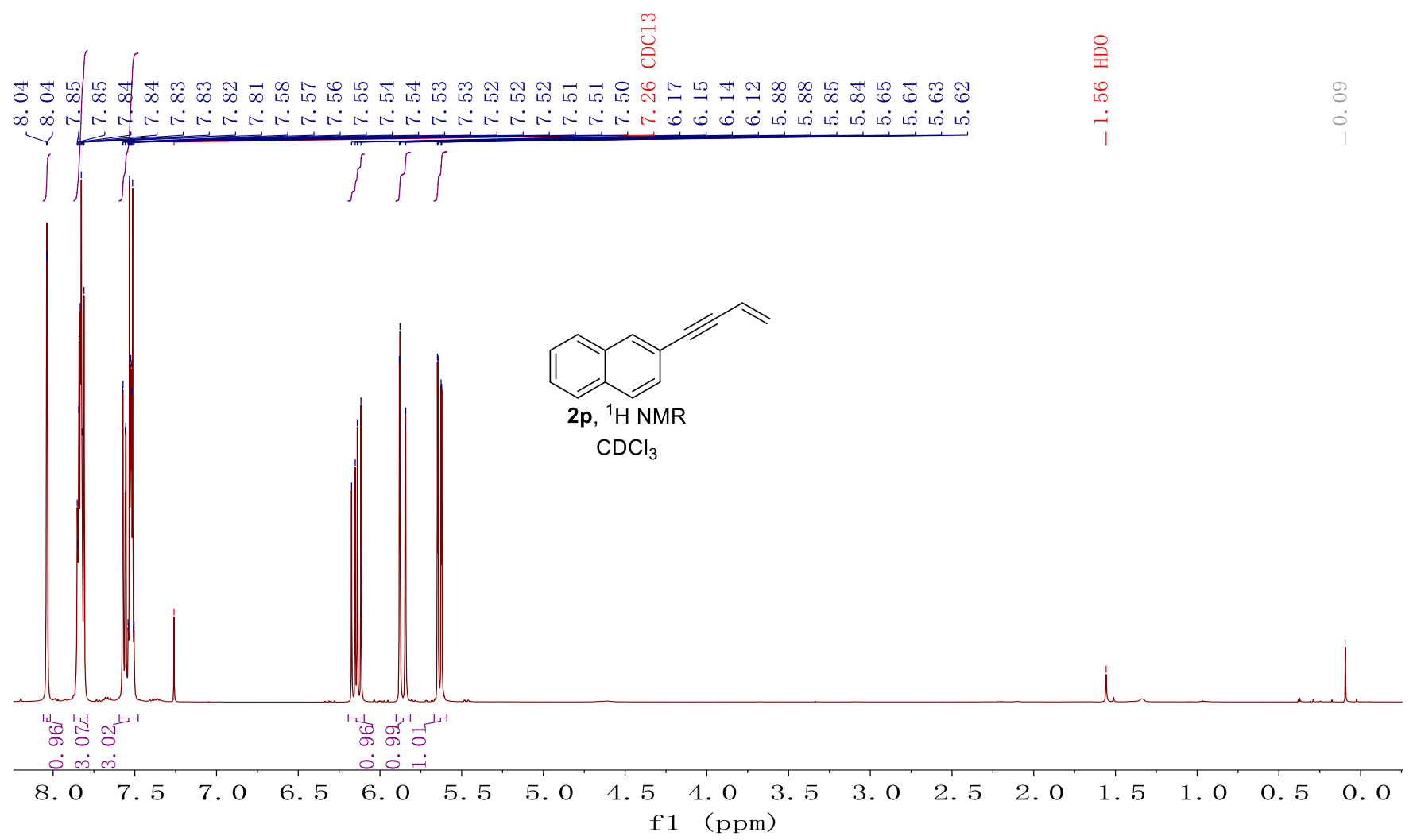




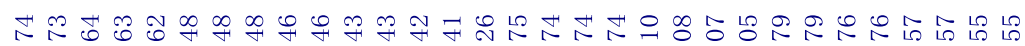

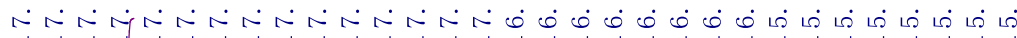
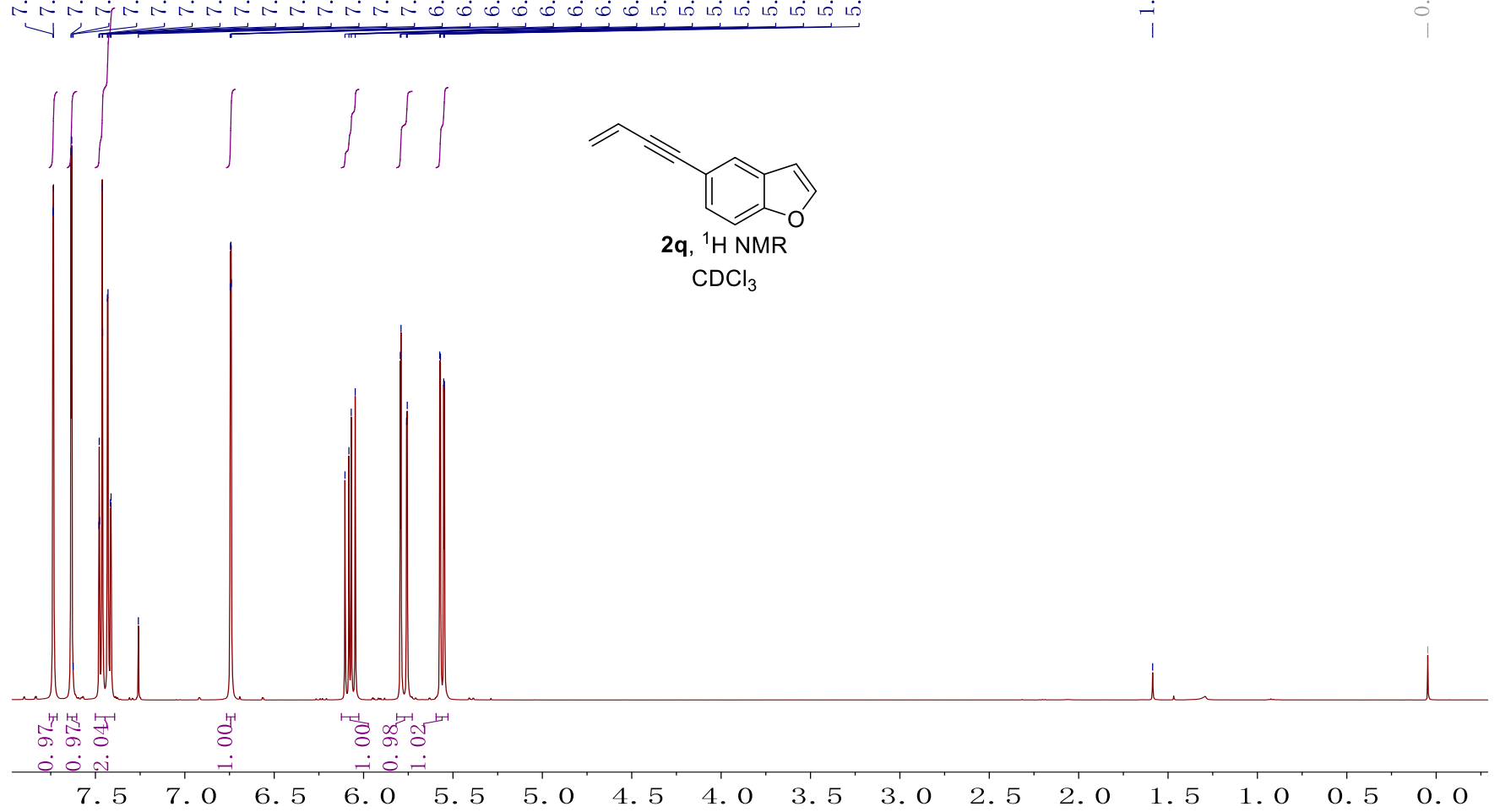

5. 04.

4. 03.5

3. 0

2. 5

2. 0 1.5

1. 0

0.50 .0

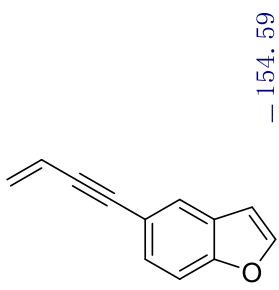

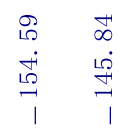

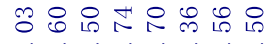

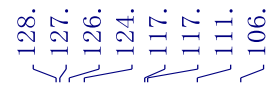

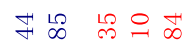

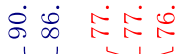

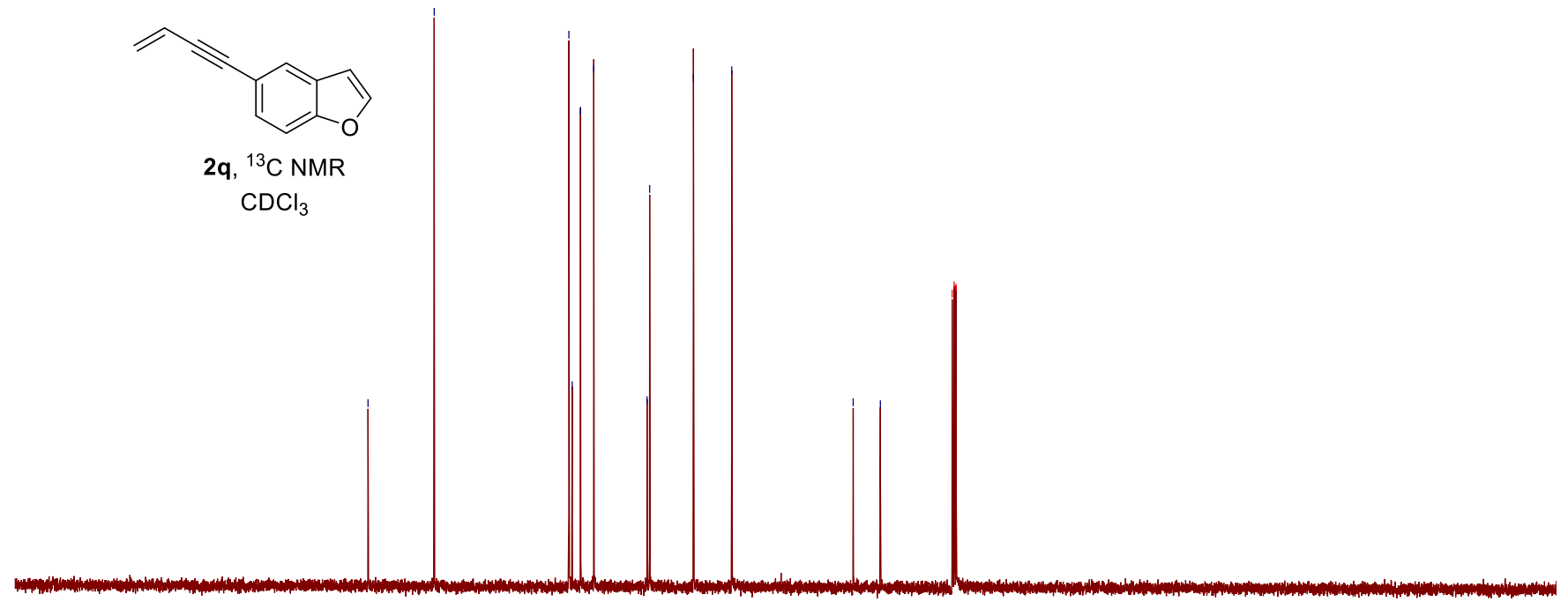

$\begin{array}{lllllllllllllllllllllll}100 & 190 & 180 & 170 & 160 & 150 & 140 & 130 & 120 & 110 & 100 & 90 & 80 & 70 & 60 & 50 & 40 & 30 & 20 & 10 & 0\end{array}$ f1 (ppm) 


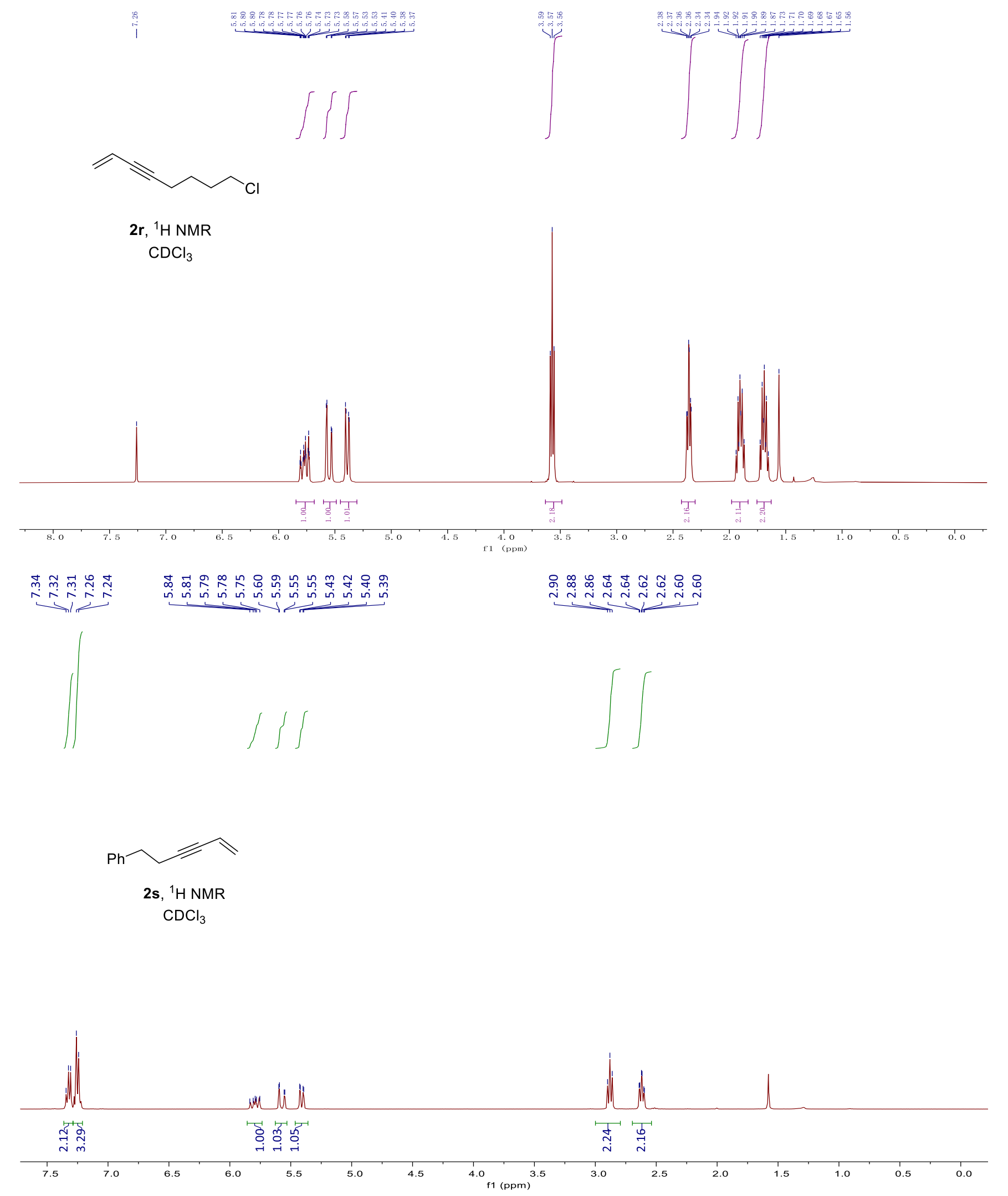


ค゚ㅅำ

ம்

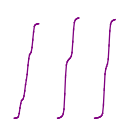

ีㅜㅇ

$\dot{ल \dot{m}}$

舟

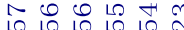

ง.ง

inin

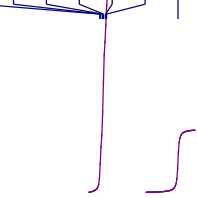

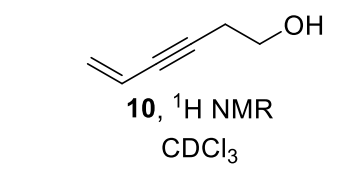

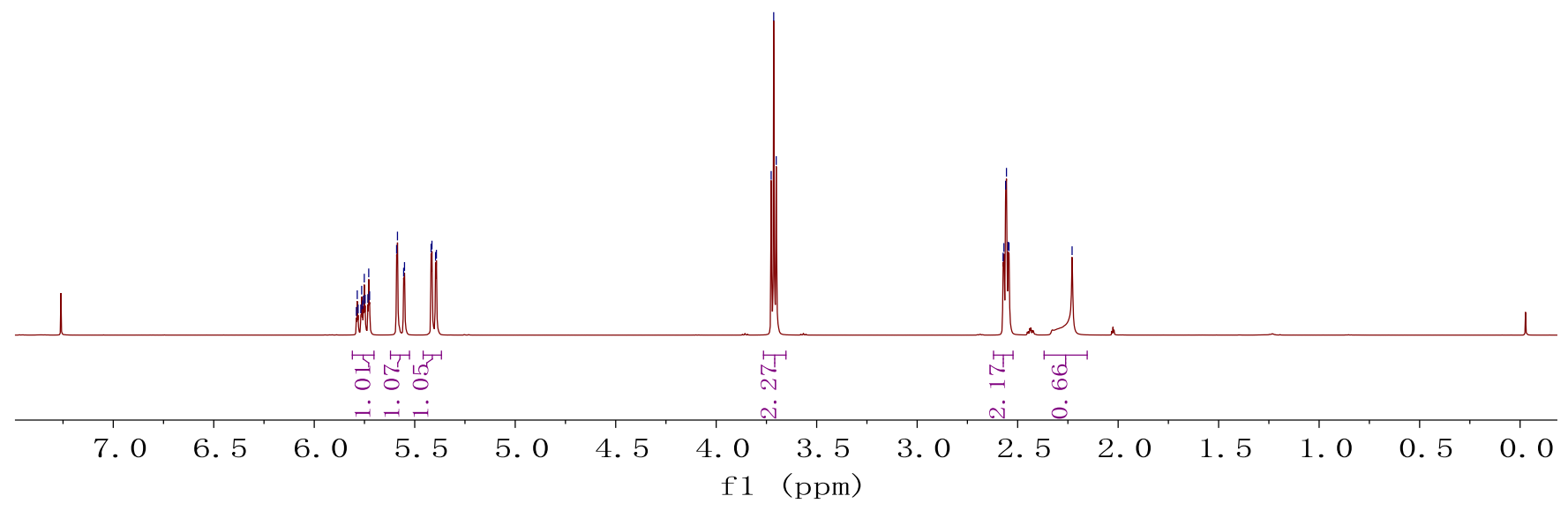



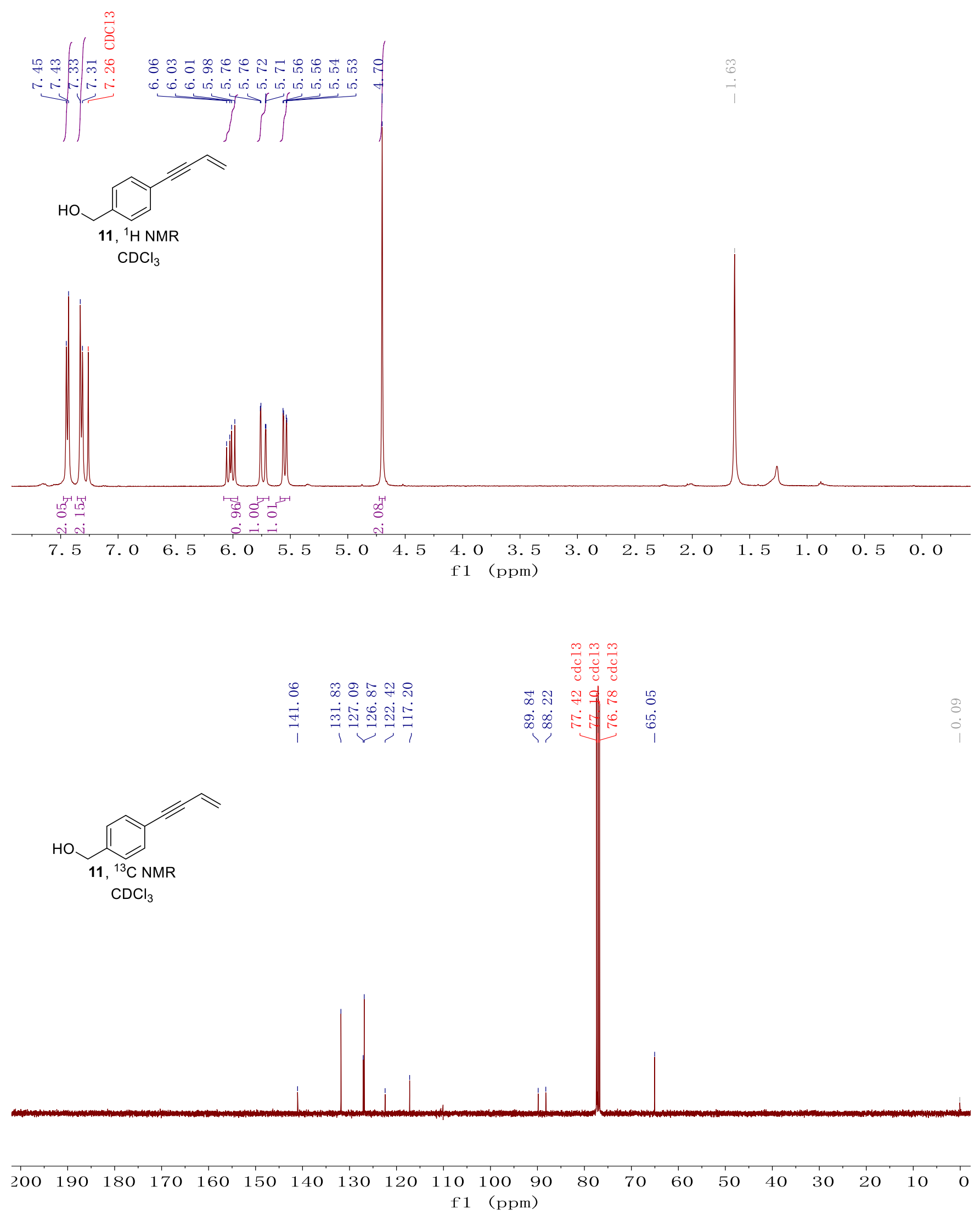
$\stackrel{m}{\tilde{U}}$

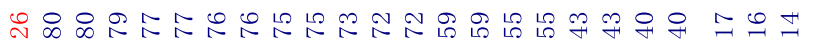

त ம்

$\left.\int\right\}$

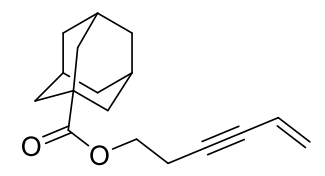

2t, ${ }^{1} \mathrm{H}$ NMR $\mathrm{CDCl}_{3}$

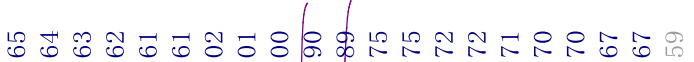

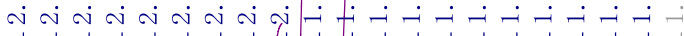

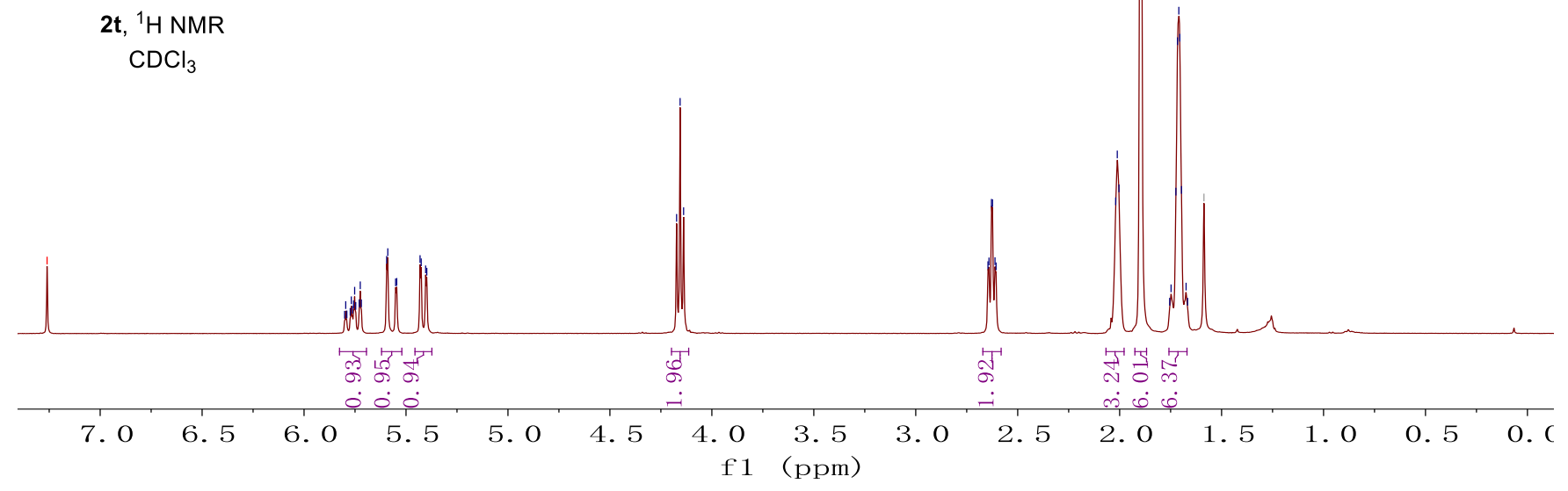

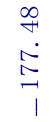

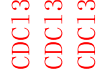

$\stackrel{\sim}{\sim} \stackrel{\circ}{\circ} \stackrel{\circ}{\circ}\llcorner$

$\dot{\infty} \dot{\infty} \sim \dot{\sim}$

$\llcorner 0$
$\dot{0}$
$\dot{0}$
1

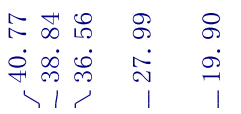

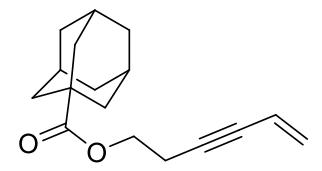

2t, ${ }^{13} \mathrm{C}$ NMR $\mathrm{CDCl}_{3}$

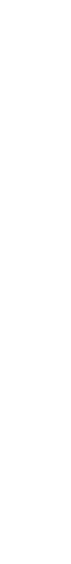


웅요 운

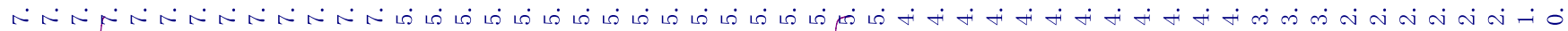
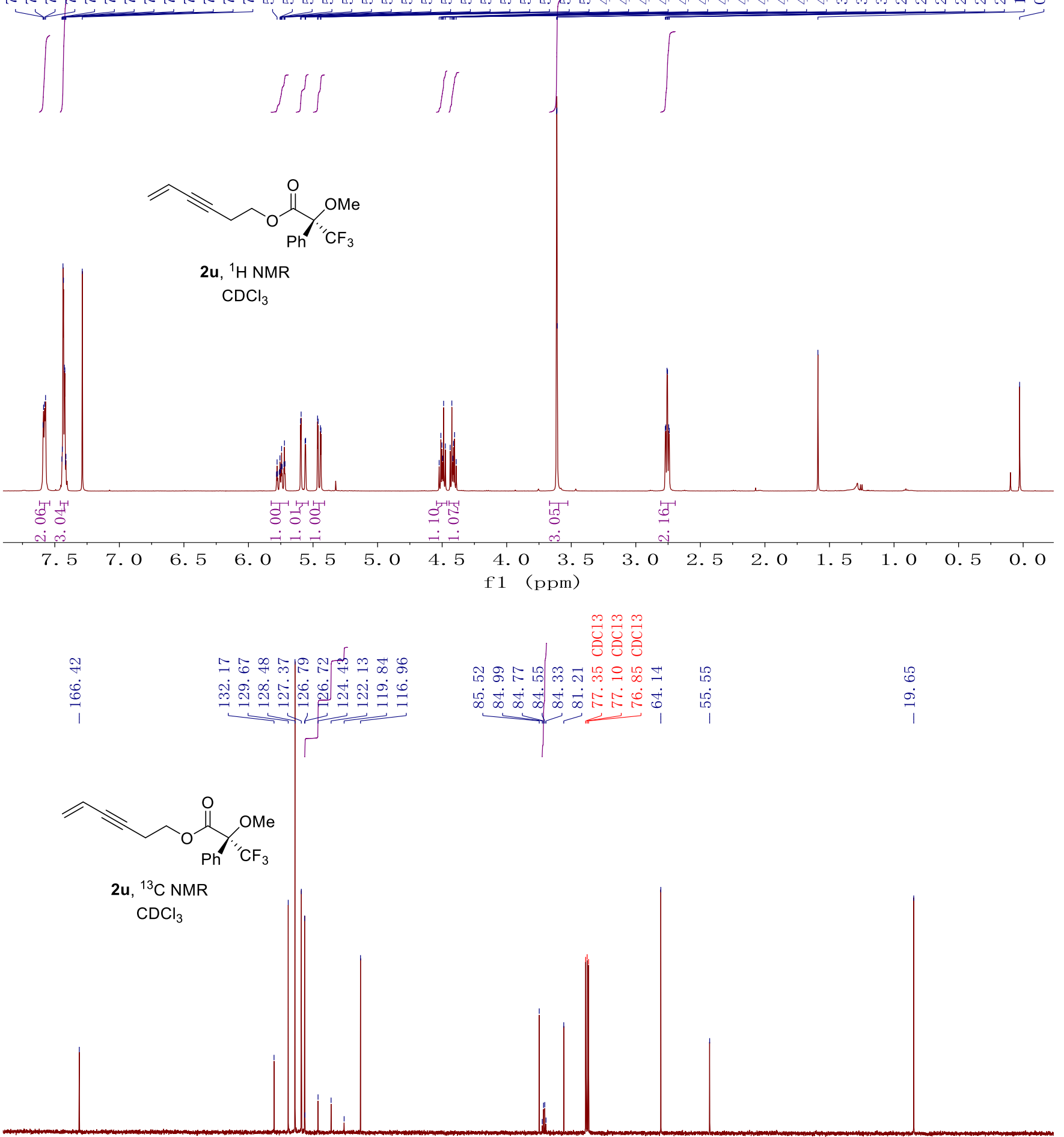

$\begin{array}{llllllllllllllllll}170 & 160 & 150 & 140 & 130 & 120 & 110 & 100 & 90 & 80 & 70 & 60 & 50 & 40 & 30 & 20 & 10 & 0\end{array}$ 


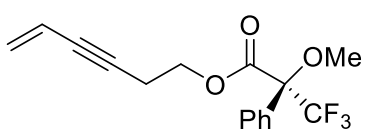

2u, ${ }^{19} \mathrm{~F}$ NMR $\mathrm{CDCl}_{3}$

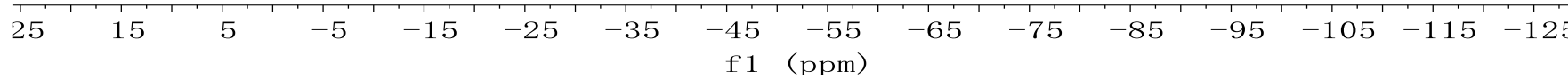




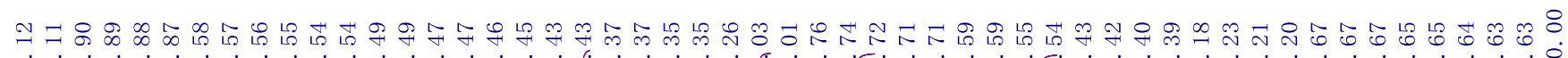

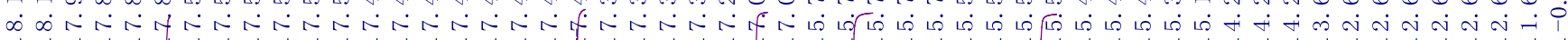
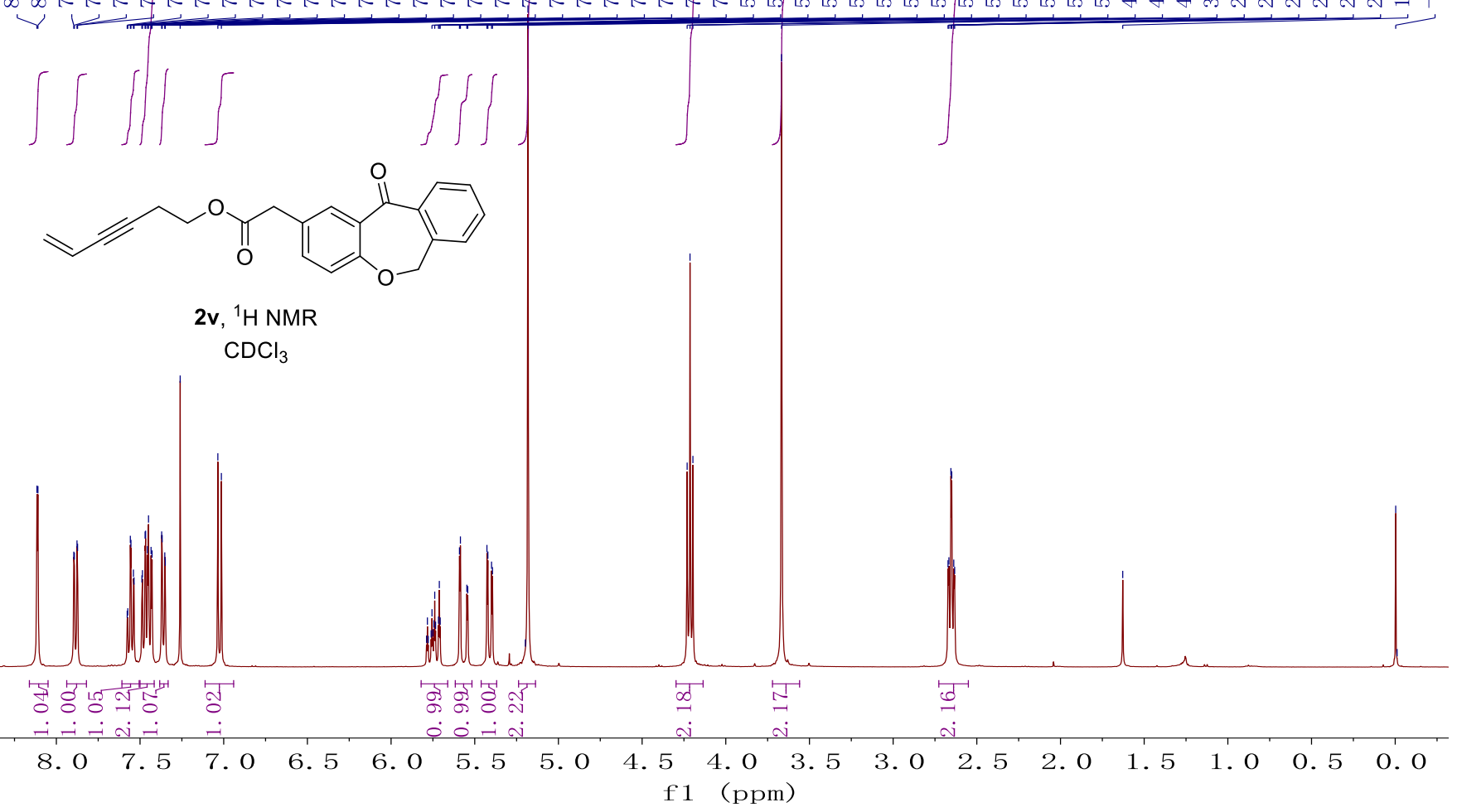

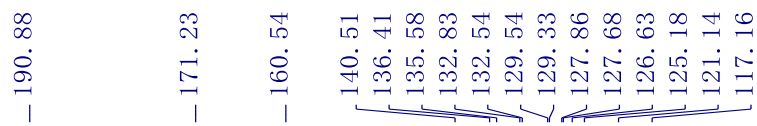

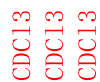

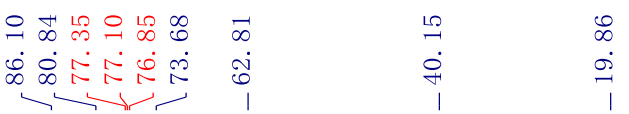<smiles>C=CC#CCCOC(=O)Cc1ccc2c(c1)C(=O)c1ccccc1CO2</smiles>

2v, ${ }^{13} \mathrm{C}$ NMR

$\mathrm{CDCl}_{3}$

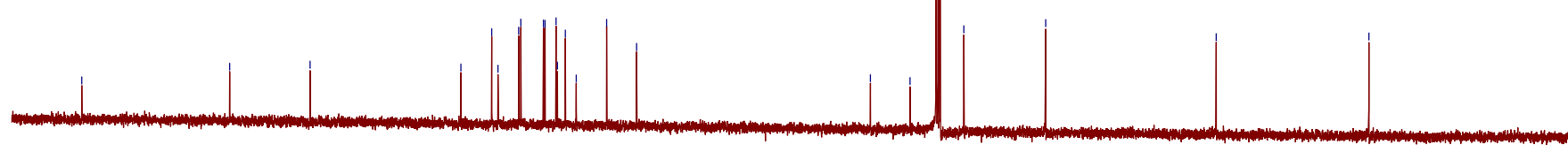

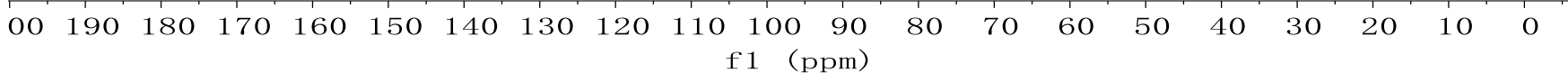



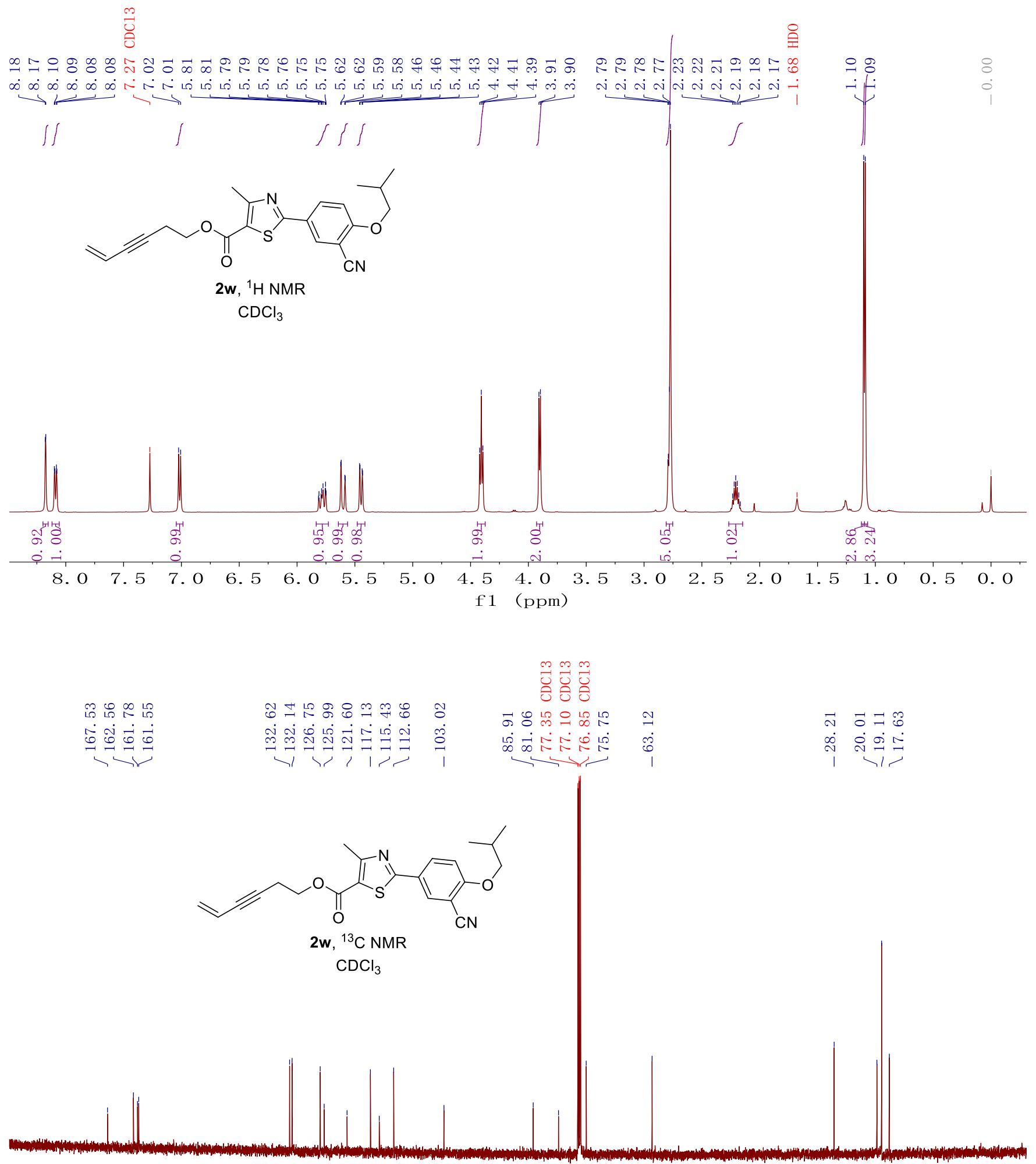

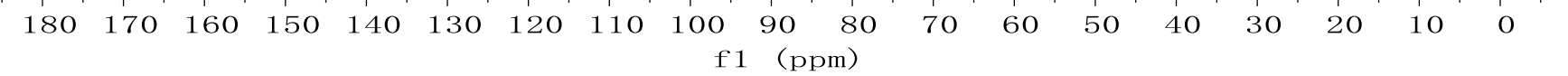




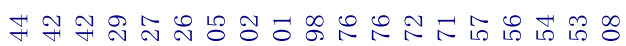

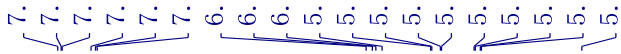

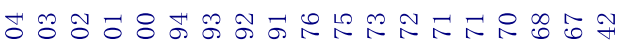

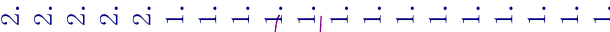

$\iint$
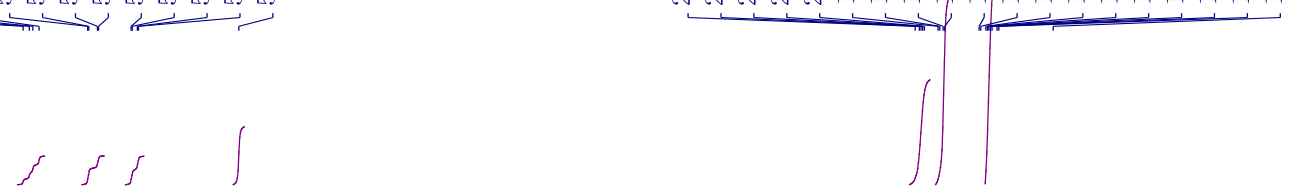

4<smiles>O=COCc1ccccc1</smiles>

2x, ${ }^{1} \mathrm{H}$ NMR

$\mathrm{CDCl}_{3}$

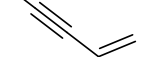

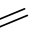
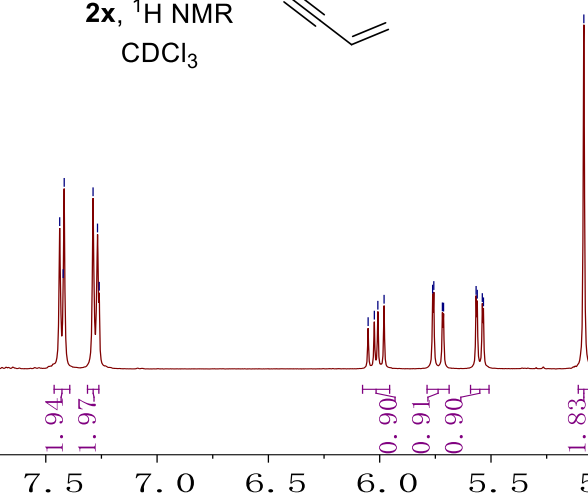

丽

5. 0

4. 5

4. $\mathrm{O} 3.5$

3. 0

2. 5

f1 (ppm)

논

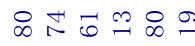

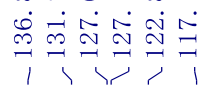
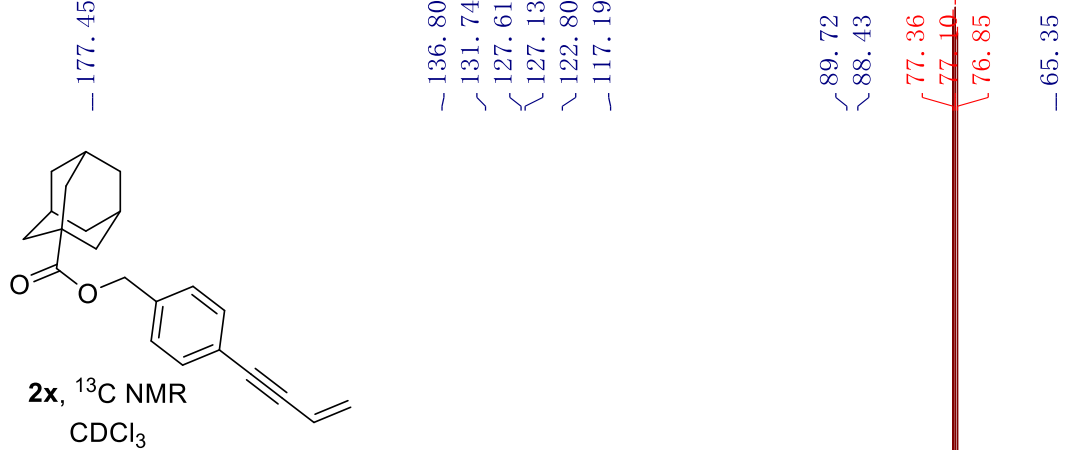

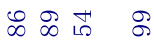

$\dot{+} \dot{\infty} \dot{\infty} \dot{\infty} \hat{\sim}$

गु'न बू बूँ

के เ⿺辶

2. $\begin{array}{lllll}1.5 & 1.0 & 0.5 & 0.1\end{array}$

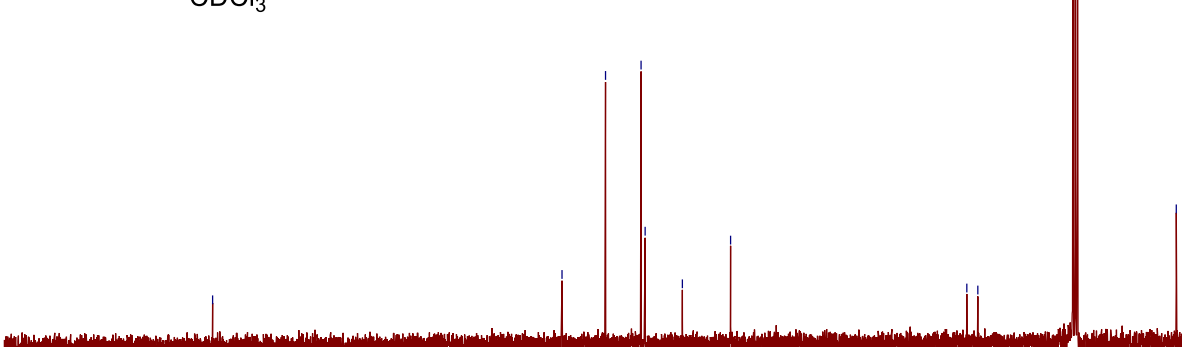

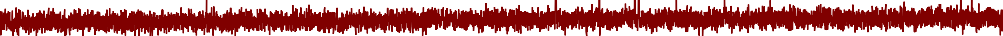

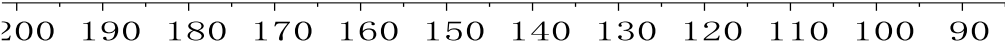

$80 \quad 70$

$60 \quad 50$

40

$3020 \quad 10 \quad 0$ f1 (ppm) 


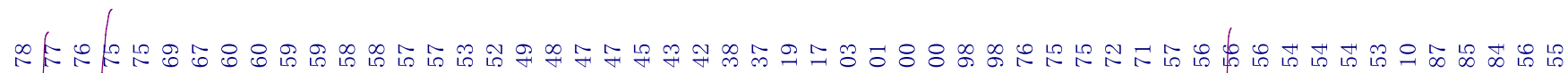

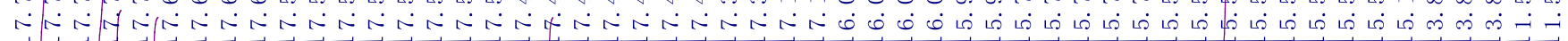

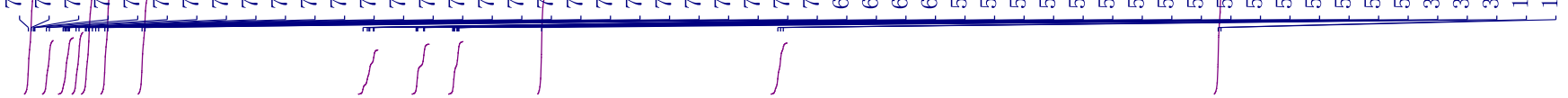<smiles>C=CC#Cc1ccc(COC(=O)[C@H](C)c2cccc(C(=O)c3ccccc3)c2)cc1</smiles>

2y, ${ }^{1} \mathrm{H}$ NMR $\mathrm{CDCl}_{3}$
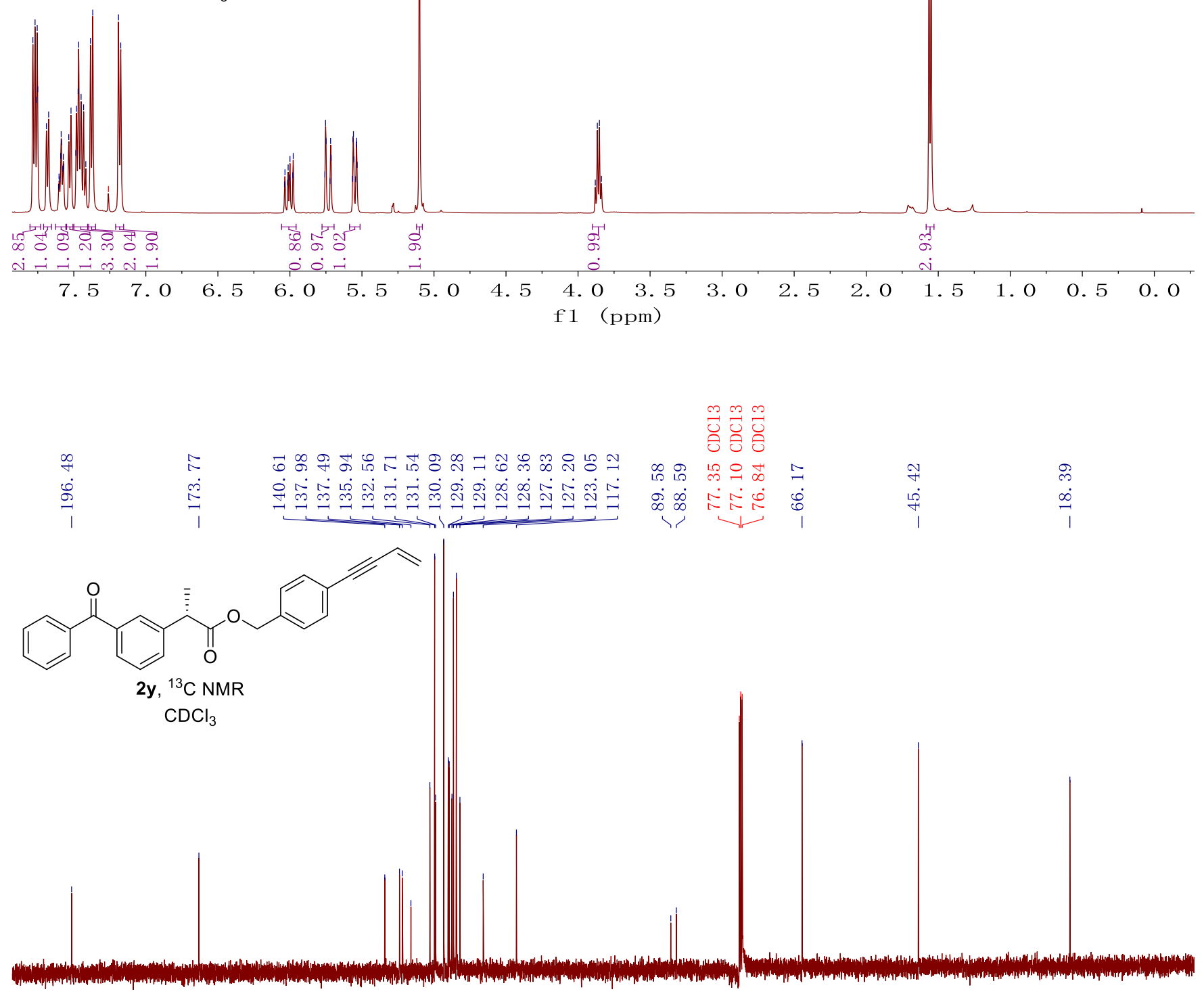
$\frac{\pi}{3}$

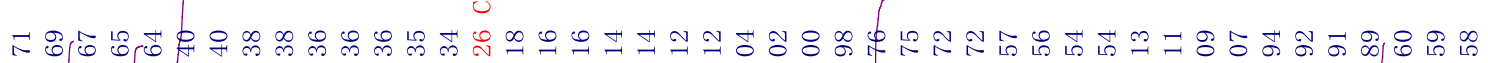

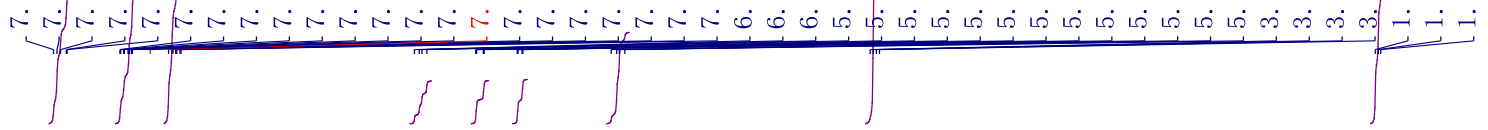
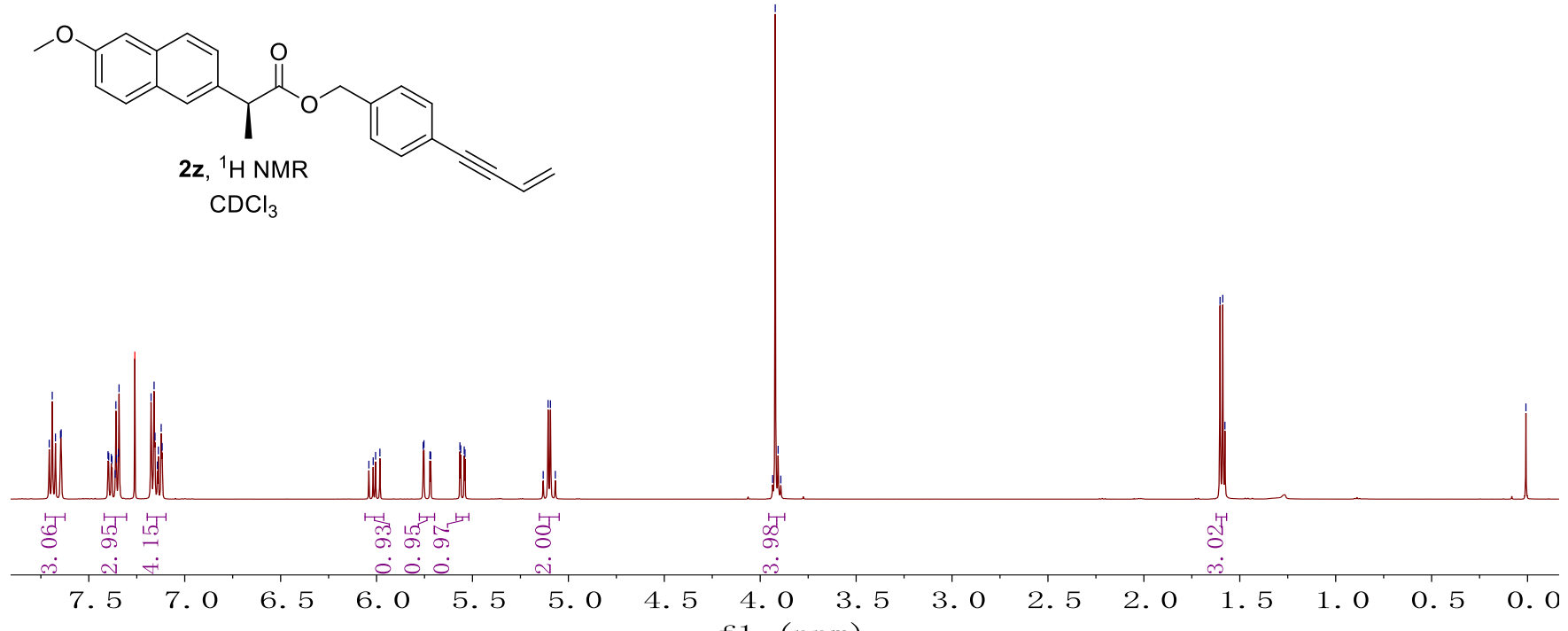

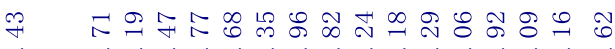

莣 $\quad \underbrace{\hat{0}}$

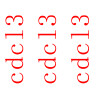

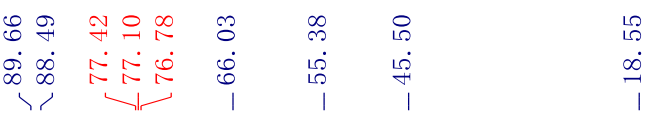<smiles>C=CC#Cc1ccc(COC(=O)C(Cc2ccc3cc(OC)ccc3c2)NOC(C)(C)C)cc1</smiles>

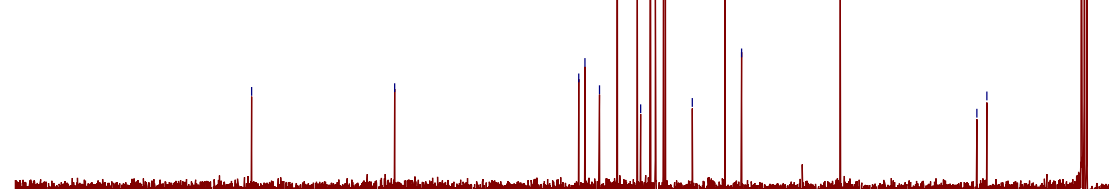

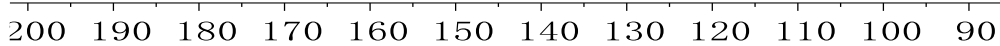

$80 \quad 70$

60

$50 \quad 40$

f1 (ppm) 
우

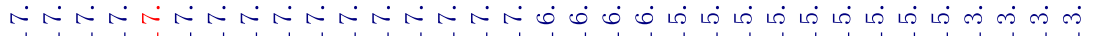

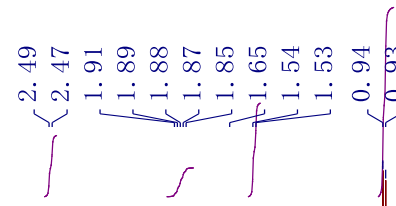<smiles>C=CC#Cc1ccc(COC(=O)[C@H](C)c2ccc(C)cc2)cc1</smiles><smiles>[R]CNCCCCCCC(C)C</smiles>
$\mathrm{CDCl}_{3}$
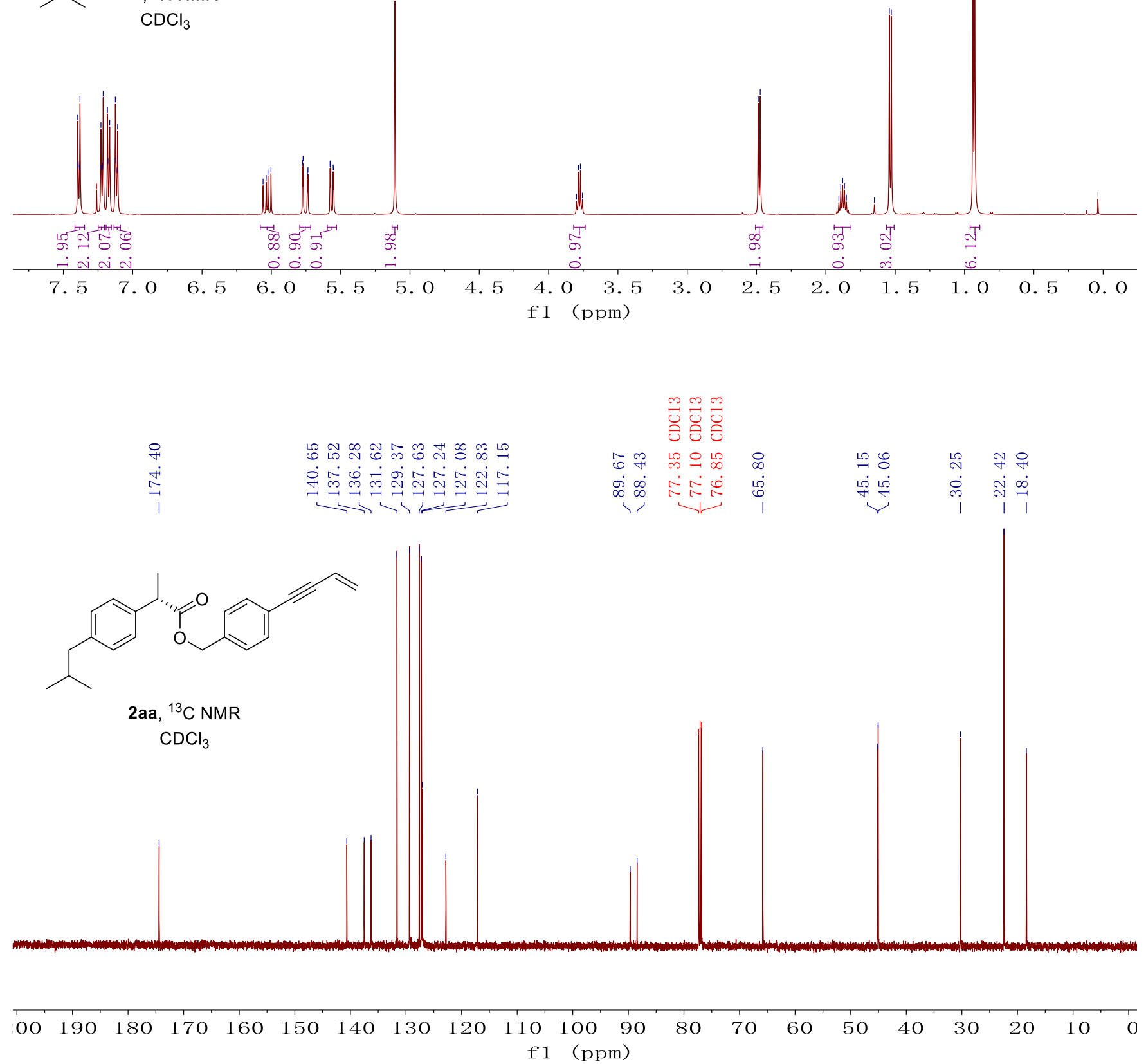
అ

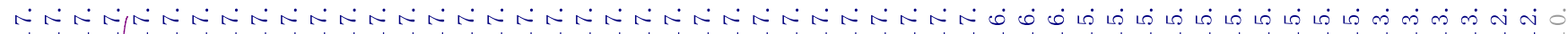
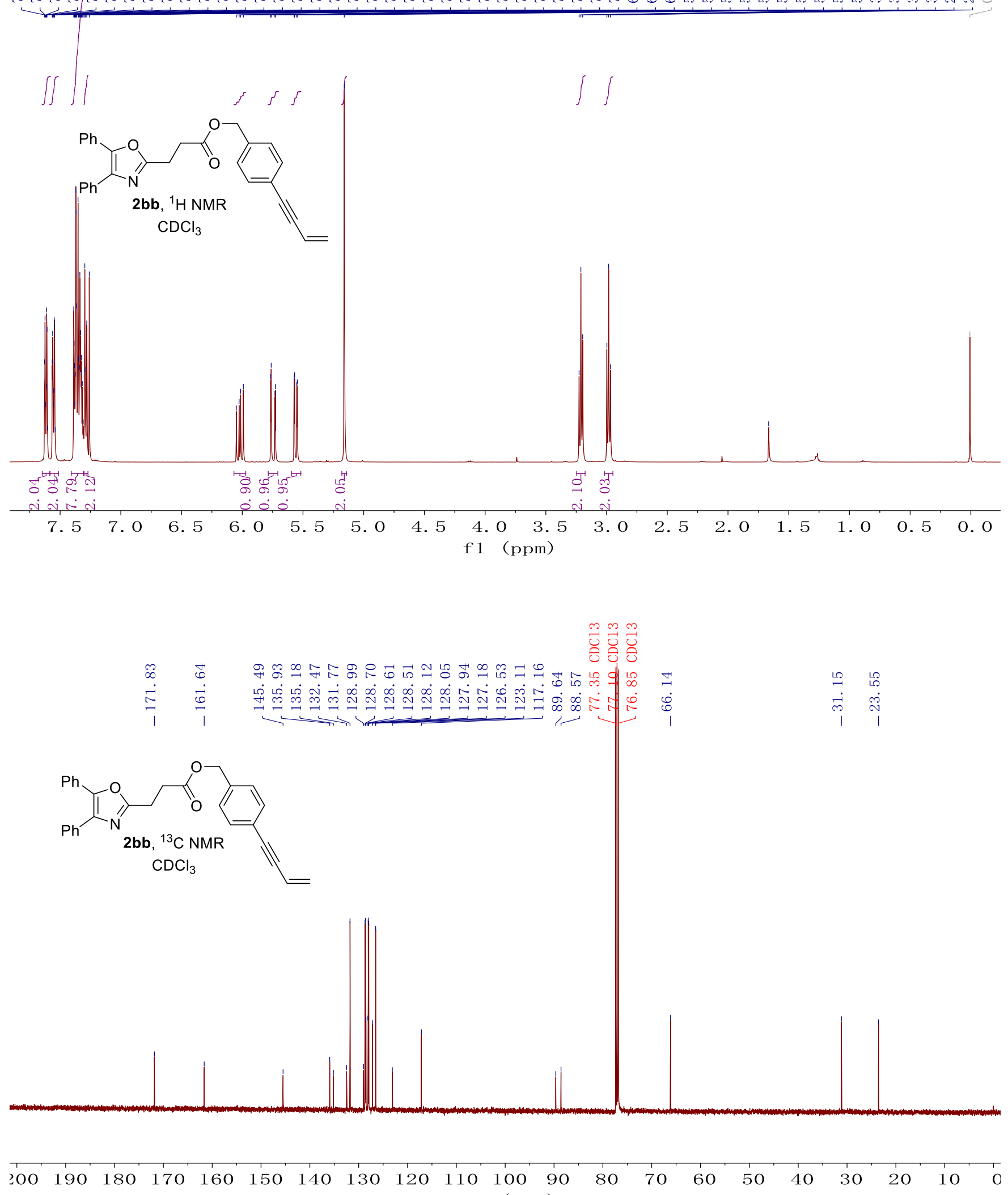
f1 (ppm) 
岕

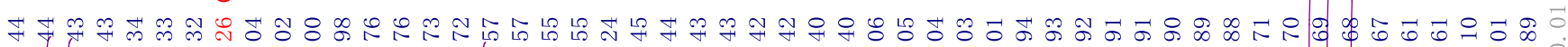

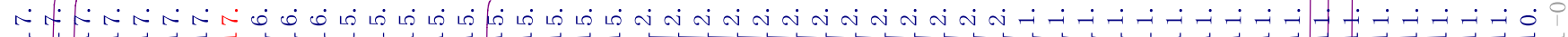
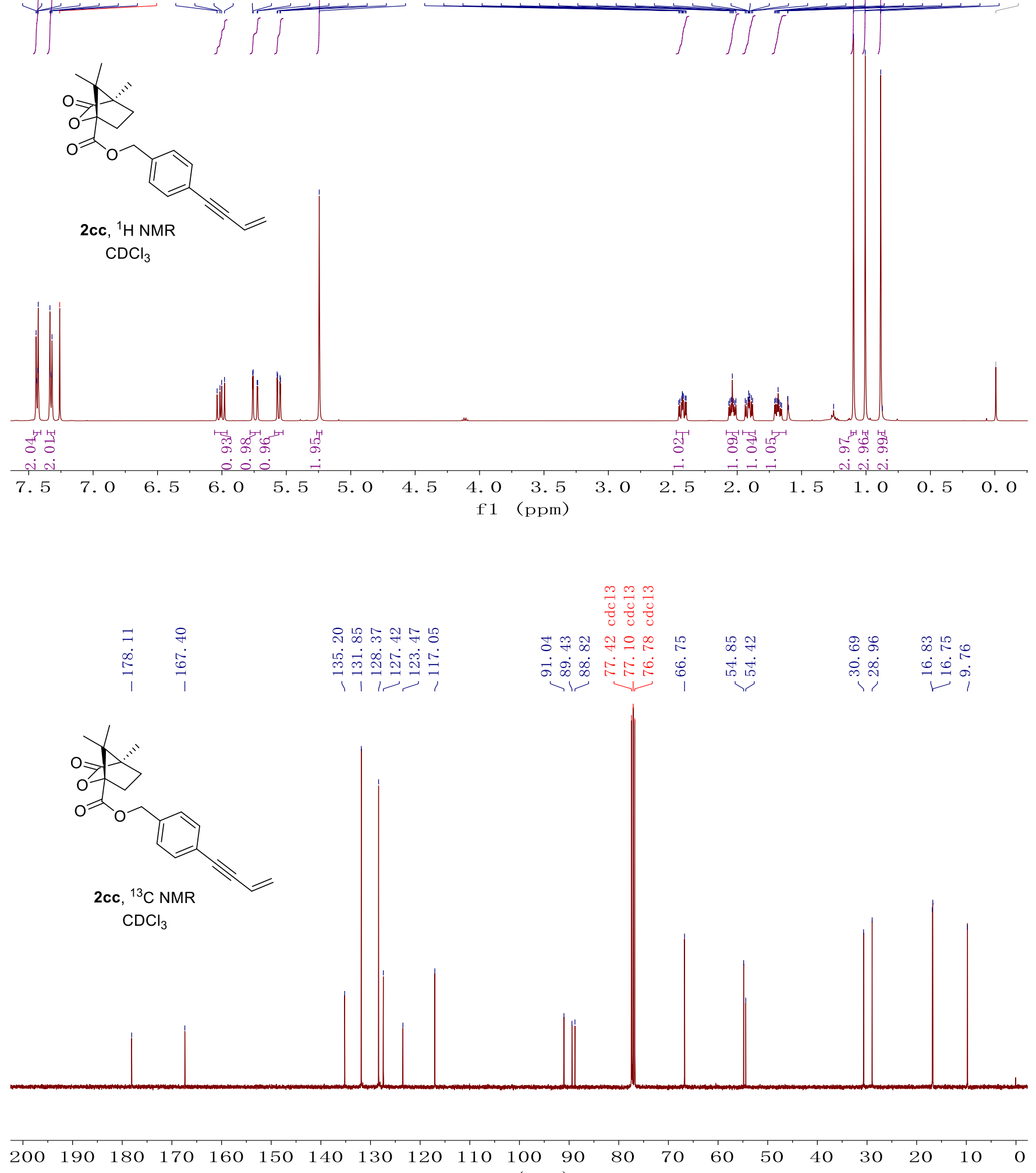
f1 (ppm) 

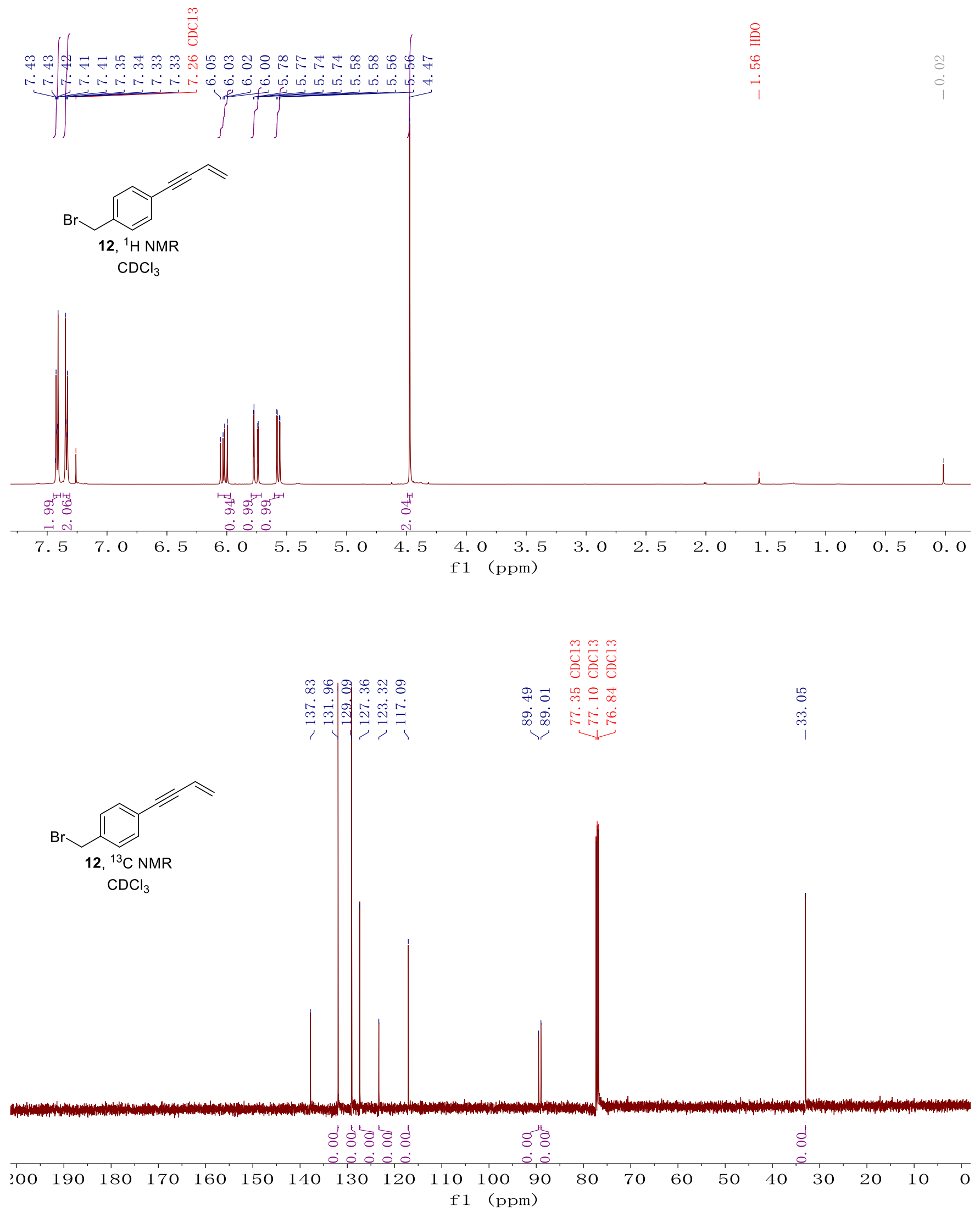


\section{离}

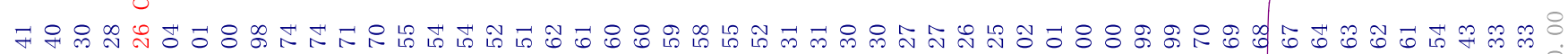

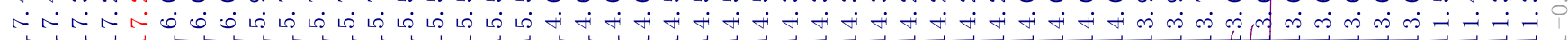

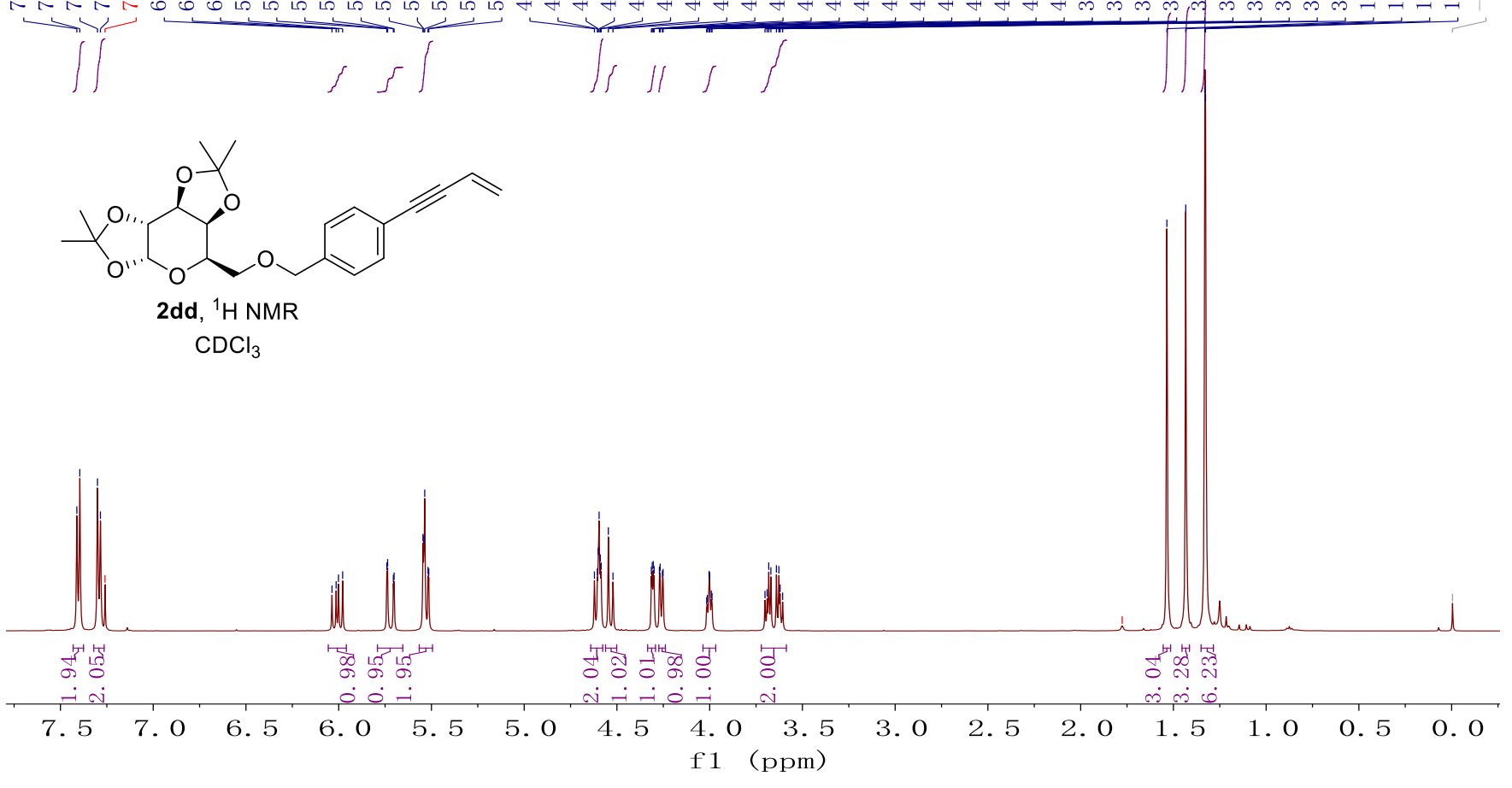
f1 (ppm)

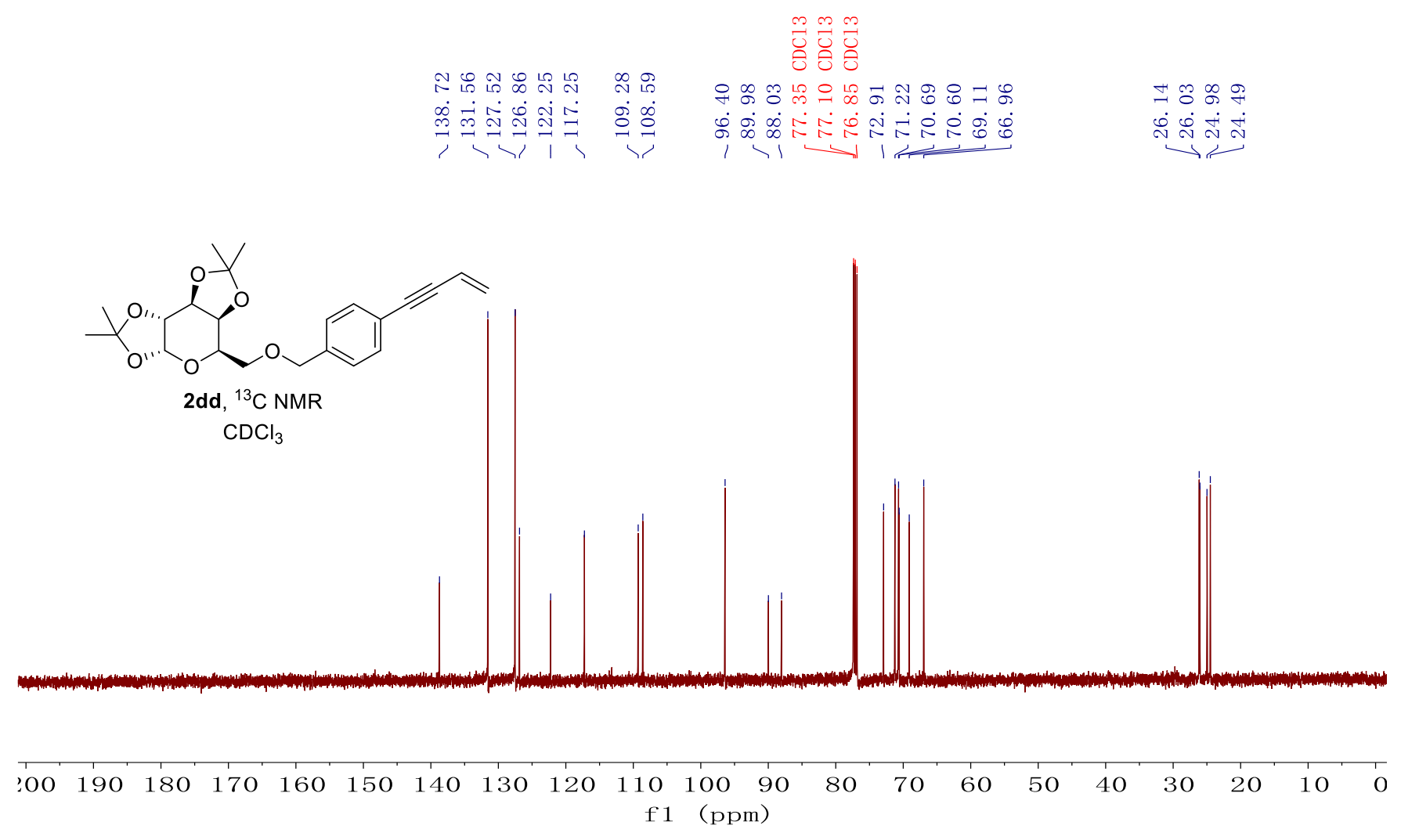



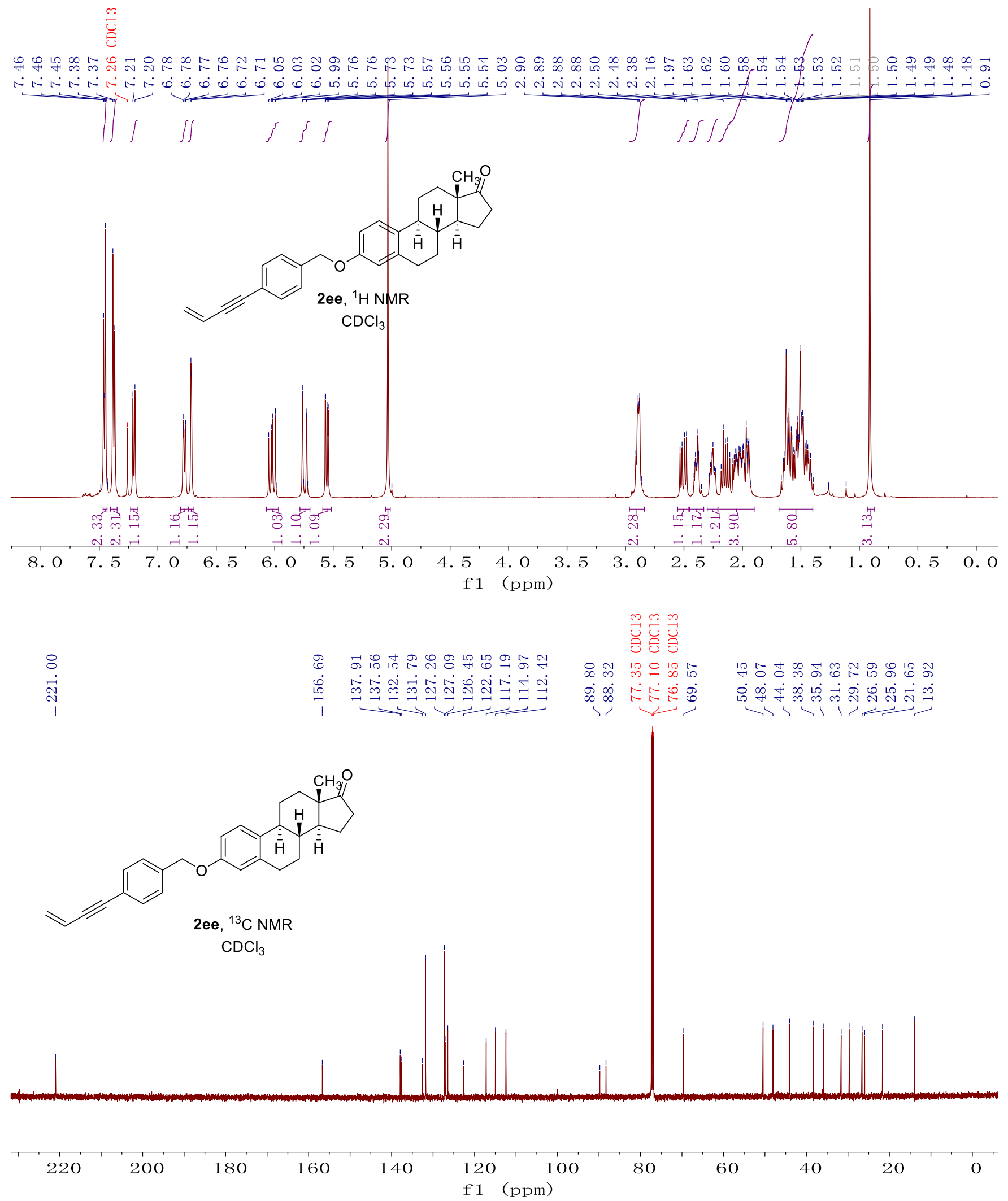

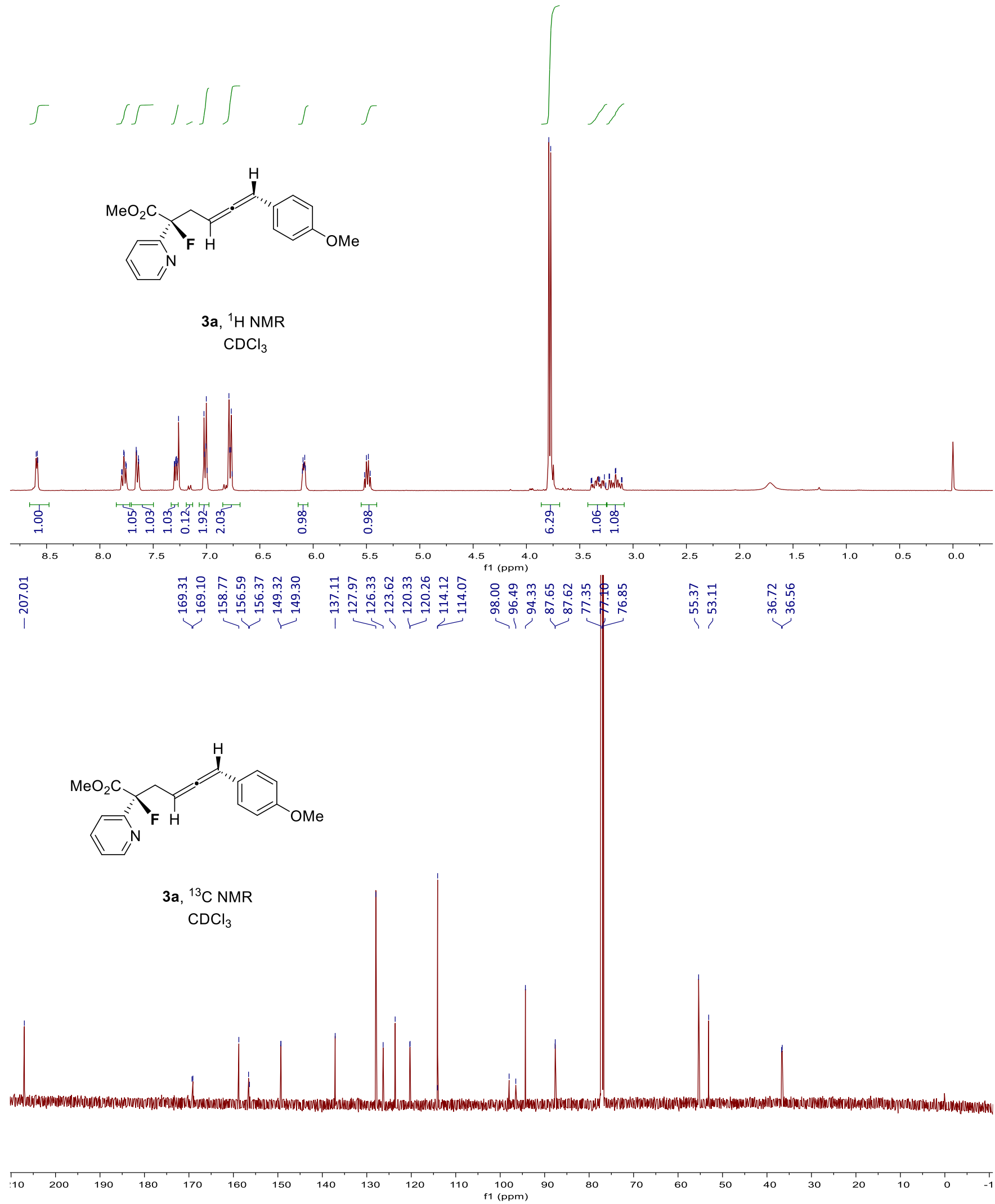
융 ₹

मี่

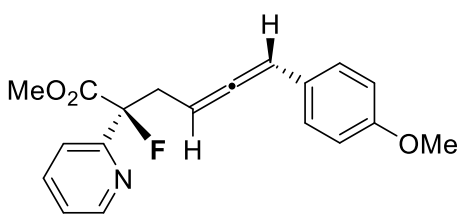

3a, ${ }^{19} \mathrm{~F}$ NMR

$\mathrm{CDCl}_{3}$

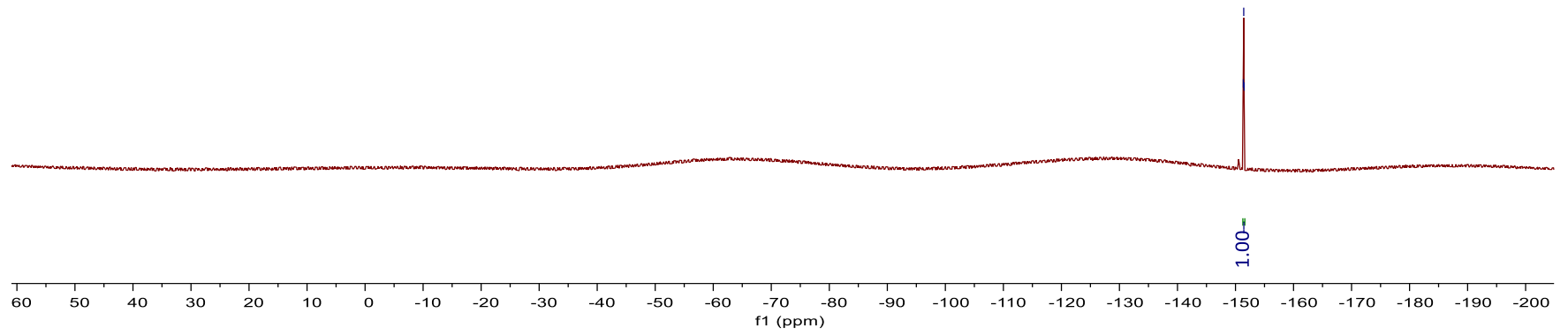




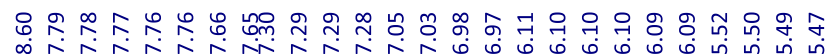

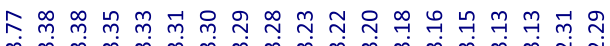

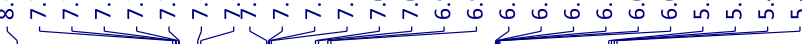

$\dot{m} \dot{m} \dot{m} \dot{m} \dot{m} \dot{m} \dot{m} m \dot{m} \dot{m} \dot{m} \dot{m} \dot{m} \dot{m} \ddot{N}$

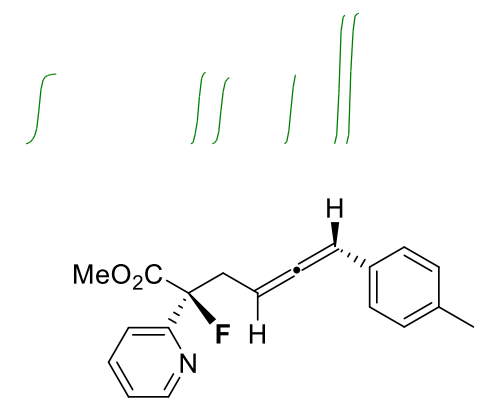

3b, ${ }^{1} \mathrm{H}$ NMR

$\mathrm{CDCl}_{3}$
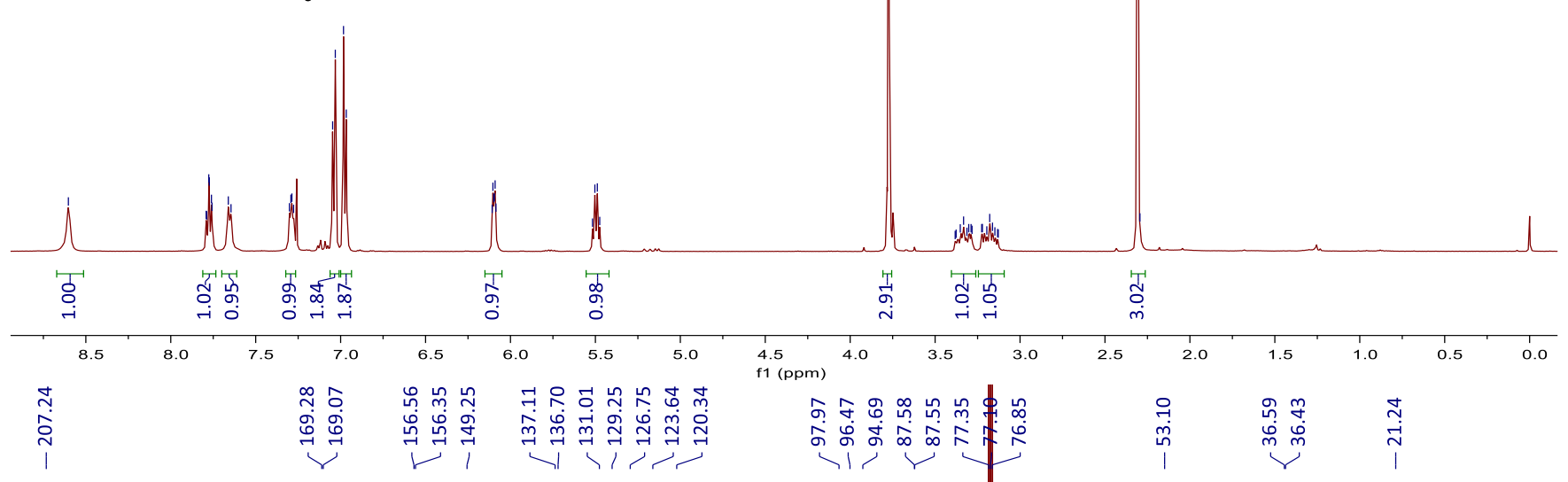

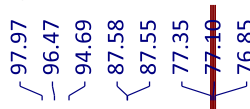

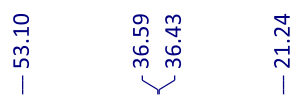
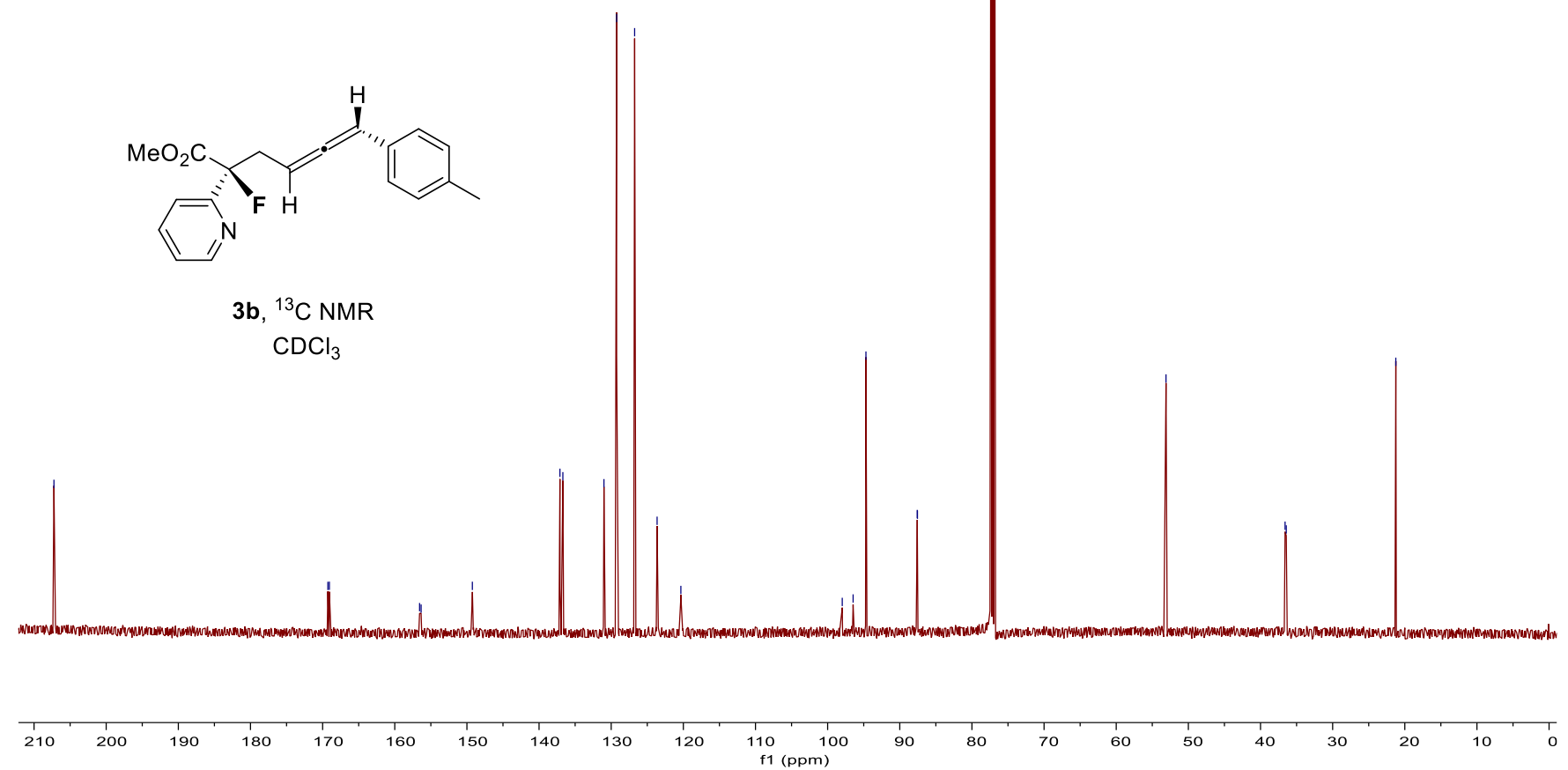
움 구 굴

링

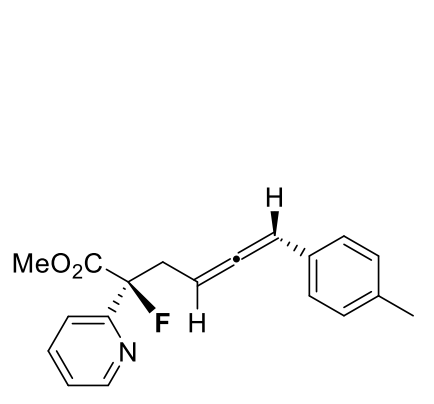

3b, ${ }^{19} \mathrm{~F}$ NMR

$\mathrm{CDCl}_{3}$

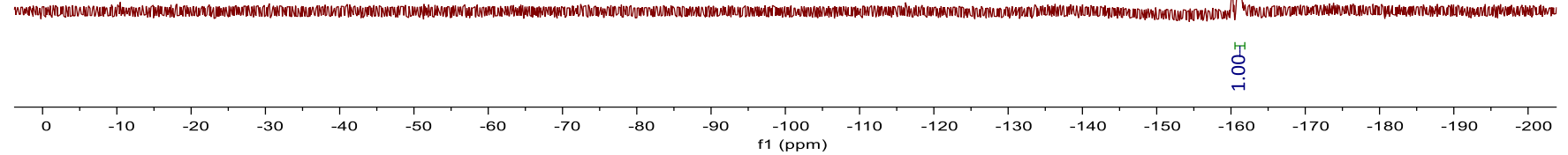




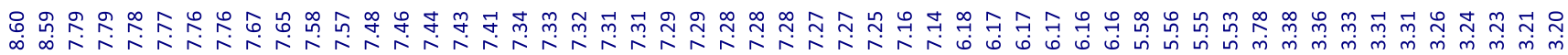
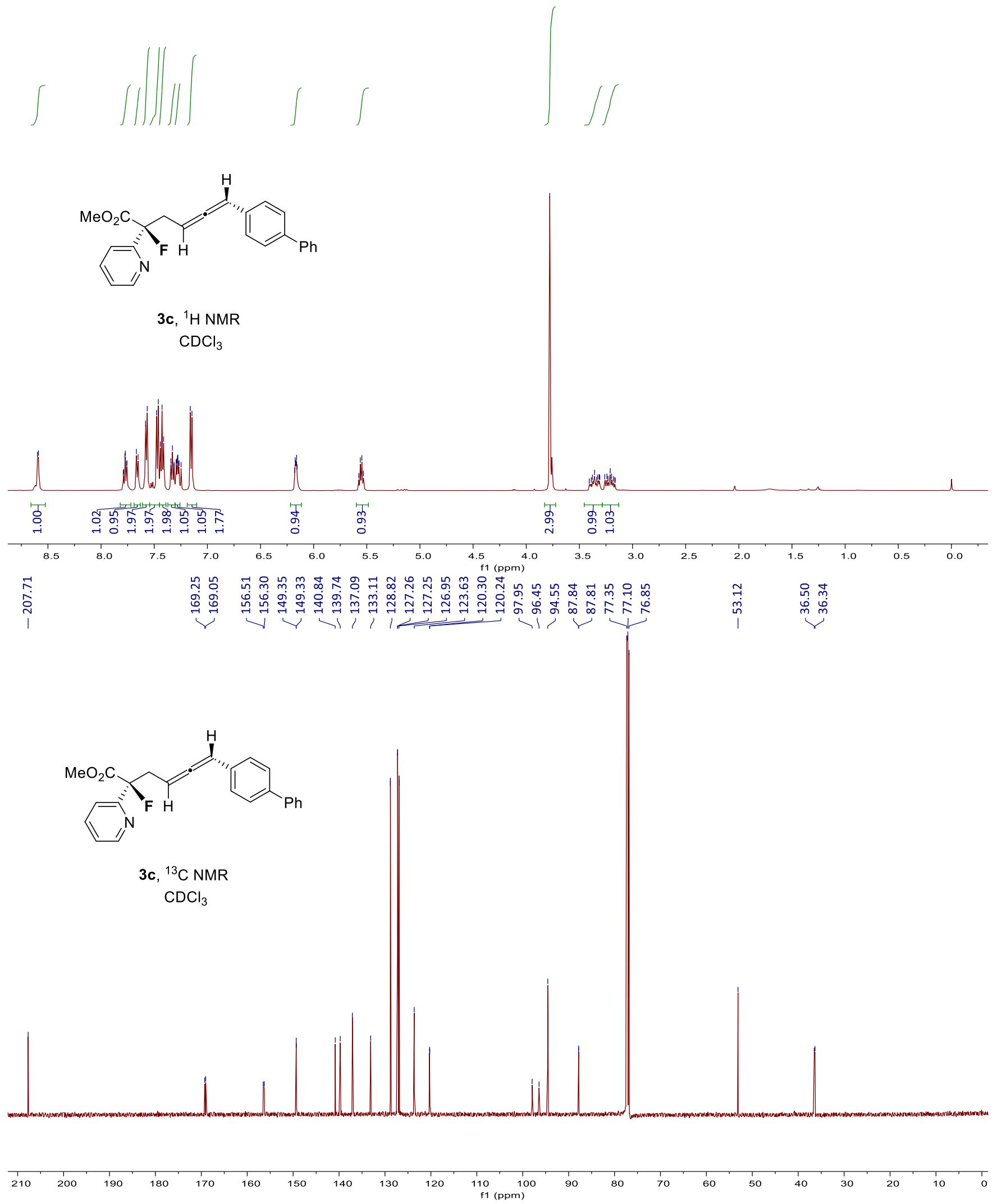


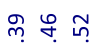

过宫

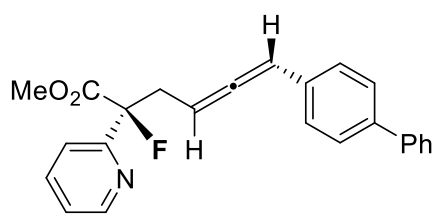

3c, ${ }^{19} \mathrm{~F}$ NMR $\mathrm{CDCl}_{3}$

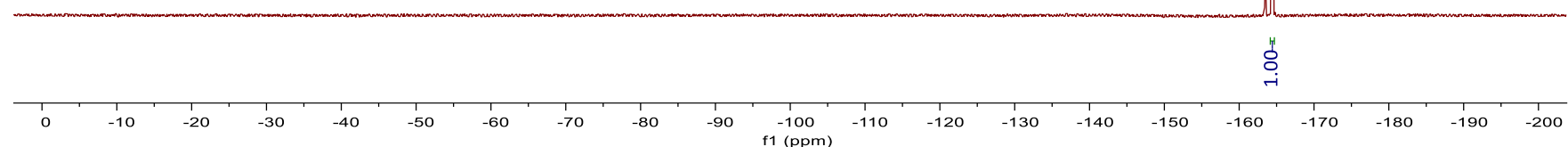




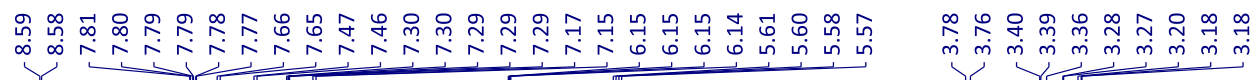
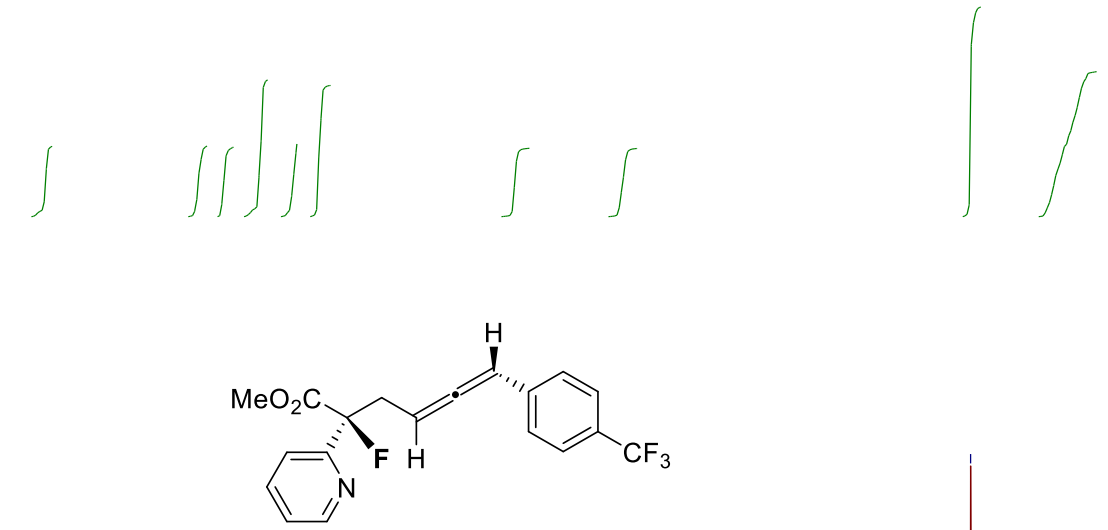

3d, ${ }^{1} \mathrm{H}$ NMR $\mathrm{CDCl}_{3}$

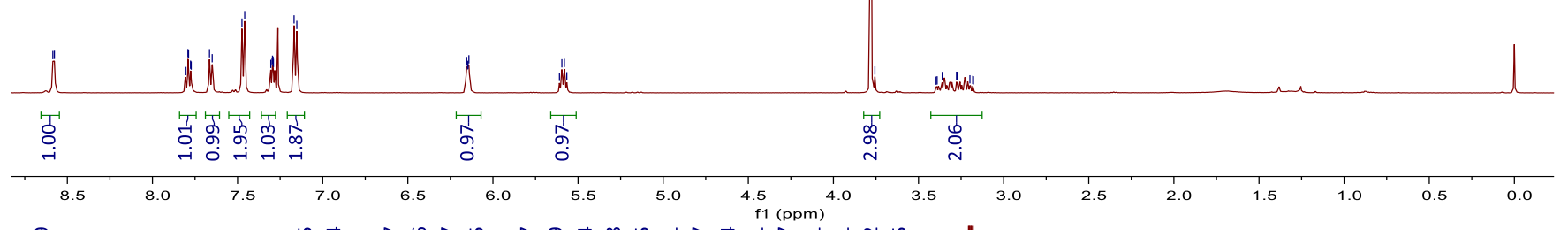

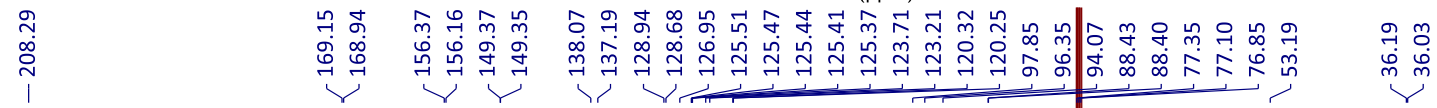

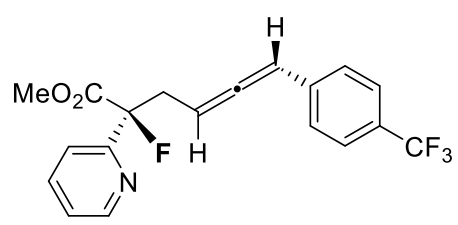

3d, ${ }^{13} \mathrm{C}$ NMR

$\mathrm{CDCl}_{3}$

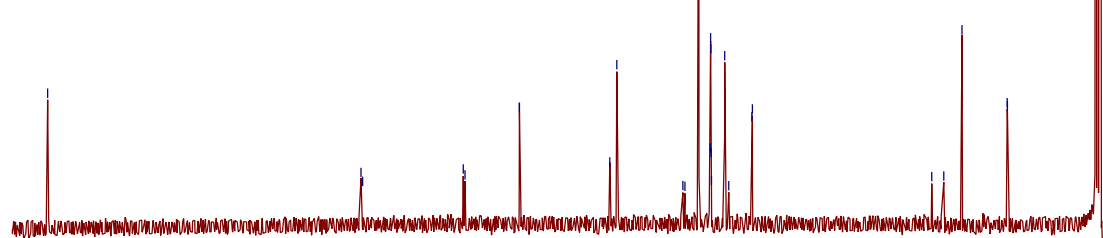

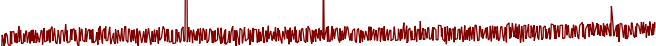

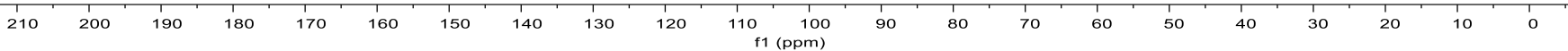




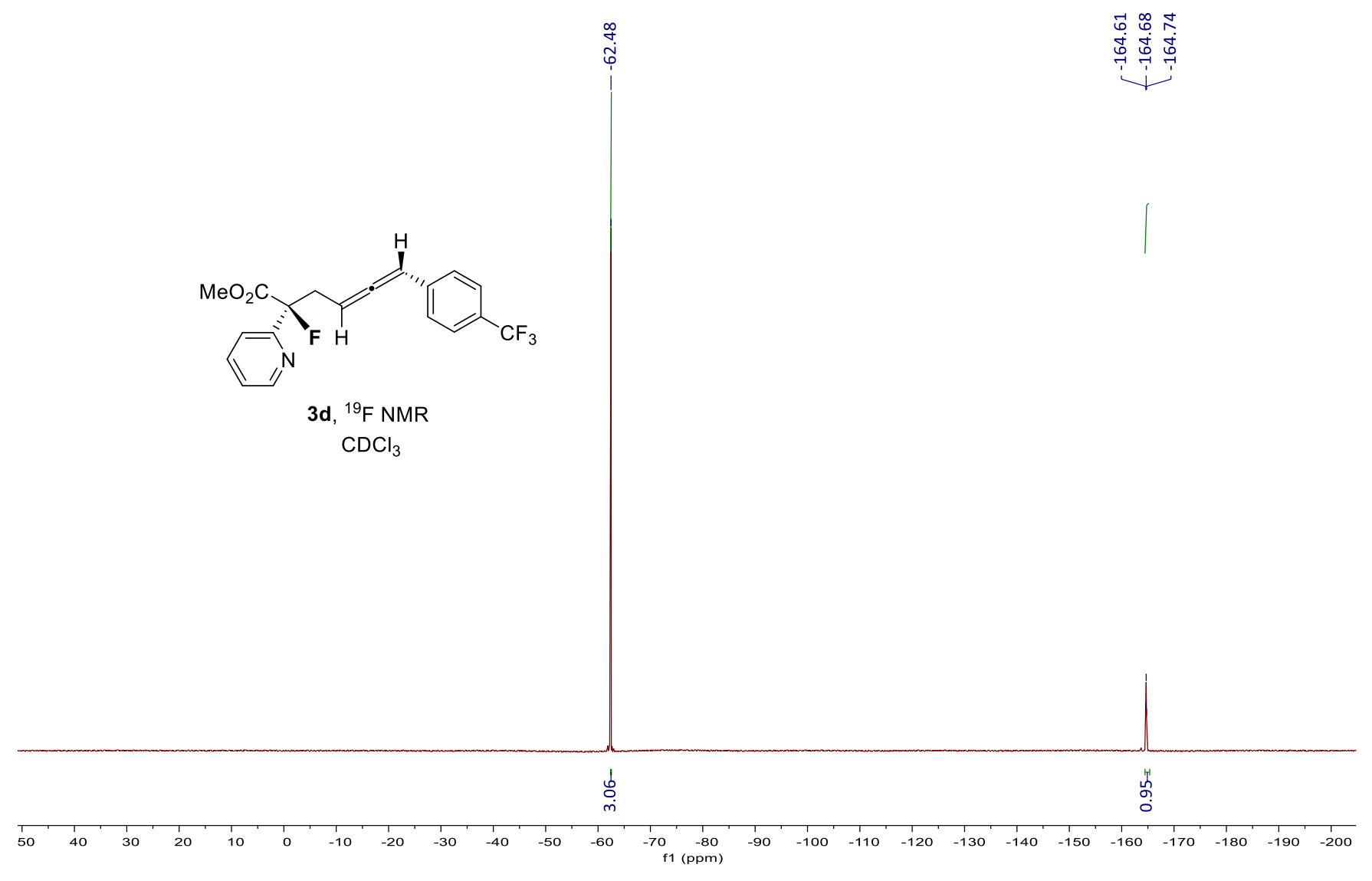



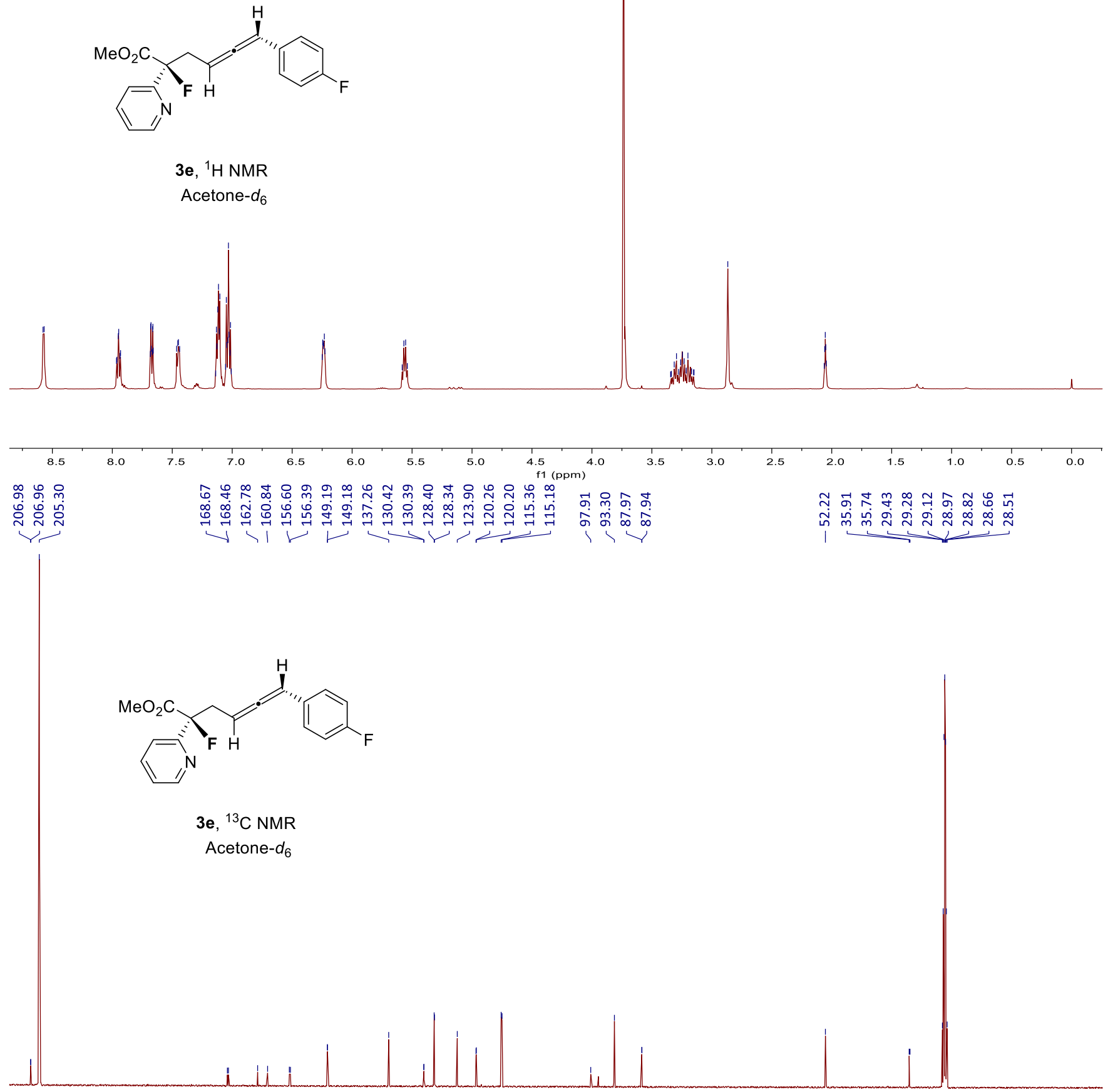

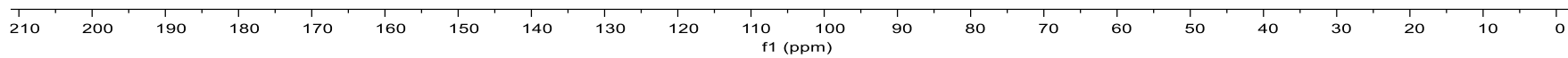



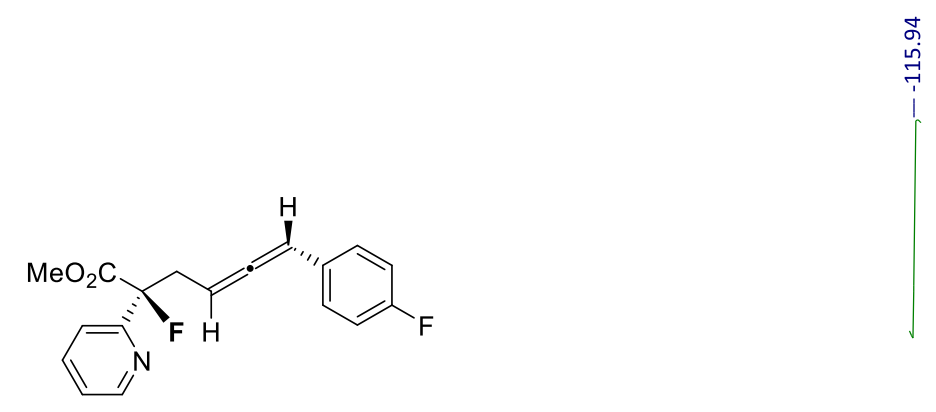

3e, ${ }^{19} \mathrm{~F}$ NMR

Acetone- $d_{6}$

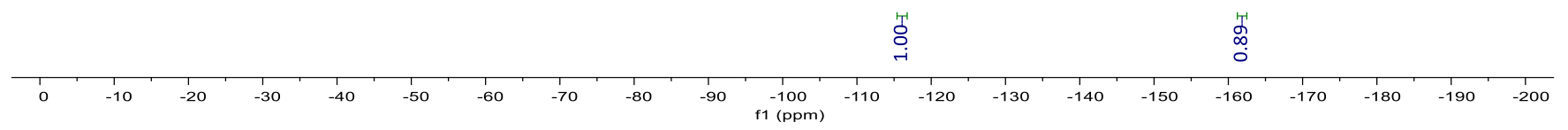



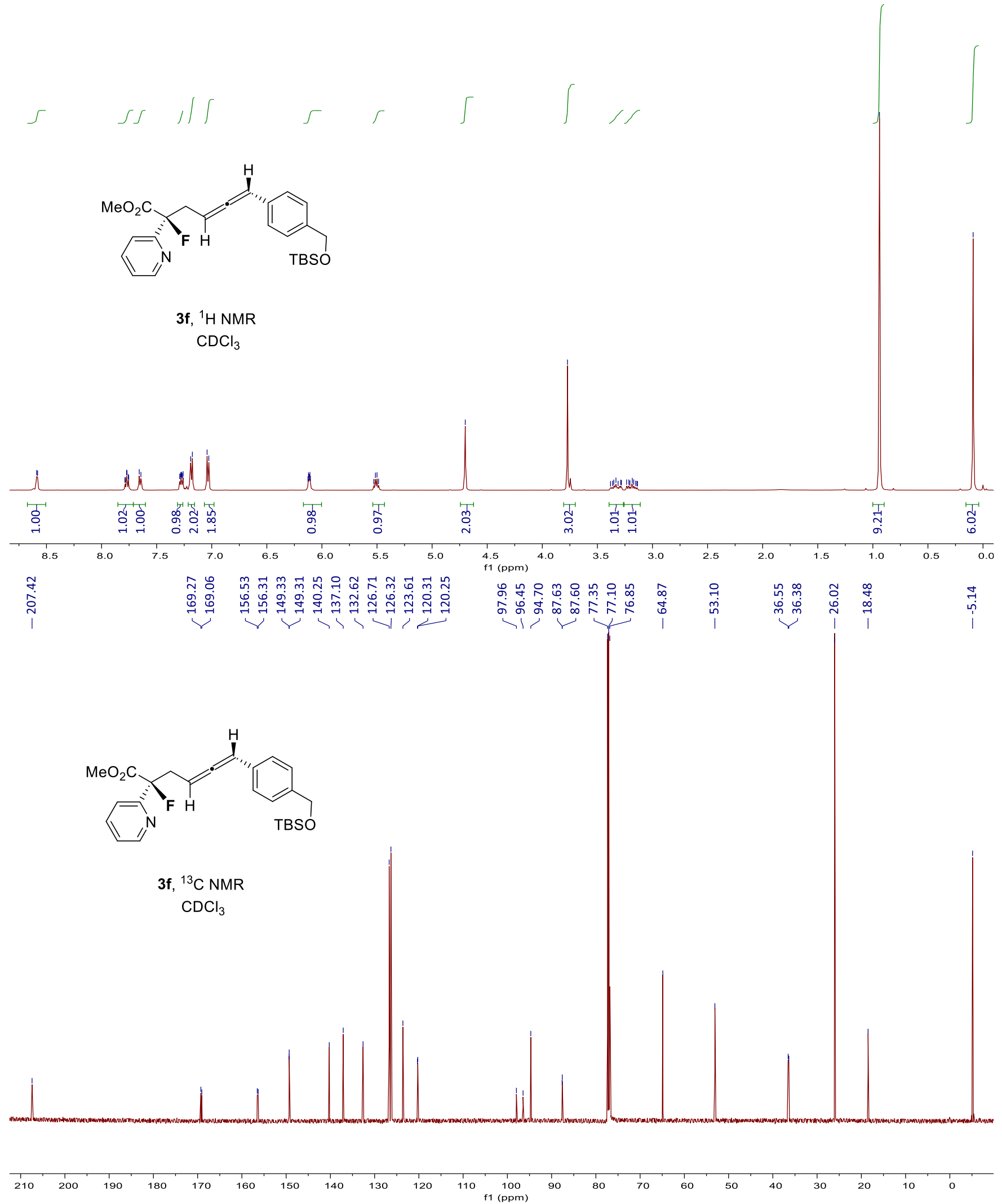
คํำ

นุ่
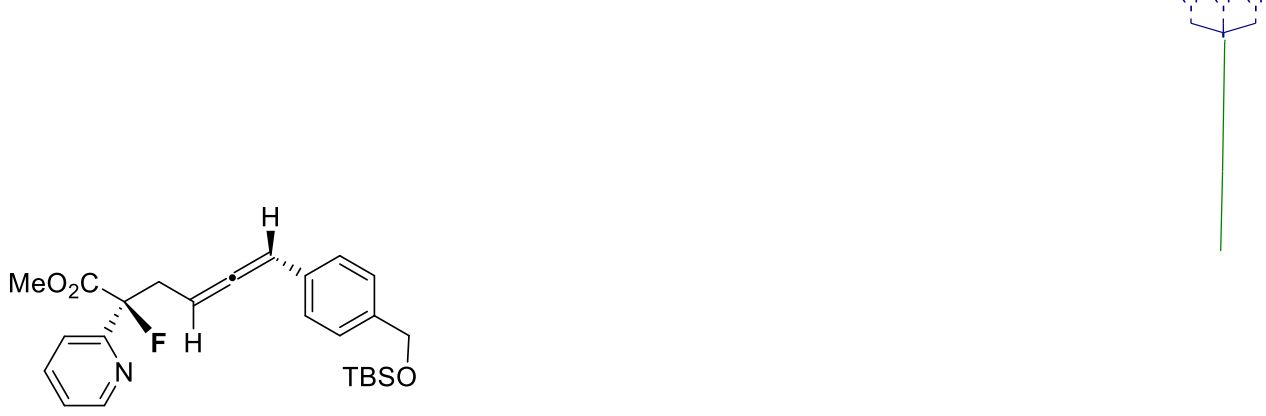

3f, ${ }^{19} \mathrm{~F}$ NMR $\mathrm{CDCl}_{3}$

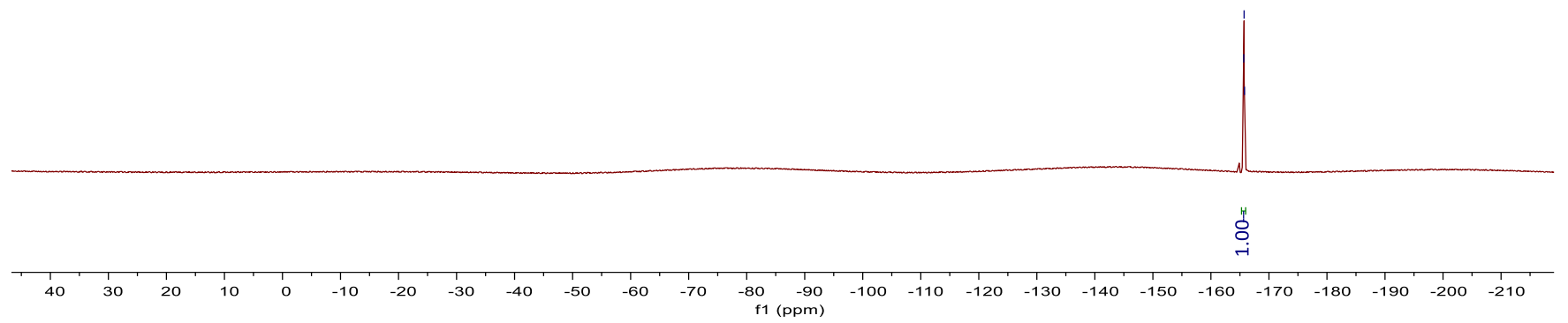



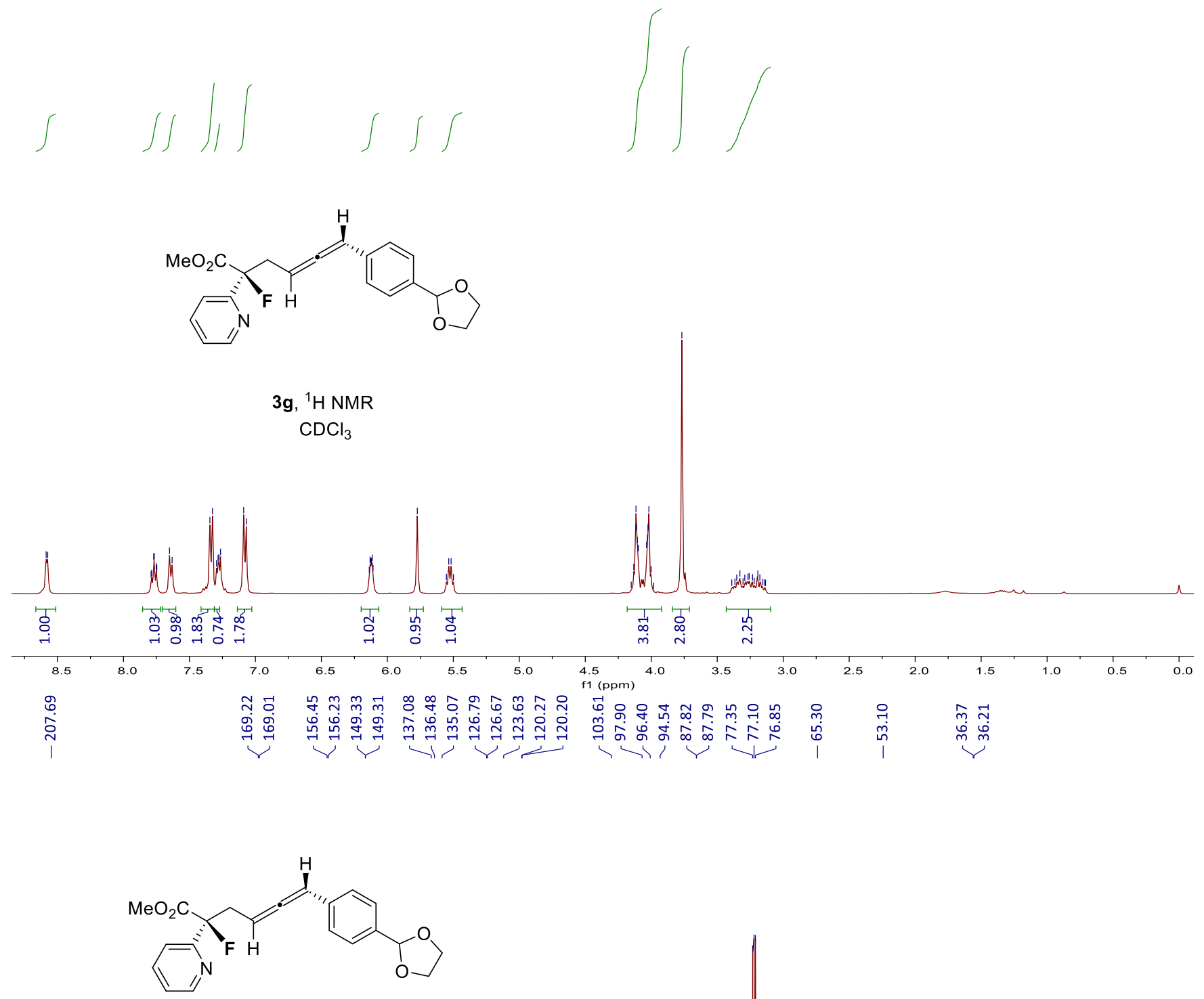

$3 \mathbf{g},{ }^{13} \mathrm{C}$ NMR $\mathrm{CDCl}_{3}$

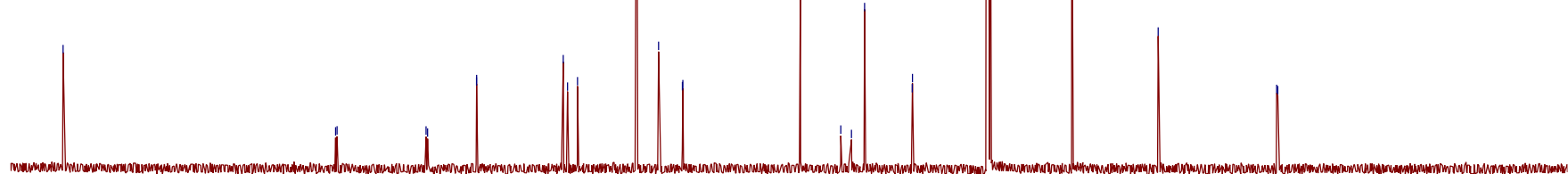

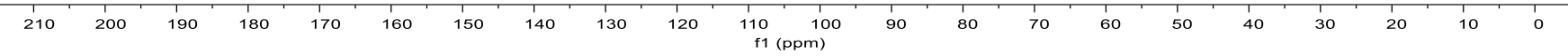


웅 용

过

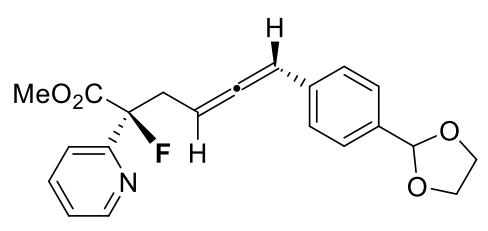

3g, ${ }^{19} \mathrm{~F}$ NMR $\mathrm{CDCl}_{3}$

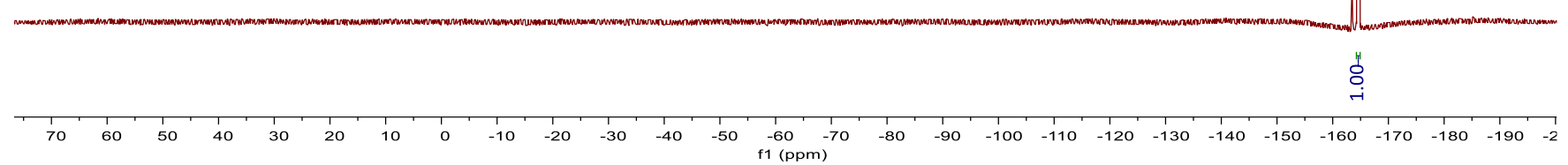




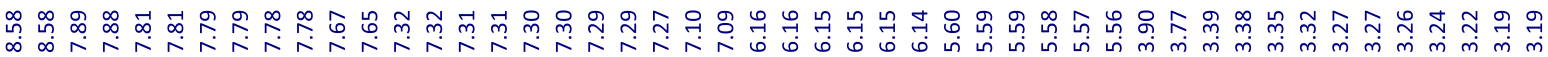
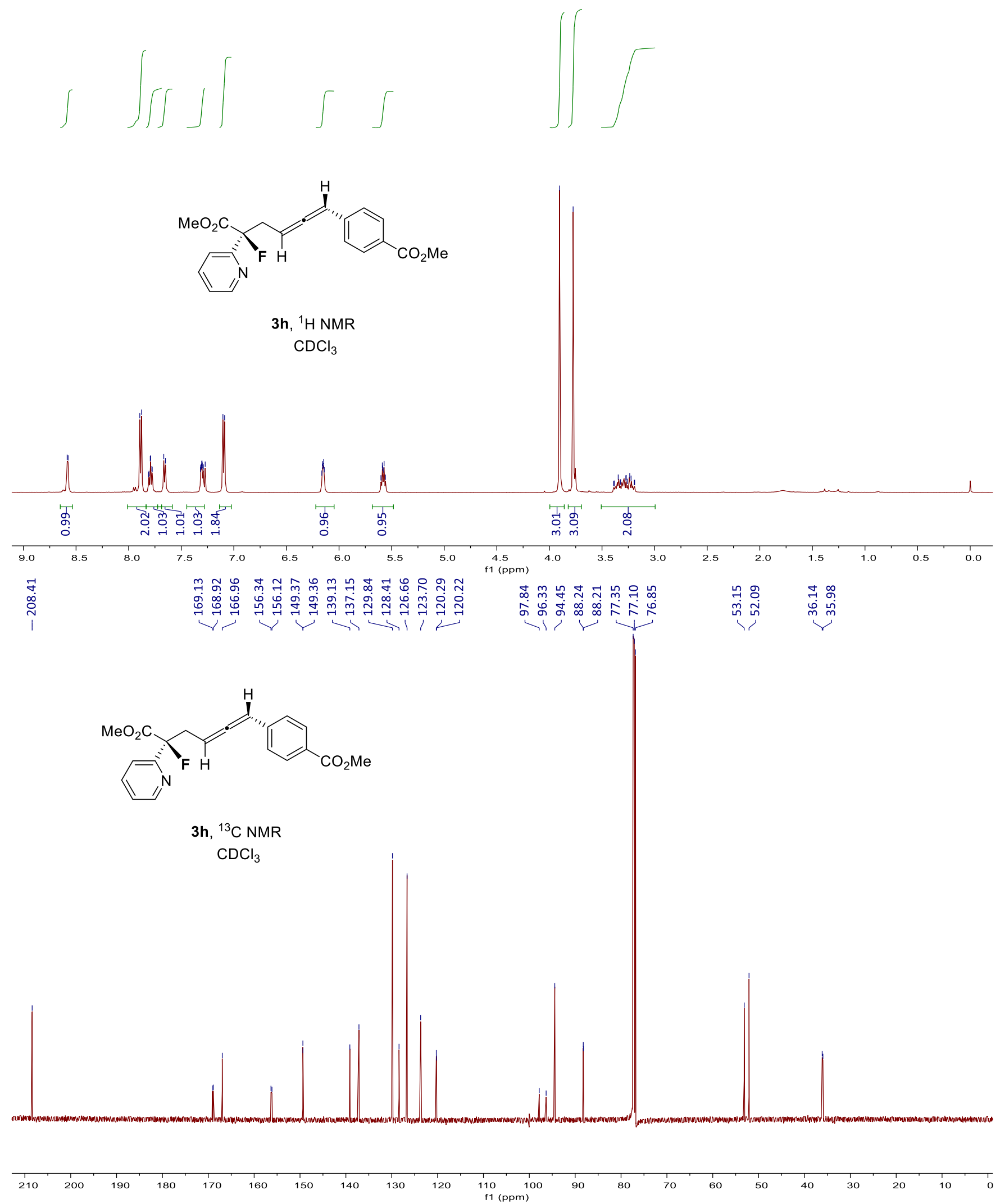


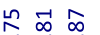

总宓宫

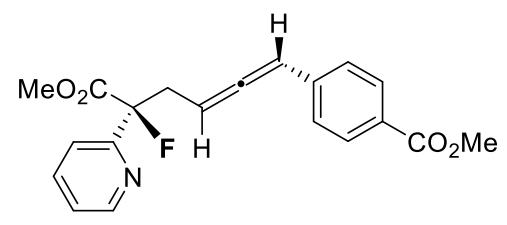

3h, ${ }^{19} \mathrm{~F}$ NMR $\mathrm{CDCl}_{3}$

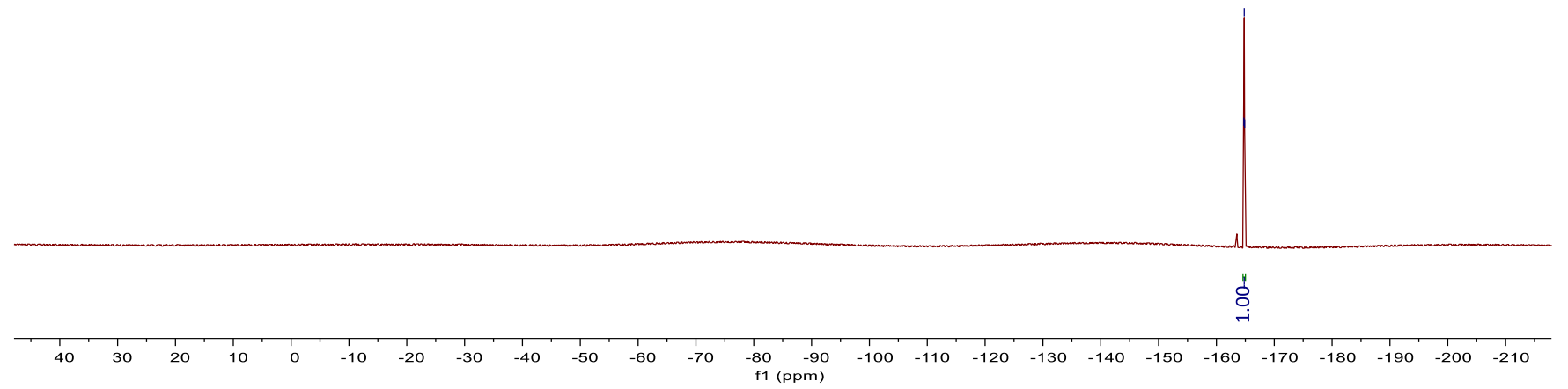



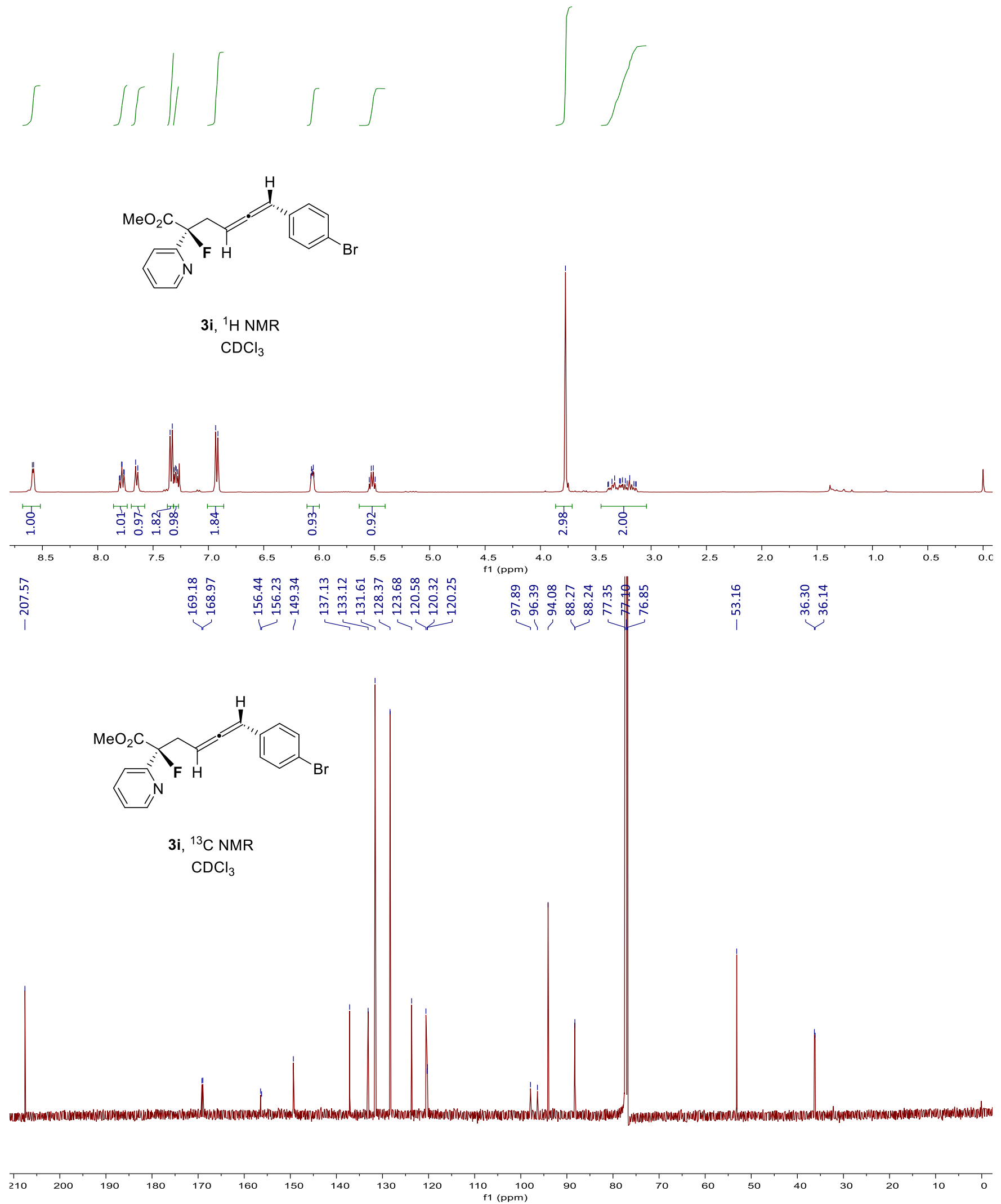
옹

它宫

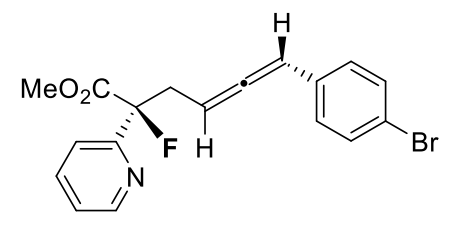

3i, ${ }^{19} \mathrm{~F}$ NMR

$\mathrm{CDCl}_{3}$

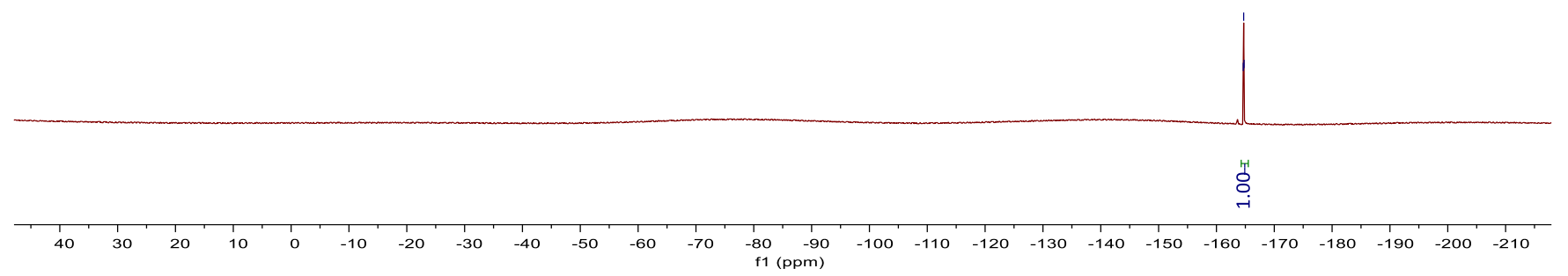




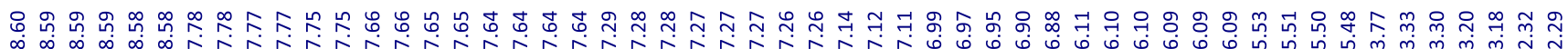
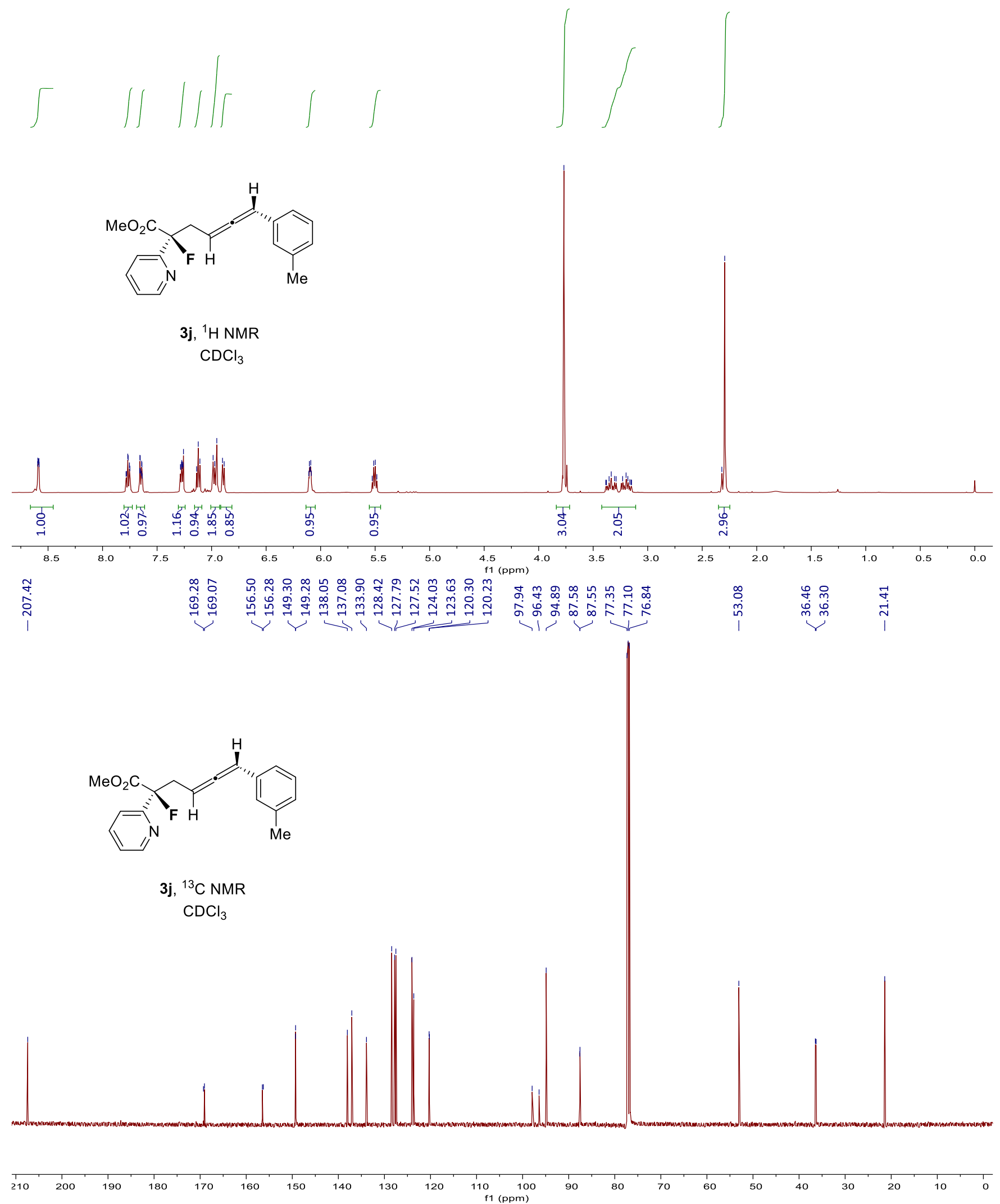

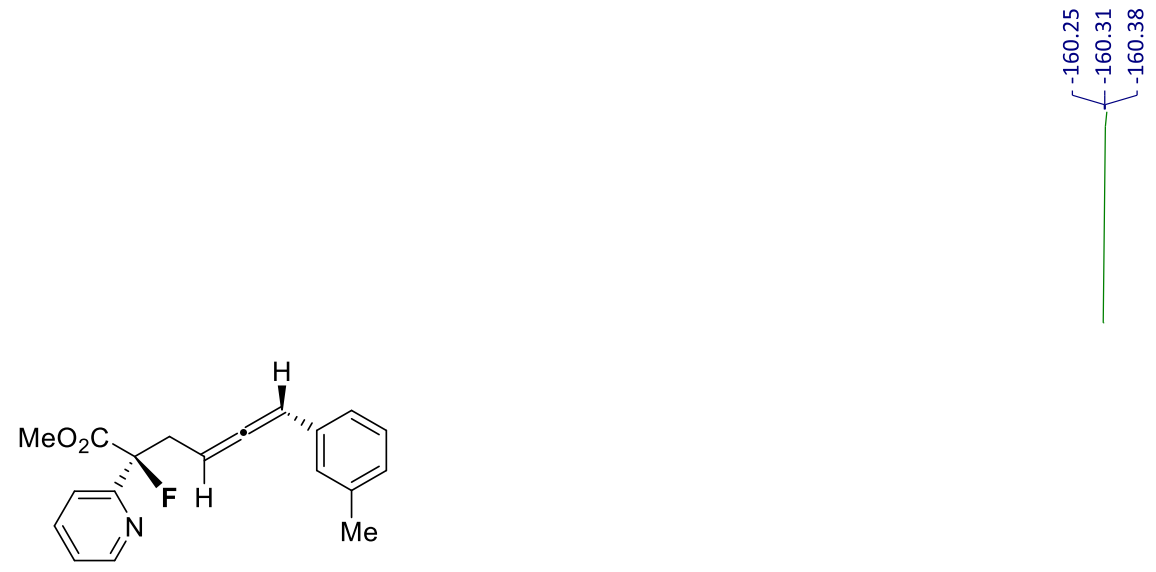

3j, ${ }^{19}$ F NMR

$\mathrm{CDCl}_{3}$

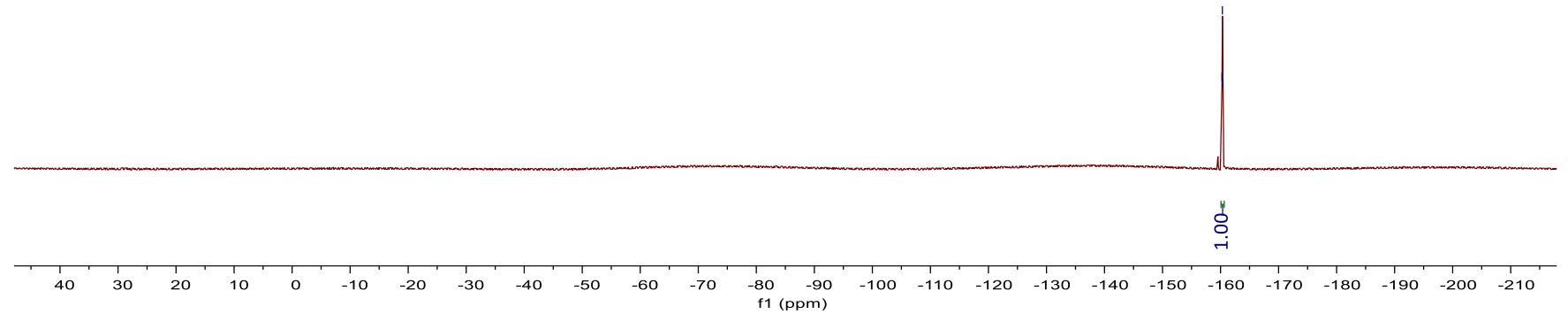



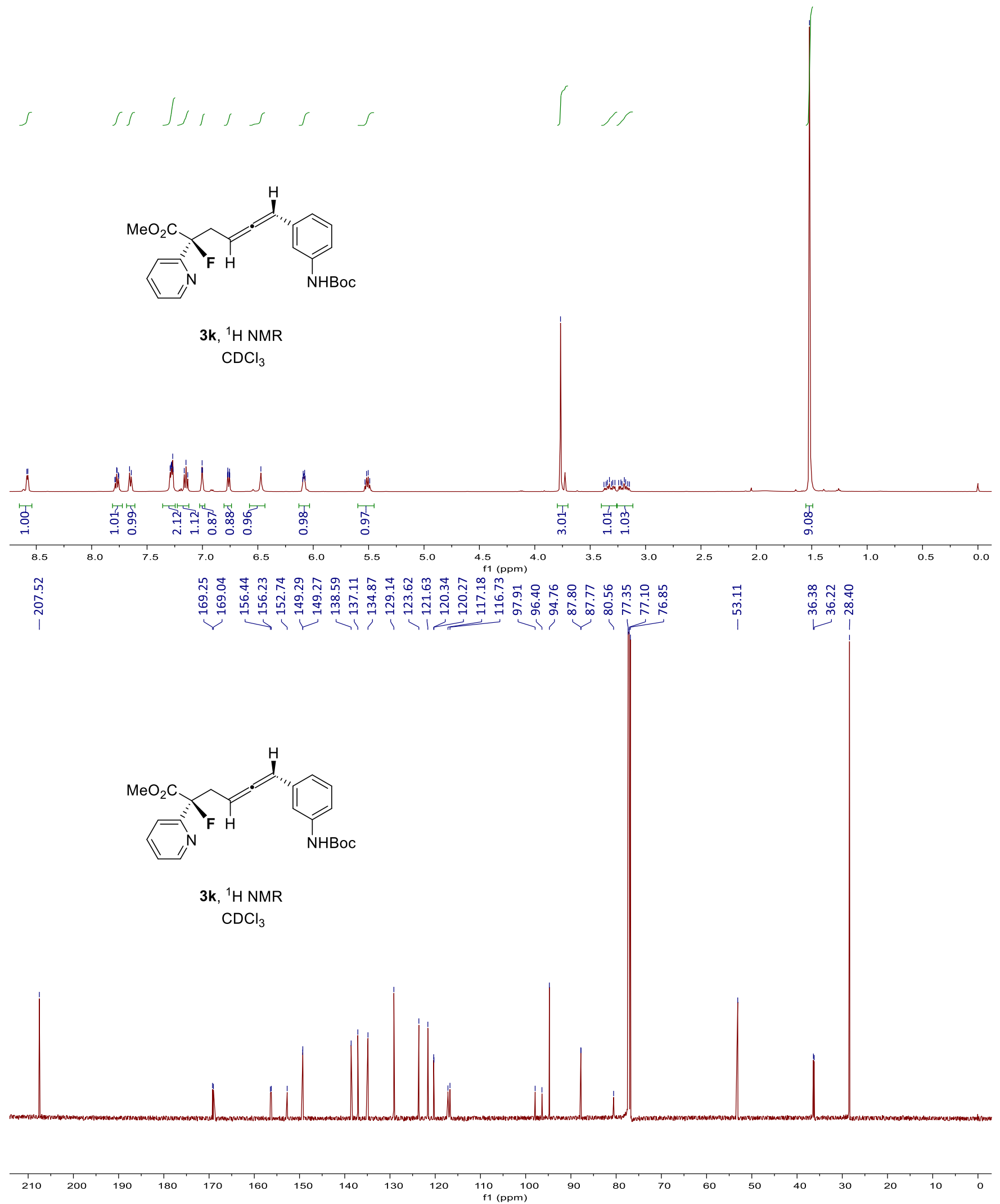


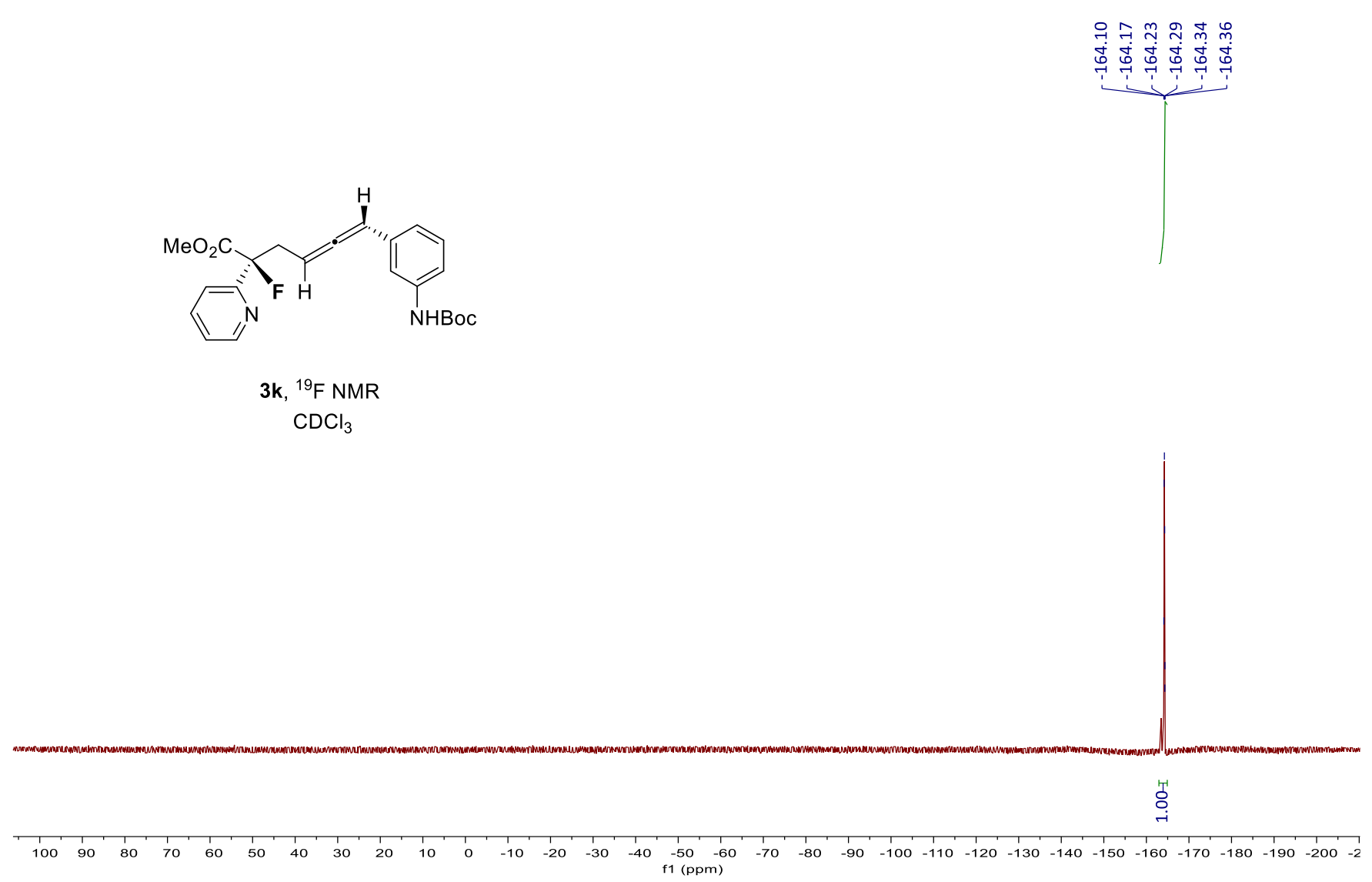




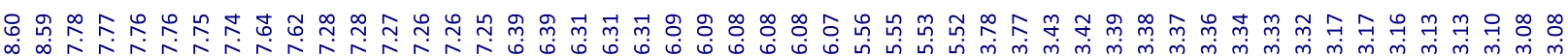

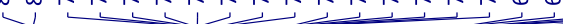
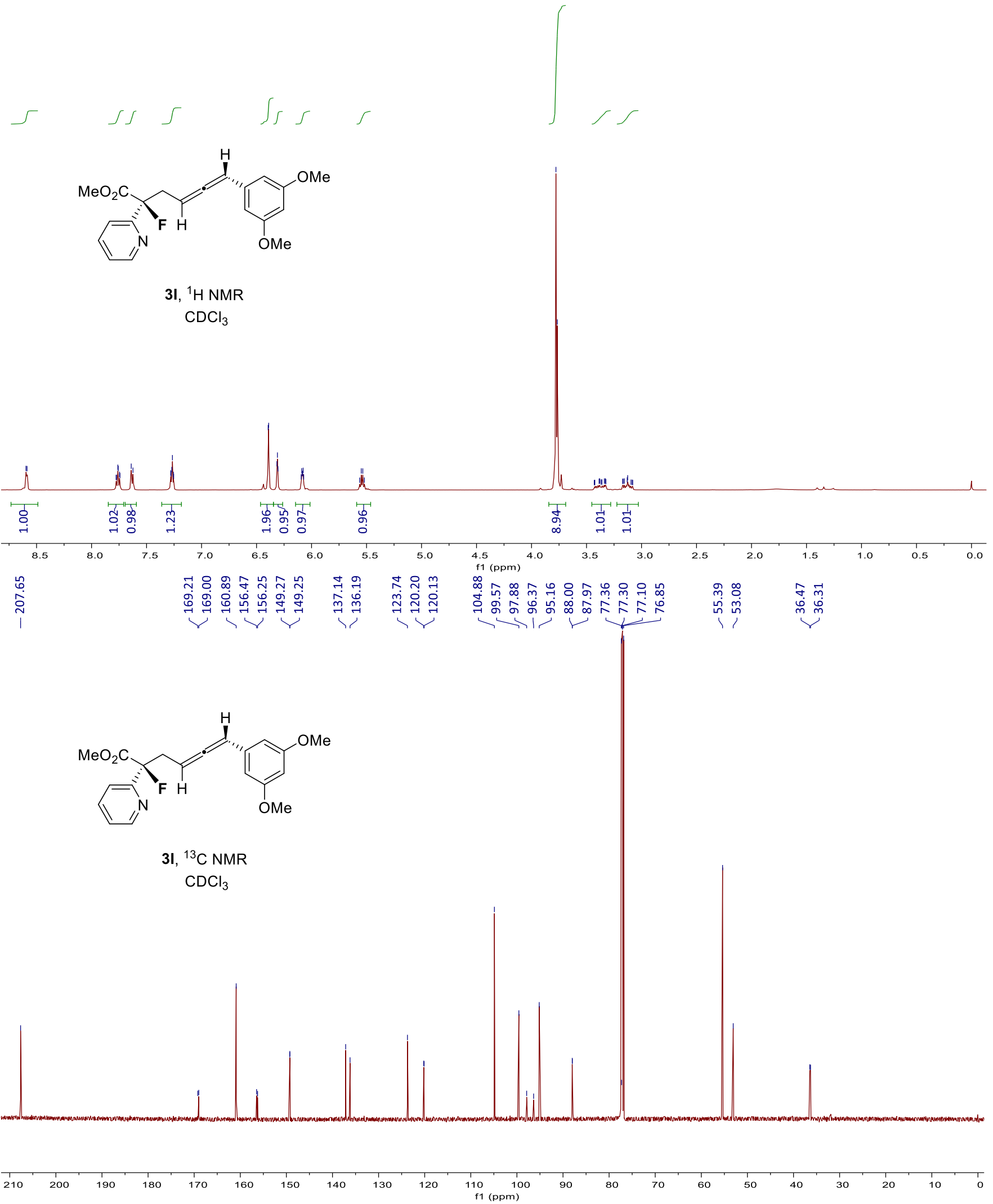
กิํํㅇํㅇㅇㅛ

过宫宫

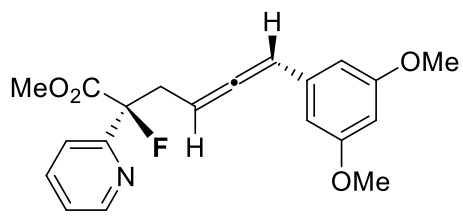

3I, ${ }^{19} \mathrm{~F}$ NMR

$\mathrm{CDCl}_{3}$

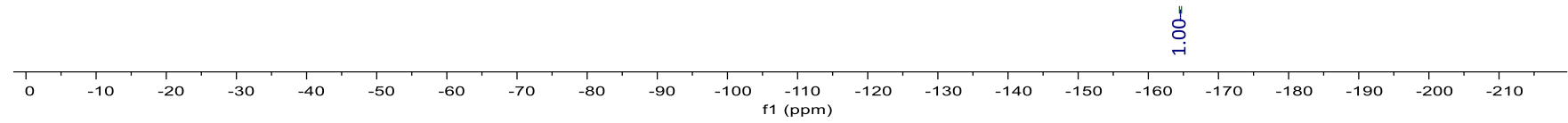




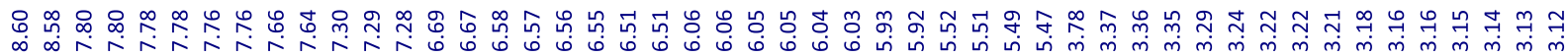

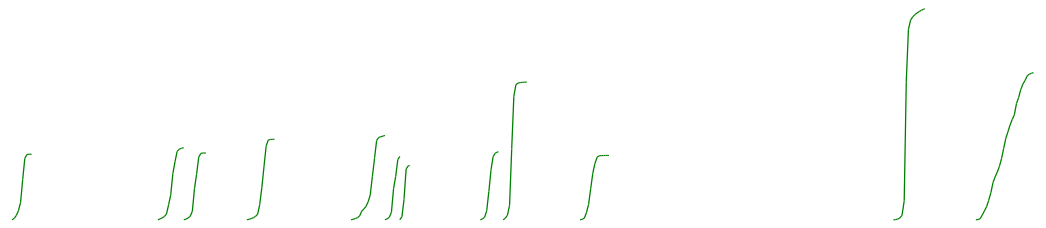<smiles>COC(=O)[C@](F)(/C=C/c1ccc2c(c1)OCO2)c1ccccn1</smiles>

$3 \mathrm{~m},{ }^{1} \mathrm{H}$ NMR

$\mathrm{CDCl}_{3}$

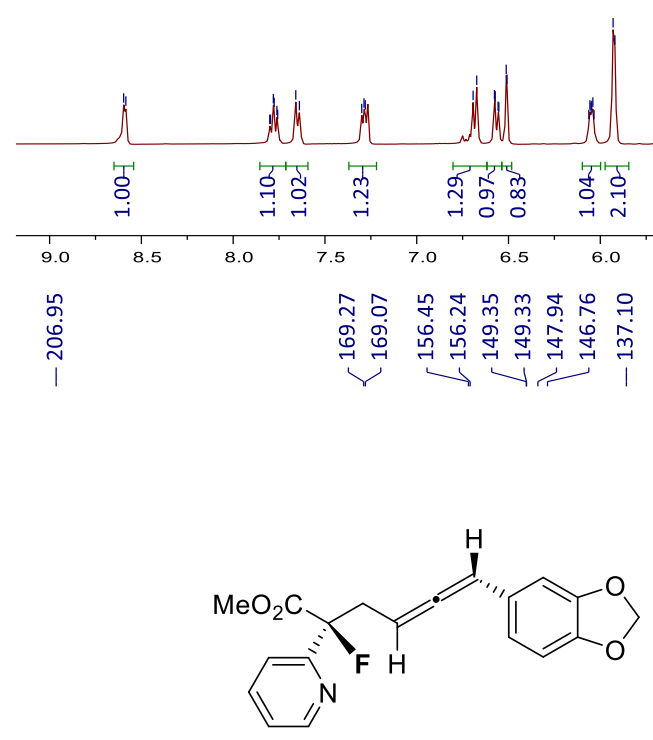

$3 \mathrm{~m},{ }^{13} \mathrm{C}$ NMR $\mathrm{CDCl}_{3}$
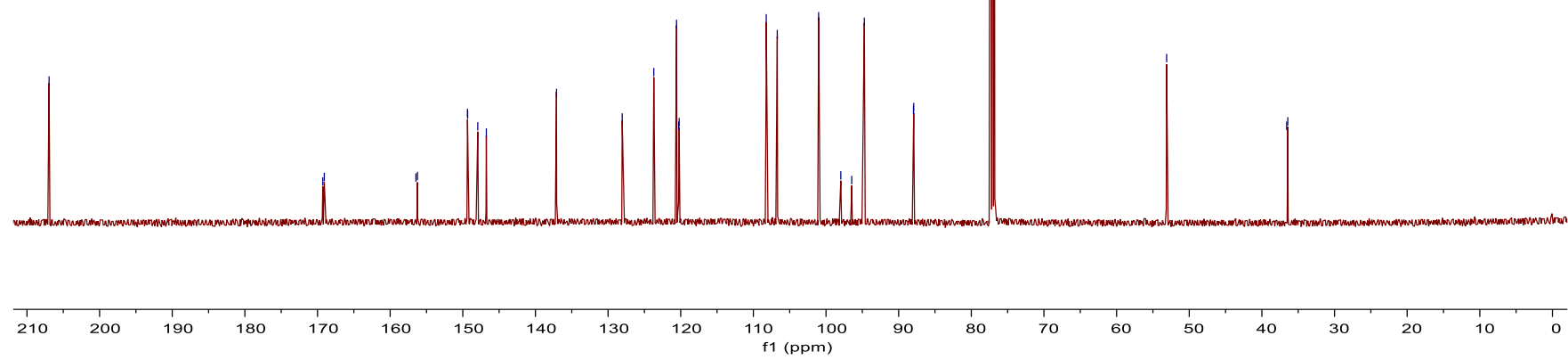
요유

它灾志

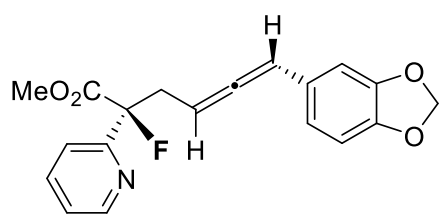

$3 \mathrm{~m},{ }^{19} \mathrm{~F} \mathrm{NMR}$

$\mathrm{CDCl}_{3}$

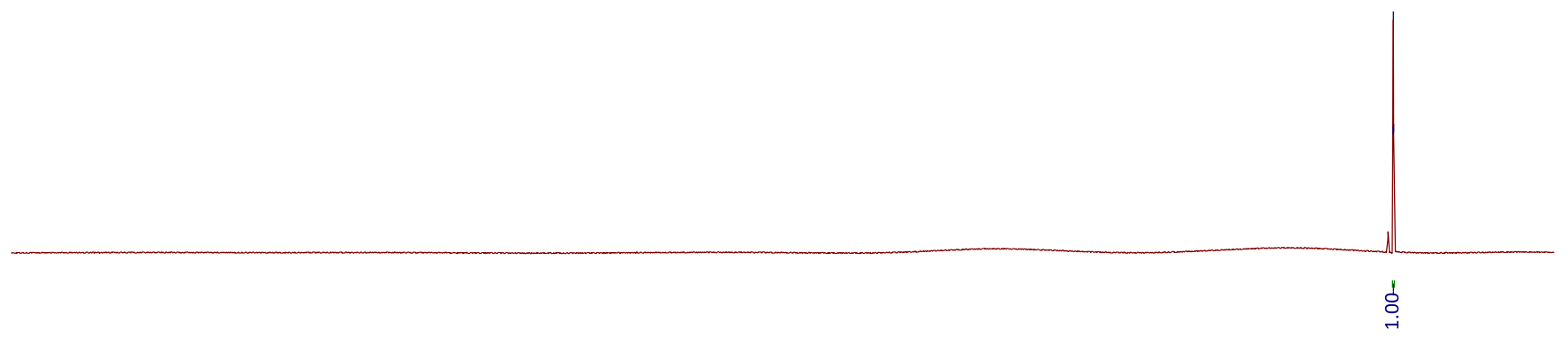

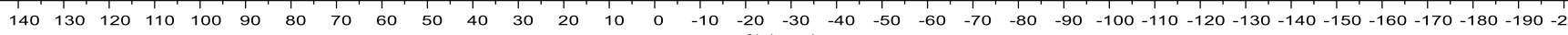
f1 (ppm) 


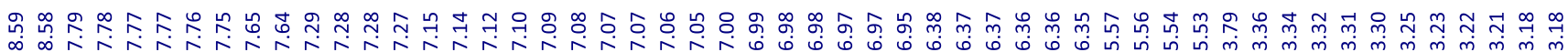

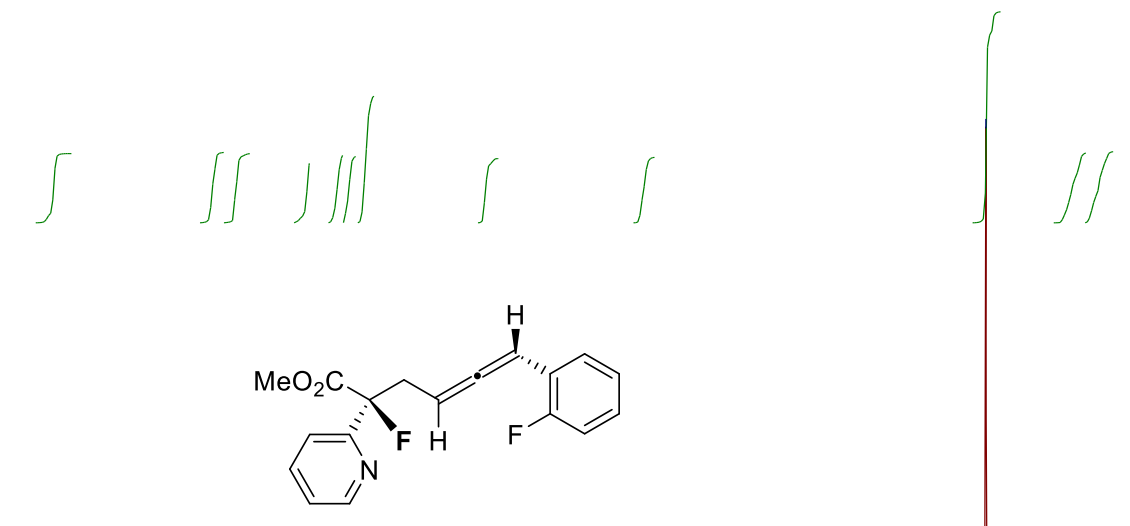

3n, ${ }^{1} \mathrm{H}$ NMR

$\mathrm{CDCl}_{3}$

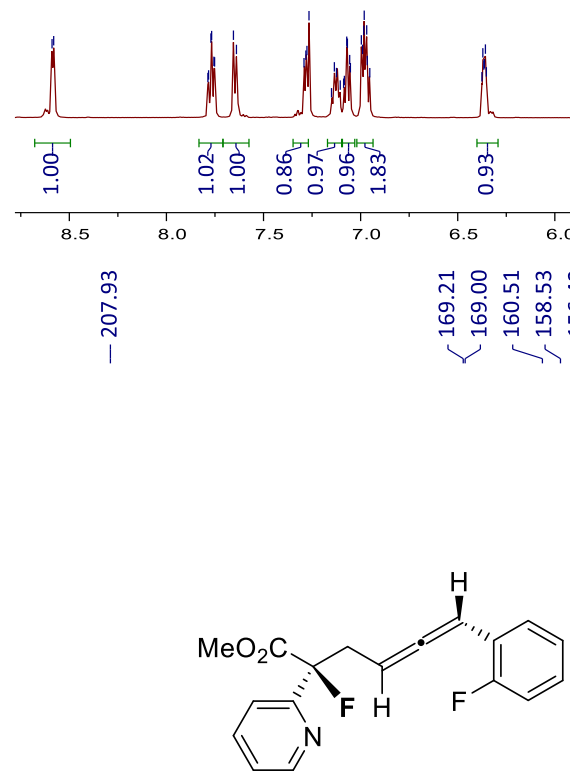

3n, ${ }^{1} \mathrm{H}$ NMR $\mathrm{CDCl}_{3}$

蝶数 密势

55
f1 (ppm)

유 శ్

우 유 우 $\approx$

3.5
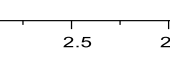

100
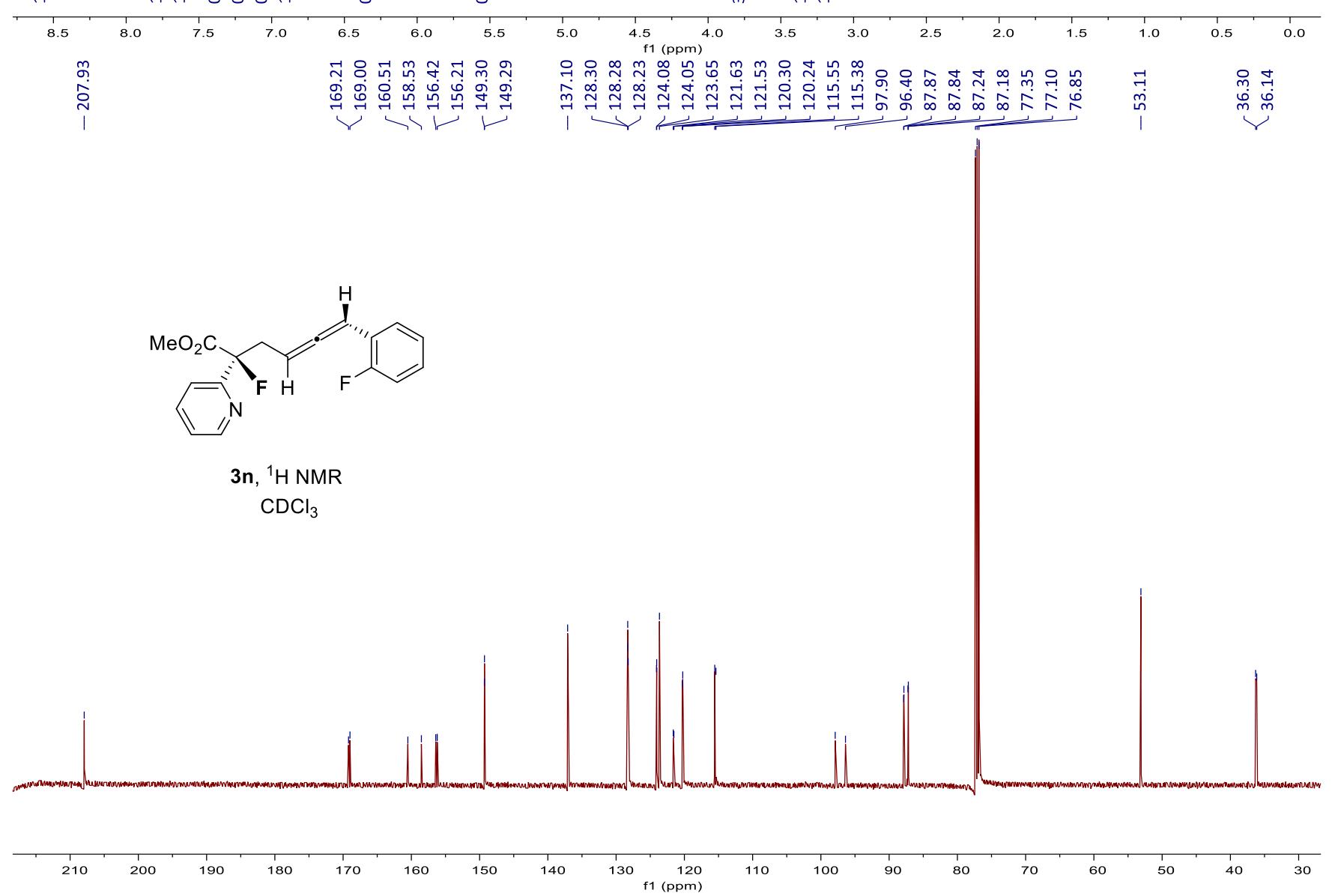


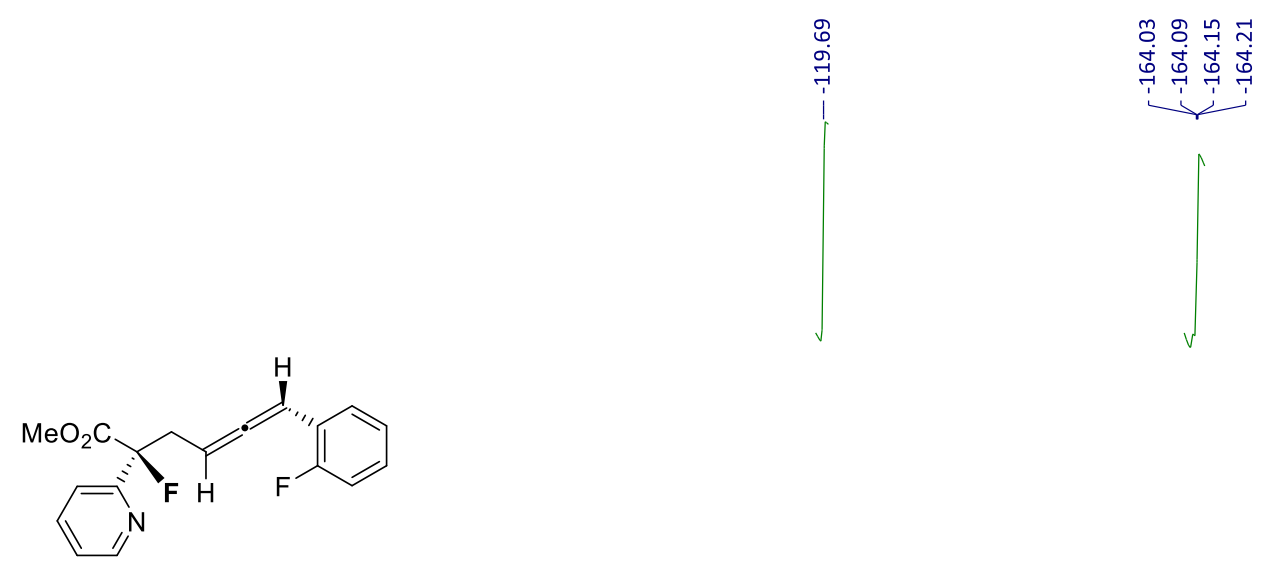

3n, ${ }^{1} \mathrm{H}$ NMR

$\mathrm{CDCl}_{3}$

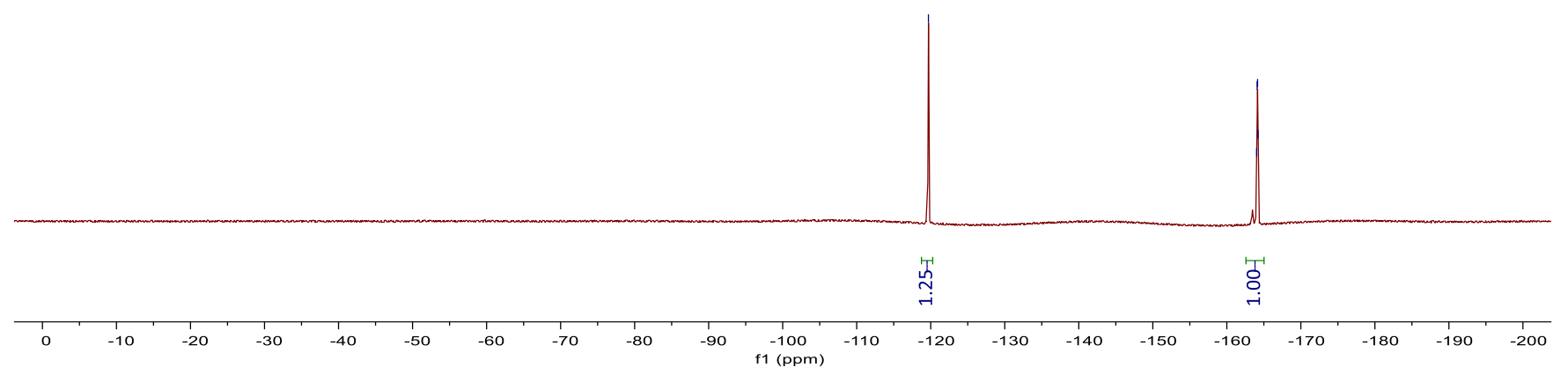




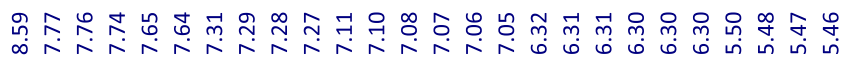

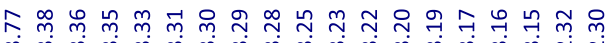

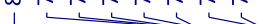

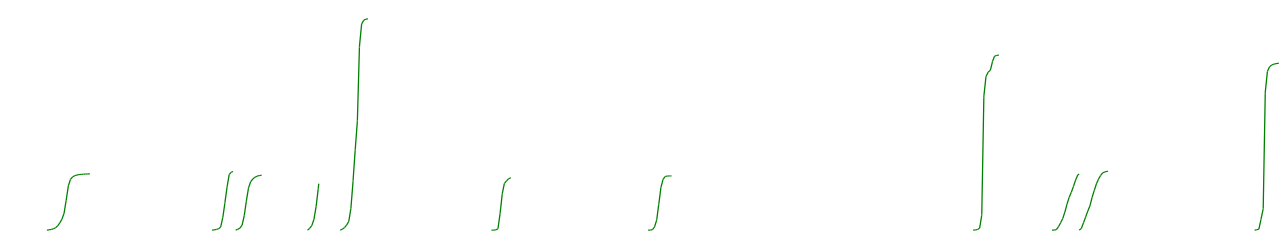<smiles>COC(=O)[C@](F)(/C=C/c1ccccc1C)c1ccccn1</smiles>

3o, ${ }^{1} \mathrm{H}$ NMR $\mathrm{CDCl}_{3}$

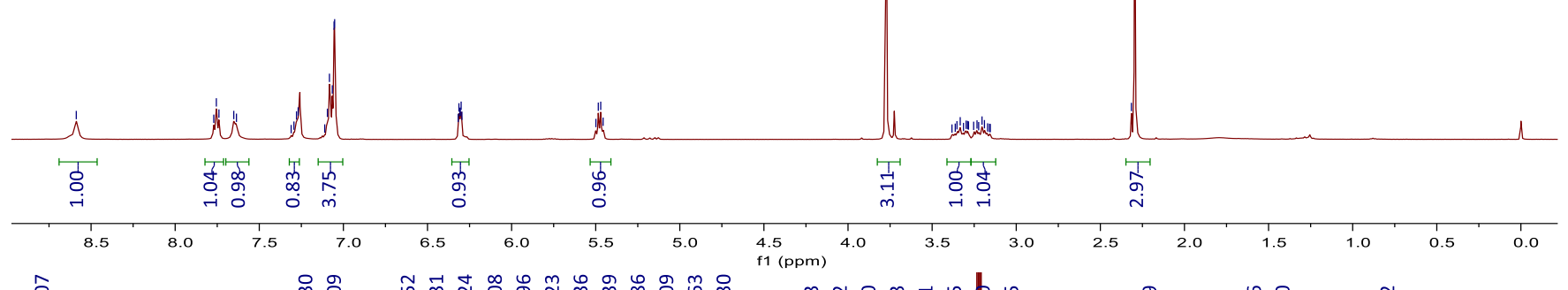
ion

3o, ${ }^{13} \mathrm{C}$ NMR $\mathrm{CDCl}_{3}$

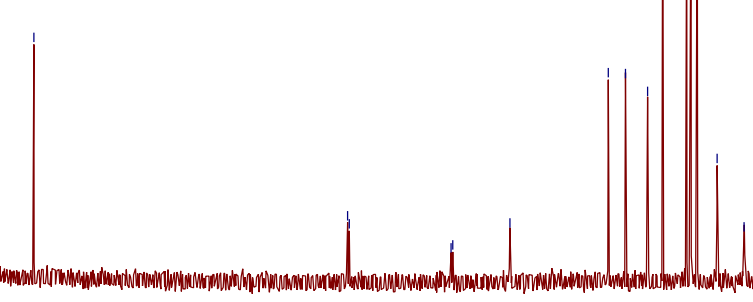

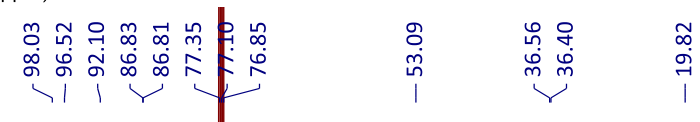

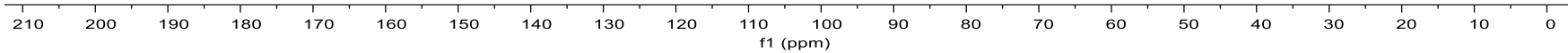


ำ ร จ

夏它
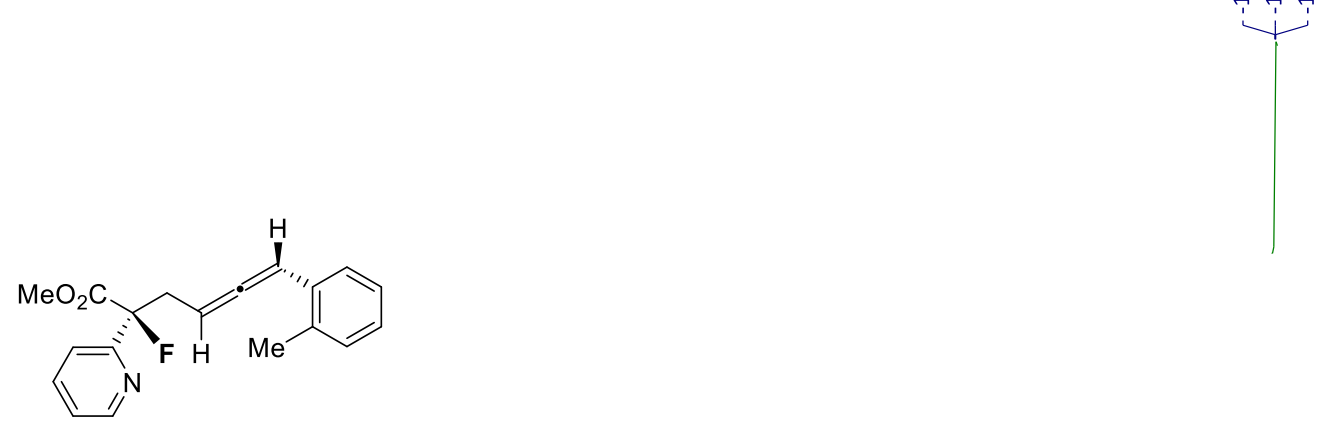

3o, ${ }^{19} \mathrm{~F}$ NMR $\mathrm{CDCl}_{3}$

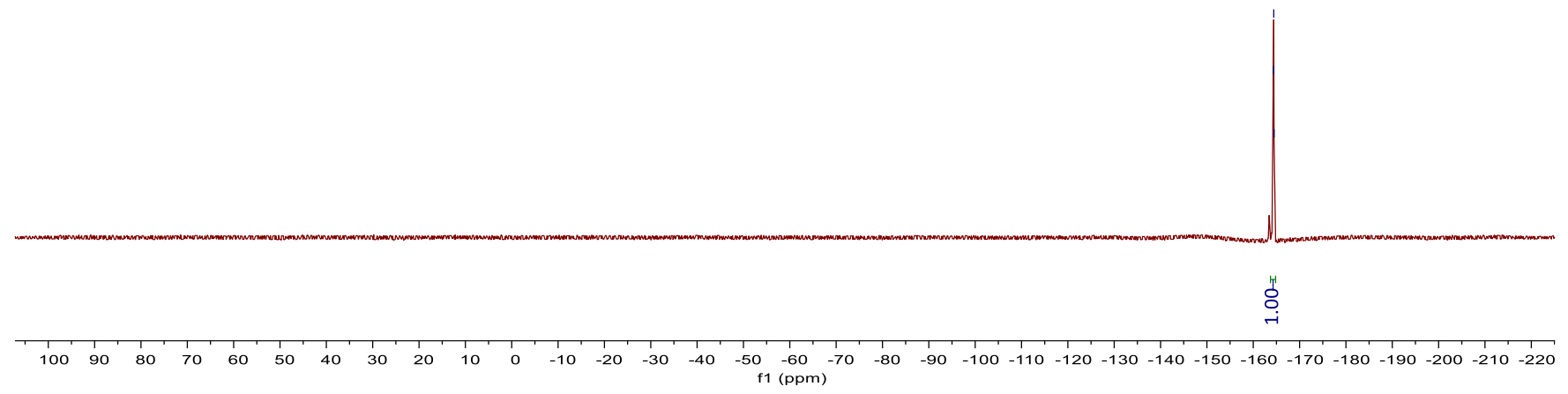


苜
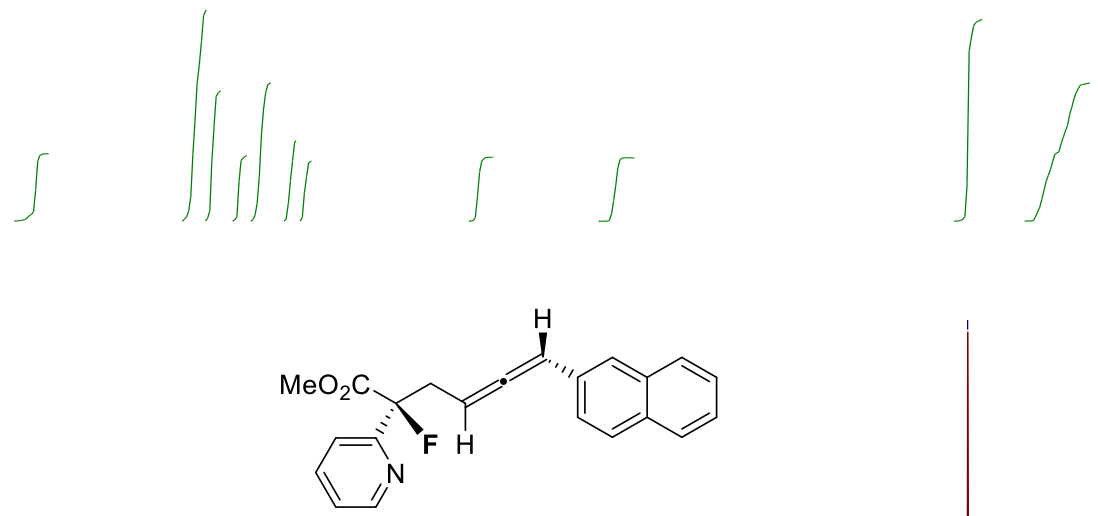

3p, ${ }^{1} \mathrm{H}$ NMR

$\mathrm{CDCl}_{3}$

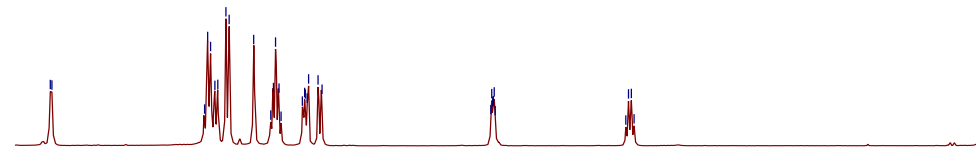

whingh

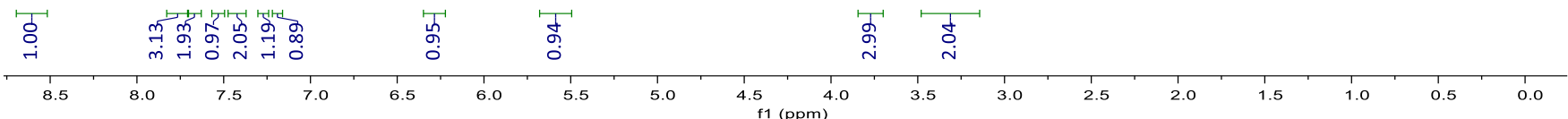

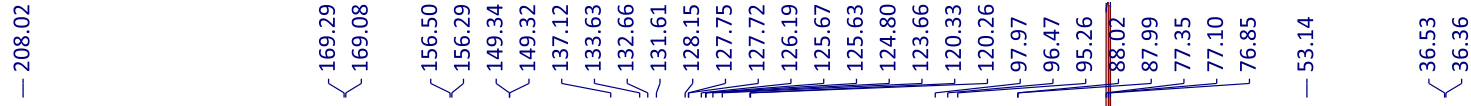<smiles>CC(=O)[C@](F)(/C=C/C=C/c1ccc2ccccc2c1)c1ccccn1</smiles>

$3 p,{ }^{13} \mathrm{C} N M R$

$\mathrm{CDCl}_{3}$

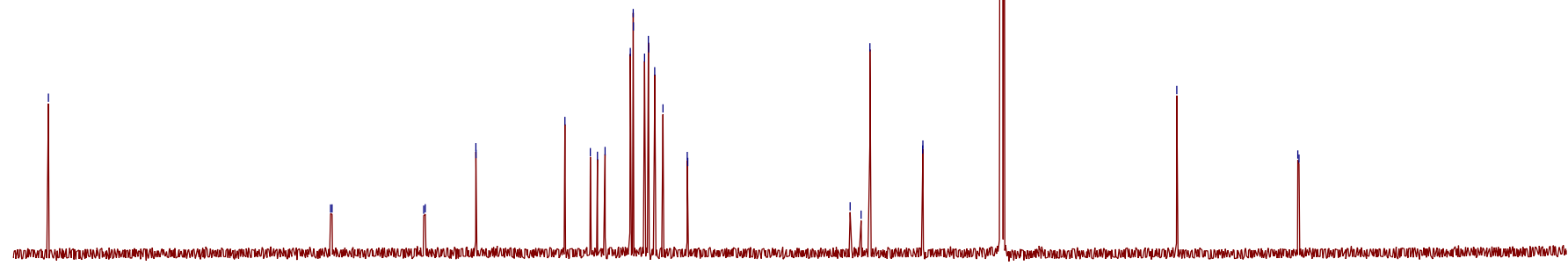

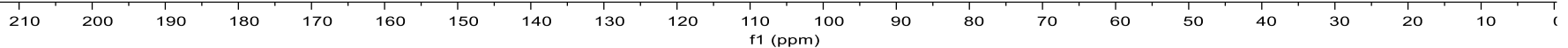





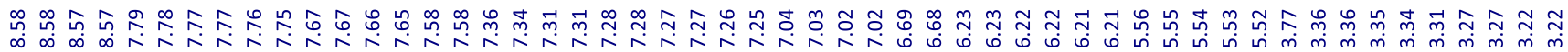
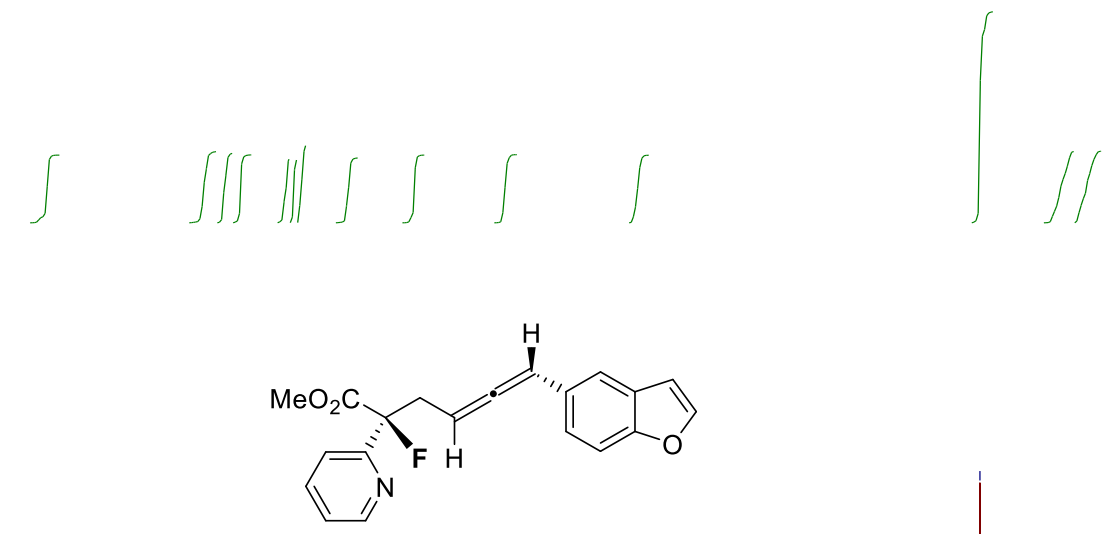

3q, ${ }^{1} \mathrm{H}$ NMR

$\mathrm{CDCl}_{3}$

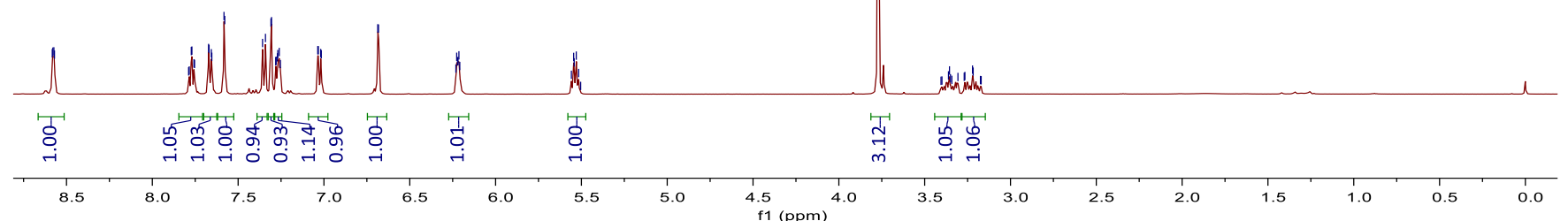

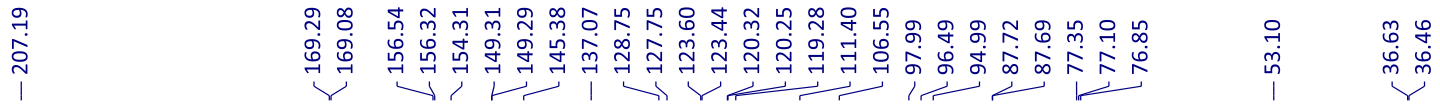

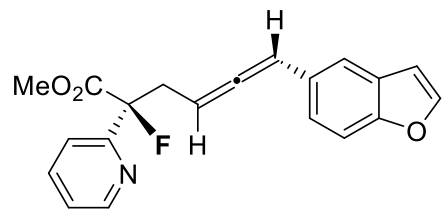

3q, ${ }^{13} \mathrm{C}$ NMR

$\mathrm{CDCl}_{3}$
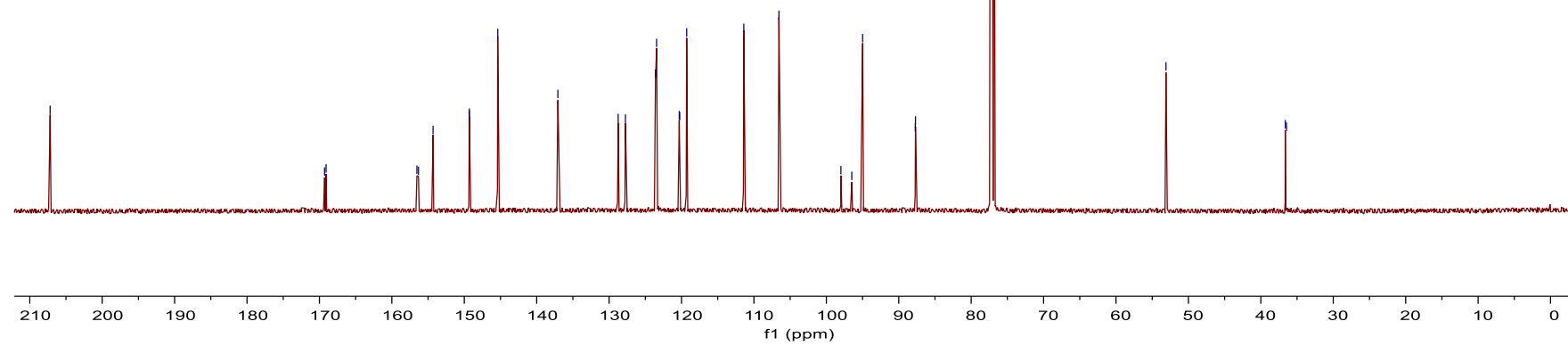
可败

它志

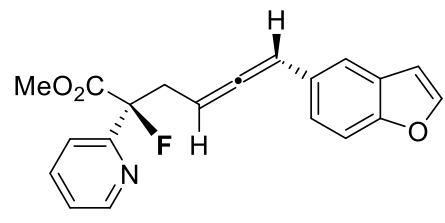

3q, ${ }^{19} \mathrm{~F} \mathrm{NMR}$

$\mathrm{CDCl}_{3}$

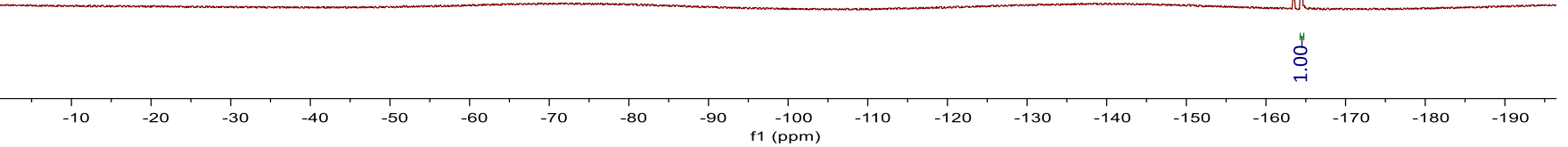




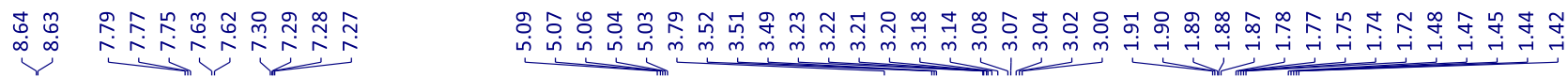

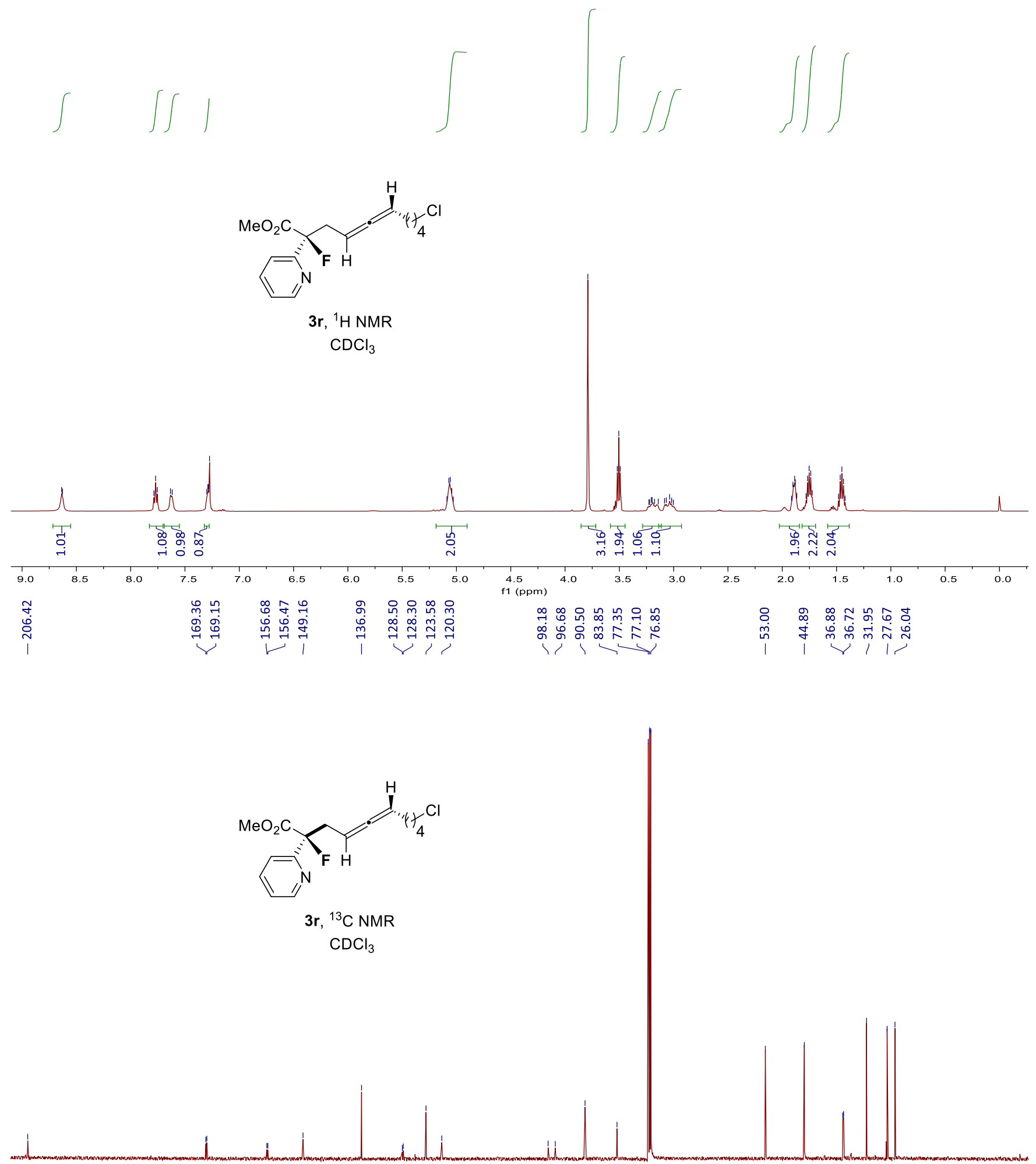

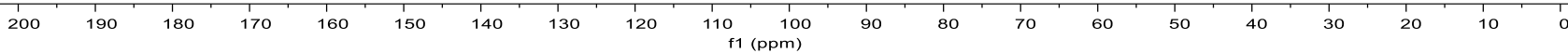


$\exists \stackrel{\circ}{\circ}$ 足

志志

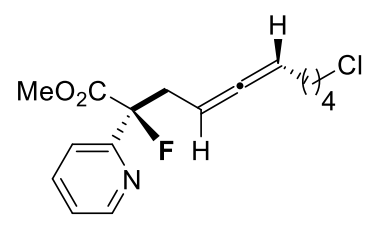

3r, ${ }^{19} \mathrm{~F}$ NMR

$\mathrm{CDCl}_{3}$

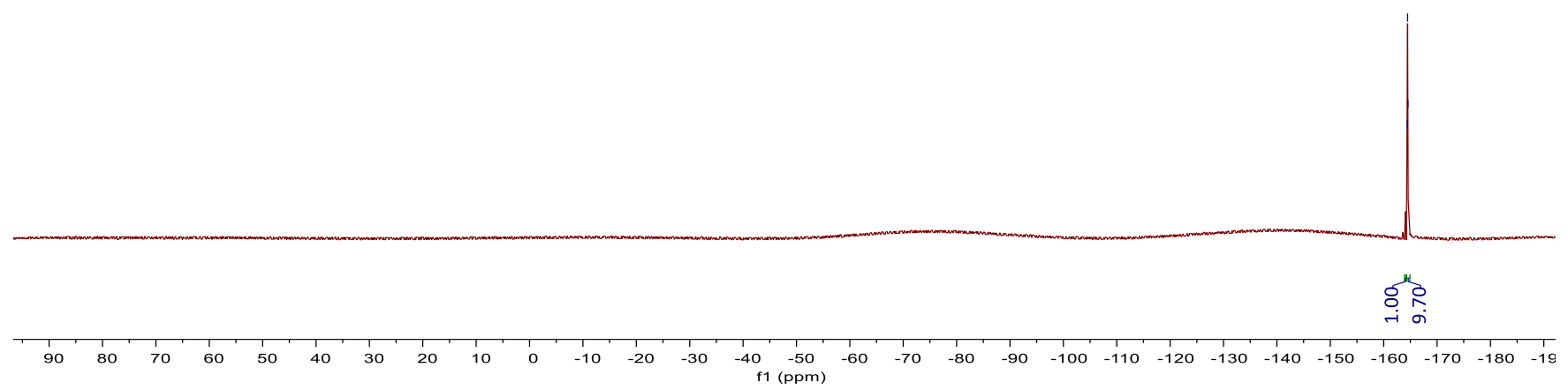




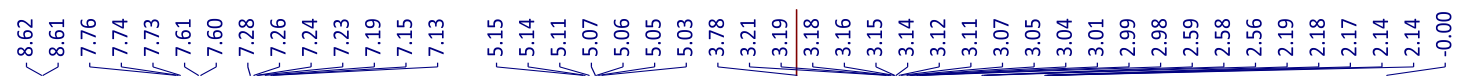

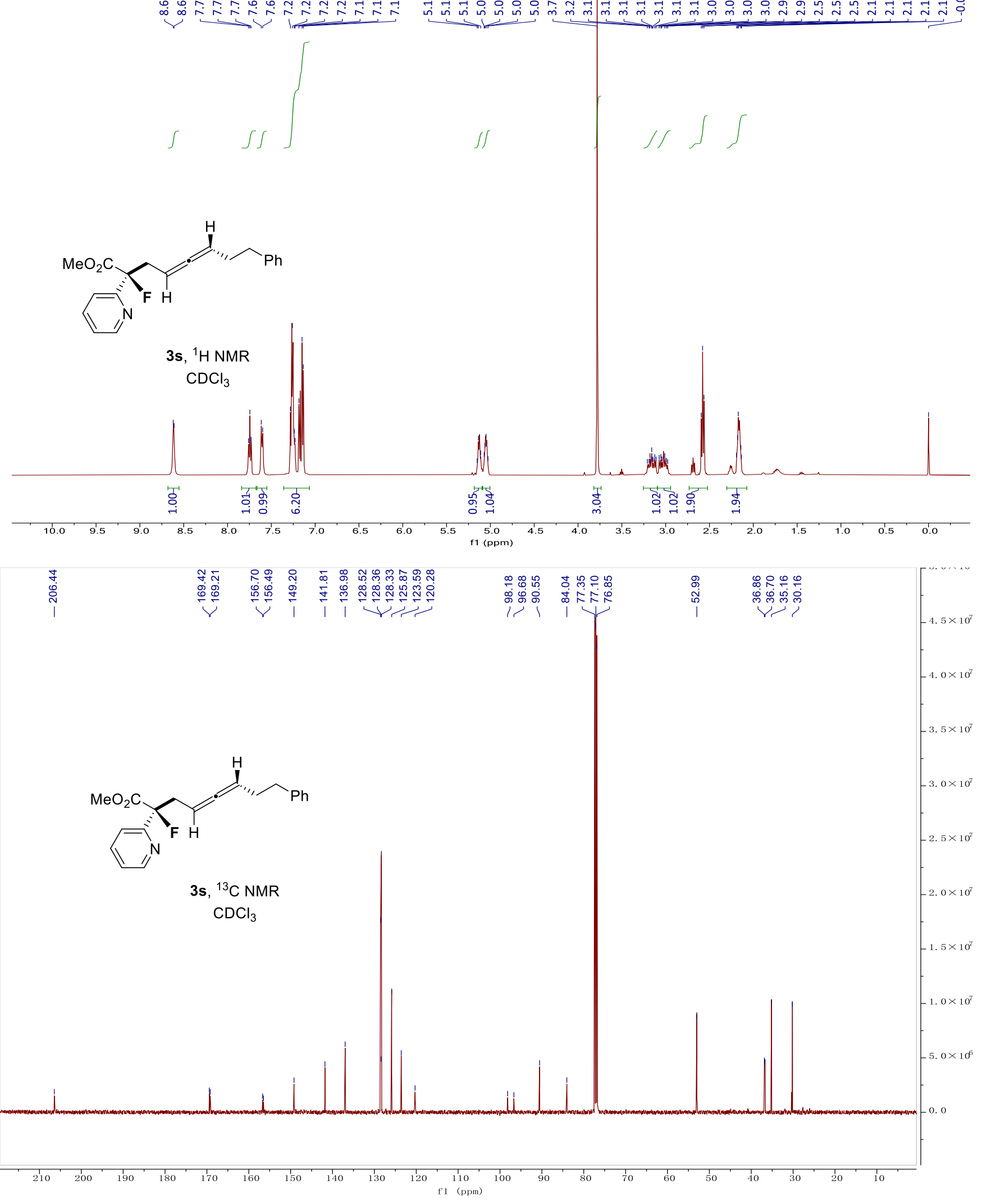


\%ㅇํㅇ

总

17

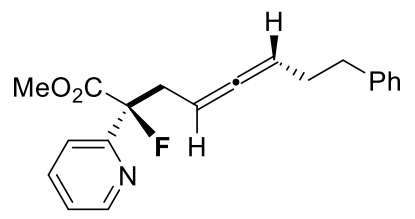

3s, ${ }^{19} \mathrm{~F}$ NMR

$\mathrm{CDCl}_{3}$

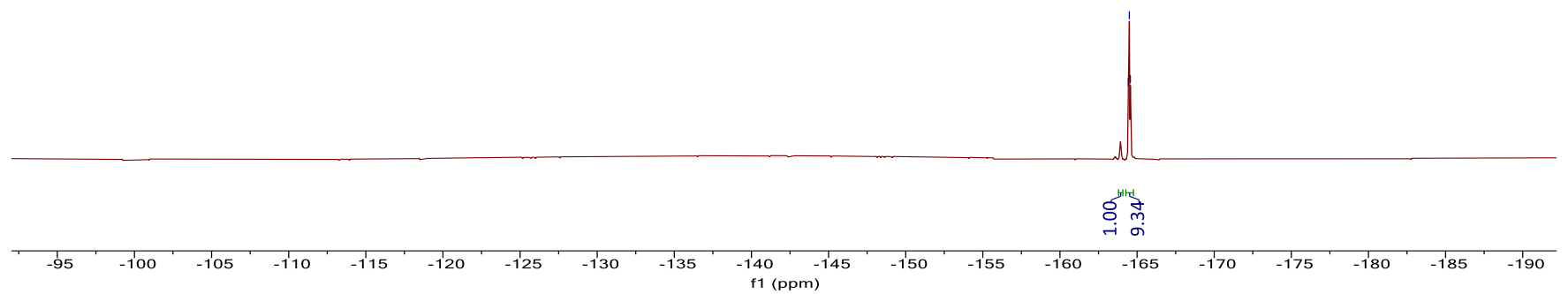


芯芯定
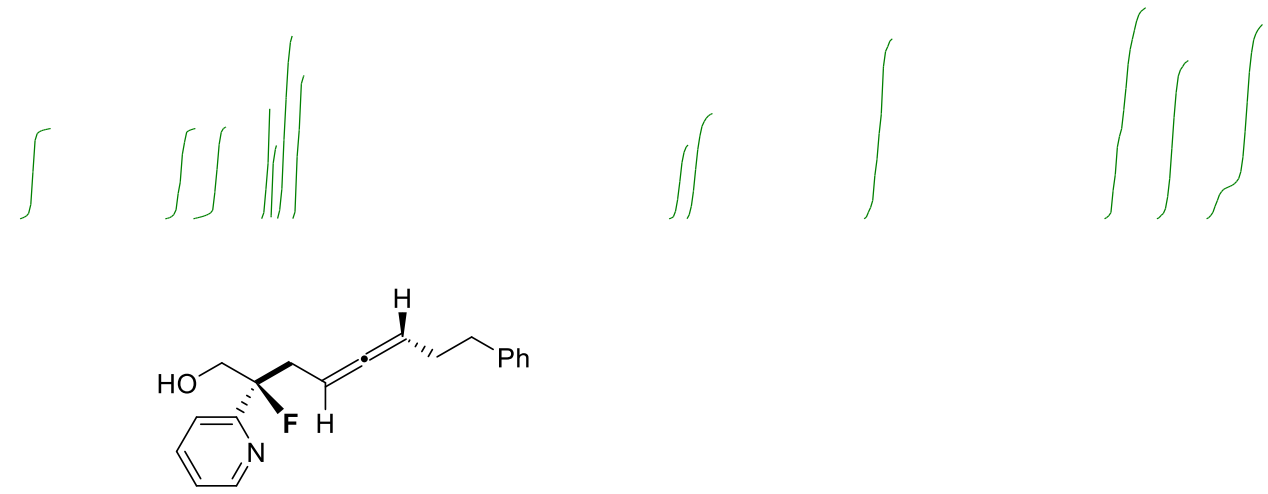

$3 \mathbf{s}^{\prime},{ }^{1} \mathrm{H}$ NMR
$\mathrm{CDCl}_{3}$
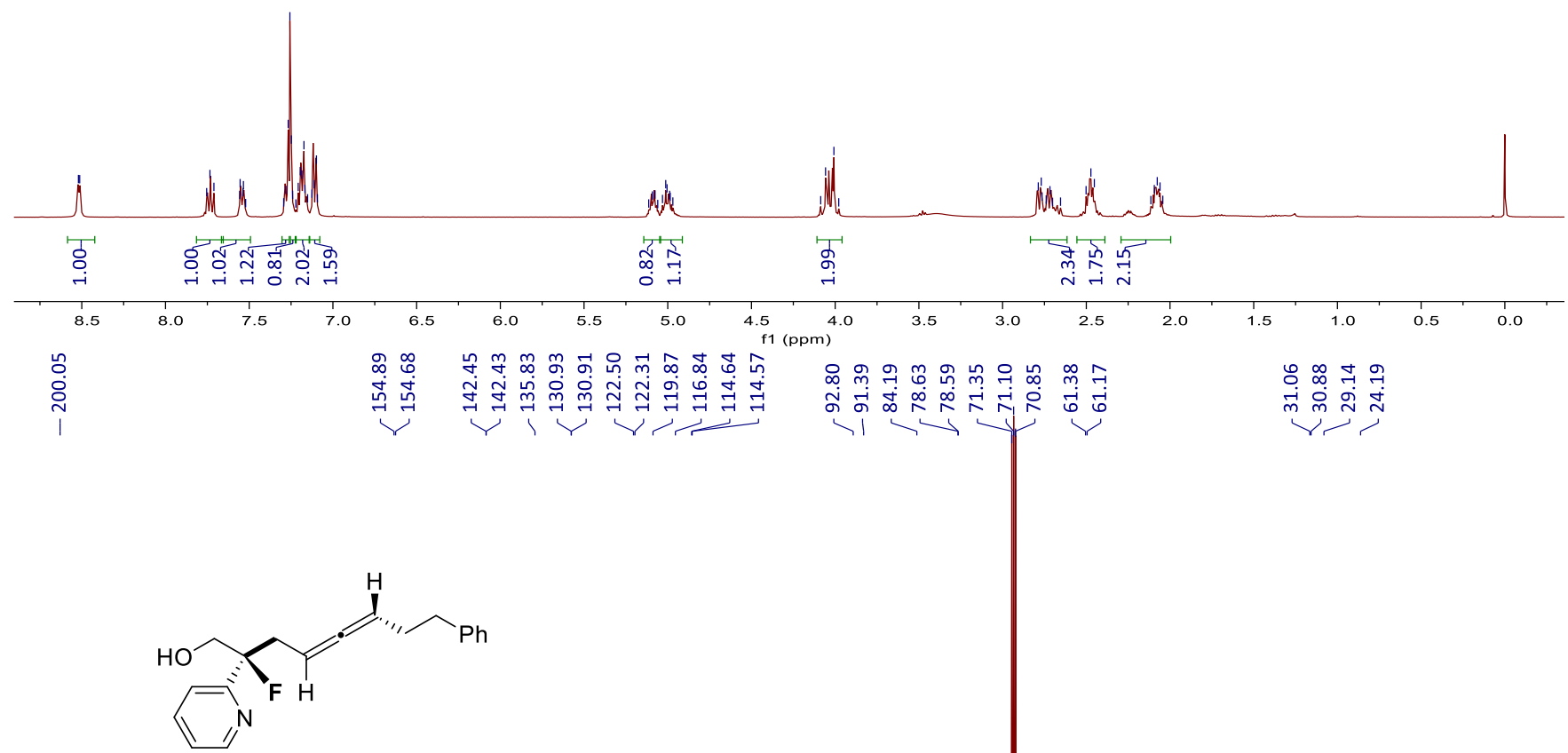

3s', ${ }^{13} \mathrm{C}$ NMR
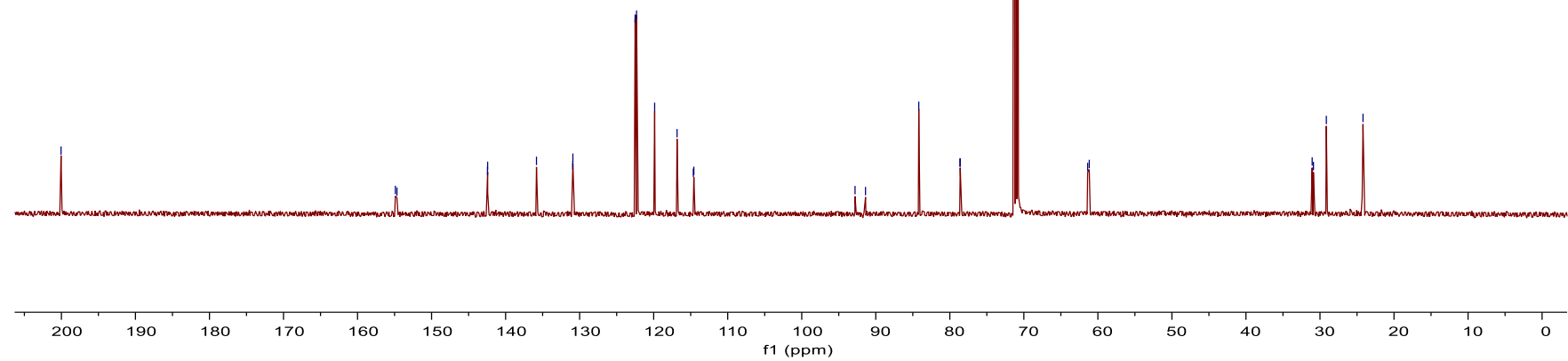


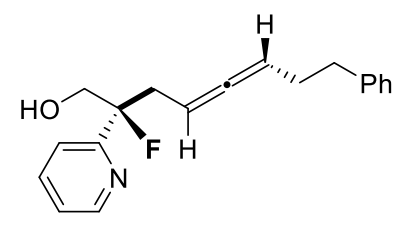

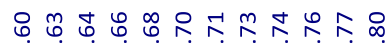

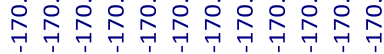

$3 \mathbf{s}^{\prime},{ }^{19} \mathrm{~F}$ NMR

$\mathrm{CDCl}_{3}$

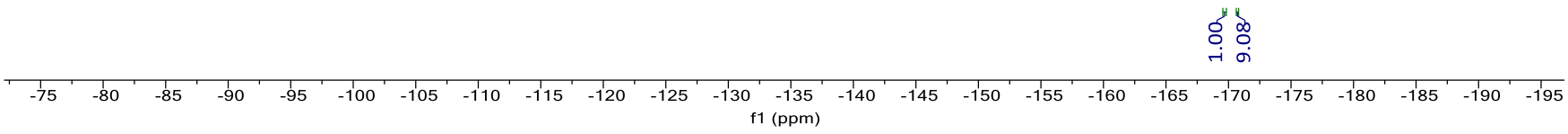



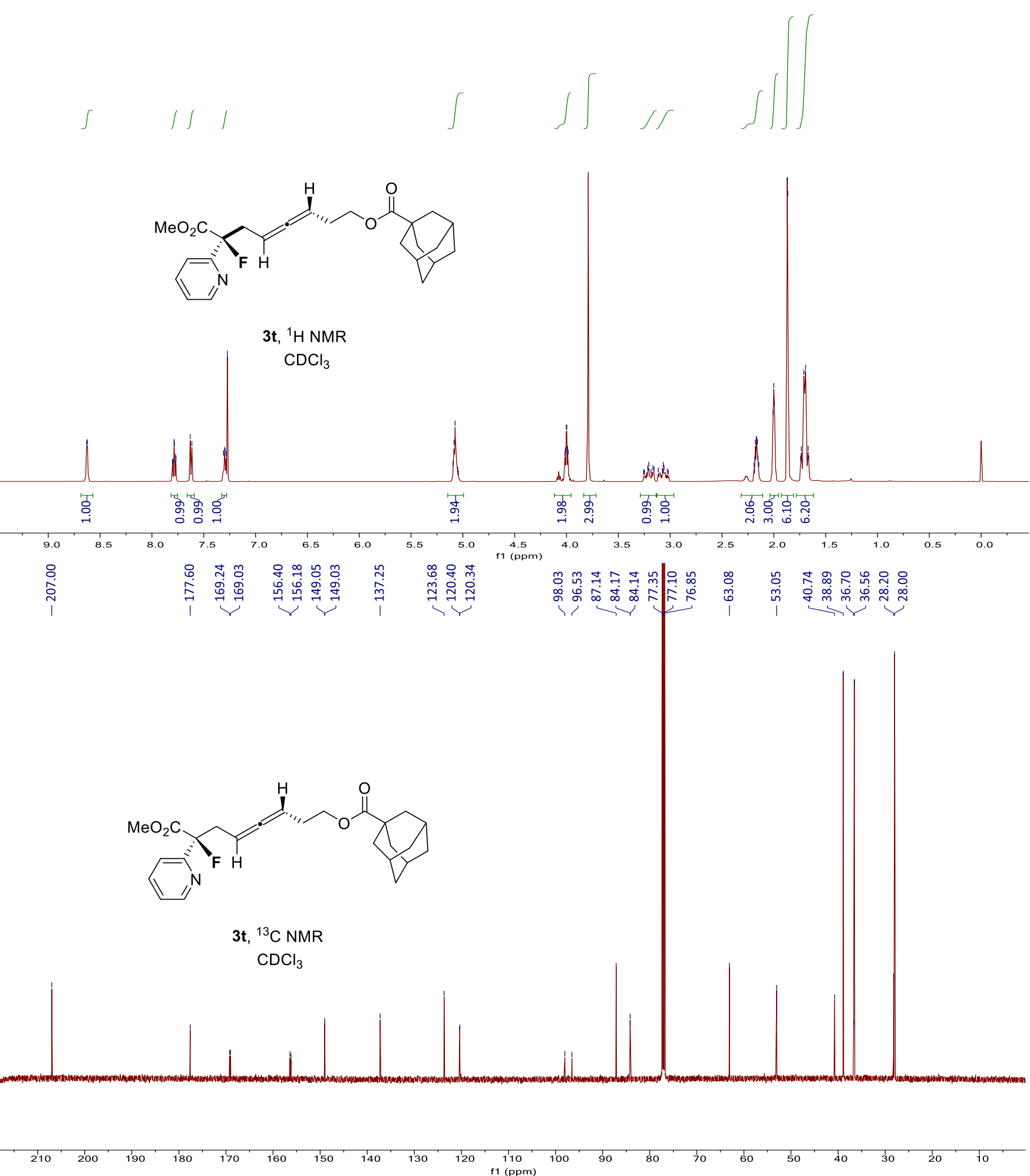
ำ 웅ำ

它宫昰

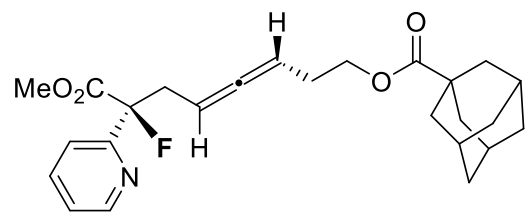

3t, ${ }^{19} \mathrm{~F}$ NMR

$\mathrm{CDCl}_{3}$

त्र

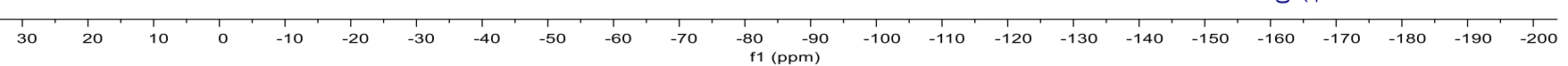


bơ

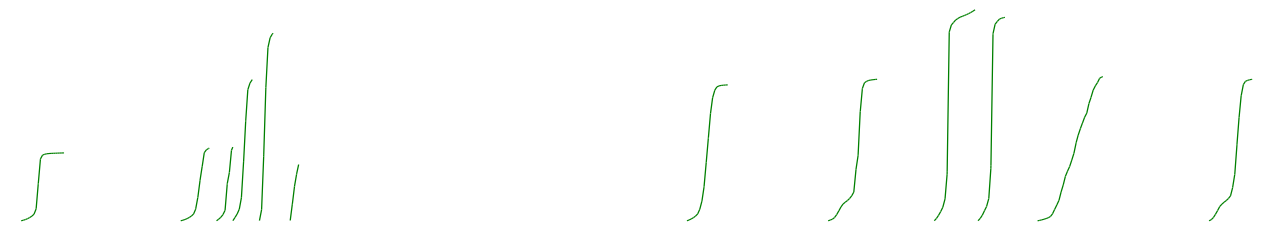<smiles>COC(=O)[C@](F)(C/C=C/CCOC(=O)[C@](F)(c1ccccc1)C(F)(F)F)c1ccccn1</smiles>

$3 \mathrm{u},{ }^{1} \mathrm{H}$ NMR

$\mathrm{CDCl}_{3}$

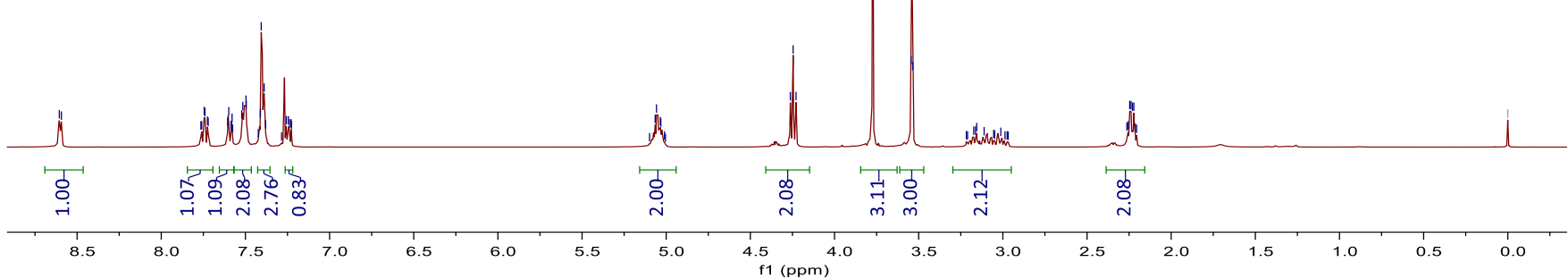

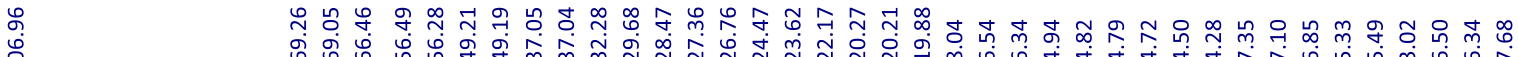

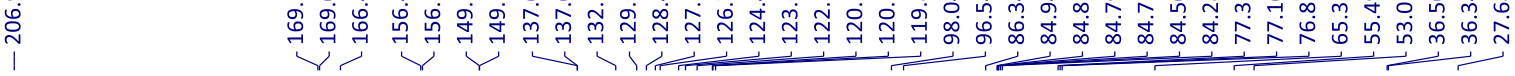
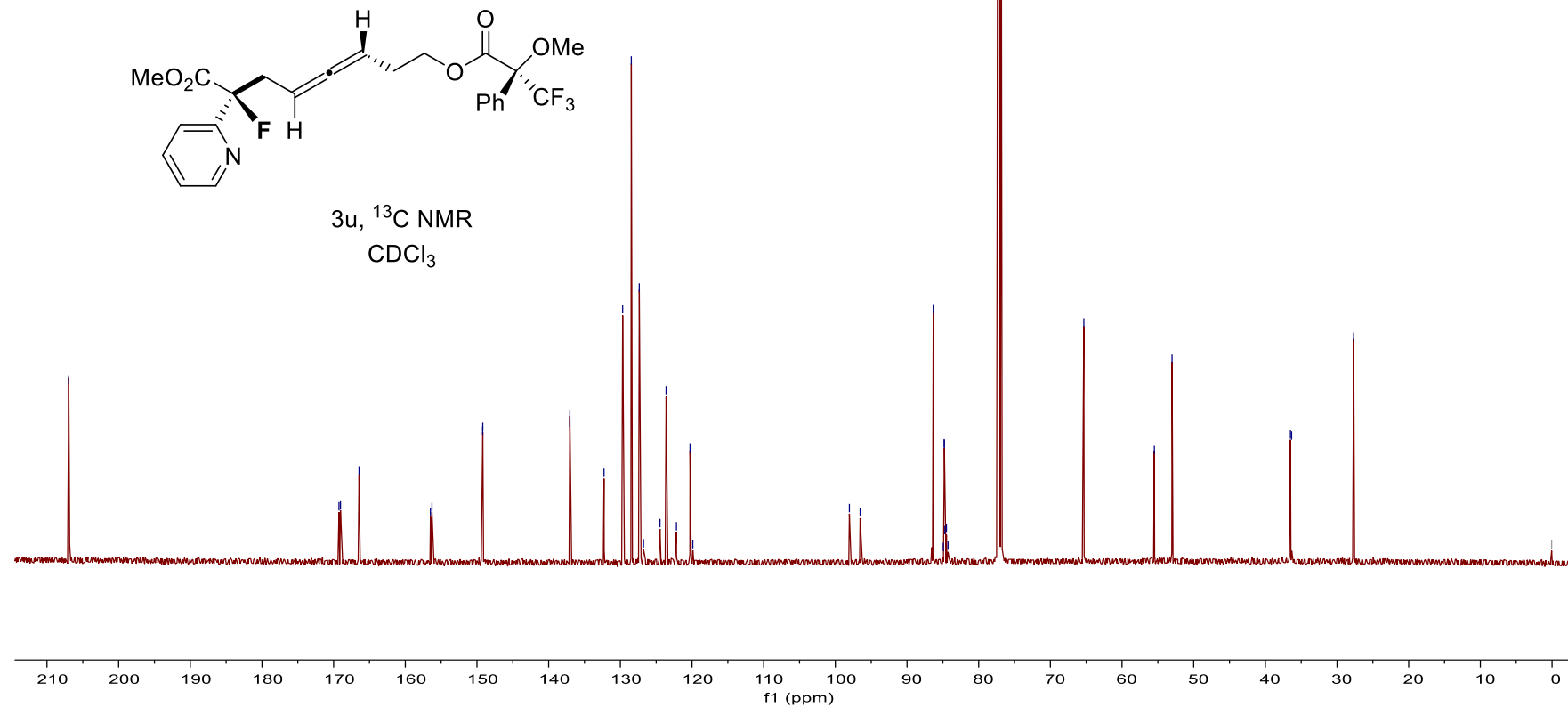


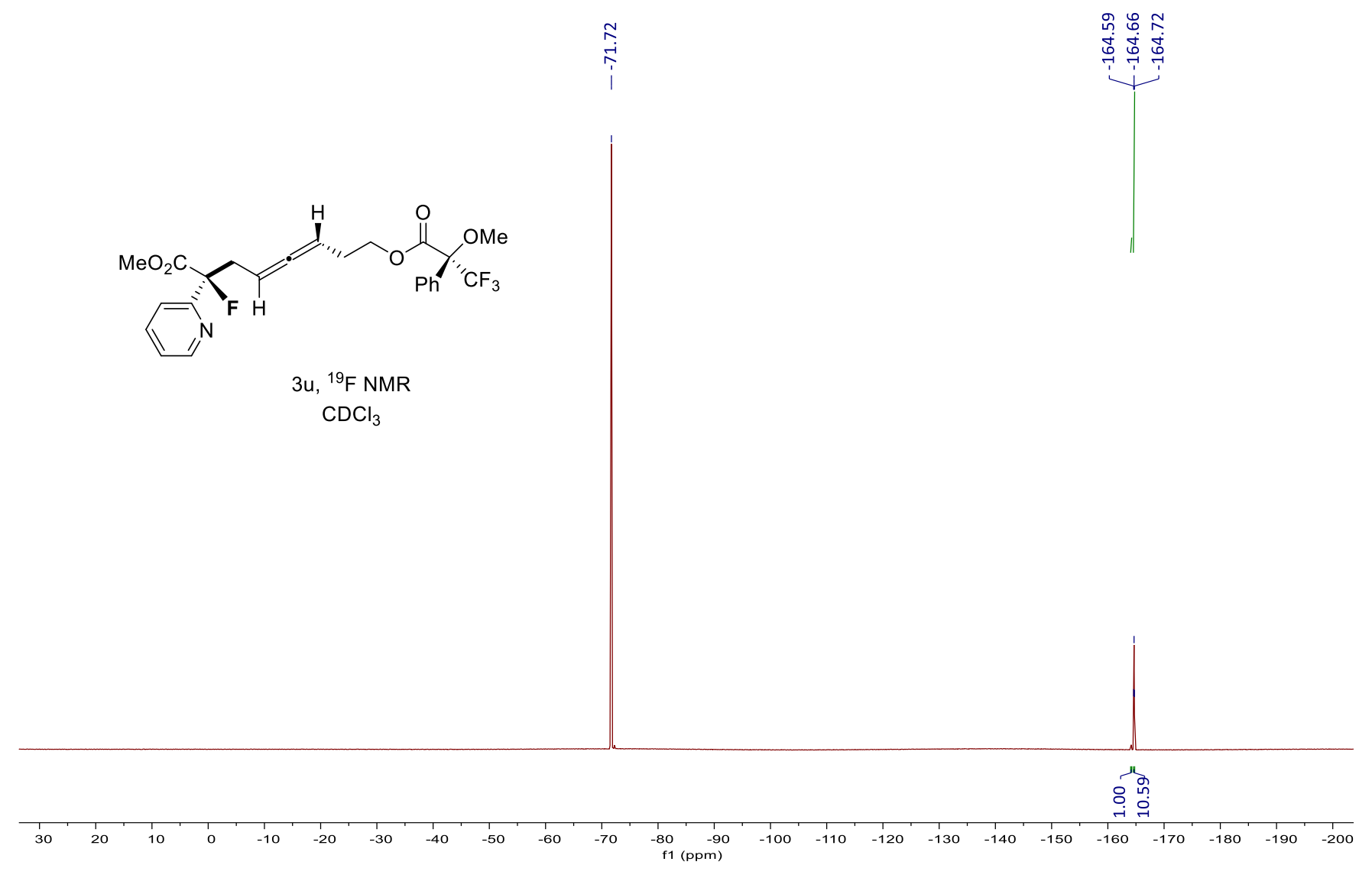




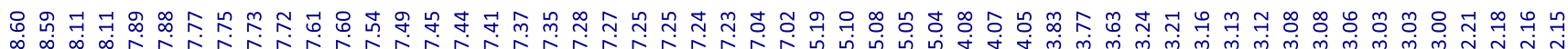
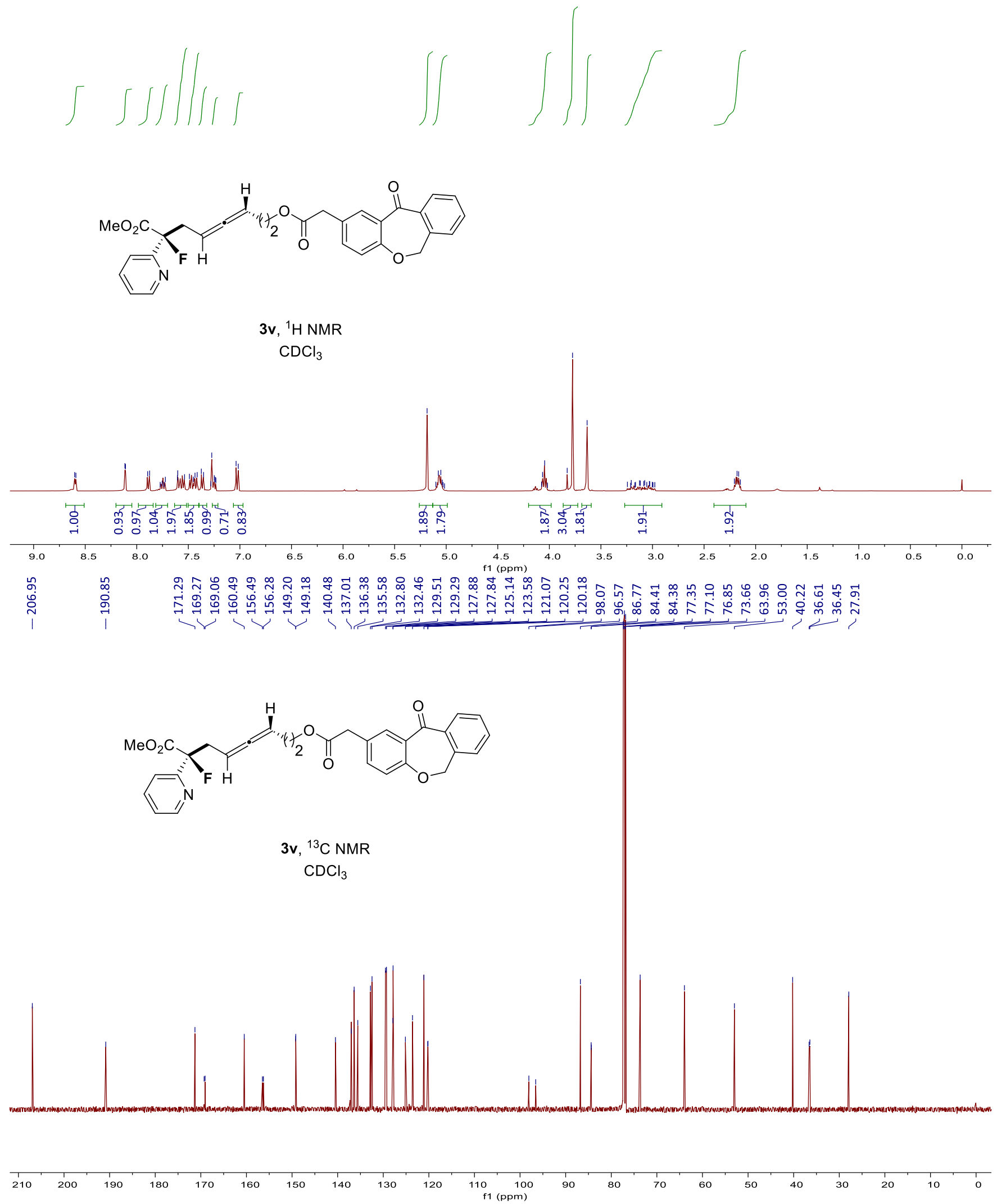
考打

总热

7

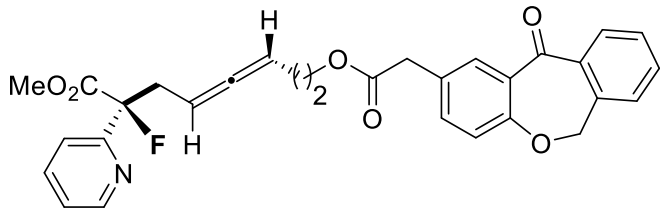

3v, ${ }^{19} \mathrm{~F}$ NMR

$\mathrm{CDCl}_{3}$

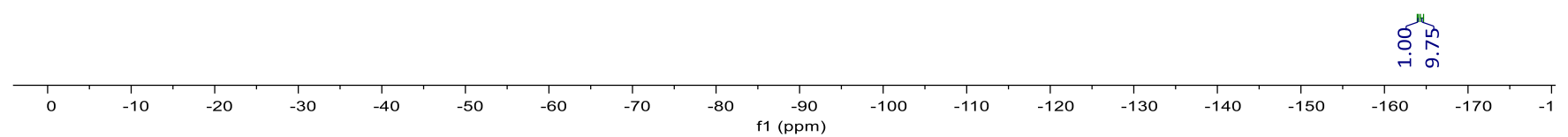



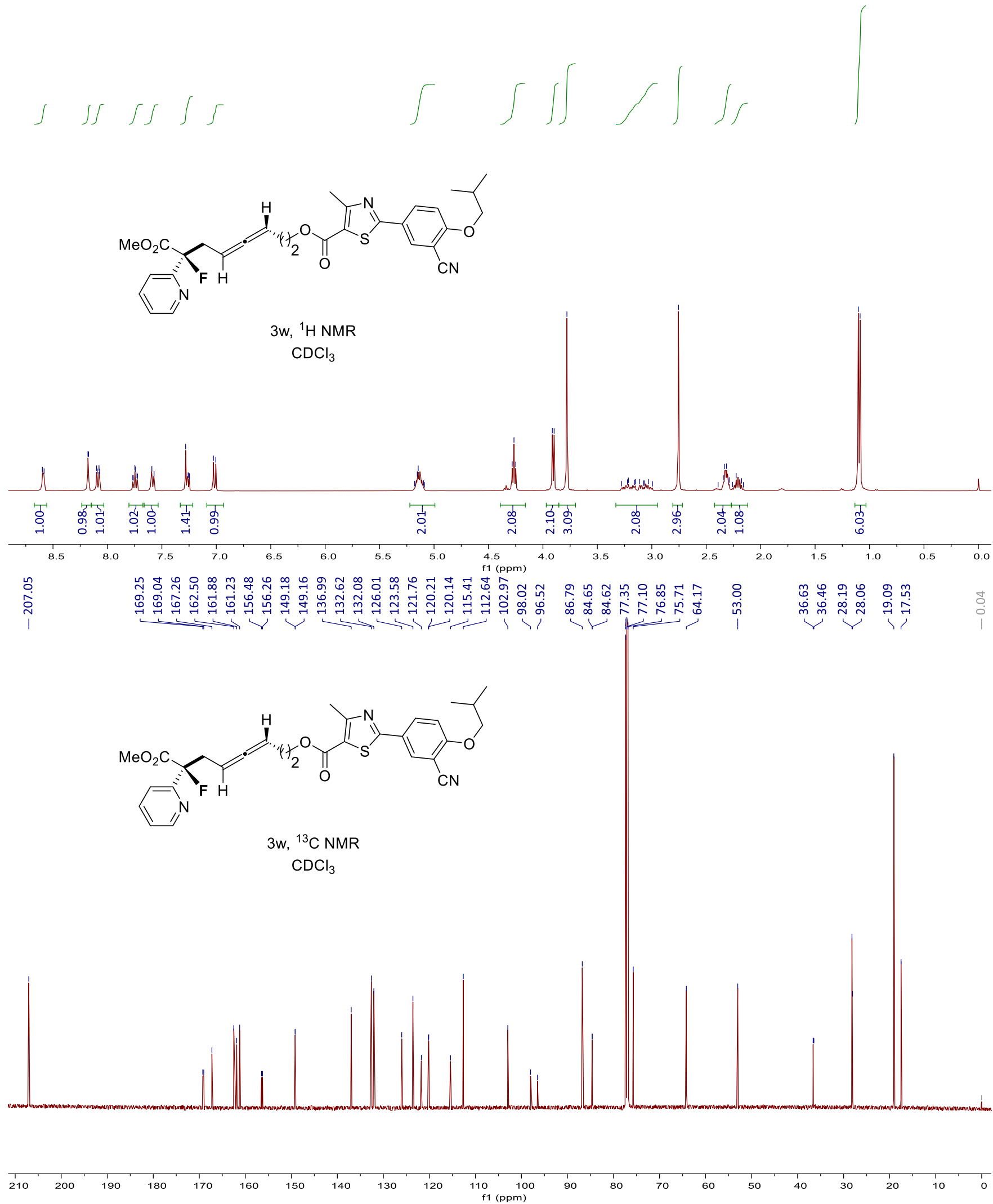
ळ

过

i
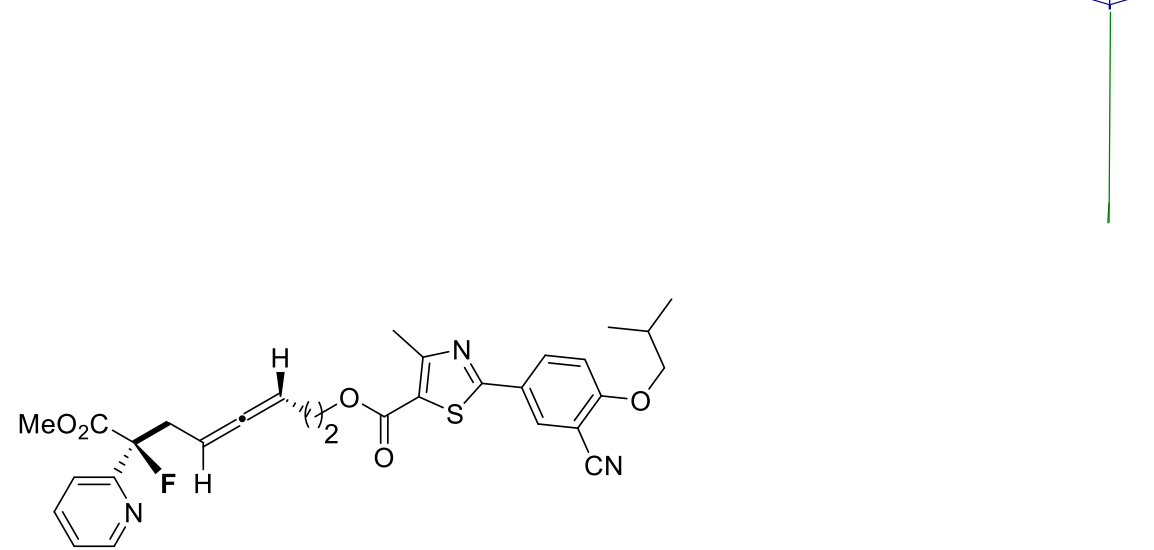

$3 w,{ }^{19} \mathrm{~F}$ NMR

$\mathrm{CDCl}_{3}$

\section{8 究}

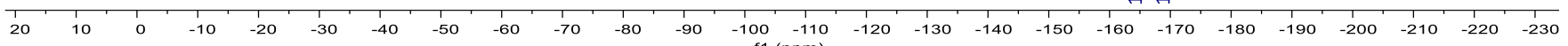




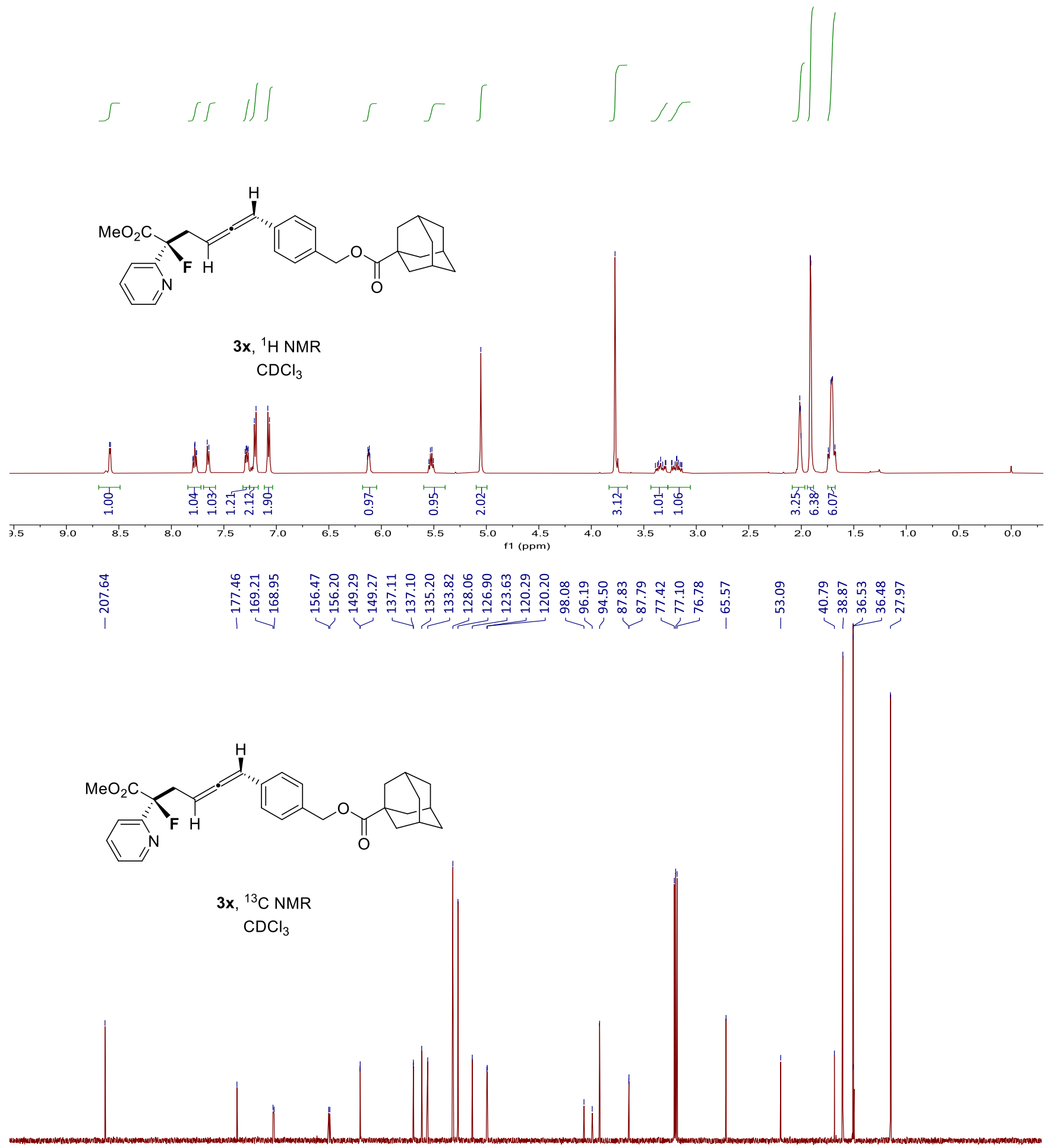


นกำ

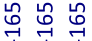

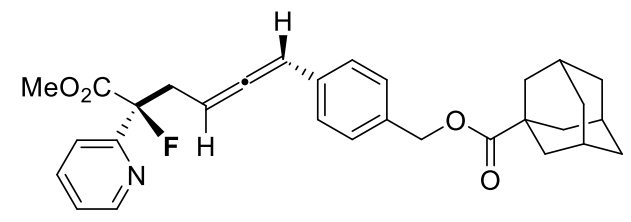

3x, ${ }^{19} \mathrm{~F}$ NMR

$\mathrm{CDCl}_{3}$

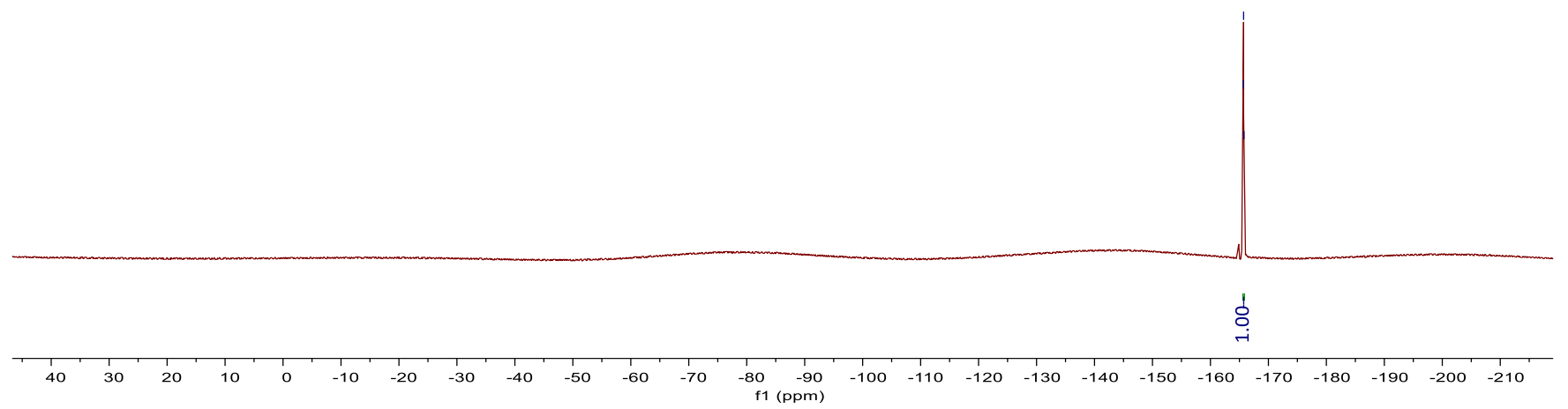




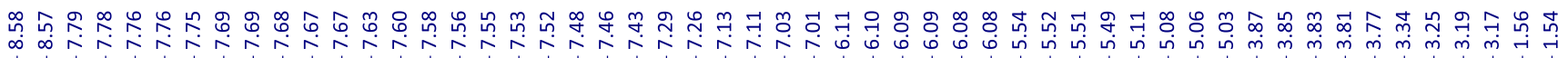

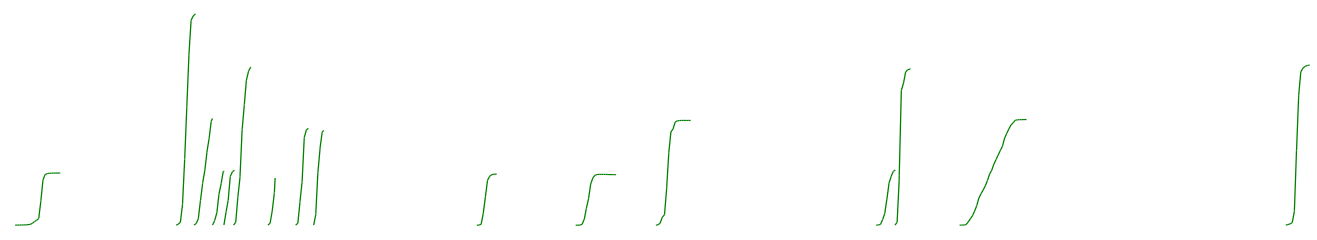<smiles>COC(=O)C(F)(C/C=C/c1ccc(COC(=O)C(C)c2cccc(C(=O)c3ccccc3)c2)cc1)c1ccccn1</smiles>

3y, ${ }^{1} \mathrm{H}$ NMR

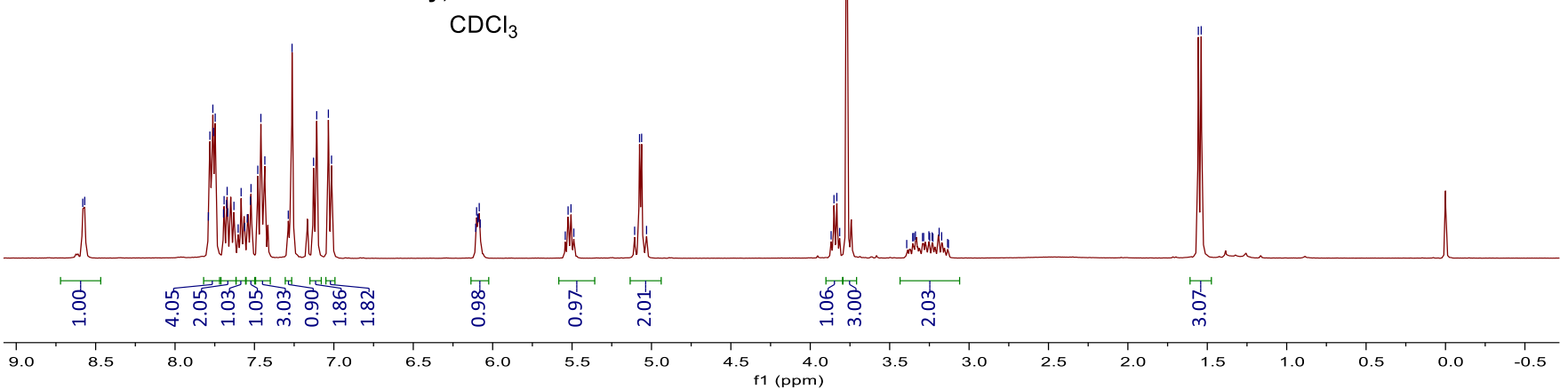

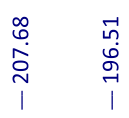

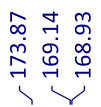

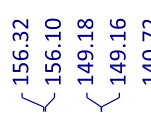

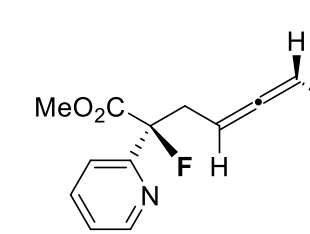<smiles>CC(C)C(=O)OCc1ccc(I)cc1</smiles>

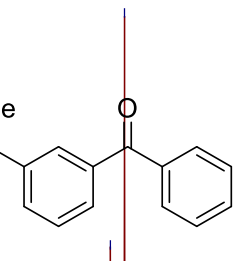

3y, ${ }^{13} \mathrm{C}$ NMR

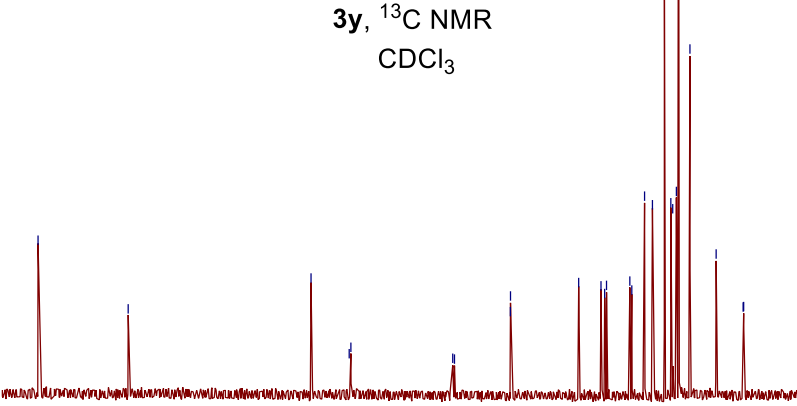

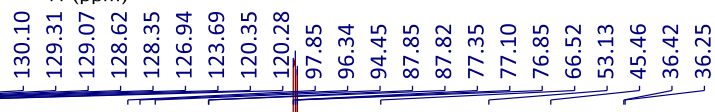

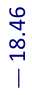

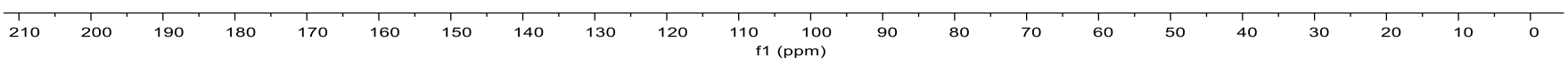

S165 
ํ. ํํㅇ

吉吉志

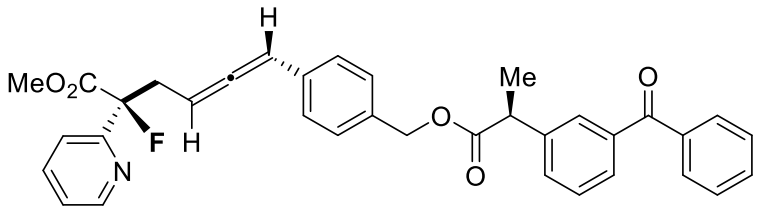

3y, ${ }^{19} \mathrm{~F}$ NMR $\mathrm{CDCl}_{3}$

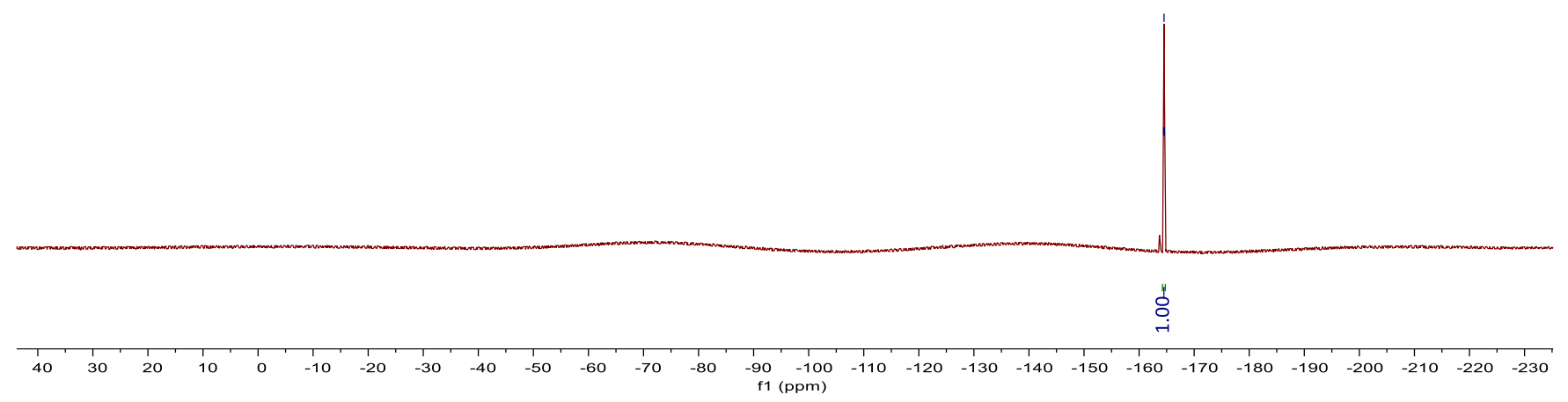




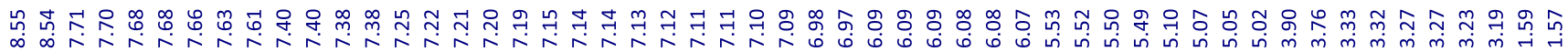

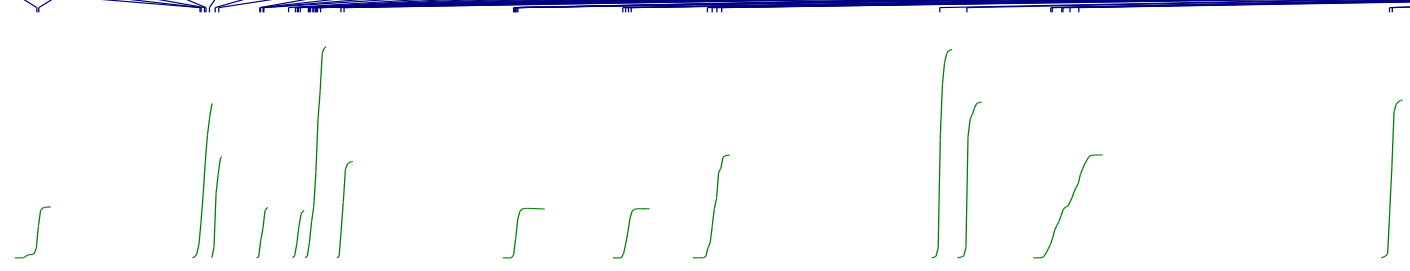

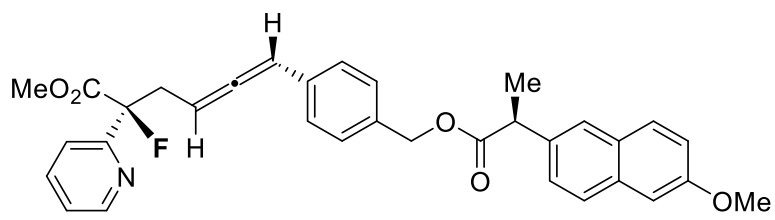

3z, ${ }^{1} \mathrm{H}$ NMR $\mathrm{CDCl}_{3}$

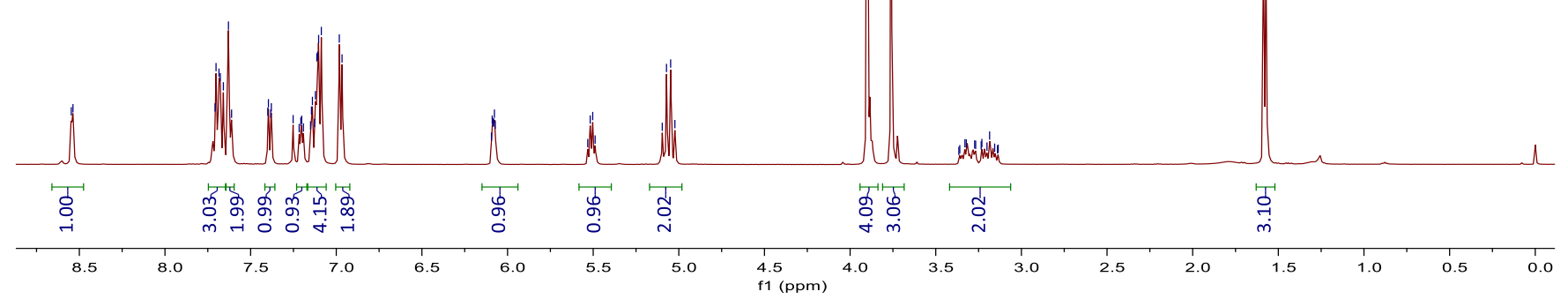

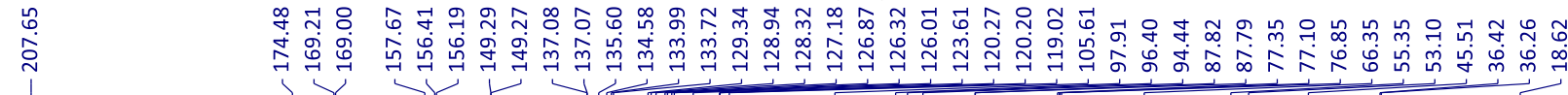
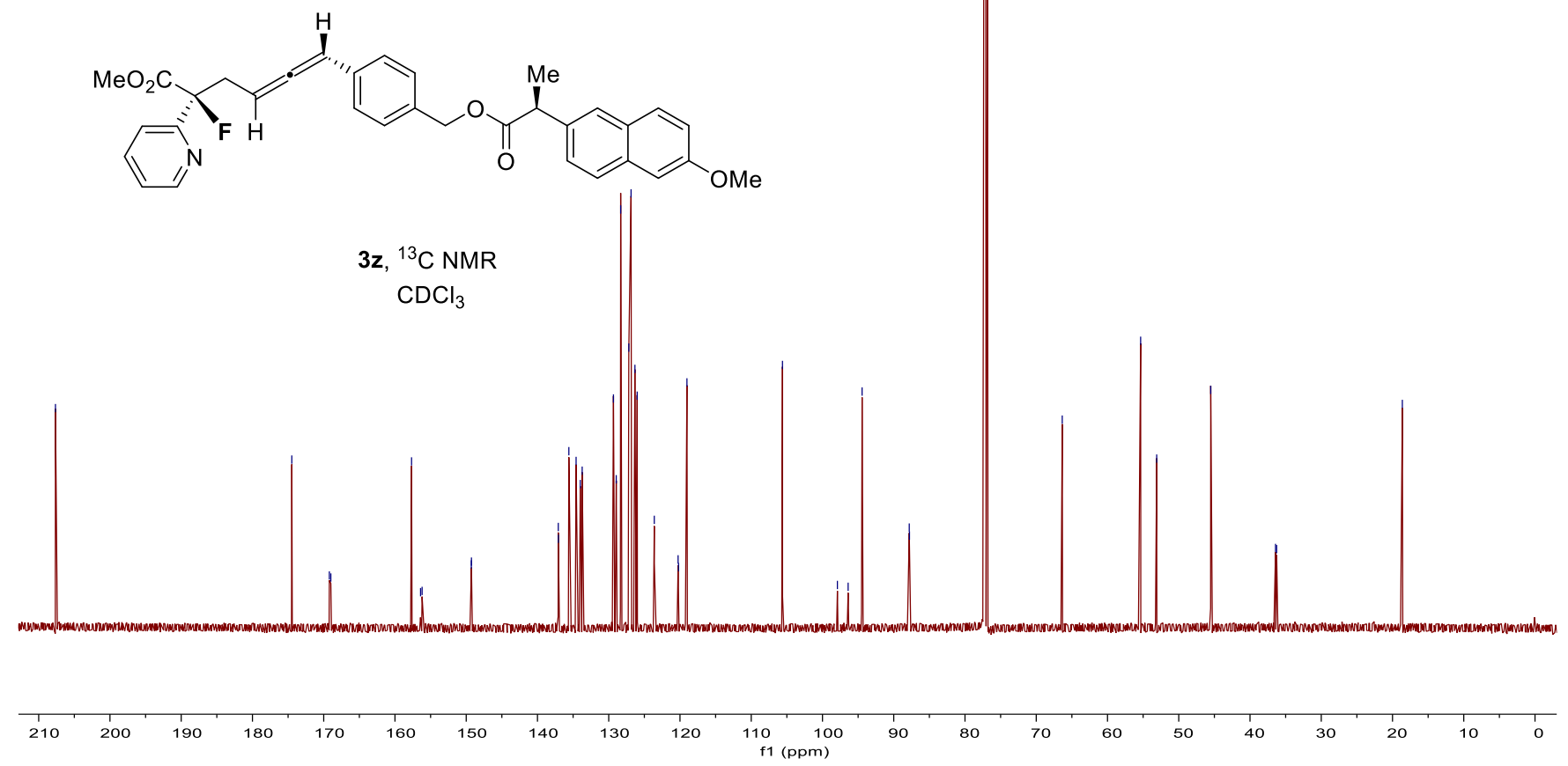
ㅇํㄴㅇㅛ

पे 它
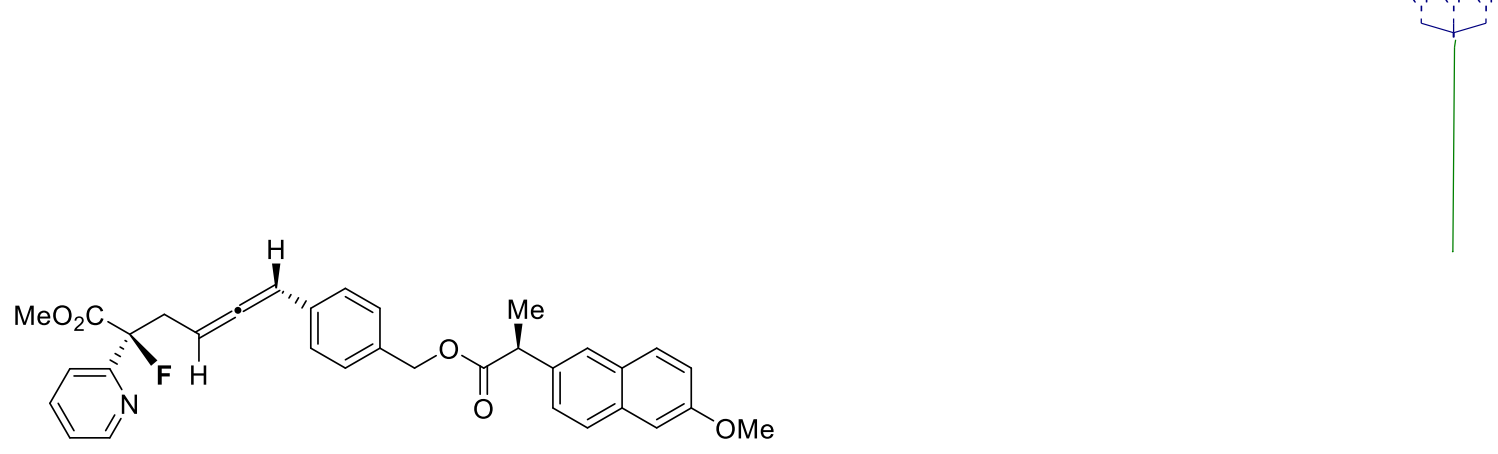

$3 \mathbf{z},{ }^{19} \mathrm{~F}$ NMR

$\mathrm{CDCl}_{3}$ 


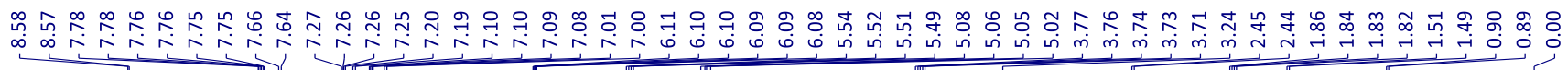
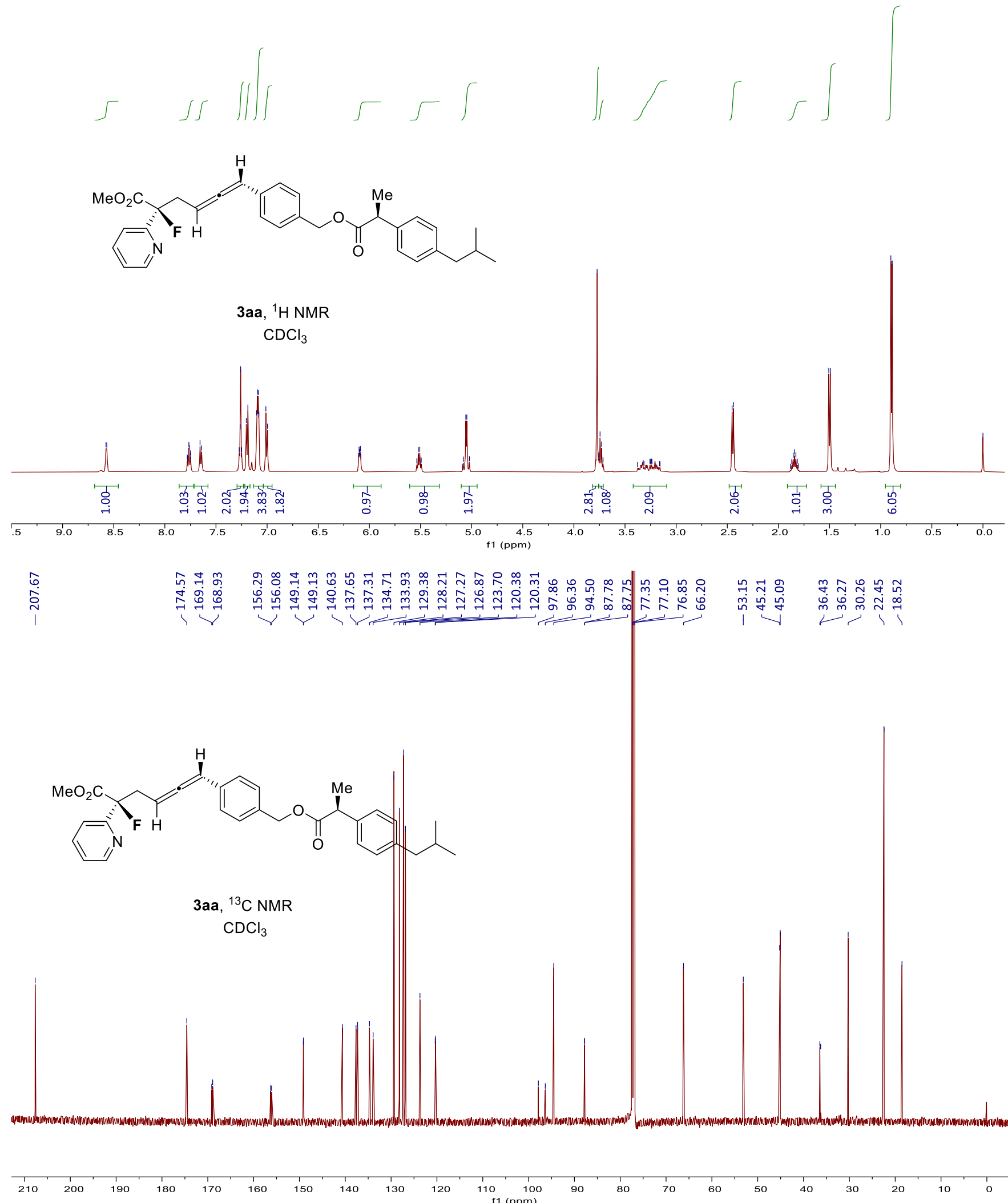


\section{유 ₹ 워}

엉

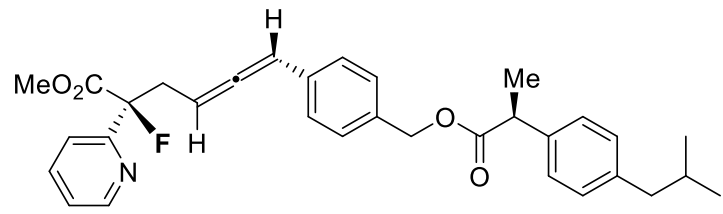

3aa, ${ }^{19} \mathrm{~F}$ NMR $\mathrm{CDCl}_{3}$

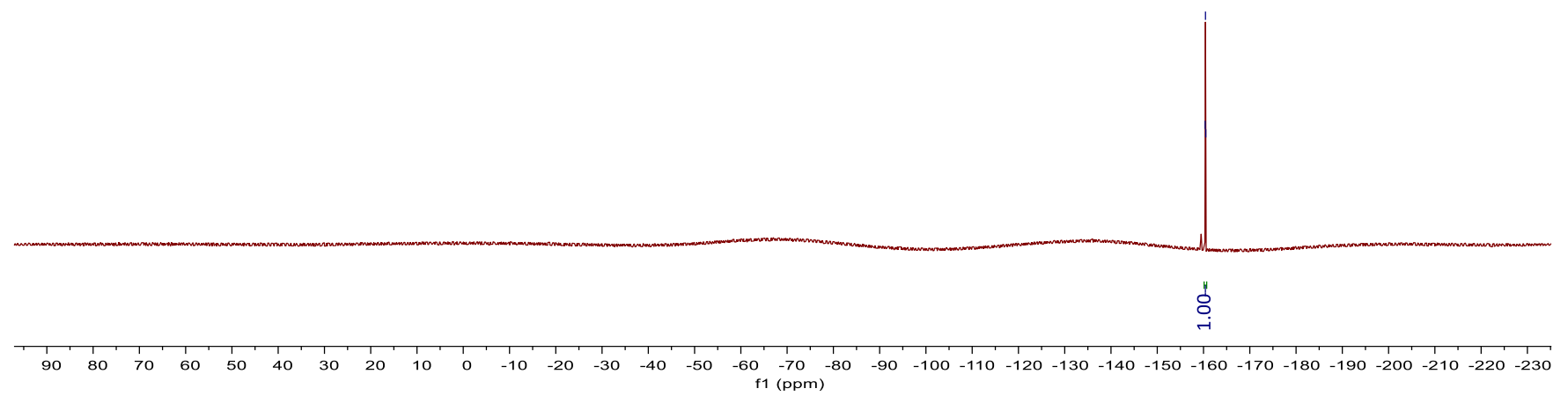




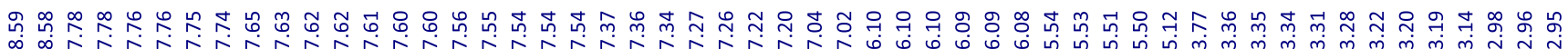

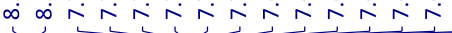

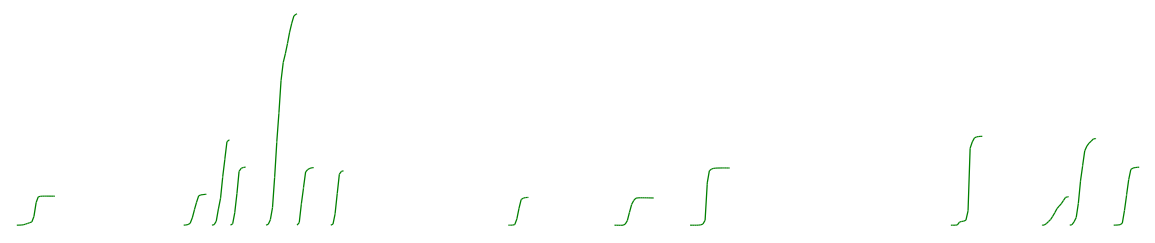<smiles>CC(=O)C(CC(=C=Cc1ccc(COC(=O)CCc2nc(-c3ccccc3)c(-c3ccccc3)o2)cc1)C(F)(F)c1ccccn1)C(F)(F)c1ccccc1</smiles>

3bb, ${ }^{1} \mathrm{H}$ NMR $\mathrm{CDCl}_{3}$

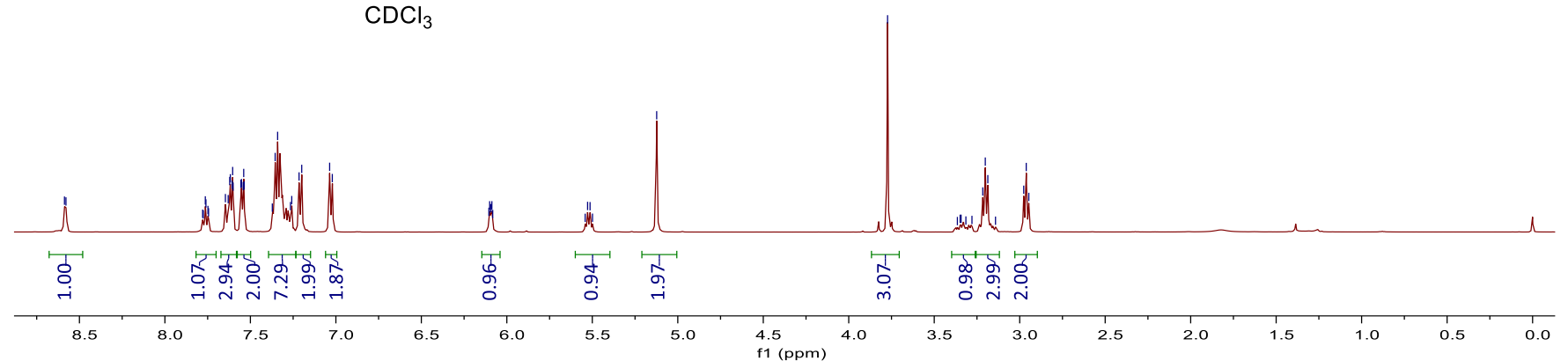

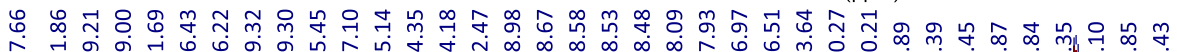

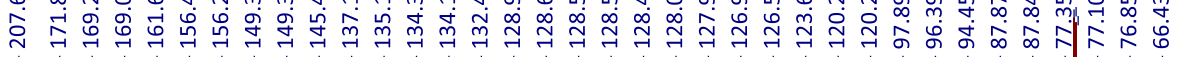<smiles>CC(=O)C(CC=Cc1ccc(COC(=O)CCc2nc(-c3ccccc3)c(-c3ccccc3)o2)cc1)(c1ccccn1)C(F)(F)c1ccccc1</smiles>

3bb, ${ }^{13} \mathrm{C}$ NMR $\mathrm{CDCl}_{3}$
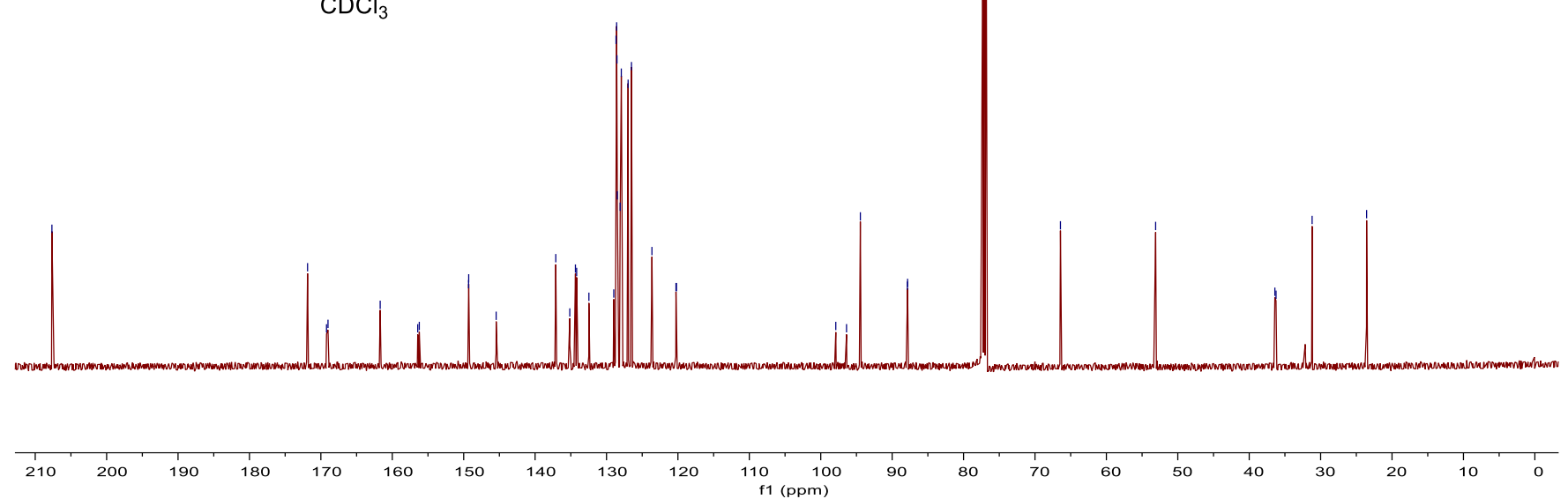
눙오

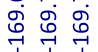

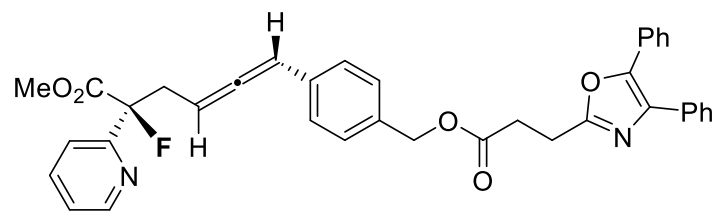

3bb, ${ }^{19} \mathrm{~F}$ NMR $\mathrm{CDCl}_{3}$

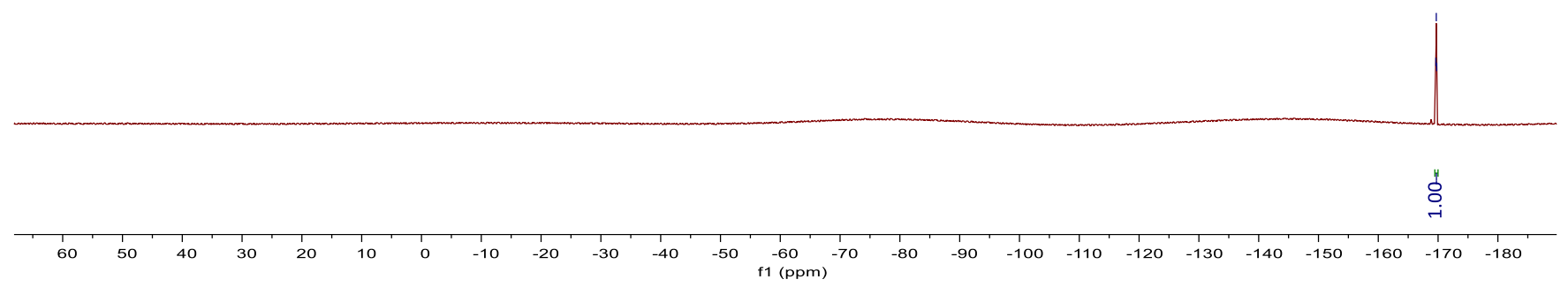




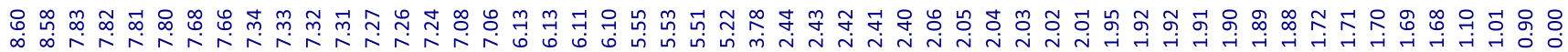
on

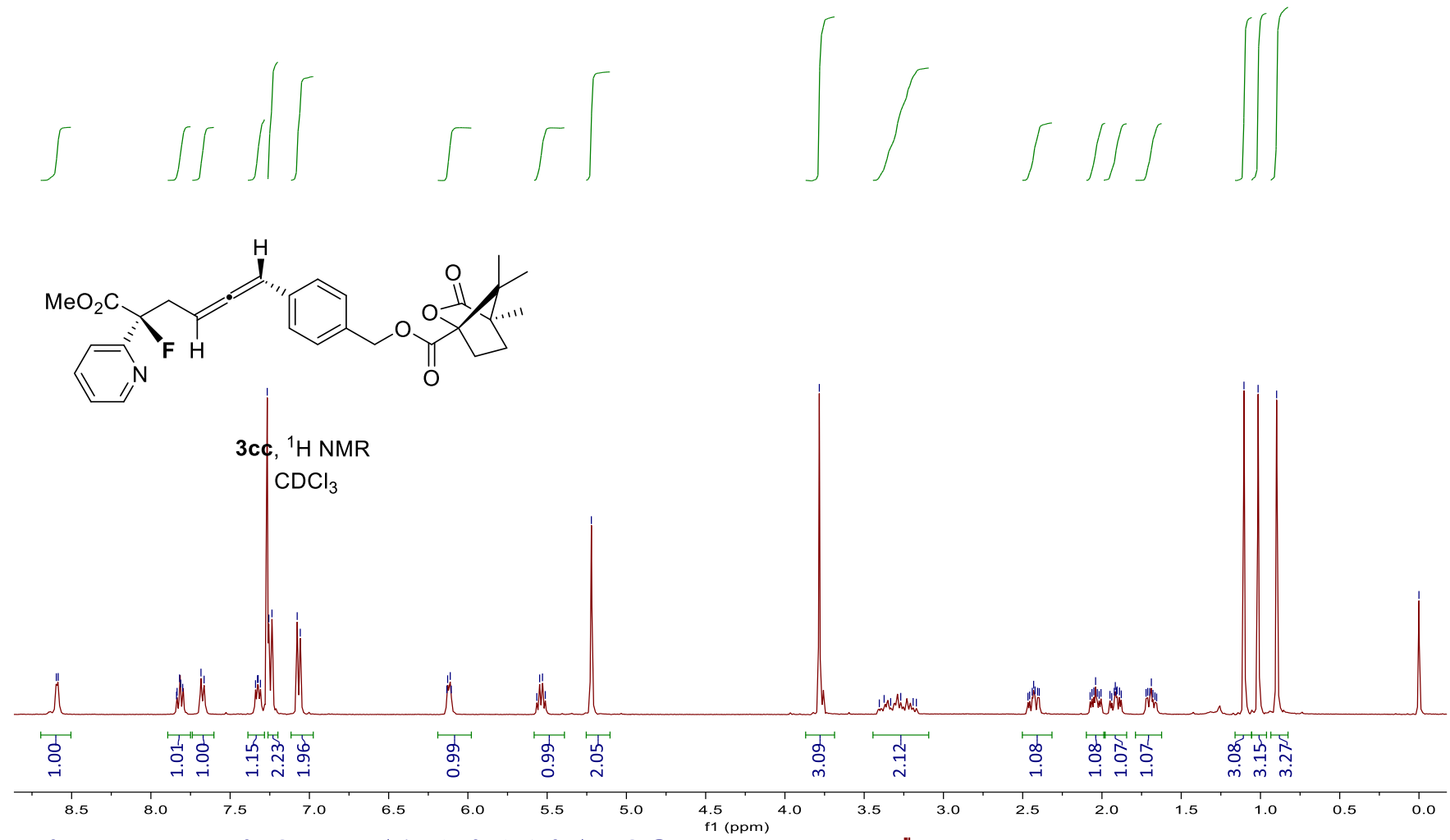

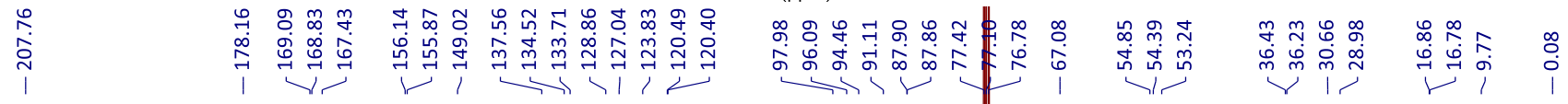<smiles>CC(=O)C(F)(Cc1ccc(COC(=O)C23CCC(C)(C(=O)O2)C3(C)C)cc1)c1ccccn1</smiles>

3cc, ${ }^{13} \mathrm{C}$ NMR $\mathrm{CDCl}_{3}$

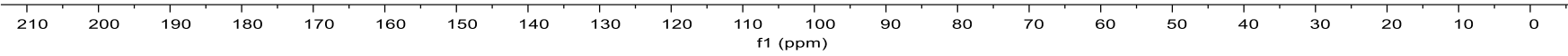




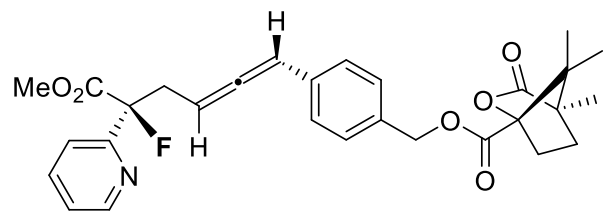

3cc, ${ }^{13} \mathrm{~F}$ NMR

$\mathrm{CDCl}_{3}$

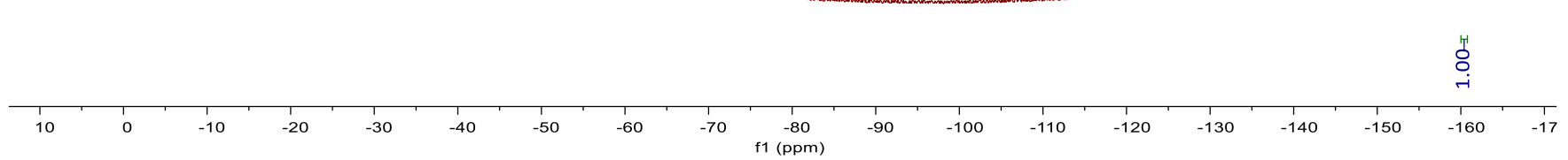




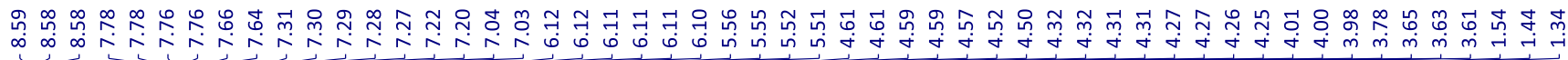
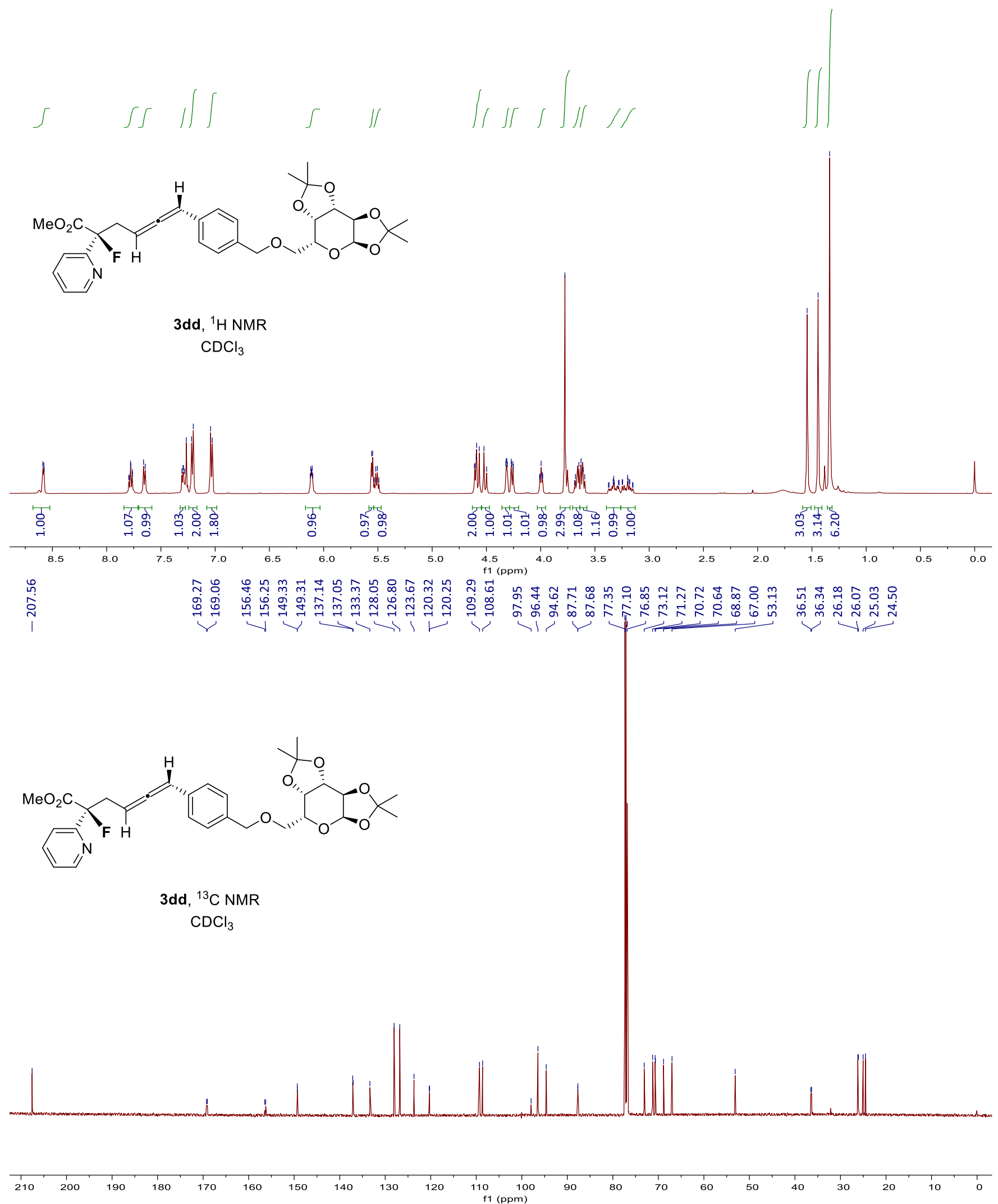
ㅇํำ

它志

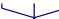

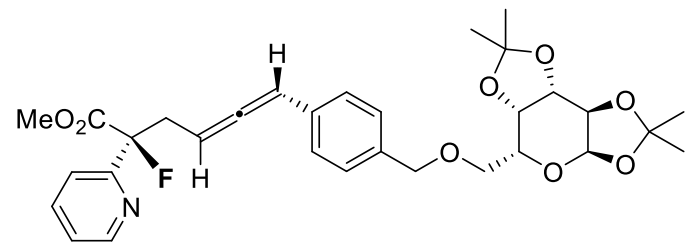

3dd, ${ }^{19} \mathrm{~F}$ NMR

$\mathrm{CDCl}_{3}$

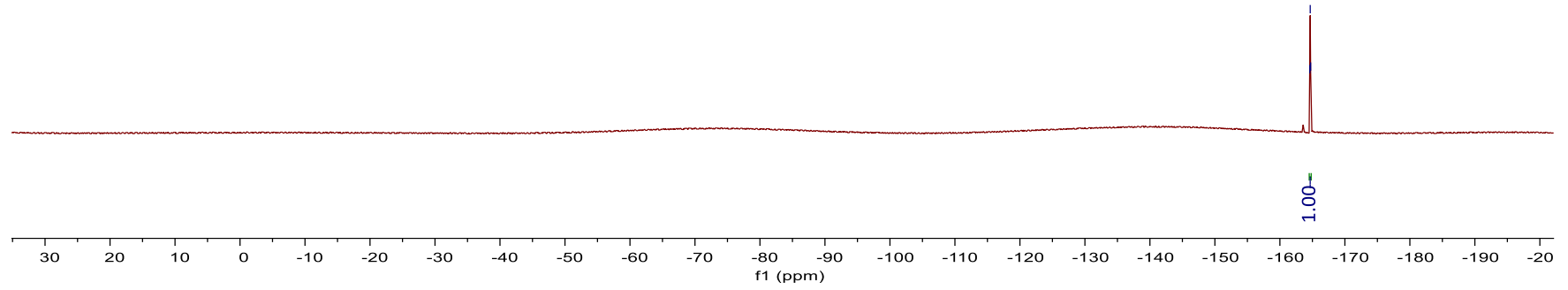




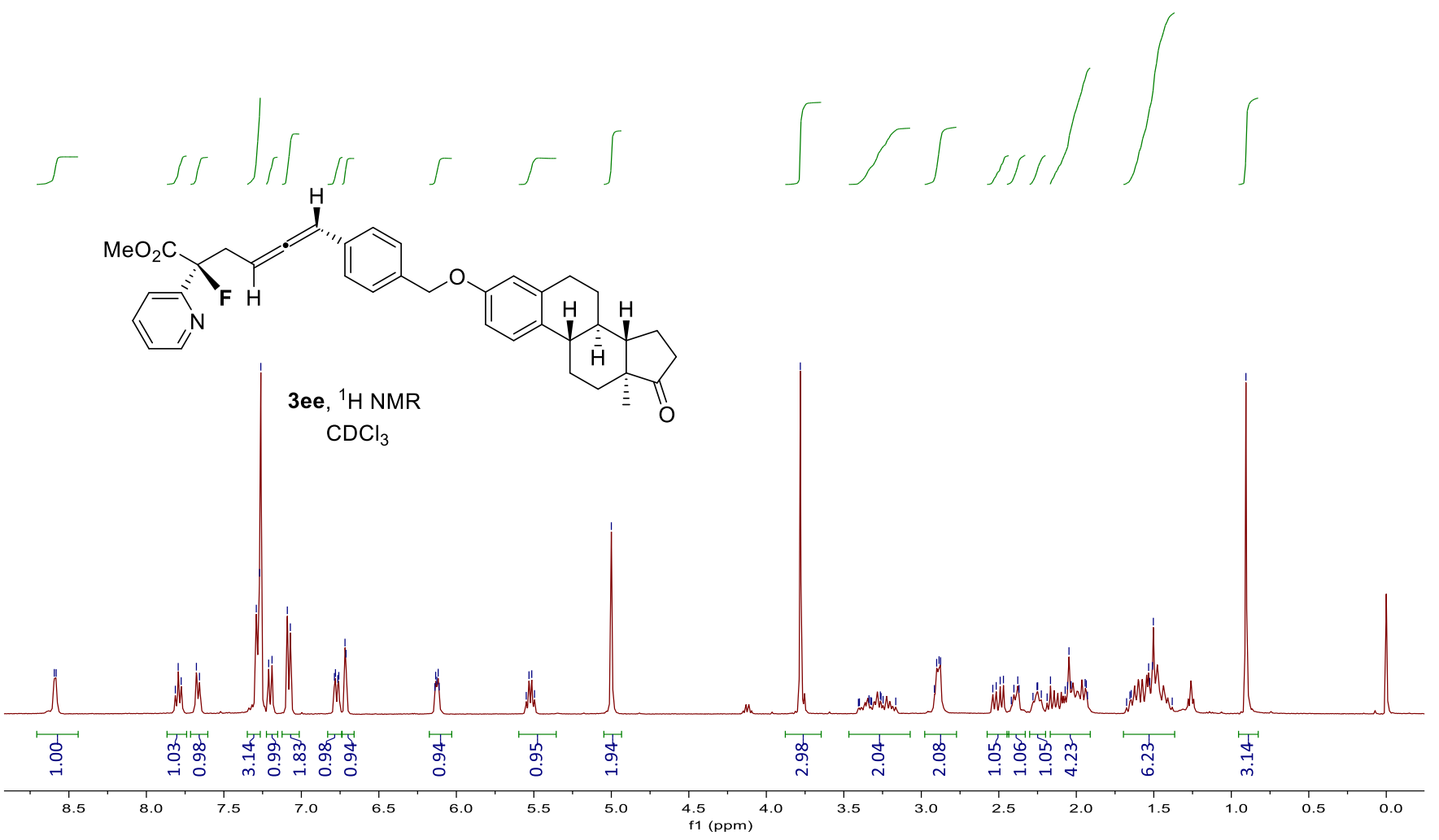

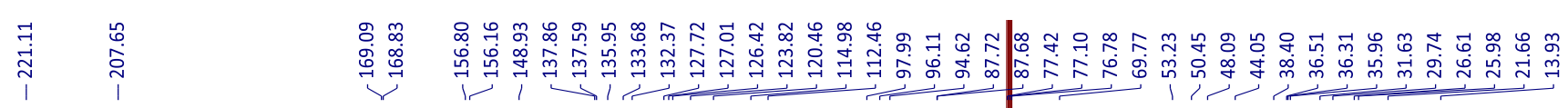

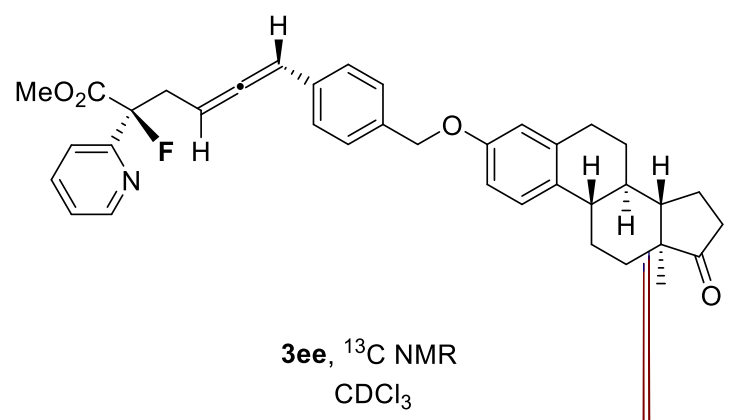

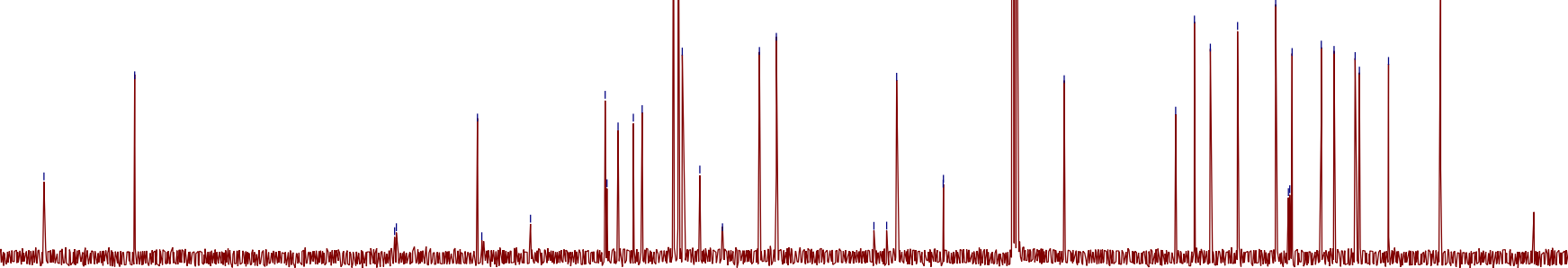


ㅇํㅇ

灾灾

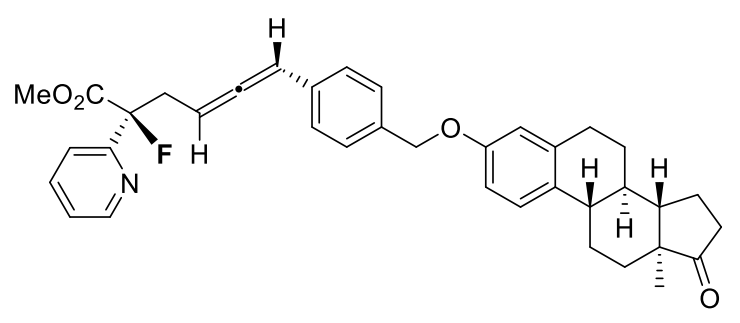

3ee, ${ }^{19} \mathrm{~F} \mathrm{NMR}$

$\mathrm{CDCl}_{3}$ 


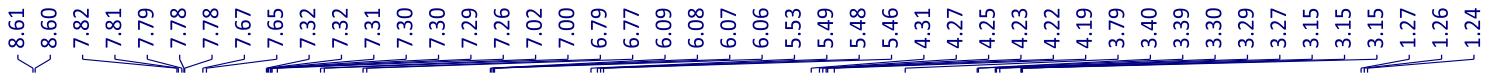
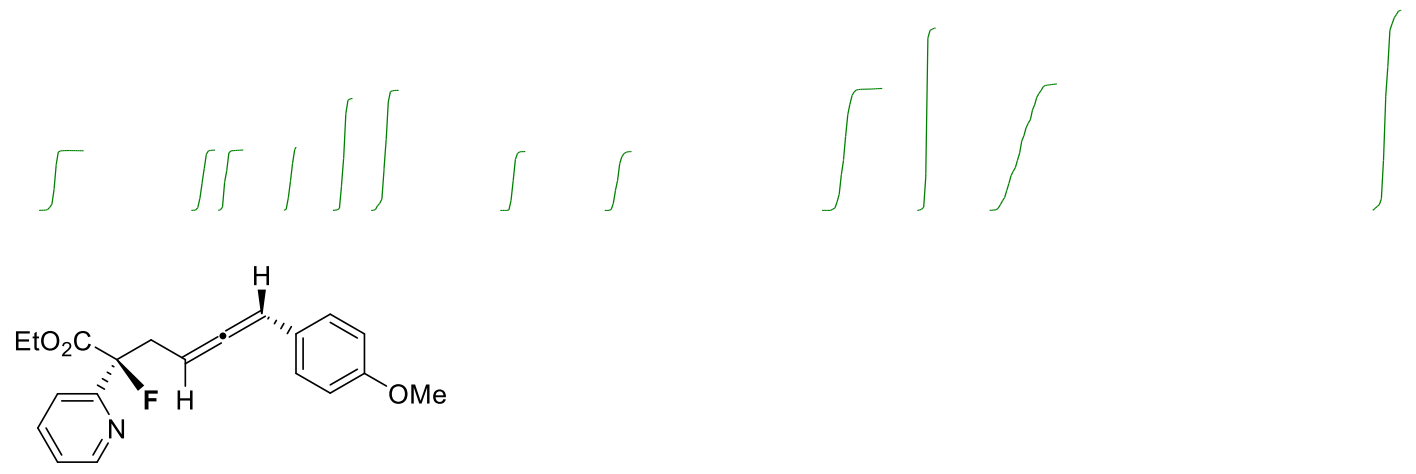

4b, ${ }^{1} \mathrm{H}$ NMR $\mathrm{CDCl}_{3}$
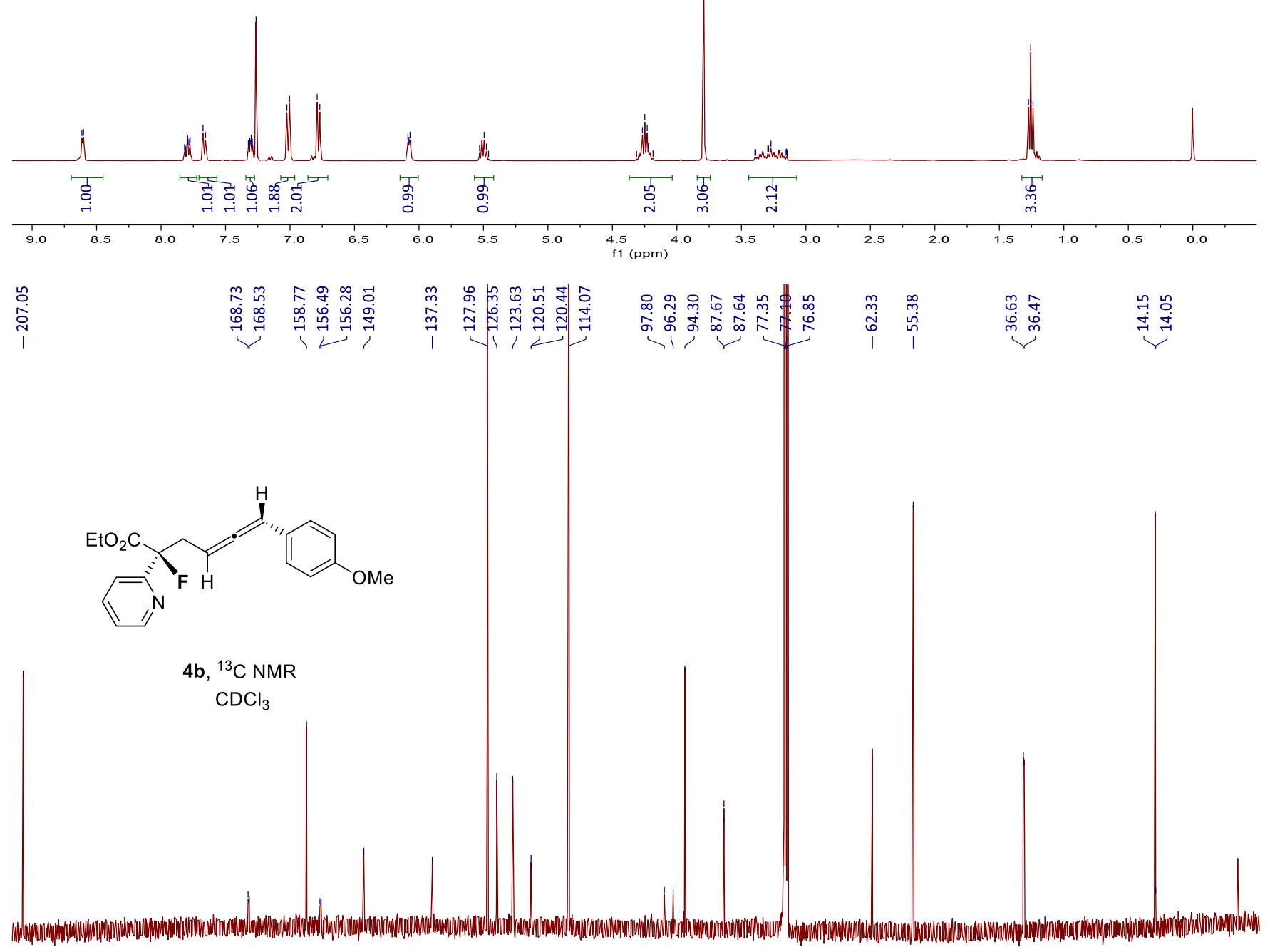


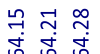

党总

i

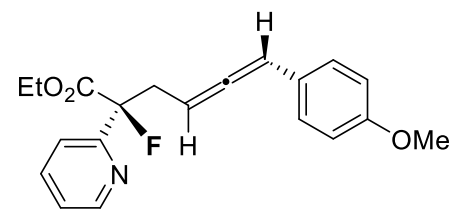

4b, ${ }^{19} \mathrm{~F}$ NMR

$\mathrm{CDCl}_{3}$

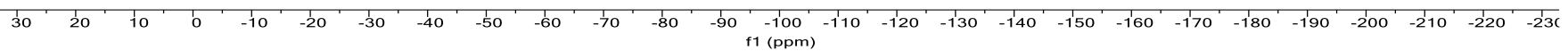




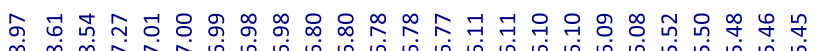

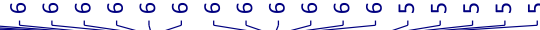

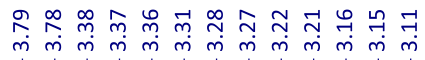
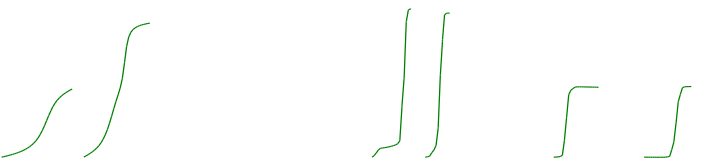

ले

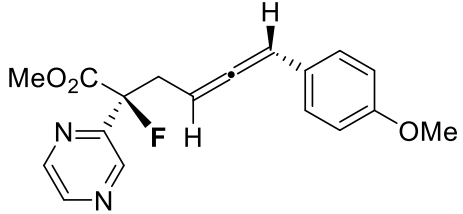

4c, ${ }^{1} \mathrm{H}$ NMR

$\mathrm{CDCl}_{3}$

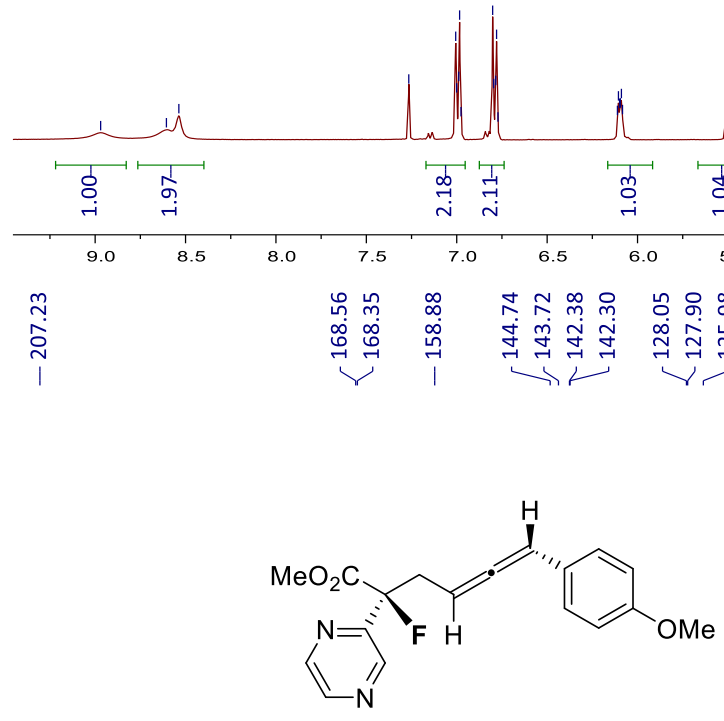

4c, ${ }^{13} \mathrm{C}$ NMR $\mathrm{CDCl}_{3}$

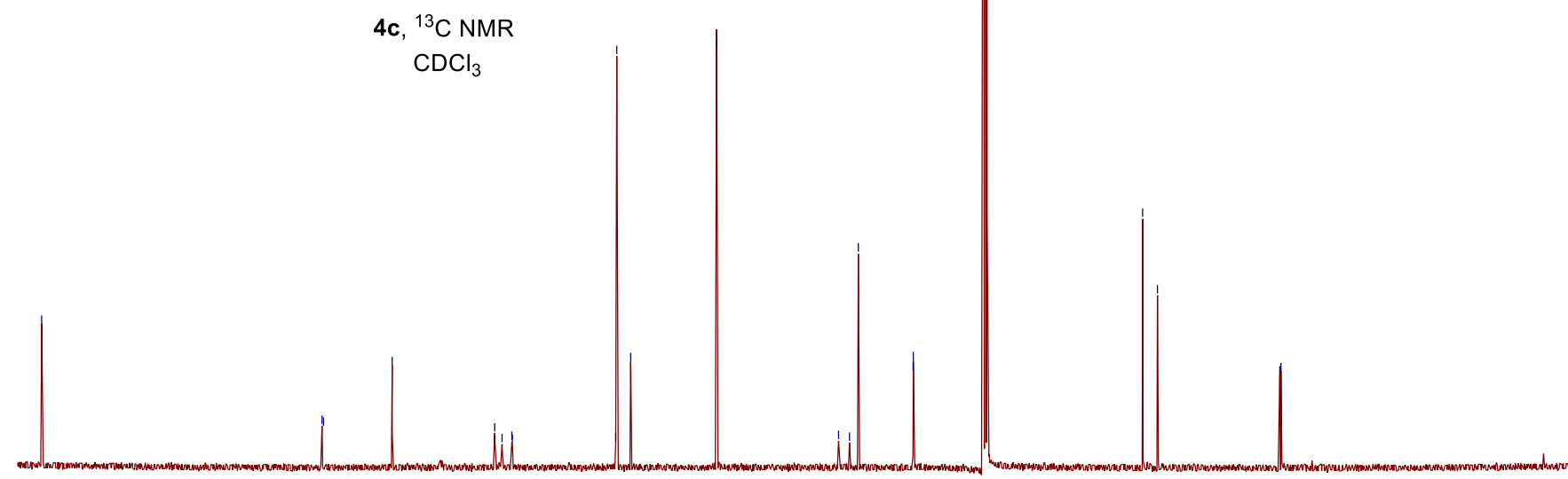

MNwhin

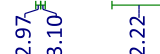

1.
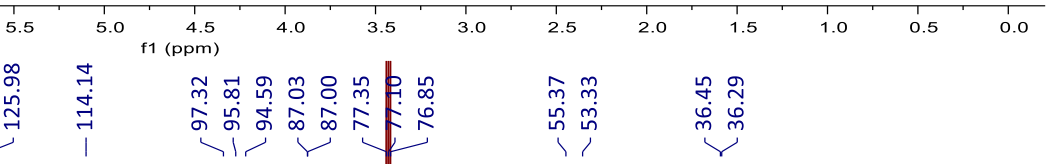

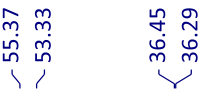

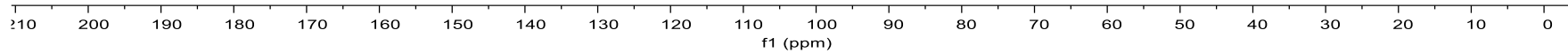




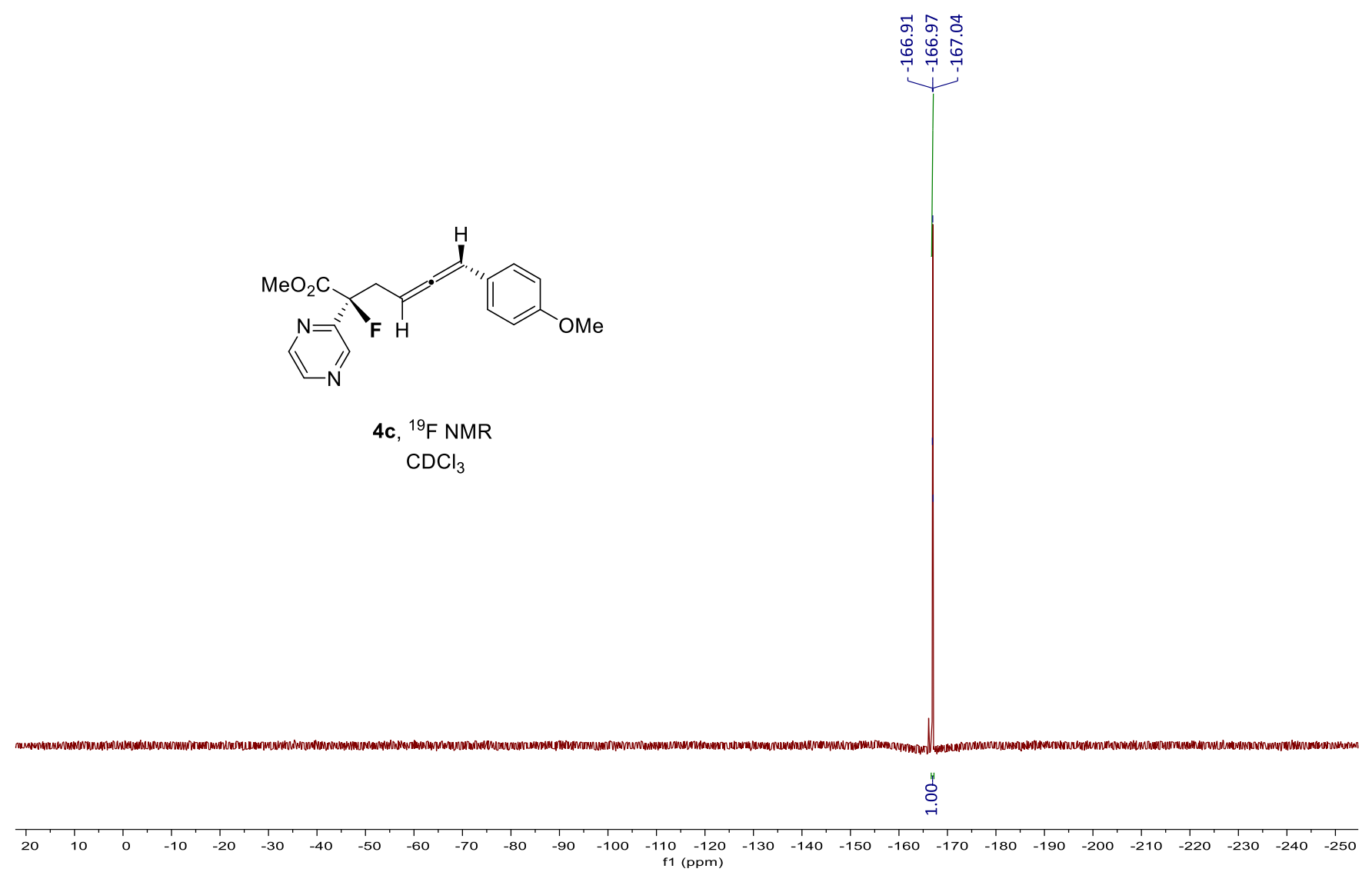



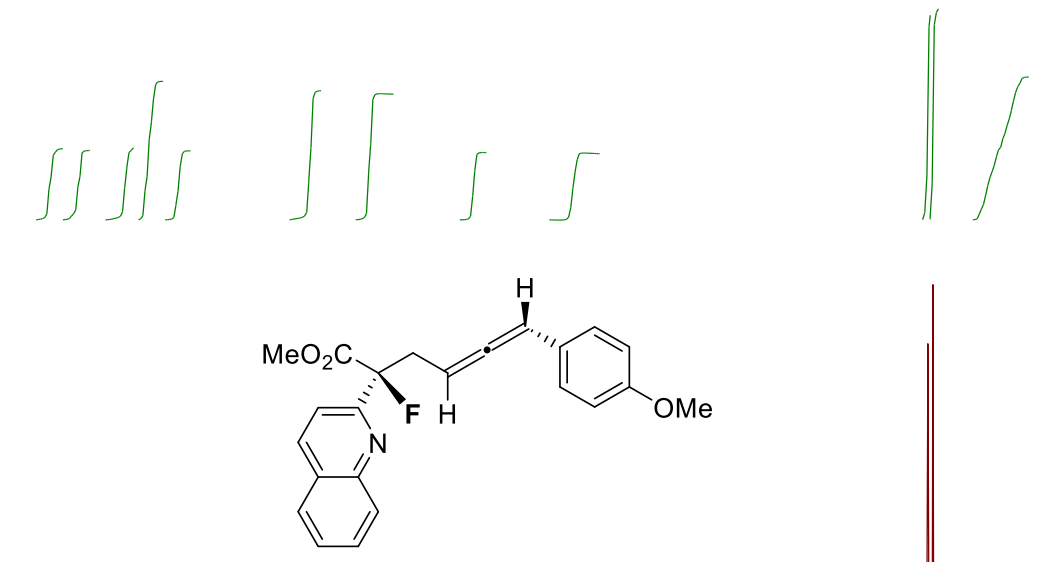

4d, ${ }^{1} \mathrm{H} \mathrm{NMR}$

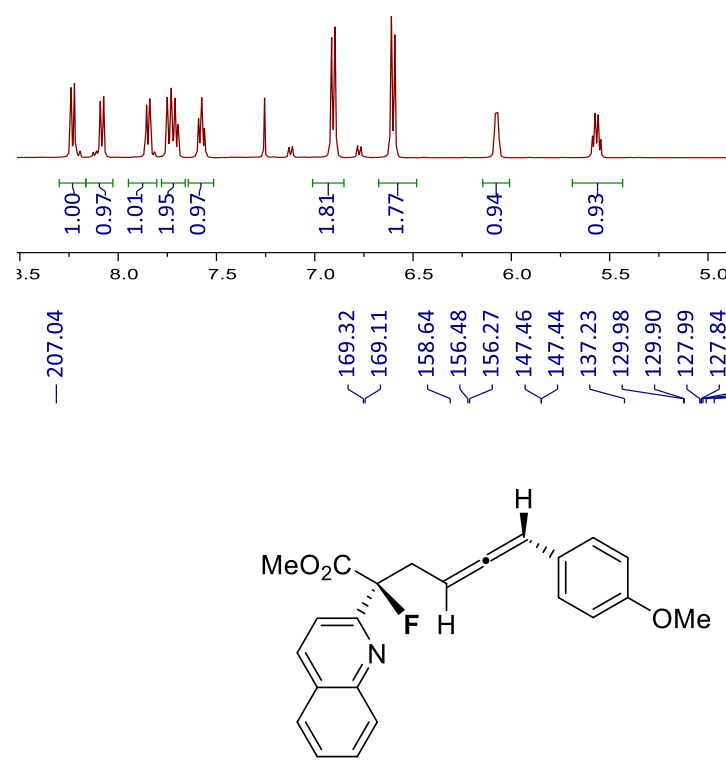

4d, ${ }^{13} \mathrm{C}$ NMR

$\mathrm{CDCl}_{3}$
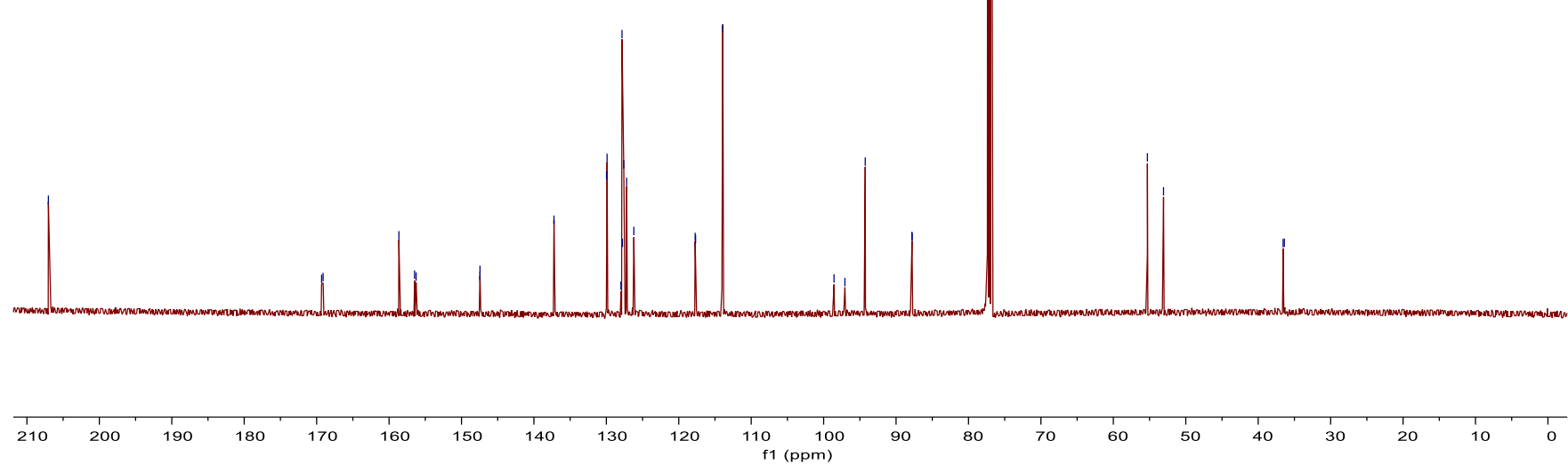
깡 웅

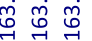

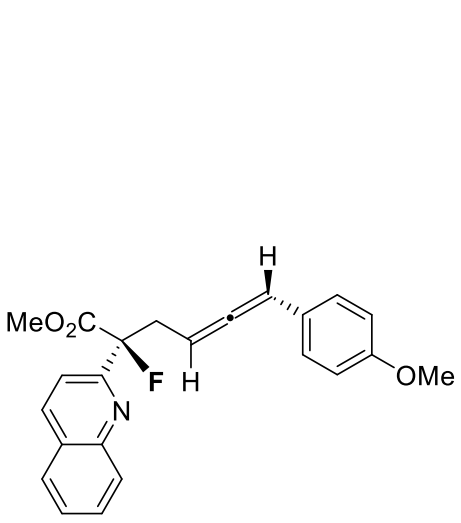

4d, ${ }^{19} \mathrm{~F}$ NMR

$\mathrm{CDCl}_{3}$

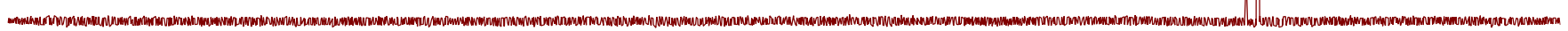

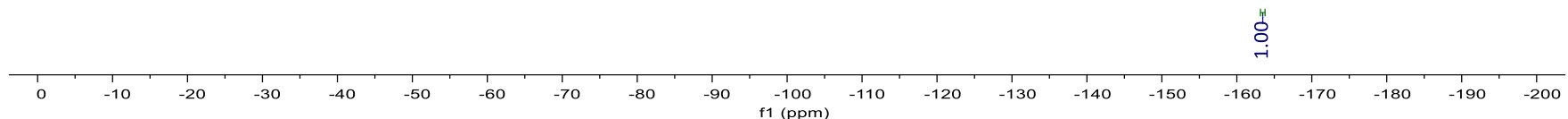




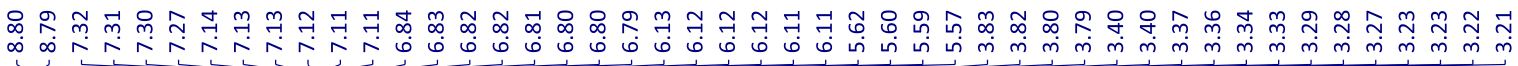

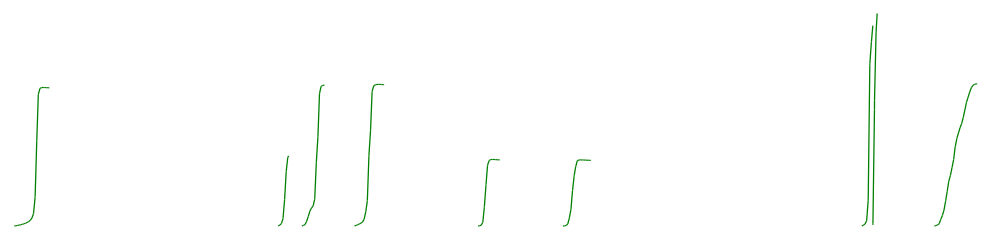<smiles>COc1ccc(/C=C/C=C/CC(F)(C(C)=O)c2ncccn2)cc1</smiles>

4e, ${ }^{1} \mathrm{H}$ NMR

$\mathrm{CDCl}_{3}$

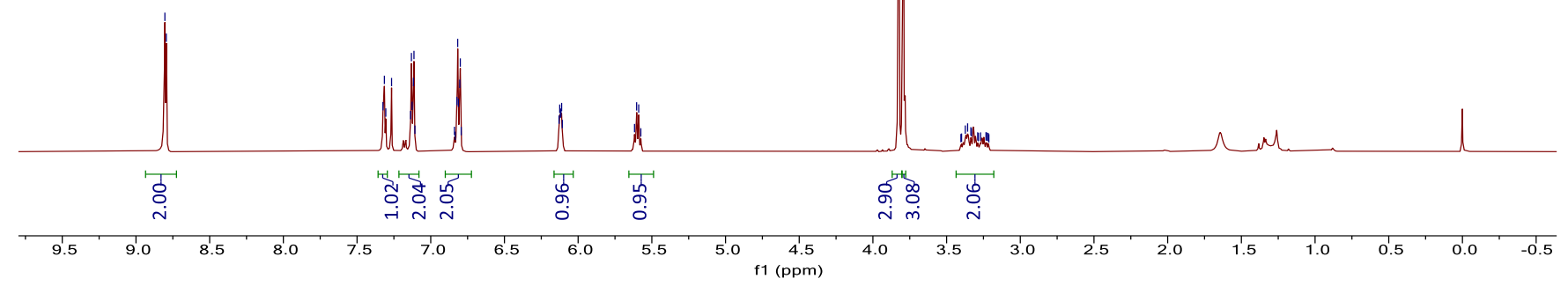

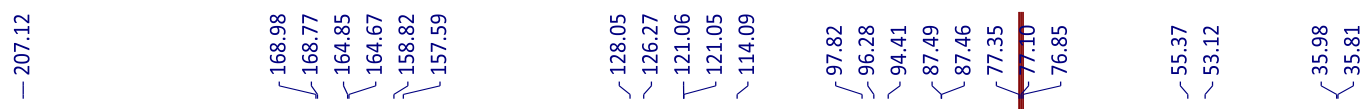<smiles>COc1ccc(/C=C/C2=C=C(CC(F)(C(C)=O)c3ncccn3)C=C2)cc1</smiles>

4e, ${ }^{13} \mathrm{C}$ NMR

$\mathrm{CDCl}_{3}$

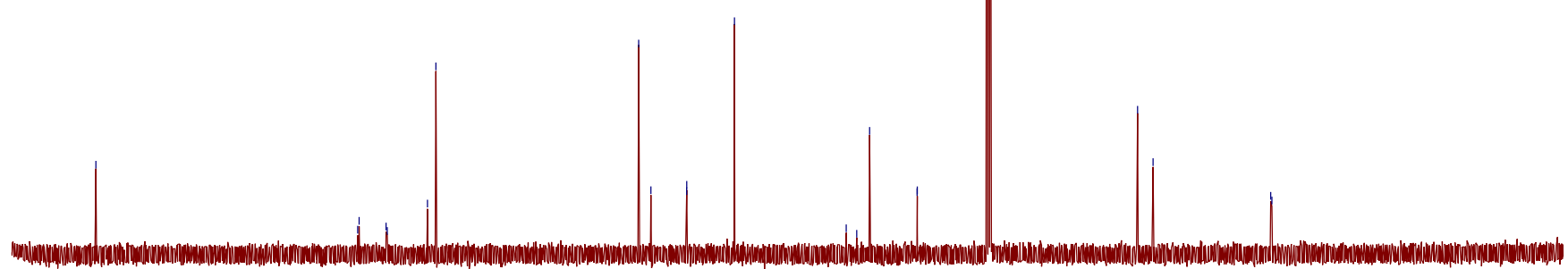




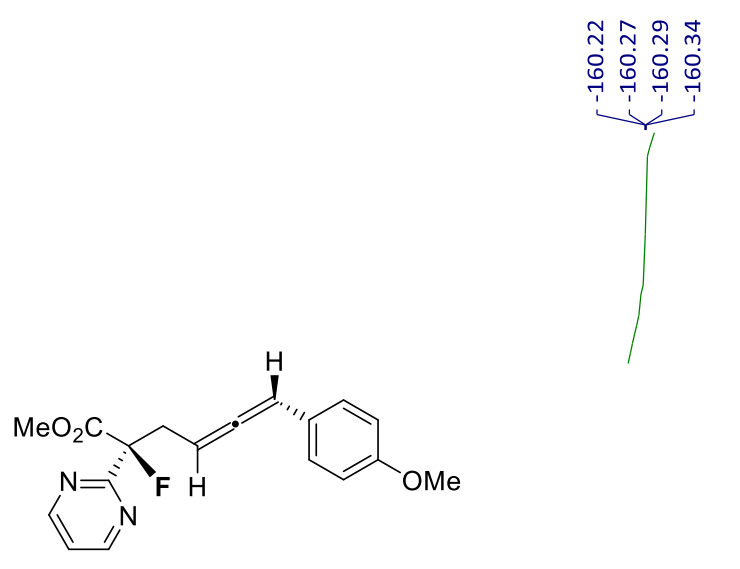

4e, ${ }^{19} \mathrm{~F}$ NMR

$\mathrm{CDCl}_{3}$

\begin{tabular}{|c|c|c|c|c|c|c|c|c|c|c|c|c|c|c|c|c|c|c|c|c|}
\hline & & & & & & & & & 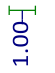 & & & & & & & & & & & \\
\hline $\begin{array}{l}1 \\
-70\end{array}$ & $\begin{array}{l}1 \\
-80\end{array}$ & $\begin{array}{c}1 \\
-90\end{array}$ & $\begin{array}{c}1 \\
-100\end{array}$ & -110 & $\begin{array}{l}1 \\
-120\end{array}$ & $\begin{array}{l}1 \\
-130\end{array}$ & $\begin{array}{l}1 \\
-140\end{array}$ & $\begin{array}{c}1 \\
-150\end{array}$ & $\begin{array}{c}160 \\
-160\end{array}$ & $\begin{array}{c}-170 \\
1(\mathrm{ppm})\end{array}$ & $\begin{array}{c} \\
-180\end{array}$ & $\begin{array}{l}190 \\
-190\end{array}$ & $\begin{array}{l}1 \\
-200\end{array}$ & $\begin{array}{l}1 \\
-210\end{array}$ & $\begin{array}{l}1 \\
-220\end{array}$ & $\begin{array}{l}1 \\
-230\end{array}$ & $\begin{array}{l}1 \\
-240\end{array}$ & $\begin{array}{l}1 \\
-250\end{array}$ & $\begin{array}{c}1 \\
-260\end{array}$ & $\frac{1}{-271}$ \\
\hline
\end{tabular}




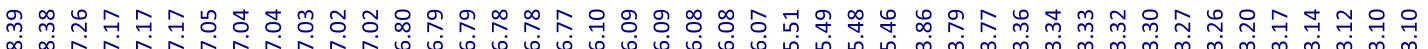

$\infty$ 舟
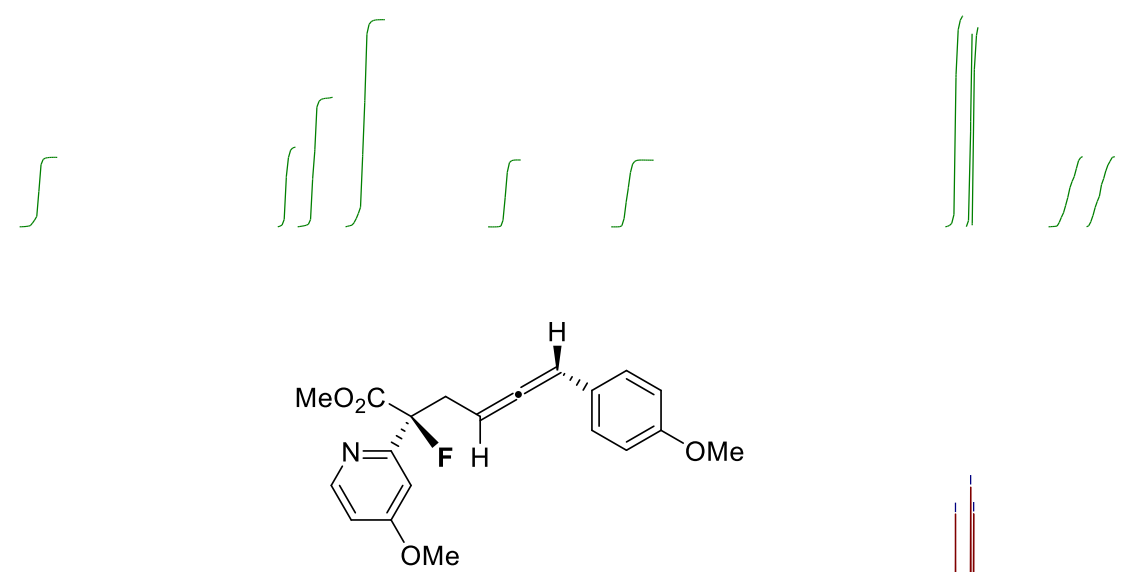

4f, ${ }^{1} \mathrm{H}$ NMR

$\mathrm{CDCl}_{3}$
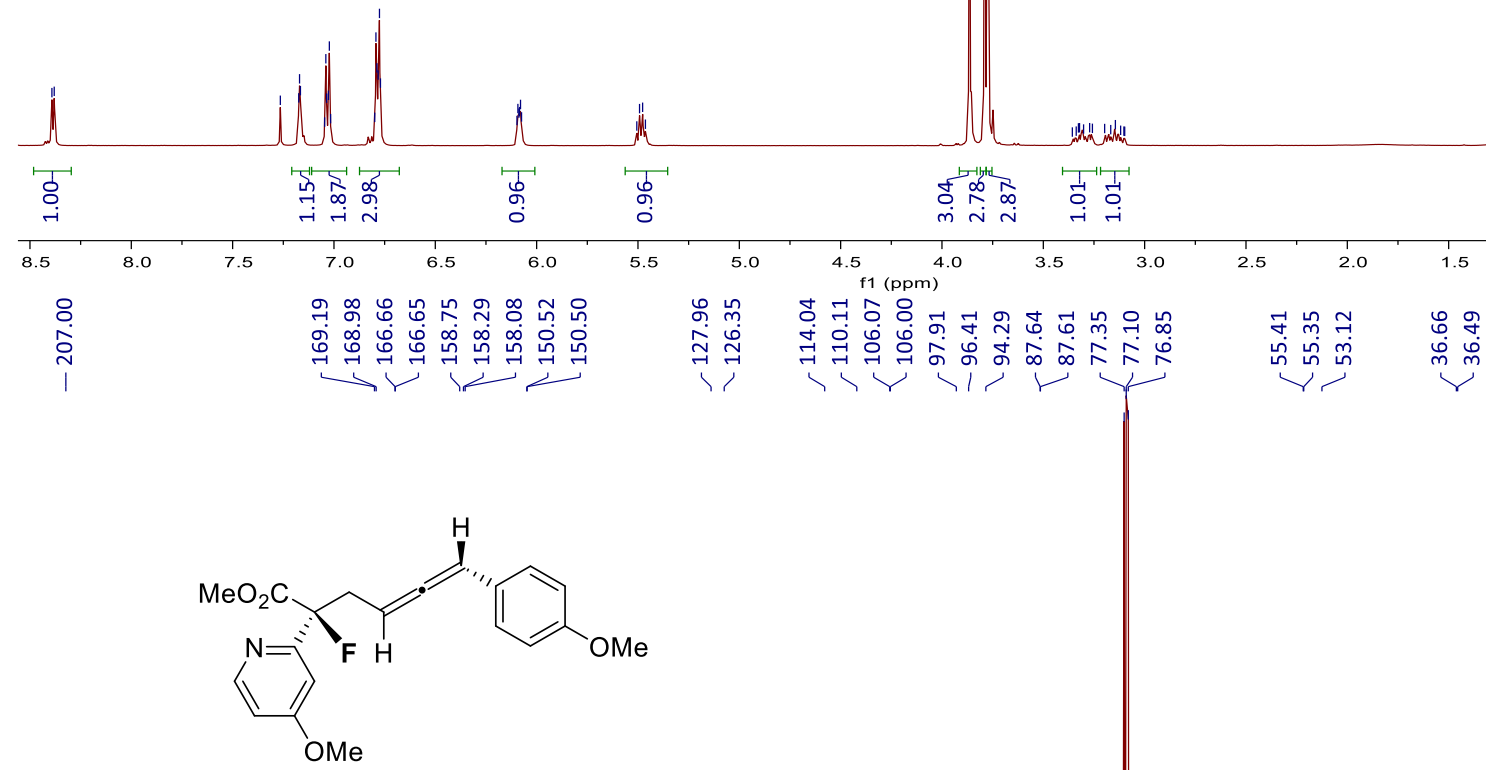

4f, ${ }^{13} \mathrm{C}$ NMR $\mathrm{CDCl}_{3}$
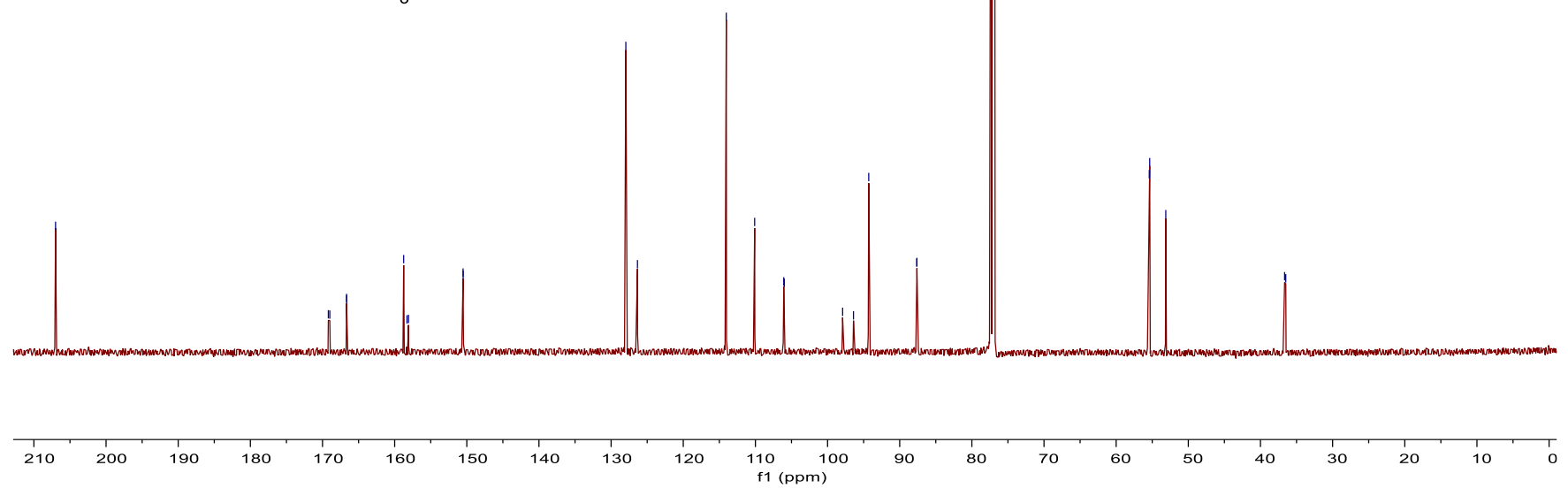
मेन के

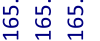
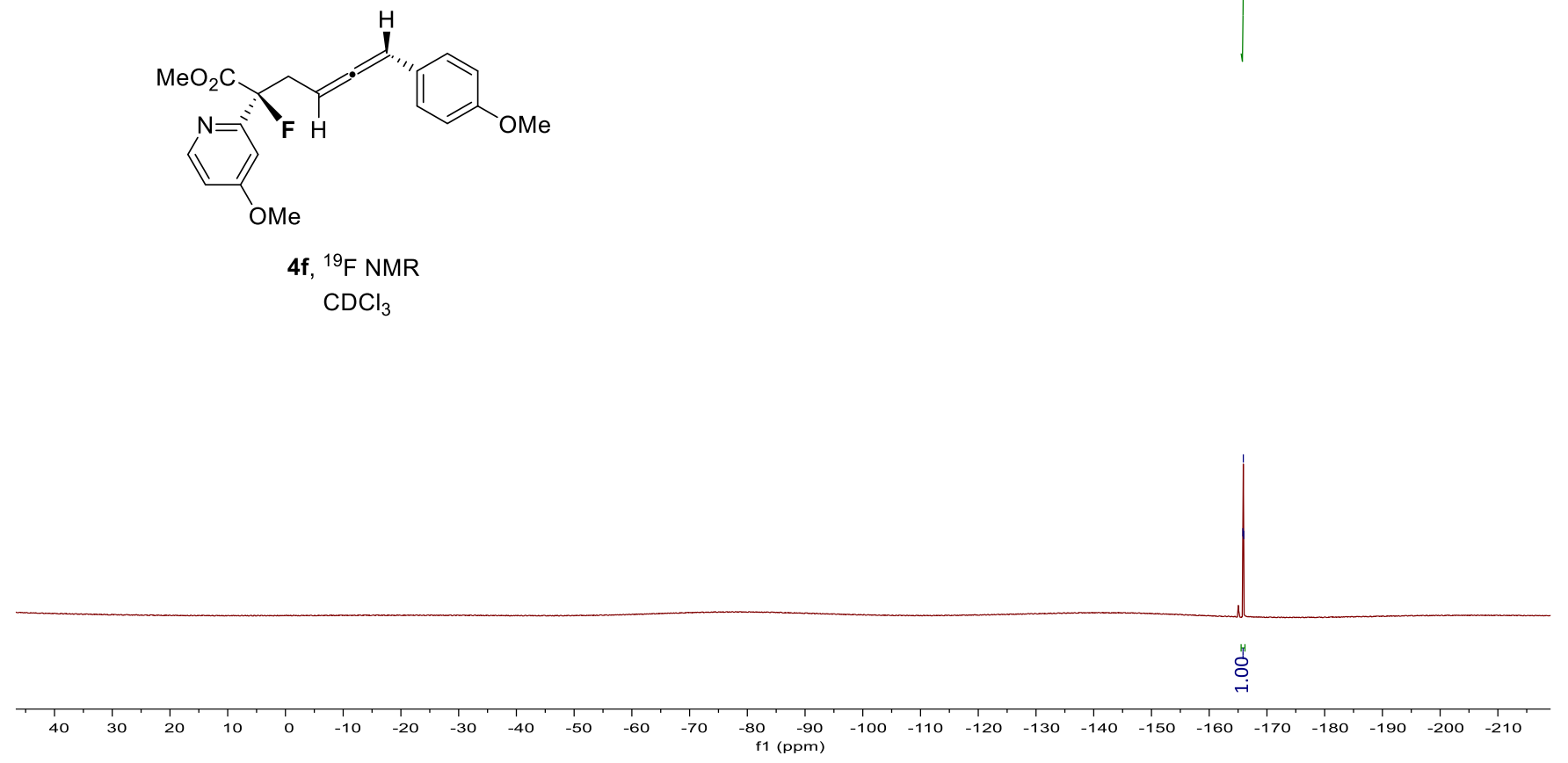


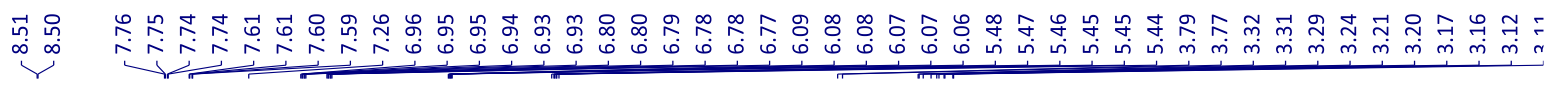

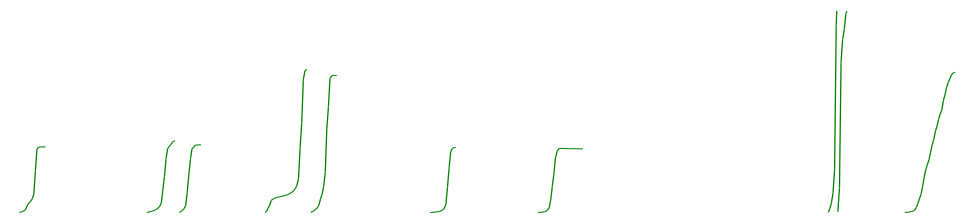<smiles>COc1ccc(/C=C/CC(F)(C(C)=O)c2ccc(Cl)cn2)cc1</smiles>

4g, ${ }^{1} \mathrm{H}$ NMR $\mathrm{CDCl}_{3}$

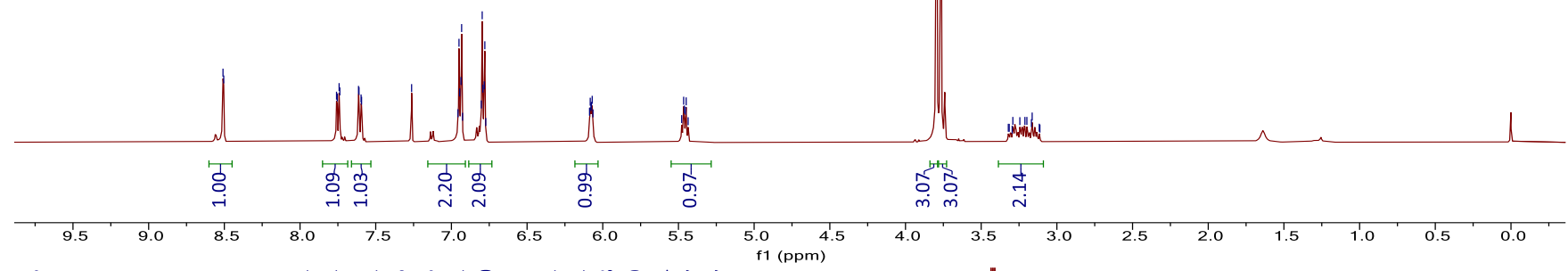

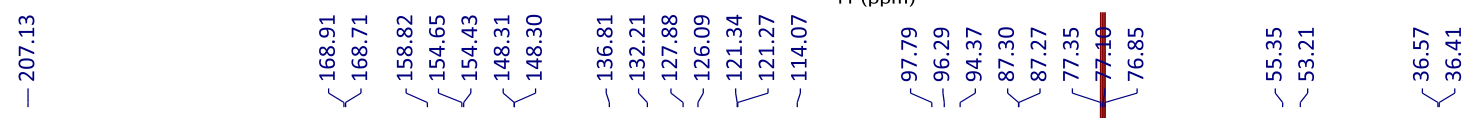

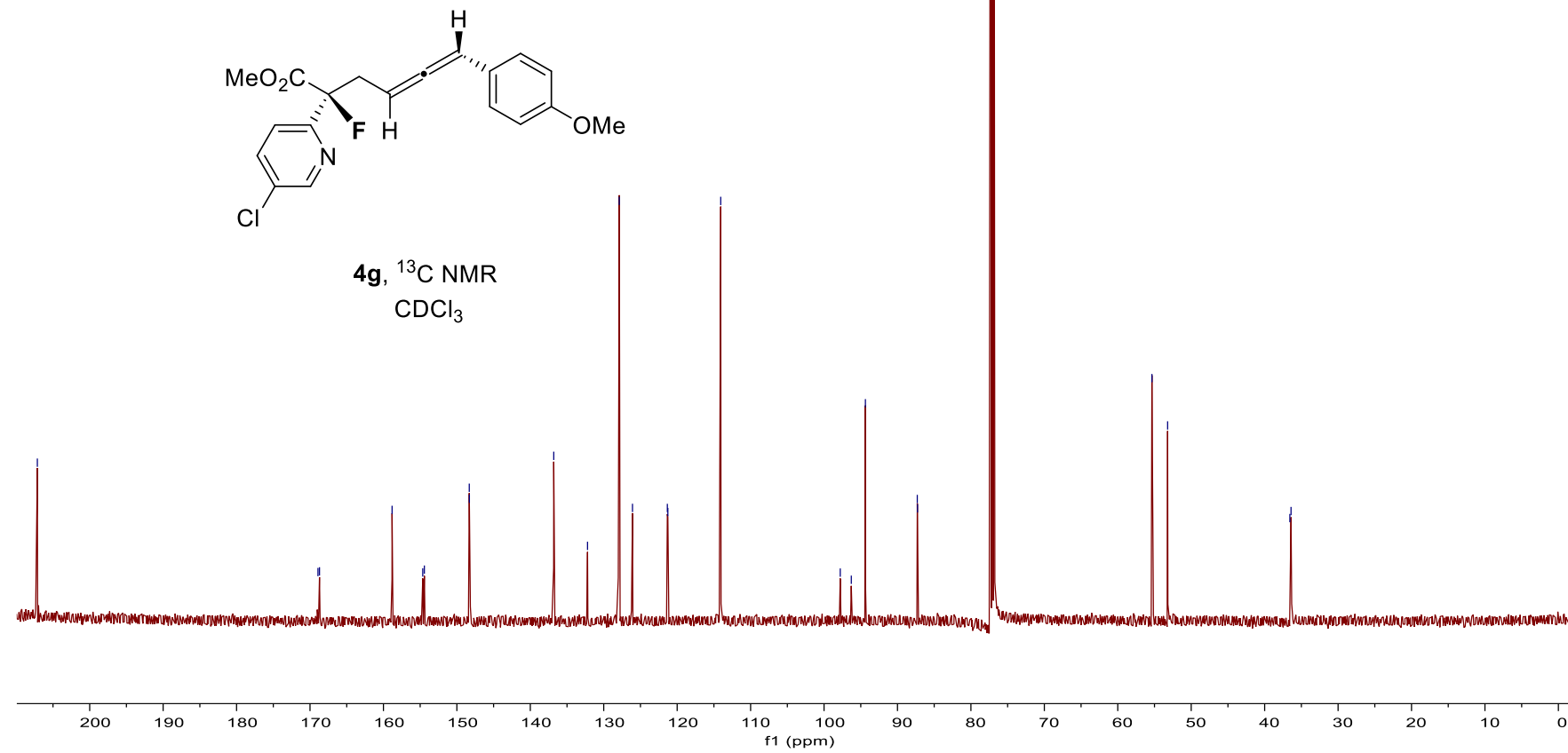


$\therefore \stackrel{\infty}{\wedge}$

氙兽兽

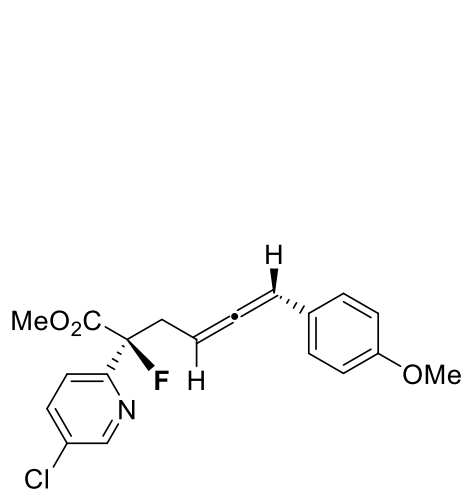

4g, ${ }^{19}$ F NMR

$\mathrm{CDCl}_{3}$

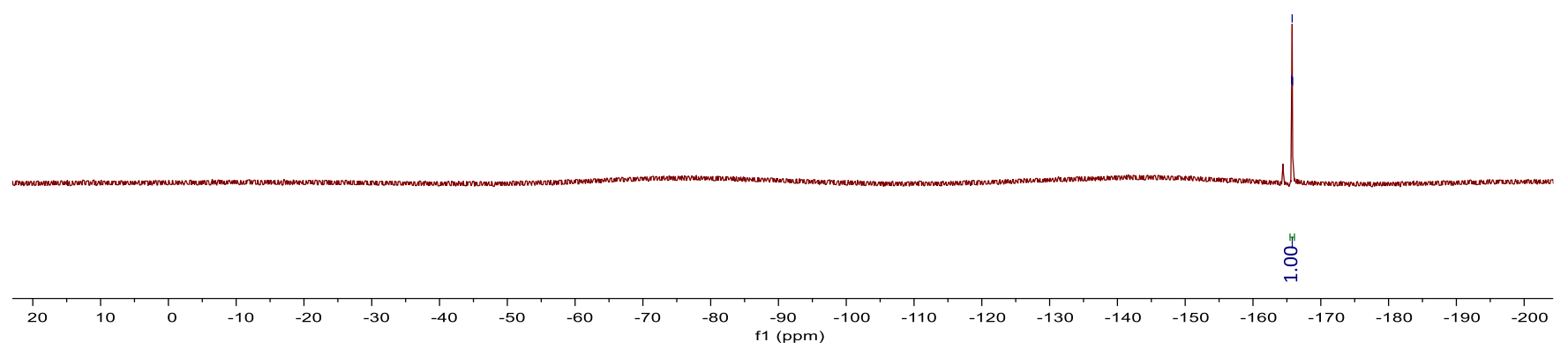




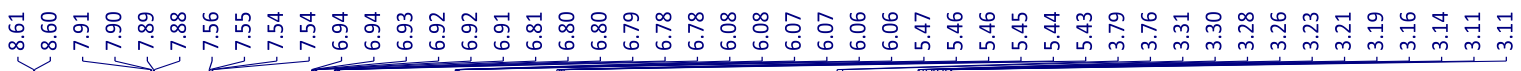
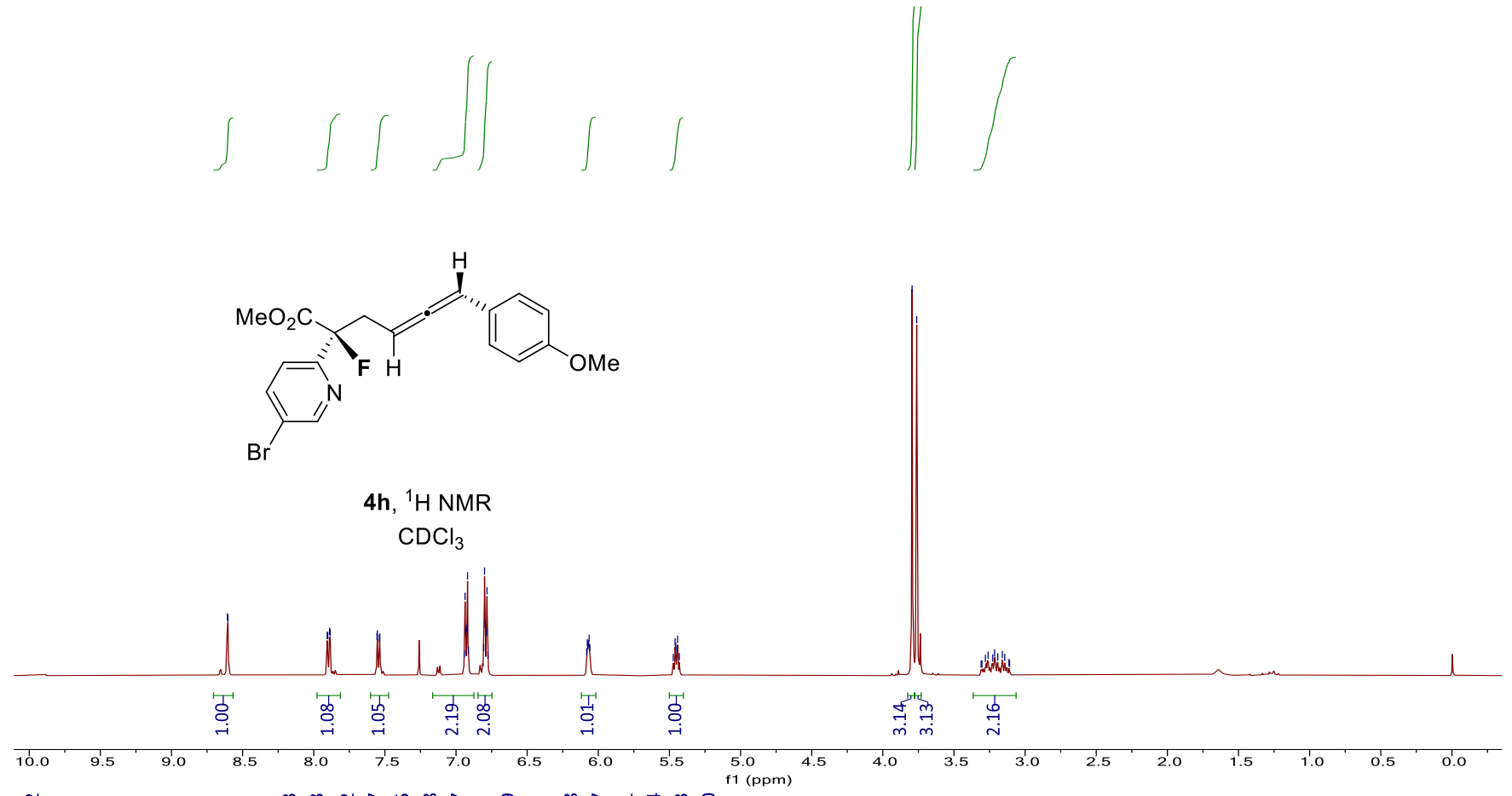

苟

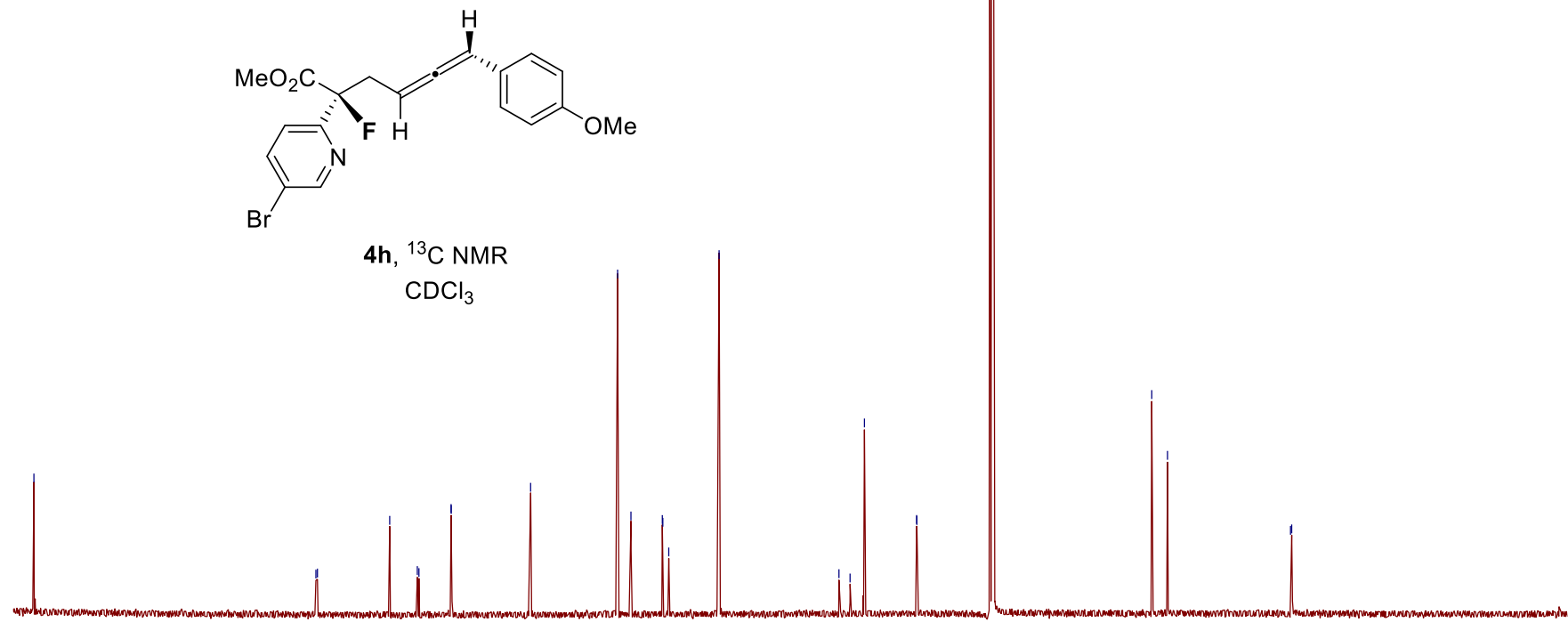


$\stackrel{2}{2} \sim \infty$

它志志

7

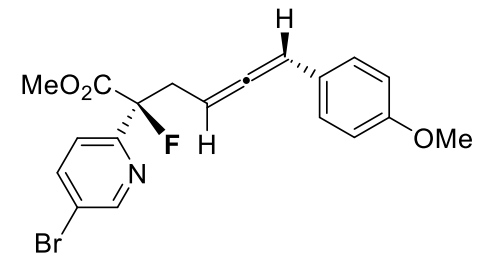

4h, ${ }^{19} \mathrm{~F}$ NMR

$\mathrm{CDCl}_{3}$

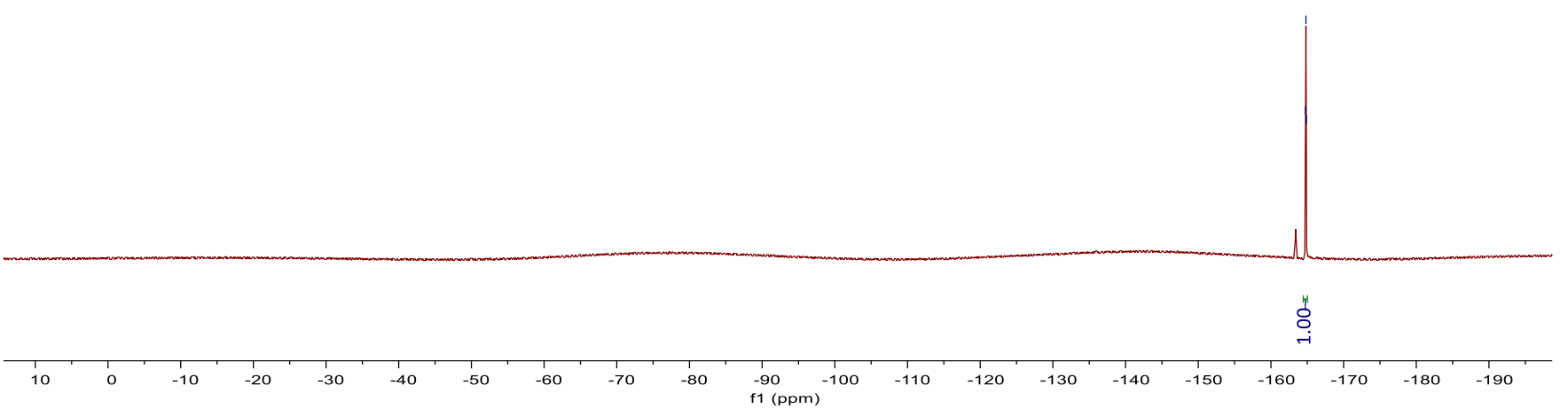



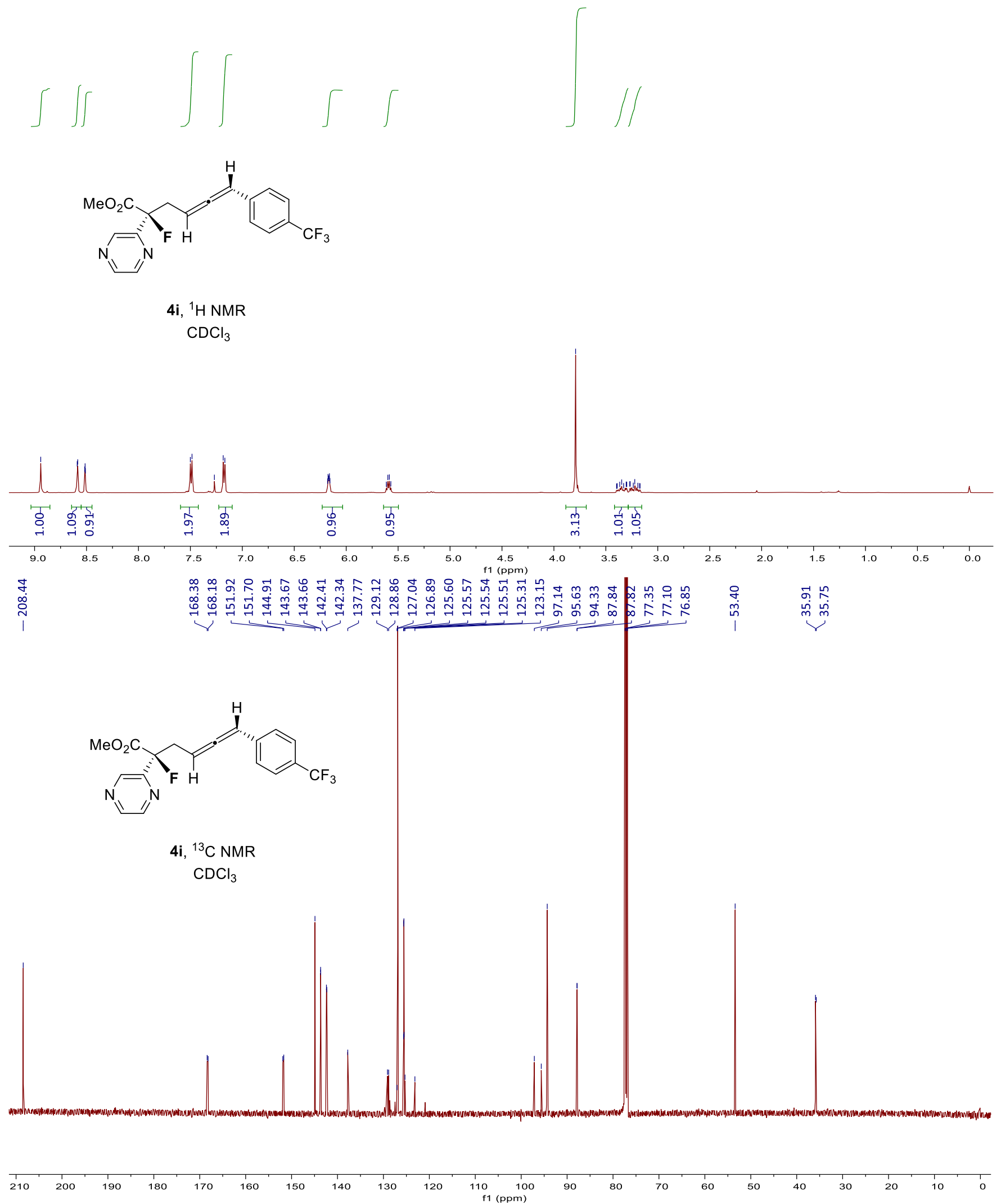


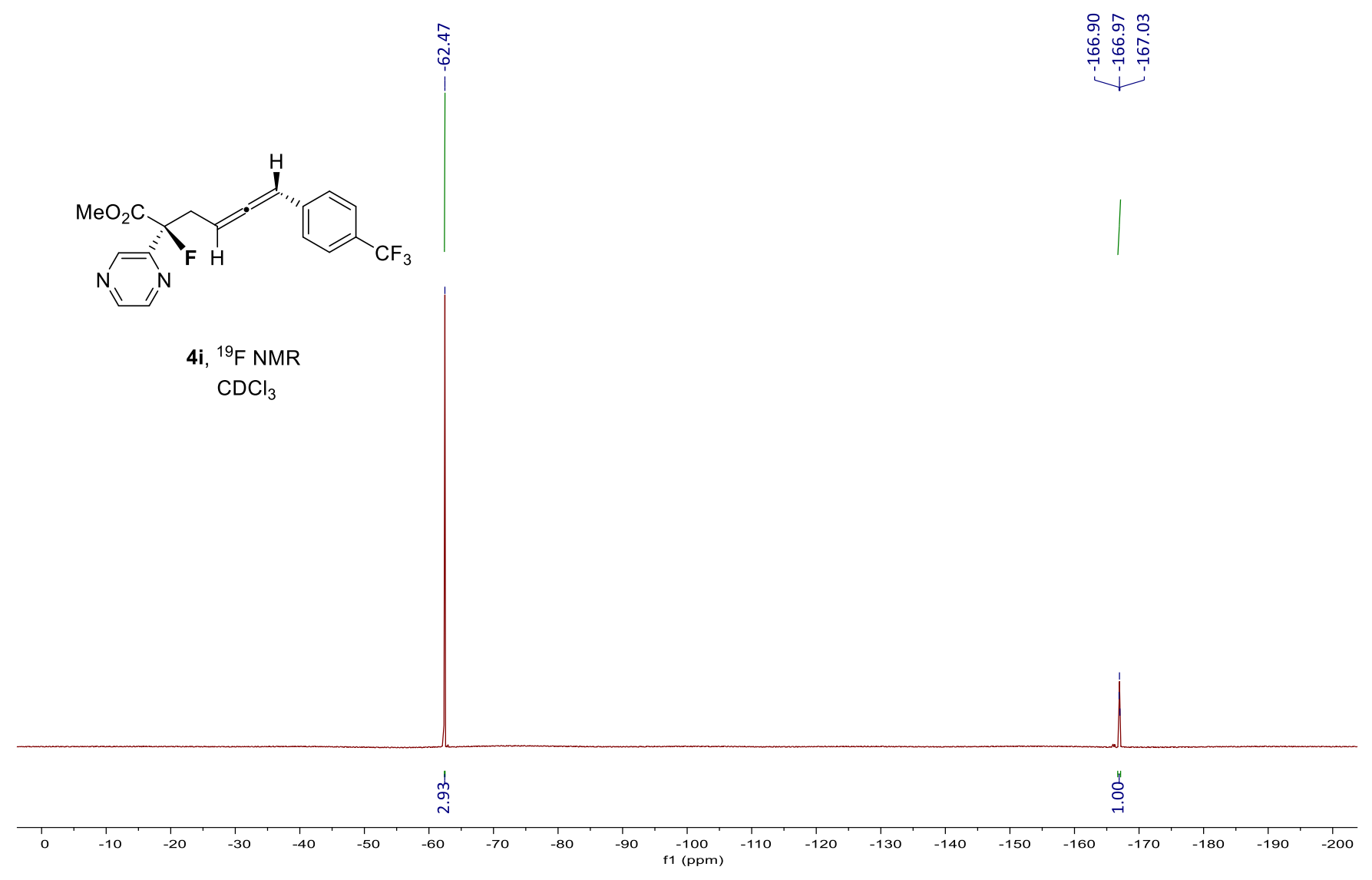




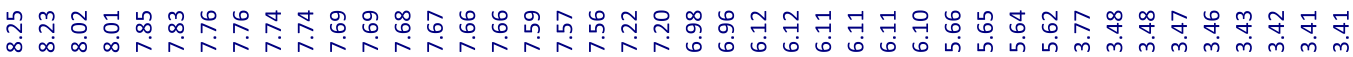
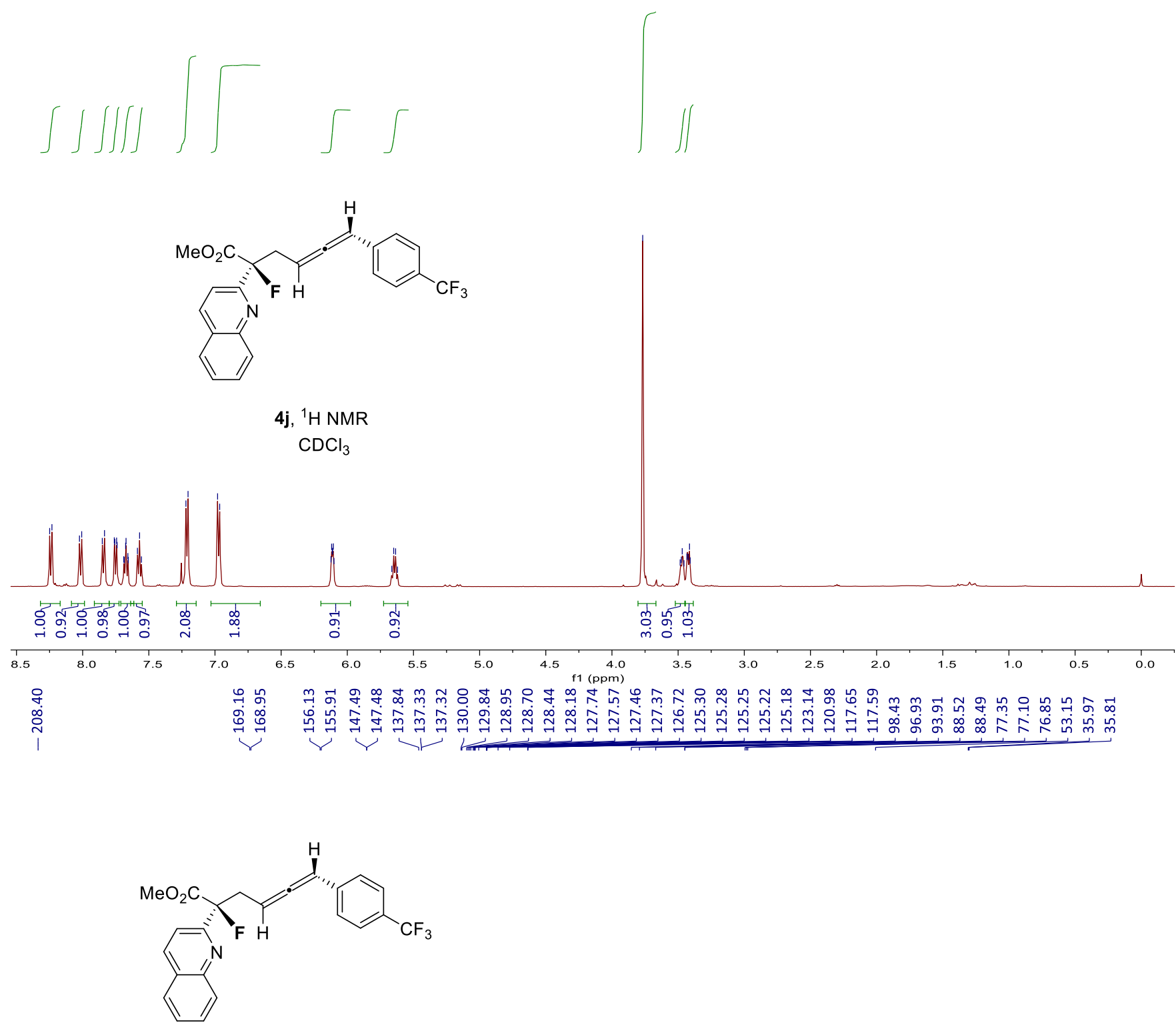

4j, ${ }^{13} \mathrm{C}$ NMR $\mathrm{CDCl}_{3}$

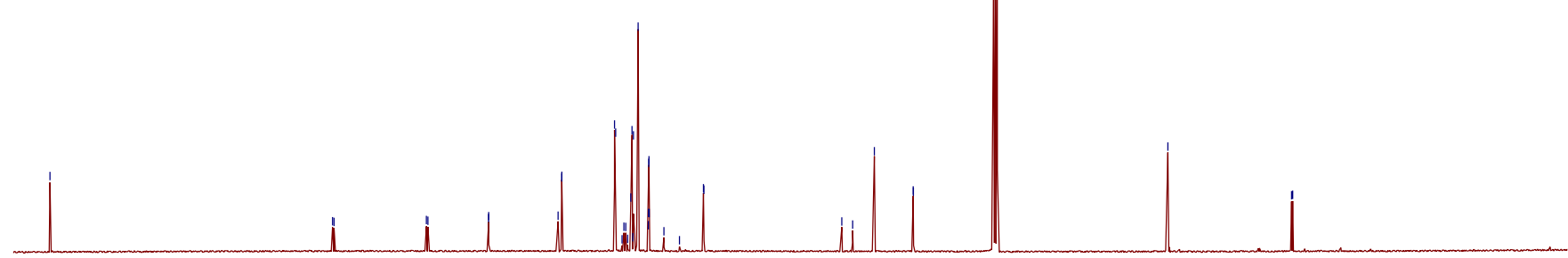

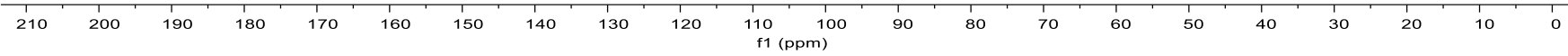




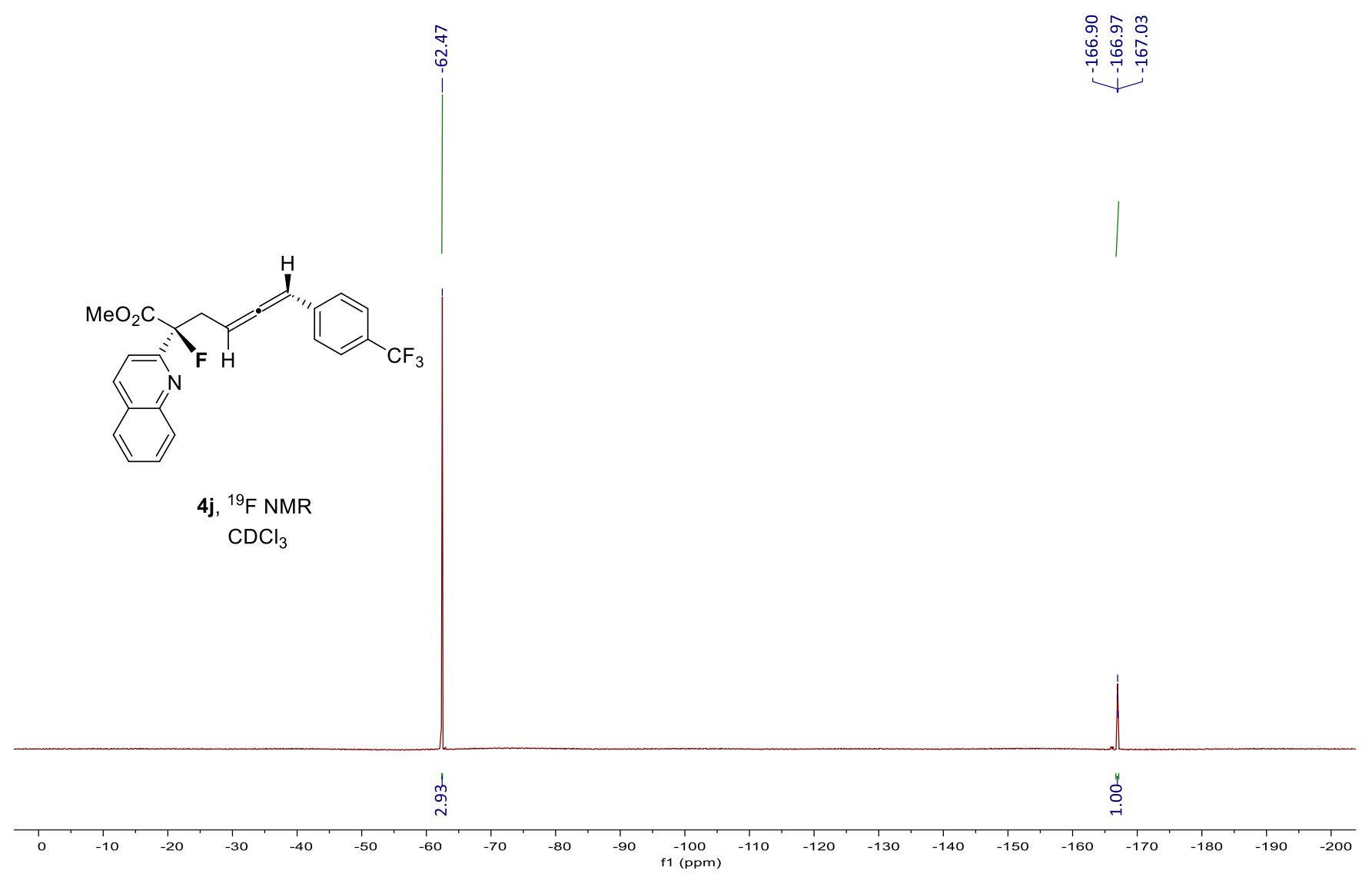




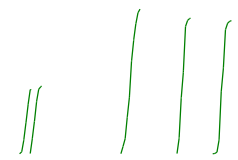<smiles>CCCCI</smiles>
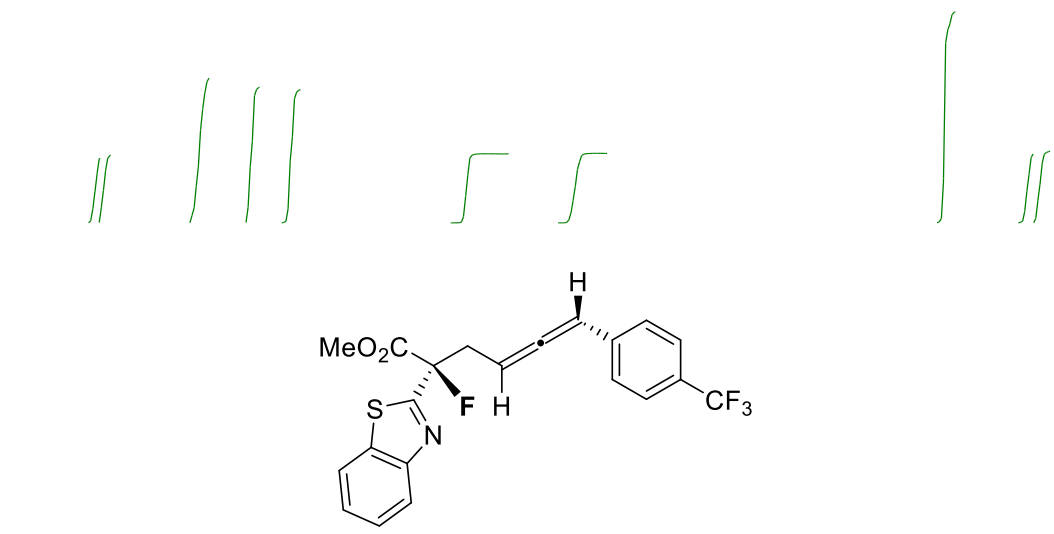

$$
\begin{aligned}
& \text { 4k, }{ }^{1} \mathrm{H} \mathrm{NMR} \\
& \mathrm{CDCl}_{3}
\end{aligned}
$$

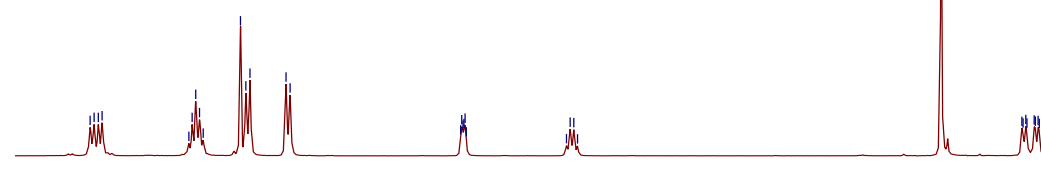

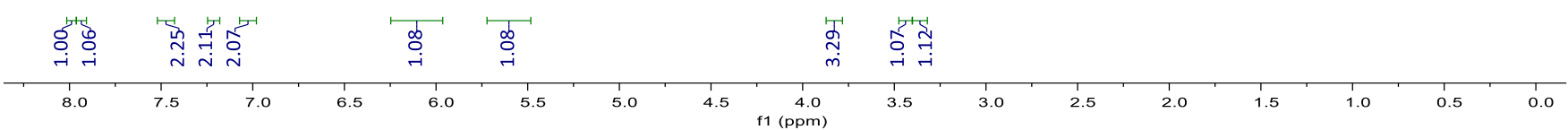
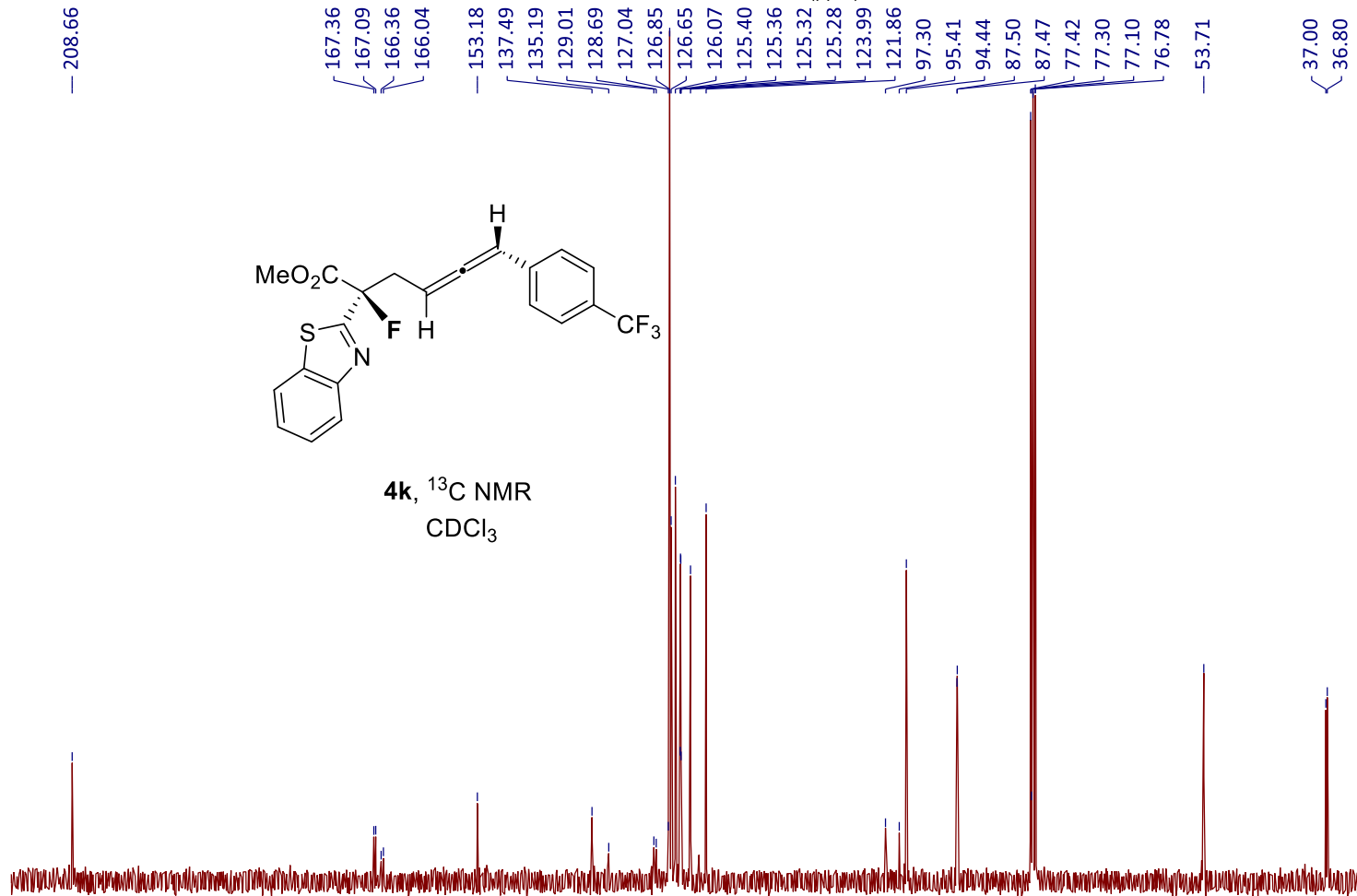

4k, ${ }^{13} \mathrm{C}$ NMR $\mathrm{CDCl}_{3}$

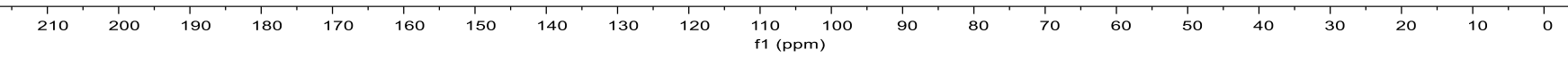




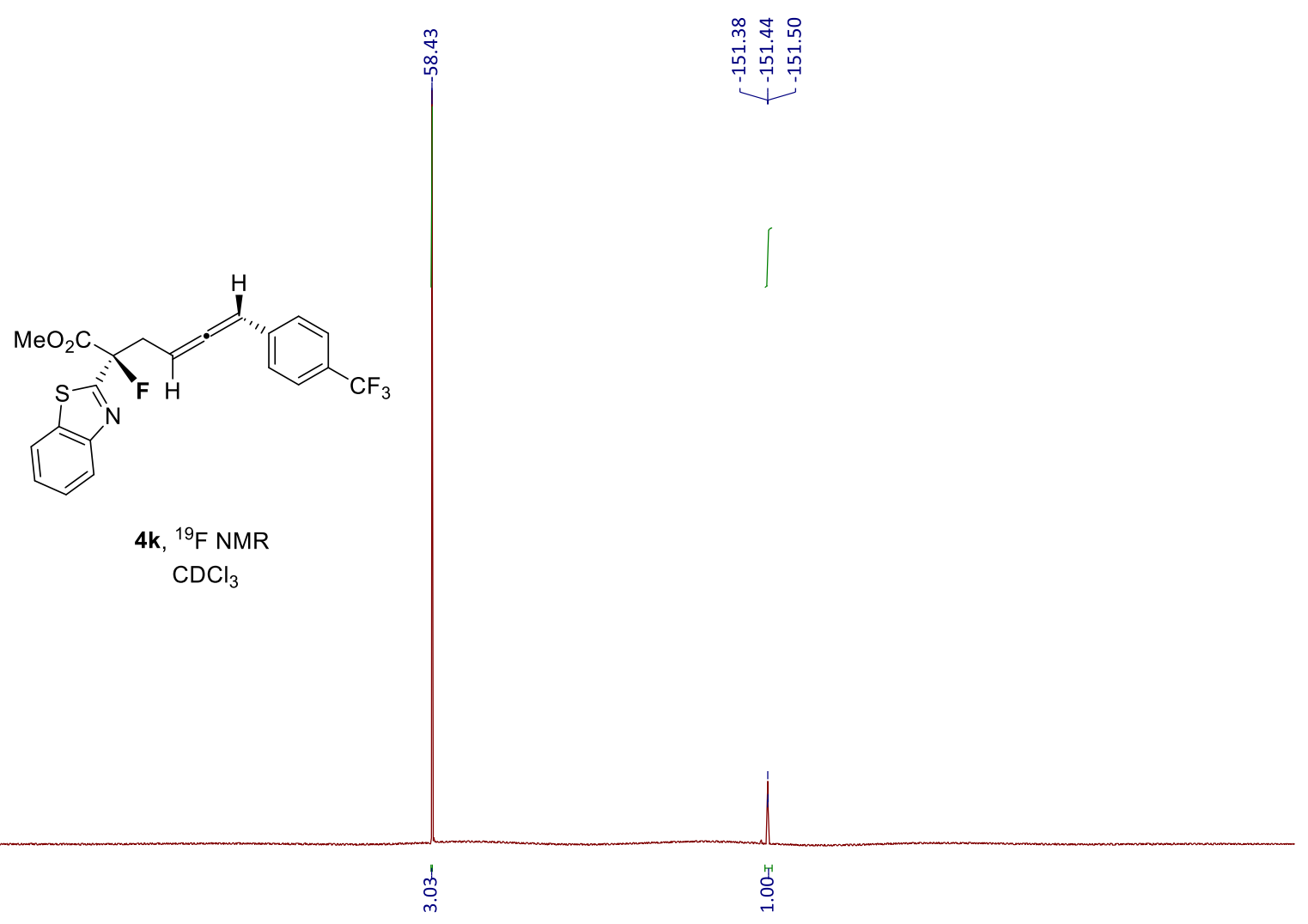

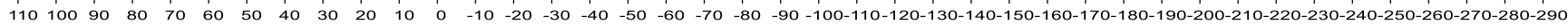
f1 (ppm) 


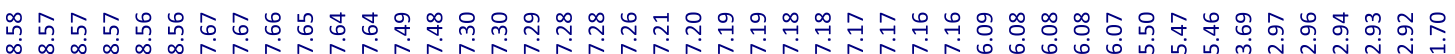
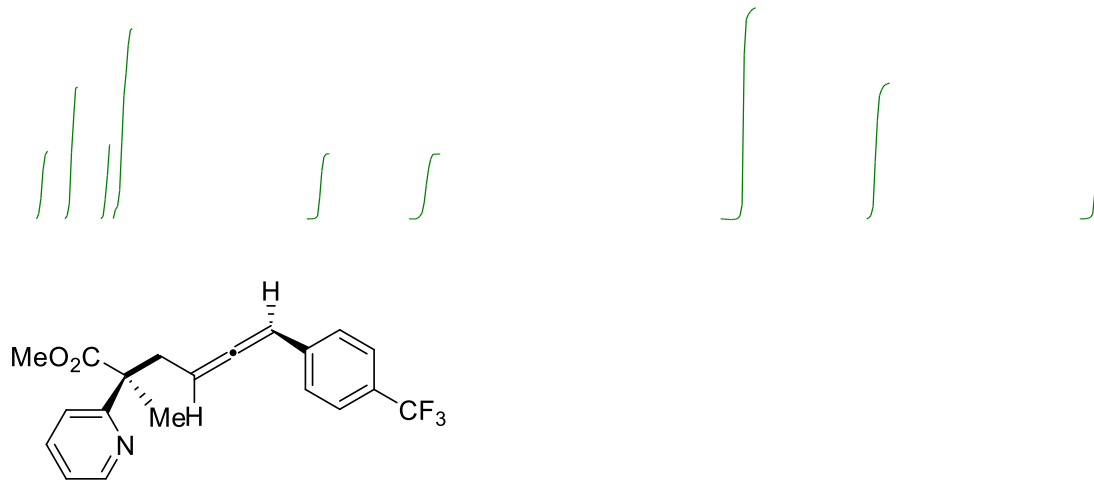

4I, ${ }^{1} \mathrm{H}$ NMR

$\mathrm{CDCl}_{3}$
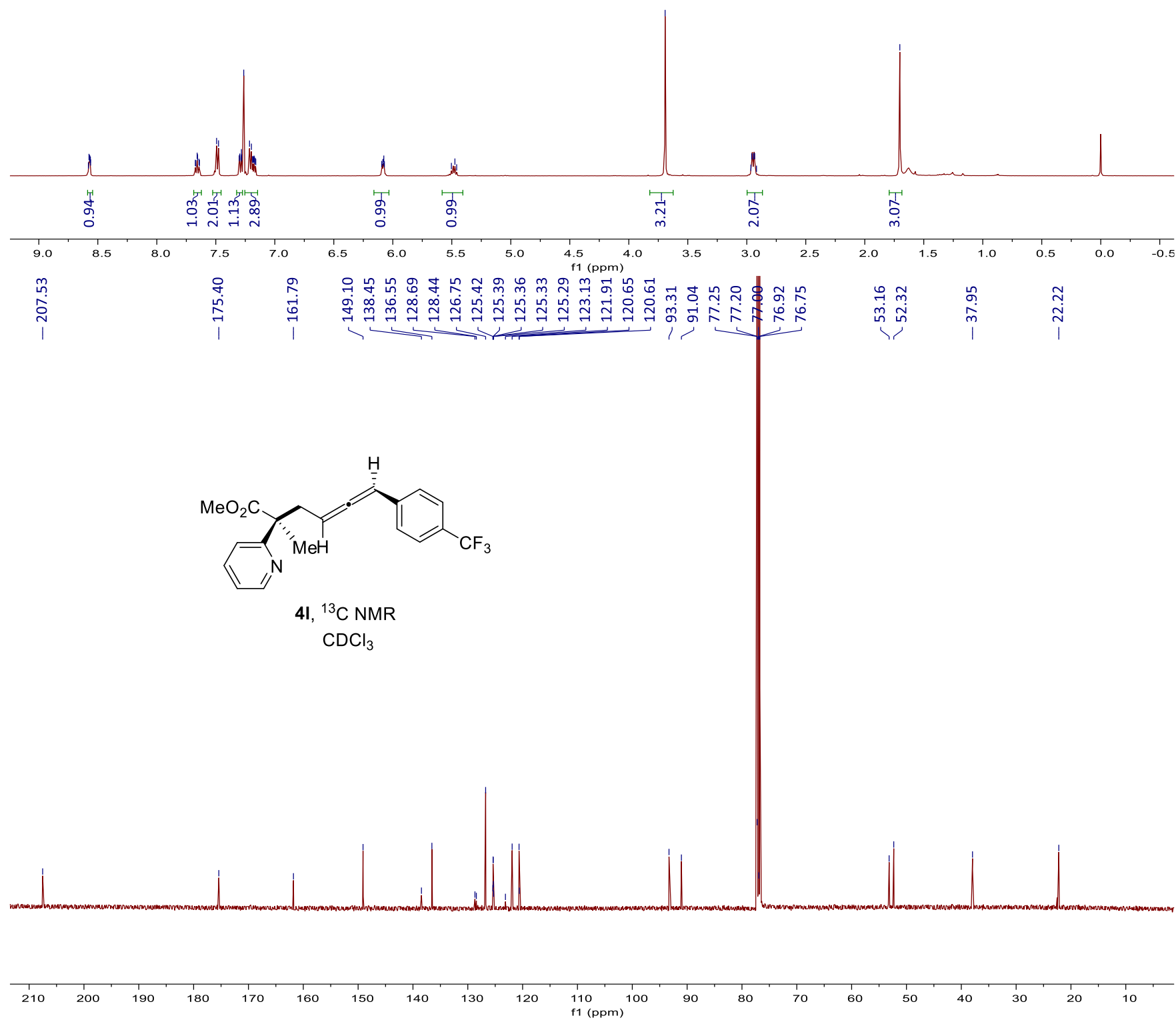


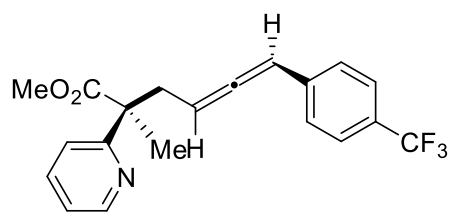

4I, ${ }^{19} \mathrm{~F}$ NMR

$\mathrm{CDCl}_{3}$ 

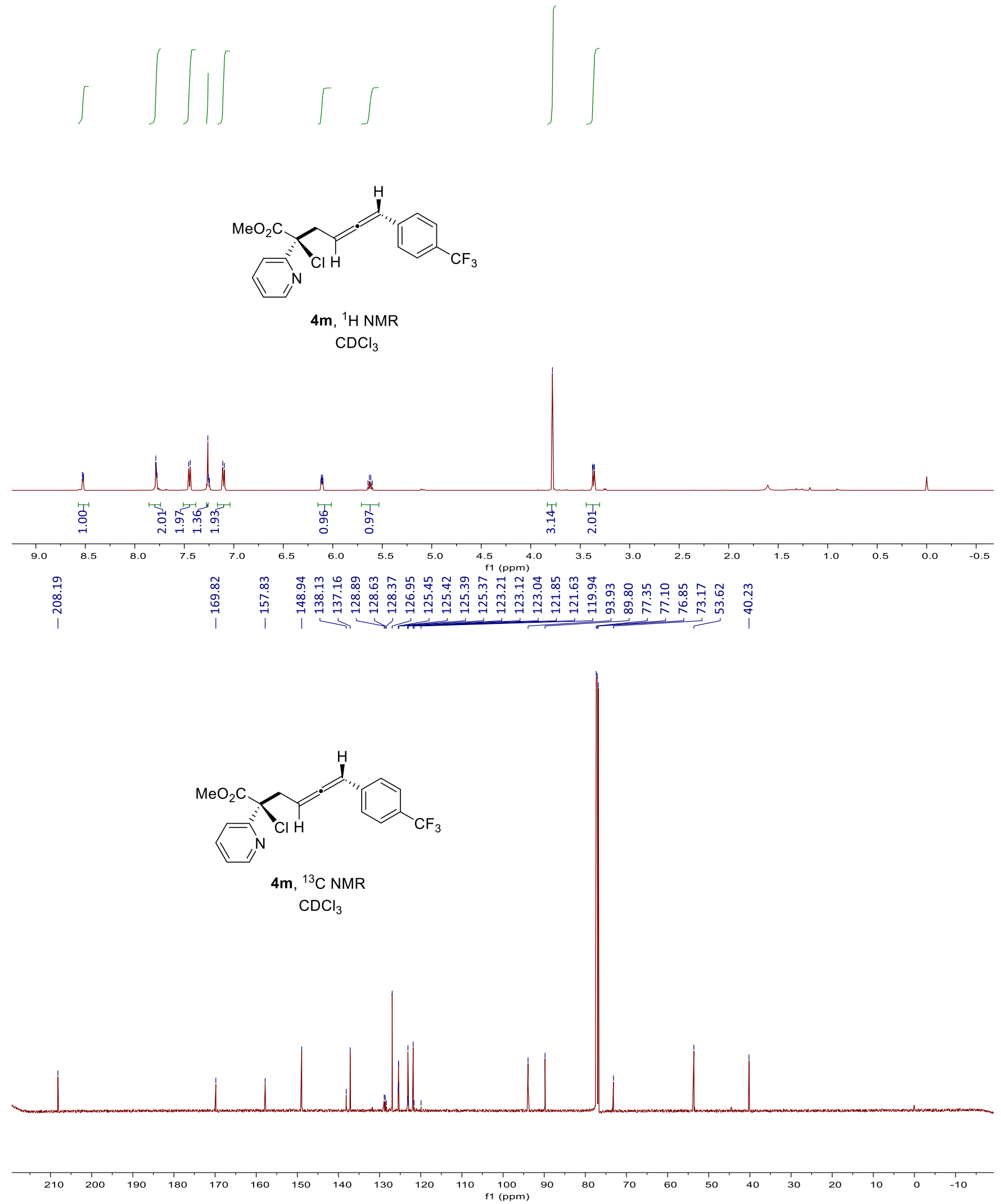


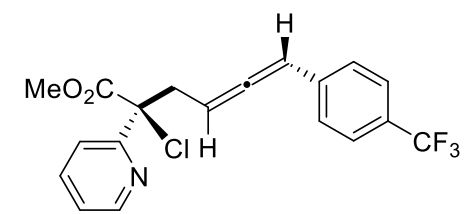

4m, ${ }^{19} \mathrm{~F}$ NMR

$\mathrm{CDCl}_{3}$ 
ఫُ

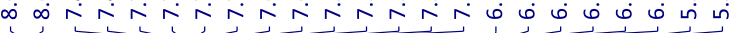

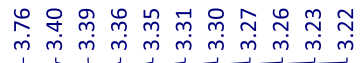

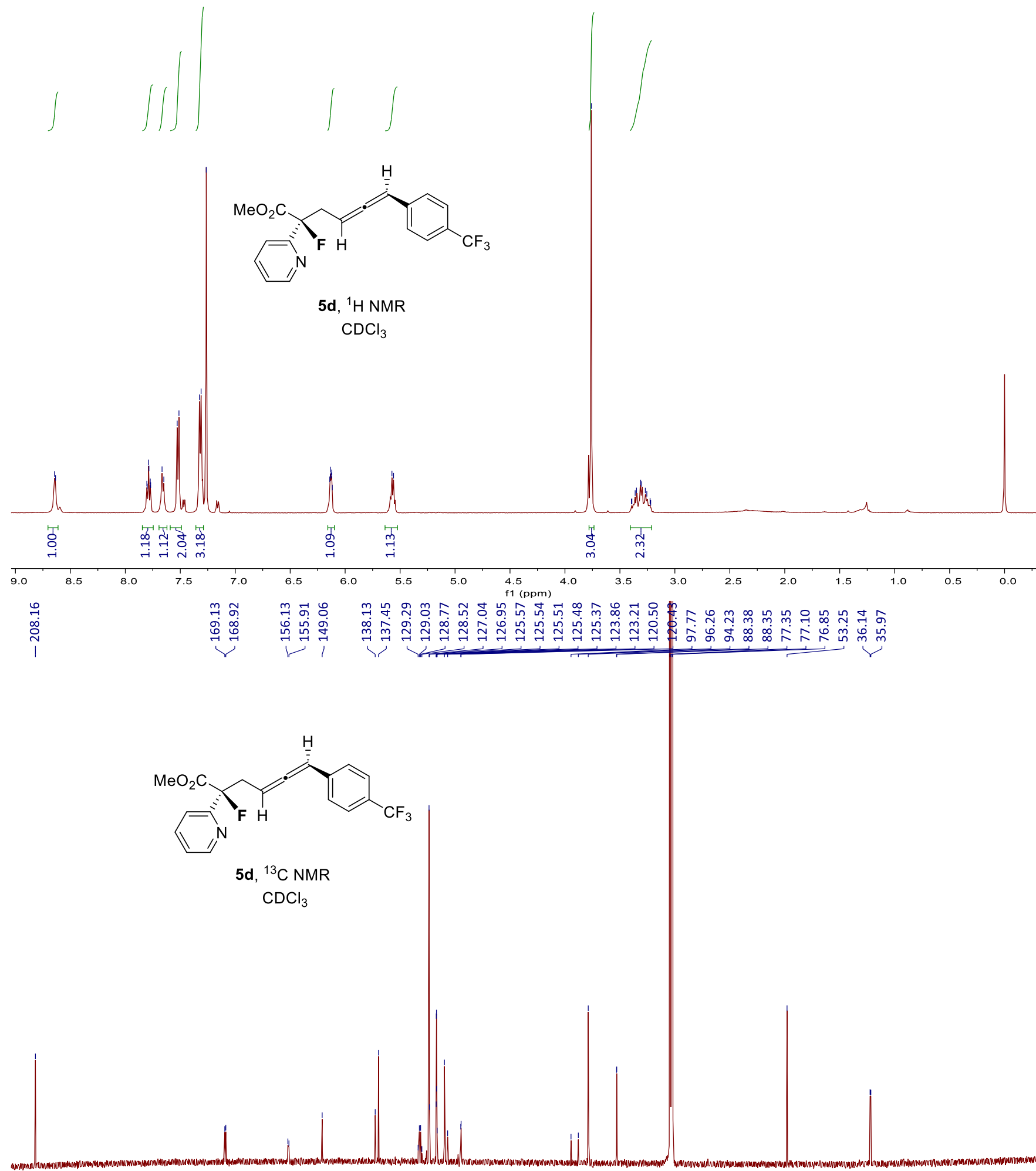

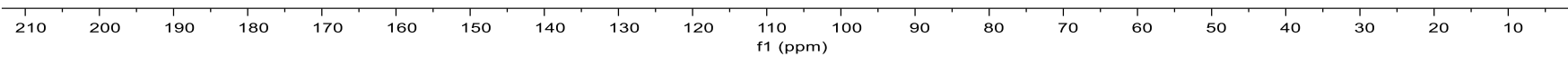




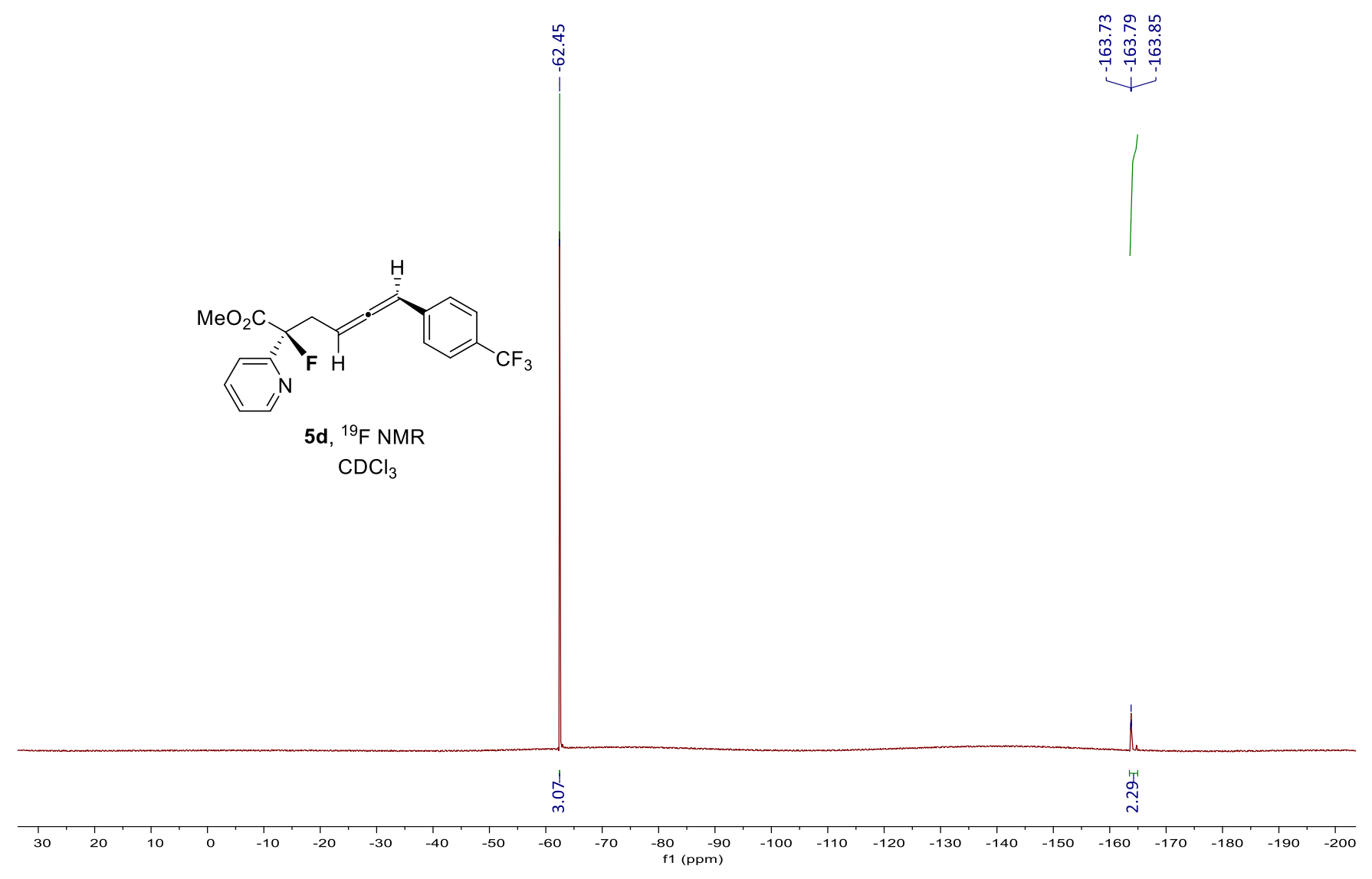




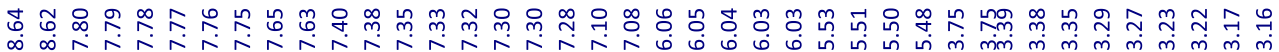

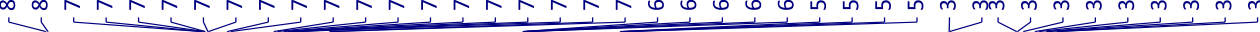
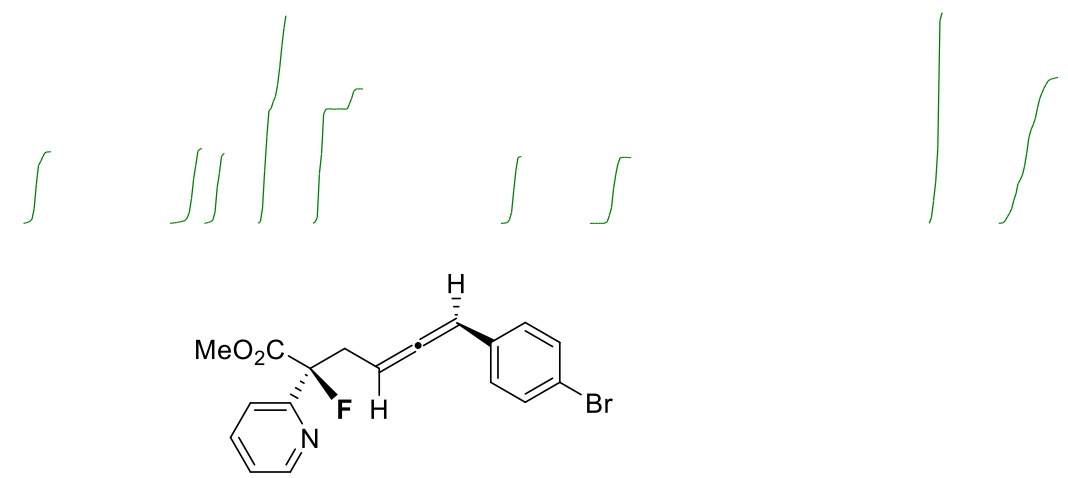

5i, ${ }^{1} \mathrm{H}$ NMR

$\mathrm{CDCl}_{3}$

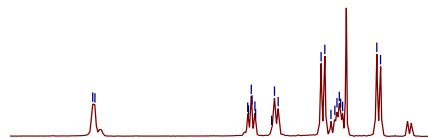

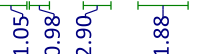

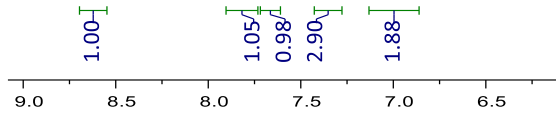

(1)

$\stackrel{\infty}{+}$

กุ

ֻุ

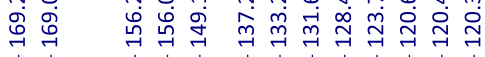

蔺,

œ

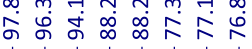

रा,

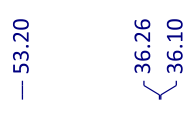

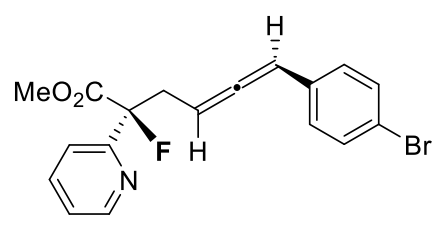

5i, ${ }^{13} \mathrm{C}$ NMR

$\mathrm{CDCl}_{3}$

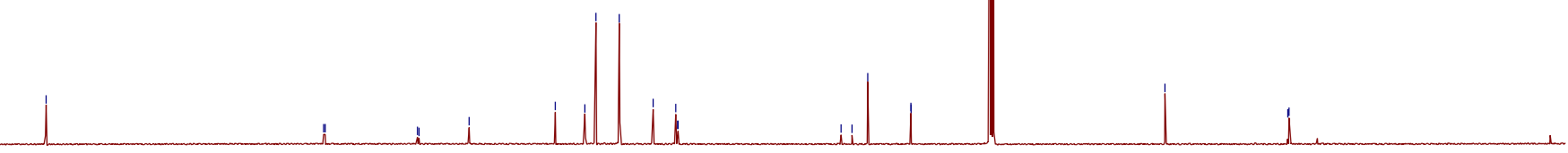

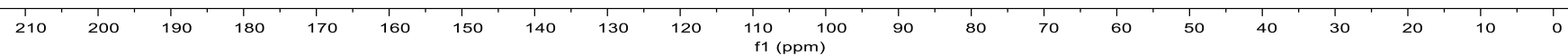


ํㅜㅇㅛ.

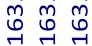

i'i's

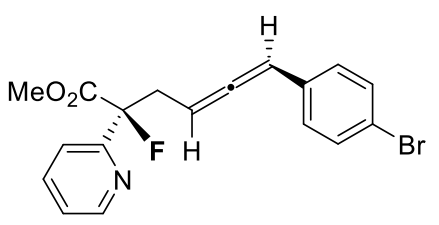

5i, ${ }^{19} \mathrm{~F} \mathrm{NMR}$

$\mathrm{CDCl}_{3}$

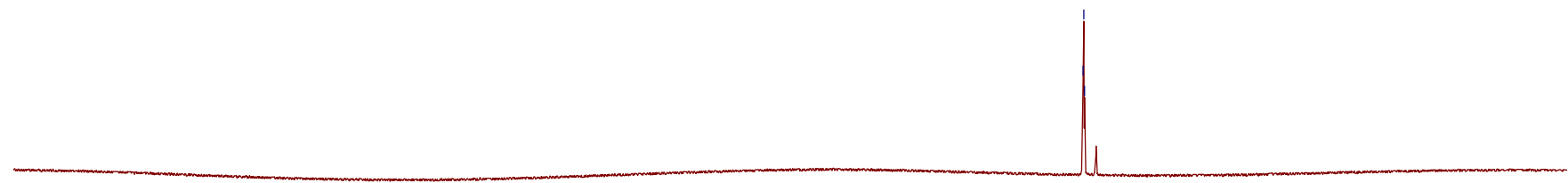

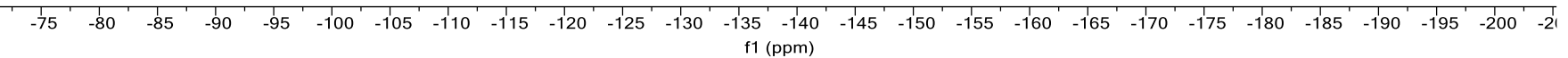




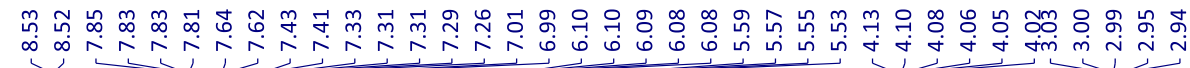
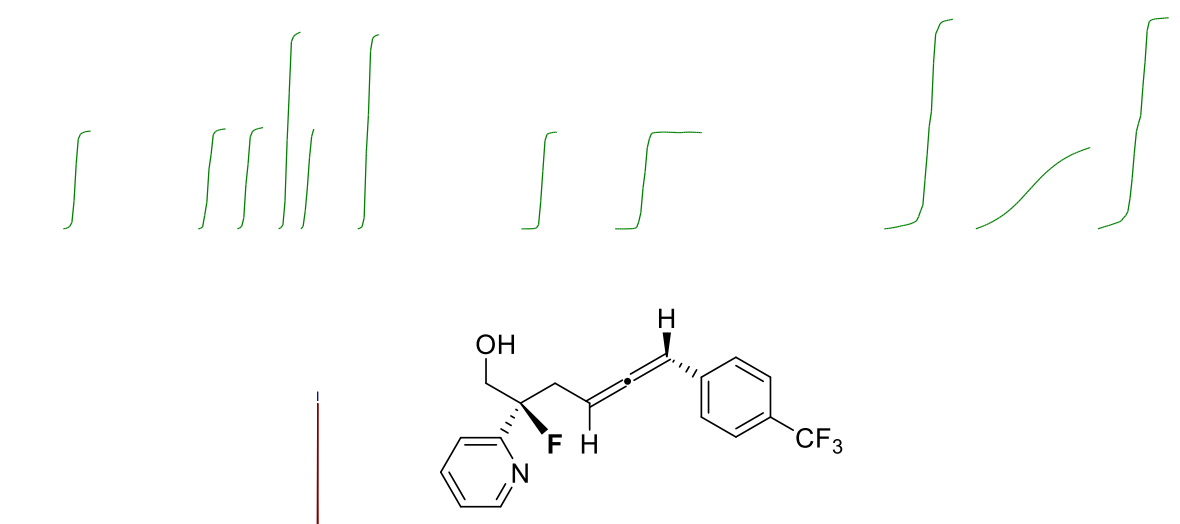

$(S, R)-8,{ }^{1} \mathrm{H}$ NMR

$\mathrm{CDCl}_{3}$
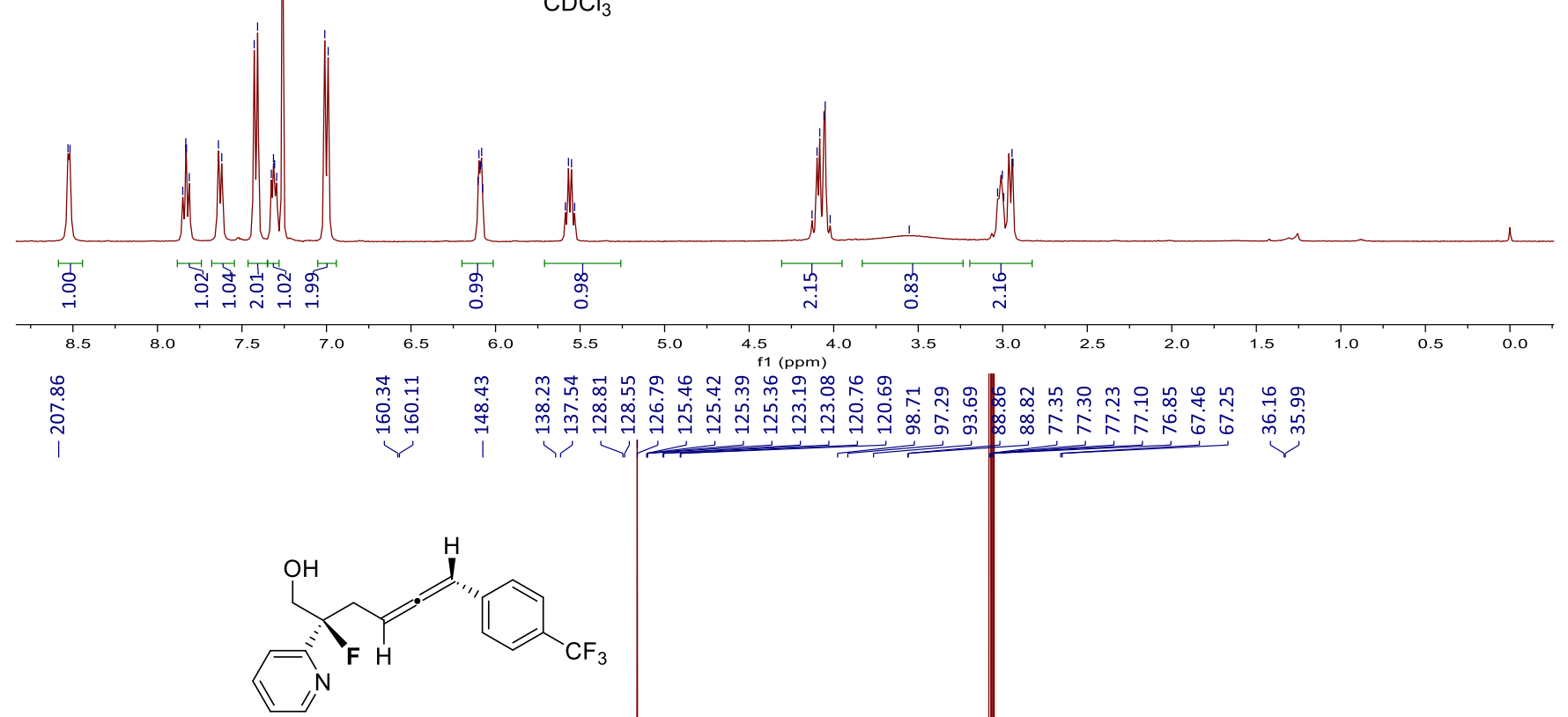

$(S, R)-8,{ }^{13} \mathrm{C}$ NMR $\mathrm{CDCl}_{3}$

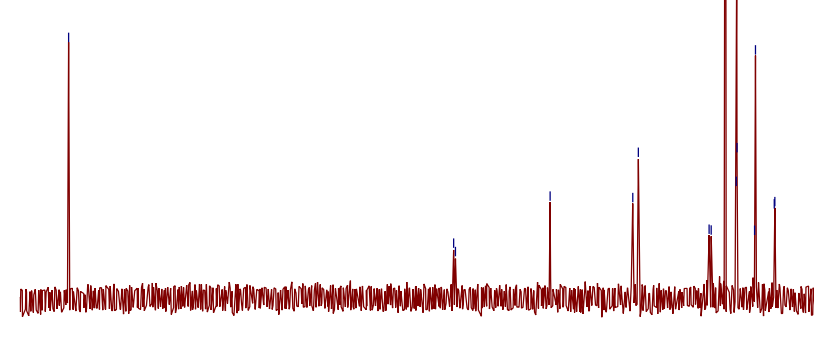

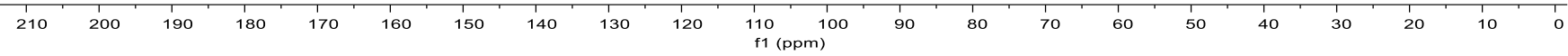




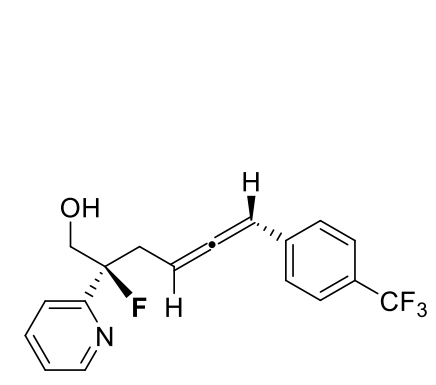

$(S, R)-8,{ }^{19} \mathrm{~F}$ NMR $\mathrm{CDCl}_{3}$

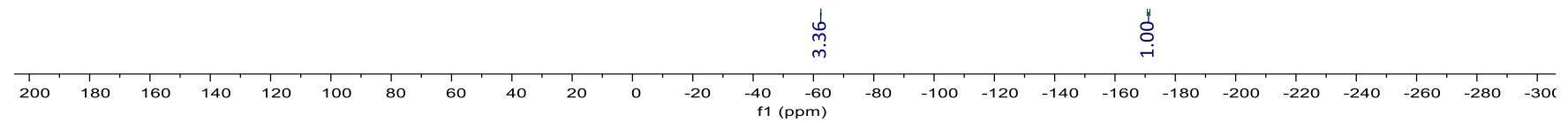




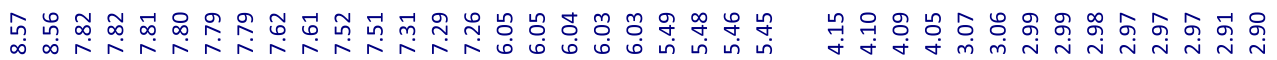

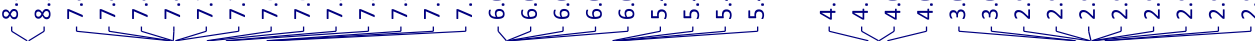
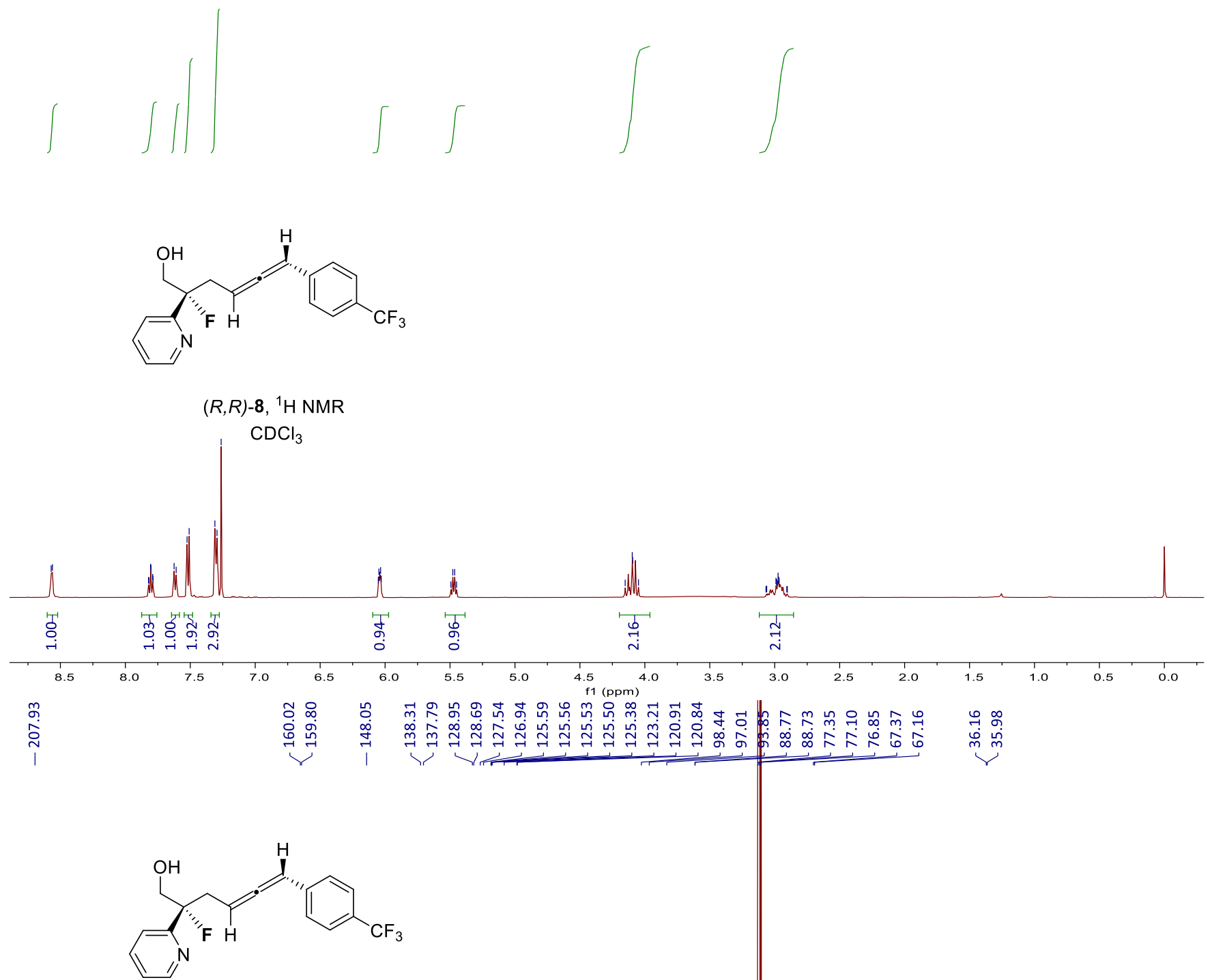

$(R, R)-8,{ }^{13} \mathrm{C}$ NMR $\mathrm{CDCl}_{3}$
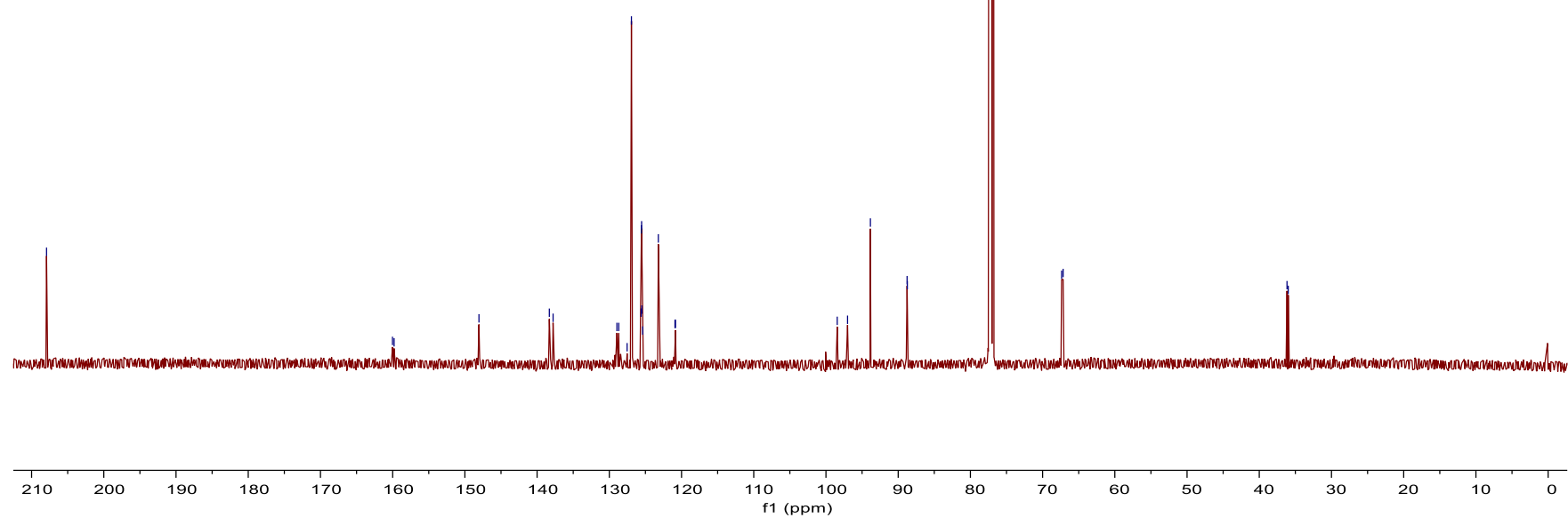


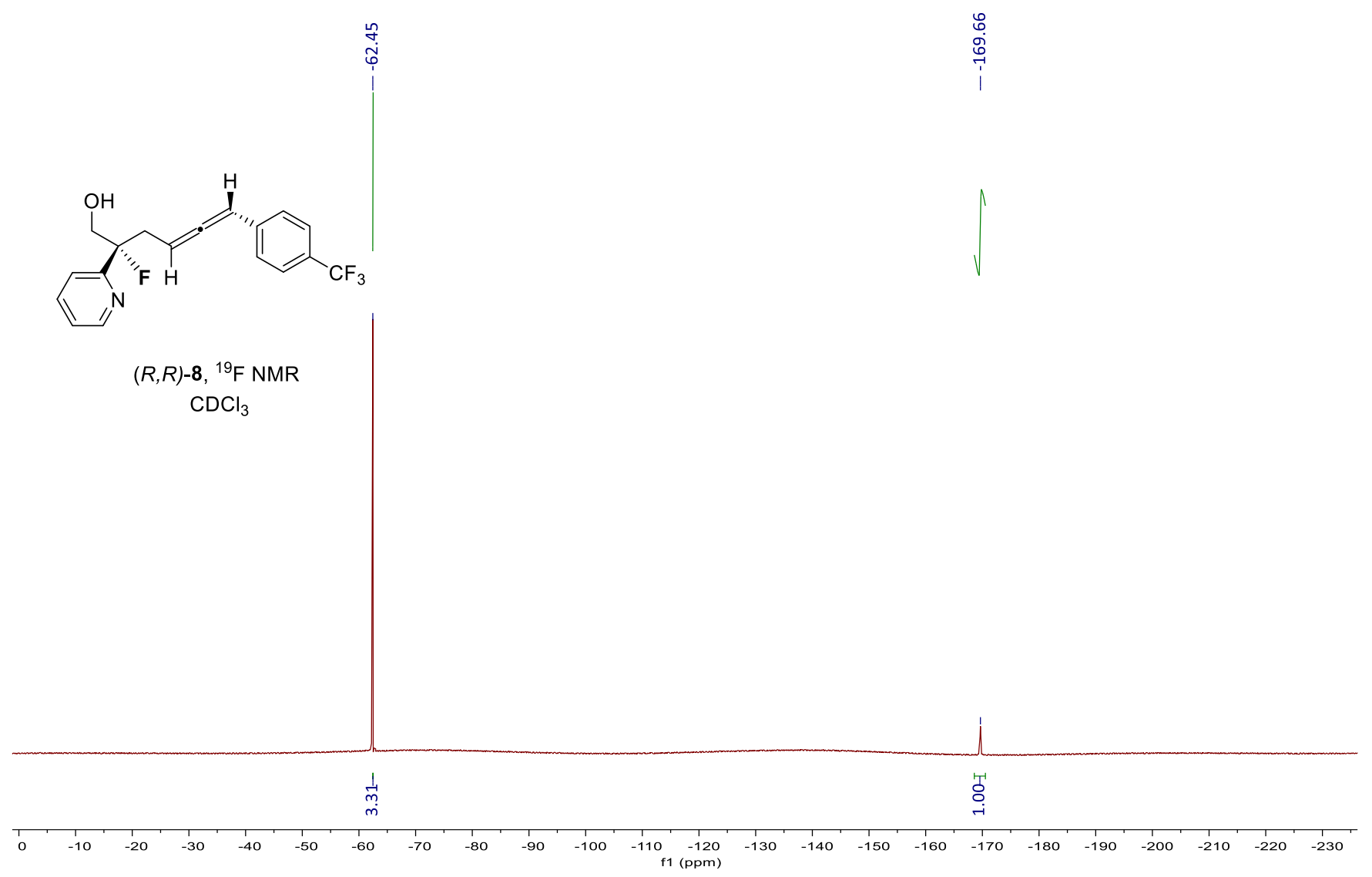




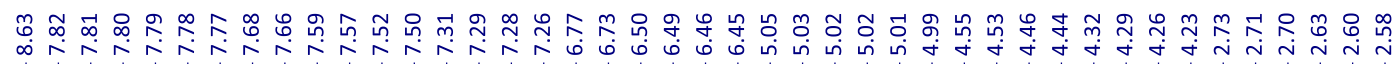
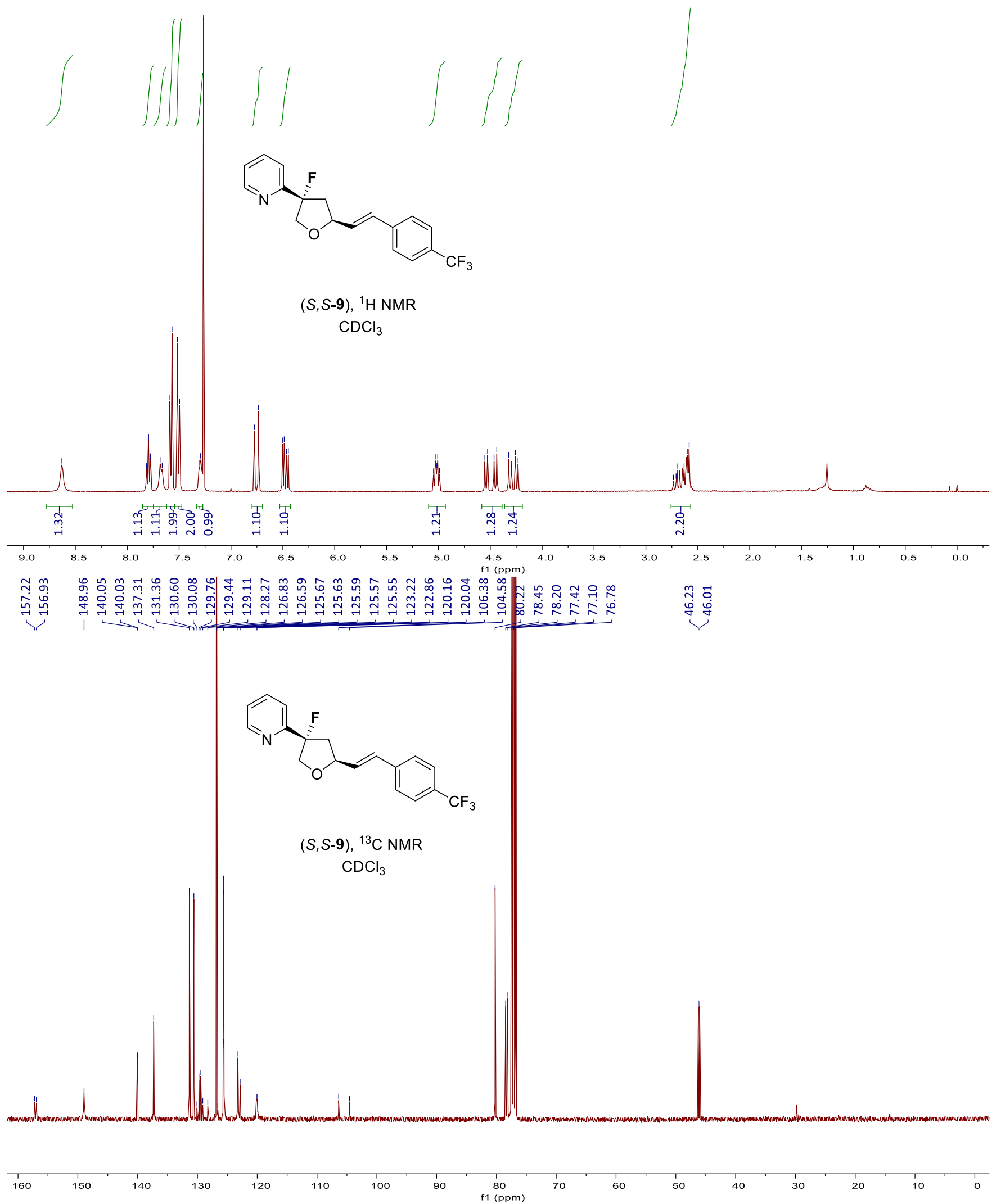


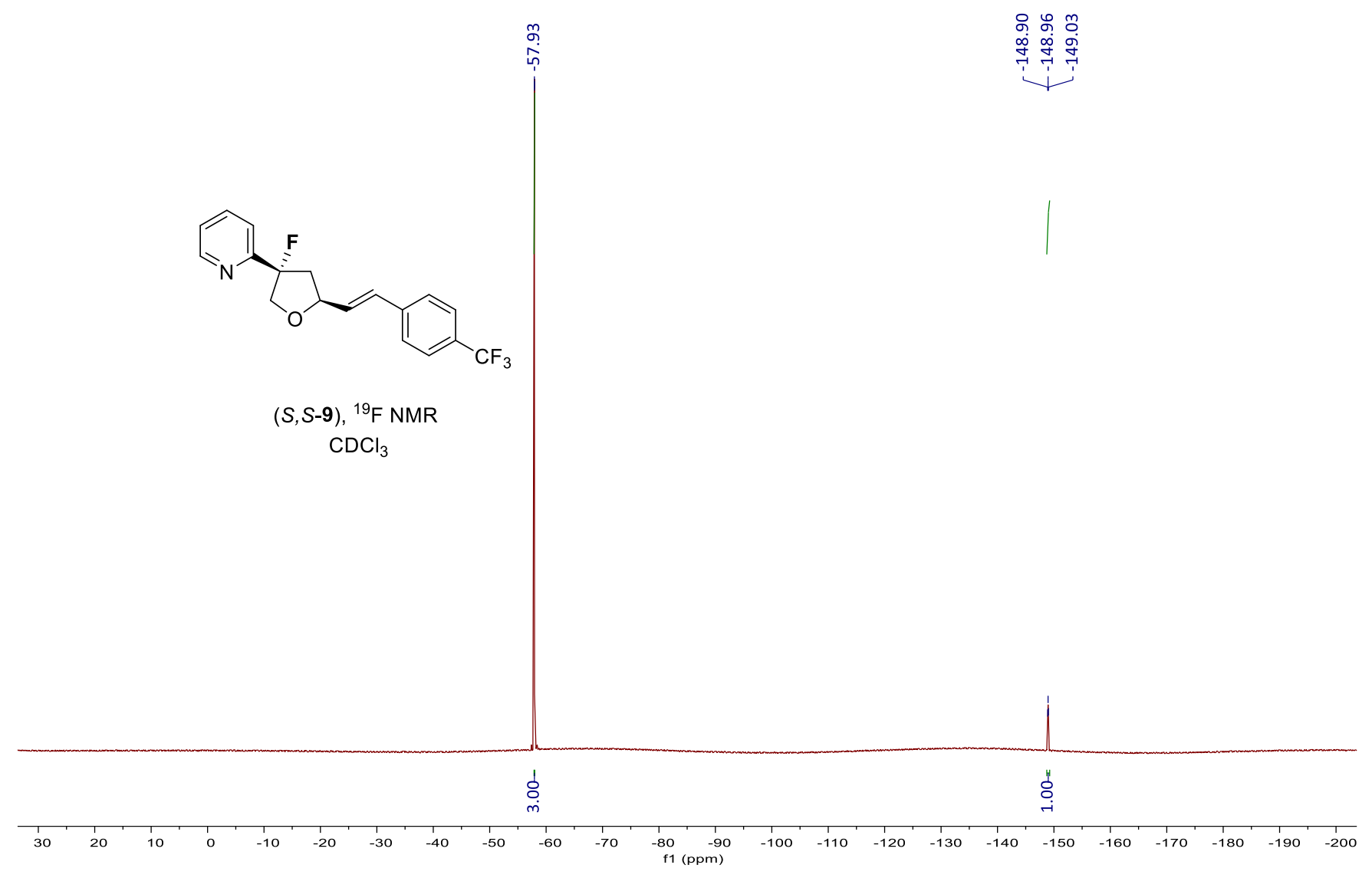


bo

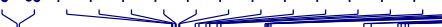
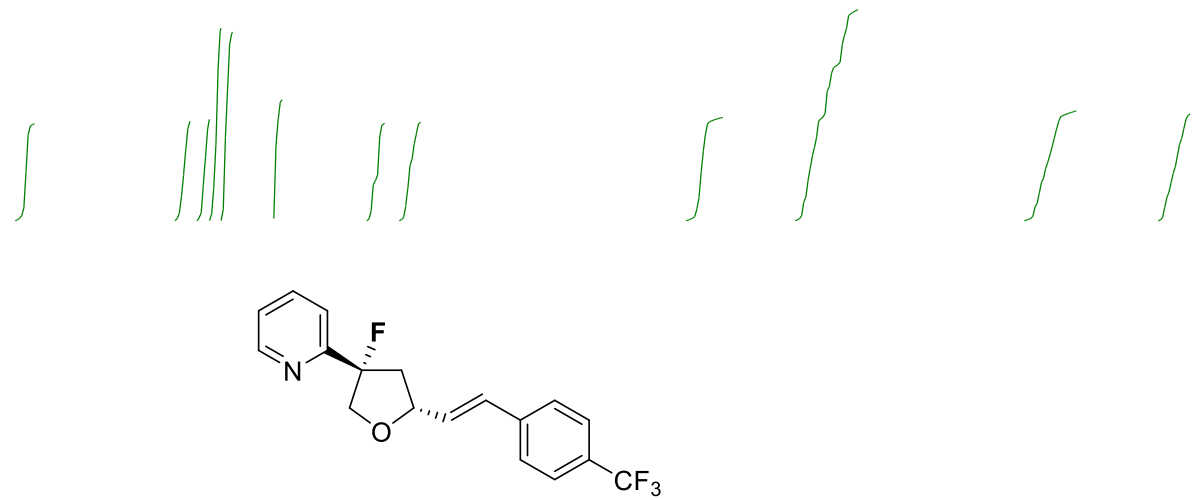

$(S, R)-9,{ }^{1} \mathrm{H}$ NMR

$\mathrm{CDCl}_{3}$

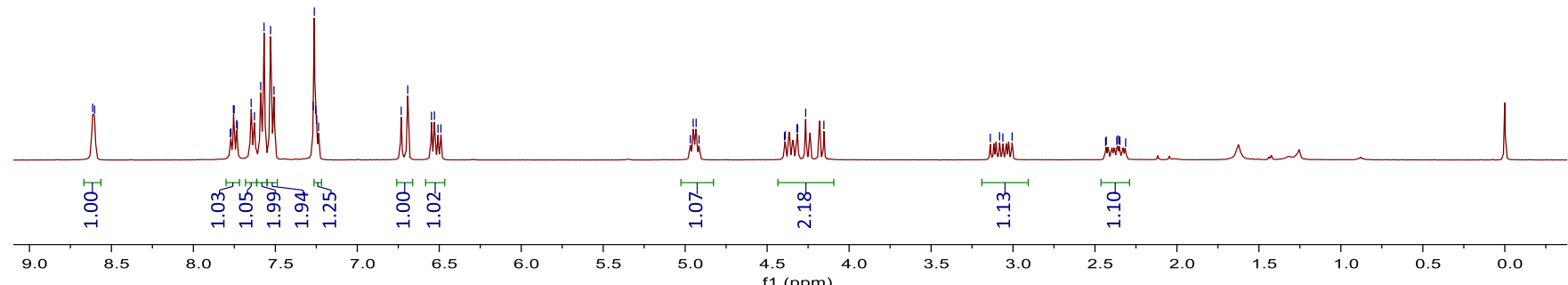

ర大

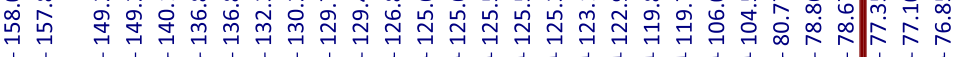

党

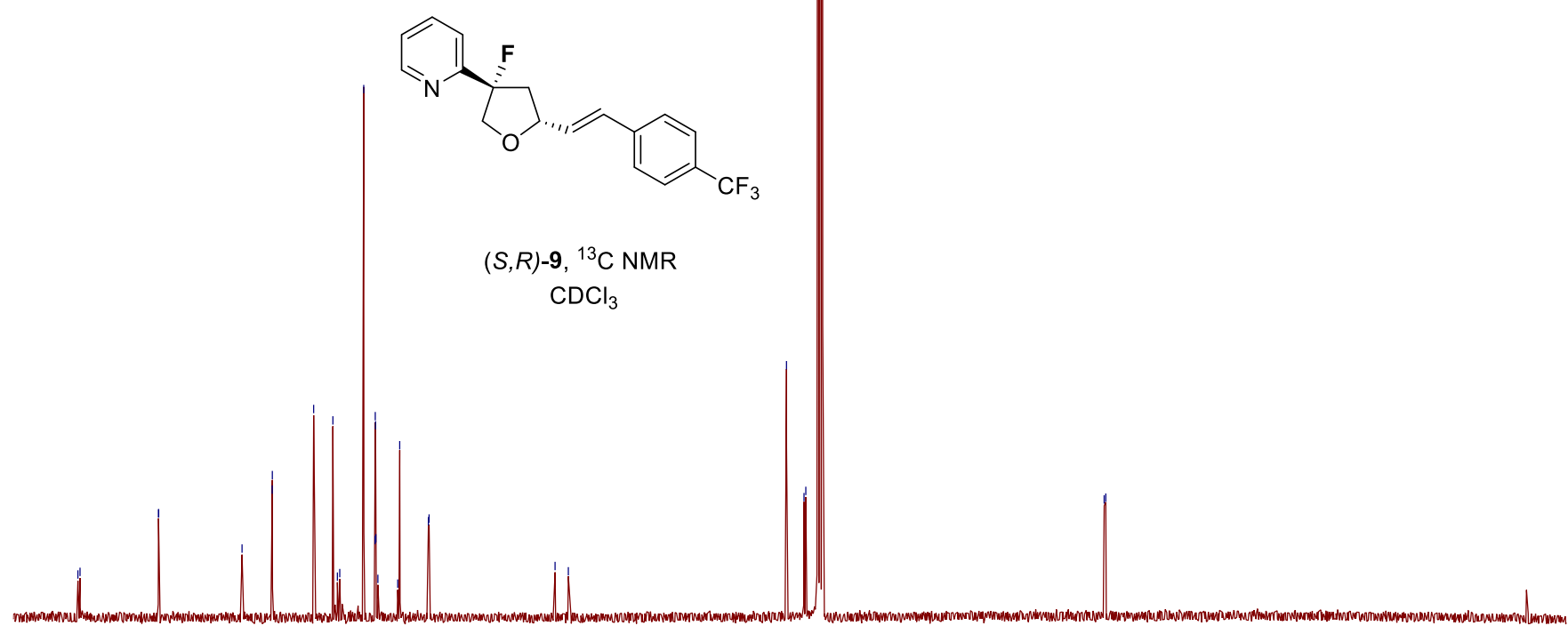

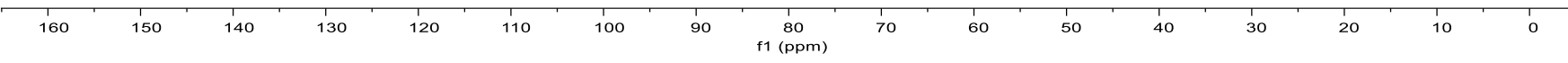




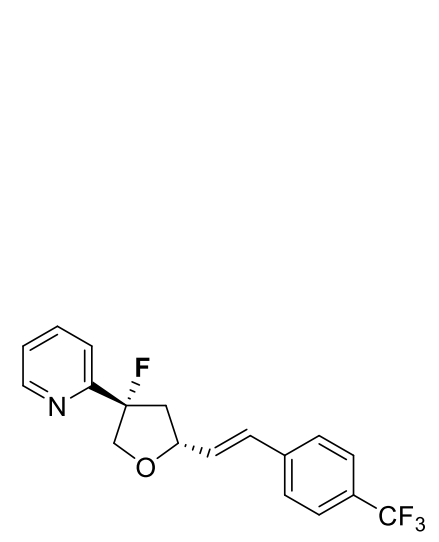

于敌

นั้

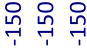

i

$(S, R)-9,{ }^{19} \mathrm{~F}$ NMR $\mathrm{CDCl}_{3}$

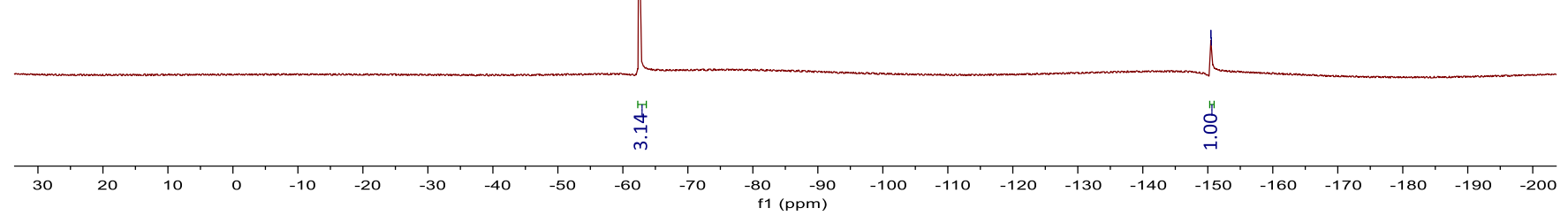

